\title{
Theoretical Studies on Palladium-Mediated Enantioselective C-H Iodination
}

\author{
Mei-Juan Zhou, Ti-Long Yang, Li Dang* \\ Department of Chemistry, South University of Science and Technology of China, Shenzhen, \\ 518055, P. R. China
}

Corresponding Author Email Addresses: dangl@sustc.edu.cn

Table of content

1. The energy profiles and optimized structures and geometric parameters of the pathways for aryl $\mathrm{C}-\mathrm{H}$ bond activation of $R$-benzylamine.

2. The energy profiles and optimized structures and geometric parameters of the pathways for iodation reaction of $R$-benzylamine.

3. The optimized structures and geometric parameters of the pathways for generation of the $(R)$-iodinated product and the regeneration of the catalyst

4. The energy profiles and optimized structures and geometric parameters of the pathwaysfor aryl C-H bond activation of $S$-benzylamine

5. The energy profiles and optimized structures and geometric parameters of the pathways for iodation reaction of $S$-benzylamine

6. The energy profiles and the optimized structures and geometric parameters of the pathways for generation of the $(S)$-iodinated product and the regeneration of the catalyst

7. The energy profiles and optimized structures and geometric parameters of pathways $R$-Me and $S$-Me for aryl $\mathrm{C}-\mathrm{H}$ bond activation of benzylamine.

8. The relative free energies and electronic energies in gas phase and the single-point energies and relative free energies in DMSO solvent of the favorable pathways for aryl $\mathrm{C}-\mathrm{H}$ bond activation of $R(S)$-benzylamine computed by different computation methods

9. Cartesian coordinates for complexes calculated in this study.

10. The relative free energies and electronic energies in gas phase and the single-point energies in DMSO solvent for complexes calculated in this study..... 
1. The energy profiles and optimized structures and geometric parameters of the pathways for aryl $\mathbf{C}-\mathbf{H}$ bond activation of $\boldsymbol{R}$-benzylamine

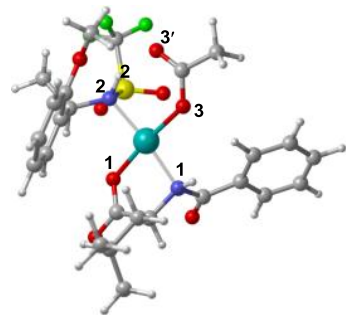

$\mathbf{R}_{1 \mathbf{a}}$

$P d-N^{2}=2.157$

$P d-N^{1}=2.122$

$\mathrm{Pd}-\mathrm{O}^{1}=1.991$

$\mathrm{Pd}-\mathrm{O}^{3}=\mathbf{2 . 0 4 9}$

$\mathrm{N}^{2}-\mathrm{H}^{2}=1.050$

$\mathrm{O}^{3}-\mathrm{H}^{2}=1.767$

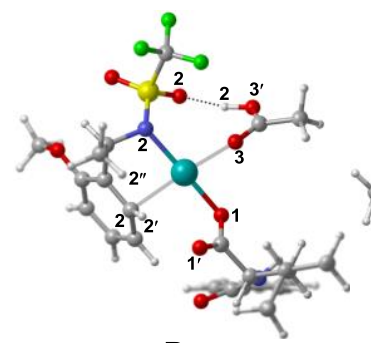

$\mathbf{R}_{\mathbf{3 a}}$

Pd- $\mathrm{N}^{2}=2.068$

$\mathrm{Pd}-\mathrm{C}^{2}=2.292$

$\mathrm{Pd}-\mathrm{O}^{1}=\mathbf{2 . 0 2 0}$

$\mathrm{Pd}-\mathrm{O}^{3}=\mathbf{2 . 1 6 3}$

$\mathrm{Pd}-\mathrm{H}^{2 "}=\mathbf{3 . 2 7 4}$

$\mathrm{C}^{2}-\mathrm{H}^{2}=1.098$

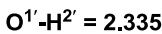

$\mathrm{O}^{2}-\mathrm{H}^{2}=1.672$

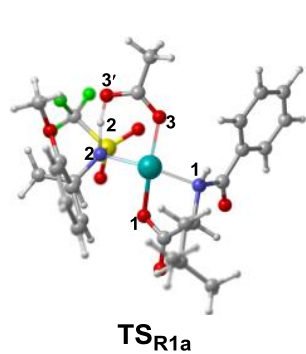

$\mathrm{Pd}-\mathrm{N}^{2}=2.13$

$\mathbf{P d}-\mathbf{N}^{1}=2.136$

$\mathrm{Pd}-\mathrm{O}^{1}=1.981$

$\mathrm{Pd}-\mathrm{O}^{3}=2.086$

$\mathrm{N}^{2}-\mathrm{H}^{2}=1.369$

$\mathrm{O}^{3^{\prime}-\mathrm{H}^{2}}=1.162$

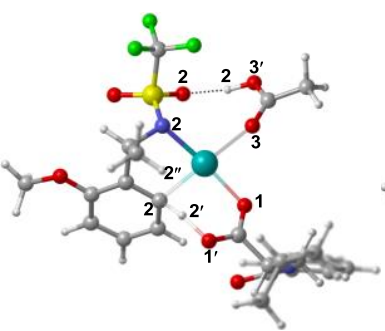

$\mathrm{TS}_{\mathrm{R} 2 \mathrm{a}}$

$\mathrm{Pd}-\mathrm{N}^{2}=2.049$

$\mathrm{Pd}-\mathrm{C}^{2}=2.096$

$\mathrm{Pd}-\mathrm{O}^{1}=2.079$

$\mathrm{Pd}-\mathrm{O}^{3}=2.189$

$\mathrm{Pd}-\mathrm{H}^{2 "}=3.066$

$\mathrm{C}^{2}-\mathrm{H}^{2^{\prime}}=\mathbf{1 . 3 1 4}$

$\mathrm{O}^{11^{\prime}-\mathrm{H}^{2}}=1.403$

$\mathrm{O}^{2}-\mathrm{H}^{2}=1.655$

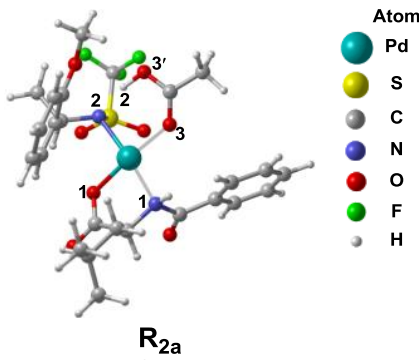

$\mathrm{Pd}-\mathrm{N}^{2}=2.128$

$\mathrm{Pd}-\mathrm{N}^{1}=\mathbf{2 . 1 4 3}$

$\mathrm{Pd}-\mathrm{O}^{1}=1.979$

$\mathrm{Pd}-\mathrm{O}^{3}=\mathbf{2 . 1 0 6}$

$\mathrm{N}^{2}-\mathrm{H}^{2}=1.654$

$\mathrm{O}^{3}-\mathrm{H}^{2}=1.039$

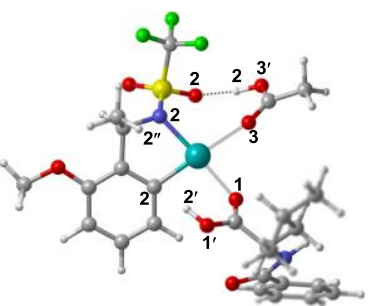

$\mathbf{R}_{\mathbf{4 a}}$

$\mathrm{Pd}-\mathrm{N}^{2}=\mathbf{2 . 0 4 3}$

$\mathrm{Pd}-\mathrm{C}^{2}=1.982$

$\mathrm{Pd}-\mathrm{O}^{1}=2.146$

$\mathrm{Pd}-\mathrm{O}^{3}=2.281$

$\mathrm{Pd}-\mathrm{H}^{2 "}=\mathbf{3 . 3 6 1}$

$\mathrm{C}^{2}-\mathrm{H}^{2}=\mathbf{3}=\mathbf{3 7 2}$

$\mathrm{O}^{1^{\prime}}-\mathrm{H}^{2^{\prime}}=0.988$

$\mathrm{O}^{2}-\mathrm{H}^{2}=1.653$

Figure S1. Optimized structures and geometric parameters for selected species of pathway a for aryl C-H bond activation of $R$-benzylamine in Figure 1 . 


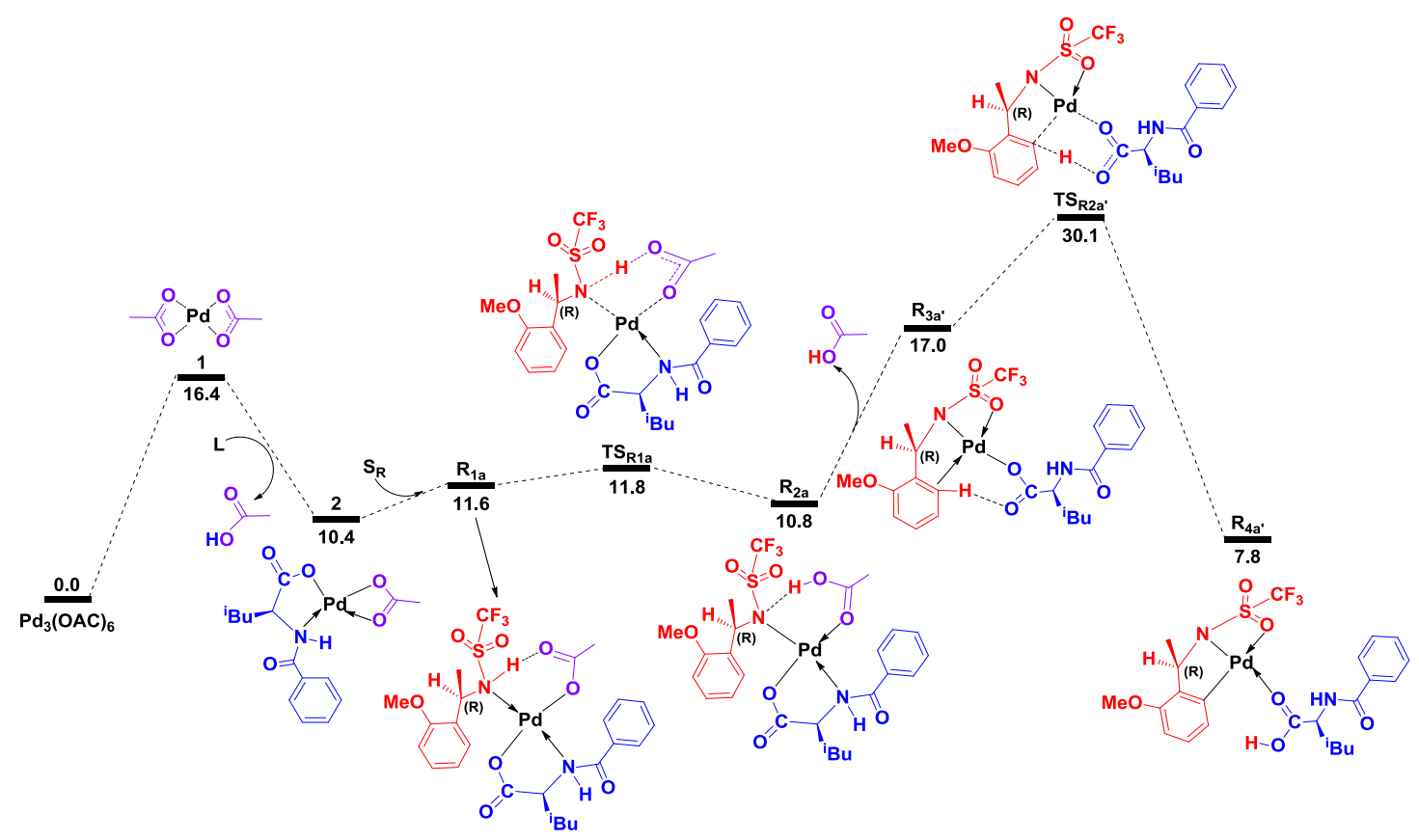

Figure S2. Energy profile of pathway a' for aryl C-H bond activation of $R$-benzylamine. The relative free energies in dimethyl sulfoxide (DMSO) are given in $\mathrm{kcal} / \mathrm{mol}$
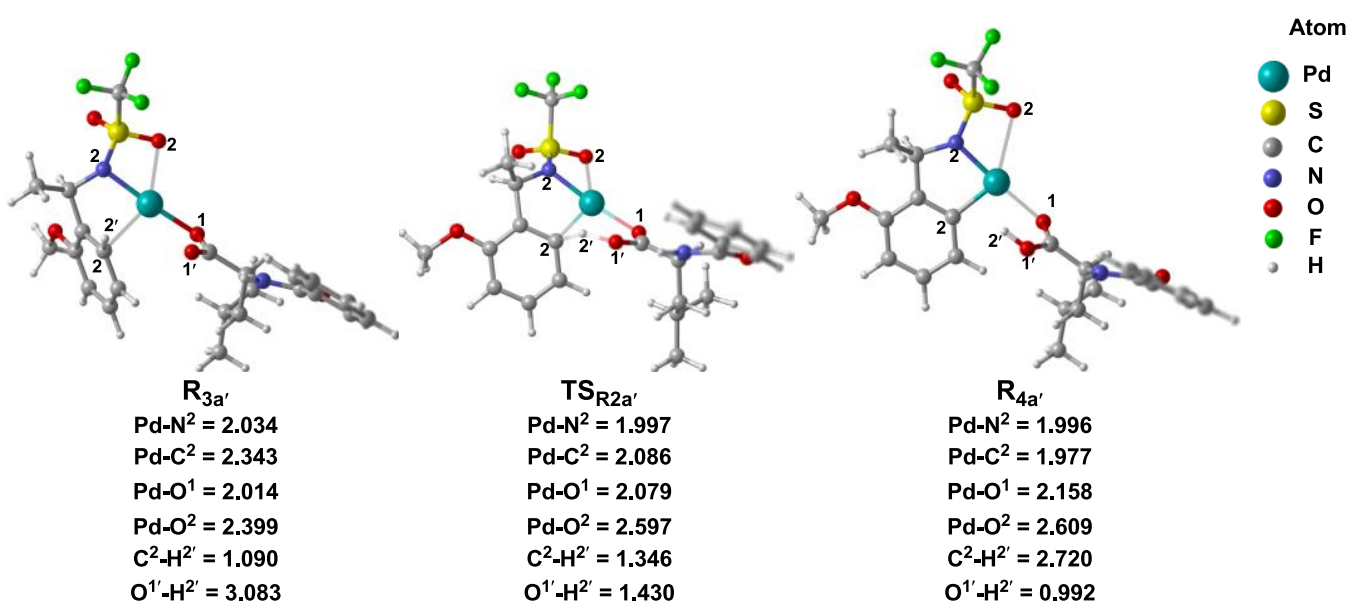

Figure S3. Optimized structures and geometric parameters for selected species of pathway a' for aryl C-H bond activation of $R$-benzylamine in Figure $\mathrm{S} 2$. 


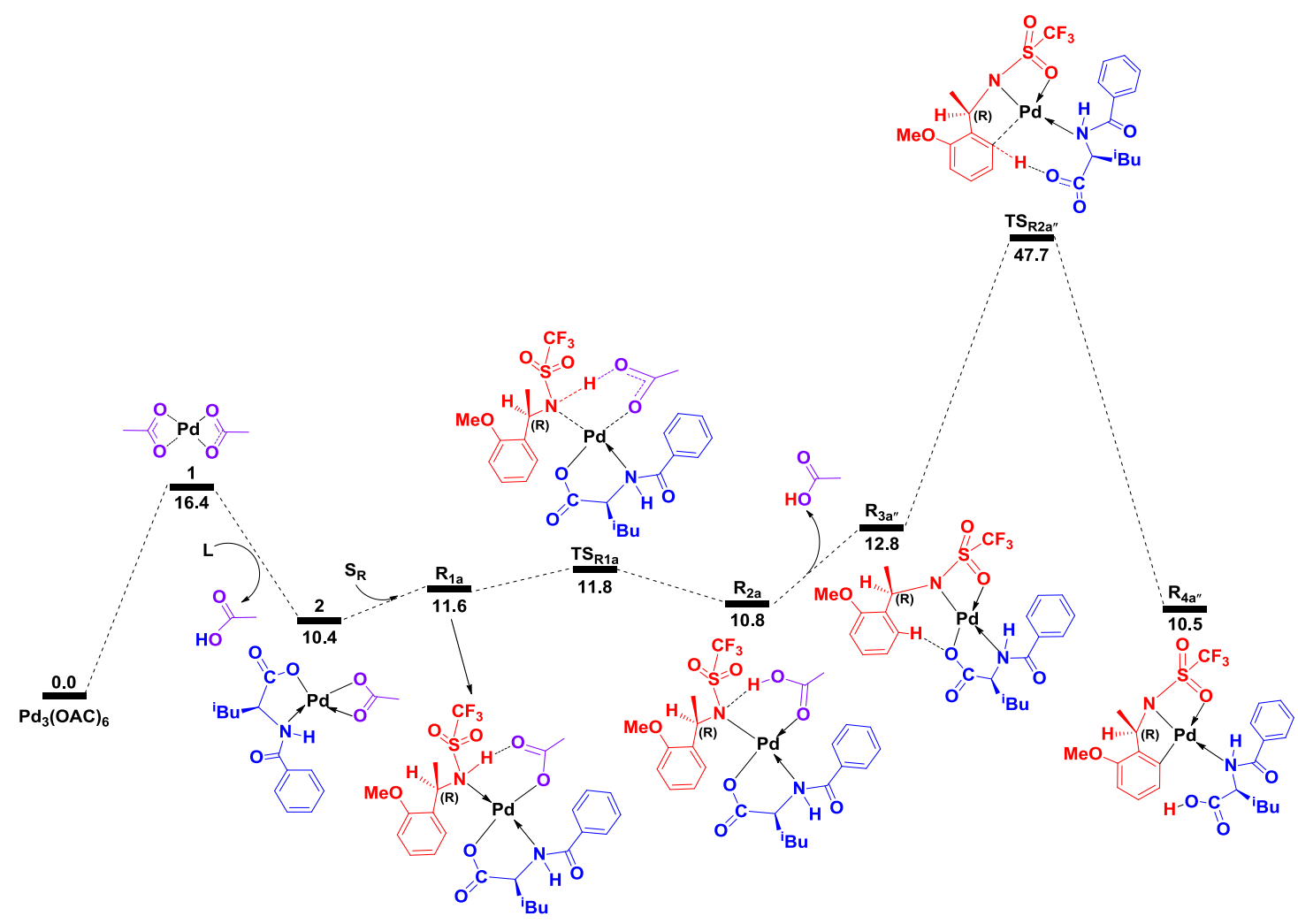

Figure S4. Energy profile of pathway a" for aryl C-H bond activation of $R$-benzylamine. The relative free energies in dimethyl sulfoxide (DMSO) are given in $\mathrm{kcal} / \mathrm{mol}$

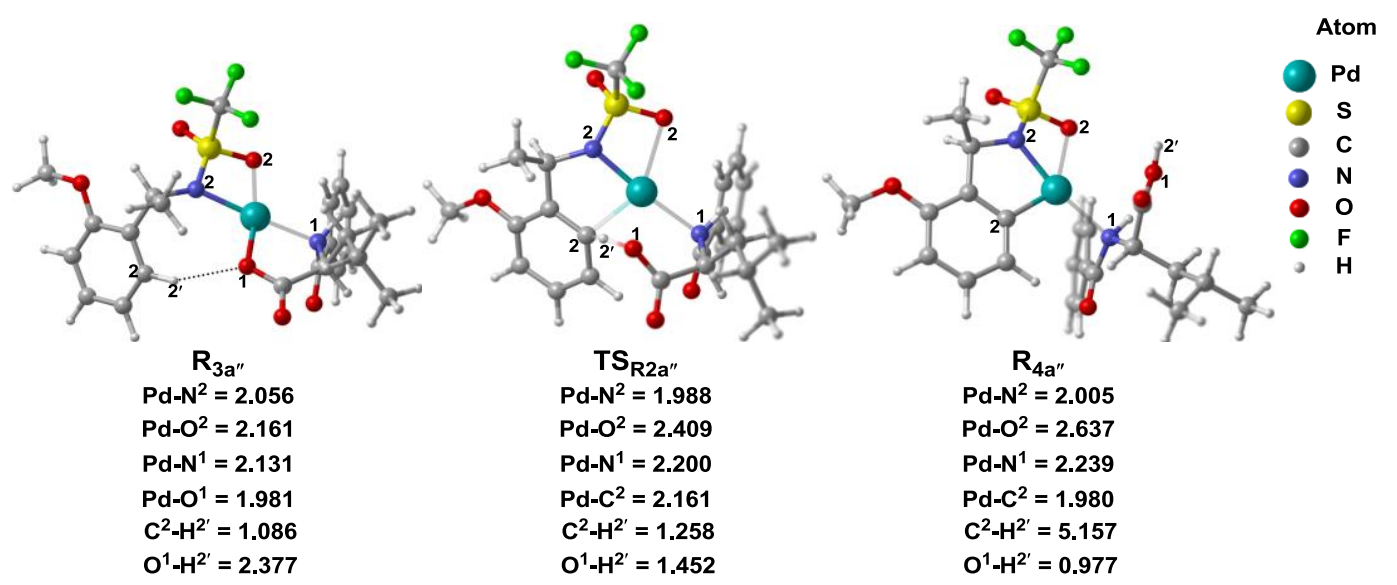

Figure S5. Optimized structures and geometric parameters for selected species of pathway a" for aryl C-H bond activation of $R$-benzylamine in Figure $\mathrm{S} 4$. 


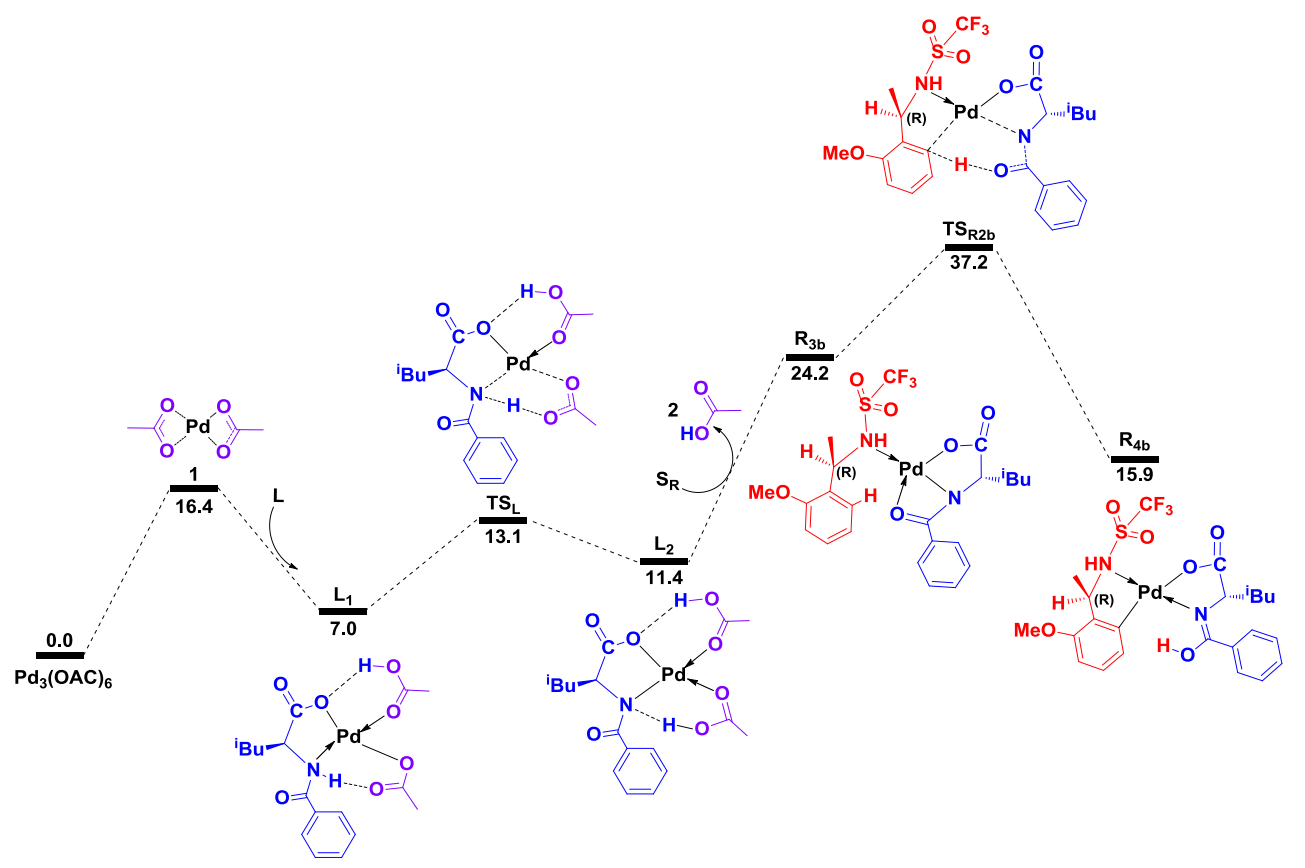

Figure S6. Energy profile of pathway b for aryl C-H bond activation of $R$-benzylamine. The relative free energies in dimethyl sulfoxide (DMSO) are given in $\mathrm{kcal} / \mathrm{mol}$

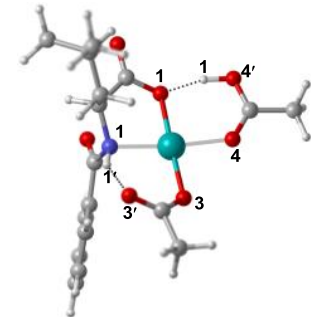

$\mathrm{L}_{1}$

$\mathrm{Pd}-\mathrm{N}^{1}=\mathbf{2 . 0 9 2}$

$\mathrm{Pd}-\mathrm{O}^{1}=2.009$

$\mathrm{Pd}-\mathrm{O}^{4}=2.100$

$\mathrm{Pd}-\mathrm{O}^{3}=2.018$

$\mathrm{O}^{1}-\mathrm{H}^{1}=1.575$

$N^{1}-H^{1}=1.039$

$\mathrm{O}^{3^{\prime}-\mathrm{H}^{1}}=1.832$

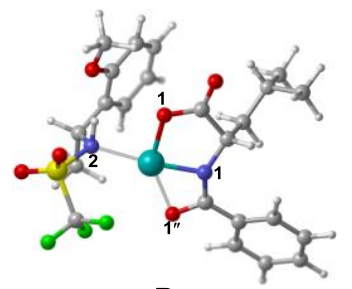

$\mathbf{R}_{\mathbf{3 b}}$

$\mathrm{Pd}-\mathrm{N}^{2}=2.209$

$\mathrm{Pd}-\mathrm{O}^{1}=2.005$

$\mathrm{Pd}-\mathrm{N}^{1}=1.931$

$P d-0^{1 "}=2.258$

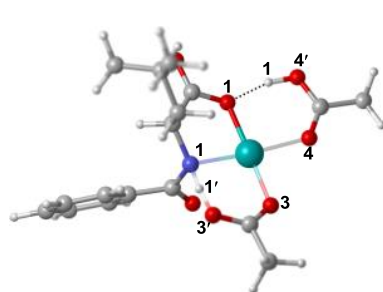

$\mathrm{TS}_{\mathrm{L}}$

$\mathrm{Pd}-\mathrm{N}^{1}=\mathbf{2 . 0 3 6}$

$\mathrm{Pd}^{-\mathrm{O}^{1}}=2.002$

$\mathrm{Pd}-\mathrm{O}^{4}=2.119$

$\mathrm{Pd}-\mathrm{O}^{3}=2.062$

$\mathrm{O}^{1}-\mathrm{H}^{1}=1.602$

$\mathrm{N}^{1}-\mathrm{H}^{1}=1.294$

$\mathrm{O}^{3} \cdot \mathrm{H}^{1{ }^{\prime}}=1.213$

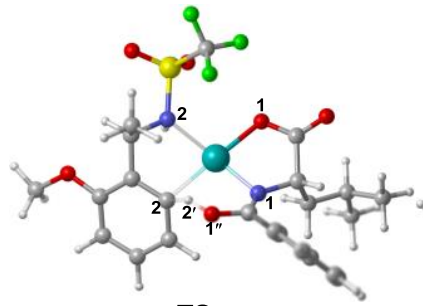

$\mathrm{TS}_{\mathrm{R} 2 \mathrm{~b}}$

$\mathrm{Pd}-\mathrm{N}^{2}=2.190$

$\mathrm{Pd}-\mathrm{O}^{1}=\mathbf{2 . 0 1 2}$

$\mathrm{Pd}-\mathrm{N}^{1}=1.997$

$\mathrm{Pd}-\mathrm{C}^{2}=2.190$

$\mathrm{C}^{2}-\mathrm{H}^{2}=1.329$

$\mathrm{O}^{1 "} \cdot \mathrm{H}^{2^{\prime}}=1.381$

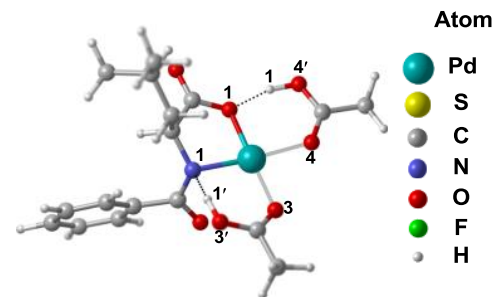

$\mathrm{L}_{2}$

$\mathrm{Pd}-\mathrm{N}^{1}=\mathbf{2 . 0 2 6}$

$\mathrm{Pd}-\mathrm{O}^{1}=1.989$

$\mathrm{Pd}-\mathrm{O}^{4}=2.128$

$\mathrm{Pd}-\mathrm{O}^{3}=2.096$

$\mathrm{O}^{1}-\mathrm{H}^{1}=1.601$

$\mathrm{N}^{1}-\mathrm{H}^{1}=1.695$

$\mathrm{O}^{3^{\prime}}-\mathrm{H}^{1^{\prime}}=1.024$

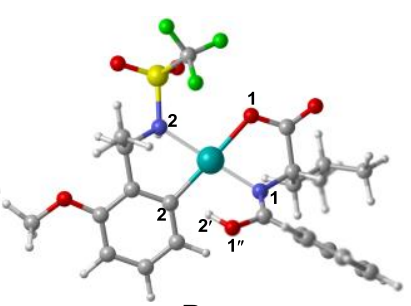

$\mathbf{R}_{\mathbf{4 b}}$

$\mathrm{Pd}-\mathrm{N}^{2}=2.152$

$\mathrm{Pd}-\mathrm{O}^{1}=2.080$

Pd- $\mathbf{N}^{1}=2.054$

$\mathrm{Pd}-\mathrm{C}^{2}=2.026$

$\mathrm{C}^{2}-\mathrm{H}^{2}=2.266$

$\mathrm{O}^{1 "}-\mathrm{H}^{2^{\prime}}=0.986$

Figure S7. Optimized structures and geometric parameters for selected species of pathway b for aryl C-H bond activation of $R$-benzylamine in Figure S6. 


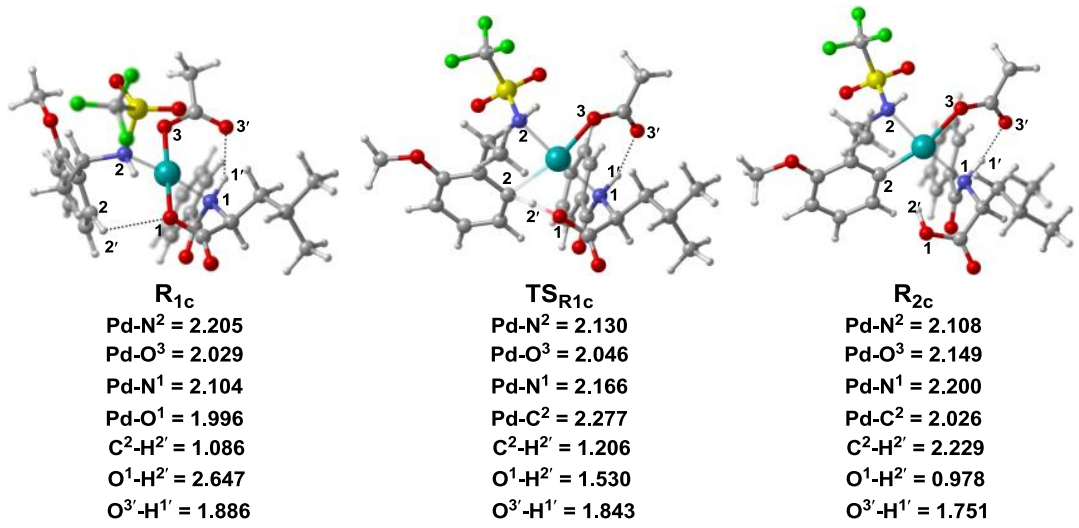

Figure S8. Optimized structures and geometric parameters for selected species of pathway c for aryl C-H bond activation of $R$-benzylamine in Figure 2

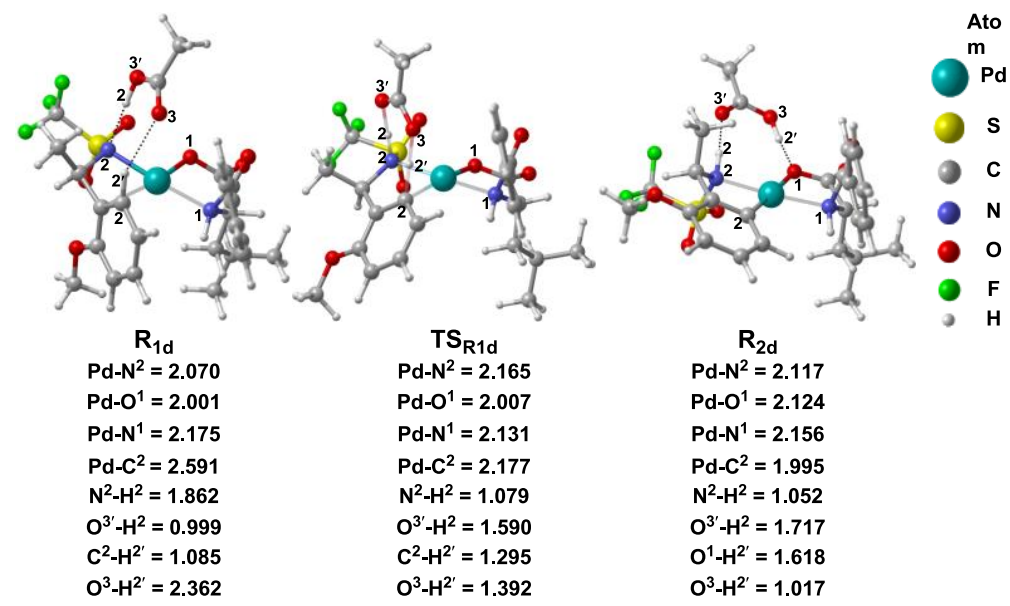

Figure S9. Optimized structures and geometric parameters for selected species of pathway d for aryl C-H bond activation of $R$-benzylamine in Figure 3 


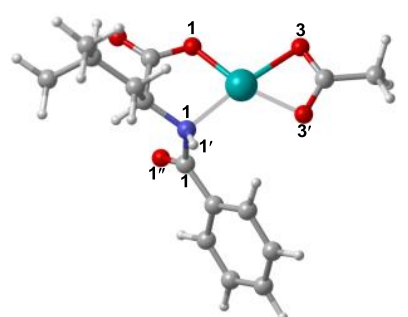

2

Pd- $\mathrm{N}^{1}=\mathbf{2 . 1 1 2}$

$\mathrm{Pd}-\mathrm{O}^{1}=1.971$

$\mathrm{Pd}-\mathrm{O}^{3}=\mathbf{2 . 0 9 0}$

$\mathrm{Pd}-\mathrm{O}^{3^{\prime}}=\mathbf{2 . 1 0 7}$

$H^{1}-N^{1}-C^{1}-O^{1 "}=148.5^{\circ}$

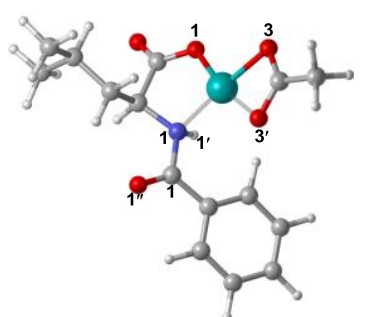

3

Atom

$\mathrm{Pd}-\mathrm{N}^{1}=\mathbf{2 . 1 1 8}$

$\mathrm{Pd}-\mathrm{O}^{1}=1.994$

$\mathrm{Pd}-\mathrm{O}^{3}=2.085$

$\mathrm{Pd}-\mathrm{O}^{3^{\prime}}=2.093$

$H^{11}-N^{1}-C^{1}-O^{1 \prime \prime}=-143.4^{\circ}$

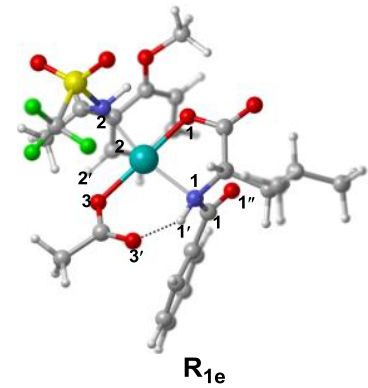

$P d-N^{2}=2.183$

$\mathrm{Pd}-\mathrm{O}^{1}=1.993$

$\mathbf{P d}-\mathrm{N}^{1}=\mathbf{2 . 1 0 1}$

$\mathrm{Pd}-\mathrm{O}^{3}=\mathbf{2 . 0 4 3}$

$\mathrm{C}^{2}-\mathrm{H}^{2^{\prime}}=1.084$

$\mathrm{O}^{3}-\mathrm{H}^{2}=3.876$

$\mathrm{O}^{3^{\prime}-\mathrm{H}^{1}}=1.844$

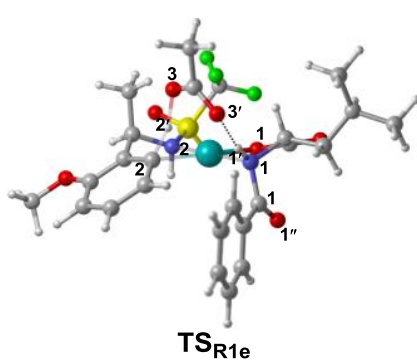

$\mathrm{Pd}^{2}=2$

${\mathrm{Pd}-\mathrm{O}^{1}=1.999}$

Pd-N ${ }^{1}=2.106$

$\mathrm{Pd}-\mathrm{C}^{2}=\mathbf{2 . 2 1 2}$

$\mathrm{C}^{2}-\mathrm{H}^{2}=1.278$

$\mathrm{O}^{3}-\mathrm{H}^{2^{\prime}}=1.428$

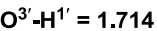

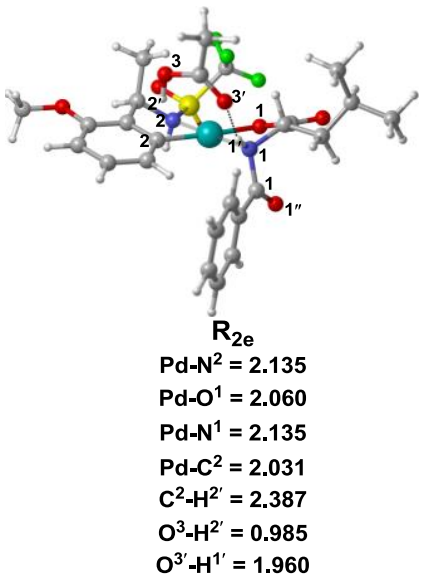

Figure S10. Optimized structures and geometric parameters for selected species of pathway e for aryl C-H bond activation of $R$-benzylamine in Figure 4 
2. The energy profiles and optimized structures and geometric parameters of the pathways for iodation reaction of $\boldsymbol{R}$-benzylamine

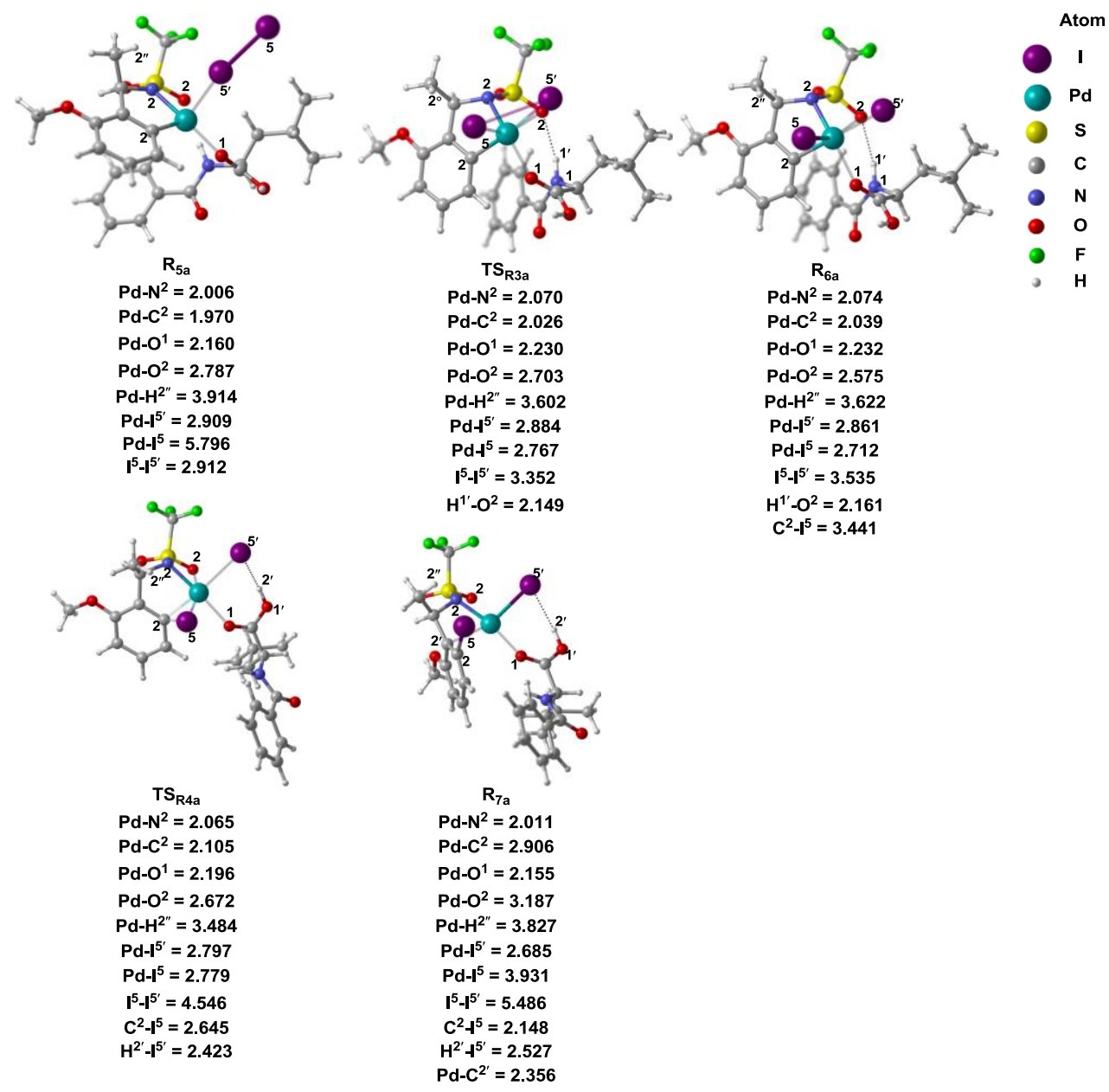

Figure S11. Optimized structures and geometric parameters for selected species of the stepwise reaction pathway a of $R$-benzylamine in Figure 5 


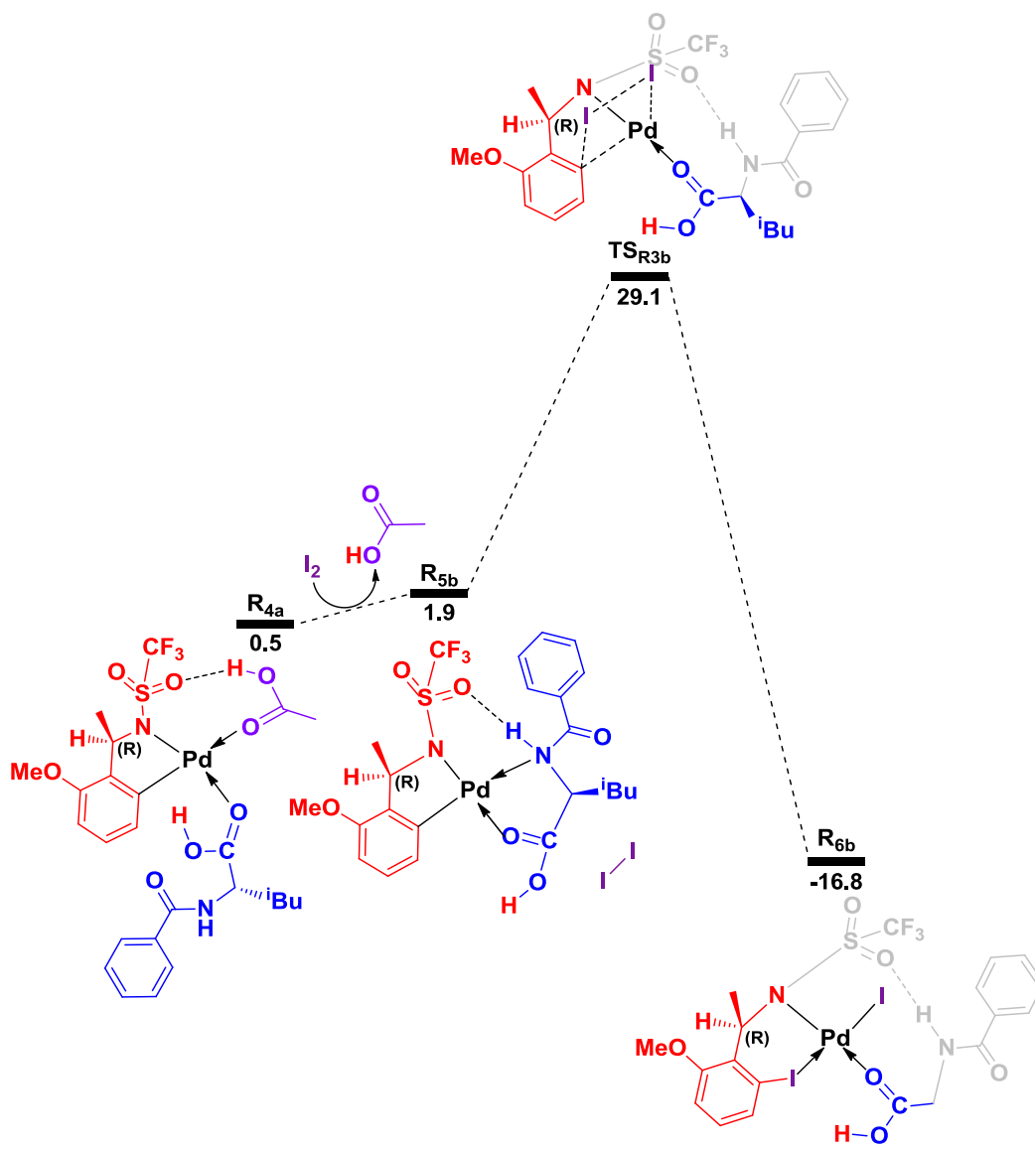

Figure S12. Energy profiles of pathway b for metathesis reaction of $R$-benzylamine. The relative free energies in dimethyl sulfoxide (DMSO) are given in $\mathrm{kcal} / \mathrm{mol}$

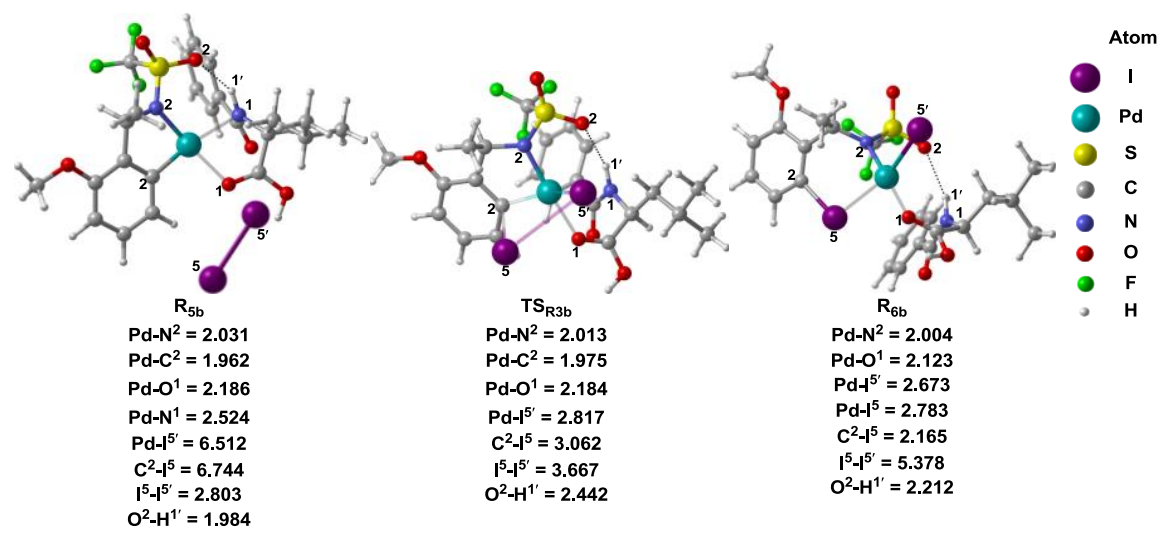

Figure S13.Optimized structures and geometric parameters for selected species of pathway b for metathesis reaction of $R$-benzylamine in Figure $\mathrm{S} 12$ 


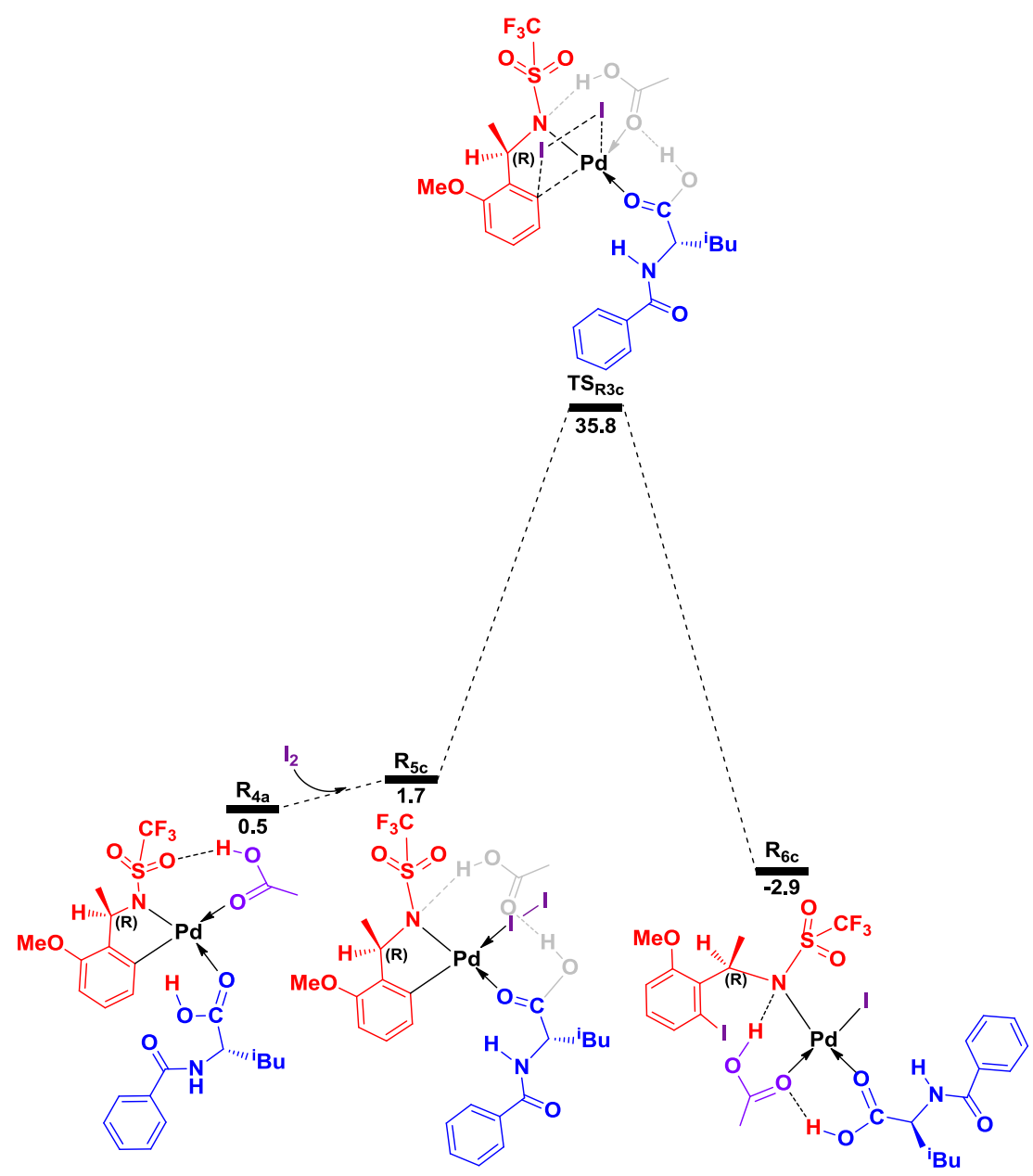

Figure S14. Energy profiles of pathway c for metathes is reaction of $R$-benzylamine. The relative free energies in dimethyl sulfoxide (DMSO) are given in $\mathrm{kcal} / \mathrm{mol}$

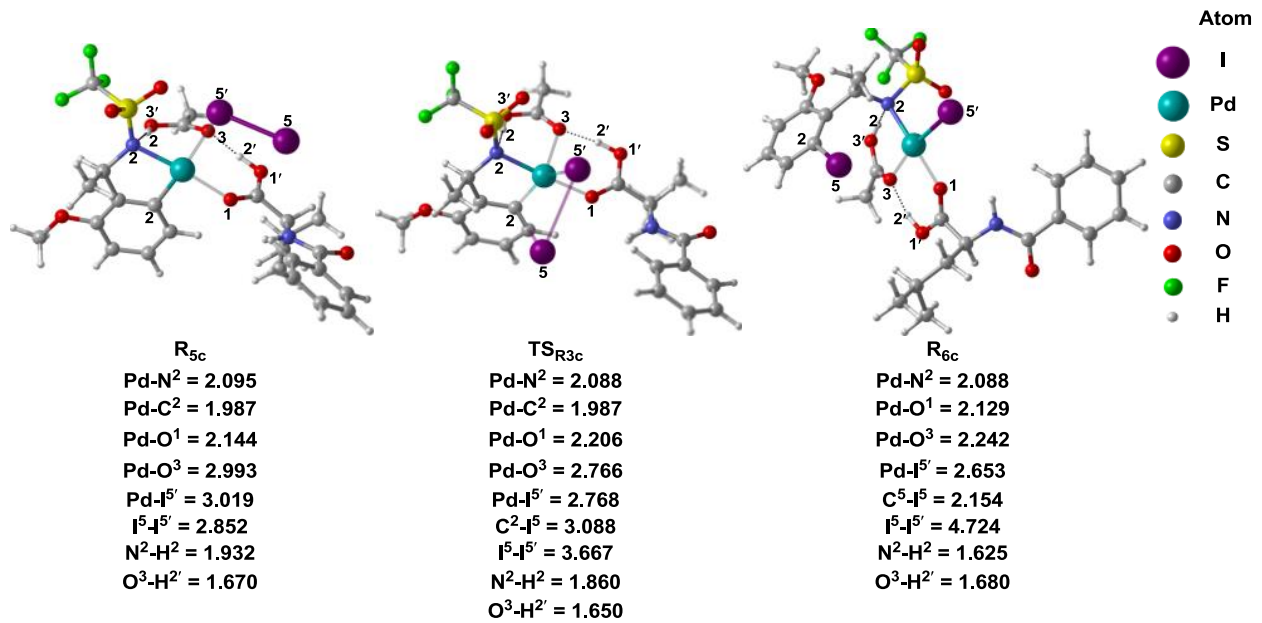

Figure S15. Optimized structures and geometric parameters for selected species of pathway c for metathesis reaction of $R$-benzylamine in Figure $\mathrm{S} 14$ 
3. The optimized structures and geometric parameters of the pathways for generation of the $(R)$-iodinated product and the regeneration of the catalyst

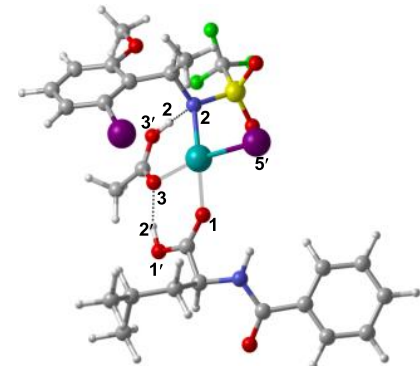

$\mathbf{R}_{8 \mathbf{a}}$

$\mathrm{Pd}-\mathrm{N}^{2}=\mathbf{2 . 0 8 8}$

$P d-1^{5^{\prime}}=2.653$

$P d-O^{1}=2.129$

$\mathrm{Pd}-\mathrm{O}^{3}=2.242$

$\mathrm{O}^{3}-\mathrm{H}^{2}=1.680$

$\mathrm{N}^{2}-\mathrm{H}^{2}=1.625$

$\mathrm{O}^{3}-\mathrm{H}^{2}=1.048$

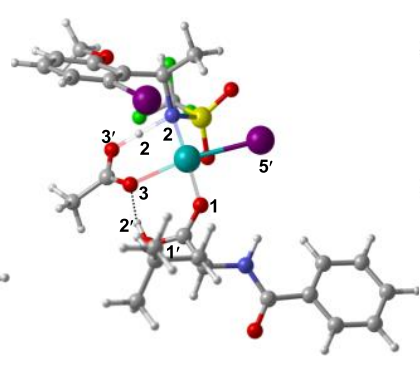

$\mathrm{TS}_{\mathrm{R} 5 \mathrm{a}}$

$\mathrm{Pd}-\mathrm{N}^{2}=\mathbf{2 . 1 1 0}$

$\mathrm{Pd}-\mathrm{I}^{5^{\prime}}=\mathbf{2 . 6 5 8}$

$\mathrm{Pd}-\mathrm{O}^{1}=2.117$

$\mathrm{Pd}-\mathrm{O}^{3}=\mathbf{2 . 2 0 1}$

$\mathrm{O}^{3}-\mathrm{H}^{2}=1.646$

$\mathrm{N}^{2}-\mathrm{H}^{2}=1.397$

$\mathrm{O}^{3}-\mathrm{H}^{2}=1.146$

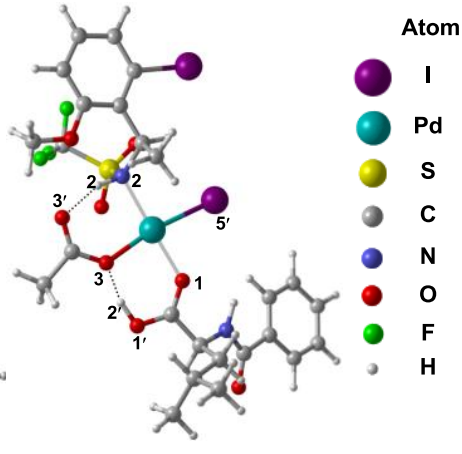

$R_{9 a}$

Pd- $\mathrm{N}^{2}=2.167$

$\mathrm{Pd}-\mathrm{I}^{5^{\prime}}=\mathbf{2 . 6 6 2}$

$\mathrm{Pd}-\mathrm{O}^{1}=2.088$

$\mathrm{Pd}-\mathrm{O}^{3}=2.123$

$\mathrm{O}^{3}-\mathrm{H}^{2}=1.530$

$\mathrm{N}^{2}-\mathrm{H}^{2}=1.053$

$\mathrm{O}^{3} \cdot \mathrm{H}^{2}=1.775$

Figure S16. Optimized structures and geometric parameters for selected species of pathway a for generation of the $(R)$-iodinated product and the regeneration of the catalyst in Figure 6

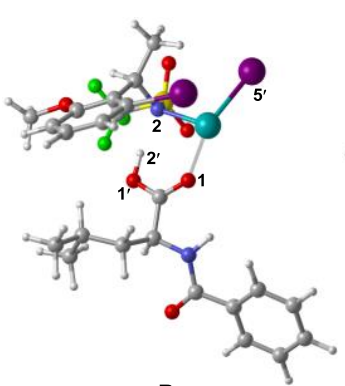

$R_{8 b}$

$P d-N^{2}=2.070$

$P d-1^{5^{\prime}}=2.592$

$\mathrm{Pd}-\mathrm{O}^{1}=2.163$

$\mathrm{O}^{1}-\mathrm{H}^{2^{\prime}}=1.021$

$\mathrm{N}^{2}-\mathrm{H}^{2}=1.732$

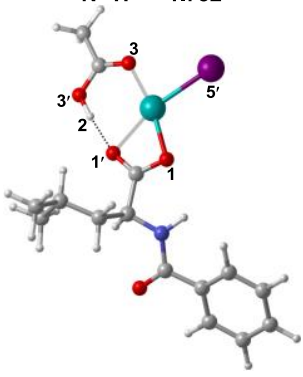

10

$P d-1^{5^{\prime}}=2.620$

$\mathrm{Pd}-\mathrm{O}^{1}=2.059$

$\mathrm{Pd}-\mathrm{O}^{1}=\mathbf{2} .196$

$\mathrm{Pd}-\mathrm{O}^{3}=\mathbf{2 . 0 7 3}$

$\mathrm{O}^{1}-\mathrm{H}^{2}=1.827$

$\mathrm{O}^{3}-\mathrm{H}^{2}=0.989$

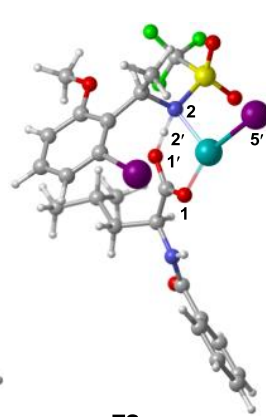

$\mathrm{TS}_{\mathrm{R} 5 \mathrm{~b}}$

$\mathrm{Pd}-\mathrm{N}^{2}=2.113$

$P d-I^{5^{2}}=2.601$

$\mathrm{Pd}-\mathrm{O}^{1}=2.093$

$\mathrm{O}^{1}-\mathrm{H}^{2}=1.217$

$\mathrm{N}^{2}-\mathrm{H}^{2}=1.298$

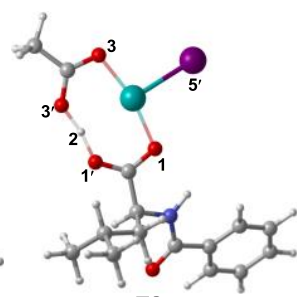

$\mathrm{TS}_{6}$

$\mathrm{Pd}-\mathrm{I}^{5^{\prime}}=\mathbf{2 . 6 0 1}$

$\mathrm{Pd}-\mathrm{O}^{1}=\mathbf{2 . 0 3 1}$

$\mathrm{Pd}-\mathrm{O}^{3}=2.027$

$\mathrm{O}^{1}-\mathrm{H}^{2}=1.232$

$\mathrm{O}^{3}-\mathrm{H}^{2}=1.215$

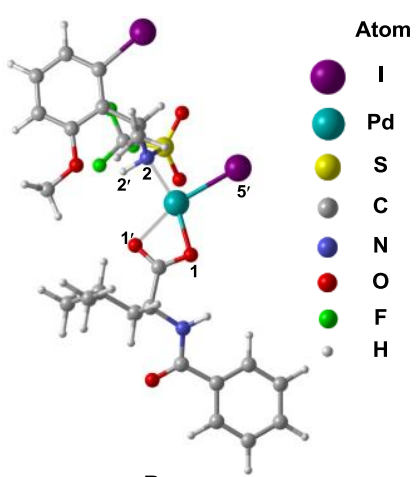

$\mathbf{R}_{\mathbf{9 b}}$

$\mathrm{Pd}-\mathrm{N}^{2}=2.149$

$P d-1^{5^{\prime}}=2.642$

$\mathrm{Pd}-\mathrm{O}^{1}=2.066$

$\mathrm{Pd}-\mathrm{O}^{1}=\mathbf{2} .182$

$\mathrm{O}^{1}-\mathrm{H}^{2}=2.890$

$\mathrm{N}^{2}-\mathrm{H}^{2}=1.031$

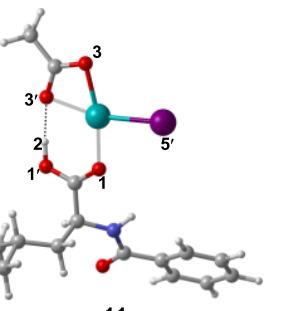

11

$P d-1^{5^{\prime}}=2.624$

$\mathrm{Pd}-\mathrm{O}^{1}=2.077$

$\mathrm{Pd}-\mathrm{O}^{3}=2.055$

$\mathrm{Pd}-\mathrm{O}^{3^{\prime}}=\mathbf{2 . 1 7 3}$

$\mathrm{O}^{1} \cdot \mathrm{H}^{2}=0.991$

$\mathrm{O}^{3}-\mathrm{H}^{2}=1.819$

Figure S17. Optimized structures and geometric parameters for selected species of pathway $b$ for generation of the $(R)$-iodinatedproduct and the regeneration of the catalyst in Figure 7 


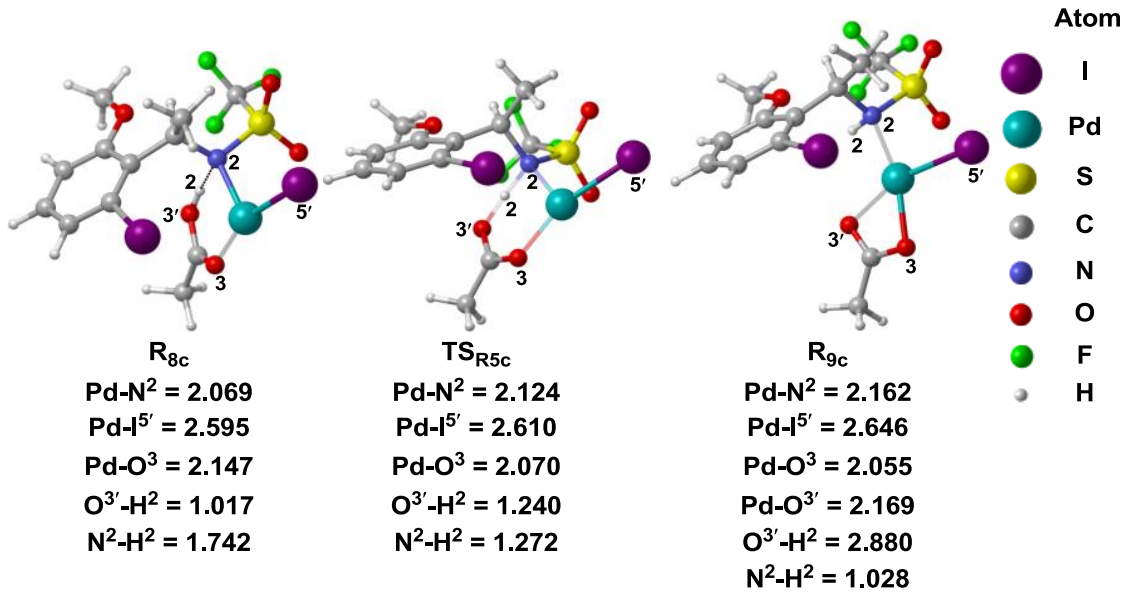

Figure S18. Optimized structures and geometric parameters for selected species of pathway c for generation of the $(R)$-iodinated product and the regeneration of the catalyst in Figure 8 
4. The energy profiles and optimized structures and geometric parameters of the pathwaysfor aryl $\mathrm{C}-\mathrm{H}$ bond activation of $S$-benzylamine

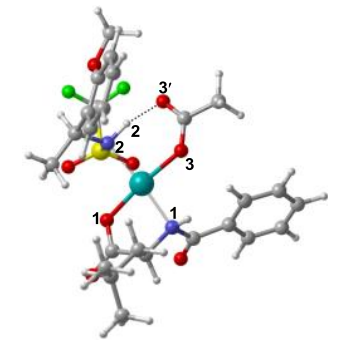

$S_{1 A}$

$P d-N^{2}=2.164$ Pd- $\mathbf{N}^{1}=2.123$ $\mathrm{Pd}-\mathrm{O}^{1}=1.988$ $\mathrm{Pd}-\mathrm{O}^{3}=\mathbf{2 . 0 5 1}$ $\mathrm{N}^{2}-\mathrm{H}^{2}=1.065$ $\mathrm{O}^{3^{\prime}-\mathrm{H}^{2}}=1.659$

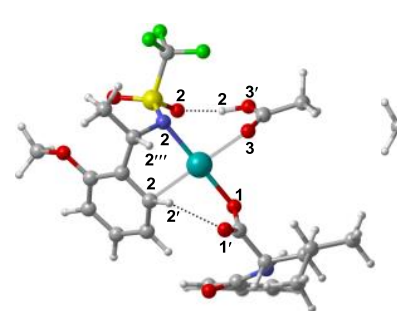

$\mathrm{S}_{3 \mathrm{~A}}$

Pd-N ${ }^{2}=2.073$

$\mathrm{Pd}-\mathrm{C}^{2}=2.293$

$P d-O^{1}=2.020$

$\mathrm{Pd}-\mathrm{O}^{3}=2.164$

$\mathrm{Pd}-\mathrm{H}^{2 \prime \prime \prime}=\mathbf{2 . 8 9 2}$

$\mathrm{C}^{2}-\mathrm{H}^{2}=1.104$

$\mathrm{O}^{1}-\mathrm{H}^{2^{\prime}}=\mathbf{2 . 2 4 1}$

$\mathrm{O}^{2}-\mathrm{H}^{2}=1.670$

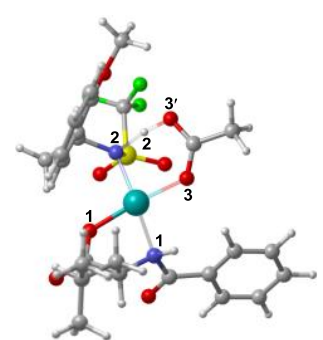

$\mathrm{TS}_{\mathrm{S} 1 \mathrm{~A}}$

Pd- $\mathrm{N}^{2}=2.138$

Pd-N ${ }^{1}=2.134$

$\mathrm{Pd}-\mathrm{O}^{1}=1.984$

$\mathrm{Pd}-\mathrm{O}^{3}=\mathbf{2 . 0 9 1}$

$\mathrm{N}^{2}-\mathrm{H}^{2}=1.385$

$\mathrm{O}^{3} \cdot \mathrm{H}^{2}=1.148$

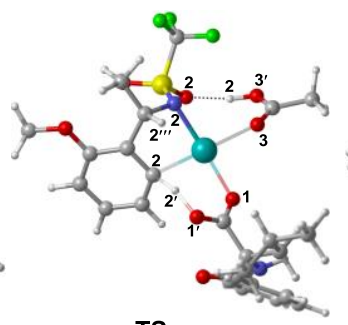

$\mathrm{TS}_{\mathrm{S} 2 \mathrm{~A}}$

$\mathrm{Pd}-\mathrm{N}^{2}=2.057$

Pd-C ${ }^{2}=2.098$

$\mathrm{Pd}-\mathrm{O}^{1}=\mathbf{2 . 0 8 1}$

$\mathrm{Pd}-\mathrm{O}^{3}=\mathbf{2 . 1 8 4}$

$\mathrm{Pd}-\mathrm{H}^{2 \text { "' }}=\mathbf{2 . 8 0 7}$

$\mathrm{C}^{2}-\mathrm{H}^{2}=1.326$

$\mathrm{O}^{1^{\prime}-\mathrm{H}^{2}}=1.377$

$\mathrm{O}^{2}-\mathrm{H}^{2}=1.663$

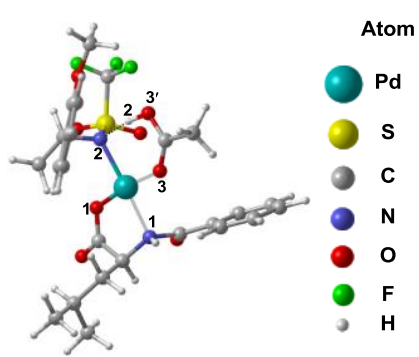

$S_{2 A}$

Pd- $\mathrm{N}^{2}=\mathbf{2 . 1 1 3}$

$\mathrm{Pd}-\mathrm{N}^{1}=\mathbf{2 . 1 4 4}$

$\mathrm{Pd}-\mathrm{O}^{1}=1.977$

$\mathrm{Pd}-\mathrm{O}^{3}=2.105$

$\mathrm{N}^{2}-\mathrm{H}^{2}=1.600$

$\mathrm{O}^{3^{\prime}}-\mathrm{H}^{2}=1.051$

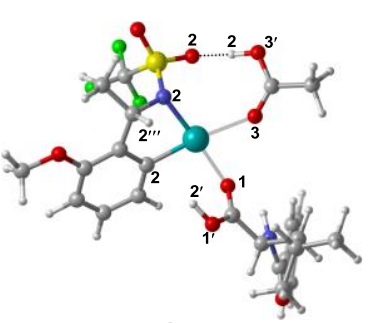

$\mathrm{S}_{4 \mathrm{~A}}$

$$
\begin{aligned}
\mathrm{Pd}-\mathrm{N}^{2} & =2.036 \\
\mathrm{Pd}-\mathrm{C}^{2} & =1.980 \\
\mathrm{Pd}-\mathrm{O}^{1} & =2.158 \\
\mathrm{Pd}-\mathrm{O}^{3} & =2.274 \\
\mathrm{Pd}-\mathrm{H}^{2 \prime \prime} & =3.078 \\
\mathrm{C}^{2}-\mathrm{H}^{2} & =2.420 \\
\mathrm{O}^{1}-\mathrm{H}^{2} & =0.989
\end{aligned}
$$$$
\mathrm{O}^{2}-\mathrm{H}^{2}=1.630
$$

Figure S19. Optimized structures and geometric parameters for selected species of pathway A for aryl C-H bond activation of $S$-benzylamine in Figure 9 


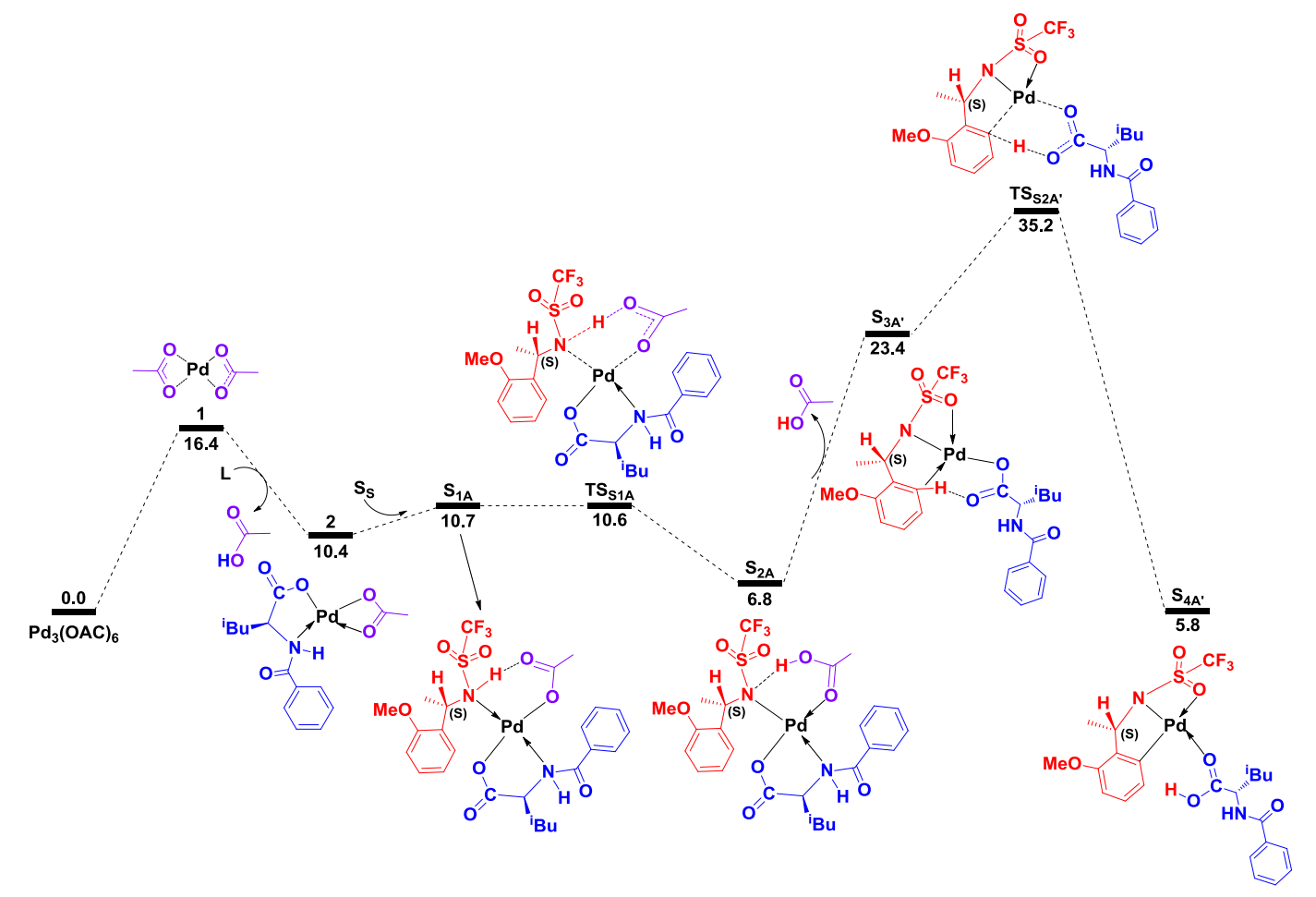

Figure S20. Energy profile of pathway $\mathrm{A}^{\prime}$ for aryl $\mathrm{C}-\mathrm{H}$ bond activation of $S$-benzylamine. The relative free energies in dimethyl sulfoxide (DMSO) are given in $\mathrm{kcal} / \mathrm{mol}$
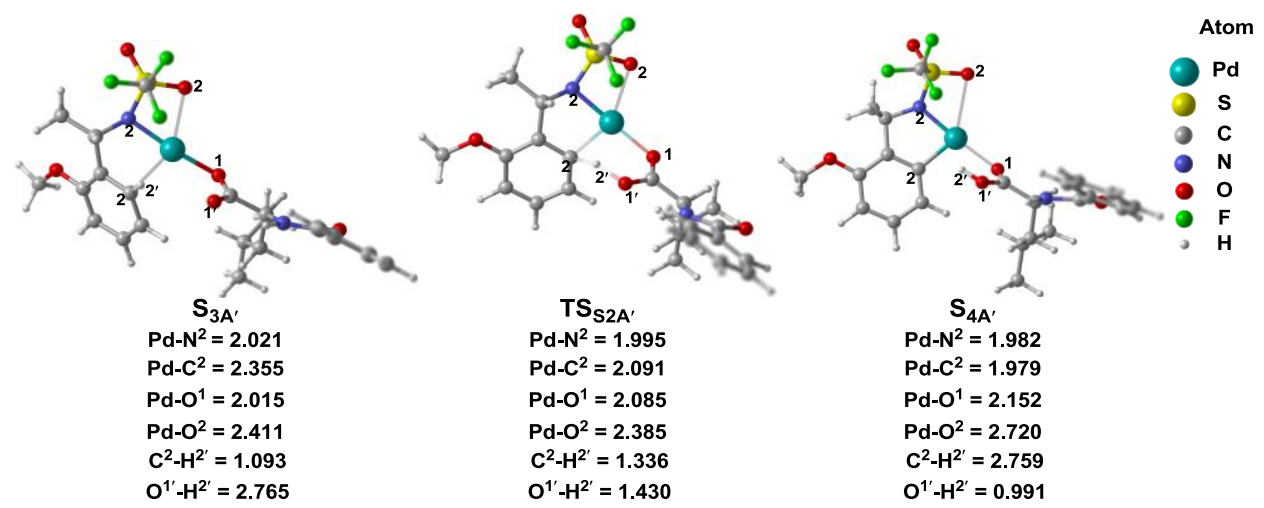

Figure S21. Optimized structures and geometric parameters for selected species of pathway A' for aryl C-H bond activation of $S$-benzylamine in Figure S20 


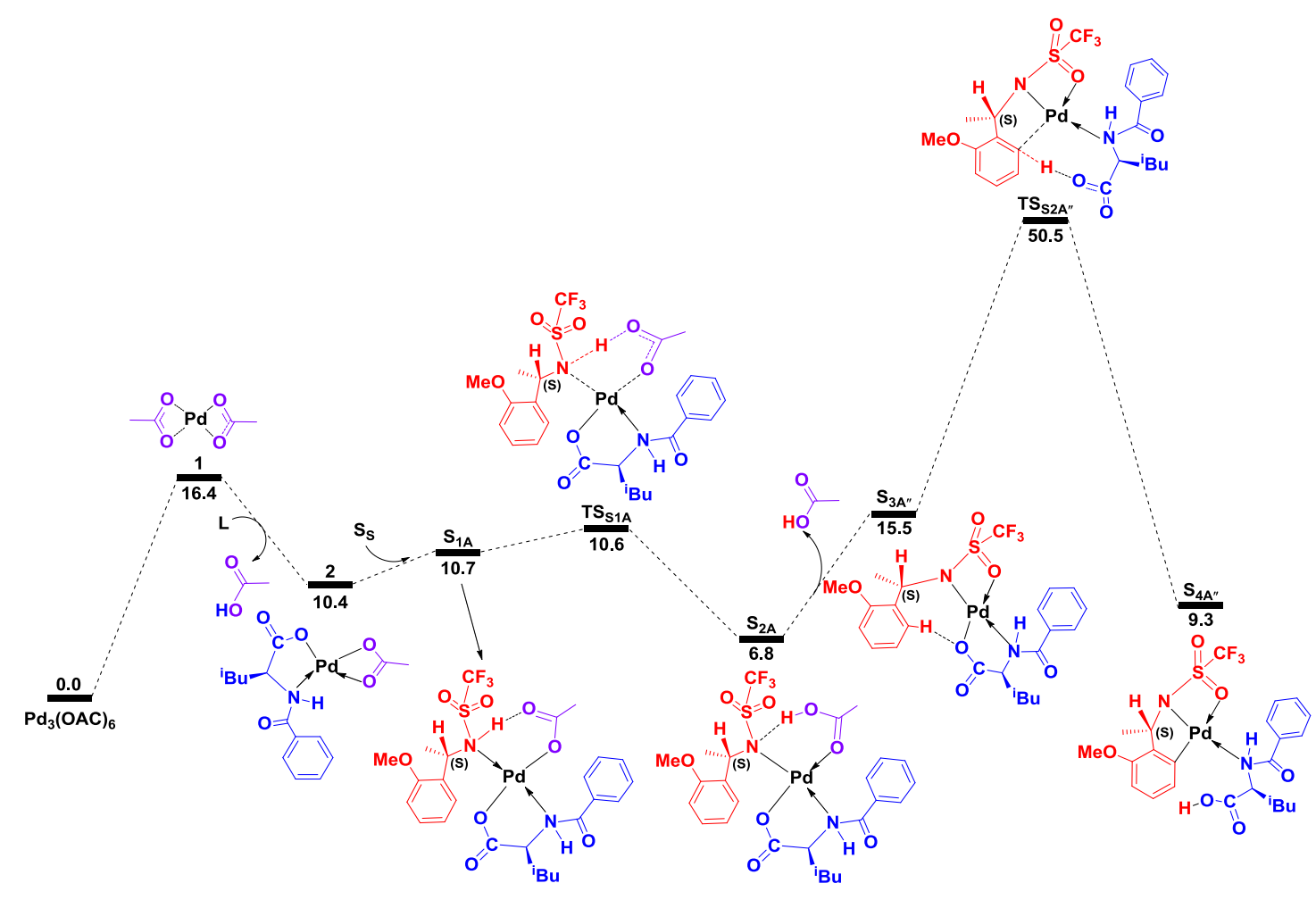

Figure S22. Energy profile of pathway A" for aryl C-H bond activation of $S$-benzylamine. The relative free energies in dimethyl sulfoxide (DMSO) are given in $\mathrm{kcal} / \mathrm{mol}$

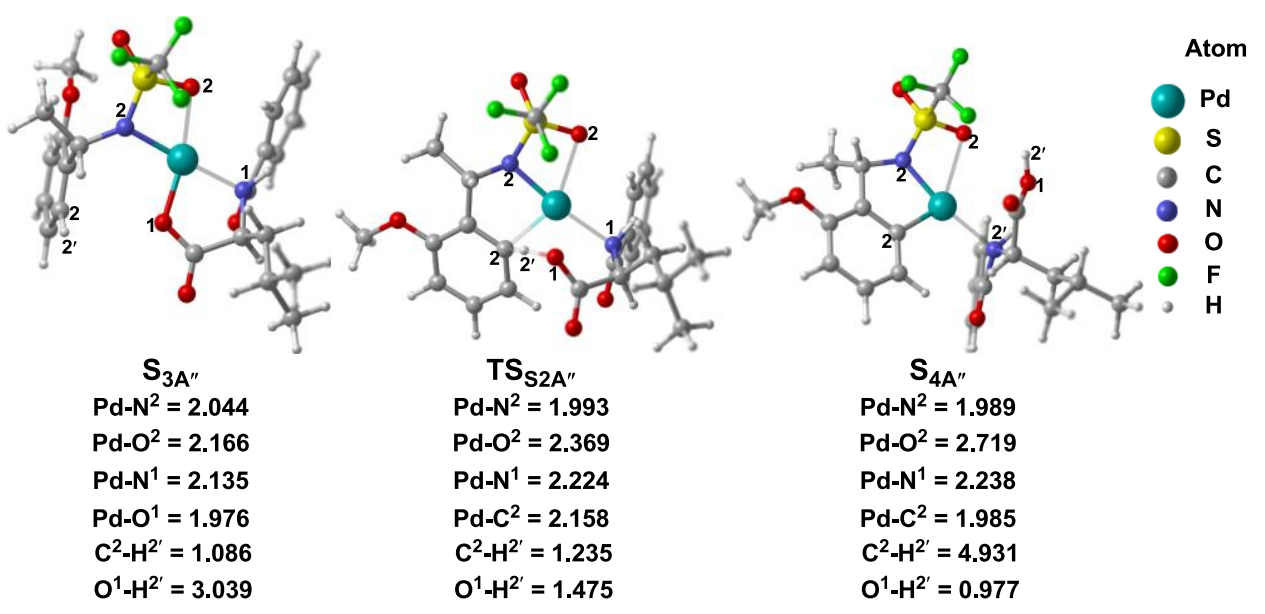

Figure S23. Optimized structures and geometric parameters for selected species of pathway A" for aryl C-H bond activation of $S$-benzylamine in Figure $\mathrm{S} 22$ 


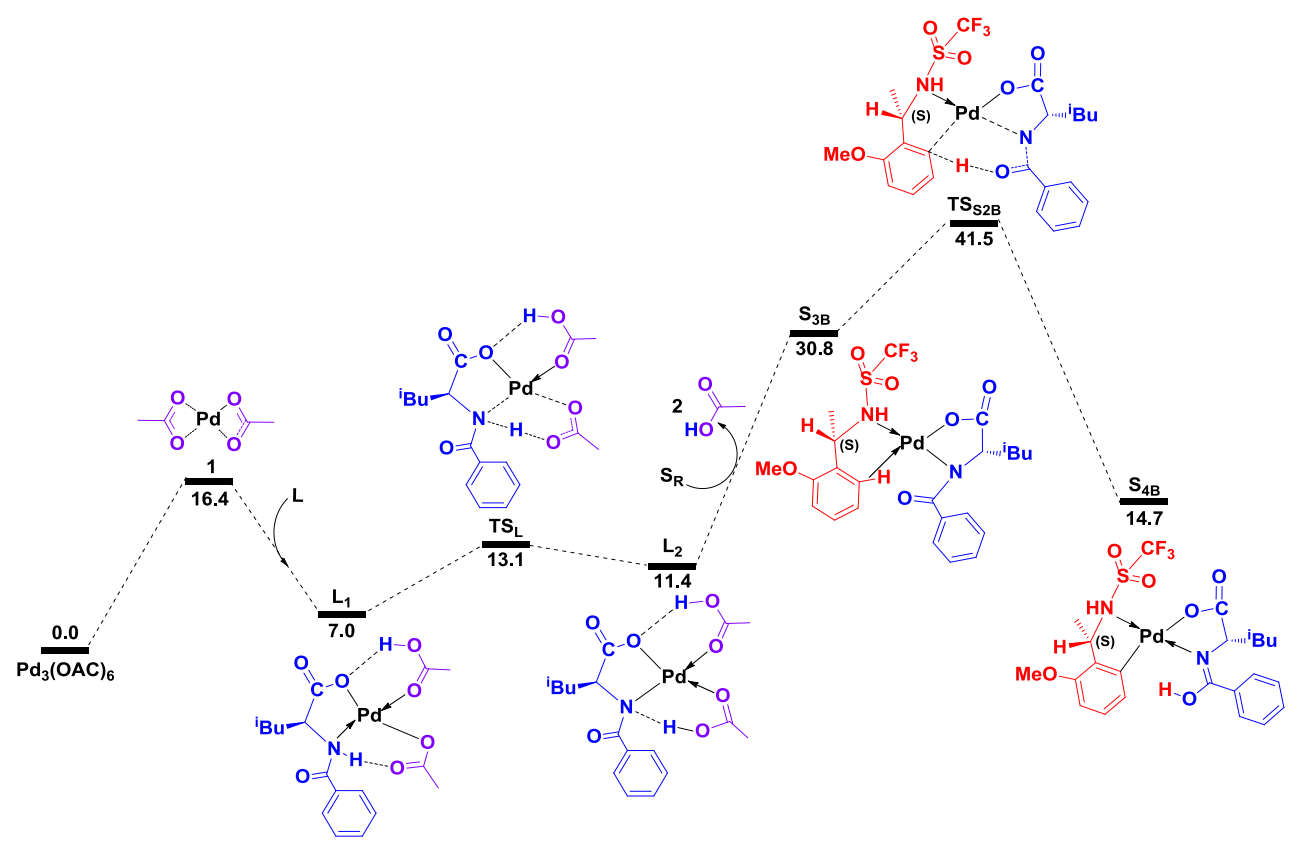

Figure S24. Energy profile of pathway B for aryl C-H bond activation of $S$-benzylamine. The relative free energies in dimethyl sulfoxide (DMSO) are given in $\mathrm{kcal} / \mathrm{mol}$

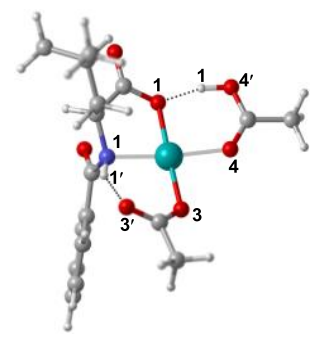

$\mathrm{L}_{1}$

$\mathrm{Pd}-\mathrm{N}^{1}=2.092$

$\mathrm{Pd}-\mathrm{O}^{1}=\mathbf{2 . 0 0 9}$

$\mathrm{Pd}-\mathrm{O}^{4}=\mathbf{2 . 1 0 0}$

$\mathrm{Pd}-\mathrm{O}^{3}=2.018$

$\mathrm{O}^{1}-\mathrm{H}^{1}=1.575$

$\mathrm{N}^{1}-\mathrm{H}^{1^{\prime}}=1.039$

$\mathrm{O}^{3^{\prime}}-\mathrm{H}^{1^{\prime}}=1.832$

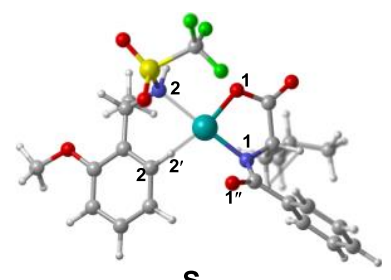

$\mathrm{S}_{3 \mathrm{~B}}$

$\mathrm{Pd}-\mathrm{N}^{2}=2.186$

$\mathrm{Pd}-\mathrm{O}^{1}=1.980$

Pd-N ${ }^{1}=1.977$

$\mathrm{Pd}-\mathrm{H}^{2}=\mathbf{2} .029$

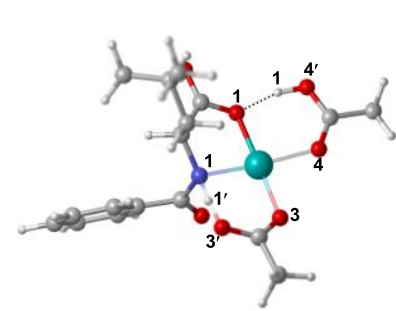

$\mathrm{TS}_{\mathrm{L}}$

$\mathrm{Pd}-\mathrm{N}^{1}=\mathbf{2 . 0 3 6}$

$\mathrm{Pd}-\mathrm{O}^{1}=\mathbf{2 . 0 0 2}$

$\mathrm{Pd}-\mathrm{O}^{4}=2.119$

$\mathrm{Pd}-\mathrm{O}^{3}=2.062$

$\mathrm{O}^{1}-\mathrm{H}^{1}=1.602$

$\mathrm{N}^{1}-\mathrm{H}^{11}=1.294$

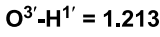

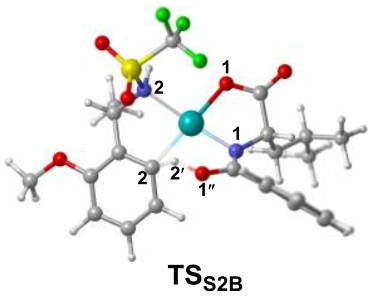

$\mathrm{Pd}-\mathrm{N}^{2}=2.142$

$\mathrm{Pd}-\mathrm{O}^{1}=\mathbf{2 . 0 1 1}$

$\mathrm{Pd}-\mathrm{N}^{1}=1.994$

$\mathrm{Pd}-\mathrm{C}^{2}=\mathbf{2 . 1 8 3}$

$\mathrm{C}^{2}-\mathrm{H}^{2}=1.364$

$\mathrm{O}^{1 "}-\mathrm{H}^{2^{\prime}}=1.444$

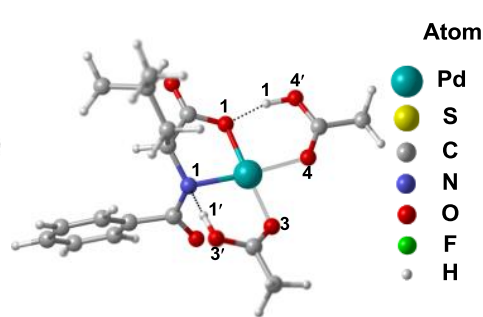

$\mathbf{L}_{2}$

$\mathrm{Pd}-\mathrm{N}^{1}=2.026$

$\mathrm{Pd}-\mathrm{O}^{1}=1.989$

$\mathrm{Pd}-\mathrm{O}^{4}=2.128$

$\mathrm{Pd}-\mathrm{O}^{3}=2.096$

$\mathrm{O}^{1}-\mathrm{H}^{1}=1.601$

$\mathrm{N}^{1}-\mathrm{H}^{1^{\prime}}=1.695$

$\mathrm{O}^{3^{\prime}-\mathrm{H}^{1}}=1.024$

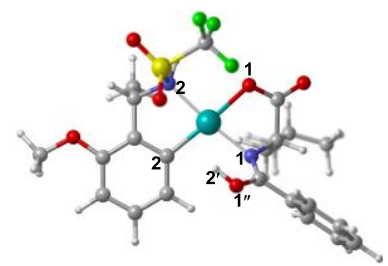

$\mathrm{S}_{4 \mathrm{~B}}$

$\mathrm{Pd}-\mathrm{N}^{2}=2.112$

$\mathrm{Pd}-\mathrm{O}^{1}=2.093$

Pd- $\mathrm{N}^{1}=\mathbf{2 . 0 5 1}$

$\mathrm{Pd}-\mathrm{C}^{2}=2.018$

$\mathrm{C}^{2}-\mathrm{H}^{2}=2.568$

$\mathrm{O}^{1 "} \cdot \mathrm{H}^{2^{\prime}}=0.985$

Figure S25. Optimized structures and geometric parameters for selected species of pathway B for aryl C-H bond activation of $S$-benzylamine in Figure S24 


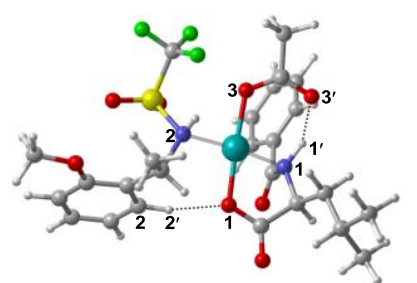

$S_{1 C}$

$\mathrm{Pd}-\mathrm{N}^{2}=\mathbf{2 . 1 8 1}$

$\mathrm{Pd}-\mathrm{O}^{3}=2.041$

Pd- $\mathrm{N}^{1}=\mathbf{2 . 0 9 8}$

$\mathrm{Pd}-\mathrm{O}^{1}=\mathbf{2 . 0 0 0}$

$\mathrm{C}^{2}-\mathrm{H}^{2}=1.085$

$\mathrm{O}^{1}-\mathrm{H}^{2^{\prime}}=\mathbf{2 . 1 8 6}$

$\mathrm{O}^{3^{\prime}}-\mathrm{H}^{1^{\prime}}=1.825$

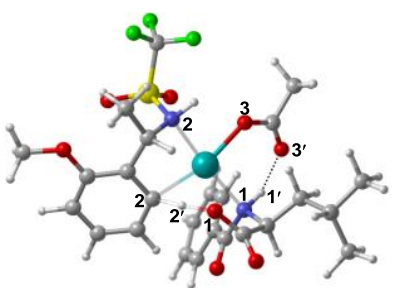

$\mathrm{TS}_{\mathrm{S} 1 \mathrm{C}}$

$\mathrm{Pd}-\mathrm{N}^{2}=2.130$

$\mathrm{Pd}-\mathrm{O}^{3}=2.052$

$\mathrm{Pd}-\mathrm{N}^{1}=2.160$

$\mathrm{Pd}-\mathrm{C}^{2}=2.273$

$\mathrm{C}^{2}-\mathrm{H}^{2}=1.209$

$\mathrm{O}^{1}-\mathrm{H}^{2^{\prime}}=1.518$

$\mathrm{O}^{3^{\prime}}-\mathrm{H}^{1^{\prime}}=1.844$

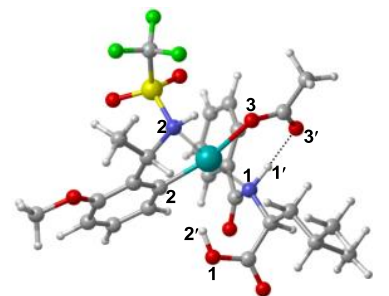

$\mathrm{S}_{2 \mathrm{C}}$

$\mathrm{Pd}-\mathrm{N}^{2}=2.093$

$\mathrm{Pd}-\mathrm{O}^{3}=2.159$

Pd-N ${ }^{1}=2.205$

$\mathrm{Pd}-\mathrm{C}^{2}=2.025$

$\mathrm{C}^{2}-\mathrm{H}^{2}=2.197$

$\mathrm{O}^{1}-\mathrm{H}^{2}=0.978$

$\mathrm{O}^{3^{\prime}-\mathrm{H}^{1}}=1.751$

Figure S26. Optimized structures and geometric parameters for selected species of pathway C for aryl C-H bond activation of $S$-benzylamine in Figure 10

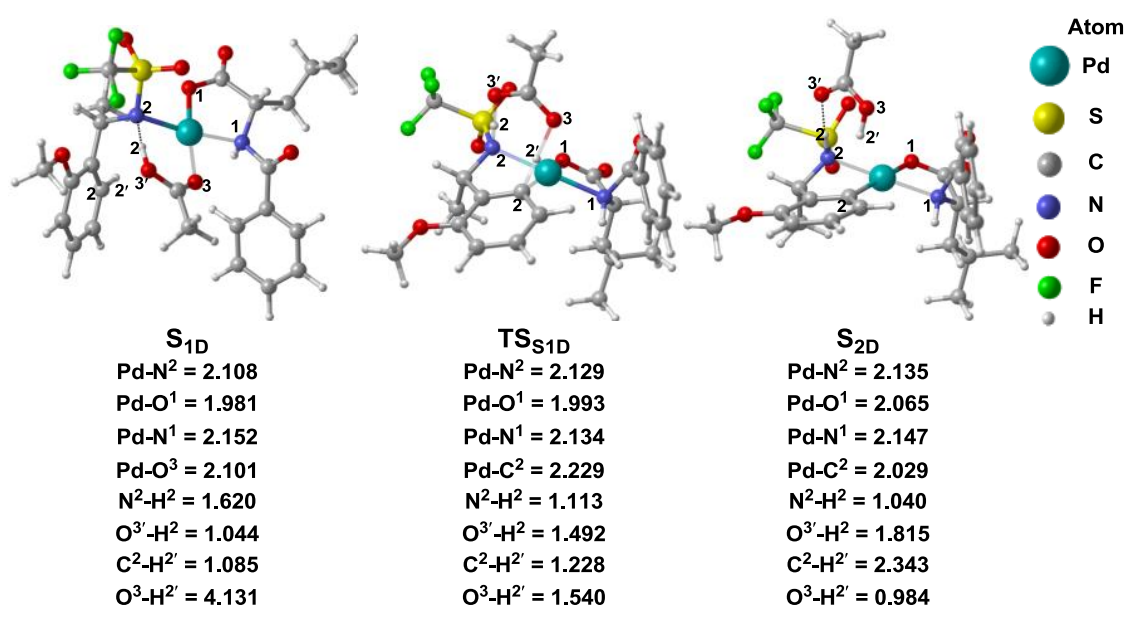

Figure S27. Optimized structures and geometric parameters for selected species of pathway D for aryl C-H bond activation of $S$-benzylamine in Figure 11 


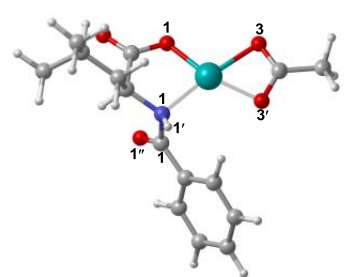

2

$\mathrm{Pd}-\mathbf{N}^{1}=\mathbf{2 . 1 1 2}$

$\mathrm{Pd}-\mathrm{O}^{1}=1.971$

$\mathrm{Pd}-\mathrm{O}^{3}=\mathbf{2 . 0 9 0}$

$\mathrm{Pd}-\mathrm{O}^{3^{\prime}}=2.107$

$\mathrm{H}^{1}-\mathrm{N}^{1}-\mathrm{C}^{1}-\mathrm{O}^{1 "}=148.5^{\circ}$

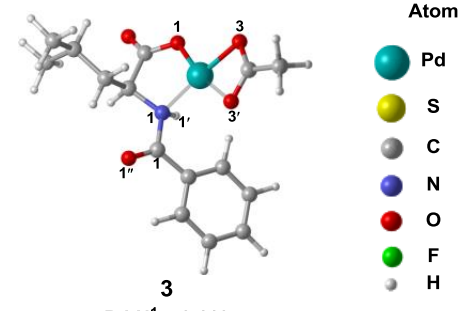

Pd-N ${ }^{1}=2.118$

$\mathrm{Pd}-\mathrm{O}^{1}=1.994$

$\mathrm{Pd}-\mathrm{O}^{3}=2.085$

$\mathrm{Pd}-\mathrm{O}^{3^{\prime}}=2.093$

$H^{1}-N^{1}-C^{1}-O^{1 "}=-143.4^{\circ}$
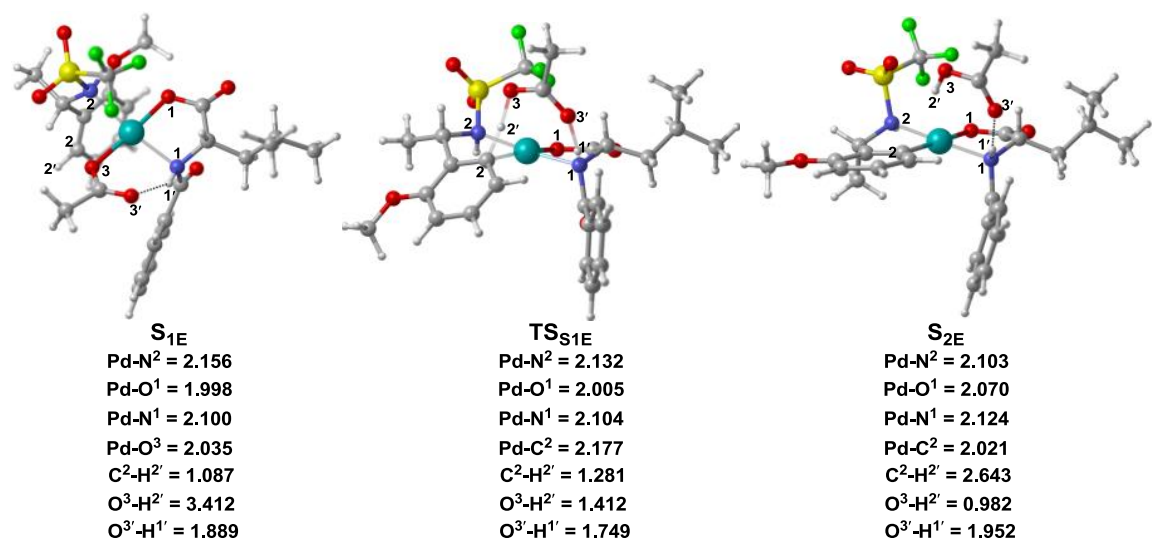

Figure S28. Optimized structures and geometric parameters for selected species of pathway E for aryl C-H bond activation of $S$-benzylamine in Figure 12 
5. The energy profiles and optimized structures and geometric parameters of the pathways for iodation reaction of $S$-benzylamine

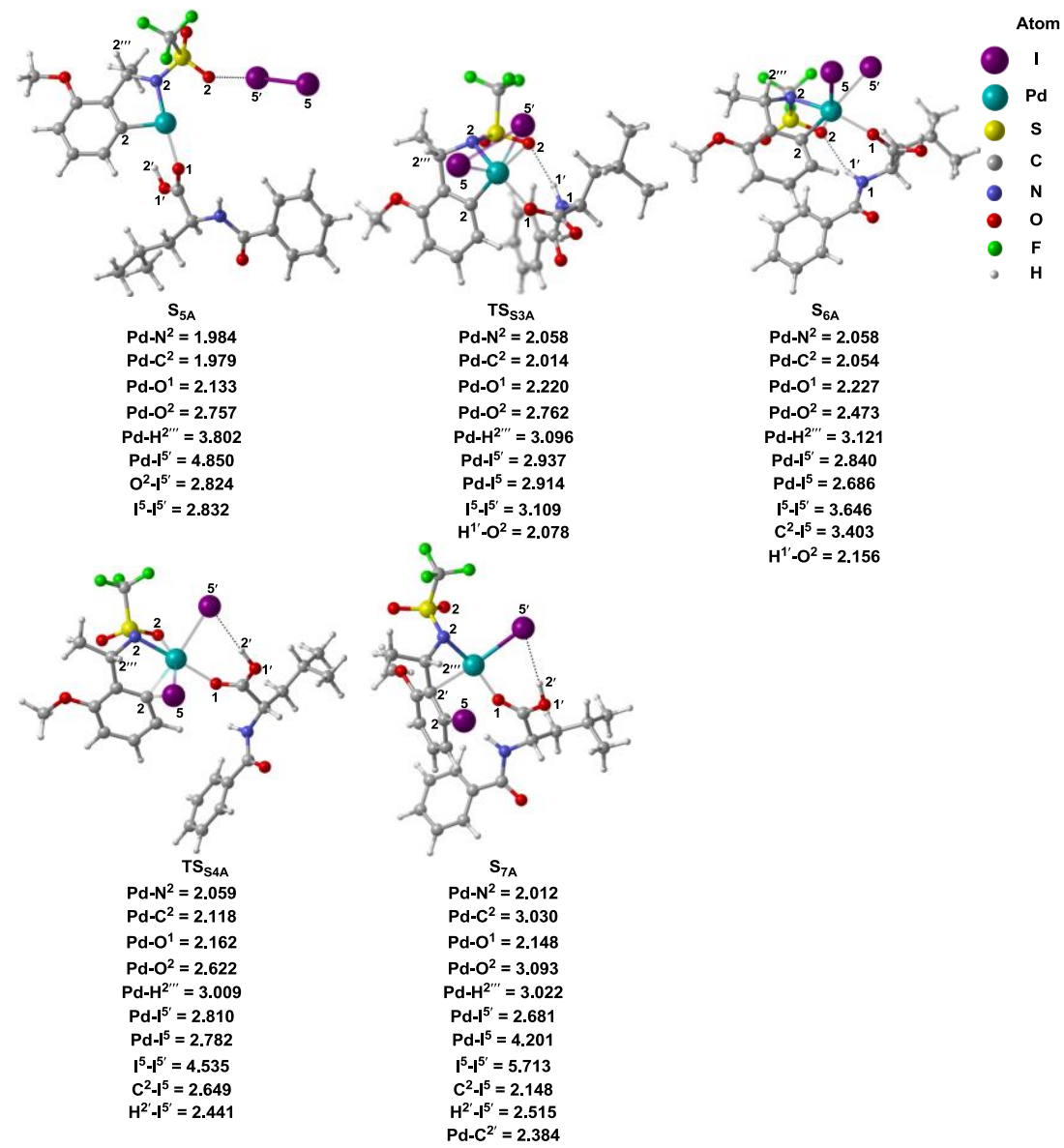

Figure S29. Optimized structures and geometric parameters for selected species of the stepwise reaction pathway A of $S$-benzylamine in Figure 13 


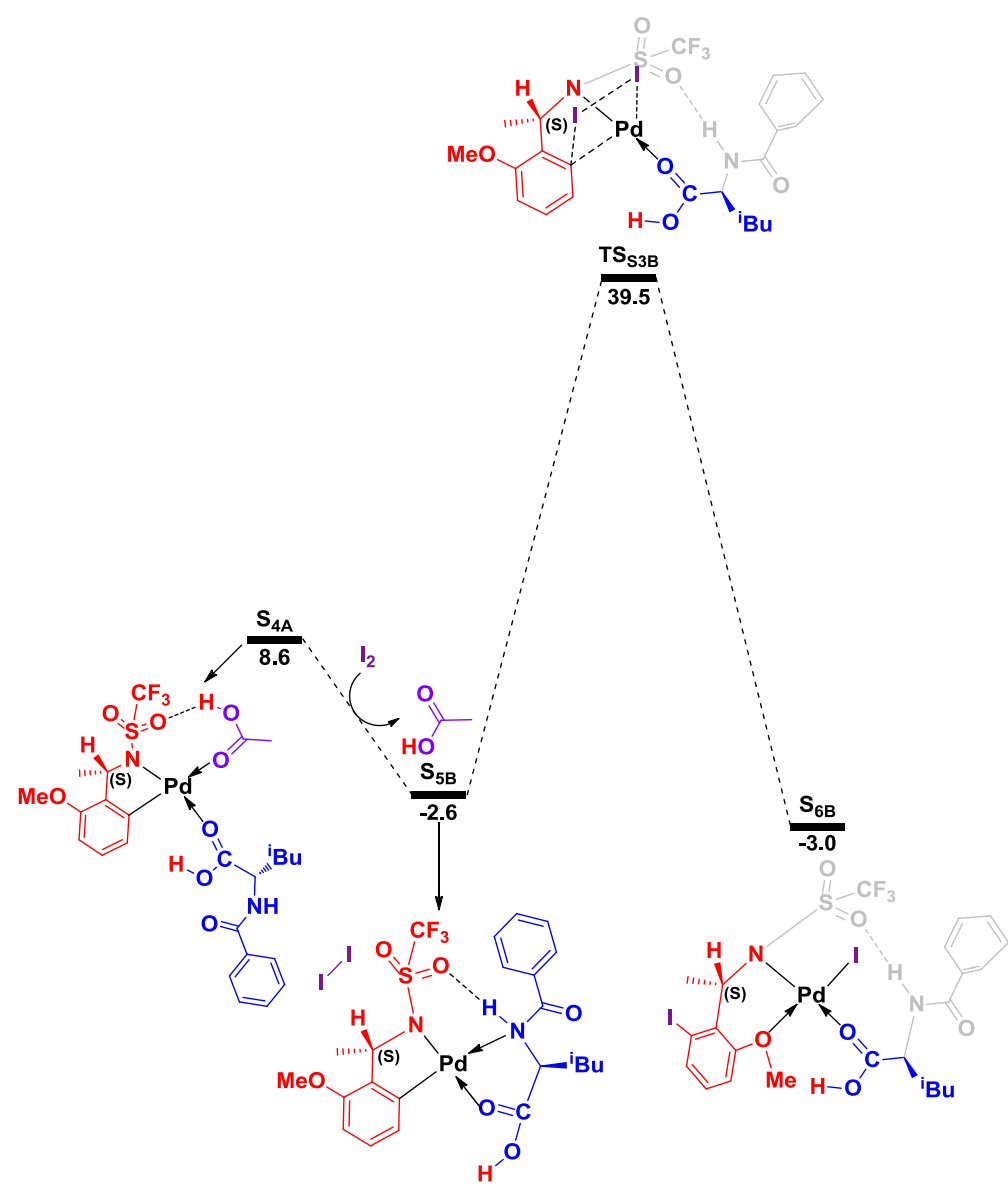

Figure S30. Energy profiles of pathway B for metathesis reaction of $S$-benzylamine. The relative free energies in dimethyl sulfoxide (DMSO) are given in $\mathrm{kcal} / \mathrm{mol}$
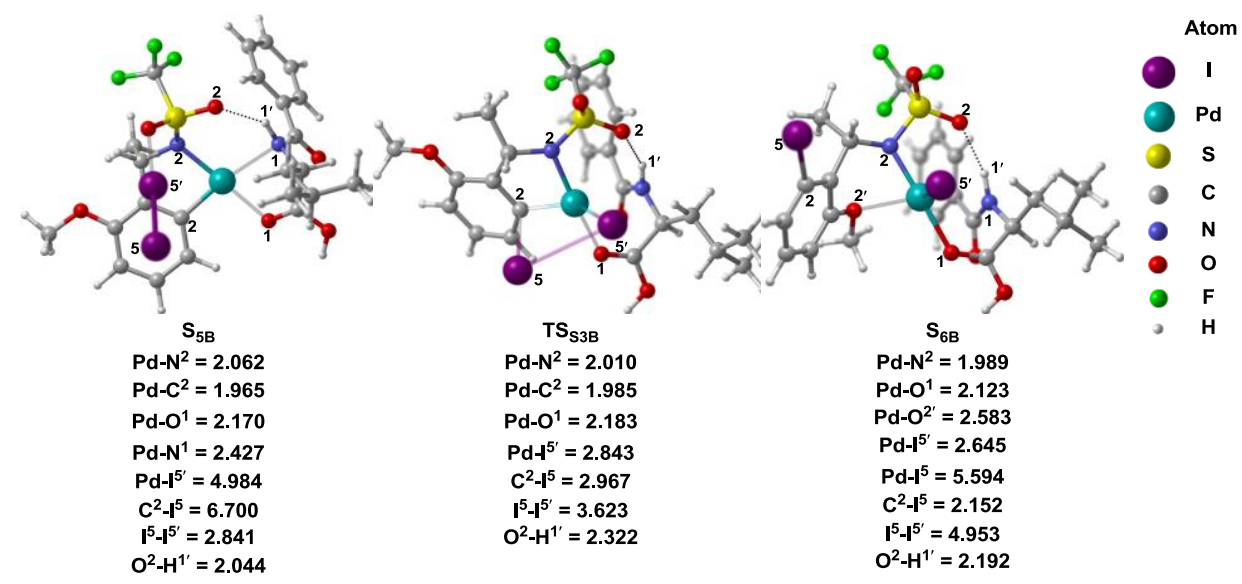

Figure S31. Optimized structures and geometric parameters for selected species of pathway B for metathesis reaction of $S$-benzylamine in Figure S30 


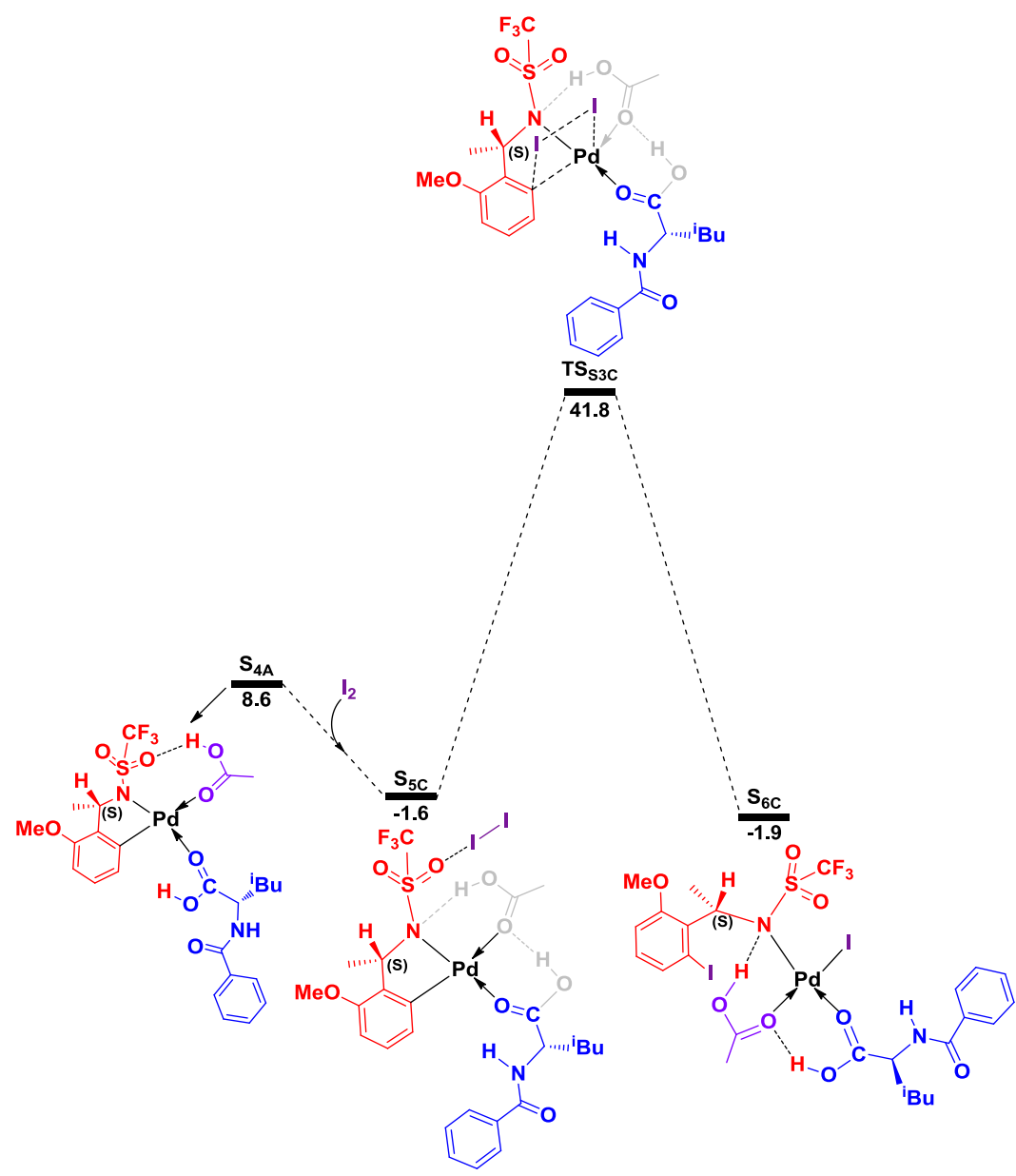

Figure S32. Energy profiles of pathway $\mathrm{C}$ for metathes is reaction of $S$-benzylamine. The relative free energies in dimethyl sulfoxide (DMSO) are given in $\mathrm{kcal} / \mathrm{mol}$

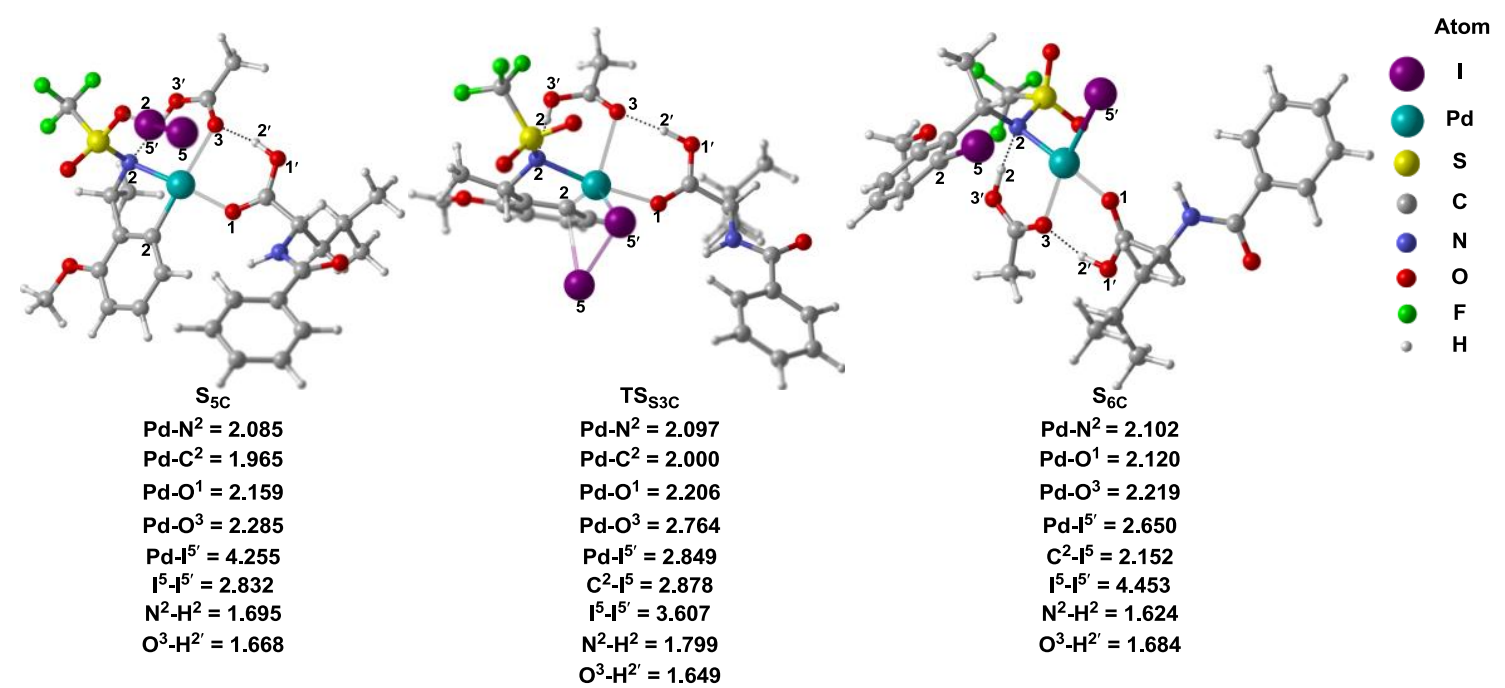

Figure S33. Optimized structures and geometric parameters for selected species of pathway $\mathrm{C}$ for metathesis reaction of $S$-benzylamine in Figure $S 32$ 
6. The energy profiles and the optimized structures and geometric parameters of the pathways for generation of the $(S)$-iodinated product and the regeneration of the catalyst

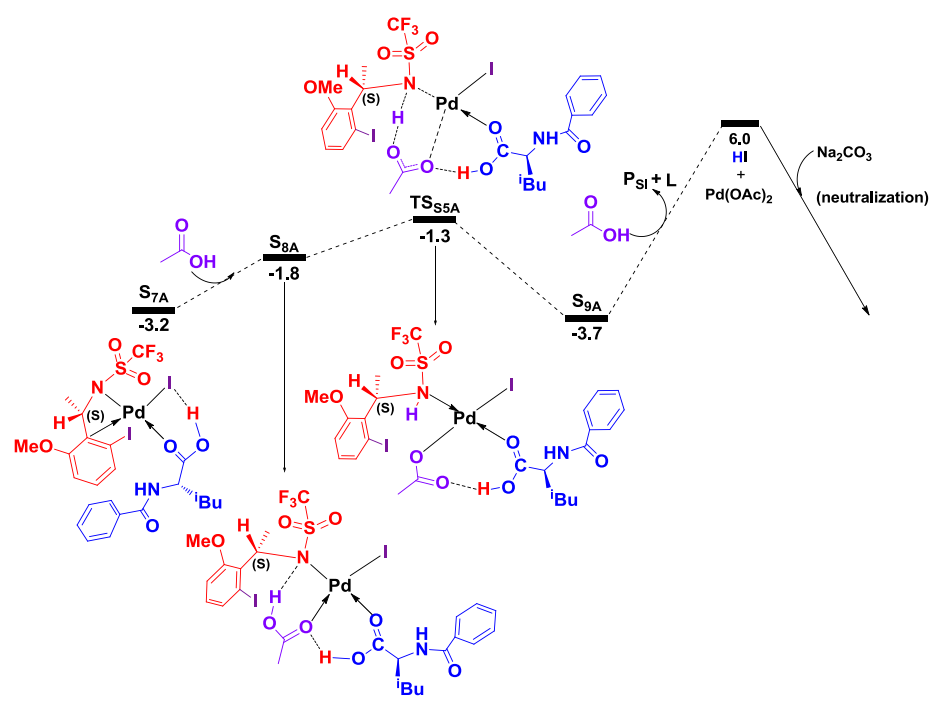

Figure S34. Energy profile of pathwayA for generation of the $(S)$-iodinated product and the regeneration of the catalyst. The relative free energies in dimethyl sulfoxide (DMSO) are given in $\mathrm{kcal} / \mathrm{mol}$

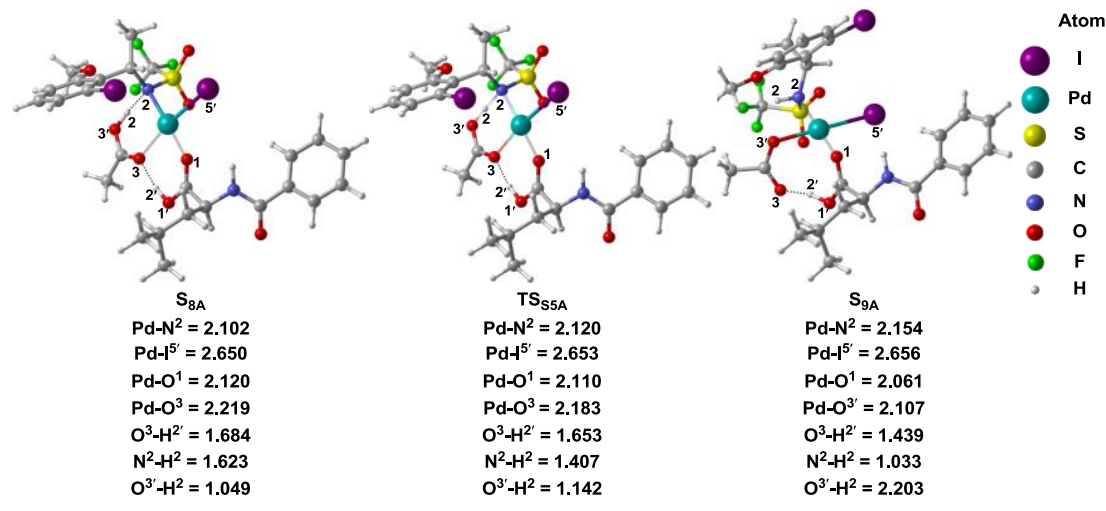

Figure S35. Optimized structures and geometric parameters for selected species of pathway A for generation of the $(S)$-iodinatedproduct and the regeneration of the catalyst in Figure S34 


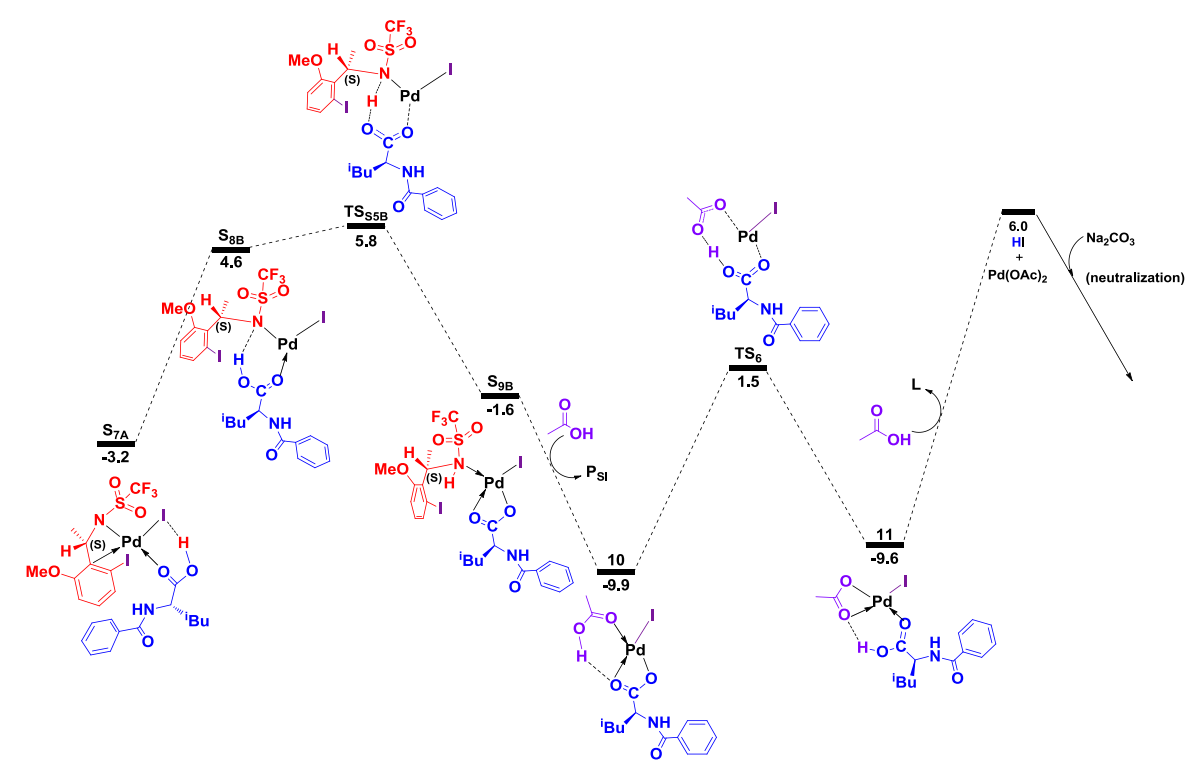

Figure S36. Energy profile of pathway B for generation of the $(S)$-iodinated product and the w3tregeneration of the catalyst. The relative free energies in dimethyl sulfoxide (DMSO) are given in $\mathrm{kcal} / \mathrm{mol}$

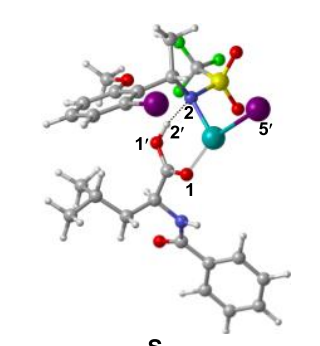

$S_{8 B}$

\section{$\mathrm{Pd}-\mathrm{N}^{2}=2.075$}

Pd-1 - $^{\prime \prime}=2.594$

$\mathrm{Pd}-\mathrm{O}^{1}=2.145$

$\mathrm{O}^{1}-\mathrm{H}^{2^{\prime}}=1.015$

$\mathrm{N}^{2}-\mathrm{H}^{2}=1.797$

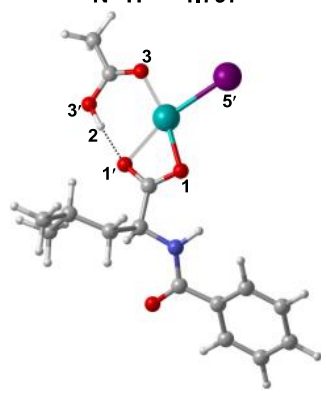

10

$P d-I^{5^{\prime}}=2.620$

$\mathrm{Pd}-\mathrm{O}^{1}=2.059$

$\mathrm{Pd}^{10^{\prime}}=2.196$

$\mathrm{Pd}-\mathrm{O}^{3}=2.073$

$\mathrm{O}^{1}-\mathrm{H}^{2}=1.827$

$\mathrm{O}^{3}-\mathrm{H}^{2}=0.989$

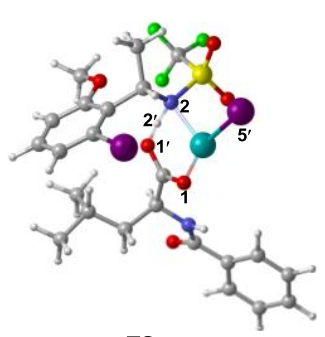

$\mathrm{TS}_{\mathrm{S} 5 \mathrm{~B}}$

$\mathrm{Pd}-\mathrm{N}^{2}=2.114$

$\mathrm{Pd}-\mathrm{F}^{5^{\prime}}=2.606$

$\mathrm{Pd}-\mathrm{O}^{1}=\mathbf{2 . 0 7 9}$

$\mathrm{O}^{1}-\mathrm{H}^{2^{\prime}}=1.221$

$\mathrm{N}^{2}-\mathrm{H}^{2}=1.300$

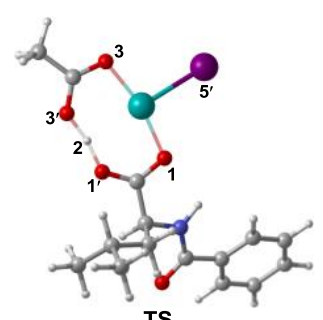

$\mathrm{TS}_{6}$

$\mathrm{Pd}-\mathrm{I}^{5^{\prime}}=\mathbf{2 . 6 0 1}$

$\mathrm{Pd}-\mathrm{O}^{1}=2.031$

$\mathrm{Pd}-\mathrm{O}^{3}=\mathbf{2 . 0 2 7}$

$\mathrm{O}^{1}-\mathrm{H}^{2}=1.232$

$\mathrm{O}^{3} \cdot \mathrm{H}^{2}=1.215$

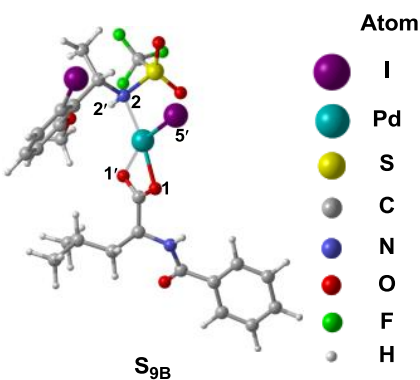

$\mathrm{S}_{9 \mathrm{~B}}$

$\mathrm{Pd}-\mathrm{N}^{2}=2.149$

$\mathrm{Pd}-\mathrm{I}^{5^{\prime}}=2.637$

$\mathrm{Pd}-\mathrm{O}^{1}=2.056$

$P d-O^{1}=2.209$

$\mathrm{O}^{1}-\mathrm{H}^{2}=3.036$

$\mathrm{N}^{2}-\mathrm{H}^{2}=1.032$

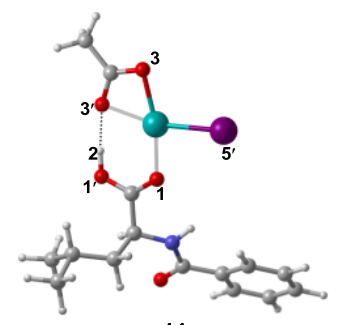

11

$P d-1^{5^{\prime}}=2.624$

$\mathrm{Pd}-\mathrm{O}^{1}=2.077$

$\mathrm{Pd}-\mathrm{O}^{3}=2.055$

$\mathrm{Pd}-\mathrm{O}^{3^{\prime}}=\mathbf{2 . 1 7 3}$

$\mathrm{O}^{1}-\mathrm{H}^{2}=0.991$

$\mathrm{O}^{3} \cdot \mathrm{H}^{2}=1.819$

Figure S37. Optimized structures and geometric parameters for selected species of pathway B for generation of the $(S)$-iodinatedproduct in Figure S36 


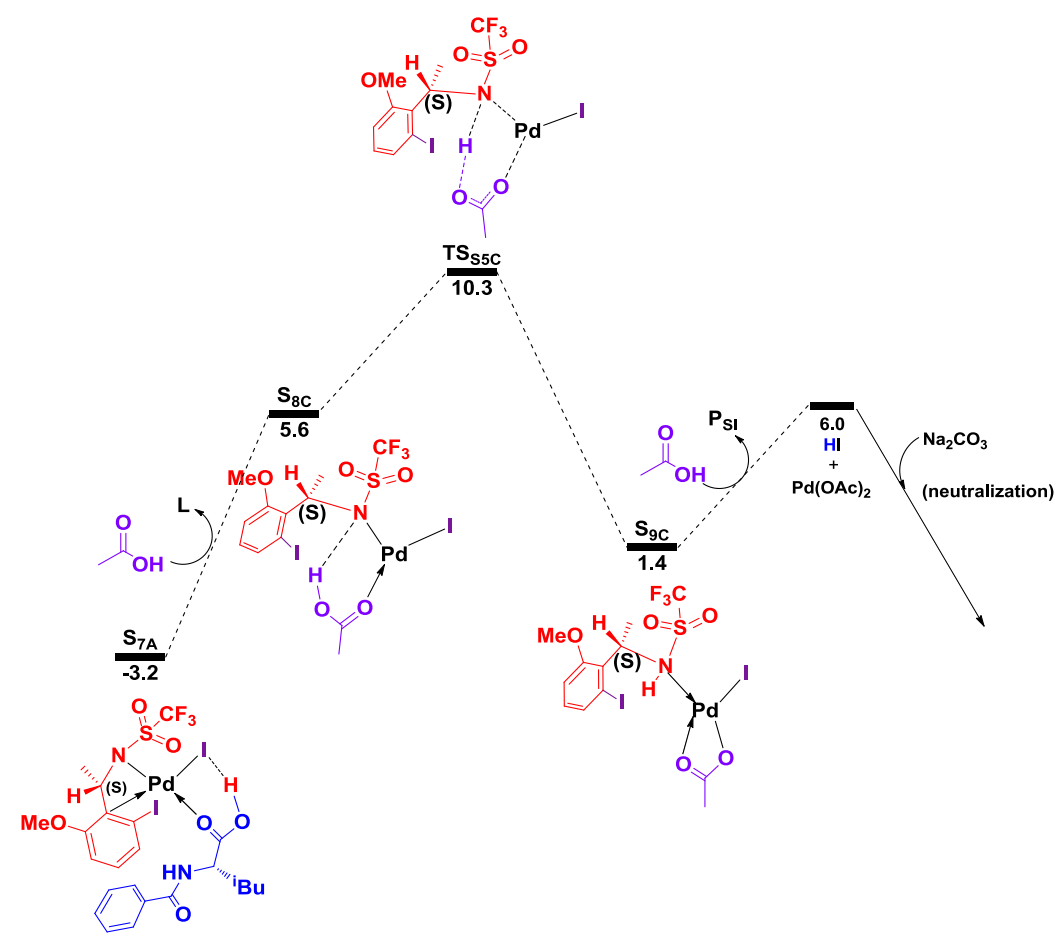

Figure S38. Energy profile of pathway $\mathrm{C}$ for generation of the $(S)$-iodinated product and the regeneration of the catalyst. The relative free energies in dimethyl sulfoxide (DMSO) are given in $\mathrm{kcal} / \mathrm{mol}$
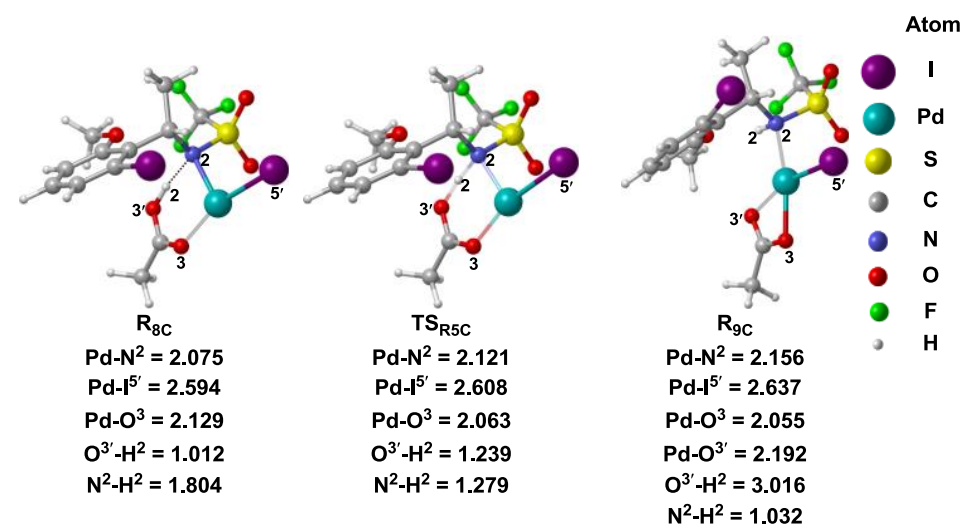

Figure S39. Optimized structures and geometric parameters for selected species of pathway $\mathrm{C}$ for generation of the $(S)$-iodinated product and the regeneration of the catalyst in Figure S38 
7. The energy profiles and optimized structures and geometric parameters of pathways $R$-Me and $S$-Me for aryl $\mathrm{C}-\mathrm{H}$ bond activation of benzylamine

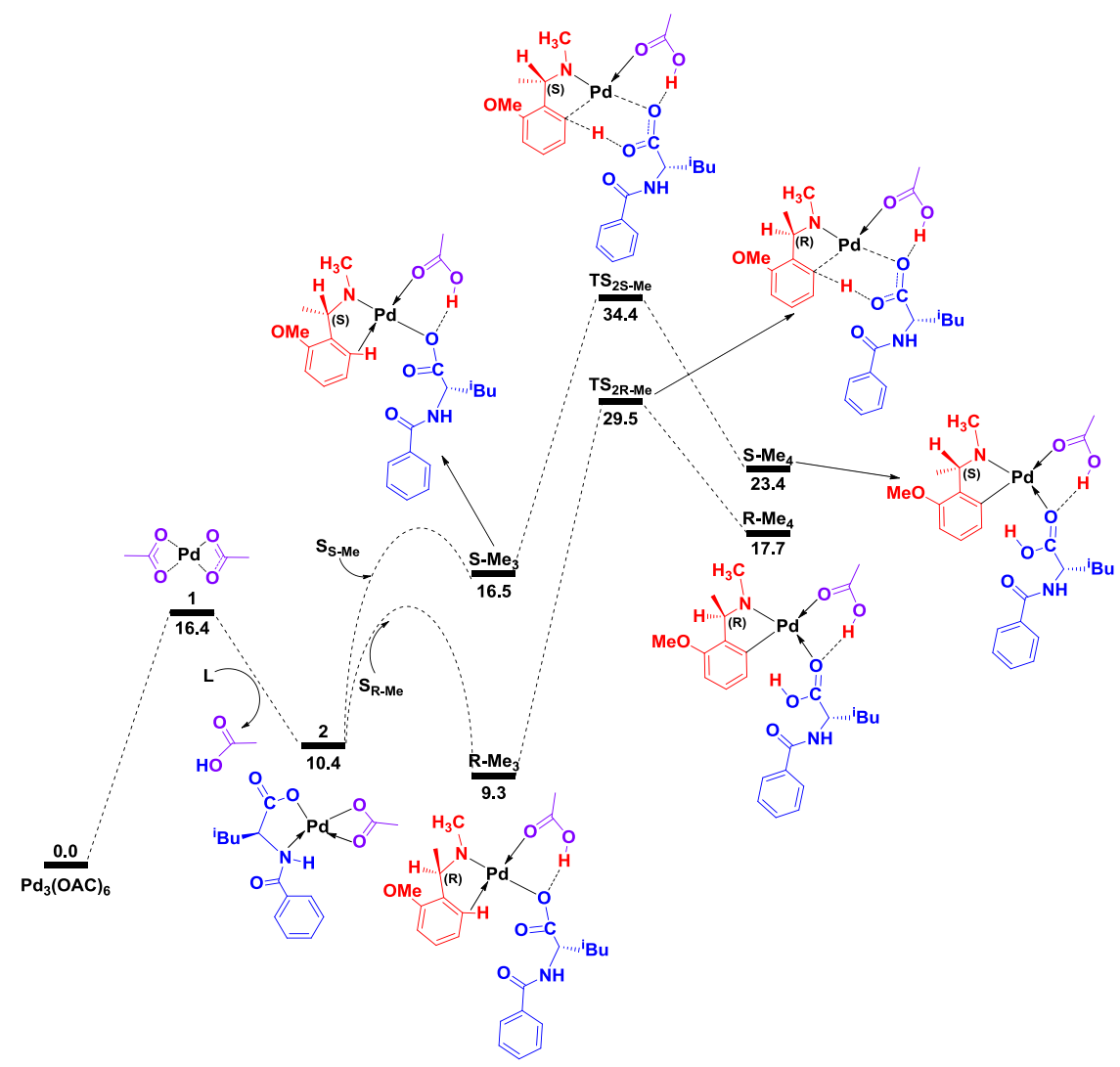

Figure S40. Energy profiles of pathways $R-\mathrm{Me}$ and $S$-Me for aryl $\mathrm{C}-\mathrm{H}$ bond activation ofbenzylamine. The relative free energies in dimethyl sulfoxide (DMSO) are given in $\mathrm{kcal} / \mathrm{mol}$

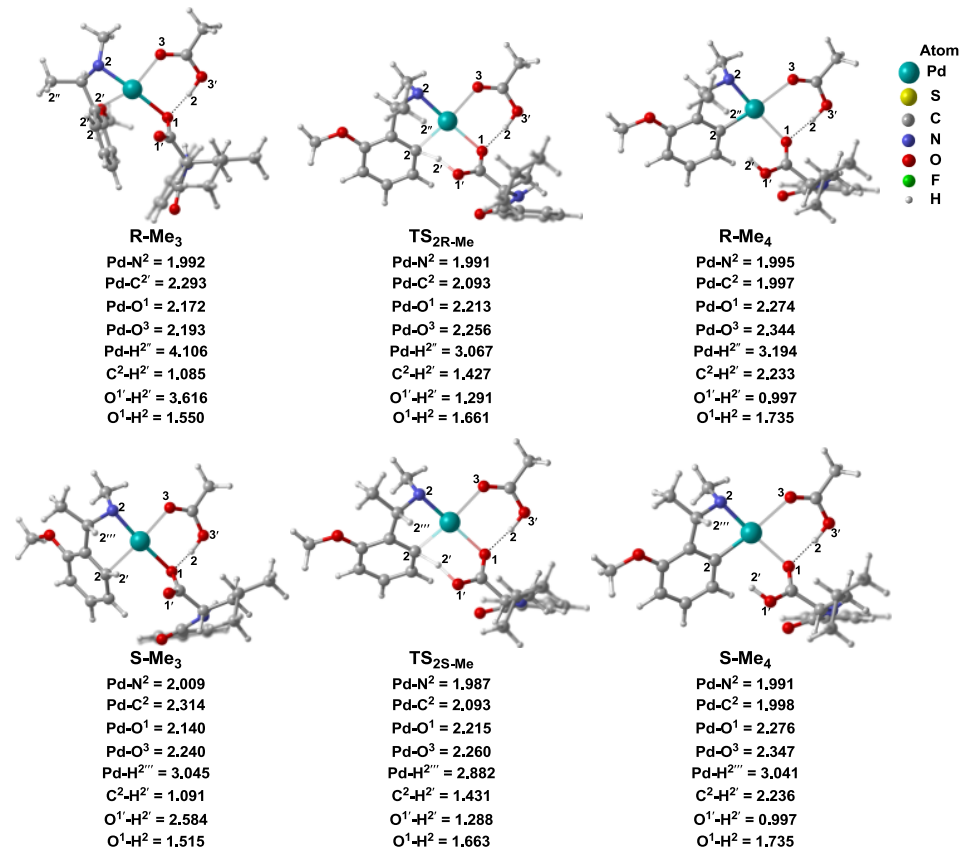

Figure S41.The optimized structures and geometric parameters for selected species of pathways $R$-Me and $S$-Me for aryl C-H bond activation of benzylamine in Figure S40 
9. The relative free energies and electronic energies in gas phase and the single-point energies and relative free energies in DMSO solvent of the favorable pathways for aryl $\mathrm{C}-\mathrm{H}$ bond activation of $R(S)$-benzylamine computed by different computation methods

Table S1. The relative free energiesand electronic energies in gas phase and the single-point energies and relative free energies in DMSO solventof the favorable pathways for the aryl $\mathrm{C}-\mathrm{H}$ bond activation of $R(S)$-benzylamine computed by different computation methods

\begin{tabular}{|c|c|c|c|c|c|c|c|c|}
\hline Computational Method & Energy & $\mathrm{Pd}(\mathrm{OAc})_{2}$ & $\mathrm{R}_{3 \mathrm{a}}$ & $\mathrm{TS}_{\mathrm{R} 2 \mathrm{a}}$ & $\mathrm{R}_{4 \mathrm{a}}$ & $\mathrm{S}_{3 \mathrm{~A}}$ & $\mathrm{TS}_{\mathrm{S} 2 \mathrm{~A}}$ & $\mathrm{~S}_{4 \mathrm{~A}}$ \\
\hline \multirow{4}{*}{ B3LYP } & $\Delta \mathrm{G}_{\mathrm{gas}}(\mathrm{kcal} / \mathrm{mol})$ & 13.3 & 41.9 & 47.3 & 31.3 & 48.5 & 54.8 & 38.7 \\
\hline & $\Delta \mathrm{E}_{\mathrm{gas}}(\mathrm{kca} / \mathrm{mol})$ & 23.9 & 38.3 & 45.9 & 27.2 & 44.0 & 52.0 & 35.3 \\
\hline & $\Delta \mathrm{E}_{\mathrm{DMSO}}(\mathrm{kcal} / \mathrm{mol})$ & 26.9 & 10.7 & 19.1 & -3.5 & 16.3 & 25.0 & 5.1 \\
\hline & $\Delta \mathrm{G}_{\mathrm{DMSO}}(\mathrm{kcal} / \mathrm{mol})$ & 16.3 & 14.3 & 20.5 & 0.6 & 20.9 & 27.8 & 8.5 \\
\hline \multirow{4}{*}{ TPSSTPSS } & $\Delta \mathrm{G}_{\mathrm{gas}}(\mathrm{kcal} / \mathrm{mol})$ & 11.9 & 32.5 & 36.0 & 25.3 & 38.8 & 42.5 & 32.8 \\
\hline & $\Delta \mathrm{E}_{\mathrm{gas}}(\mathrm{kcal} / \mathrm{mol})$ & 22.7 & 30.0 & 35.1 & 22.0 & 34.9 & 40.7 & 29.3 \\
\hline & $\Delta \mathrm{E}_{\mathrm{DMSO}}(\mathrm{kcal} / \mathrm{mol})$ & 27.1 & 11.7 & 19.5 & -3.1 & 17.0 & 25.4 & 5.5 \\
\hline & $\Delta \mathrm{G}_{\mathrm{DMSO}}(\mathrm{kcal} / \mathrm{mol})$ & 16.3 & 14.2 & 20.4 & 0.2 & 20.9 & 27.2 & 9.0 \\
\hline \multirow{4}{*}{$\omega-\mathrm{B} 97 \mathrm{XD}$} & $\Delta \mathrm{G}_{\mathrm{gas}}(\mathrm{kcal} / \mathrm{mol})$ & 19.9 & 26.6 & 40.9 & 24.0 & 40.0 & 48.3 & 33.3 \\
\hline & $\Delta \mathrm{E}_{\mathrm{gas}}(\mathrm{kca} / \mathrm{mol})$ & 31.2 & 21.3 & 39.1 & 20.0 & 34.3 & 45.2 & 28.5 \\
\hline & $\Delta \mathrm{E}_{\mathrm{DMSO}}(\mathrm{kcal} / \mathrm{mol})$ & 28.4 & 5.5 & 23.3 & 1.3 & 19.6 & 30.3 & 10.5 \\
\hline & $\Delta \mathrm{G}_{\text {DMSO }}(\mathrm{kca} 1 / \mathrm{mol})$ & 17.1 & 10.8 & 25.1 & 5.3 & 25.3 & 33.4 & 15.3 \\
\hline
\end{tabular}




\section{Cartesian coordinates for complexes calculated in this study}

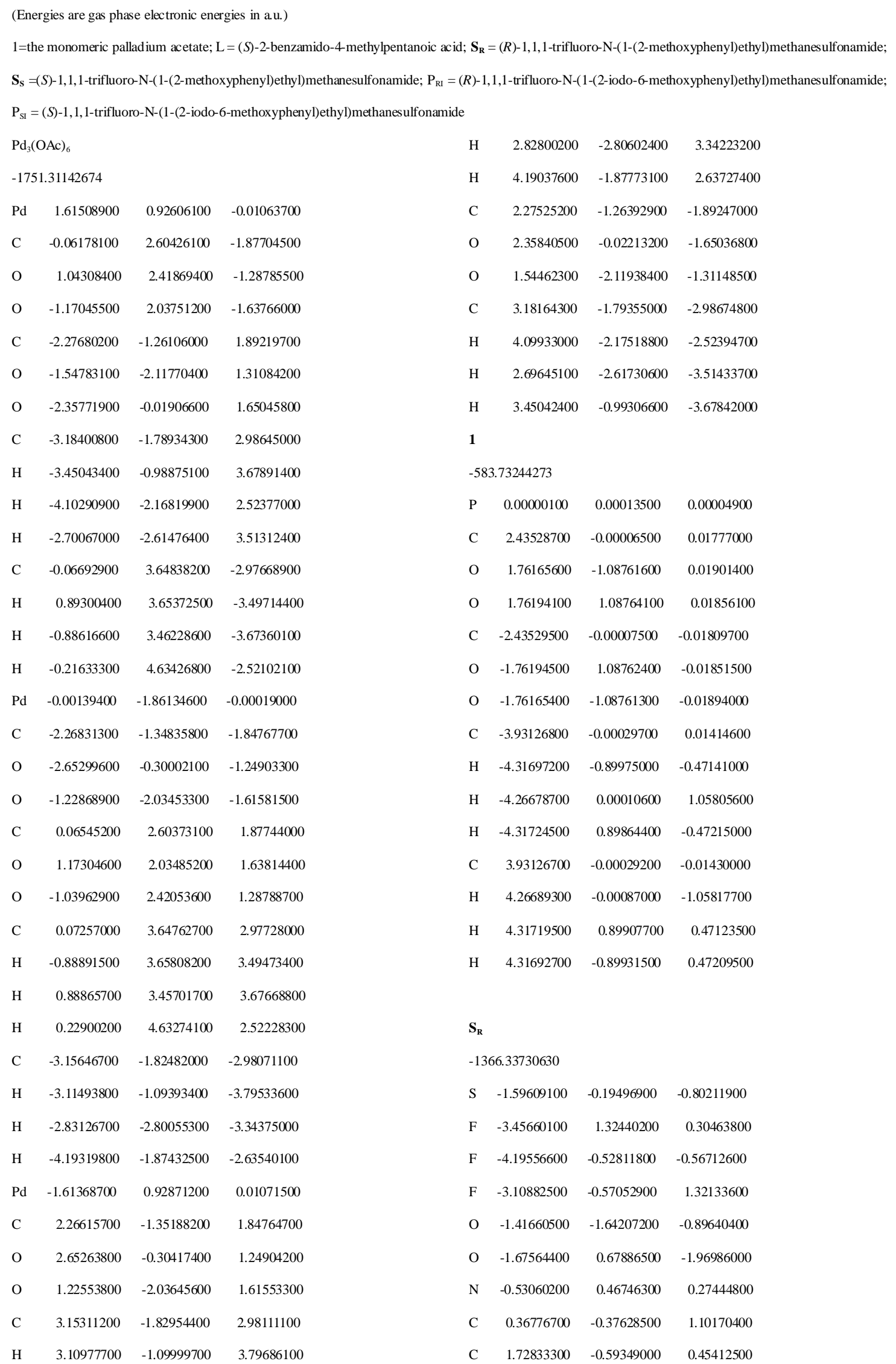




\begin{tabular}{lrrr}
$\mathrm{C}$ & 2.23672800 & -1.88549400 & 0.31627000 \\
$\mathrm{C}$ & 3.49727400 & -2.12368700 & -0.23695700 \\
$\mathrm{C}$ & 4.26430300 & -1.04746700 & -0.67121800 \\
$\mathrm{C}$ & 3.77931800 & 0.25866900 & -0.55769600 \\
$\mathrm{C}$ & 2.52040600 & 0.48387700 & 0.00335900 \\
$\mathrm{C}$ & -3.20081200 & 0.02712700 & 0.12578800 \\
$\mathrm{H}$ & 3.86697000 & -3.14002000 & -0.33304600 \\
$\mathrm{H}$ & 5.24492700 & -1.21243900 & -1.10897100 \\
$\mathrm{H}$ & 4.38541300 & 1.08584200 & -0.90811400 \\
$\mathrm{C}$ & 0.45929600 & 0.22897000 & 2.51094100 \\
$\mathrm{H}$ & 0.91220900 & 1.22377500 & 2.48253300 \\
$\mathrm{H}$ & -0.54220100 & 0.31036100 & 2.94476100 \\
$\mathrm{H}$ & 1.07361700 & -0.41100900 & 3.15306300 \\
$\mathrm{H}$ & -0.12099700 & -1.35045700 & 1.17424600 \\
$\mathrm{H}$ & -0.09258700 & 1.30766700 & -0.09751700 \\
$\mathrm{O}$ & 1.96440000 & 1.73644200 & 0.14470300 \\
$\mathrm{C}$ & 2.70375600 & 2.86636300 & -0.30534900 \\
$\mathrm{H}$ & 2.90248800 & 2.81047100 & -1.38253200 \\
$\mathrm{H}$ & 2.07743800 & 3.73506000 & -0.09609500 \\
$\mathrm{H}$ & 3.65198000 & 2.96184200 & 0.23739600 \\
\hline & 1.62592100 & -2.72300200 & 0.64409100 \\
\hline
\end{tabular}

$\mathbf{S}_{\mathrm{s}}$

$-1366.33730630$

\begin{tabular}{lrrr} 
S & 1.59609100 & -0.19496900 & -0.80211900 \\
F & 3.45660100 & 1.32440200 & 0.30463900 \\
F & 3.10882500 & -0.57052900 & 1.32133600 \\
F & 4.19556600 & -0.52811700 & -0.56712600 \\
O & 1.67564400 & 0.67886600 & -1.96986000 \\
O & 1.41660500 & -1.64207200 & -0.89640500 \\
N & 0.53060200 & 0.46746200 & 0.27444900 \\
C & -0.36776700 & -0.37628600 & 1.10170400 \\
C & -1.72833300 & -0.59349000 & 0.45412500 \\
C & -2.23672800 & -1.88549500 & 0.31627000 \\
C & -3.49727400 & -2.12368700 & -0.23695800 \\
C & -4.26430300 & -1.04746700 & -0.67121800 \\
C & -3.77931800 & 0.25866900 & -0.55769600 \\
C & -2.52040600 & 0.48387700 & 0.00336000 \\
C & 3.20081200 & 0.02712700 & 0.12578800 \\
H & -3.86697000 & -3.14002000 & -0.33304700 \\
H & -5.24492700 & -1.21243800 & -1.10897100 \\
H & -4.38541300 & 1.08584300 & -0.90811300 \\
H & 0.09258700 & 1.30766700 & -0.09751600 \\
O & -1.96440000 & 1.73644200 & 0.14470400 \\
\hline & & & \\
\hline
\end{tabular}

$\begin{array}{lrrr}\mathrm{C} & -2.70375500 & 2.86636300 & -0.30534900 \\ \mathrm{H} & -3.65197900 & 2.96184300 & 0.23739500 \\ \mathrm{H} & -2.07743600 & 3.73506000 & -0.09609600 \\ \mathrm{H} & -2.90248700 & 2.81047100 & -1.38253200 \\ \mathrm{H} & 0.12099700 & -1.35045800 & 1.17424600 \\ \mathrm{C} & -0.45929600 & 0.22896900 & 2.51094100 \\ \mathrm{H} & -0.91220900 & 1.22377400 & 2.48253400 \\ \mathrm{H} & -1.07361700 & -0.41101000 & 3.15306300 \\ \mathrm{H} & 0.54220100 & 0.31036000 & 2.94476100 \\ \mathrm{H} & -1.62592100 & -2.72300200 & 0.64409000\end{array}$

L

$-786.07985391$

\begin{tabular}{|c|c|c|c|}
\hline $\mathrm{C}$ & -3.68577500 & -0.73783800 & 0.21071900 \\
\hline $\mathrm{C}$ & -2.16106600 & -0.65969100 & 0.43779200 \\
\hline $\mathrm{C}$ & -1.37186300 & 0.12790100 & -0.62633300 \\
\hline $\mathrm{C}$ & -1.57170500 & 1.64503900 & -0.48598700 \\
\hline $\mathrm{O}$ & -0.68025400 & 2.33615300 & -1.23845100 \\
\hline $\mathrm{H}$ & -0.88836500 & 3.27851400 & -1.09555800 \\
\hline $\mathrm{C}$ & -4.05537100 & -1.56681400 & -1.02976900 \\
\hline $\mathrm{C}$ & -4.36254000 & -1.31961700 & 1.46101400 \\
\hline $\mathrm{O}$ & -2.45334300 & 2.18589800 & 0.13992000 \\
\hline$N$ & 0.04565400 & -0.23388900 & -0.65804800 \\
\hline $\mathrm{C}$ & 0.89545900 & 0.18855200 & 0.33585300 \\
\hline $\mathrm{C}$ & 5.08317600 & -0.70565800 & -0.06105300 \\
\hline $\mathrm{C}$ & 4.64157200 & 0.33009400 & 0.76657500 \\
\hline $\mathrm{C}$ & 3.27993500 & 0.59768700 & 0.88398000 \\
\hline $\mathrm{C}$ & 2.34526900 & -0.15858700 & 0.16430700 \\
\hline $\mathrm{C}$ & 2.79332300 & -1.20482000 & -0.65477500 \\
\hline $\mathrm{C}$ & 4.15693300 & -1.47640000 & -0.76613000 \\
\hline $\mathrm{O}$ & 0.48365000 & 0.81034500 & 1.31168400 \\
\hline $\mathrm{H}$ & -4.05864100 & 0.28386100 & 0.07334700 \\
\hline $\mathrm{H}$ & -1.74930300 & -1.67737800 & 0.45600300 \\
\hline $\mathrm{H}$ & -1.95259000 & -0.21141700 & 1.41303900 \\
\hline $\mathrm{H}$ & -1.75629900 & -0.12512100 & -1.62206500 \\
\hline $\mathrm{H}$ & -5.14262300 & -1.59764300 & -1.16677300 \\
\hline $\mathrm{H}$ & -3.62776800 & -1.15964700 & -1.95442500 \\
\hline $\mathrm{H}$ & -3.70454200 & -2.60271500 & -0.92947900 \\
\hline $\mathrm{H}$ & -5.45055700 & -1.36881600 & 1.33456600 \\
\hline $\mathrm{H}$ & -4.15351500 & -0.70709600 & 2.34525900 \\
\hline $\mathrm{H}$ & -4.00539200 & -2.33784500 & 1.66569300 \\
\hline $\mathrm{H}$ & 6.14546100 & -0.91702900 & -0.14964000 \\
\hline $\mathrm{H}$ & 5.35987300 & 0.92659300 & 1.32236300 \\
\hline $\mathrm{H}$ & 2.91430100 & 1.38923900 & 1.52977300 \\
\hline
\end{tabular}




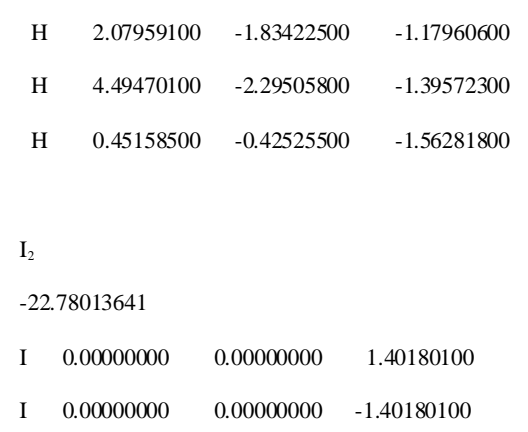

$\begin{array}{cccc}\mathrm{H} & -0.17991300 & -0.77227600 & 0.76997700 \\ \mathrm{H} & -1.48717500 & 1.84062300 & 0.39940900 \\ \mathrm{O} & 0.14777300 & 3.03861600 & 0.58448100 \\ \mathrm{C} & 0.18957100 & 4.45438100 & 0.43492500 \\ \mathrm{H} & 0.19438100 & 4.74393900 & -0.62260100 \\ \mathrm{H} & -0.71832000 & 4.82881600 & 0.91014000 \\ \mathrm{H} & 1.06648700 & 4.87911100 & 0.93776100 \\ \mathrm{I} & 2.18408600 & -2.03088400 & 0.07968700\end{array}$

$\mathrm{P}_{\mathrm{SI}}$

$-1377.09389441$

$\begin{array}{llll}\text { S } & 2.21879700 & 0.12167800 & -0.82432100\end{array}$

$\begin{array}{llll}\text { F } & 4.35472900 & -0.03002200 & 0.72433200\end{array}$

F $\quad 2.93714200 \quad-1.66754400 \quad 0.96330100$

$\begin{array}{llll}\text { F } & 4.21928500 & -1.58047900 & -0.79717600\end{array}$

$\begin{array}{llll}\text { O } & 2.91602700 & 1.17644000 & -1.55608500\end{array}$

$\begin{array}{llll}\text { O } & 1.38040700 & -0.88242500 & -1.47245400\end{array}$

$\begin{array}{llll}\mathrm{N} & 1.44237600 & 0.82594300 & 0.46004900\end{array}$

$\begin{array}{llll}\text { C } & 0.15735300 & 0.29851500 & 0.97026900\end{array}$

$\begin{array}{llll}\text { C } & -1.06257600 & 0.88321600 & 0.25866300\end{array}$

$\begin{array}{lrrr}\text { C } & -2.12639700 & 0.10160400 & -0.20555500\end{array}$

$\begin{array}{llll}\text { C } & -3.23609800 & 0.64986100 & -0.85554100\end{array}$

$\begin{array}{lrrr}\text { C } & -3.29735400 & 2.02513000 & -1.04398000\end{array}$

$\begin{array}{llll}\text { C } & -2.27521500 & 2.85097200 & -0.57680100\end{array}$

$\begin{array}{lrrr}\text { C } & -1.17944400 & 2.28503600 & 0.07545400\end{array}$

$\begin{array}{llll}\text { C } & 3.52662400 & -0.85236300 & 0.07870900\end{array}$

$\begin{array}{llll}\mathrm{H} & -4.03585900 & 0.01053900 & -1.20937200\end{array}$

H $\quad-4.15061100 \quad 2.46254400 \quad-1.55438300$

$\begin{array}{llll}\mathrm{H} & -2.34244400 & 3.92216700 & -0.72108600\end{array}$

$\begin{array}{llll}\mathrm{H} & 1.48717100 & 1.84062800 & 0.39939700\end{array}$

$\begin{array}{llll}\text { O } & -0.14778000 & 3.03861800 & 0.58447400\end{array}$

$\begin{array}{lrrr}\text { C } & -0.18959200 & 4.45438400 & 0.43493400\end{array}$

$\begin{array}{llll}\text { H } & -1.06651400 & 4.87909900 & 0.93777100\end{array}$

$\begin{array}{llll}\mathrm{H} & 0.71829500 & 4.82882300 & 0.91015600\end{array}$

$\begin{array}{llll}\mathrm{H} & -0.19440100 & 4.74395300 & -0.62258800\end{array}$

H $\quad 0.17991600 \quad-0.77227300 \quad 0.76997300$

$\begin{array}{llll}\text { C } & 0.10148700 & 0.50240200 & 2.49220500\end{array}$

$\begin{array}{llll}\mathrm{H} & 0.10603900 & 1.56563100 & 2.74855500\end{array}$

$\begin{array}{llll}\mathrm{H} & -0.81205800 & 0.05315000 & 2.89522700\end{array}$

$\begin{array}{llll}\mathrm{H} & 0.96696500 & 0.02316400 & 2.95989000\end{array}$

$\begin{array}{llll}\text { I } & -2.18408000 & -2.03088800 & 0.07968900\end{array}$

HI

$-11.97931602$ 
$\begin{array}{cccc}\text { I } & 0.00000000 & 0.00000000 & 0.03032500 \\ \text { H } & 0.00000000 & 0.00000000 & -1.60723000\end{array}$

$-1140.71906839$

$\begin{array}{llll} & 0.20838800 & 1.31509300 & -0.25982400 \\ \text { O } & 0.95189800 & 3.26148800 & -0.42550700\end{array}$

$\begin{array}{llll}\text { C } & 2.95474800 & 3.98464400 & 0.72362200\end{array}$

$\begin{array}{llll}\text { C } & 1.90173900 & 2.97559800 & 0.37729600\end{array}$

$\begin{array}{llll}\text { O } & 1.93377200 & 1.78632800 & 0.85374200\end{array}$

$\begin{array}{llll}\text { C } & -1.83199400 & -0.77388400 & -0.23332900\end{array}$

$\begin{array}{llll}\text { C } & -2.20346300 & 0.26935100 & -1.30185800\end{array}$

$\begin{array}{llll}\mathrm{H} & -2.02841500 & -1.76138700 & -0.65319000\end{array}$

$\begin{array}{llll}\mathrm{H} & 2.50960400 & 4.98027900 & 0.79572000\end{array}$

$\begin{array}{llll}\mathrm{H} & 3.44674100 & 3.71349800 & 1.66020600\end{array}$

$\begin{array}{llll}\mathrm{H} & 3.70534900 & 4.00686000 & -0.07524300\end{array}$

$\begin{array}{llll}\mathrm{N} & -0.35670200 & -0.69087900 & 0.08002400\end{array}$

$\begin{array}{llll}\mathrm{H} & -0.18998800 & -0.87037800 & 1.07062300\end{array}$

$\begin{array}{llll}\text { C } & 0.48635200 & -1.55346000 & -0.74602600\end{array}$

$\begin{array}{llll}\text { O } & 0.07492200 & -1.91538300 & -1.82634800\end{array}$

$\begin{array}{llll}\text { C } & 1.79541900 & -1.97367400 & -0.17310300\end{array}$

$\begin{array}{llll}\text { C } & 2.35436300 & -1.43785100 & 0.99901900\end{array}$

$\begin{array}{llll}\text { C } & 2.48229000 & -2.98476200 & -0.86707400\end{array}$

$\begin{array}{llll}\text { C } & 3.57558700 & -1.91817300 & 1.47034700\end{array}$

$\begin{array}{llll}\mathrm{H} & 1.88135200 & -0.61726200 & 1.52749100\end{array}$

$\begin{array}{llll}\text { C } & 3.69817900 & -3.46257300 & -0.38953500\end{array}$

$\begin{array}{llll}\mathrm{H} & 2.04085600 & -3.38203300 & -1.77468800\end{array}$

$\begin{array}{llll}\text { C } & 4.24563800 & -2.93160500 & 0.78234400\end{array}$

$\begin{array}{llll}\mathrm{H} & 4.00663100 & -1.49175300 & 2.37124300\end{array}$

$\mathrm{H} \quad 4.22009900 \quad-4.24728300 \quad-0.92936300$

$\begin{array}{llll}\mathrm{H} & 5.19616900 & -3.30320300 & 1.15511700\end{array}$

$\begin{array}{llll}\text { C } & -2.61877500 & -0.57203600 & 1.07645300\end{array}$

$\begin{array}{llll}\mathrm{H} & -2.28724500 & -1.34322800 & 1.79105500\end{array}$

$\begin{array}{llll}\mathrm{H} & -2.34355900 & 0.40120400 & 1.50700600\end{array}$

$\begin{array}{llll}\text { C } & -4.15579800 & -0.64860700 & 0.95567000\end{array}$

$\begin{array}{llll}\text { C } & -4.78297000 & -0.41850600 & 2.33898200\end{array}$

$\begin{array}{llll}\text { C } & -4.63364800 & -1.97276400 & 0.34140500\end{array}$

$\begin{array}{llll}\text { H } & -4.47850800 & 0.16066200 & 0.29074100\end{array}$

$\begin{array}{llll}\text { H } & -4.46216400 & 0.53574400 & 2.77441400\end{array}$

$\begin{array}{llll}\mathrm{H} & -5.87646200 & -0.40368400 & 2.27095900\end{array}$

$\begin{array}{llll}\mathrm{H} & -4.50505000 & -1.21770100 & 3.03927700\end{array}$

$\begin{array}{llll}\mathrm{H} & -4.32253700 & -2.05882200 & -0.70370900\end{array}$

$\begin{array}{llll}\mathrm{H} & -4.24775600 & -2.83704600 & 0.89994900\end{array}$

$\begin{array}{llll}\mathrm{H} & -5.72801000 & -2.02926600 & 0.36269800\end{array}$

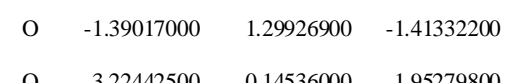

$\mathrm{R}_{1 \mathrm{a}}$

$-2507.05518503$

$\begin{array}{llll}\mathrm{Pd} & -0.32806000 & -0.13534200 & -0.12713900\end{array}$

O $\quad 1.94163200 \quad-1.40626400 \quad 1.93689900$

$\begin{array}{llll}\text { C } & 0.43207700 & -2.38827100 & 3.52610900\end{array}$

$\begin{array}{llll}\text { C } & 0.76249100 & -1.57107100 & 2.28457100\end{array}$

O $\quad-0.28585000 \quad-1.10470100 \quad 1.67714100$

$\begin{array}{llll}\text { C } & -2.75340300 & 1.30659000 & -0.71307100\end{array}$

C $\quad-1.73541800 \quad 1.59537300 \quad-1.83538100$

$\mathrm{H} \quad-3.73712000 \quad 1.23334200 \quad-1.18045900$

H $\quad-0.08736600 \quad-3.30540900 \quad 3.22604800$

$\begin{array}{llll}\mathrm{H} & 1.34740500 & -2.65027800 & 4.05956000\end{array}$

H $\quad-0.23924400 \quad-1.82854000 \quad 4.18554100$

N $\quad-2.44589200 \quad-0.02389800 \quad-0.06825500$

$\begin{array}{llll}\mathrm{H} & -2.70715400 & -0.02990200 & 0.91769500\end{array}$

$\begin{array}{llll}\text { C } & -3.02913600 & -1.15909400 & -0.78246500\end{array}$

O $\quad-3.25379200 \quad-1.04027200 \quad-1.96751000$

$\begin{array}{llll}\text { C } & -3.38038900 & -2.37608800 & 0.00087500\end{array}$

$\begin{array}{llll}\text { C } & -3.03419900 & -2.58150800 & 1.34605200\end{array}$

C $\quad-4.13600800 \quad-3.34904500 \quad-0.67658900$

$\begin{array}{llll}\text { C } & -3.45201800 & -3.74048900 & 2.00032100\end{array}$

$\mathrm{H} \quad-2.38863000 \quad-1.88411900 \quad 1.86845100$

C $\quad-4.55008500 \quad-4.50138400 \quad-0.01719100$

H $\quad-4.38840200 \quad-3.17723400 \quad-1.71724600$

C $\quad-4.21230700 \quad-4.69725100 \quad 1.32530900$

H $\quad-3.17559300 \quad-3.89829700 \quad 3.03901200$

H $\quad-5.13537000 \quad-5.24688500 \quad-0.54765900$

$\mathrm{H} \quad-4.53648900 \quad-5.59669600 \quad 1.84174600$

$\begin{array}{llll}\text { C } & -2.75755100 & 2.39113300 & 0.38160200\end{array}$

$\mathrm{H} \quad-3.47370400 \quad 2.07870200 \quad 1.15936800$

H $\quad-1.76683800 \quad 2.42309600 \quad 0.85508700$

$\begin{array}{llll}\text { C } & -3.13764300 & 3.81374700 & -0.08068700\end{array}$

C $\quad-3.09730200 \quad 4.76239800 \quad 1.12727200$

$\begin{array}{llll}\text { C } & -4.50295800 & 3.86596400 & -0.78170500\end{array}$

$\mathrm{H} \quad-2.38146900 \quad 4.14120400 \quad-0.80291300$

$\mathrm{H} \quad-2.11446300 \quad 4.74954100 \quad 1.61327700$

$\begin{array}{llll}\mathrm{H} & -3.30784300 & 5.79297900 & 0.81908400\end{array}$

H $\quad-3.84836700 \quad 4.48187400 \quad 1.87802300$

$\mathrm{H} \quad-4.48120700 \quad 3.32696700 \quad-1.73352400$

$\mathrm{H} \quad-5.29482600 \quad 3.43887800 \quad-0.15061300$

H $\quad-4.78067700 \quad 4.90363000 \quad-1.00029100$ 


\begin{tabular}{|c|c|c|c|}
\hline $\mathrm{O}$ & -0.60655000 & 0.91817200 & -1.79378900 \\
\hline $\mathrm{O}$ & -1.97904100 & 2.44447000 & -2.67526700 \\
\hline S & 2.09948300 & -1.54534200 & -1.45609100 \\
\hline & 4.67618400 & -2.00534700 & -1.90633800 \\
\hline $\mathrm{F}$ & 4.25335800 & -1.53241200 & 0.17479600 \\
\hline $\mathrm{F}$ & 3.70820200 & -3.47635800 & -0.63397700 \\
\hline $\mathrm{O}$ & 2.22445900 & -1.10867100 & -2.84006600 \\
\hline $\mathrm{O}$ & 1.16376600 & -2.57938300 & -1.03555000 \\
\hline$N$ & 1.80314900 & -0.13112700 & -0.45873600 \\
\hline 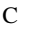 & 2.32668600 & 1.18794400 & -1.00649700 \\
\hline $\mathrm{C}$ & 1.89081900 & 2.32520500 & -0.09653900 \\
\hline $\mathrm{C}$ & 1.14112700 & 3.37958400 & -0.62543800 \\
\hline 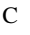 & 0.79986500 & 4.49060200 & 0.15127200 \\
\hline C & 1.20309200 & 4.54439900 & 1.48382300 \\
\hline C & 1.94616100 & 3.50070100 & 2.04235800 \\
\hline $\mathrm{C}$ & 2.29673400 & 2.39942500 & 1.25453800 \\
\hline C & 3.81778000 & -2.18295200 & -0.90300600 \\
\hline $\mathrm{H}$ & 0.80725800 & 3.31933200 & -1.65705000 \\
\hline $\mathrm{H}$ & 0.22410600 & 5.30089200 & -0.28523300 \\
\hline $\mathrm{H}$ & 0.94652000 & 5.40173500 & 2.10055600 \\
\hline $\mathrm{H}$ & 2.25883300 & 3.55702000 & 3.07871800 \\
\hline $\mathrm{H}$ & 2.11673500 & -0.37378900 & 0.51404500 \\
\hline $\mathrm{O}$ & 3.03262100 & 1.34295000 & 1.70822300 \\
\hline C & 3.30185000 & 1.22900100 & 3.09964000 \\
\hline 11 & 2.37597700 & 1.27970800 & 3.68493100 \\
\hline $\mathrm{H}$ & 3.75411000 & 0.24652200 & 3.23009700 \\
\hline $\mathrm{H}$ & 3.99248200 & 2.01292900 & 3.43562800 \\
\hline$C$ & 3.84551500 & 1.20819500 & -1.24908800 \\
\hline $\mathrm{H}$ & 4.40436900 & 1.06699200 & -0.32264800 \\
\hline $\mathrm{H}$ & 4.14960600 & 0.45678800 & -1.98107400 \\
\hline 11 & 4.10533800 & 2.18771800 & -1.66290200 \\
\hline & .80793700 & 1.30567400 & -1.95794300 \\
\hline
\end{tabular}

$\mathrm{TS}_{\text {Rla }}$

$-2507.04626098$

$\begin{array}{lrrr}\mathrm{Pd} & 0.36077200 & 0.14303500 & -0.13551400 \\ \mathrm{O} & -2.04411900 & 1.08052200 & 1.86413700 \\ \mathrm{C} & -0.81241500 & 2.19074900 & 3.56948400 \\ \mathrm{C} & -0.88105000 & 1.39371400 & 2.28978000 \\ \mathrm{O} & 0.20309200 & 1.10276000 & 1.70977400 \\ \mathrm{C} & 2.84430700 & -1.23799600 & -0.72661400 \\ \mathrm{C} & 1.83641500 & -1.51980400 & -1.85759700 \\ \mathrm{H} & 3.82349600 & -1.11228300 & -1.19247800 \\ \mathrm{H} & -0.81511700 & 3.25671200 & 3.31242400\end{array}$

\begin{tabular}{|c|c|c|c|}
\hline $\mathrm{H}$ & -1.68442700 & 1.98623100 & 4.19367500 \\
\hline $\mathrm{H}$ & 0.10994600 & 1.97118900 & 4.11121700 \\
\hline $\mathrm{N}$ & 2.49260600 & 0.05172900 & -0.02895300 \\
\hline $\mathrm{H}$ & 2.74046300 & 0.01466300 & 0.95912000 \\
\hline $\mathrm{C}$ & 3.04528000 & 1.23642600 & -0.67631200 \\
\hline $\mathrm{O}$ & 3.30021000 & 1.18347100 & -1.86003200 \\
\hline $\mathrm{C}$ & 3.31169500 & 2.43701000 & 0.16547700 \\
\hline $\mathrm{C}$ & 3.03429300 & 2.52481100 & 1.53903600 \\
\hline $\mathrm{C}$ & 3.91752700 & 3.52537200 & -0.48590500 \\
\hline $\mathrm{C}$ & 3.36768500 & 3.68003200 & 2.24537700 \\
\hline $\mathrm{H}$ & 2.51771400 & 1.72718500 & 2.06036000 \\
\hline $\mathrm{C}$ & 4.24513100 & 4.67624800 & 0.22284500 \\
\hline $\mathrm{H}$ & 4.12252600 & 3.44308500 & -1.54757300 \\
\hline $\mathrm{C}$ & 3.97433700 & 4.75429500 & 1.59206200 \\
\hline $\mathrm{H}$ & 3.14764700 & 3.74218700 & 3.30732600 \\
\hline 1 & 4.71144500 & 5.51224600 & -0.29020300 \\
\hline $\mathrm{H}$ & 4.23211700 & 5.65197700 & 2. 14737500 \\
\hline $\mathrm{C}$ & 2.88921800 & -2.36436700 & 0.32425900 \\
\hline $\mathrm{H}$ & 3.59451300 & -2.05618800 & 1.11389100 \\
\hline $\mathrm{H}$ & 1.90074300 & -2.45261600 & 0.79505300 \\
\hline $\mathrm{C}$ & 3.32284100 & -3.75253600 & -0.19279300 \\
\hline $\mathrm{C}$ & 3.32021900 & -4.74787900 & 0.97775100 \\
\hline $\mathrm{C}$ & 4.68852900 & -3.72483700 & -0.89439500 \\
\hline $\mathrm{H}$ & 2.57960000 & -4.08059800 & -0.92817700 \\
\hline $\mathrm{H}$ & 2.33987800 & -4.78810600 & 1.46755300 \\
\hline $\mathrm{H}$ & 3.56466300 & -5.75798300 & 0.62965800 \\
\hline $\mathrm{H}$ & 4.06433000 & -4.47052700 & 1.73658600 \\
\hline $\mathrm{H}$ & 4.64471000 & -3.15338600 & -1.82627600 \\
\hline $\mathrm{H}$ & 5.46316700 & -3.28936600 & -0.24779200 \\
\hline $\mathrm{H}$ & 5.00711700 & -4.74203100 & -1.14993700 \\
\hline $\mathrm{O}$ & 0.68530200 & -0.88019600 & -1.80093700 \\
\hline $\mathrm{O}$ & 2.10408600 & -2.33440800 & -2.72331300 \\
\hline S & -1.99376700 & 1.50275200 & -1.47502800 \\
\hline $\mathrm{F}$ & -4.59201200 & 1.80574500 & -2.05182600 \\
\hline $\mathrm{F}$ & -4.19838600 & 1.62975200 & 0.08178900 \\
\hline $\mathrm{F}$ & -3.69530400 & 3.46180900 & -0.97513500 \\
\hline $\mathrm{O}$ & -2.01892300 & 1.13614900 & -2.88864700 \\
\hline $\mathrm{O}$ & -1.13067500 & 2.57025600 & -0.96211200 \\
\hline $\mathrm{N}$ & -1.75336100 & 0.15644400 & -0.45750900 \\
\hline $\mathrm{C}$ & -2.24675600 & -1.17017700 & -1.00342400 \\
\hline $\mathrm{C}$ & -1.86306900 & -2.29718400 & -0.05316700 \\
\hline $\mathrm{C}$ & -0.99905000 & -3.29954400 & -0.50590400 \\
\hline $\mathrm{C}$ & -0.67019100 & -4.40296100 & 0.28760100 \\
\hline $\mathrm{C}$ & -1.20768400 & -4.50711300 & 1.56764200 \\
\hline
\end{tabular}




\begin{tabular}{|c|c|c|c|c|c|c|c|}
\hline $\mathrm{C}$ & -2.07753500 & -3.52500100 & 2.04895900 & $\mathrm{H}$ & 2.34079800 & 6.42812700 & -0.21860900 \\
\hline C & -2.41550900 & -2.43227100 & 1.24187600 & $\mathrm{H}$ & 2.44779800 & 6.35277600 & 2.26616700 \\
\hline C & -3.74926600 & 2.13519800 & -1.07219400 & $\mathrm{C}$ & 3.42043600 & -1.67412300 & 0.25837800 \\
\hline $\mathrm{H}$ & -0.57338500 & -3.20717400 & -1.50051000 & $\mathrm{H}$ & 4.05802200 & -1.19113000 & 1.01752900 \\
\hline $\mathrm{H}$ & -0.00600300 & -5.17031100 & -0.09855900 & $\mathrm{H}$ & 2.50436400 & -2.00090400 & 0.76923700 \\
\hline $\mathrm{H}$ & -0.96435500 & -5.35692900 & 2.20009900 & $\mathrm{C}$ & 4.16435600 & -2.91721600 & -0.27357300 \\
\hline $\mathrm{H}$ & -2.50074500 & -3.62637400 & 3.04168400 & $\mathrm{C}$ & 4.45147800 & -3.87056500 & 0.89660800 \\
\hline $\mathrm{H}$ & -2.01144900 & 0.56888500 & 0.82164900 & $\mathrm{C}$ & 5.45363000 & -2.55960900 & -1.02676500 \\
\hline $\mathrm{O}$ & -3.28586400 & -1.45425700 & 1.62915800 & $\mathrm{H}$ & 3.49877700 & -3.42656300 & -0.97957900 \\
\hline $\mathrm{C}$ & -3.80977800 & -1.47677800 & 2.94639800 & $\mathrm{H}$ & 3.53119500 & -4.14568600 & 1.42573100 \\
\hline $\mathrm{H}$ & -3.00974300 & -1.44701700 & 3.69726100 & $\mathrm{H}$ & 4.92294600 & -4.79296200 & 0.53877800 \\
\hline $\mathrm{H}$ & -4.42075400 & -0.57781800 & 3.03638400 & $\mathrm{H}$ & 5.13371300 & -3.41199700 & 1.62518900 \\
\hline $\mathrm{H}$ & -4.43290000 & -2.36461000 & 3.11571400 & $\mathrm{H}$ & 5.23503100 & -2.01855800 & -1.95199100 \\
\hline $\mathrm{C}$ & -3.74927600 & -1.19587400 & -1.33860600 & $\mathrm{H}$ & 6.12338700 & -1.94713800 & -0.40702300 \\
\hline $\mathrm{H}$ & -4.36539100 & -0.95754500 & -0.47094500 & $\mathrm{H}$ & 6.00001000 & -3.46921300 & -1.30155100 \\
\hline $\mathrm{H}$ & -3.98386500 & -0.51288800 & -2.15702100 & $\mathrm{O}$ & 0.84197100 & -0.83076200 & -1.80260700 \\
\hline $\mathrm{H}$ & -4.00324300 & -2.20614100 & -1.67616400 & $\mathrm{O}$ & 2.56624700 & -1.87394000 & -2.75578200 \\
\hline $\mathrm{H}$ & -1.69159000 & -1.33263600 & -1.92954500 & $\mathrm{~S}$ & -2.26001000 & 0.85370700 & -1.64304600 \\
\hline $\mathrm{R}_{2 \mathrm{a}}$ & & & & $\mathrm{F}$ & -4.84801400 & 0.37567800 & -1.03316200 \\
\hline & .04666528 & & & $\mathrm{~F}$ & -3.92106400 & 1.97319800 & 0.10975000 \\
\hline $\mathrm{Pd}$ & 0.29439700 & 0.17658700 & -0.18997900 & $\mathrm{~F}$ & -4.42233900 & 2.28213600 & -1.98474400 \\
\hline $\mathrm{O}$ & -2.22774600 & 0.70779300 & 1.92143400 & $\mathrm{O}$ & -2.44021600 & 0.30326900 & -2.98709500 \\
\hline $\mathrm{C}$ & -1.20303600 & 2.16978800 & 3.47642800 & $\mathrm{O}$ & -1.48153600 & 2.07811800 & -1.41497900 \\
\hline $\mathrm{C}$ & -1.12904100 & 1.32076600 & 2.23636700 & $\mathrm{~N}$ & -1.75554800 & -0.29420500 & -0.51253100 \\
\hline $\mathrm{O}$ & -0.06149800 & 1.23487000 & 1.59528000 & $\mathrm{C}$ & -1.92201600 & -1.74056100 & -0.94275100 \\
\hline $\mathrm{C}$ & 3.05012500 & -0.60247300 & -0.78616300 & $\mathrm{C}$ & -1.27420400 & -2.66006100 & 0.08665000 \\
\hline $\mathrm{C}$ & 2.11840400 & -1.14710800 & -1.88646000 & $\mathrm{C}$ & -0.20378500 & -3.46912100 & -0.30904100 \\
\hline $\mathrm{H}$ & 3.94570700 & -0.22518100 & -1.28333700 & $\mathrm{C}$ & 0.38252900 & -4.39714500 & 0.55727100 \\
\hline $\mathrm{H}$ & -1.45450500 & 1.54195900 & 4.33776500 & $\mathrm{C}$ & -0.10287400 & -4.51876400 & 1.85609200 \\
\hline $\mathrm{H}$ & -0.25303600 & 2.67669200 & 3.64546800 & $\mathrm{C}$ & -1.17407200 & -3.72936000 & 2.28319200 \\
\hline $\mathrm{H}$ & -2.00468200 & 2.90616800 & 3.36161100 & $\mathrm{C}$ & -1.76513400 & -2.81423800 & 1.40411900 \\
\hline $\mathrm{N}$ & 2.40084100 & 0.55027000 & -0.06737500 & $\mathrm{C}$ & -3.98132700 & 1.39158300 & -1.09427300 \\
\hline $\mathrm{H}$ & 2.62243000 & 0.54029000 & 0.92653700 & $\mathrm{H}$ & 0.17699500 & -3.36310100 & -1.32027700 \\
\hline $\mathrm{C}$ & 2.63575200 & 1.85148100 & -0.66881200 & $\mathrm{H}$ & 1.20314600 & -5.01896000 & 0.21189800 \\
\hline $\mathrm{O}$ & 2.89090200 & 1.91300000 & -1.85211900 & $\mathrm{H}$ & 0.33831400 & -5.23394300 & 2.54548400 \\
\hline $\mathrm{C}$ & 2.54774400 & 3.05268400 & 0.21144500 & $\mathrm{H}$ & -1.55197300 & -3.84539800 & 3.29239300 \\
\hline $\mathrm{C}$ & 2.59898400 & 3.01718700 & 1.61373100 & $\mathrm{H}$ & -2.13414400 & 0.20205500 & 1.01876000 \\
\hline $\mathrm{C}$ & 2.46347100 & 4.29444500 & -0.44016200 & $\mathrm{O}$ & -2.83531900 & -2.03409200 & 1.74696600 \\
\hline $\mathrm{C}$ & 2.57053300 & 4.20237300 & 2.34752700 & $\mathrm{C}$ & -3.37100600 & -2.13772100 & 3.05562500 \\
\hline $\mathrm{H}$ & 2.66452300 & 2.07872400 & 2.15546800 & $\mathrm{H}$ & -2.62612800 & -1.87482500 & 3.81814500 \\
\hline $\mathrm{C}$ & 2.41782800 & 5.47460600 & 0.29545000 & $\mathrm{H}$ & -4.19392600 & -1.42285500 & 3.09703000 \\
\hline $\mathrm{H}$ & 2.43152200 & 4.30788500 & -1.52404600 & $\mathrm{H}$ & -3.75097200 & -3.14802600 & 3.25476000 \\
\hline $\mathrm{C}$ & 2.47481200 & 5.43126800 & 1.69113200 & $\mathrm{C}$ & -3.37509900 & -2.16447300 & -1.22722600 \\
\hline $\mathrm{H}$ & 2.62697800 & 4.16558200 & 3.43185600 & $\mathrm{H}$ & -4.02171200 & -2.00616800 & -0.36358900 \\
\hline
\end{tabular}




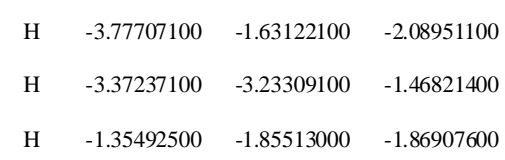

$\mathrm{R}_{3 \mathrm{a}}$

$-2507.04476022$

$\begin{array}{llll}\text { C } & -3.45631100 & -1.20519000 & 1.03766700\end{array}$

$\begin{array}{llll}\text { C } & -1.92975100 & -1.01671100 & 1.18663700\end{array}$

$\mathrm{H} \quad-3.88256200 \quad-0.91845700 \quad 2.00088600$

$\begin{array}{llll}\mathrm{N} & -4.05350000 & -0.34006500 & 0.02837900\end{array}$

$\begin{array}{llll}\mathrm{H} & -4.02188600 & -0.67401100 & -0.92559700\end{array}$

$\begin{array}{llll}\text { C } & -4.10341400 & 1.01593900 & 0.21102600\end{array}$

$\begin{array}{llll}\mathrm{O} & -3.74658300 & 1.54992400 & 1.26255700\end{array}$

$\begin{array}{llll}\text { C } & -4.64948000 & 1.82050200 & -0.93485500\end{array}$

$\begin{array}{llll}\text { C } & -5.46315300 & 1.27338400 & -1.93773200\end{array}$

$\begin{array}{llll}\text { C } & -4.34461900 & 3.18770100 & -0.97296600\end{array}$

$\begin{array}{lrrr}\text { C } & -5.94533400 & 2.07747700 & -2.97097000\end{array}$

$\begin{array}{llll}\mathrm{H} & -5.75302400 & 0.22709400 & -1.89643100\end{array}$

$\begin{array}{llll}\text { C } & -4.82058200 & 3.98908300 & -2.00815100\end{array}$

$\begin{array}{llll}\mathrm{H} & -3.73519900 & 3.60065200 & -0.17610200\end{array}$

$\begin{array}{llll}\text { C } & -5.62007900 & 3.43479700 & -3.01160300\end{array}$

$\begin{array}{llll}\mathrm{H} & -6.58236700 & 1.64602600 & -3.73829500\end{array}$

$\begin{array}{llll}\mathrm{H} & -4.57186100 & 5.04663400 & -2.03273100\end{array}$

$\begin{array}{llll}\mathrm{H} & -5.99510800 & 4.05997500 & -3.81750800\end{array}$

$\begin{array}{lllll}\text { C } & -3.81309400 & -2.67019400 & 0.71061300\end{array}$

$\begin{array}{llll}\mathrm{H} & -4.90726000 & -2.72492700 & 0.61737900\end{array}$

$\mathrm{H} \quad-3.40042000 \quad-2.91145300 \quad-0.28103900$

$\begin{array}{llll}\text { C } & -3.33906100 & -3.73788700 & 1.71706200\end{array}$

$\begin{array}{llll}\text { C } & -3.73711000 & -5.13212000 & 1.20831700\end{array}$

$\begin{array}{llll}\text { C } & -3.88307200 & -3.49153800 & 3.13219200\end{array}$

$\begin{array}{llll}\mathrm{H} & -2.24409100 & -3.69387500 & 1.77121300\end{array}$

$\begin{array}{llll}\mathrm{H} & -3.33323400 & -5.32709400 & 0.20671900\end{array}$

$\begin{array}{llll}\mathrm{H} & -3.36266800 & -5.91508900 & 1.87784700\end{array}$

$\begin{array}{llll}\mathrm{H} & -4.82891800 & -5.23678600 & 1.15318900\end{array}$

$\begin{array}{llll}\mathrm{H} & -3.47820700 & -2.56789200 & 3.55525200\end{array}$

$\begin{array}{llll}\mathrm{H} & -4.97986700 & -3.42723100 & 3.13131300\end{array}$

$\mathrm{H} \quad-3.60012300 \quad-4.31295400 \quad 3.80111000$

$\begin{array}{llll}\text { O } & -1.32876000 & -0.62259000 & 0.10020800\end{array}$

$\begin{array}{llll}\mathrm{O} & -1.38778200 & -1.25935000 & 2.26623500\end{array}$

$\begin{array}{llll}\mathrm{S} & 3.53484000 & 0.18402500 & -0.95296100\end{array}$

F $\quad 5.28665900 \quad-1.64001700 \quad-0.20889200$

F $\quad 3.68176600 \quad-2.41636000 \quad-1.46163400$

F $\quad 5.27181200 \quad-1.22432400 \quad-2.34697600$

\begin{abstract}
$\begin{array}{llll}\mathrm{O} & 4.55238500 & 1.22711200 & -0.80586500\end{array}$
$\begin{array}{llll}\text { O } & 2.60962300 & 0.26262300 & -2.11352000\end{array}$

$\begin{array}{llll}\mathrm{N} & 2.72272800 & -0.17010700 & 0.40569500\end{array}$

$\begin{array}{llll}\text { C } & 3.06746100 & 0.61921100 & 1.62676800\end{array}$

$\begin{array}{llll}\text { C } & 1.93372500 & 1.62627400 & 1.74447400\end{array}$

$\begin{array}{llll}\text { C } & 0.60740000 & 1.14761500 & 1.92203800\end{array}$

$\begin{array}{llll}\text { C } & -0.50307000 & 2.01785500 & 1.79650100\end{array}$

$\begin{array}{llll}\text { C } & -0.28013100 & 3.35730900 & 1.51514200\end{array}$

$\begin{array}{llll}\text { C } & 1.02448500 & 3.84773400 & 1.37460200\end{array}$

$\begin{array}{llll}\text { C } & 2.13168500 & 2.99369800 & 1.48000200\end{array}$

$\begin{array}{llll}\text { C } & 4.50830500 & -1.37994200 & -1.26310000\end{array}$

$\begin{array}{llll}\mathrm{H} & 0.43452600 & 0.19105800 & 2.43236100\end{array}$

$\begin{array}{llll}\mathrm{H} & -1.51062900 & 1.64266000 & 1.93747400\end{array}$

$\begin{array}{llll}\mathrm{H} & -1.11914300 & 4.03982200 & 1.41971300\end{array}$

$\begin{array}{llll}\mathrm{H} & 1.17308500 & 4.90263700 & 1.17090700\end{array}$

$\begin{array}{llll}\text { O } & 3.42261000 & 3.39999000 & 1.34673600\end{array}$

$\begin{array}{llll}\text { C } & 3.68779800 & 4.70904300 & 0.86319100\end{array}$

$\begin{array}{llll}\mathrm{H} & 3.37226700 & 5.47648800 & 1.58226300\end{array}$

$\begin{array}{llll}\mathrm{H} & 4.76919400 & 4.76071100 & 0.73108200\end{array}$

$\begin{array}{llll}\mathrm{H} & 3.19520100 & 4.88434200 & -0.10133500\end{array}$

$\begin{array}{llll}\text { C } & 3.23323100 & -0.31211300 & 2.82920500\end{array}$

$\begin{array}{llll}\mathrm{H} & 2.33128100 & -0.90107700 & 3.02361800\end{array}$

$\begin{array}{llll}\mathrm{H} & 4.05506000 & -1.00840800 & 2.63613100\end{array}$

$\begin{array}{llll}\text { H } & 3.46785700 & 0.26942700 & 3.72806100\end{array}$

$\mathrm{H} \quad 4.00078600 \quad 1.15678200 \quad 1.45764900$

$\begin{array}{llll}\text { C } & 0.61951200 & -2.43874600 & -2.14204100\end{array}$

$\begin{array}{llll}\mathrm{O} & 0.70582400 & -2.23223100 & -0.92478100\end{array}$

$\begin{array}{llll}\mathrm{O} & 0.96058500 & -1.58281100 & -3.07667100\end{array}$

$\begin{array}{llll}\mathrm{H} & 1.46326900 & -0.81221300 & -2.68428200\end{array}$

$\begin{array}{llll}\text { C } & 0.11913500 & -3.75410200 & -2.67367100\end{array}$

$\begin{array}{llll}\mathrm{H} & -0.60297300 & -3.57877700 & -3.47656900\end{array}$

$\begin{array}{llll}\mathrm{H} & 0.96027600 & -4.30988700 & -3.10302400\end{array}$

$\begin{array}{llll}\mathrm{H} & -0.33231200 & -4.33666300 & -1.87070300\end{array}$
\end{abstract}

$\mathrm{TS}_{\text {R2a }}$

$-2507.03270403$

$\begin{array}{lrrr}\text { Pd } & -0.54999400 & 0.31004600 & 0.26509000 \\ \mathrm{C} & 3.42612900 & -0.82336500 & 1.50599300 \\ \mathrm{C} & 1.92573500 & -0.77091200 & 1.16915900 \\ \mathrm{H} & 3.69002800 & -1.88134700 & 1.55344600 \\ \mathrm{~N} & 4.23604500 & -0.20991300 & 0.46425600 \\ \mathrm{H} & 4.40554500 & 0.78334300 & 0.55413600 \\ \mathrm{C} & 4.20165000 & -0.72210200 & -0.81099200 \\ \mathrm{O} & 3.59716300 & -1.76163600 & -1.07105600\end{array}$




\begin{tabular}{|c|c|c|c|}
\hline $\mathrm{C}$ & 4.95628400 & 0.04439000 & -1.85596800 \\
\hline $\mathrm{C}$ & 5.99737000 & 0.93319700 & -1.55004800 \\
\hline C & 4.60238500 & -0.16350600 & -3.19608600 \\
\hline 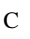 & 6.66119100 & 1.61604000 & -2.56947500 \\
\hline 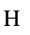 & 6.31634800 & 1.06806100 & -0.52010900 \\
\hline C & 5.26085300 & 0.52477400 & -4.21256200 \\
\hline $\mathrm{H}$ & 3.80785000 & -0.86856800 & -3.41664000 \\
\hline 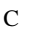 & 6.28996500 & 1.41775200 & -3.90108800 \\
\hline $\mathrm{H}$ & 7.47301200 & 2.29552600 & -2.32471400 \\
\hline $\mathrm{H}$ & 4.97353700 & 0.36501700 & -5.24814600 \\
\hline $\mathrm{H}$ & 6.80602300 & 1.95201600 & -4.69430300 \\
\hline $\mathrm{C}$ & 3.72467600 & -0.13014600 & 2.85126300 \\
\hline $\mathrm{H}$ & 4.81302100 & -0.17147800 & 2.99867300 \\
\hline $\mathrm{H}$ & 3.45709800 & 0.93252800 & 2.75439600 \\
\hline $\mathrm{C}$ & 3.02466200 & -0.70907500 & 4.09716300 \\
\hline $\mathrm{C}$ & 3.38361100 & 0.14214700 & 5.32523200 \\
\hline C & 3.37351700 & -2.18622900 & 4.33411000 \\
\hline $\mathrm{H}$ & 1.93963500 & -0.64134300 & 3.94457200 \\
\hline $\mathrm{H}$ & 3.10980000 & 1.19437900 & 5.18052300 \\
\hline $\mathrm{H}$ & 2.86092600 & -0.22013700 & 6.21778500 \\
\hline $\mathrm{H}$ & 4.46128900 & 0.10179400 & 5.53217300 \\
\hline $\mathrm{H}$ & 3.00635700 & -2.82328500 & 3.52374800 \\
\hline $\mathrm{H}$ & 4.45941300 & -2.32660200 & 4.42211800 \\
\hline $\mathrm{H}$ & 2.91719900 & -2.54380100 & 5.26447900 \\
\hline $\mathrm{O}$ & 1.51593200 & 0.21713900 & 0.48299200 \\
\hline $\mathrm{O}$ & 1.19228900 & -1.70449600 & 1.61014000 \\
\hline S & .40194700 & 0.90376200 & -0.93908400 \\
\hline $\mathrm{F}$ & -5.29379900 & 1.40711900 & 0.82869800 \\
\hline $\mathrm{F}$ & -3.81068100 & 2.99303000 & 0.63143200 \\
\hline $\mathrm{F}$ & -5.32329600 & 2.72053100 & -0.90887000 \\
\hline $\mathrm{O}$ & -4.30125900 & 0.02016500 & -1.68588500 \\
\hline $\mathrm{O}$ & -2.49683500 & 1.80355700 & -1.69965800 \\
\hline$N$ & -2.59688600 & 0.20942800 & 0.28126300 \\
\hline $\mathrm{C}$ & -3.01898400 & 17672500 & 0.65378800 \\
\hline $\mathrm{C}$ & -2.09902700 & -2.10050000 & -0.11261900 \\
\hline $\mathrm{C}$ & -0.73564900 & -1.71703700 & -0.23390000 \\
\hline $\mathrm{C}$ & 0.09648600 & -2.37676800 & -1.17140600 \\
\hline $\mathrm{C}$ & -0.40450200 & -3.44941700 & -1.89839100 \\
\hline $\mathrm{C}$ & -1.72779600 & -3.87086500 & -1.72821400 \\
\hline $\mathrm{C}$ & -2.57845500 & -3.20275100 & -0.83611200 \\
\hline $\mathrm{C}$ & -4.53376500 & 2.08516300 & -0.04048600 \\
\hline $\mathrm{H}$ & 0.02434400 & -1.64591700 & 0.83505200 \\
\hline $\mathrm{H}$ & 1.14126400 & -2.09513300 & -1.26451200 \\
\hline $\mathrm{H}$ & 0.23593300 & -3.97927900 & 59784600 \\
\hline
\end{tabular}

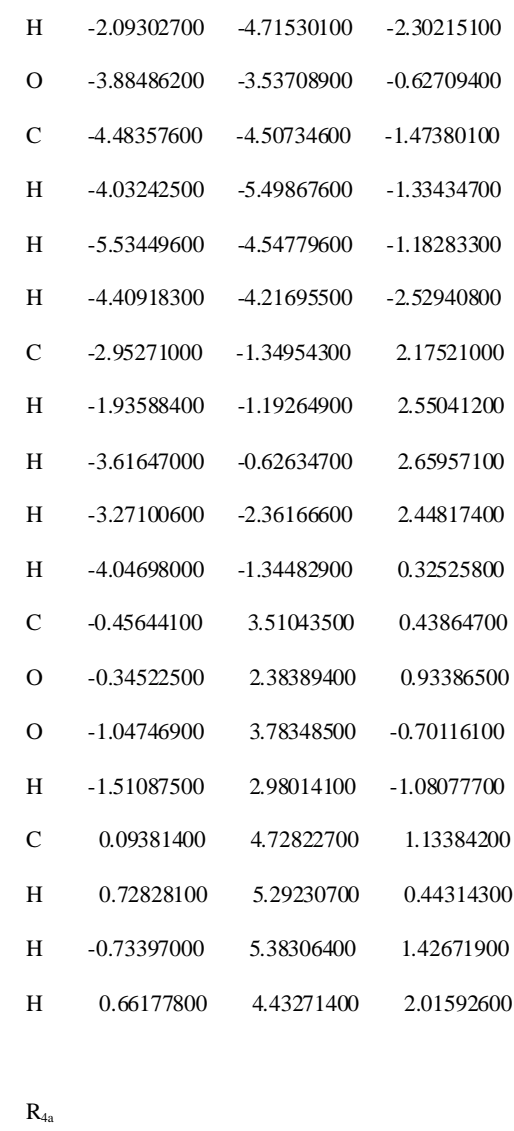

$\mathrm{R}_{4 \mathrm{a}}$

$-2507.06243349$

$\begin{array}{llll}\mathrm{Pd} & 0.66565100 & -0.19787100 & 0.03088900 \\ \mathrm{C} & -3.46687900 & -0.19952300 & 1.68732100 \\ \mathrm{C} & -2.00903300 & 0.13690000 & 1.38101000 \\ \mathrm{H} & -3.84318500 & 0.58682100 & 2.34498400 \\ \mathrm{~N} & -4.26064100 & -0.19687100 & 0.47124000 \\ \mathrm{H} & -4.38651000 & -1.08441800 & 0.00293300 \\ \mathrm{C} & -4.29215100 & 0.94709700 & -0.29698700 \\ \mathrm{O} & -3.72994600 & 1.97194900 & 0.08505900 \\ \mathrm{C} & -5.04389600 & 0.87027700 & -1.58756400 \\ \mathrm{C} & -6.03467600 & -0.09090700 & -1.83719100 \\ \mathrm{C} & -4.74032300 & 1.82025100 & -2.57268300 \\ \mathrm{C} & -6.69987700 & -0.11019900 & -3.06286900 \\ \mathrm{H} & -6.31482300 & -0.80257400 & -1.06522600 \\ \mathrm{C} & -5.40058100 & 1.79436000 & -3.79881200 \\ \mathrm{H} & -3.98280600 & 2.56627900 & -2.35690700 \\ \mathrm{H} & -6.37941000 & 0.82790100 & -4.04673800 \\ \mathrm{H} & -7.47277400 & -0.85126900 & -3.24660500 \\ \mathrm{H} & -5.15310500 & 2.52712500 & -4.56161500 \\ \mathrm{H} & -6.89608700 & 0.80981900 & -5.00246300 \\ \mathrm{H} & -3.58073200 & -1.58084600 & 2.36951400 \\ \mathrm{H} & -4.65353800 & -1.78638700 & 2.48686300 \\ \mathrm{H} & -2.334466600 & 1.67550500\end{array}$




\begin{tabular}{|c|c|c|c|c|c|c|c|}
\hline $\mathrm{C}$ & -2.88479500 & -1.73959600 & 3.73670500 & $\mathrm{H}$ & 1.49042500 & -2.56998700 & -2.03961300 \\
\hline $\mathrm{C}$ & -3.02702700 & -3.19464500 & 4.21068200 & $\mathrm{C}$ & -0.10689000 & -4.78598400 & -0.29244400 \\
\hline $\mathrm{C}$ & -3.43062600 & -0.76598300 & 4.79246400 & $\mathrm{H}$ & -0.69627700 & -5.20025700 & -1.11588700 \\
\hline $\mathrm{H}$ & -1.81312300 & -1.53554000 & 3.60741200 & $\mathrm{H}$ & 0.72286000 & -5.47657700 & -0.10504400 \\
\hline $\mathrm{H}$ & -2.60750300 & -3.89816900 & 3.48152400 & $\mathrm{H}$ & -0.71638500 & -4.69740100 & 0.60736900 \\
\hline $\mathrm{H}$ & -2.50508300 & -3.34764300 & 5.16170500 & & & & \\
\hline $\mathrm{H}$ & -4.08171800 & -3.45811000 & 4.36368200 & $\mathrm{R}_{3}$ & & & \\
\hline $\mathrm{H}$ & -3.24168800 & 0.27913900 & 4.52709300 & & 7.93470121 & & \\
\hline $\mathrm{H}$ & -4.51293600 & -0.89589200 & 4.92480100 & $\mathrm{Pd}$ & 1.19540700 & -0.22104000 & -0.15739100 \\
\hline $\mathrm{H}$ & -2.95432200 & -0.94660600 & 5.76265300 & $\mathrm{C}$ & -2.88138300 & 0.75981600 & -0.74735600 \\
\hline $\mathrm{O}$ & -1.45544200 & -0.25621700 & 0.35140200 & $\mathrm{C}$ & -1.54347700 & 0.26592100 & -0.18834800 \\
\hline $\mathrm{O}$ & -1.40258500 & 0.82079400 & 2.33430100 & $\mathrm{H}$ & -2.92500400 & 0.55051100 & -1.81941500 \\
\hline $\mathrm{S}$ & 3.40010400 & -0.57862200 & -1.49666800 & $\mathrm{~N}$ & -3.92367500 & -0.00813200 & -0.08480000 \\
\hline $\mathrm{F}$ & 5.33543800 & -1.61273200 & -0.02015800 & $\mathrm{H}$ & -3.65254200 & -0.42964400 & 0.79386900 \\
\hline $\mathrm{F}$ & 3.68512600 & -2.98162800 & -0.41148700 & $\mathrm{C}$ & -5.17914800 & -0.13601900 & -0.59612200 \\
\hline $\mathrm{F}$ & 5.12221100 & -2.53229400 & -1.98212900 & $\mathrm{O}$ & -5.53043800 & 0.44461500 & -1.62469900 \\
\hline $\mathrm{O}$ & 4.35850200 & 0.35697300 & -2.09037500 & $\mathrm{C}$ & -6.12858400 & -1.01589200 & 0.17384200 \\
\hline $\mathrm{O}$ & 2.39399500 & -1.22729400 & -2.37792900 & $\mathrm{C}$ & -7.49742800 & -0.84993100 & -0.07612200 \\
\hline $\mathrm{N}$ & 2.69697400 & -0.07333600 & -0.14563700 & $\mathrm{C}$ & -5.71543100 & -1.98984000 & 1.09432100 \\
\hline $\mathrm{C}$ & 3.26998900 & 1.11703000 & 0.55302000 & $\mathrm{C}$ & -8.43847700 & -1.62502300 & 0.59801900 \\
\hline $\mathrm{C}$ & 2.19447800 & 2.17469900 & 0.50832300 & $\mathrm{H}$ & -7.79650500 & -0.10735200 & -0.80844600 \\
\hline $\mathrm{C}$ & 0.88106800 & 1.76260300 & 0.22866200 & $\mathrm{C}$ & -6.65793300 & -2.77110200 & 1.76411200 \\
\hline $\mathrm{C}$ & -0.14927000 & 2.70218600 & 0.08117400 & $\mathrm{H}$ & -4.65867200 & -2.17094700 & 1.27219100 \\
\hline $\mathrm{C}$ & 0.14382600 & 4.05729100 & 0.25427800 & $\mathrm{C}$ & -8.02076700 & -2.58622700 & 1.52251100 \\
\hline $\mathrm{C}$ & 1.43733400 & 4.48572600 & 0.55958600 & $\mathrm{H}$ & -9.49782300 & -1.48279500 & 0.40132800 \\
\hline $\mathrm{C}$ & 2.46524200 & 3.54282000 & 0.68554700 & $\mathrm{H}$ & -6.32662100 & -3.52894100 & 2.46901400 \\
\hline $\mathrm{C}$ & 4.45463400 & -2.01882800 & -0.94580400 & $\mathrm{H}$ & -8.75353800 & -3.19414400 & 2.04672000 \\
\hline $\mathrm{H}$ & -0.49165000 & 1.04827300 & 2.02602300 & $\mathrm{C}$ & -3.07902600 & 2.28294900 & -0.52063000 \\
\hline $\mathrm{H}$ & -1.16308700 & 2.40211500 & -0.16327400 & $\mathrm{H}$ & -4.10861900 & 2.49769300 & -0.83055900 \\
\hline $\mathrm{H}$ & -0.64935200 & 4.79331700 & 0.14993600 & $\mathrm{H}$ & -3.02480600 & 2.47109100 & 0.56122800 \\
\hline $\mathrm{H}$ & 1.63590700 & 5.54394700 & 0.68572800 & $\mathrm{C}$ & -2.12061900 & 3.23586400 & -1.26165200 \\
\hline $\mathrm{O}$ & 3.76894600 & 3.85520600 & 0.96535800 & $\mathrm{C}$ & -2.44205100 & 4.68725100 & -0.86922000 \\
\hline $\mathrm{C}$ & 4.13090500 & 5.22326800 & 1.05835800 & $\mathrm{C}$ & -2.18196000 & 3.06073000 & -2.78704400 \\
\hline $\mathrm{H}$ & 3.61499600 & 5.72272800 & 1.88939400 & $\mathrm{H}$ & -1.09410900 & 3.01660300 & -0.94025300 \\
\hline $\mathrm{H}$ & 5.20671200 & 5.23631400 & 1.24310300 & $\mathrm{H}$ & -2.37742600 & 4.83569100 & 0.21656000 \\
\hline $\mathrm{H}$ & 3.91600300 & 5.76077100 & 0.12560900 & $\mathrm{H}$ & -1.74755700 & 5.38866600 & -1.34750700 \\
\hline $\mathrm{C}$ & 3.69219400 & 0.73670200 & 1.97933900 & $\mathrm{H}$ & -3.45817400 & 4.96371700 & -1.17909800 \\
\hline $\mathrm{H}$ & 2.83731100 & 0.34085500 & 2.53935100 & $\mathrm{H}$ & -1.82226100 & 2.07409600 & -3.09502600 \\
\hline $\mathrm{H}$ & 4.47221200 & -0.03035300 & 1.95009700 & $\mathrm{H}$ & -3.20847900 & 3.18087400 & -3.15650700 \\
\hline $\mathrm{H}$ & 4.07948600 & 1.61720600 & 2.50203300 & $\mathrm{H}$ & -1.55544200 & 3.80895900 & -3.28779800 \\
\hline $\mathrm{H}$ & 4.15090200 & 1.47195300 & 0.00913900 & $\mathrm{O}$ & -0.50918500 & 0.51889500 & -0.93436700 \\
\hline $\mathrm{C}$ & 0.44925900 & -3.43830700 & -0.67968400 & $\mathrm{O}$ & -1.47844900 & -0.30546500 & 0.90910000 \\
\hline $\mathrm{O}$ & 0.35410900 & -2.45715300 & 0.05993100 & $\mathrm{~S}$ & 3.49992700 & -1.61984800 & -0.94198800 \\
\hline $\mathrm{O}$ & 1.03666900 & -3.44493400 & -1.85662400 & $\mathrm{~F}$ & 4.43895600 & -3.69615400 & 0.35978800 \\
\hline
\end{tabular}




\begin{tabular}{|c|c|c|c|}
\hline $\mathrm{F}$ & 2.35850400 & -3.90228600 & -0.25485200 \\
\hline $\mathrm{F}$ & 3.95040800 & -4.08101600 & -1.72985500 \\
\hline $\mathrm{O}$ & 4.85220900 & -1.23720700 & -1.34361500 \\
\hline $\mathrm{O}$ & 2.34731000 & -1.42596600 & -1.88221600 \\
\hline $\mathrm{N}$ & 2.95402700 & -1.02158800 & 0.47747400 \\
\hline $\mathrm{C}$ & 3.76138200 & -0.00621000 & 1.21471100 \\
\hline $\mathrm{C}$ & 2.81133600 & 1.18075800 & 1.36809600 \\
\hline $\mathrm{C}$ & 1.48757500 & 0.96165500 & 1.84415000 \\
\hline $\mathrm{C}$ & 0.56890100 & 2.03078300 & 1.94617800 \\
\hline 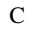 & 0.97295000 & 3.30587000 & 1.58622700 \\
\hline C & 2.27836000 & 3.54124000 & 1.12758200 \\
\hline $\mathrm{C}$ & 3.19331400 & 2.49114000 & 1.00207100 \\
\hline $\mathrm{C}$ & 3.56029500 & -3.45996000 & -0.61827600 \\
\hline 2 & 1.24263100 & 0.01329800 & 2.32219800 \\
\hline $\mathrm{x}$ & -0.43707400 & 1.82894800 & 2.29503000 \\
\hline $\mathrm{H}$ & 0.28206000 & 4.14038800 & 1.65851000 \\
\hline $\mathrm{H}$ & 2.56806100 & 4.55067800 & 0.85901800 \\
\hline $\mathrm{O}$ & 4.46939200 & 2.63335500 & 0.55500700 \\
\hline $\mathrm{C}$ & 4.89312900 & 3.90677100 & 0.08142500 \\
\hline $\mathrm{H}$ & 4.88472400 & 4.65447800 & 0.88447000 \\
\hline $\mathrm{H}$ & 5.91539500 & 3.76467000 & -0.27082700 \\
\hline $\mathrm{H}$ & 4.26453900 & 4.25117200 & -0.74897900 \\
\hline 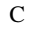 & 4.25574600 & -0.57675200 & 2.54497200 \\
\hline $\mathrm{H}$ & 4.83219300 & 0.17853700 & 3.09160600 \\
\hline $\mathrm{H}$ & 3.41885100 & -0.90189900 & 3.17151400 \\
\hline $\mathrm{H}$ & 4.90123200 & -1.44185600 & 2.36136800 \\
\hline $\mathrm{H}$ & 4.61720300 & 0.30747300 & 0.61281600 \\
\hline
\end{tabular}

$\mathrm{TS}_{\mathrm{R} 2 \mathrm{a}^{\prime}}$

$-2277.90936001$

$\begin{array}{lrrr}\mathrm{Pd} & -1.16407500 & -0.04170300 & 0.84649400 \\ \mathrm{C} & 3.06730400 & 0.50810800 & 1.04419400 \\ \mathrm{C} & 1.60028500 & 0.42075100 & 0.63261300 \\ \mathrm{H} & 3.20252600 & -0.02876700 & 1.98432600 \\ \mathrm{~N} & 3.87043200 & -0.15745600 & 0.03065600 \\ \mathrm{H} & 3.53709300 & -0.09475200 & -0.92085300 \\ \mathrm{C} & 5.11296000 & -0.65202300 & 0.31599200 \\ \mathrm{O} & 5.60505500 & -0.55983900 & 1.43996600 \\ \mathrm{C} & 5.84646400 & -1.31076000 & -0.81943200 \\ \mathrm{C} & 7.23265100 & -1.46399800 & -0.68086200 \\ \mathrm{C} & 5.21730600 & -1.78926300 & -1.97797800 \\ \mathrm{C} & 7.98115300 & -2.06283200 & -1.69167700 \\ \mathrm{H} & 7.69899500 & -1.10798400 & 0.23178200 \\ \mathrm{C} & 5.96678600 & -2.39457900 & -2.98724000\end{array}$

\begin{tabular}{|c|c|c|c|}
\hline $\mathrm{H}$ & 4.13781600 & -1.72690200 & -2.08785100 \\
\hline C & 7.35004400 & -2.52693400 & -2.84897600 \\
\hline & 9.05632600 & -2.17082900 & -1.57706700 \\
\hline & 5.46885600 & -2.77005100 & -3.87706600 \\
\hline & 7.93253300 & -2.99727500 & -3.63671400 \\
\hline & 3.49853800 & 1.98884800 & 1.23181400 \\
\hline & 4.57537100 & 1.95665800 & 1.43666600 \\
\hline & 3.36938900 & 2.50070600 & 0.26791500 \\
\hline 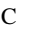 & 2.79571000 & 2.78857200 & 2.34676900 \\
\hline C & 3.30181600 & 4.24007700 & 2.32858900 \\
\hline $\mathrm{C}$ & 2.99470100 & 2.15976500 & 3.73457800 \\
\hline & 1.71590000 & 2.80626400 & 2.14137800 \\
\hline & 3.14112800 & 4.71242700 & 1.35136800 \\
\hline H & 2.78571500 & 4.84473800 & 3.08336500 \\
\hline H & 4.37686300 & 4.28343600 & 2.54582700 \\
\hline H & 2.52411200 & 1.17415100 & 3.80601100 \\
\hline $\mathrm{H}$ & 4.06172700 & 2.04633200 & 3.96506400 \\
\hline $\mathrm{H}$ & 2.54872200 & 2.79199900 & 4.51123300 \\
\hline $\mathrm{O}$ & 0.73159800 & 0.41890200 & 1.56449300 \\
\hline $\mathrm{O}$ & 1.32863500 & 0.40740900 & -0.60880000 \\
\hline S & -3.83315600 & -1.15281800 & 1.09344200 \\
\hline $\mathrm{F}$ & -4.68351600 & -3.15542500 & -0.38981000 \\
\hline $\mathrm{F}$ & -2.99972700 & -3.65743100 & 0.89827100 \\
\hline $\mathrm{F}$ & -4.98834800 & -3.42715300 & 1.75148300 \\
\hline $\mathrm{O}$ & -5.15825700 & -0.53549100 & 1.01813500 \\
\hline $\mathrm{O}$ & -3.01709800 & -1.08755200 & 2.33535200 \\
\hline $\mathrm{N}$ & -2.78560100 & -0.76111900 & -0.07068700 \\
\hline C & -3.15368200 & -0.15096700 & -1.36744600 \\
\hline $\mathrm{C}$ & -2.39166100 & 1.15546000 & -1.45840400 \\
\hline $\mathrm{C}$ & -1.20460700 & 1.35836700 & -0.69930400 \\
\hline C & 0.66415900 & 2.66171000 & -0.58915200 \\
\hline $\mathrm{C}$ & 1.21555200 & 3.70926800 & -1.31378100 \\
\hline C & 2.33267900 & 3.49780000 & -2.12666000 \\
\hline C & -2.92267400 & 2.22947100 & -2.19608600 \\
\hline C & -4.14314700 & -2.97459900 & 0.82552200 \\
\hline $\mathrm{H}$ & -0.07808100 & 0.62331700 & -0.74599900 \\
\hline $\mathrm{H}$ & 0.22144600 & 2.82592400 & 0.01354400 \\
\hline $\mathrm{H}$ & -0.77811300 & 4.70173700 & -1.25581000 \\
\hline $\mathrm{H}$ & -2.74948100 & 4.32800100 & -2.68529000 \\
\hline $\mathrm{O}$ & -4.04062900 & 1.94627800 & -2.92337100 \\
\hline C & -4.70874500 & 3.00621200 & -3.59649700 \\
\hline $\mathrm{H}$ & -4.07423100 & 3.45043100 & -4.37386600 \\
\hline $\mathrm{H}$ & -5.58604800 & 2.55446900 & -4.06147400 \\
\hline $\mathrm{H}$ & & 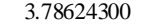 & 47400 \\
\hline
\end{tabular}




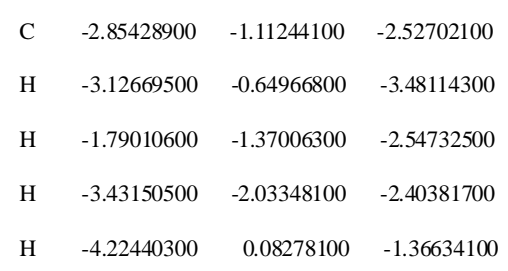

$\mathrm{R}_{4 \mathrm{a}^{\prime}}$

$-2277.94490261$

Pd $\quad 1.17461500 \quad-0.08100400 \quad-0.64264400$

$\begin{array}{llll}\text { C } & -3.15285600 & 0.87285800 & -0.71890900\end{array}$

$\begin{array}{llll}\text { C } & -1.70913400 & 0.59265000 & -0.35902300\end{array}$

$\begin{array}{llll}\mathrm{H} & -3.31448500 & 0.52366100 & -1.73915800\end{array}$

$\begin{array}{llll}\text { N } & -4.03061500 & 0.09198700 & 0.13873300\end{array}$

$\begin{array}{llll}\mathrm{H} & -3.86706300 & 0.12748200 & 1.13501800\end{array}$

$\begin{array}{llll}\text { C } & -5.25014600 & -0.34315800 & -0.33221800\end{array}$

$\begin{array}{llll}\text { O } & -5.60411300 & -0.13099700 & -1.48831200\end{array}$

$\begin{array}{llll}\text { C } & -6.10856900 & -1.08729700 & 0.64701000\end{array}$

$\begin{array}{llll}\text { C } & -7.47907000 & -1.17189900 & 0.36517900\end{array}$

$\begin{array}{llll}\text { C } & -5.60319900 & -1.71089000 & 1.79734600\end{array}$

$\begin{array}{llll}\text { C } & -8.33492500 & -1.84709100 & 1.23225400\end{array}$

$\begin{array}{llll}\mathrm{H} & -7.84914100 & -0.70341300 & -0.54075600\end{array}$

$\begin{array}{llll}\text { C } & -6.46055600 & -2.39297100 & 2.66084000\end{array}$

$\begin{array}{llll}\mathrm{H} & -4.53673400 & -1.70317400 & 2.00659000\end{array}$

$\begin{array}{llll}\text { C } & -7.82772000 & -2.45649600 & 2.38322500\end{array}$

$\begin{array}{llll}\mathrm{H} & -9.39695800 & -1.90206300 & 1.00993400\end{array}$

$\begin{array}{llll}\mathrm{H} & -6.05955600 & -2.88234400 & 3.54401200\end{array}$

$\begin{array}{llll}\mathrm{H} & -8.49448700 & -2.98707100 & 3.05749300\end{array}$

$\begin{array}{llll}\text { C } & -3.45987100 & 2.39500500 & -0.64676900\end{array}$

$\begin{array}{llll}\mathrm{H} & -4.53010200 & 2.48232300 & -0.87026200\end{array}$

$\begin{array}{llll}\mathrm{H} & -3.31909300 & 2.72528500 & 0.39144600\end{array}$

$\begin{array}{llll}\text { C } & -2.66643800 & 3.31120100 & -1.60044000\end{array}$

$\begin{array}{llll}\text { C } & -3.07734100 & 4.77223500 & -1.35629500\end{array}$

$\begin{array}{llll}\text { C } & -2.85550900 & 2.92807600 & -3.07660500\end{array}$

$\begin{array}{llll}\mathrm{H} & -1.59550700 & 3.22372800 & -1.36632000\end{array}$

$\begin{array}{llll}\mathrm{H} & -2.92211800 & 5.06681400 & -0.31121800\end{array}$

H $\quad-2.49405700 \quad 5.45191400 \quad-1.98751700$

$\mathrm{H} \quad-4.13797600 \quad 4.92528200 \quad-1.59295800$

$\begin{array}{llll}\mathrm{H} & -2.44166800 & 1.93967000 & -3.30127700\end{array}$

$\begin{array}{llll}\mathrm{H} & -3.91864800 & 2.92214200 & -3.34858000\end{array}$

$\begin{array}{llll}\mathrm{H} & -2.34696400 & 3.64855900 & -3.72695900\end{array}$

$\begin{array}{llll}\text { O } & -0.80814000 & 0.55862900 & -1.20360300\end{array}$

$\begin{array}{llll}\text { O } & -1.48398500 & 0.43955700 & 0.93893300\end{array}$

$\begin{array}{llll}\text { S } & 3.33221500 & -1.94607400 & -1.17920200\end{array}$

$\begin{array}{llll}\text { F } & 4.34172100 & -3.65592600 & 0.58305700\end{array}$

\begin{tabular}{|c|c|c|c|}
\hline $\mathrm{F}$ & 2.18064900 & -3.78164900 & 0.34615700 \\
\hline $\mathrm{F}$ & 3.49414900 & -4.57324200 & -1.19861600 \\
\hline 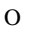 & 4.68422900 & -1.81653400 & -1.72601200 \\
\hline $\mathrm{O}$ & 2.14030700 & -2.04636200 & -2.06176400 \\
\hline $\mathrm{N}$ & 2.92123800 & -0.85641500 & -0.06705400 \\
\hline $\mathrm{C}$ & 3.88243500 & 0.00732000 & 0.66465100 \\
\hline $\mathrm{C}$ & 3.16625300 & 1.33140900 & 0.83873100 \\
\hline $\mathrm{C}$ & 1.84391300 & 1.47100800 & 0.38289100 \\
\hline $\mathrm{C}$ & 1.14158600 & 2.67159400 & 0.52742500 \\
\hline $\mathrm{C}$ & 1.77428400 & 3.75484000 & 1.13719100 \\
\hline $\mathrm{C}$ & 3.09502700 & 3.65733100 & 1.57728900 \\
\hline C & 3.79388200 & 2.45484900 & 1.41802100 \\
\hline $\mathrm{C}$ & 3.33561200 & -3.59737100 & -0.30183500 \\
\hline $\mathrm{H}$ & -0.51099100 & 0.29619000 & 1.06844000 \\
\hline $\mathrm{H}$ & 0.12975000 & 2.77991500 & 0.15291500 \\
\hline $\mathrm{H}$ & 1.24089900 & 4.69448700 & 1.25666000 \\
\hline $\mathrm{H}$ & 3.57338000 & 4.51878800 & 2.02806100 \\
\hline $\mathrm{O}$ & 5.10126700 & 2.28321900 & 1.77886400 \\
\hline $\mathrm{C}$ & 5.81581600 & 3.39279300 & 2.30173600 \\
\hline $\mathrm{H}$ & 5.38428900 & 3.74087800 & 3.24932400 \\
\hline $\mathrm{H}$ & 6.83184100 & 3.03554200 & 2.47815000 \\
\hline $\mathrm{H}$ & 5.84167900 & 4.22571500 & 1.58755800 \\
\hline $\mathrm{C}$ & 4.27980100 & -0.64846200 & 1.99805700 \\
\hline $\mathrm{H}$ & 4.96635500 & -0.00415600 & 2.55162200 \\
\hline $\mathrm{H}$ & 3.38656200 & -0.82713500 & 2.60646800 \\
\hline $\mathrm{H}$ & 4.77039400 & -1.60778200 & 1.81481200 \\
\hline $\mathrm{H}$ & 4.78347500 & 0.15520900 & 0.05484100 \\
\hline
\end{tabular}

$\mathrm{R}_{3 \mathrm{a} "}$

$-2277.94970393$

$\begin{array}{llll}\mathrm{Pd} & 0.42601800 & -0.06512200 & 0.06269700 \\ \mathrm{C} & 3.02030900 & -1.12647000 & -0.72962200 \\ \mathrm{C} & 1.92924200 & -1.85943900 & -1.52924700 \\ \mathrm{H} & 3.86258800 & -0.96040400 & -1.40298800 \\ \mathrm{~N} & 2.51062700 & 0.21670000 & -0.27562100 \\ \mathrm{H} & 2.92084600 & 0.47288900 & 0.62268600 \\ \mathrm{C} & 2.68893000 & 1.28790900 & -1.25130700 \\ \mathrm{O} & 2.82765900 & 0.99790600 & -2.41985700 \\ \mathrm{C} & 2.72507500 & 2.68287800 & -0.73262400 \\ \mathrm{C} & 2.41197300 & 3.04281600 & 0.58868800 \\ \mathrm{C} & 3.11192000 & 3.67771000 & -1.64710400 \\ \mathrm{C} & 2.49466500 & 4.37685500 & 0.98542600 \\ \mathrm{H} & 2.05718300 & 2.31051500 & 1.30600300 \\ \mathrm{C} & 3.19741400 & 5.00608800 & -1.24412600\end{array}$




\begin{tabular}{|c|c|c|c|c|c|c|c|}
\hline $\mathrm{H}$ & 3.34244000 & 3.38434600 & -2.66553800 & $\mathrm{C}$ & -2.25803000 & -2.60377500 & 0.19465400 \\
\hline $\mathrm{C}$ & 2.89089800 & 5.35756700 & 0.07402800 & $\mathrm{H}$ & -1.39527800 & -2.80973900 & -0.44432100 \\
\hline $\mathrm{H}$ & 2.23741700 & 4.64894400 & 2.00461700 & $\mathrm{H}$ & -1.98376400 & -2.82813800 & 1.23123000 \\
\hline $\mathrm{H}$ & 3.50036400 & 5.76831400 & -1.95604600 & $\mathrm{H}$ & -3.07872100 & -3.26620200 & -0.10012700 \\
\hline $\mathrm{H}$ & 2.95394200 & 6.39569900 & 0.38825100 & $\mathrm{H}$ & -3.51699500 & -0.96148600 & 0.77538000 \\
\hline $\mathrm{C}$ & 3.46541400 & -1.92355300 & 0.51280900 & & & & \\
\hline $\mathrm{H}$ & 4.23907200 & -1.33345000 & 1.03097400 & \multicolumn{4}{|c|}{$\mathrm{TS}_{\mathrm{R} 2 \mathrm{a}^{\prime}}$} \\
\hline $\mathrm{H}$ & 2.61405400 & -2.00661500 & 1.20294500 & \multicolumn{4}{|c|}{-2277.87414106} \\
\hline $\mathrm{C}$ & 4.03004000 & -3.33433800 & 0.24141900 & $\mathrm{Pd}$ & 0.01566400 & 0.18742500 & 0.07992100 \\
\hline $\mathrm{C}$ & 4.40425300 & -3.99486700 & 1.57704000 & $\mathrm{C}$ & -2.96634500 & 0.13926600 & -0.98359900 \\
\hline $\mathrm{C}$ & 5.22469500 & -3.31485000 & -0.72339800 & $\mathrm{C}$ & -2.18471900 & 0.00696900 & -2.32458200 \\
\hline $\mathrm{H}$ & 3.23577900 & -3.92868300 & -0.22556000 & $\mathrm{H}$ & -3.78874500 & -0.57519000 & -1.02049100 \\
\hline $\mathrm{H}$ & 3.54901000 & -4.03261400 & 2.26283100 & $\mathrm{~N}$ & -2.14029500 & -0.22001800 & 0.24245900 \\
\hline $\mathrm{H}$ & 4.75019300 & -5.02220900 & 1.41755400 & $\mathrm{H}$ & -2.35735300 & 0.44762800 & 0.98473000 \\
\hline $\mathrm{H}$ & 5.21288400 & -3.44692200 & 2.07913100 & $\mathrm{C}$ & -2.32889500 & -1.56292400 & 0.74447800 \\
\hline $\mathrm{H}$ & 4.92030200 & -2.99420800 & -1.72391700 & $\mathrm{O}$ & -2.67681500 & -2.44765100 & -0.01177900 \\
\hline $\mathrm{H}$ & 6.01970800 & -2.64737700 & -0.36287800 & $\mathrm{C}$ & -2.08738800 & -1.79020600 & 2.20227000 \\
\hline $\mathrm{H}$ & 5.65481800 & -4.31844600 & -0.81780600 & $\mathrm{C}$ & -1.53555000 & -0.83671000 & 3.07514400 \\
\hline $\mathrm{O}$ & 2.22015500 & -2.75142400 & -2.30324900 & $\mathrm{C}$ & -2.44945900 & -3.05176500 & 2.70377000 \\
\hline $\mathrm{O}$ & 0.68306700 & -1.49854100 & -1.28083000 & $\mathrm{C}$ & -1.35499300 & -1.14437300 & 4.42343600 \\
\hline S & -1.58949000 & 0.72820400 & 1.71994700 & $\mathrm{H}$ & -1.20267500 & 0.13544300 & 2.72491500 \\
\hline $\mathrm{F}$ & -2.55067000 & -1.03071400 & 3.42230200 & $\mathrm{C}$ & -2.27410900 & -3.35124000 & 4.05115500 \\
\hline $\mathrm{F}$ & -0.38171700 & -1.08286900 & 3.23997400 & $\mathrm{H}$ & -2.86858900 & -3.77962500 & 2.01759500 \\
\hline $\mathrm{F}$ & -1.33641000 & 0.52240300 & 4.35786600 & $\mathrm{C}$ & -1.72674000 & -2.39739000 & 4.91419900 \\
\hline $\mathrm{O}$ & -2.72481800 & 1.60852000 & 1.95770600 & $\mathrm{H}$ & -0.91516600 & -0.40439300 & 5.08523800 \\
\hline $\mathrm{O}$ & -0.18950600 & 1.33316600 & 1.59075200 & $\mathrm{H}$ & -2.56186000 & -4.32803500 & 4.42928200 \\
\hline $\mathrm{N}$ & -1.58031000 & -0.24106100 & 0.47520300 & $\mathrm{H}$ & -1.58597100 & -2.63184300 & 5.96573500 \\
\hline $\mathrm{C}$ & -2.70009100 & -1.13994100 & 0.07230100 & $\mathrm{C}$ & -3.48188100 & 1.57522900 & -0.79333300 \\
\hline $\mathrm{C}$ & -3.19970800 & -0.76768600 & -1.31507200 & $\mathrm{H}$ & -4.07651400 & 1.61867500 & 0.13457300 \\
\hline $\mathrm{C}$ & -2.42426900 & -0.98848600 & -2.45653300 & $\mathrm{H}$ & -2.61622400 & 2.23838400 & -0.66174500 \\
\hline $\mathrm{C}$ & -2.88521500 & -0.63052000 & -3.72602800 & $\mathrm{C}$ & -4.34425600 & 2.11687700 & -1.95330000 \\
\hline $\mathrm{C}$ & -4.14249000 & -0.04673300 & -3.85996600 & $\mathrm{C}$ & -4.77834100 & 3.55684100 & -1.64120300 \\
\hline $\mathrm{C}$ & -4.93988700 & 0.18279800 & -2.73633100 & $\mathrm{C}$ & -5.55682800 & 1.22304500 & -2.25586700 \\
\hline $\mathrm{C}$ & -4.47175000 & -0.17422700 & -1.46652200 & $\mathrm{H}$ & -3.71246300 & 2.14155800 & -2.85091900 \\
\hline $\mathrm{C}$ & -1.44933300 & -0.27755100 & 3.29854700 & $\mathrm{H}$ & -3.91488100 & 4.20535000 & -1.44940800 \\
\hline $\mathrm{H}$ & -1.43787800 & -1.43092300 & -2.35125300 & $\mathrm{H}$ & -5.33901300 & 3.98483800 & -2.48005200 \\
\hline $\mathrm{H}$ & -2.26270000 & -0.80811600 & -4.59794200 & $\mathrm{H}$ & -5.42787700 & 3.59149600 & -0.75621800 \\
\hline $\mathrm{H}$ & -4.51538300 & 0.23554800 & -4.84118600 & $\mathrm{H}$ & -5.24488700 & 0.24273200 & -2.62815500 \\
\hline $\mathrm{H}$ & -5.91666300 & 0.63692000 & -2.85721000 & $\mathrm{H}$ & -6.17826100 & 1.08035400 & -1.36084100 \\
\hline $\mathrm{O}$ & -5.19217900 & 0.01109100 & -0.31652800 & $\mathrm{H}$ & -6.18778300 & 1.68352600 & -3.02504500 \\
\hline $\mathrm{C}$ & -6.44221100 & 0.67392500 & -0.39423100 & $\mathrm{O}$ & -2.72707500 & -0.61326400 & -3.23522500 \\
\hline $\mathrm{H}$ & -7.16459200 & 0.10591700 & -0.99575000 & $\mathrm{O}$ & -1.03734900 & 0.61648600 & -2.36178800 \\
\hline $\mathrm{H}$ & -6.80586600 & 0.74676300 & 0.63239100 & $\mathrm{~S}$ & 2.12190500 & 1.54365800 & 1.35340700 \\
\hline $\mathrm{H}$ & -6.33685800 & 1.68278700 & -0.81381900 & $\mathrm{~F}$ & 3.55289200 & 3.47434800 & 0.27847600 \\
\hline
\end{tabular}




\begin{tabular}{|c|c|c|c|}
\hline $\mathrm{F}$ & 1.40416300 & 3.82995700 & 0.23154100 \\
\hline $\mathrm{F}$ & 2.49887700 & 4.03705100 & 2.10144000 \\
\hline $\mathrm{O}$ & 3.27660500 & 1.14299800 & 2.15413800 \\
\hline $\mathrm{O}$ & 0.73785600 & 1.52957800 & 1.94491000 \\
\hline $\mathrm{N}$ & 1.89640100 & 0.81740600 & -0.05257100 \\
\hline $\mathrm{C}$ & 2.87262300 & 0.04436600 & -0.84887600 \\
\hline C & 2.12786900 & -1.18618300 & -1.33097300 \\
\hline $\mathrm{C}$ & 0.70472200 & -1.19791100 & -1.42849300 \\
\hline $\mathrm{C}$ & 0.03405100 & -2.40262500 & -1.74504200 \\
\hline $\mathrm{C}$ & 0.76334700 & -3.54542300 & -2.04283600 \\
\hline $\mathrm{C}$ & 2.16087400 & -3.52628600 & -1.99542200 \\
\hline $\mathrm{C}$ & 2.84315600 & -2.35508000 & -1.63971400 \\
\hline $\mathrm{C}$ & 2.40857700 & 3.34982200 & 0.96244900 \\
\hline $\mathrm{H}$ & 0.04164000 & -0.26087800 & -1.94285400 \\
\hline $\mathrm{H}$ & -1.04783300 & -2.41547300 & -1.80864800 \\
\hline $\mathrm{H}$ & 0.25129100 & -4.46222100 & -2.31961800 \\
\hline $\mathrm{H}$ & 2.71231800 & -4.43018700 & -2.22826500 \\
\hline $\mathrm{O}$ & 4.20273200 & -2.26055800 & -1.54584700 \\
\hline $\mathrm{C}$ & 4.98758200 & -3.42493300 & -1.76619800 \\
\hline $\mathrm{H}$ & 4.87479900 & -3.79742600 & -2.79231900 \\
\hline $\mathrm{H}$ & 6.02248000 & -3.11918700 & -1.60542400 \\
\hline $\mathrm{H}$ & 4.72955700 & -4.22242400 & -1.05774000 \\
\hline $\mathrm{C}$ & 3.44443000 & 0.88527000 & -1.99956300 \\
\hline $\mathrm{H}$ & 2.64000900 & 1.22305200 & -2.66042900 \\
\hline $\mathrm{H}$ & 3.96441800 & 1.76328600 & -1.60452400 \\
\hline $\mathrm{H}$ & 4.15664900 & 0.28899500 & -2.57837700 \\
\hline $\mathrm{H}$ & 3.69176200 & -0.28095100 & -0.19685300 \\
\hline
\end{tabular}

$\mathrm{R}_{4 \mathrm{a}}$

$-2277.93913266$

$\begin{array}{lrrr}\mathrm{Pd} & 0.15771000 & 0.15696800 & -0.05530300 \\ \mathrm{C} & -2.73705900 & 0.00970900 & -1.20448100 \\ \mathrm{C} & -2.00554000 & 0.80011700 & -2.28079200 \\ \mathrm{H} & -2.61504500 & -1.04648700 & -1.43489400 \\ \mathrm{~N} & -2.07344300 & 0.24746000 & 0.11369800 \\ \mathrm{H} & -2.13193400 & 1.23841300 & 0.35376800 \\ \mathrm{C} & -2.53477000 & -0.57033300 & 1.20636500 \\ \mathrm{O} & -3.07536300 & -1.63644900 & 0.97281400 \\ \mathrm{C} & -2.32429700 & -0.04268600 & 2.58520700 \\ \mathrm{C} & -1.52480700 & 1.07213600 & 2.89364300 \\ \mathrm{C} & -2.98568600 & -0.72484700 & 3.62022600 \\ \mathrm{C} & -1.39972400 & 1.49413600 & 4.21656000 \\ \mathrm{H} & -0.95291700 & 1.59716300 & 2.13397800 \\ \mathrm{C} & -2.86184900 & -0.29462500 & 4.93766000\end{array}$

\begin{tabular}{|c|c|c|c|}
\hline $\mathrm{H}$ & -3.59145800 & -1.58847500 & 3.36799600 \\
\hline $\mathrm{C}$ & -2.06928100 & 0.81684200 & 5.23795100 \\
\hline & 0.76628300 & 2.34564200 & 4.44570100 \\
\hline$U$ & -3.37956900 & -0.82657400 & 5.73066700 \\
\hline $\mathrm{H}$ & -1.96746400 & 1.15006000 & 6.26710200 \\
\hline$C$ & -4.23794700 & 0.38796600 & -1.17791600 \\
\hline $\mathrm{H}$ & -4.70759900 & -0.21086700 & -0.38957300 \\
\hline $\mathrm{H}$ & -4.32319900 & 1.44349800 & -0.88347200 \\
\hline $\mathrm{C}$ & -5.00801500 & 0.16673300 & -2.49785500 \\
\hline C & -6.46489400 & 0.62002300 & -2.31429400 \\
\hline $\mathrm{C}$ & -4.94092800 & -1.29082700 & -2.98013100 \\
\hline $\mathrm{H}$ & -4.55507500 & 0.79852200 & -3.27409800 \\
\hline $\mathrm{H}$ & -6.52522400 & 1.66767900 & -1.99553100 \\
\hline $\mathrm{H}$ & -7.02292700 & 0.52201800 & -3.25218800 \\
\hline $\mathrm{H}$ & -6.97499800 & 0.00956200 & -1.55803800 \\
\hline $\mathrm{H}$ & -3.92342000 & -1.57898600 & -3.26256300 \\
\hline $\mathrm{H}$ & -5.29370400 & -1.98123400 & -2.20300600 \\
\hline $\mathrm{H}$ & -5.57531800 & -1.43006900 & -3.86279600 \\
\hline $\mathrm{O}$ & -1.83627200 & 0.42908700 & -3.41657900 \\
\hline $\mathrm{O}$ & -1.62934200 & 2.02665000 & -1.82897600 \\
\hline S & 2.49157700 & 1.79632300 & 0.51725200 \\
\hline $\mathrm{F}$ & 4.29166500 & 2.55254800 & -1.28589100 \\
\hline $\mathrm{F}$ & 2.23569300 & 3.04627800 & -1.80743800 \\
\hline $\mathrm{F}$ & 3.27497100 & 4.18696100 & -0.27241400 \\
\hline $\mathrm{O}$ & 3.58141600 & 1.72996100 & 1.49407500 \\
\hline $\mathrm{O}$ & 1.17857400 & 2.40399500 & 0.87396400 \\
\hline $\mathrm{N}$ & 2.13588300 & 0.42620300 & -0.23690600 \\
\hline $\mathrm{C}$ & 3.02139700 & -0.76584700 & -0.28519700 \\
\hline $\mathrm{C}$ & 2.07973500 & -1.95229400 & -0.23229500 \\
\hline $\mathrm{C}$ & 0.68976400 & -1.74335000 & -0.21235800 \\
\hline $\mathrm{C}$ & -0.21560200 & -2.80517100 & -0.20878900 \\
\hline $\mathrm{C}$ & 0.28080100 & -4.10922000 & -0.20796100 \\
\hline $\mathrm{C}$ & 1.65455200 & -4.35563500 & -0.19066700 \\
\hline 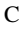 & 2.55133500 & -3.28183600 & -0.19234100 \\
\hline $\mathrm{C}$ & 3.11510300 & 2.96963000 & -0.79905300 \\
\hline $\mathrm{H}$ & 1.14549300 & 2.46958400 & -2.55267000 \\
\hline $\mathrm{H}$ & -1.28671200 & -2.63991500 & -0.17554000 \\
\hline $\mathrm{H}$ & -0.41159800 & -4.94727800 & -0.20461700 \\
\hline $\mathrm{H}$ & 2.01504300 & -5.37729600 & -0.16755000 \\
\hline 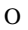 & 3.91224200 & -3.42735900 & -0.13973300 \\
\hline$C$ & 4.45232400 & -4.73605700 & -0.05351300 \\
\hline $\mathrm{H}$ & 4.20125100 & -5.33642800 & -0.93793800 \\
\hline $\mathrm{H}$ & 5.53515600 & -4.60873600 & 0.00001600 \\
\hline $\mathrm{H}$ & 10285900 & -5.25705800 & 84736 \\
\hline
\end{tabular}

$0.81684200-5.23795100$

$\begin{array}{llll}\mathrm{H} & -0.76628300 & 2.34564200 & 4.44570100\end{array}$

$\begin{array}{lll}-3.37956900 & -0.82657400 & 5.73066700\end{array}$

$\begin{array}{llll}\text { C } & -4.23794700 & 0.38796600 & -1.17791600\end{array}$

$\begin{array}{llll}-4.70759900 & -0.21086700 & -0.38957300\end{array}$

$\begin{array}{llll}H & -4.32319900 & 1.44349800 & -0.88347200\end{array}$

$\begin{array}{llll}\text { C } & -4.94092800 & -1.29082700 & -2.98013100\end{array}$

$\begin{array}{llll}-7.02292700 & 0.52201800 & -3.25218800\end{array}$

$\begin{array}{llll}-3.92342000 & -1.57898600 & -3.26256300\end{array}$

$\begin{array}{llll}-1.83627200 & 0.42908700 & -3.41657900\end{array}$

$\begin{array}{lll}2.49157700 & 1.79632300 & 0.51725200\end{array}$

$2.23569300-3.04627800-1.80743800$

$\begin{array}{llll} & 3.27497100 & 4.18696100 & -0.27241400\end{array}$

$\begin{array}{llll}\text { O } & 3.58141600 & 1.72996100 & 1.49407500\end{array}$

$\begin{array}{llll} & 1.17857400 & 2.40399500 & 0.87396400\end{array}$

$\begin{array}{llll}2.13588300 & 0.42620300 & -0.23690600\end{array}$

$\begin{array}{lll}3.02139700 & -0.76584700 & -0.28519700\end{array}$

$\begin{array}{llll}\text { C } & 0.68976400 & -1.74335000 & -0.21235800\end{array}$

$\begin{array}{llll}\text { C } & 1.65455200 & -4.35563500 & -0.19066700\end{array}$

$3.11510300-296963000-0.79905300$

$\begin{array}{lll}-128671200 & -2.63991500 & -0.17554000\end{array}$

\begin{tabular}{l}
$2.01504300-5.37729600-0.16755000$ \\
\hline
\end{tabular}

$\begin{array}{llll}\text { O } & 3.91224200 & -3.42735900 & -0.13973300\end{array}$

$\begin{array}{llll}\text { C } & 4.45232400 & -4.73605700 & -0.05351300\end{array}$

$\begin{array}{lll}4.20125100 & -5.33642800 & -0.93793800\end{array}$

H $\quad 4.10285900 \quad-5.25705800 \quad 0.84736300$ 


$$
\begin{array}{llll}
\mathrm{C} & 3.88355500 & -0.73510200 & -1.55867400 \\
\mathrm{H} & 3.23956800 & -0.69531900 & -2.44366100 \\
\mathrm{H} & 4.52711500 & 0.14857900 & -1.55789800 \\
\mathrm{H} & 4.51244700 & -1.62609700 & -1.61448600 \\
\mathrm{H} & 3.67954500 & -0.77315800 & 0.59369100
\end{array}
$$

\begin{tabular}{|c|c|c|c|}
\hline $\mathrm{Pd}$ & 0.34136400 & -0.98624700 & 0.02438300 \\
\hline $\mathrm{O}$ & -0.99943900 & 0.42401400 & 2.48351200 \\
\hline 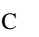 & -1.92279500 & -1.48589000 & 3.61934600 \\
\hline $\mathrm{C}$ & -1.19666000 & -0.80539100 & 2.46967900 \\
\hline 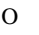 & -0.86571500 & -1.61697500 & 1.51303700 \\
\hline$c$ & 0.92868300 & 1.76904300 & -0.45208400 \\
\hline $\mathrm{C}$ & 1.84377700 & 0.96234800 & -1.39378200 \\
\hline $\mathrm{O}$ & 1.63639400 & -0.35549900 & -1.37573200 \\
\hline 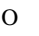 & 2.71121700 & 1.48938500 & -2.05570100 \\
\hline $\mathrm{H}$ & 0.64485400 & 2.67933100 & -0.98388300 \\
\hline $\mathrm{H}$ & -2.95570900 & -1.69339100 & 3.31545300 \\
\hline $\mathrm{H}$ & -1.45238600 & -2.44209700 & 3.86252500 \\
\hline $\mathrm{H}$ & -1.93426000 & -0.83084500 & 4.49259000 \\
\hline $\mathrm{N}$ & -0.32359400 & 0.98917200 & -0.15669100 \\
\hline $\mathrm{H}$ & -0.67289100 & 1.10578300 & 0.81458300 \\
\hline 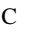 & -1.31222700 & 1.05950200 & -1.22245800 \\
\hline $\mathrm{O}$ & -0.92590900 & 1.31721200 & -2.34523700 \\
\hline $\mathrm{C}$ & 2.16678900 & -3.31676300 & -0.56729300 \\
\hline $\mathrm{C}$ & 2.64078000 & -4.73442300 & -0.40324000 \\
\hline $\mathrm{H}$ & 3.70013100 & -4.73608600 & -0.12764200 \\
\hline $\mathrm{H}$ & 2.05025400 & -5.24441300 & 0.35753900 \\
\hline $\mathrm{H}$ & 2.55110600 & -5.25769100 & -1.36114600 \\
\hline 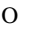 & 1.18775300 & -2.90654800 & 0.08847400 \\
\hline $\mathrm{O}$ & 2.85518100 & -2.61384900 & -1.42462500 \\
\hline $\mathrm{H}$ & 2.50123200 & -1.66466800 & -1.51803600 \\
\hline C & -2.74744800 & 0.81227900 & -0.90643800 \\
\hline C & -3.30973800 & 0.91945400 & 0.37509300 \\
\hline $\mathrm{C}$ & -3.57756700 & 0.52110500 & -2.00378400 \\
\hline $\mathrm{C}$ & -4.68096100 & 0.72887500 & 0.54943400 \\
\hline$\Pi$ & -2.69827500 & 1.14423600 & 1.24150000 \\
\hline C & -4.94098700 & 0.31816200 & -1.82017000 \\
\hline $\mathrm{H}$ & -3.13159900 & 0.46049300 & -2.99054400 \\
\hline$C$ & -5.49637100 & 0.42318200 & -0.54122800 \\
\hline$\Pi$ & -5.11068800 & 0.82148500 & 1.54280500 \\
\hline $\mathrm{H}$ & -5.57205600 & 0.08243100 & -2.67227700 \\
\hline $\mathrm{H}$ & -6.56252400 & 0.27020800 & -0.39695700 \\
\hline
\end{tabular}

$\mathrm{L}_{1}$

$-1369.83383907$

\begin{tabular}{|c|c|c|c|}
\hline $\mathrm{Pd}$ & -0.50979800 & -0.43262000 & 0.03437900 \\
\hline $\mathrm{O}$ & 2.43434500 & -1.25642900 & -0.72535800 \\
\hline $\mathrm{C}$ & 2.36891800 & -3.62824300 & -0.52292000 \\
\hline $\mathrm{C}$ & 1.71628600 & -2.27078800 & -0.41658000 \\
\hline $\mathrm{O}$ & 0.51513100 & -2.22096900 & -0.02156100 \\
\hline $\mathrm{C}$ & 0.81530800 & 1.92986600 & -0.79670300 \\
\hline $\mathrm{C}$ & -0.66106200 & 2.32510100 & -0.56376400 \\
\hline $\mathrm{O}$ & -1.42617800 & 1.34358800 & -0.08260700 \\
\hline $\mathrm{O}$ & -1.08740200 & 3.43242500 & -0.81904000 \\
\hline $\mathrm{H}$ & 1.42444800 & 2.78126700 & -0.48625800 \\
\hline $\mathrm{H}$ & 3.13021500 & -3.71802200 & 0.26000700 \\
\hline $\mathrm{H}$ & 1.63100400 & -4.42245900 & -0.40519300 \\
\hline $\mathrm{H}$ & 2.87731400 & -3.71674400 & -1.48742900 \\
\hline $\mathrm{N}$ & 1.15534100 & 0.73929700 & 0.04419900 \\
\hline $\mathrm{H}$ & 1.88074600 & -0.21366900 & -0.44528600 \\
\hline $\mathrm{C}$ & 1.62504700 & 0.98281100 & 1.37851500 \\
\hline $\mathrm{O}$ & 1.16833400 & 0.35898900 & 2.32067200 \\
\hline $\mathrm{C}$ & -3.47407700 & -1.04358300 & -0.11103100 \\
\hline $\mathrm{C}$ & -4.67686800 & -1.94669800 & -0.10570000 \\
\hline $\mathrm{H}$ & -5.30956500 & -1.72450300 & -0.97044400 \\
\hline $\mathrm{H}$ & -4.36374800 & -2.99052800 & -0.12032700 \\
\hline $\mathrm{H}$ & -5.27037600 & -1.74938400 & 0.79365500 \\
\hline $\mathrm{O}$ & -2.32719900 & -1.51968300 & -0.02906900 \\
\hline $\mathrm{O}$ & -3.76196200 & 0.22985900 & -0.19995200 \\
\hline $\mathrm{H}$ & -2.93426000 & 0.81332300 & -0.18301300 \\
\hline $\mathrm{C}$ & 2.75422100 & 1.94899500 & 1.56540400 \\
\hline $\mathrm{C}$ & 3.72926500 & 2.17732800 & 0.58202200 \\
\hline $\mathrm{C}$ & .87019100 & 2.58322000 & 2.81193900 \\
\hline
\end{tabular}

$\begin{array}{llll}\mathrm{C} & 1.62485800 & 2.12009200 & 0.87992600 \\ \mathrm{H} & 0.90528100 & 2.68281200 & 1.49148500 \\ \mathrm{H} & 1.82851800 & 1.19133700 & 1.42924200 \\ \mathrm{C} & 2.92773800 & 2.93852700 & 0.76330300 \\ \mathrm{C} & 3.49287700 & 3.18518200 & 2.17087300 \\ \mathrm{C} & 2.73125700 & 4.26189300 & 0.00837000 \\ \mathrm{H} & 3.65697300 & 2.34192700 & 0.20179200 \\ \mathrm{H} & 3.65612000 & 2.24456800 & 2.71086200 \\ \mathrm{H} & 4.45235200 & 3.71219600 & 2.11824300 \\ \mathrm{H} & 2.80806600 & 3.79913200 & 2.77047900 \\ \mathrm{H} & 2.48331400 & 4.08833600 & -1.04309900 \\ \mathrm{H} & 1.93501300 & 4.86649100 & 0.46398700 \\ \mathrm{H} & 3.65169400 & 4.85659400 & 0.03262900\end{array}$

$\mathrm{TS}_{\mathrm{L}}$

$-1369.81928295$ 


\begin{tabular}{|c|c|c|c|}
\hline C & 4.79800300 & 3.03502300 & 0.84108300 \\
\hline $\mathrm{H}$ & 3.66985700 & 1.66544600 & -0.37376100 \\
\hline C & 3.92833200 & 3.45357900 & 3.06066400 \\
\hline I & 2.12277100 & 2.38017600 & 3.57185300 \\
\hline 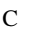 & 4.89416200 & 3.68040200 & 2.07591100 \\
\hline I & 5.55587600 & 3.19729300 & 0.07988200 \\
\hline $\mathrm{H}$ & 4.00411200 & 3.95120900 & 4.02334000 \\
\hline $\mathrm{H}$ & 5.72302200 & 4.35501100 & 2.27249000 \\
\hline $\mathrm{C}$ & 1.09316600 & 1.61637900 & -2.28324700 \\
\hline $\mathrm{H}$ & 2.15786500 & 1.35472700 & -2.36880700 \\
\hline $\mathrm{H}$ & 0.53220200 & 0.71168600 & -2.55665900 \\
\hline $\mathrm{C}$ & 0.77165200 & 2.73490600 & -3.29566100 \\
\hline $\mathrm{C}$ & 1.09082600 & 2.24067200 & -4.71540300 \\
\hline $\mathrm{C}$ & 1.51508100 & 4.04300200 & -2.98615000 \\
\hline$\Pi$ & -0.30341300 & 2.94565000 & -3.24201900 \\
\hline $\mathrm{H}$ & 0.54364200 & 1.32057000 & -4.95473500 \\
\hline $\mathrm{H}$ & 0.81867100 & 2.99586100 & -5.46170500 \\
\hline $\mathrm{H}$ & 2.16254000 & 2.03055100 & -4.83061600 \\
\hline $\mathrm{H}$ & 1.16904300 & 4.48287000 & -2.04605100 \\
\hline $\mathrm{H}$ & 2.59965500 & 3.87964600 & -2.92095100 \\
\hline & 1.33946200 & 4.78102400 & -3.77748400 \\
\hline
\end{tabular}

$\mathrm{L}_{2}$

$-1369.82226551$

\begin{tabular}{llll}
$\mathrm{Pd}$ & 1.46530400 & -0.40108900 & -0.08922600 \\
$\mathrm{O}$ & -0.16019400 & -1.77494500 & 2.33312800 \\
$\mathrm{C}$ & 1.30206900 & -3.51675900 & 3.00401300 \\
$\mathrm{C}$ & 0.98557100 & -2.36170600 & 2.09686100 \\
$\mathrm{O}$ & 1.77691000 & -2.02500400 & 1.19824900 \\
$\mathrm{C}$ & -0.93140100 & 1.14054200 & -0.03312600 \\
$\mathrm{C}$ & 0.00737200 & 1.83410200 & -1.04096200 \\
$\mathrm{O}$ & 1.17356600 & 1.20651100 & -1.22272000 \\
$\mathrm{O}$ & -0.26978100 & 2.87131500 & -1.60615100 \\
$\mathrm{H}$ & -1.93884200 & 1.20723100 & -0.44853900 \\
$\mathrm{H}$ & 0.65800200 & -4.36189900 & 2.73557200 \\
$\mathrm{H}$ & 2.34665100 & -3.80763000 & 2.89232100 \\
$\mathrm{H}$ & 1.08313900 & -3.24943500 & 4.04157200 \\
$\mathrm{~N}$ & -0.54615000 & -0.28619900 & 0.11938200 \\
$\mathrm{H}$ & -0.36309300 & -1.08743000 & 1.60223600 \\
$\mathrm{C}$ & -1.20803100 & -1.28020900 & -0.61045300 \\
$\mathrm{O}$ & -0.60981700 & -2.27434800 & -1.01441800 \\
$\mathrm{C}$ & 4.20854000 & 0.43636700 & -1.10770400 \\
$\mathrm{C}$ & 5.69792800 & 0.29714500 & -1.26718300 \\
\hline & 6.18697800 & 1.24029700 & -1.00446500
\end{tabular}

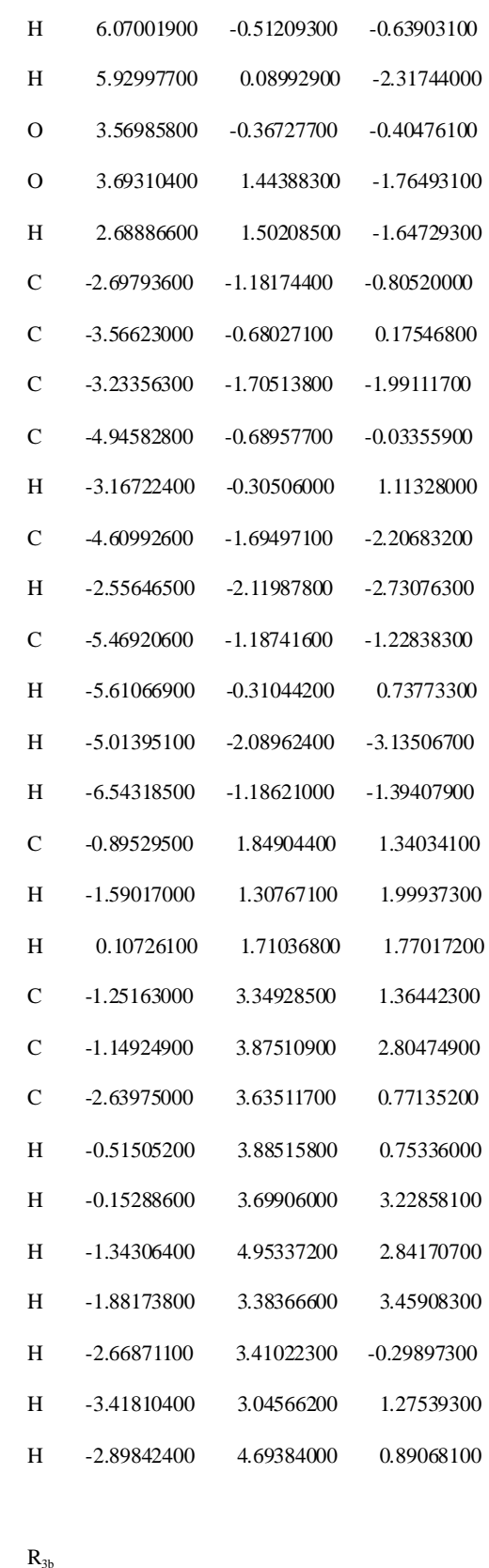

\section{$-2277.93520796$}

$\begin{array}{llll}\text { Pd } & -0.32314200 & -0.43616100 & 0.31968600\end{array}$

$\begin{array}{llll}\text { C } & 2.16576100 & 0.58720900 & 1.27873500\end{array}$

$\begin{array}{llll}\text { C } & 1.02031600 & 1.46931400 & 1.86534000\end{array}$

$\begin{array}{llll}\text { O } & -0.22203800 & 1.17882900 & 1.50325300\end{array}$

$\begin{array}{llll}\text { O } & 1.27574800 & 2.41287800 & 2.59439400\end{array}$

$\begin{array}{llll}\mathrm{H} & 2.82743300 & 0.33569600 & 2.11486300\end{array}$

$\begin{array}{llll}\mathrm{N} & 1.55004700 & -0.63015600 & 0.74506200\end{array}$

$\begin{array}{llll}\text { C } & 1.84977300 & -1.61431900 & -0.10171200\end{array}$

$\begin{array}{llll}\text { O } & 0.81578800 & -2.10169700 & -0.69483000\end{array}$

$\begin{array}{llll}\text { C } & 3.19472500 & -2.18257000 & -0.34437100\end{array}$

$\begin{array}{llll}\text { C } & 4.24560700 & -2.01457600 & 0.57030700\end{array}$

$\begin{array}{llll}\text { C } & 3.40284300 & -2.93759200 & -1.50864000\end{array}$ 


\begin{tabular}{|c|c|c|c|c|c|c|c|}
\hline $\mathrm{C}$ & 5.49192600 & -2.58436900 & 0.31531100 & $\mathrm{O}$ & -2.68984700 & 2.68831300 & 0.26632400 \\
\hline $\mathrm{H}$ & 4.08287900 & -1.46105500 & 1.48946800 & $\mathrm{C}$ & -2.55101900 & 3.51415100 & 1.43224500 \\
\hline $\mathrm{C}$ & 4.65223700 & -3.49818000 & -1.76343300 & $\mathrm{H}$ & -2.77369600 & 4.56013200 & 1.19420200 \\
\hline $\mathrm{H}$ & 2.57579000 & -3.07403400 & -2.19712000 & $\mathrm{H}$ & -3.29195000 & 3.13920400 & 2.13947300 \\
\hline $\mathrm{C}$ & 5.69838500 & -3.32159000 & -0.85345100 & $\mathrm{H}$ & -1.54695700 & 3.40870400 & 1.85340700 \\
\hline $\mathrm{H}$ & 6.29954900 & -2.45805500 & 1.03072200 & $\mathrm{H}$ & -3.71115800 & 0.92630300 & -1.56566500 \\
\hline $\mathrm{H}$ & 4.81060000 & -4.07626800 & -2.66953200 & $\mathrm{H}$ & -2.50447600 & 0.82816800 & 0.48822000 \\
\hline $\mathrm{H}$ & 6.67148500 & -3.76264800 & -1.05176600 & & & & \\
\hline $\mathrm{C}$ & 2.95410500 & 1.35927200 & 0.19004600 & \multicolumn{4}{|c|}{$\mathrm{TS}_{\mathrm{R} 2 \mathrm{~b}}$} \\
\hline $\mathrm{H}$ & 3.70711200 & 0.67206700 & -0.21836400 & \multicolumn{4}{|c|}{-2277.90396691} \\
\hline $\mathrm{H}$ & 2.25700400 & 1.58322300 & -0.62866200 & $\mathrm{Pd}$ & 0.25005500 & -0.08408100 & -0.25435200 \\
\hline $\mathrm{C}$ & 3.65885400 & 2.65945600 & 0.62900400 & $\mathrm{C}$ & -2.54342500 & -0.75878600 & -0.55133500 \\
\hline $\mathrm{C}$ & 4.35854000 & 3.28801300 & -0.58651400 & $\mathrm{C}$ & -1.75646100 & -2.08351000 & -0.39687800 \\
\hline $\mathrm{C}$ & 4.65090000 & 2.43410300 & 1.77981300 & $\mathrm{O}$ & -0.44128300 & -1.96794500 & -0.40162700 \\
\hline $\mathrm{H}$ & 2.89340600 & 3.35789700 & 0.98462600 & $\mathrm{O}$ & -2.33550200 & -3.15454500 & -0.33202500 \\
\hline $\mathrm{H}$ & 3.65092300 & 3.47312700 & -1.40410300 & $\mathrm{H}$ & -3.45178500 & -0.83160400 & 0.05124700 \\
\hline $\mathrm{H}$ & 4.82122300 & 4.24547200 & -0.31953300 & $\mathrm{~N}$ & -1.69249400 & 0.33121000 & -0.05388200 \\
\hline $\mathrm{H}$ & 5.15056800 & 2.63251700 & -0.97280500 & $\mathrm{C}$ & -1.97017900 & 1.22292100 & 0.88856500 \\
\hline $\mathrm{H}$ & 4.13252000 & 2.14327600 & 2.69877000 & $\mathrm{O}$ & -1.04132400 & 1.94277100 & 1.40643500 \\
\hline $\mathrm{H}$ & 5.38595900 & 1.65695400 & 1.52806100 & $\mathrm{C}$ & -3.35069700 & 1.45911200 & 1.41751400 \\
\hline $\mathrm{H}$ & 5.20585700 & 3.35461300 & 1.99629800 & $\mathrm{C}$ & -4.48290200 & 1.52597100 & 0.59378100 \\
\hline $\mathrm{S}$ & -3.70685600 & -1.01088000 & 0.49035700 & $\mathrm{C}$ & -3.49284300 & 1.67621700 & 2.79726300 \\
\hline $\mathrm{F}$ & -2.33685100 & -2.98060500 & -0.65254500 & $\mathrm{C}$ & -5.73838100 & 1.78862400 & 1.14351000 \\
\hline $\mathrm{F}$ & -1.99797800 & -2.77694700 & 1.49184100 & $\mathrm{H}$ & -4.38389000 & 1.39921200 & -0.47855300 \\
\hline $\mathrm{F}$ & -3.87808300 & -3.59072600 & 0.76024800 & $\mathrm{C}$ & -4.75029100 & 1.91969400 & 3.34475400 \\
\hline $\mathrm{O}$ & -4.80770500 & -1.10196500 & -0.46401900 & $\mathrm{H}$ & -2.61030800 & 1.65210600 & 3.42739800 \\
\hline $\mathrm{O}$ & -3.89981000 & -0.61077700 & 1.87876800 & $\mathrm{C}$ & -5.87645400 & 1.97690400 & 2.51940400 \\
\hline$N$ & -2.43630400 & 0.01130300 & -0.14428100 & $\mathrm{H}$ & -6.60715100 & 1.84753900 & 0.49384800 \\
\hline $\mathrm{C}$ & -2.67274100 & 0.57621700 & -1.54592400 & $\mathrm{H}$ & -4.85078000 & 2.07278300 & 4.41567500 \\
\hline $\mathrm{C}$ & -1.74635000 & 1.78113500 & -1.69957300 & $\mathrm{H}$ & -6.85593400 & 2.17392600 & 2.94655000 \\
\hline $\mathrm{C}$ & -0.84698500 & 1.89284000 & -2.76436000 & $\mathrm{C}$ & -2.90145200 & -0.52902300 & -2.04038600 \\
\hline $\mathrm{C}$ & -0.01573400 & 3.00639600 & -2.90790700 & $\mathrm{H}$ & -3.43550800 & 0.42942700 & -2.11610200 \\
\hline $\mathrm{C}$ & -0.07030400 & 4.02907700 & -1.96435500 & $\mathrm{H}$ & -1.96049300 & -0.39641500 & -2.59147900 \\
\hline $\mathrm{C}$ & -0.95226700 & 3.94609100 & -0.88560000 & $\mathrm{C}$ & -3.74058300 & -1.62700600 & -2.72524900 \\
\hline $\mathrm{C}$ & -1.79080000 & 2.83709400 & -0.75855000 & $\mathrm{C}$ & -3.98131500 & -1.24406800 & -4.19376300 \\
\hline $\mathrm{C}$ & -2.90499600 & -2.71496700 & 0.52243100 & $\mathrm{C}$ & -5.06703900 & -1.89708400 & -1.99935600 \\
\hline $\mathrm{H}$ & -0.78696700 & 1.09776600 & -3.49852400 & $\mathrm{H}$ & -3.16079700 & -2.55764300 & -2.70584000 \\
\hline $\mathrm{H}$ & 0.66755600 & 3.06657800 & -3.74954300 & $\mathrm{H}$ & -3.03687100 & -1.07585700 & -4.72586400 \\
\hline $\mathrm{H}$ & 0.57452000 & 4.89844700 & -2.05806100 & $\mathrm{H}$ & -4.52555600 & -2.03652800 & -4.72059300 \\
\hline $\mathrm{H}$ & -0.97801300 & 4.73868200 & -0.14780700 & $\mathrm{H}$ & -4.57684800 & -0.32423200 & -4.27205100 \\
\hline $\mathrm{C}$ & -2.49578800 & -0.50838100 & -2.60385300 & $\mathrm{H}$ & -4.89249000 & -2.32115400 & -1.00615800 \\
\hline $\mathrm{H}$ & -1.48411100 & -0.92625900 & -2.58809800 & $\mathrm{H}$ & -5.66040700 & -0.97804800 & -1.89233000 \\
\hline $\mathrm{H}$ & -3.21224500 & -1.31593400 & -2.44700400 & $\mathrm{H}$ & -5.67379000 & -2.61668300 & -2.56173700 \\
\hline $\mathrm{H}$ & -2.68959800 & -0.08821500 & -3.59520800 & $\mathrm{~S}$ & 3.17192200 & -2.05170500 & 0.10055300 \\
\hline
\end{tabular}




\begin{tabular}{|c|c|c|c|}
\hline $\mathrm{F}$ & 1.30361500 & -2.09665700 & 2.03099200 \\
\hline $\mathrm{F}$ & 1.30609300 & -3.83887700 & 0.71100600 \\
\hline $\mathrm{F}$ & 2.87731000 & -3.58481000 & 2.18441500 \\
\hline $\mathrm{O}$ & 4.43040500 & -1.81219900 & 0.80151100 \\
\hline $\mathrm{O}$ & 3.10066500 & -2.75509000 & -1.17322100 \\
\hline $\mathrm{N}$ & 2.40294000 & -0.48001200 & -0.19581200 \\
\hline $\mathrm{C}$ & 3.01114900 & 0.71405100 & 0.54209100 \\
\hline $\mathrm{C}$ & 2.40160100 & 1.90972900 & -0.16009400 \\
\hline $\mathrm{C}$ & 0.99790300 & 1.97301900 & -0.31290900 \\
\hline 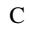 & 0.44712900 & 2.99538300 & -1.10919600 \\
\hline $\mathrm{C}$ & 1.26823200 & 3.94886500 & -1.70388300 \\
\hline $\mathrm{C}$ & 2.65302000 & 3.90134700 & -1.53056800 \\
\hline $\mathrm{C}$ & 3.22686500 & 2.87631800 & -0.76573200 \\
\hline $\mathrm{C}$ & 2.04804400 & -2.96302200 & 1.34711200 \\
\hline $\mathrm{H}$ & 0.09827400 & 1.77112800 & 0.64469400 \\
\hline $\mathrm{H}$ & -0.63065400 & 3.05734400 & -1.22274700 \\
\hline $\mathrm{H}$ & 0.83676500 & 4.74363500 & -2.30645000 \\
\hline $\mathrm{H}$ & 3.27652000 & 4.65473600 & -1.99803900 \\
\hline $\mathrm{C}$ & 2.71769400 & 0.67955900 & 2.04303000 \\
\hline $\mathrm{H}$ & 1.64426600 & 0.71180600 & 2.24870300 \\
\hline $\mathrm{H}$ & 3.15149000 & -0.20465100 & 2.51622000 \\
\hline 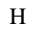 & 3.17966500 & 1.56203100 & 2.49675100 \\
\hline $\mathrm{O}$ & 4.57112800 & 2.73560800 & -0.56791300 \\
\hline $\mathrm{C}$ & 5.45469400 & 3.66457400 & -1.18166600 \\
\hline $\mathrm{H}$ & 5.27893700 & 4.68469400 & -0.81767900 \\
\hline $\mathrm{H}$ & 6.45996700 & 3.34822500 & -0.89935300 \\
\hline $\mathrm{H}$ & 5.36065100 & 3.64639800 & -2.27497500 \\
\hline $\mathrm{H}$ & 4.08988200 & 0.68914200 & 0.38511000 \\
\hline 11 & 2.60868300 & -0.36912100 & -1.19608700 \\
\hline
\end{tabular}

$\mathrm{R}_{4 \mathrm{~b}}$

$\begin{array}{llll}-2277.93475246 & & \\ \text { Pd } & -0.28053600 & -0.06314900 & 0.14943300 \\ \text { C } & 2.49028000 & -0.81612900 & 0.43769100 \\ \text { C } & 1.74086900 & -2.10799700 & -0.00416600 \\ \text { O } & 0.45373900 & -1.98535000 & -0.15147300 \\ \text { O } & 2.38601300 & -3.13839100 & -0.14773600 \\ \text { H } & 3.47867700 & -0.83315000 & -0.02415100 \\ \text { N } & 1.72146200 & 0.34715000 & -0.05541100 \\ \text { C } & 2.19797500 & 1.26065400 & -0.83889300 \\ \text { O } & 1.38107200 & 2.14965300 & -1.41732800 \\ \text { C } & 3.61586000 & 1.46855800 & -1.22995300 \\ \text { C } & 4.65956500 & 1.42285500 & -0.29388800 \\ \text { C } & 3.90418000 & 1.76442800 & -2.57211100\end{array}$

\begin{tabular}{|c|c|c|c|}
\hline C & 5.97373100 & 1.65128000 & -0.69970000 \\
\hline & 4.44369200 & 1.23284900 & 0.75182700 \\
\hline & 5.22082900 & 1.97732400 & -2.97422700 \\
\hline 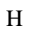 & 3.09500700 & 1.81868100 & -3.29245100 \\
\hline $\mathrm{C}$ & 6.25765900 & 1.92113900 & -2.03979300 \\
\hline I & 6.77452300 & 1.62045300 & 0.03330000 \\
\hline $\mathrm{H}$ & 5.43662700 & 2.19128000 & -4.01701000 \\
\hline $\mathrm{H}$ & 7.28336600 & 2.09225800 & -2.35429300 \\
\hline C & 2.61677700 & -0.74873100 & 1.97630400 \\
\hline $\mathrm{H}$ & 3.14632800 & 0.18025300 & 2.23866600 \\
\hline $\mathrm{H}$ & 1.60537500 & -0.64967300 & 2.39343300 \\
\hline C & 3.32748800 & -1.93986200 & 2.65075700 \\
\hline C & 3.33515200 & -1.73292000 & 4.17319500 \\
\hline $\mathrm{C}$ & 4.74941800 & -2.16149800 & 2.11369300 \\
\hline $\mathrm{H}$ & 2.74931800 & -2.84517400 & 2.43020700 \\
\hline $\mathrm{H}$ & 2.31977200 & -1.60034700 & 4.56653500 \\
\hline $\mathrm{H}$ & 3.78098700 & -2.59517100 & 4.68237200 \\
\hline $\mathrm{H}$ & 3.91939100 & -0.84500200 & 4.45123200 \\
\hline $\mathrm{H}$ & 4.72643700 & -2.47023700 & 1.06468800 \\
\hline $\mathrm{H}$ & 5.35832100 & -1.25078100 & 2.20575800 \\
\hline $\mathrm{H}$ & 5.25509200 & -2.95293300 & 2.67947900 \\
\hline S & -3.09421100 & -2.12457100 & 0.06056000 \\
\hline F & -1.59758600 & -1.87173700 & -2.16932400 \\
\hline F & -1.44418300 & -3.80375600 & -1.15898000 \\
\hline F & -3.23466500 & -3.29022400 & -2.27101200 \\
\hline $\mathrm{O}$ & 4.49750300 & -1.93055800 & -0.29401800 \\
\hline $\mathrm{O}$ & -2.67814300 & -2.92150300 & 1.20571200 \\
\hline $\mathrm{N}$ & -2.37500800 & -0.52224400 & 0.33114200 \\
\hline $\mathrm{C}$ & -3.16809700 & 0.67341600 & -0.18318500 \\
\hline$C$ & -2.43235400 & 1.86276600 & 0.39267400 \\
\hline $\mathrm{C}$ & -1.03987200 & 1.76681800 & 0.57391500 \\
\hline $\mathrm{C}$ & -0.35701800 & 2.85197400 & 1.14675900 \\
\hline $\mathrm{C}$ & -1.05239900 & 4.00840800 & 1.50281700 \\
\hline $\mathrm{C}$ & -2.43142200 & 4.11303100 & 1.30466400 \\
\hline $\mathrm{C}$ & -3.12742700 & 3.03345600 & 0.74946700 \\
\hline $\mathrm{C}$ & -2.24573500 & -2.82487700 & -1.50731600 \\
\hline $\mathrm{H}$ & 0.46183600 & 1.97008400 & -1.11041400 \\
\hline $\mathrm{H}$ & 0.71356700 & 2.79808300 & 1.32114900 \\
\hline $\mathrm{H}$ & -0.51789100 & 4.84654300 & 1.94342900 \\
\hline $\mathrm{H}$ & -2.94857100 & 5.02286200 & 1.58646800 \\
\hline $\mathrm{C}$ & -3.23372000 & 0.70962800 & -1.71156100 \\
\hline $\mathrm{H}$ & -2.23046500 & 0.76533700 & -2.14397600 \\
\hline $\mathrm{H}$ & -3.76184000 & -0.15516800 & -2.12208200 \\
\hline $\mathrm{H}$ & .78708700 & 1.60847000 & 1.9993410 \\
\hline
\end{tabular}




$\begin{array}{llll}\mathrm{O} & -4.47539400 & 3.02172300 & 0.51241500 \\ \mathrm{C} & -5.24011500 & 4.15707600 & 0.89176500 \\ \mathrm{H} & -4.92426800 & 5.05459800 & 0.34466600 \\ \mathrm{H} & -6.27366200 & 3.92100500 & 0.63272000 \\ \mathrm{H} & -5.16917900 & 4.34673600 & 1.97044800 \\ \mathrm{H} & -4.18318200 & 0.60550000 & 0.21587500 \\ \mathrm{H} & -2.40543300 & -0.49167200 & 1.35827200\end{array}$

$\mathrm{R}_{\mathrm{lc}}$

$-2507.04994504$

$\begin{array}{llll}\mathrm{Pd} & 0.12428100 & -0.32190500 & -0.05366000\end{array}$

$\begin{array}{llll}\text { C } & 1.98472700 & -2.38788700 & -0.67297300\end{array}$

$\begin{array}{llll}\text { C } & 0.95371300 & -2.34783800 & -1.82037000\end{array}$

$\begin{array}{llll}\mathrm{H} & 2.95096800 & -2.63587300 & -1.11664400\end{array}$

$\begin{array}{llll}\mathrm{N} & 2.10599200 & -1.02800300 & -0.02921100\end{array}$

$\begin{array}{llll}\mathrm{H} & 2.22501800 & -1.06022600 & 0.99823500\end{array}$

$\begin{array}{llll}\text { C } & 2.97293200 & -0.12105300 & -0.75419900\end{array}$

$\begin{array}{llll}\text { O } & 3.08507000 & -0.27070900 & -1.95641400\end{array}$

$\begin{array}{llll}\text { C } & 3.71666200 & 0.93982500 & -0.01321700\end{array}$

$\begin{array}{llll}\text { C } & 3.82696100 & 1.01326700 & 1.38397900\end{array}$

$\begin{array}{llll}\text { C } & 4.39296500 & 1.88322900 & -0.80773600\end{array}$

$\begin{array}{llll}\text { C } & 4.59582700 & 2.02081000 & 1.96864100\end{array}$

$\begin{array}{llll}\mathrm{H} & 3.31738500 & 0.30628100 & 2.02884700\end{array}$

$\begin{array}{llll}\text { C } & 5.15195300 & 2.88825300 & -0.21873800\end{array}$

H $\quad 4.31459300 \quad 1.80095900 \quad-1.88601500$

$\begin{array}{llll}\text { C } & 5.25516100 & 2.95928900 & 1.17406200\end{array}$

$\begin{array}{llll}\mathrm{H} & 4.67859300 & 2.06766600 & 3.05078200\end{array}$

H $\quad 5.66805200 \quad 3.61275400 \quad-0.84255500$

$\begin{array}{llll}\mathrm{H} & 5.85165600 & 3.74120900 & 1.63663200\end{array}$

$\begin{array}{llll}\text { C } & 1.61311300 & -3.41829400 & 0.41338500\end{array}$

$\begin{array}{llll}\mathrm{H} & 2.39669100 & -3.38622400 & 1.18434000\end{array}$

$\begin{array}{llll}\text { H } & 0.68542400 & -3.09815900 & 0.90531400\end{array}$

$\begin{array}{llll}\text { C } & 1.45479800 & -4.87415100 & -0.07304500\end{array}$

$\begin{array}{llll}\text { C } & 1.07587300 & -5.76387800 & 1.12107800\end{array}$

$\begin{array}{llll}\text { C } & 2.71149400 & -5.40367900 & -0.78046000\end{array}$

H $\quad 0.63092000 \quad-4.90324600 \quad-0.79665900$

$\begin{array}{llll}\mathrm{H} & 0.16715800 & -5.40400400 & 1.61839200\end{array}$

$\begin{array}{llll}\mathrm{H} & 0.89654900 & -6.79542700 & 0.79666800\end{array}$

$\begin{array}{llll}\mathrm{H} & 1.87961600 & -5.78486900 & 1.86885200\end{array}$

$\begin{array}{llll}\mathrm{H} & 2.89100300 & -4.87440600 & -1.72104100\end{array}$

$\mathrm{H} \quad 3.60043100 \quad-5.30325000 \quad-0.14225100$

$\begin{array}{llll}\mathrm{H} & 2.59663300 & -6.46735700 & -1.02007100\end{array}$

$\begin{array}{llll}\text { O } & 0.98851300 & -3.15964600 & -2.72472300\end{array}$

$\begin{array}{lllll}\text { O } & 0.01269300 & -1.41868000 & -1.71706000\end{array}$

\begin{tabular}{|c|c|c|c|}
\hline S & -2.94125800 & -0.44468200 & 0.92175000 \\
\hline $\mathrm{F}$ & -5.27307900 & 0.03992300 & -0.25983700 \\
\hline $\mathrm{F}$ & -4.33487900 & -1.84157700 & -0.83653500 \\
\hline $\mathrm{F}$ & -5.26942500 & -1.64057800 & 1.12397700 \\
\hline $\mathrm{O}$ & -3.21685200 & 0.74683800 & 1.70597300 \\
\hline $\mathrm{O}$ & -2.33300100 & -1.65447300 & 1.45450400 \\
\hline $\mathrm{N}$ & -2.01367300 & 0.00625200 & -0.47968600 \\
\hline $\mathrm{C}$ & -2.42302800 & 1.29300900 & -1.21553500 \\
\hline $\mathrm{C}$ & -1.23829100 & 2.22493800 & -1.38148000 \\
\hline E & -0.18212100 & 1.92567300 & -2.24976400 \\
\hline 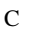 & 0.89912900 & 2.79571200 & -2.40378400 \\
\hline $\mathrm{C}$ & 0.92384200 & 3.98687500 & -1.68143900 \\
\hline C & -0.11937300 & 4.31429200 & -0.81360100 \\
\hline $\mathrm{C}$ & -1.20145000 & 3.43891400 & -0.66069600 \\
\hline $\mathrm{C}$ & -4.57787700 & -1.01008600 & 0.18392800 \\
\hline H & -0.18732800 & 0.98879800 & -2.79950800 \\
\hline $\mathrm{H}$ & 1.71016000 & 2.53417400 & -3.07573400 \\
\hline $\mathrm{H}$ & 1.75918500 & 4.67366800 & -1.78751900 \\
\hline $\mathrm{H}$ & -0.08356400 & 5.24594800 & -0.26105900 \\
\hline $\mathrm{O}$ & -2.26875000 & 3.68962500 & 0.15022700 \\
\hline $\mathrm{C}$ & -2.23072800 & 4.82090500 & 1.00649800 \\
\hline $\mathrm{H}$ & -2.21976500 & 5.75843500 & 0.43515100 \\
\hline $\mathrm{H}$ & -3.14292900 & 4.77154400 & 1.60306300 \\
\hline $\mathrm{H}$ & -1.35869100 & 4.78744400 & 1.67097700 \\
\hline $\mathrm{C}$ & -3.07628600 & 0.91944200 & -2.55242000 \\
\hline $\mathrm{H}$ & -2.38122300 & 0.37142700 & -3.19738800 \\
\hline $\mathrm{H}$ & -3.97187500 & 0.30915700 & -2.40870500 \\
\hline $\mathrm{H}$ & -3.36924200 & 1.83371500 & -3.07677200 \\
\hline $\mathrm{H}$ & -3.14641100 & 1.78738000 & -0.56852100 \\
\hline $\mathrm{H}$ & -2.02275900 & -0.79512200 & -1.12623300 \\
\hline 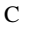 & 0.68344200 & 0.21695200 & 2.73050600 \\
\hline $\mathrm{O}$ & 1.51714400 & -0.70600800 & 2.70965900 \\
\hline $\mathrm{O}$ & 0.07691700 & 0.70956100 & 1.69259700 \\
\hline$C$ & 0.29880700 & 0.89620700 & 4.03507300 \\
\hline $\mathrm{H}$ & 0.54520700 & 1.96233000 & 3.98329700 \\
\hline $\mathrm{H}$ & -0.78269300 & 0.81945900 & 4. 18526800 \\
\hline $\mathrm{H}$ & 0.82728700 & 0.43428300 & 4.87080100 \\
\hline
\end{tabular}

$\mathrm{TS}_{\mathrm{Rlc}}$

$-2506.98359295$

$\begin{array}{llll}\mathrm{Pd} & 0.29443400 & -0.26730700 & -0.02632200 \\ \mathrm{C} & 3.24155800 & 0.01332000 & -0.93724700 \\ \mathrm{C} & 2.65692600 & 0.07190700 & -2.38268200 \\ \mathrm{H} & 4.08874900 & 0.70092300 & -0.93099900\end{array}$ 


\begin{tabular}{|c|c|c|c|c|c|c|c|}
\hline$N$ & 2.31530100 & 0.48954500 & 0.16206200 & $\mathrm{C}$ & -3.96025400 & -2.21908200 & 0.95391000 \\
\hline $\mathrm{H}$ & 2.50675300 & -0.01231500 & 1.05045500 & $\mathrm{H}$ & 0.31719900 & 0.62927200 & -1.83570800 \\
\hline $\mathrm{C}$ & 2.30072500 & 1.92505400 & 0.32280600 & $\mathrm{H}$ & 0.58748900 & 3.04289300 & -1.30489300 \\
\hline $\mathrm{O}$ & 2.64500400 & 2.62281800 & -0.61522400 & $\mathrm{H}$ & -1.28922500 & 4.59127600 & -1.77435100 \\
\hline $\mathrm{C}$ & 1.84136900 & 2.51452100 & 1.61698000 & $\mathrm{H}$ & -3.54114700 & 3.71820700 & -2.24293200 \\
\hline $\mathrm{C}$ & 1.45069800 & 1.76915800 & 2.74140800 & $\mathrm{O}$ & -4.19487400 & 1.07892600 & -2.24738500 \\
\hline $\mathrm{C}$ & 1.81499400 & 3.91952500 & 1.67956600 & $\mathrm{C}$ & -5.31483000 & 1.92855400 & -2.45901300 \\
\hline $\mathrm{C}$ & 1.03215700 & 2.42559500 & 3.89942300 & $\mathrm{H}$ & -5.18844600 & 2.54178700 & -3.36011200 \\
\hline $\mathrm{H}$ & 1.48727200 & 0.68566700 & 2.73656500 & $\mathrm{H}$ & -6.16979600 & 1.26414100 & -2.59387600 \\
\hline $\mathrm{C}$ & 1.39731100 & 4.56707000 & 2.83723100 & $\mathrm{H}$ & -5.49171100 & 2.57936000 & -1.59374400 \\
\hline $\mathrm{H}$ & 2.13233900 & 4.48284500 & 0.80889600 & $\mathrm{C}$ & -1.58938700 & -1.57910000 & -2.66697000 \\
\hline $\mathrm{C}$ & 1.00055200 & 3.81986500 & 3.95056600 & $\mathrm{H}$ & -0.50875400 & -1.42486100 & -2.74750800 \\
\hline $\mathrm{H}$ & 0.73290800 & 1.84020500 & 4.76405600 & $\mathrm{H}$ & -1.80463500 & -2.64230900 & -2.50083500 \\
\hline $\mathrm{H}$ & 1.38172900 & 5.65276800 & 2.87385000 & $\mathrm{H}$ & -2.04491800 & -1.29090900 & -3.61937200 \\
\hline $\mathrm{H}$ & 0.67261600 & 4.32407800 & 4.85574000 & $\mathrm{H}$ & -3.29115000 & -0.88371000 & -1.54250500 \\
\hline $\mathrm{C}$ & 3.71674400 & -1.41686400 & -0.60803100 & $\mathrm{H}$ & -1.36508000 & -2.14937200 & -0.20560500 \\
\hline $\mathrm{H}$ & 4.11871900 & -1.42546100 & 0.41482600 & $\mathrm{C}$ & 1.50214900 & -2.13042300 & 1.99336800 \\
\hline $\mathrm{H}$ & 2.84655600 & -2.08513000 & -0.60995600 & $\mathrm{O}$ & 2.30465800 & -1.27126200 & 2.38171000 \\
\hline $\mathrm{C}$ & 4.78916500 & -1.99742100 & -1.55513300 & $\mathrm{O}$ & 0.68194300 & -1.99928300 & 0.99111500 \\
\hline $\mathrm{C}$ & 5.11411100 & -3.43621500 & -1.12360100 & $\mathrm{C}$ & 1.39365500 & -3.48052300 & 2.68381100 \\
\hline $\mathrm{C}$ & 6.06109700 & -1.13831400 & -1.60756000 & $\mathrm{H}$ & 0.41641900 & -3.55554600 & 3.17343000 \\
\hline $\mathrm{H}$ & 4.37053000 & -2.02749000 & -2.56768500 & $\mathrm{H}$ & 1.46005400 & -4.28935800 & 1.94961300 \\
\hline $\mathrm{H}$ & 4.21516500 & -4.06543800 & -1.10675200 & $\mathrm{H}$ & 2.18571000 & -3.58316100 & 3.42729800 \\
\hline $\mathrm{H}$ & 5.83228500 & -3.89784500 & -1.81148400 & & & & \\
\hline $\mathrm{H}$ & 5.55621600 & -3.45888100 & -0.11824800 & $\mathrm{R}_{2 \mathrm{c}}$ & & & \\
\hline $\mathrm{H}$ & 5.85684700 & -0.16950600 & -2.07142600 & -25 & .03169603 & & \\
\hline $\mathrm{H}$ & 6.47403600 & -0.97422100 & -0.60213400 & $\mathrm{Pd}$ & 0.18164100 & -0.16348800 & -0.18505700 \\
\hline $\mathrm{H}$ & 6.83532000 & -1.63428900 & -2.20536900 & $\mathrm{C}$ & 3.33042800 & 0.52328100 & -0.46210600 \\
\hline $\mathrm{O}$ & 3.48025300 & 0.25717400 & -3.27671200 & $\mathrm{C}$ & 3.28202000 & 2.02739900 & -0.76379000 \\
\hline $\mathrm{O}$ & 1.40037500 & -0.18977600 & -2.54004400 & $\mathrm{H}$ & 4.28888600 & 0.37555000 & 0.04108500 \\
\hline S & -2.64238000 & -0.88628100 & 1.18990200 & $\mathrm{~N}$ & 2.27345000 & -0.00286500 & 0.47719500 \\
\hline $\mathrm{F}$ & -4.84923700 & -1.84384100 & 0.03540900 & $\mathrm{H}$ & 2.34757300 & -1.05248500 & 0.47090300 \\
\hline $\mathrm{F}$ & -3.36232900 & -3.34993700 & 0.55623900 & $\mathrm{C}$ & 2.38719600 & 0.55328900 & 1.80686000 \\
\hline $\mathrm{F}$ & -4.56666900 & -2.41465300 & 2.11580900 & $\mathrm{O}$ & 2.93986600 & 1.63150800 & 1.94213100 \\
\hline $\mathrm{O}$ & -3.28590400 & 0.40763100 & 1.04202600 & $\mathrm{C}$ & 1.79339600 & -0.18236700 & 2.96042600 \\
\hline $\mathrm{O}$ & -1.89724700 & -1.29016500 & 2.36863200 & $\mathrm{C}$ & 1.29731200 & -1.49487500 & 2.89838000 \\
\hline $\mathrm{N}$ & -1.63111400 & -1.15792700 & -0.22026200 & $\mathrm{C}$ & 1.75820800 & 0.51235900 & 4.18301200 \\
\hline $\mathrm{C}$ & -2.21329100 & -0.71901000 & -1.56018200 & $\mathrm{C}$ & 0.76379700 & -2.08939000 & 4.04214900 \\
\hline $\mathrm{C}$ & -1.93269800 & 0.76025900 & -1.68821600 & $\mathrm{H}$ & 1.34993800 & -2.07170400 & 1.98179900 \\
\hline $\mathrm{C}$ & -0.62928300 & 1.26207400 & -1.43813600 & $\mathrm{C}$ & 1.22114200 & -0.08544500 & 5.31770500 \\
\hline $\mathrm{C}$ & -0.41206000 & 2.65249400 & -1.45559000 & $\mathrm{H}$ & 2.15893300 & 1.51941400 & 4.21932200 \\
\hline $\mathrm{C}$ & -1.46128900 & 3.51915000 & -1.74385700 & $\mathrm{C}$ & 0.71864600 & -1.38853800 & 5.24815300 \\
\hline $\mathrm{C}$ & -2.73867900 & 3.02590100 & -2.01540900 & $\mathrm{H}$ & 0.38412500 & -3.10531400 & 3.98578600 \\
\hline $\mathrm{C}$ & -2.97890500 & 1.64568000 & -1.99236300 & $\mathrm{H}$ & 1.19391000 & 0.46178800 & 6.25572800 \\
\hline
\end{tabular}




\begin{tabular}{|c|c|c|c|}
\hline $\mathrm{H}$ & 0.29777600 & -1.85696700 & 6.13387300 \\
\hline $\mathrm{C}$ & 3.30734700 & -0.28111200 & -1.78104500 \\
\hline $\mathrm{H}$ & 3.40805000 & -1.34061800 & -1.52141900 \\
\hline I & 2.31977800 & -0.16358900 & -2.24524400 \\
\hline $\mathrm{C}$ & 4.39756000 & 0.08145500 & -2.81245800 \\
\hline C & 4.18711200 & -0.76653900 & -4.07716600 \\
\hline$c$ & 5.81928800 & -0.10198600 & -2.25951800 \\
\hline $\mathrm{H}$ & 4.28133400 & 1.13638800 & -3.09124200 \\
\hline $\mathrm{H}$ & 3.18436400 & -0.62131500 & -4.49798800 \\
\hline $\mathrm{H}$ & 4.91687100 & -0.50043100 & -4.85046200 \\
\hline $\mathrm{H}$ & 4.30657500 & -1.83622900 & -3.86046600 \\
\hline $\mathrm{H}$ & 6.03017800 & 0.61137200 & -1.45769500 \\
\hline I & 5.96626700 & -1.12018200 & -1.87439500 \\
\hline $\mathrm{H}$ & 6.56135800 & 0.06389500 & -3.04932500 \\
\hline $\mathrm{O}$ & 4.30394500 & 2.65079200 & -0.92727500 \\
\hline $\mathrm{O}$ & 2.08553900 & 2.59577100 & -1.00413100 \\
\hline $\mathrm{S}$ & 2.76729000 & -1.33389800 & 0.28638600 \\
\hline $\mathrm{F}$ & -4.90807500 & -1.13374500 & -1.28390500 \\
\hline $\mathrm{F}$ & -3.47629900 & -2.66136400 & -1.88654000 \\
\hline $\mathrm{F}$ & -4.75941500 & -2.98844200 & -0.15283400 \\
\hline $\mathrm{O}$ & -3.41917600 & -0.34238000 & 1.12368700 \\
\hline $\mathrm{O}$ & -2.00729700 & -2.45915100 & 0.80109300 \\
\hline $\mathrm{N}$ & -1.77568600 & -0.49262500 & -0.89654100 \\
\hline $\mathrm{C}$ & -2.32145300 & 0.83938200 & -1.38662800 \\
\hline $\mathrm{C}$ & -1.79550900 & 1.88313600 & -0.43378300 \\
\hline$C$ & -0.60758400 & 1.64077800 & 0.28874400 \\
\hline $\mathrm{C}$ & -0.17339300 & 2.59509600 & 1.21554500 \\
\hline $\mathrm{C}$ & -0.89862700 & 3.77473700 & 1.39499600 \\
\hline $\mathrm{C}$ & -2.06295900 & 4.03010300 & 0.67052200 \\
\hline $\mathrm{C}$ & -2.51198100 & 3.07876600 & -0.24919500 \\
\hline $\mathrm{C}$ & -4.08072200 & -2.08183100 & -0.84125900 \\
\hline $\mathrm{H}$ & 1.34194200 & 2.02718900 & -0.72039200 \\
\hline $\mathrm{H}$ & 0.73907800 & 2.45956700 & 1.78217800 \\
\hline $\mathrm{H}$ & -0.54395200 & 4.51538400 & 2.10691900 \\
\hline $\mathrm{H}$ & -2.60486700 & 4.95551600 & 0.82595800 \\
\hline $\mathrm{O}$ & -3.64032800 & 3.21200700 & -1.01615100 \\
\hline $\mathrm{C}$ & -4.45915400 & 4.35626200 & -0.81766800 \\
\hline $\mathrm{H}$ & -3.92349200 & 5.28090900 & -1.06754900 \\
\hline $\mathrm{H}$ & -5.30818500 & 4.23742200 & -1.49324700 \\
\hline $\mathrm{H}$ & -4.82128600 & 4.41432500 & 0.21646300 \\
\hline $\mathrm{C}$ & -1.87560000 & 1.02915600 & -2.84058300 \\
\hline $\mathrm{H}$ & -0.78375000 & 1.02235700 & -2.92243000 \\
\hline $\mathrm{H}$ & -2.29102700 & 0.25025100 & -3.49241000 \\
\hline $\mathrm{H}$ & 2.24105400 & .99820400 & -3.19070100 \\
\hline
\end{tabular}

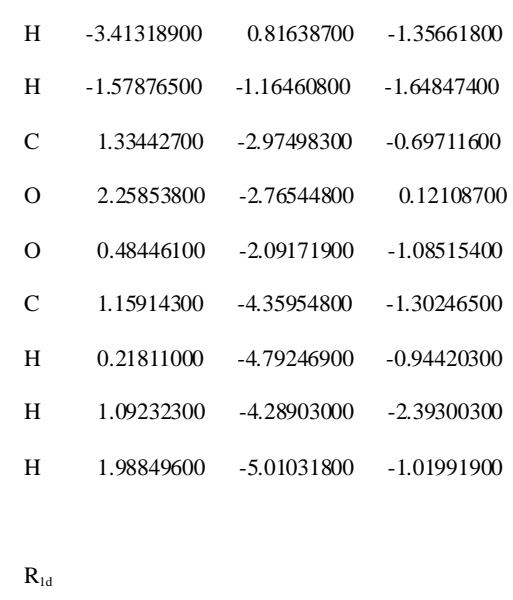




\begin{tabular}{|c|c|c|c|c|c|c|c|}
\hline $\mathrm{H}$ & -0.69650000 & 4.41137500 & -1.70856900 & $\mathrm{C}$ & 0.23013200 & -3.99241000 & -2.97558200 \\
\hline $\mathrm{H}$ & -0.52905800 & 5.33927800 & 0.61404200 & $\mathrm{C}$ & 0.54799500 & -3.00600500 & -1.86097200 \\
\hline $\mathrm{H}$ & -1.21420900 & 6.52804200 & -0.50436100 & $\mathrm{O}$ & -0.37877900 & -2.82817400 & -0.98905100 \\
\hline $\mathrm{H}$ & -2.27557500 & 5.65951800 & 0.61525100 & $\mathrm{C}$ & -2.32684500 & 2.19770300 & -0.45489100 \\
\hline $\mathrm{H}$ & -2.82653200 & 4.10263200 & -2.85362200 & $\mathrm{C}$ & -1.18668500 & 2.65641700 & -1.38128600 \\
\hline $\mathrm{H}$ & -3.73573800 & 4.75182400 & -1.46168100 & $\mathrm{H}$ & -3.27351200 & 2.39880900 & -0.96161000 \\
\hline $\mathrm{H}$ & -2.67758000 & 5.80467300 & -2.41307300 & $\mathrm{H}$ & -0.17372400 & -3.43630500 & -3.83063000 \\
\hline $\mathrm{O}$ & 0.17625300 & 0.89386300 & -1.94310000 & $\mathrm{H}$ & 1.14436900 & -4.49143300 & -3.30604600 \\
\hline $\mathrm{O}$ & -0.76867600 & 2.35751800 & -3.33662300 & $\mathrm{H}$ & -0.51410100 & -4.72516800 & -2.65513000 \\
\hline S & 3.13360300 & 0.52783300 & -0.40524600 & $\mathrm{~N}$ & -2.23477600 & 0.70704600 & -0.21695400 \\
\hline $\mathrm{F}$ & 4.99228300 & -0.69057300 & 1.09661000 & $\mathrm{H}$ & -2.55173300 & 0.46332900 & 0.72047100 \\
\hline $\mathrm{F}$ & 4.58173100 & -1.67867900 & -0.79976200 & $\mathrm{C}$ & -2.89444300 & -0.10875000 & -1.23211900 \\
\hline $\mathrm{F}$ & 5.70931400 & 0.18330600 & -0.76202500 & $\mathrm{O}$ & -2.78659500 & 0.18891700 & -2.39883100 \\
\hline $\mathrm{O}$ & 3.28875300 & 1.64147400 & 0.53782900 & $\mathrm{C}$ & -3.68754700 & -1.27363700 & -0.74699300 \\
\hline $\mathrm{O}$ & 3.03182900 & 0.74988600 & -1.83912800 & $\mathrm{C}$ & -3.71573500 & -2.42242200 & -1.55168300 \\
\hline $\mathrm{N}$ & 1.95979900 & -0.58387600 & 0.06747400 & $\mathrm{C}$ & -4.46182400 & -1.22674800 & 0.42350000 \\
\hline $\mathrm{C}$ & 1.96357100 & -0.93548100 & 1.52641100 & $\mathrm{C}$ & -4.48728100 & -3.51794800 & -1.17511500 \\
\hline $\mathrm{C}$ & 0.56039400 & -0.58010900 & 2.05079200 & $\mathrm{H}$ & -3.11488000 & -2.44450100 & -2.45343800 \\
\hline $\mathrm{C}$ & -0.53415100 & -1.46232200 & 1.89715900 & $\mathrm{C}$ & -5.25031100 & -2.31914500 & 0.78535100 \\
\hline $\mathrm{C}$ & -1.76234300 & -1.21527600 & 2.53442700 & $\mathrm{H}$ & -4.48994100 & -0.32975600 & 1.03814000 \\
\hline $\mathrm{C}$ & -1.90805100 & -0.07685100 & 3.31567400 & $\mathrm{C}$ & -5.25641400 & -3.46770000 & -0.00902300 \\
\hline $\mathrm{C}$ & -0.85087600 & 0.83192000 & 3.47993900 & $\mathrm{H}$ & -4.49014400 & -4.41221200 & -1.79113900 \\
\hline $\mathrm{C}$ & 0.38015400 & 0.58528800 & 2.87146300 & $\mathrm{H}$ & -5.86205300 & -2.27028500 & 1.68150800 \\
\hline $\mathrm{C}$ & 4.70463200 & -0.48859700 & -0.20223300 & $\mathrm{H}$ & -5.86376200 & -4.32140800 & 0.27840100 \\
\hline $\mathrm{H}$ & -0.40297200 & -2.36802900 & 1.31498700 & $\mathrm{C}$ & -2.28208100 & 2.90889100 & 0.91055600 \\
\hline $\mathrm{H}$ & -2.57814000 & -1.91970400 & 2.42195200 & $\mathrm{H}$ & -3.11600900 & 2.52619900 & 1.52254300 \\
\hline $\mathrm{H}$ & -2.84977500 & 0.11657300 & 3.82303500 & $\mathrm{H}$ & -1.35291900 & 2.62091000 & 1.42209600 \\
\hline $\mathrm{H}$ & -0.98673700 & 1.70384600 & 4.10907700 & $\mathrm{C}$ & -2.37465600 & 4.44900600 & 0.86931900 \\
\hline $\mathrm{H}$ & 1.79905600 & -1.94841700 & -1.18891000 & $\mathrm{C}$ & -2.29119900 & 4.99857800 & 2.30144500 \\
\hline $\mathrm{O}$ & 1.47138200 & 1.35821400 & 3.02521700 & $\mathrm{C}$ & -3.64114300 & 4.94378400 & 0.15540300 \\
\hline $\mathrm{C}$ & 1.36628000 & 2.60202800 & 3.70849800 & $\mathrm{H}$ & -1.51014100 & 4.81947200 & 0.30674000 \\
\hline $\mathrm{H}$ & 1.12073600 & 2.45516400 & 4.76773900 & $\mathrm{H}$ & -1.37568800 & 4.66820200 & 2.80721500 \\
\hline $\mathrm{H}$ & 2.34794500 & 3.06636000 & 3.61684900 & $\mathrm{H}$ & -2.29316900 & 6.09430800 & 2.29636400 \\
\hline $\mathrm{H}$ & 0.61163800 & 3.24459100 & 3.23921100 & $\mathrm{H}$ & -3.14722800 & 4.66820400 & 2.90551400 \\
\hline $\mathrm{C}$ & 2.32256700 & -2.41115200 & 1.72571300 & $\mathrm{H}$ & -3.61725500 & 4.69301200 & -0.90913200 \\
\hline $\mathrm{H}$ & 2.23205100 & -2.68322700 & 2.78406800 & $\mathrm{H}$ & -4.54811500 & 4.51344300 & 0.60316000 \\
\hline $\mathrm{H}$ & 1.67272400 & -3.06541100 & 1.13834400 & $\mathrm{H}$ & -3.72159000 & 6.03414200 & 0.23286600 \\
\hline $\mathrm{H}$ & 3.35365400 & -2.58911300 & 1.41277900 & $\mathrm{O}$ & -0.11613600 & 1.90030300 & -1.39492600 \\
\hline \multirow[t]{2}{*}{$\mathrm{H}$} & 2.67487900 & -0.30918700 & 2.06632900 & $\mathrm{O}$ & -1.29415100 & 3.69931800 & -2.00254000 \\
\hline & & & & $\mathrm{S}$ & 2.83872800 & 1.06051100 & -1.05907600 \\
\hline \multicolumn{4}{|c|}{$\mathrm{TS}_{\mathrm{RId}}$} & $\mathrm{F}$ & 5.17033000 & 0.43267600 & 0.09415200 \\
\hline \multicolumn{4}{|c|}{-2506.98860287} & $\mathrm{~F}$ & 4.73840800 & -0.68388000 & -1.73084100 \\
\hline $\mathrm{Pd}$ & -0.15682400 & 0.23936100 & -0.26835800 & $\mathrm{~F}$ & 5.29520900 & 1.41697700 & -1.84027500 \\
\hline $\mathrm{O}$ & 1.66091400 & -2.42676000 & -1.88101000 & $\mathrm{O}$ & 2.82175400 & 2.15594800 & -0.09928900 \\
\hline
\end{tabular}




\begin{tabular}{|c|c|c|c|}
\hline $\mathrm{O}$ & 2.43946300 & 1.15085300 & -2.44967900 \\
\hline$N$ & 1.94647200 & -0.26368500 & -0.35937800 \\
\hline 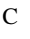 & 2.40580600 & -0.75614400 & 1.00486600 \\
\hline C & 1.17982600 & -1.08607000 & 1.83258700 \\
\hline $\mathrm{C}$ & -0.05599400 & -1.35723900 & 1.20753700 \\
\hline 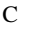 & -1.16398300 & -1.71178200 & 2.01098400 \\
\hline 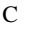 & -1.04272900 & -1.78464600 & 3.39224700 \\
\hline C & 0.17713100 & -1.48703500 & 4.01145000 \\
\hline $\mathrm{C}$ & 1.28876300 & -1.14046500 & 3.23649800 \\
\hline C & 4.63829800 & 0.50307900 & -1.13202400 \\
\hline $\mathrm{H}$ & -0.10877900 & -1.95244700 & 0.05870100 \\
\hline $\mathrm{H}$ & -2.10562900 & -1.96776800 & 1.53562100 \\
\hline I & -1.89145200 & -2.07428300 & 4.00633700 \\
\hline $\mathrm{H}$ & 0.25426700 & -1.53691800 & 5.09164300 \\
\hline $\mathrm{H}$ & 1.89565400 & -1.09142500 & -1.04985400 \\
\hline 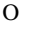 & 2.51768800 & -0.83876800 & 3.74971800 \\
\hline $\mathrm{C}$ & 2.68206100 & -0.80897400 & 5.16133200 \\
\hline $\mathrm{H}$ & 2.50344100 & -1.79592200 & 5.60690100 \\
\hline $\mathrm{H}$ & 3.71905400 & -0.51701100 & 5.33332700 \\
\hline $\mathrm{H}$ & 2.01439400 & -0.07249900 & 5.62610800 \\
\hline C & 3.31729800 & -1.99254800 & 0.89823300 \\
\hline $\mathrm{H}$ & 3.59485900 & -2.30606300 & 1.90828600 \\
\hline $\mathrm{H}$ & 2.79304400 & -2.81026400 & 0.39886800 \\
\hline $\mathrm{H}$ & 4.23370300 & -1.78617500 & 0.34590700 \\
\hline $\mathrm{H}$ & 94197800 & 0.06769300 & 1.48210300 \\
\hline
\end{tabular}

$\mathrm{R}_{2 \mathrm{~d}}$

$-2507.05312709$

$\begin{array}{lrrr}\mathrm{Pd} & -0.32475700 & 0.15855000 & 0.02548200 \\ \mathrm{O} & 2.39107200 & 1.59526200 & -2.36962400 \\ \mathrm{C} & 2.91700400 & 3.43533800 & -3.82395300 \\ \mathrm{C} & 2.10417400 & 2.73002200 & -2.76263000 \\ \mathrm{O} & 1.09617200 & 3.45071800 & -2.33416700 \\ \mathrm{C} & -2.67887300 & 1.90926200 & 0.31079900 \\ \mathrm{C} & -1.65056900 & 2.73356500 & -0.49508000 \\ \mathrm{H} & -3.66865800 & 2.15388500 & -0.08170500 \\ \mathrm{H} & 3.28099900 & 4.39171400 & -3.43450500 \\ \mathrm{H} & 3.75728400 & 2.81174100 & -4.13110700 \\ \mathrm{H} & 2.28123900 & 3.65908900 & -4.68698500 \\ \mathrm{~N} & -2.46238600 & 0.42648800 & 0.11930500 \\ \mathrm{H} & -2.78868100 & -0.09785500 & 0.92875400 \\ \mathrm{C} & -3.00456700 & -0.09861800 & -1.11909800 \\ \mathrm{O} & -2.95745900 & 0.57575900 & -2.12525300 \\ \mathrm{C} & -3.60593600 & -1.46452500 & -1.08956500\end{array}$

$\begin{array}{llll}\text { C } & -3.58620200 & -2.19432700 & -2.28869300\end{array}$

$\begin{array}{llll}\text { C } & -4.25442800 & -2.00317000 & 0.03163700\end{array}$

$\begin{array}{llll}\text { C } & -4.17170400 & -3.45512200 & -2.35320800\end{array}$

$\begin{array}{llll}\mathrm{H} & -3.10681100 & -1.75474100 & -3.15701800\end{array}$

$\begin{array}{llll}\text { C } & -4.85885800 & -3.25792200 & -0.04153800\end{array}$

$\begin{array}{llll}\mathrm{H} & -4.33175700 & -1.44083900 & 0.95884300\end{array}$

$\begin{array}{llll}\text { C } & -4.80912300 & -3.98922800 & -1.22936800\end{array}$

$\begin{array}{llll}\mathrm{H} & -4.13785900 & -4.01949900 & -3.28058000\end{array}$

$\begin{array}{llll}\mathrm{H} & -5.37109400 & -3.66074500 & 0.82733000\end{array}$

$\begin{array}{llll}\mathrm{H} & -5.27345300 & -4.97001900 & -1.28207300\end{array}$

$\begin{array}{llll}\text { C } & -2.60635800 & 2.21856700 & 1.81807600\end{array}$

$\begin{array}{llll}\mathrm{H} & -3.35989000 & 1.59837100 & 2.33232200\end{array}$

$\begin{array}{llll}\mathrm{H} & -1.62373200 & 1.90203300 & 2.19362900\end{array}$

$\begin{array}{llll}\text { C } & -2.84224200 & 3.69258700 & 2.20982200\end{array}$

$\begin{array}{llll}\text { C } & -2.71241900 & 3.83872400 & 3.73371600\end{array}$

$\begin{array}{llll}\text { C } & -4.19351000 & 4.22769100 & 1.71314000\end{array}$

$\begin{array}{llll}\mathrm{H} & -2.05491400 & 4.29278500 & 1.73887100\end{array}$

$\begin{array}{llll}\mathrm{H} & -1.73869600 & 3.48350600 & 4.09204000\end{array}$

$\begin{array}{llll}\mathrm{H} & -2.81621500 & 4.88798600 & 4.03260200\end{array}$

$\begin{array}{llll}\mathrm{H} & -3.49170500 & 3.26695400 & 4.25585100\end{array}$

$\begin{array}{llll}\text { H } & -4.21740200 & 4.28495200 & 0.62111700\end{array}$

$\begin{array}{llll}\mathrm{H} & -5.02529900 & 3.59612400 & 2.05605600\end{array}$

$\begin{array}{llll}\mathrm{H} & -4.36809800 & 5.23873500 & 2.09889800\end{array}$

$\begin{array}{llll}\text { O } & -0.49213200 & 2.15505300 & -0.68095600\end{array}$

$\begin{array}{llll}\text { O } & -1.94590200 & 3.86407200 & -0.84827000\end{array}$

$\begin{array}{llll}\text { S } & 2.56442500 & 0.65377800 & 1.18410700\end{array}$

$\begin{array}{llll}\text { F } & 4.88843000 & -0.63454600 & 0.96588300\end{array}$

$\begin{array}{llll}\text { F } & 4.53792700 & 0.83989500 & -0.59724000\end{array}$

F $\quad 5.03874200 \quad 1.48657000 \quad 1.42299000$

$\begin{array}{llll}\text { O } & 2.40595200 & -0.24629700 & 2.31583900\end{array}$

$\begin{array}{llll}\text { O } & 2.22167600 & 2.06651500 & 1.22509000\end{array}$

$\begin{array}{llll}\mathrm{N} & 1.77109600 & -0.02294900 & -0.21036500\end{array}$

$\begin{array}{llll}\text { C } & 2.08545900 & -1.48292800 & -0.50754900\end{array}$

$\begin{array}{llll}\text { C } & 1.09669900 & -2.31102500 & 0.27165100\end{array}$

$\begin{array}{llll}\text { C } & -0.12330200 & -1.72672500 & 0.64745400\end{array}$

C $\quad-1.02264100 \quad-2.46200100 \quad 1.42691100$

$\begin{array}{llll}\text { C } & -0.71793700 & -3.77357200 & 1.79308900\end{array}$

$\begin{array}{llll}\text { C } & 0.48034500 & -4.37322600 & 1.39860900\end{array}$

$\begin{array}{llll}\text { C } & 1.39239900 & -3.63603000 & 0.63701400\end{array}$

$\begin{array}{llll}\text { C } & 4.39267800 & 0.57696100 & 0.70097100\end{array}$

$\begin{array}{llll}\mathrm{H} & 0.51374700 & 2.95970500 & -1.66056400\end{array}$

$\begin{array}{llll}\mathrm{H} & -1.96178500 & -2.03143400 & 1.76173100\end{array}$

$\begin{array}{llll}\mathrm{H} & -1.42153700 & -4.34373000 & 2.39493400\end{array}$

$\begin{array}{llll}\mathrm{H} & 0.69503000 & -5.39379900 & 1.69334500\end{array}$ 


$\begin{array}{llll}\mathrm{H} & 1.98662300 & 0.61098600 & -1.02191500 \\ \mathrm{O} & 2.60291000 & -4.11069100 & 0.20282700 \\ \mathrm{C} & 2.99886000 & -5.41407300 & 0.60139300 \\ \mathrm{H} & 2.31450800 & -6.17890100 & 0.21146000 \\ \mathrm{H} & 3.99209400 & -5.56911800 & 0.17588300 \\ \mathrm{H} & 3.05333000 & -5.50069300 & 1.69415500 \\ \mathrm{C} & 2.00550500 & -1.69020500 & -2.02514500 \\ \mathrm{H} & 2.23311300 & -2.73803700 & -2.24075400 \\ \mathrm{H} & 0.99852600 & -1.46914000 & -2.39387900 \\ \mathrm{H} & 2.72162800 & -1.05233000 & -2.55214600 \\ \mathrm{H} & 3.10556400 & -1.70675900 & -0.18438200\end{array}$

3

$-1140.71215618$

\begin{tabular}{|c|c|c|c|}
\hline $\mathrm{Pd}$ & 0.00281500 & -1.24325900 & -0.31518200 \\
\hline $\mathrm{O}$ & -0.28139000 & -3.29399100 & -0.06651900 \\
\hline $\mathrm{C}$ & -1.98838800 & -4.18749900 & 1.39946600 \\
\hline $\mathrm{C}$ & -1.22864000 & -3.06517300 & 0.76061800 \\
\hline $\mathrm{O}$ & -1.50903800 & -1.84008800 & 1.00400100 \\
\hline $\mathrm{C}$ & 1.65986900 & 1.08368200 & -0.55327400 \\
\hline $\mathrm{C}$ & 2.13600600 & 0.08279200 & -1.63038500 \\
\hline $\mathrm{H}$ & 1.83656500 & 2.10507400 & -0.90081800 \\
\hline $\mathrm{H}$ & -1.29137000 & -4.95369000 & 1.75084400 \\
\hline $\mathrm{H}$ & -2.59373600 & -3.81286500 & 2.22711300 \\
\hline $\mathrm{H}$ & -2.64343400 & -4.65093800 & 0.65281300 \\
\hline $\mathrm{N}$ & 0.16086100 & 0.86328800 & -0.46974900 \\
\hline $\mathrm{H}$ & -0.21642200 & 0.98122700 & -1.41231100 \\
\hline $\mathrm{C}$ & -0.64672400 & 1.66743400 & 0.43694400 \\
\hline $\mathrm{O}$ & -0.13945000 & 2.24334800 & 1.37418100 \\
\hline $\mathrm{C}$ & -2.10162200 & 1.75714400 & 0.11595600 \\
\hline $\mathrm{C}$ & -2.74159500 & 1.00783100 & -0.88579200 \\
\hline $\mathrm{C}$ & -2.85213700 & 2.66979700 & 0.87635500 \\
\hline $\mathrm{C}$ & -4.10426900 & 1.17823800 & -1.12447400 \\
\hline $\mathrm{H}$ & -2.20121200 & 0.26512300 & -1.46523000 \\
\hline $\mathrm{C}$ & -4.21180600 & 2.83694900 & 0.63443300 \\
\hline $\mathrm{H}$ & -2.34708400 & 3.23836500 & 1.64958600 \\
\hline $\mathrm{C}$ & -4.84016100 & 2.09288500 & -0.36825100 \\
\hline $\mathrm{H}$ & -4.59114600 & 0.59018300 & -1.89674400 \\
\hline $\mathrm{H}$ & -4.78254300 & 3.54648900 & 1.22635400 \\
\hline $\mathrm{H}$ & -5.90217800 & 2.22237700 & -0.55724900 \\
\hline $\mathrm{C}$ & 2.39244700 & 0.83760400 & 0.78354200 \\
\hline $\mathrm{H}$ & 2.05572500 & 1.58471500 & 1.50526000 \\
\hline $\mathrm{H}$ & 2.08255400 & -0.14295600 & 1.17103100 \\
\hline $\mathrm{C}$ & 3.93419900 & 0.87597600 & 0.69016600 \\
\hline
\end{tabular}

$\begin{array}{llll}\mathrm{C} & 4.52328800 & 0.57739800 & 2.07759400 \\ \mathrm{C} & 4.45907500 & 2.21378600 & 0.14564400 \\ \mathrm{H} & 4.26292900 & 0.08522900 & 0.00428000 \\ \mathrm{H} & 4.17568300 & -0.38857900 & 2.46385300 \\ \mathrm{H} & 5.61815400 & 0.54721200 & 2.03732000 \\ \mathrm{H} & 4.23725800 & 1.35038400 & 2.80294900 \\ \mathrm{H} & 4.17219800 & 2.35749900 & -0.90053700 \\ \mathrm{H} & 4.08117900 & 3.05927300 & 0.73665000 \\ \mathrm{H} & 5.55400600 & 2.24171500 & 0.19062700 \\ \mathrm{O} & 1.48138800 & -1.06276500 & -1.64124300 \\ \mathrm{O} & 3.06100500 & 0.34699800 & -2.37672200\end{array}$

$\mathrm{R}_{\mathrm{le}}$

$-2507.05825055$

\begin{tabular}{|c|c|c|c|}
\hline $\mathrm{Pd}$ & 0.21072600 & -0.15788300 & 0.2079890 \\
\hline & .96908600 & 1.86491200 & 0.396240 \\
\hline & .11126100 & 1.77923600 & 1.6810260 \\
\hline & 0.00655700 & -1.06541300 & -1.5528430 \\
\hline & -1.40031900 & -2.37133800 & -2.7039690 \\
\hline & 1.46223500 & -2.58086300 & 0.2609940 \\
\hline & -1.84685400 & -0.56000100 & 0.3494600 \\
\hline & -2.56711200 & 0.55524700 & -0.2330990 \\
\hline & -2.76902700 & 0.55710900 & -1.4314500 \\
\hline & -3.02299300 & 1.66543400 & 0.653071 \\
\hline & -3.21755700 & 1.54797900 & 2.038014 \\
\hline & -3.34087800 & 2.87550700 & 0.011254 \\
\hline & -3.71736000 & 2.63005900 & 2.764058 \\
\hline & -2.97801100 & 0.63204300 & 2.565493 \\
\hline & -3.82639200 & 3.95406100 & 0.742760 \\
\hline & -3.19735800 & 2.94494200 & -1.061396 \\
\hline & -4.01821200 & 3.83239700 & 2.122636 \\
\hline & -3.87297000 & 2.52868100 & 3.834334 \\
\hline & -4.06116400 & 4.88768000 & 0.239265 \\
\hline & -4.40429900 & 4.67198200 & 2.694480 \\
\hline & -3.41798200 & -2.31322700 & -0.63247200 \\
\hline & -4.02607000 & -2.00963300 & 0.233398 \\
\hline & -3.80607600 & -1.78327000 & -1.50593200 \\
\hline & -3.57311300 & -3.83644300 & -0.84213500 \\
\hline & -4.97010700 & -4.14169900 & -1.4030880 \\
\hline & -3.32539800 & -4.63676600 & 0.446150 \\
\hline & -2.83526800 & -4.13792700 & -1.594819 \\
\hline & -5.13547700 & -3.62665200 & -2.3561750 \\
\hline & -5.09850300 & -5.21695000 & -1.5744970 \\
\hline & 62100 & 1800 & -0.705434 \\
\hline
\end{tabular}




\begin{tabular}{|c|c|c|c|}
\hline I & -2.32439300 & -4.47068300 & 0.86280100 \\
\hline $\mathrm{H}$ & -4.05629800 & -4.37022700 & 1.22145400 \\
\hline $\mathrm{H}$ & -3.41989100 & -5.71249300 & 0.25766400 \\
\hline $\mathrm{S}$ & 3.52271200 & -1.06035400 & 0.16496700 \\
\hline $\mathrm{F}$ & 3.17865300 & -0.14220800 & 2.64738600 \\
\hline $\mathrm{F}$ & 1.97882600 & -1.90127800 & 2.16349700 \\
\hline $\mathrm{F}$ & 4.12391100 & -2.09082800 & 2.45512600 \\
\hline $\mathrm{O}$ & 4.83396500 & -0.42207100 & 0.10628700 \\
\hline $\mathrm{O}$ & 3.23708000 & -2.33757700 & -0.47523500 \\
\hline $\mathrm{N}$ & 2.29163200 & 0.05610800 & -0.41696600 \\
\hline $\mathrm{C}$ & 2.77066000 & 1.48426300 & -0.70487500 \\
\hline $\mathrm{C}$ & 1.72559000 & 2. 10449600 & -1.62303100 \\
\hline C & 1.01420400 & 3.25826100 & -1.28283800 \\
\hline $\mathrm{C}$ & 0.07105000 & 3.81261200 & -2.15163200 \\
\hline $\mathrm{C}$ & -0.17985100 & 3.19783500 & -3.37583600 \\
\hline C & 0.50819800 & 2.03932600 & -3.74132500 \\
\hline C & 1.46215200 & 1.50171200 & -2.87581200 \\
\hline $\mathrm{C}$ & 3.15321100 & -1.30182300 & 1.99670200 \\
\hline $\mathrm{H}$ & 1.19272100 & 3.73378700 & -0.32482000 \\
\hline $\mathrm{H}$ & -0.46315400 & 4.71376500 & -1.86626200 \\
\hline $\mathrm{H}$ & -0.91875600 & 3.61171400 & -4.05631600 \\
\hline $\mathrm{H}$ & 0.29110700 & 1.55916200 & -4.68776400 \\
\hline C & 3.05074000 & 2.25107400 & 0.58208000 \\
\hline $\mathrm{H}$ & 2.17515900 & 2.28106100 & 1.23470400 \\
\hline $\mathrm{H}$ & 3.87668500 & 1.78840000 & 1.12517700 \\
\hline $\mathrm{H}$ & 3.35537800 & 3.27181000 & 0.33145600 \\
\hline $\mathrm{O}$ & 2.19386700 & 0.37711500 & -3.15189500 \\
\hline $\mathrm{C}$ & 1.78566500 & -0.46729600 & -4.23671600 \\
\hline $\mathrm{H}$ & 1.88284300 & 0.05719400 & -5.19377500 \\
\hline $\mathrm{H}$ & 2.47388300 & -1.31321400 & -4.21710900 \\
\hline $\mathrm{H}$ & 0.76260000 & -0.82147600 & -4.08080700 \\
\hline $\mathrm{H}$ & 3.70457700 & 1.37252400 & -1.26626400 \\
\hline $\mathrm{H}$ & 2.10420300 & -0.35703900 & -1.35024900 \\
\hline $\mathrm{C}$ & -0.17628100 & 0.28287100 & 3.05774300 \\
\hline $\mathrm{O}$ & -1.08436500 & -0.56610100 & 3.02776400 \\
\hline $\mathrm{O}$ & 0.46892000 & 0.73862700 & 2.02588800 \\
\hline $\mathrm{C}$ & 0.26807200 & 0.90171900 & 4.37403000 \\
\hline $\mathrm{H}$ & 0.07312900 & 1.97970900 & 4.35842600 \\
\hline $\mathrm{H}$ & -0.26767100 & 0.44340500 & 5.20703400 \\
\hline $\mathrm{H}$ & 1.34662100 & 0.76718500 & 4.50163600 \\
\hline $\mathrm{H}$ & -1.90271300 & 0.66284600 & 1.37817900 \\
\hline
\end{tabular}

$\mathrm{TS}_{\text {Rle }}$

$-2506.99297788$

\begin{tabular}{|c|c|c|c|}
\hline $\mathrm{Pd}$ & -0.28904900 & 0.14910700 & -0.32292300 \\
\hline $\mathrm{C}$ & 2.21350500 & 1.50340800 & -0.10931400 \\
\hline $\mathrm{C}$ & 1.34508100 & 2.32972200 & -1.07823000 \\
\hline $\mathrm{O}$ & 0.12371100 & 1.86389700 & -1.26466000 \\
\hline $\mathrm{O}$ & 1.74056600 & 3.36942600 & -1.57033100 \\
\hline $\mathrm{H}$ & 1.91025900 & 1.79656500 & 0.90240900 \\
\hline $\mathrm{N}$ & 1.81005200 & 0.04870900 & -0.17975700 \\
\hline $\mathrm{C}$ & 2.34579000 & -0.66186000 & -1.33010600 \\
\hline $\mathrm{O}$ & 2.34177700 & -0.11782000 & -2.41551500 \\
\hline $\mathrm{C}$ & 2.90102900 & -2.03203400 & -1.12778900 \\
\hline $\mathrm{C}$ & 3.35075800 & -2.52255400 & 0.10991400 \\
\hline $\mathrm{C}$ & 3.04425600 & -2.82392300 & -2.28076000 \\
\hline $\mathrm{C}$ & 3.92567300 & -3.79304200 & 0.18300700 \\
\hline $\mathrm{H}$ & 3.26050200 & -1.93427100 & 1.01705100 \\
\hline $\mathrm{C}$ & 3.60450100 & -4.09413500 & -2.19655000 \\
\hline $\mathrm{H}$ & 2.71317000 & -2.42017800 & -3.23167000 \\
\hline $\mathrm{C}$ & 4.04828800 & -4.58085700 & -0.96260400 \\
\hline $\mathrm{H}$ & 4.27979700 & -4.16376500 & 1.14070000 \\
\hline $\mathrm{H}$ & 3.70228800 & -4.70292500 & -3.09096000 \\
\hline $\mathrm{H}$ & 4.49372600 & -5.57000000 & -0.89695600 \\
\hline $\mathrm{C}$ & 3.72362300 & 1.73068200 & -0.28082000 \\
\hline $\mathrm{H}$ & 4.26331800 & 0.86832400 & 0.13806500 \\
\hline $\mathrm{H}$ & 3.94841900 & 1.77030500 & -1.35074700 \\
\hline $\mathrm{C}$ & 4.23838800 & 3.01770600 & 0.40221200 \\
\hline $\mathrm{C}$ & 5.63492800 & 3.36825700 & -0.13216200 \\
\hline C & 4.26456400 & 2.89249000 & 1.93363200 \\
\hline $\mathrm{H}$ & 3.55779500 & 3.82978200 & 0.12100800 \\
\hline $\mathrm{H}$ & 5.61447000 & 3.52821200 & -1.21600700 \\
\hline $\mathrm{H}$ & 6.01749300 & 4.28263700 & 0.33659700 \\
\hline $\mathrm{H}$ & 6.35204500 & 2.56293700 & 0.07738100 \\
\hline $\mathrm{H}$ & 3.28472000 & 2.63711000 & 2.35462000 \\
\hline $\mathrm{H}$ & 4.96999700 & 2.11286200 & 2.25037600 \\
\hline $\mathrm{H}$ & 4.58123700 & 3.83542600 & 2.39470800 \\
\hline S & 3.19982700 & 1.91414000 & -1.03181800 \\
\hline $\mathrm{F}$ & 1.62784800 & 2.65564500 & 1.03105100 \\
\hline $\mathrm{F}$ & -1.62070900 & 4.01881800 & -0.67425300 \\
\hline $\mathrm{F}$ & -3.34668200 & 3.91987900 & 0.63770100 \\
\hline $\mathrm{O}$ & -4.58732800 & 1.84094900 & -0.58378700 \\
\hline $\mathrm{O}$ & -2.83805300 & 2.15825800 & -2.41836100 \\
\hline $\mathrm{N}$ & -2.41386100 & 0.36632500 & -0.60542300 \\
\hline $\mathrm{C}$ & -3.16380800 & -0.51830300 & 0.38496200 \\
\hline $\mathrm{C}$ & -2.36770100 & -1.80615100 & 0.39005000 \\
\hline $\mathrm{C}$ & -0.96575600 & -1.74846400 & 0.59043600 \\
\hline $\mathrm{C}$ & .22355600 & -2.94378600 & 0.5354210 \\
\hline
\end{tabular}




\begin{tabular}{|c|c|c|c|c|c|c|c|}
\hline $\mathrm{C}$ & -0.85725200 & -4.15894600 & 0.29436700 & $\mathrm{C}$ & -3.76220000 & 3.10465200 & -3.25334500 \\
\hline $\mathrm{C}$ & -2.23927800 & -4.21331900 & 0.10038600 & $\mathrm{H}$ & -2.72806000 & 1.29220900 & -3.81685900 \\
\hline $\mathrm{C}$ & -3.00195200 & -3.03741800 & 0.14818400 & $\mathrm{C}$ & -4.30517800 & 3.83908900 & -2.19438100 \\
\hline $\mathrm{C}$ & -2.35656400 & 3.22985900 & 0.07607000 & $\mathrm{H}$ & -4.65238100 & 3.91123200 & -0.06708400 \\
\hline $\mathrm{H}$ & -0.51676100 & -0.97360300 & 1.50150600 & $\mathrm{H}$ & -3.83384200 & 3.47934800 & -4.27041200 \\
\hline $\mathrm{H}$ & 0.84264000 & -2.91861000 & 0.72715600 & $\mathrm{H}$ & -4.80209800 & 4.78593800 & -2.38766100 \\
\hline $\mathrm{H}$ & -0.28032500 & -5.07905900 & 0.26012800 & $\mathrm{C}$ & -3.53508700 & -2.01809400 & 0.24591700 \\
\hline $\mathrm{H}$ & -2.71551100 & -5.16934300 & -0.08473900 & $\mathrm{H}$ & -4.12930100 & -1.11104400 & 0.43733600 \\
\hline $\mathrm{C}$ & -3.28436200 & 0.11250500 & 1.77576600 & $\mathrm{H}$ & -3.79240100 & -2.37381900 & -0.75595000 \\
\hline $\mathrm{H}$ & -2.30988200 & 0.28430200 & 2.23730200 & $\mathrm{C}$ & -3.92886500 & -3.09184900 & 1.28442300 \\
\hline $\mathrm{H}$ & -3.85596700 & 1.04338800 & 1.74171000 & $\mathrm{C}$ & -5.31809500 & -3.65541500 & 0.95125500 \\
\hline $\mathrm{H}$ & -3.83676500 & -0.59011500 & 2.40797300 & $\mathrm{C}$ & -3.90103500 & -2.55152200 & 2.72279300 \\
\hline $\mathrm{O}$ & -4.35556200 & -2.99878800 & -0.03146600 & $\mathrm{H}$ & -3.20258100 & -3.90669800 & 1.19213800 \\
\hline $\mathrm{C}$ & -5.04477600 & -4.21035100 & -0.31048200 & $\mathrm{H}$ & -5.33043600 & -4.10791400 & -0.04660200 \\
\hline $\mathrm{H}$ & -4.95040900 & -4.92488400 & 0.51696100 & $\mathrm{H}$ & -5.61508800 & -4.42497500 & 1.67387300 \\
\hline $\mathrm{H}$ & -6.09324500 & -3.93362900 & -0.43069500 & $\mathrm{H}$ & -6.08260100 & -2.86647500 & 0.97378300 \\
\hline $\mathrm{H}$ & -4.68230600 & -4.67443400 & -1.23660400 & $\mathrm{H}$ & -2.92023100 & -2.14844400 & 3.00246500 \\
\hline $\mathrm{H}$ & -4.16203700 & -0.68753200 & -0.02121400 & $\mathrm{H}$ & -4.63668700 & -1.74618200 & 2.85345500 \\
\hline $\mathrm{H}$ & -2.44752000 & -0.09614200 & -1.52315400 & $\mathrm{H}$ & -4.14404200 & -3.34443500 & 3.44021600 \\
\hline $\mathrm{C}$ & 1.02171300 & -0.51901100 & 3.16690500 & $\mathrm{~S}$ & 3.38550900 & -1.74743900 & -1.13197700 \\
\hline $\mathrm{O}$ & 2.00189700 & -0.76925500 & 2.42501400 & $\mathrm{~F}$ & 2.02813300 & -2.54002500 & 1.07438400 \\
\hline $\mathrm{O}$ & -0.20143500 & -0.43619300 & 2.78622300 & $\mathrm{~F}$ & 2.16067000 & -4.01990000 & -0.52855500 \\
\hline $\mathrm{C}$ & 1.25574600 & -0.29113600 & 4.65509100 & $\mathrm{~F}$ & 3.90034700 & -3.55581300 & 0.68181100 \\
\hline $\mathrm{H}$ & 0.75210000 & -1.07708500 & 5.22896900 & $\mathrm{O}$ & 4.78202400 & -1.47969800 & -0.79718900 \\
\hline $\mathrm{H}$ & 2.32304000 & -0.29638300 & 4.88424700 & $\mathrm{O}$ & 2.96275900 & -2.14360300 & -2.46576300 \\
\hline $\mathrm{H}$ & 0.81238500 & 0.66262900 & 4.95969700 & $\mathrm{~N}$ & 2.46181200 & -0.26438200 & -0.77627400 \\
\hline \multirow[t]{2}{*}{$\mathrm{H}$} & 1.96701300 & -0.40183300 & 0.75104200 & $\mathrm{C}$ & 3.12547100 & 0.73840400 & 0.15293400 \\
\hline & & & & $\mathrm{C}$ & 2.20083400 & 1.93514300 & 0.12056000 \\
\hline \multicolumn{4}{|c|}{$\mathrm{R}_{2 \mathrm{e}}$} & $\mathrm{C}$ & 0.82600500 & 1.72735400 & -0.11629200 \\
\hline \multicolumn{4}{|c|}{-2507.02697466} & $\mathrm{C}$ & -0.02188100 & 2.84957600 & -0.11591300 \\
\hline $\mathrm{Pd}$ & 0.34698600 & -0.21059800 & -0.49028500 & $\mathrm{C}$ & 0.49242000 & 4.12965900 & 0.11564100 \\
\hline $\mathrm{C}$ & -2.04165700 & -1.65522000 & 0.25982900 & $\mathrm{C}$ & 1.85279100 & 4.33041800 & 0.35066300 \\
\hline $\mathrm{C}$ & -1.13526600 & -2.67837300 & -0.47501300 & $\mathrm{C}$ & 2.71288200 & 3.22600600 & 0.35190600 \\
\hline $\mathrm{O}$ & 0.01661100 & -2.21008500 & -0.86112700 & $\mathrm{C}$ & 2.78953700 & -3.07162600 & 0.12383700 \\
\hline $\mathrm{O}$ & -1.50062300 & -3.84015100 & -0.58376800 & $\mathrm{H}$ & -0.14137900 & 2.06968700 & 2.03917000 \\
\hline $\mathrm{H}$ & -1.68082200 & -1.61587100 & 1.29353000 & $\mathrm{H}$ & -1.08134500 & 2.74329900 & -0.32056700 \\
\hline $\mathrm{N}$ & -1.77416700 & -0.25109100 & -0.24763300 & $\mathrm{H}$ & -0.17596800 & 4.98739300 & 0.10867900 \\
\hline $\mathrm{C}$ & -2.38697700 & 0.05767200 & -1.52626200 & $\mathrm{H}$ & 2.22947500 & 5.33091400 & 0.52803800 \\
\hline $\mathrm{O}$ & -2.37861400 & -0.76625400 & -2.41542100 & $\mathrm{C}$ & 3.30508100 & 0.19156300 & 1.57196900 \\
\hline $\mathrm{C}$ & -3.02161200 & 1.40089100 & -1.69338300 & $\mathrm{H}$ & 2.34057300 & -0.06779000 & 2.01721200 \\
\hline $\mathrm{C}$ & -3.57273000 & 2.13918500 & -0.63331000 & $\mathrm{H}$ & 3.96908100 & -0.67668100 & 1.59827200 \\
\hline $\mathrm{C}$ & -3.13589300 & 1.88698300 & -3.00633100 & $\mathrm{H}$ & 3.76401600 & 0.98066800 & 2.17449900 \\
\hline $\mathrm{C}$ & -4.21656600 & 3.35124700 & -0.88969200 & $\mathrm{O}$ & 4.05936500 & 3.29967500 & 0.58050700 \\
\hline $\mathrm{H}$ & -3.52428300 & 1.77711900 & 0.38880800 & C & 4.64629200 & 4.57848300 & 0.77939700 \\
\hline
\end{tabular}




$\begin{array}{lrrr}\mathrm{H} & 4.24334000 & 5.06979400 & 1.67422700 \\ \mathrm{H} & 5.71397000 & 4.39846400 & 0.91543000 \\ \mathrm{H} & 4.49433600 & 5.22881400 & -0.09131300 \\ \mathrm{H} & 4.10816900 & 0.98338300 & -0.25859100 \\ \mathrm{H} & 2.44475800 & 0.15144400 & -1.71694100 \\ \mathrm{C} & -1.50311900 & 1.45553100 & 3.22174200 \\ \mathrm{O} & -2.23745000 & 1.06631400 & 2.32521800 \\ \mathrm{O} & -0.30509300 & 2.00909600 & 3.00895700 \\ \mathrm{C} & -1.83832200 & 1.36935100 & 4.68872200 \\ \mathrm{H} & -1.82869600 & 2.37033500 & 5.13253200 \\ \mathrm{H} & -2.81924300 & 0.91174800 & 4.81721700 \\ \mathrm{H} & -1.07806300 & 0.77679600 & 5.20833900 \\ \mathrm{H} & -2.00638100 & 0.42122700 & 0.48878900\end{array}$

$\mathrm{R}_{5 \mathrm{a}}$

$-2300.74951356$

$\begin{array}{llll}\text { Pd } & -0.37575400 & 0.07149000 & -0.36794700\end{array}$

$\begin{array}{llll}\text { S } & -1.27736000 & -0.56397700 & 2.41176500\end{array}$

F $\quad-0.02729800 \quad-2.26155700 \quad 4.01879400$

F $\quad 1.22290000 \quad-0.63639200 \quad 3.28289000$

F $\quad-0.27104800 \quad-0.25289900 \quad 4.81889300$

$\begin{array}{llll}\text { O } & -2.53782300 & -1.05997700 & 2.97075200\end{array}$

$\begin{array}{llll}\text { O } & -1.06759300 & 0.89604800 & 2.20307000\end{array}$

$\begin{array}{llll}\mathrm{N} & -0.81421600 & -1.27863800 & 1.04875100\end{array}$

$\begin{array}{llll}\text { C } & -1.31364500 & -2.61596600 & 0.61726000\end{array}$

$\begin{array}{llll}\text { C } & -1.43990800 & -2.52215800 & -0.88638400\end{array}$

$\begin{array}{llll}\text { C } & -0.97438500 & -1.38502700 & -1.55224800\end{array}$

$\begin{array}{llll}\text { C } & -1.00536300 & -1.23372900 & -2.93640300\end{array}$

$\begin{array}{llll}\text { C } & -1.56052600 & -2.27203500 & -3.68449300\end{array}$

$\begin{array}{llll}\text { C } & -2.07116200 & -3.41574700 & -3.06498900\end{array}$

$\begin{array}{llll}\text { C } & -2.01832600 & -3.54223800 & -1.67223400\end{array}$

$\begin{array}{llll}\text { C } & 0.00514400 & -0.95704900 & 3.71867400\end{array}$

H $\quad-0.62249100 \quad-0.34006300 \quad-3.41415600$

$\begin{array}{llll}\mathrm{H} & -1.60434600 & -2.18861400 & -4.76715000\end{array}$

$\mathrm{H} \quad-2.50974200 \quad-4.19858700 \quad-3.67234800$

$\begin{array}{llll}\text { O } & -2.51282600 & -4.61018000 & -0.97946000\end{array}$

$\begin{array}{llll}\text { C } & -3.13371500 & -5.66245800 & -1.70431900\end{array}$

$\mathrm{H} \quad-2.42976900 \quad-6.14451400 \quad-2.39466200$

H $\quad-3.45958900 \quad-6.38657700 \quad-0.95585200$

$\begin{array}{llll}\mathrm{H} & -4.00480000 & -5.30174600 & -2.26599300\end{array}$

I $\quad 2.45715000 \quad-0.58773400 \quad-0.41625800$

I $\quad 5.36678400 \quad-0.71009200 \quad-0.43556800$

$\begin{array}{llll}\text { C } & -0.35892300 & -3.73355500 & 1.06794900\end{array}$

H $\quad 0.64047200 \quad-3.56783500 \quad 0.65391800$

\begin{tabular}{|c|c|c|c|}
\hline $\mathrm{H}$ & -0.28569600 & -3.75172600 & 2.15802500 \\
\hline $\mathrm{H}$ & -0.73077000 & -4.70284700 & 0.72718500 \\
\hline $\mathrm{H}$ & -2.30273200 & -2.78652700 & 1.06083400 \\
\hline $\mathrm{C}$ & -0.71134500 & 3.49628200 & -0.51332800 \\
\hline E & -0.16871500 & 2.78973300 & -1.75215900 \\
\hline $\mathrm{H}$ & -1.02974200 & 4.48732700 & -0.84750100 \\
\hline v & -1.86142700 & 2.77527700 & -0.00135700 \\
\hline $\mathrm{H}$ & -1.79892700 & 2.32269100 & 0.90507400 \\
\hline $\mathrm{C}$ & -2.94216000 & 2.62233700 & -0.83779900 \\
\hline $\mathrm{O}$ & -2.86631000 & 3.05092500 & -1.99260900 \\
\hline $\mathrm{C}$ & -4.16859100 & 1.94205100 & -0.31832700 \\
\hline $\mathrm{C}$ & -4.29949200 & 1.44882500 & 0.98885600 \\
\hline $\mathrm{C}$ & -5.23614800 & 1.79530800 & -1.21760100 \\
\hline $\mathrm{C}$ & -5.47469800 & 0.80899200 & 1.38237200 \\
\hline $\mathrm{H}$ & -3.49695300 & 1.53930400 & 1.71219800 \\
\hline $\mathrm{C}$ & -6.41204500 & 1.16601900 & -0.81856000 \\
\hline $\mathrm{H}$ & -5.11785200 & 2.18298300 & -2.22359600 \\
\hline $\mathrm{C}$ & -6.53259900 & 0.66864800 & 0.48226100 \\
\hline $\mathrm{H}$ & -5.55381500 & 0.41564000 & 2.39132200 \\
\hline $\mathrm{H}$ & -7.23343400 & 1.05920500 & -1.52180100 \\
\hline $\mathrm{H}$ & -7.44768700 & 0.17131700 & 0.79270300 \\
\hline $\mathrm{C}$ & 0.37771600 & 3.62276400 & 0.57624100 \\
\hline $\mathrm{H}$ & -0.10272300 & 4.09219700 & 1.44506800 \\
\hline $\mathrm{H}$ & 0.67982300 & 2.61797300 & 0.89906800 \\
\hline $\mathrm{C}$ & 1.63307200 & 4.43243700 & 0.19284000 \\
\hline $\mathrm{C}$ & 2.64715200 & 4.36826400 & 1.34589900 \\
\hline $\mathrm{C}$ & 1.30902200 & 5.89023600 & -0.16994400 \\
\hline $\mathrm{H}$ & 2.10000900 & 3.95861200 & -0.68297300 \\
\hline $\mathrm{H}$ & 2.91383000 & 3.33341100 & 1.58852600 \\
\hline $\mathrm{H}$ & 3.56825000 & 4.90000300 & 1.08319000 \\
\hline $\mathrm{H}$ & 2.24040500 & 4.83324400 & 2.25311900 \\
\hline $\mathrm{H}$ & 0.67673900 & 5.96803300 & -1.06066000 \\
\hline $\mathrm{H}$ & 0.79460500 & 6.39476400 & 0.65840100 \\
\hline $\mathrm{H}$ & 2.23007900 & 6.44727700 & -0.37514800 \\
\hline $\mathrm{O}$ & -0.01350300 & 1.57006000 & -1.88045700 \\
\hline $\mathrm{O}$ & 0.22886800 & 3.61506900 & -2.70923300 \\
\hline $\mathrm{H}$ & 0.60062700 & 3.07885400 & -3.43753500 \\
\hline
\end{tabular}

$\mathrm{TS}_{\mathrm{R} 3 \mathrm{a}}$

$-2300.71691337$

$\begin{array}{llll}\text { Pd } & 0.36637200 & -0.50032700 & -0.41484800 \\ \text { S } & -0.71487400 & -0.69046300 & 2.33115200 \\ \text { F } & -0.44282900 & -2.85227200 & 3.78673700 \\ \text { F } & 1.45275900 & -1.92259100 & 3.25690700\end{array}$ 


\begin{tabular}{|c|c|c|c|c|c|c|c|}
\hline $\mathrm{F}$ & 0.18541100 & -1.01083200 & 4.77113700 & $\mathrm{H}$ & -6.04226000 & 3.23127300 & 1.65558000 \\
\hline $\mathrm{O}$ & -2.05589200 & -0.51277700 & 2.89741600 & $\mathrm{C}$ & 2.37331900 & 2.95521900 & 0.74712600 \\
\hline $\mathrm{O}$ & 0.13237300 & 0.50784500 & 2.08265900 & $\mathrm{H}$ & 2.07054300 & 3.61731400 & 1.56972000 \\
\hline $\mathrm{N}$ & -0.63669200 & -1.63923100 & 0.99289800 & $\mathrm{H}$ & 2.31558600 & 1.92974800 & 1.12792900 \\
\hline $\mathrm{C}$ & -1.94410300 & -2.20468600 & 0.51852700 & $\mathrm{C}$ & 3.82930900 & 3.27818100 & 0.35550400 \\
\hline $\mathrm{C}$ & -2.41465500 & -1.31331800 & -0.60063900 & $\mathrm{C}$ & 4.75039600 & 2.96514400 & 1.54519200 \\
\hline $\mathrm{C}$ & -1.51886700 & -0.39110100 & -1.14799800 & $\mathrm{C}$ & 4.01261900 & 4.73292100 & -0.10482600 \\
\hline $\mathrm{C}$ & -1.86864400 & 0.48778500 & -2.16897800 & $\mathrm{H}$ & 4.12328700 & 2.61734300 & -0.47184700 \\
\hline $\mathrm{C}$ & -3.17973800 & 0.43755500 & -2.64683700 & $\mathrm{H}$ & 4.65678900 & 1.91937100 & 1.85873700 \\
\hline $\mathrm{C}$ & -4.11094700 & -0.46366300 & -2.12479700 & $\mathrm{H}$ & 5.79913900 & 3.14628800 & 1.28404400 \\
\hline $\mathrm{C}$ & -3.73216500 & -1.33738700 & -1.10013200 & $\mathrm{H}$ & 4.50737400 & 3.59907700 & 2.40784000 \\
\hline $\mathrm{C}$ & 0.19736200 & -1.69060800 & 3.62187300 & $\mathrm{H}$ & 3.44717500 & 4.95652500 & -1.01529300 \\
\hline $\mathrm{H}$ & -1.16275100 & 1.19627300 & -2.58293700 & $\mathrm{H}$ & 3.69205900 & 5.43434800 & 0.67680400 \\
\hline $\mathrm{H}$ & -3.48143500 & 1.11472100 & -3.44137700 & $\mathrm{H}$ & 5.06805300 & 4.93695400 & -0.31866700 \\
\hline $\mathrm{H}$ & -5.12212900 & -0.47249600 & -2.51449100 & $\mathrm{O}$ & 1.17325700 & 1.09214000 & -1.75102800 \\
\hline $\mathrm{O}$ & -4.56561200 & -2.24318900 & -0.50616100 & $\mathrm{O}$ & 2.30676500 & 2.84426800 & -2.49342000 \\
\hline $\mathrm{C}$ & -5.92854700 & -2.27128100 & -0.90387000 & $\mathrm{H}$ & 2.45942300 & 2.20013000 & -3.21344500 \\
\hline $\mathrm{H}$ & -6.03364600 & -2.54745500 & -1.96106000 & & & & \\
\hline $\mathrm{H}$ & -6.40431200 & -3.03259300 & -0.28328300 & $\mathrm{R}_{6 \mathrm{a}}$ & & & \\
\hline $\mathrm{H}$ & -6.41587900 & -1.30324200 & -0.73115700 & -23 & 0.71697179 & & \\
\hline I & 3.12864700 & -1.07126500 & 0.18792700 & $\mathrm{Pd}$ & 0.38401200 & -0.58490000 & -0.39042400 \\
\hline I & 1.22176200 & -2.41508400 & -2.21959800 & $\mathrm{~S}$ & -0.69147300 & -0.71932600 & 2.30399700 \\
\hline $\mathrm{C}$ & -1.76758300 & -3.68053500 & 0.14429700 & $\mathrm{~F}$ & -0.36650900 & -2.77921400 & 3.88394600 \\
\hline $\mathrm{H}$ & -1.04978800 & -3.80261800 & -0.66941700 & $\mathrm{~F}$ & 1.51009700 & -1.88395000 & 3.24145000 \\
\hline $\mathrm{H}$ & -1.40943000 & -4.23984500 & 1.01466500 & $\mathrm{~F}$ & 0.28182100 & -0.87444800 & 4.72590400 \\
\hline $\mathrm{H}$ & -2.73200800 & -4.09192000 & -0.16832400 & $\mathrm{O}$ & -2.01797500 & -0.51943600 & 2.89198900 \\
\hline $\mathrm{H}$ & -2.66215600 & -2.14967100 & 1.34377000 & $\mathrm{O}$ & 0.12996200 & 0.47452900 & 1.94285400 \\
\hline $\mathrm{C}$ & 1.30232800 & 3.13826300 & -0.35439400 & $\mathrm{~N}$ & -0.62366700 & -1.73811400 & 1.00778000 \\
\hline $\mathrm{C}$ & 1.55938700 & 2.24752000 & -1.56862300 & $\mathrm{C}$ & -1.95649400 & -2.25644500 & 0.54556200 \\
\hline $\mathrm{H}$ & 1.33514800 & 4.16021100 & -0.74029700 & $\mathrm{C}$ & -2.43235900 & -1.32073300 & -0.53420800 \\
\hline $\mathrm{N}$ & -0.01901100 & 2.90951500 & 0.19008600 & $\mathrm{C}$ & -1.52482600 & -0.41257900 & -1.08551800 \\
\hline $\mathrm{H}$ & -0.10516200 & 2.37496600 & 1.04605200 & $\mathrm{C}$ & -1.87722300 & 0.50083000 & -2.07439900 \\
\hline $\mathrm{C}$ & -1.10795600 & 3.38467600 & -0.49850200 & $\mathrm{C}$ & -3.20463900 & 0.50661500 & -2.50751100 \\
\hline $\mathrm{O}$ & -0.94999600 & 3.89265400 & -1.61148000 & $\mathrm{C}$ & -4.14864300 & -0.37595500 & -1.97625600 \\
\hline $\mathrm{C}$ & -2.45534200 & 3.30009200 & 0.15172000 & $\mathrm{C}$ & -3.76625800 & -1.28848500 & -0.98770100 \\
\hline $\mathrm{C}$ & -2.70806500 & 2.61933000 & 1.35134300 & $\mathrm{C}$ & 0.26816400 & -1.63293300 & 3.62750100 \\
\hline $\mathrm{C}$ & -3.51189300 & 3.95260000 & -0.50095100 & $\mathrm{H}$ & -1.16098100 & 1.19135600 & -2.50037400 \\
\hline $\mathrm{C}$ & -3.99641800 & 2.59003700 & 1.88493000 & $\mathrm{H}$ & -3.50869300 & 1.21294300 & -3.27520200 \\
\hline $\mathrm{H}$ & -1.92404400 & 2.08781600 & 1.87824700 & $\mathrm{H}$ & -5.17170700 & -0.34123200 & -2.33184700 \\
\hline $\mathrm{C}$ & -4.79463900 & 3.93371300 & 0.04009400 & $\mathrm{O}$ & -4.60846100 & -2.18170700 & -0.38807800 \\
\hline $\mathrm{H}$ & -3.29972700 & 4.46975400 & -1.43047700 & $\mathrm{C}$ & -5.98411000 & -2.15782500 & -0.74162400 \\
\hline $\mathrm{C}$ & -5.04030600 & 3.25062200 & 1.23469900 & $\mathrm{H}$ & -6.13096800 & -2.40390900 & -1.80114400 \\
\hline $\mathrm{H}$ & -4.17705600 & 2.04228300 & 2.80505500 & $\mathrm{H}$ & -6.46333900 & -2.91964800 & -0.12445100 \\
\hline $\mathrm{H}$ & -5.60403400 & 4.44961200 & -0.46961400 & $\mathrm{H}$ & -6.43463400 & -1.17994000 & -0.52945700 \\
\hline
\end{tabular}




\begin{tabular}{|c|c|c|c|c|c|c|c|}
\hline I & 3.13503000 & -1.10524000 & 0.19767300 & $\mathrm{Pd}$ & -0.83485500 & -0.12545800 & -0.41217800 \\
\hline I & 0.99421600 & -2.42682900 & -2.28570700 & $\mathrm{C}$ & 3.16597100 & -1.83625400 & 0.89698300 \\
\hline $\mathrm{C}$ & -1.83250100 & -3.72703900 & 0.13557800 & $\mathrm{C}$ & 1.81057600 & -1.80793500 & 0.21073400 \\
\hline $\mathrm{H}$ & -1.14556500 & -3.85373200 & -0.70269600 & $\mathrm{H}$ & 3.68219200 & -2.75876300 & 0.62827500 \\
\hline $\mathrm{H}$ & -1.46623800 & -4.31582100 & 0.98272400 & $\mathrm{~N}$ & 3.94584000 & -0.72670700 & 0.36883500 \\
\hline $\mathrm{H}$ & -2.81902400 & -4.10072300 & -0.15472800 & $\mathrm{H}$ & 3.45177400 & 0.12629500 & 0.14798100 \\
\hline $\mathrm{H}$ & -2.64975100 & -2.20377500 & 1.39225100 & $\mathrm{C}$ & 5.31710900 & -0.76542300 & 0.36893500 \\
\hline $\mathrm{C}$ & 1.37883800 & 3.10294600 & -0.40694400 & $\mathrm{O}$ & 5.93199700 & -1.72607200 & 0.82669200 \\
\hline $\mathrm{C}$ & 1.60316000 & 2.16038400 & -1.58742300 & $\mathrm{C}$ & 6.01706700 & 0.42794700 & -0.21486200 \\
\hline $\mathrm{H}$ & 1.44073900 & 4.10847200 & -0.83015900 & $\mathrm{C}$ & 5.40525000 & 1.33074100 & -1.09711100 \\
\hline$N$ & 0.05311300 & 2.93904000 & 0.15049500 & $\mathrm{C}$ & 7.36179500 & 0.61429600 & 0.13413600 \\
\hline $\mathrm{H}$ & -0.04915800 & 2.41544700 & 1.01029000 & $\mathrm{C}$ & 6.12471900 & 2.41213500 & -1.60647400 \\
\hline $\mathrm{C}$ & -1.02471800 & 3.44381800 & -0.53304900 & $\mathrm{H}$ & 4.37971600 & 1.17927000 & -1.42385300 \\
\hline $\mathrm{O}$ & -0.86461700 & 3.93692200 & -1.65192900 & $\mathrm{C}$ & 8.07679100 & 1.69812200 & -0.37005200 \\
\hline $\mathrm{C}$ & -2.36632200 & 3.41364600 & 0.13607600 & $\mathrm{H}$ & 7.82523700 & -0.10679400 & 0.79926600 \\
\hline $\mathrm{C}$ & -2.64348000 & 2.70170300 & 1.31210400 & $\mathrm{C}$ & 7.45870000 & 2.60115100 & -1.23946000 \\
\hline $\mathrm{C}$ & -3.39049000 & 4.15239300 & -0.47459100 & $\mathrm{H}$ & 5.64565700 & 3.10036300 & -2.29729300 \\
\hline $\mathrm{C}$ & -3.92345900 & 2.73052300 & 1.86567100 & $\mathrm{H}$ & 9.11705900 & 1.83772800 & -0.08910300 \\
\hline $\mathrm{H}$ & -1.88637200 & 2.09938700 & 1.80126600 & $\mathrm{H}$ & 8.01721700 & 3.44438700 & -1.63684500 \\
\hline $\mathrm{C}$ & -4.66431500 & 4.19060300 & 0.08664300 & $\mathrm{C}$ & 2.99991100 & -1.75953000 & 2.43766200 \\
\hline $\mathrm{H}$ & -3.16049900 & 4.69074000 & -1.38776700 & $\mathrm{H}$ & 4.01779900 & -1.72301100 & 2.84358300 \\
\hline $\mathrm{C}$ & -4.93405700 & 3.47860200 & 1.25878600 & $\mathrm{H}$ & 2.51936900 & -0.80105300 & 2.67584100 \\
\hline $\mathrm{H}$ & -4.12494200 & 2.16052500 & 2.76794300 & $\mathrm{C}$ & 2.21875200 & -2.90973600 & 3.10489000 \\
\hline $\mathrm{H}$ & -5.44767800 & 4.77415100 & -0.38962400 & $\mathrm{C}$ & 2.07259200 & -2.62155100 & 4.60744300 \\
\hline $\mathrm{H}$ & -5.92883600 & 3.50469600 & 1.69596700 & $\mathrm{C}$ & 2.87956800 & -4.27843600 & 2.87517100 \\
\hline $\mathrm{C}$ & 2.44942400 & 2.92709800 & 0.69650100 & $\mathrm{H}$ & 1.20645300 & -2.94363200 & 2.67553600 \\
\hline $\mathrm{H}$ & 2.16570700 & 3.62118700 & 1.49933300 & $\mathrm{H}$ & 1.57031000 & -1.66328500 & 4.78644300 \\
\hline $\mathrm{H}$ & 2.37042600 & 1.91411100 & 1.10578800 & $\mathrm{H}$ & 1.48501300 & -3.40460600 & 5.09963900 \\
\hline $\mathrm{C}$ & 3.91161900 & 3.20128700 & 0.29091800 & $\mathrm{H}$ & 3.05472300 & -2.58364800 & 5.09634900 \\
\hline $\mathrm{C}$ & 4.82793900 & 2.90188100 & 1.48776900 & $\mathrm{H}$ & 2.89086400 & -4.56049000 & 1.81724000 \\
\hline $\mathrm{C}$ & 4.12990600 & 4.63555300 & -0.21567500 & $\mathrm{H}$ & 3.91564300 & -4.27841600 & 3.23702800 \\
\hline $\mathrm{H}$ & 4.18559400 & 2.50653100 & -0.51480900 & $\mathrm{H}$ & 2.33583300 & -5.06219000 & 3.41479500 \\
\hline $\mathrm{H}$ & 4.70969700 & 1.86818400 & 1.83112100 & $\mathrm{O}$ & 1.22636400 & -0.72129000 & 0.05725300 \\
\hline $\mathrm{H}$ & 5.87998900 & 3.04954500 & 1.21892600 & $\mathrm{O}$ & 1.35193000 & -2.97496800 & -0.14960100 \\
\hline $\mathrm{H}$ & 4.60307100 & 3.56738700 & 2.33151100 & $\mathrm{~S}$ & -3.43881400 & -0.63877000 & 0.84387000 \\
\hline $\mathrm{H}$ & 3.56688800 & 4.84508800 & -1.13109300 & $\mathrm{~F}$ & -5.65121600 & -0.70176400 & -0.56417300 \\
\hline $\mathrm{H}$ & 3.83048400 & 5.36957400 & 0.54420700 & $\mathrm{~F}$ & -4.33529400 & -2.39070200 & -0.96093900 \\
\hline $\mathrm{H}$ & 5.18936800 & 4.80556100 & -0.43916900 & $\mathrm{~F}$ & -5.44883300 & -2.30283100 & 0.90252900 \\
\hline $\mathrm{O}$ & 1.17279000 & 1.01550500 & -1.73163400 & $\mathrm{O}$ & -4.14110500 & 0.10723900 & 1.89303100 \\
\hline $\mathrm{O}$ & 2.36899100 & 2.69727400 & -2.53322400 & $\mathrm{O}$ & -2.37398100 & -1.59604200 & 1.20350900 \\
\hline \multirow[t]{2}{*}{$\mathrm{H}$} & 2.50415600 & 2.02018200 & -3.22621600 & $\mathrm{~N}$ & -2.84908200 & 0.32790400 & -0.40953800 \\
\hline & & & & $\mathrm{C}$ & -3.10039300 & 1.77354200 & -0.16653300 \\
\hline \multicolumn{4}{|c|}{$\mathrm{TS}_{\mathrm{R} 4 \mathrm{a}}$} & $\mathrm{C}$ & -2.01122100 & 2.24782800 & 0.77824800 \\
\hline \multicolumn{4}{|c|}{-2300.71127869} & $\mathrm{C}$ & -0.74500100 & 1.64226100 & 0.72751100 \\
\hline
\end{tabular}




\begin{tabular}{|c|c|c|c|}
\hline $\mathrm{C}$ & 0.25201800 & 1.87159200 & 1.68797200 \\
\hline C & -0.06113000 & 2.70190300 & 2.75689900 \\
\hline C & -1.30910800 & 3.33424800 & 2.83919800 \\
\hline C & -2.28605900 & 3.10734800 & 1.86033700 \\
\hline C & -4.80694800 & -1.58603400 & -0.02285400 \\
\hline $\mathrm{H}$ & 0.49748800 & -2.87883000 & -0.66703300 \\
\hline $\mathrm{H}$ & 1.22648600 & 1.40847000 & 1.59891400 \\
\hline $\mathrm{H}$ & 0.67732400 & 2.88244000 & 3.53304800 \\
\hline 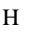 & -1.51090100 & 4.00047300 & 3.66990100 \\
\hline $\mathrm{O}$ & -3.52544400 & 3.66064400 & 1.86856900 \\
\hline C & -3.95733300 & 4.36250900 & 3.03016700 \\
\hline $\mathrm{H}$ & -3.37753000 & 5.28189600 & 3.17955900 \\
\hline $\mathrm{H}$ & -5.00116200 & 4.62073000 & 2.84774800 \\
\hline $\mathrm{H}$ & -3.88645500 & 3.73018500 & 3.92313300 \\
\hline I & -1.15057000 & -2.16945900 & -2.29515800 \\
\hline I & 0.39021500 & 2.05450900 & -1.62538200 \\
\hline $\mathrm{C}$ & -3.28960800 & 2.53280800 & -1.48138800 \\
\hline $\mathrm{H}$ & -2.43246700 & 2.43800300 & -2.14858800 \\
\hline $\mathrm{H}$ & -4.17200600 & 2.13838600 & -1.99433300 \\
\hline $\mathrm{H}$ & -3.45200400 & 3.59500500 & -1.26797600 \\
\hline & -4.04188900 & 1.85679700 & 0.38478800 \\
\hline
\end{tabular}

$\mathrm{R}_{7 \mathrm{a}}$

$-2300.73724181$

$\begin{array}{llll}\text { Pd } & 0.95451600 & -0.19637900 & -0.25950800\end{array}$

$\begin{array}{llll}\text { C } & -3.27784400 & -1.75619500 & 0.03809100\end{array}$

$\begin{array}{llll}\text { C } & -1.77285600 & -1.68311300 & 0.20293100\end{array}$

$\begin{array}{llll}\mathrm{H} & -3.73213800 & -1.87589200 & 1.02661600\end{array}$

N $\quad-3.74641600 \quad-0.50024300 \quad-0.52100400$

$\begin{array}{llll}\mathrm{H} & -3.06995600 & 0.06038300 & -1.02010500\end{array}$

$\begin{array}{llll}\text { C } & -4.95828800 & 0.02426100 & -0.15654900\end{array}$

$\begin{array}{llll}\text { O } & -5.69793200 & -0.54300500 & 0.64412900\end{array}$

$\begin{array}{llll}\text { C } & -5.32406800 & 1.33917500 & -0.78620900\end{array}$

$\begin{array}{llll}\text { C } & -4.80432800 & 1.77619400 & -2.01438800\end{array}$

$\begin{array}{llll}\text { C } & -6.25006500 & 2.14380800 & -0.10782200\end{array}$

$\begin{array}{llll}\text { C } & -5.19120000 & 3.00861500 & -2.54323200\end{array}$

$\begin{array}{llll}\mathrm{H} & -4.12946200 & 1.14087000 & -2.58180700\end{array}$

$\begin{array}{llll}\text { C } & -6.62597700 & 3.37955400 & -0.63139900\end{array}$

$\begin{array}{llll}\text { H } & -6.66489100 & 1.77758100 & 0.82563800\end{array}$

$\begin{array}{llll}\text { C } & -6.09540300 & 3.81572900 & -1.84870200\end{array}$

$\begin{array}{llll}\mathrm{H} & -4.79799000 & 3.33156100 & -3.50340000\end{array}$

$\begin{array}{llll}\mathrm{H} & -7.33782800 & 4.00026000 & -0.09444900\end{array}$

$\begin{array}{llll}\mathrm{H} & -6.39440900 & 4.77566200 & -2.26068500\end{array}$

$\begin{array}{llll}\text { C } & -3.70765300 & -2.95430700 & -0.86123500\end{array}$
$\mathrm{H} \quad-4.78355500 \quad-2.80834900 \quad-1.01848400$

$\begin{array}{llll}\mathrm{H} & -3.22568200 & -2.83264400 & -1.84048100\end{array}$

$\begin{array}{llll}\text { C } & -3.47137400 & -4.38277700 & -0.33234000\end{array}$

$\begin{array}{llll}\text { C } & -3.97740200 & -5.38989600 & -1.37848800\end{array}$

$\begin{array}{llll}\text { C } & -4.14643300 & -4.62464000 & 1.02652500\end{array}$

$\mathrm{H} \quad-2.39232700 \quad-4.54169300 \quad-0.21249200$

$\mathrm{H} \quad-3.48577000 \quad-5.24465600 \quad-2.34777400$

H $\quad-3.78155500 \quad-6.41817000 \quad-1.05444300$

H $\quad-5.05964200 \quad-5.28890700 \quad-1.53190400$

H $\quad-3.70898600 \quad-4.01013200 \quad 1.82058400$

$\mathrm{H} \quad-5.22018900 \quad-4.40236800 \quad 0.97861300$

$\mathrm{H} \quad-4.03480300 \quad-5.67202500 \quad 1.32924700$

$\begin{array}{llll}\text { O } & -1.10506400 & -0.81803800 & -0.38225800\end{array}$

$\begin{array}{llll}\text { O } & -1.26729900 & -2.61565900 & 0.97533900\end{array}$

$\begin{array}{llll}\text { S } & 3.64467900 & 0.86541000 & -1.51981600\end{array}$

F $\quad 5.74477200 \quad 0.64419100 \quad 0.05814700$

F $\quad 5.04554800 \quad-1.26142900 \quad-0.72370100$

F $\quad 6.10070300 \quad 0.12141900 \quad-2.02680200$

$\begin{array}{llll}\text { O } & 4.05857700 & 2.25995400 & -1.73246200\end{array}$

$\begin{array}{llll}\text { O } & 3.08224300 & 0.07645100 & -2.61703300\end{array}$

$\begin{array}{llll}\mathrm{N} & 2.74651400 & 0.70496400 & -0.12016500\end{array}$

$\begin{array}{llll}\text { C } & 2.38542700 & 1.98042500 & 0.55638700\end{array}$

$\begin{array}{llll}\text { C } & 0.82669700 & 1.99231900 & 0.60426400\end{array}$

$\begin{array}{llll}\text { C } & -0.04518700 & 1.70789300 & 1.69476300\end{array}$

C $\quad-1.41014400 \quad 1.98266200 \quad 1.63672000$

$\begin{array}{llll}\text { C } & -1.96390800 & 2.53945500 & 0.48248800\end{array}$

$\begin{array}{llll}\text { C } & -1.17532200 & 2.81738200 & -0.62588200\end{array}$

$\begin{array}{llll}\text { C } & 0.20177800 & 2.56597400 & -0.56757700\end{array}$

$\begin{array}{llll}\text { C } & 5.23481200 & 0.02088900 & -1.01299000\end{array}$

$\begin{array}{llll}\mathrm{H} & -0.27702800 & -2.64675100 & 0.86183700\end{array}$

H $\quad-2.04698500 \quad 1.75589400 \quad 2.48344600$

H $\quad-3.02880600 \quad 2.74931300 \quad 0.44911900$

H $\quad-1.61894000 \quad 3.25157700 \quad-1.51334300$

O $\quad 1.04946400 \quad 2.88986100 \quad-1.56109600$

$\begin{array}{llll}\text { C } & 0.55543100 & 3.32000900 & -2.82764300\end{array}$

H $\quad 0.03261700 \quad 4.27987900 \quad-2.74304900$

H $\quad 1.44193300 \quad 3.43259600 \quad-3.45093400$

$\mathrm{H} \quad-0.10960900 \quad 2.56510200 \quad-3.26231500$

I $\quad \begin{array}{llll}1.84647600 & -2.71648200 & -0.50570700\end{array}$

I $\quad 0.55296200 \quad 0.76594700 \quad 3.53063700$

$\begin{array}{llll}\text { C } & 3.18860600 & 2.16554500 & 1.84360400\end{array}$

$\mathrm{H} \quad 4.21801200 \quad 2.39528900 \quad 1.55099700$

H $\quad 2.80591200 \quad 3.00529300 \quad 2.43498100$

H $\quad 3.21182100 \quad 1.26907900 \quad 2.46141400$ 


\begin{tabular}{|c|c|c|c|c|c|c|c|}
\hline \multirow[t]{2}{*}{$\mathrm{H}$} & 2.64584300 & 2.81553000 & -0.09519400 & $\mathrm{~N}$ & -1.58755400 & -1.42538600 & 0.93281300 \\
\hline & & & & $\mathrm{C}$ & -0.72827300 & -2.64718800 & 0.96178700 \\
\hline \multicolumn{4}{|l|}{$\mathrm{R}_{5 \mathrm{~b}}$} & $\mathrm{C}$ & -0.07609000 & -2.69059800 & -0.39660500 \\
\hline \multicolumn{4}{|c|}{-2300.73854334} & $\mathrm{C}$ & -0.14382800 & -1.55436400 & -1.20817300 \\
\hline $\mathrm{Pd}$ & -1.24682200 & -0.07396100 & -0.54451600 & $\mathrm{C}$ & 0.46844900 & -1.49995200 & -2.46305900 \\
\hline $\mathrm{C}$ & -1.46241900 & 2.96379300 & -0.17751300 & $\mathrm{C}$ & 1.15764900 & -2.62646300 & -2.91296600 \\
\hline $\mathrm{C}$ & -0.66918000 & 2.63772300 & -1.43701900 & $\mathrm{C}$ & 1.23382300 & -3.78470700 & -2.13360500 \\
\hline $\mathrm{H}$ & -1.83506200 & 3.98576000 & -0.29363200 & $\mathrm{C}$ & 0.61821600 & -3.81825000 & -0.87736200 \\
\hline $\mathrm{N}$ & -2.59172300 & 2.02177100 & -0.13469800 & $\mathrm{C}$ & -4.27614600 & -2.05137300 & 1.15018700 \\
\hline $\mathrm{H}$ & -2.89236300 & 1.67920000 & 0.77958500 & $\mathrm{H}$ & 0.38832700 & 3.37121900 & -2.79807300 \\
\hline $\mathrm{C}$ & -3.54033600 & 2.23521900 & -1.15037700 & $\mathrm{H}$ & 0.40515400 & -0.60740900 & -3.07665100 \\
\hline $\mathrm{O}$ & -3.18115700 & 2.86015300 & -2.14617000 & $\mathrm{H}$ & 1.64110100 & -2.60999000 & -3.88680600 \\
\hline $\mathrm{C}$ & -4.93031600 & 1.71776800 & -1.00845500 & $\mathrm{H}$ & 1.76800900 & -4.64879300 & -2.51130800 \\
\hline $\mathrm{C}$ & -5.49608700 & 1.29888300 & 0.20461400 & $\mathrm{O}$ & 0.62851200 & -4.90529000 & -0.04420000 \\
\hline $\mathrm{C}$ & -5.71664900 & 1.71595000 & -2.17372100 & $\mathrm{C}$ & 1.28308900 & -6.08506500 & -0.48027400 \\
\hline $\mathrm{C}$ & -6.82371500 & 0.87282500 & 0.24364000 & $\mathrm{H}$ & 2.35426000 & -5.91290200 & -0.65005700 \\
\hline $\mathrm{H}$ & -4.91648000 & 1.27741000 & 1.11951800 & $\mathrm{H}$ & 1.15768400 & -6.81167400 & 0.32470800 \\
\hline $\mathrm{C}$ & -7.03889200 & 1.28709300 & -2.12982000 & $\mathrm{H}$ & 0.83176500 & -6.48056600 & -1.39982700 \\
\hline $\mathrm{H}$ & -5.26936100 & 2.05752900 & -3.10072800 & I & 4.93778700 & 1.23435800 & 1.01805200 \\
\hline $\mathrm{C}$ & -7.59564000 & 0.86436000 & -0.91871100 & I & 6.47312700 & -0.35244800 & -0.70818800 \\
\hline $\mathrm{H}$ & -7.24802200 & 0.53856700 & 1.18562400 & $\mathrm{C}$ & 0.31256600 & -2.57527300 & 2.09365200 \\
\hline $\mathrm{H}$ & -7.63623300 & 1.28192500 & -3.03721900 & $\mathrm{H}$ & 0.95851900 & -3.45775900 & 2.06097000 \\
\hline $\mathrm{H}$ & -8.62853300 & 0.52840000 & -0.88271800 & $\mathrm{H}$ & 0.93184300 & -1.67959900 & 1.97199300 \\
\hline $\mathrm{C}$ & -0.60166700 & 2.84806300 & 1.10042900 & $\mathrm{H}$ & -0.18652100 & -2.53481600 & 3.06444400 \\
\hline $\mathrm{H}$ & -1.27733100 & 2.99529400 & 1.95408700 & $\mathrm{H}$ & -1.34934900 & -3.53901200 & 1.10985100 \\
\hline $\mathrm{H}$ & -0.22385300 & 1.82056300 & 1.17752100 & & & & \\
\hline $\mathrm{C}$ & 0.57358600 & 3.84098600 & 1.21307000 & TS & & & \\
\hline $\mathrm{C}$ & 1.37146600 & 3.53157900 & 2.48990900 & & .69038046 & & \\
\hline $\mathrm{C}$ & 0.11934200 & 5.30905300 & 1.19859600 & $\mathrm{Pd}$ & 0.44880300 & -0.37746800 & 0.05545000 \\
\hline $\mathrm{H}$ & 1.24463400 & 3.68503000 & 0.35638300 & $\mathrm{C}$ & 1.84018700 & 2.84891700 & -0.89468300 \\
\hline $\mathrm{H}$ & 1.73075300 & 2.49554000 & 2.49715700 & $\mathrm{C}$ & 2.09218300 & 1.59683100 & -1.73542800 \\
\hline $\mathrm{H}$ & 2.23998600 & 4.19429500 & 2.57662600 & $\mathrm{H}$ & 2.12083600 & 3.68306800 & -1.54354000 \\
\hline $\mathrm{H}$ & 0.75325800 & 3.67462200 & 3.38528800 & $\mathrm{~N}$ & 0.43120500 & 2.98174000 & -0.57743000 \\
\hline $\mathrm{H}$ & -0.35128700 & 5.58977200 & 0.25046400 & $\mathrm{H}$ & 0.11779400 & 2.79766900 & 0.36982500 \\
\hline $\mathrm{H}$ & -0.59663600 & 5.50620100 & 2.00725900 & $\mathrm{C}$ & -0.45183400 & 3.12337600 & -1.62068000 \\
\hline $\mathrm{H}$ & 0.97592900 & 5.97657400 & 1.34577300 & $\mathrm{O}$ & -0.05325200 & 2.99947800 & -2.78066300 \\
\hline $\mathrm{O}$ & -0.48480800 & 1.49517400 & -1.86211900 & $\mathrm{C}$ & -1.87065800 & 3.46303100 & -1.29772000 \\
\hline $\mathrm{O}$ & -0.10917500 & 3.69069800 & -2.01970100 & $\mathrm{C}$ & -2.25982500 & 4.04364300 & -0.08284700 \\
\hline S & -2.78977800 & -1.25575800 & 1.96624900 & $\mathrm{C}$ & -2.83325700 & 3.22739800 & -2.28998900 \\
\hline $\mathrm{F}$ & -4.44422200 & -1.57116100 & -0.09041300 & $\mathrm{C}$ & -3.59795000 & 4.36687200 & 0.14181700 \\
\hline $\mathrm{F}$ & -4.08440900 & -3.37577000 & 1.07036200 & $\mathrm{H}$ & -1.52361300 & 4.26226700 & 0.68480500 \\
\hline $\mathrm{F}$ & -5.39130300 & -1.81583300 & 1.85456600 & $\mathrm{C}$ & -4.16913600 & 3.54630800 & -2.06185700 \\
\hline $\mathrm{O}$ & -3.16179100 & 0.17680800 & 2.04649700 & $\mathrm{H}$ & -2.50911200 & 2.80007200 & -3.23305800 \\
\hline $\mathrm{O}$ & -2.62379100 & -2.02659900 & 3.20168600 & $\mathrm{C}$ & -4.55378300 & 4.11602200 & -0.84440200 \\
\hline
\end{tabular}




\begin{tabular}{|c|c|c|c|c|c|c|c|}
\hline $\mathrm{H}$ & -3.89153100 & 4.81715300 & 1.08551200 & $\mathrm{C}$ & -1.11204400 & -3.38699300 & 1.84096400 \\
\hline $\mathrm{H}$ & -4.91057800 & 3.35657200 & -2.83318200 & $\mathrm{H}$ & -1.82094100 & -4.19017800 & 1.61337600 \\
\hline $\mathrm{H}$ & -5.59563600 & 4.36947500 & -0.66749000 & $\mathrm{H}$ & -0.15094300 & -3.61778100 & 1.37441500 \\
\hline $\mathrm{C}$ & 2.68072600 & 2.89076200 & 0.40140800 & $\mathrm{H}$ & -0.97578300 & -3.32774400 & 2.92461100 \\
\hline $\mathrm{H}$ & 2.40851300 & 3.82855500 & 0.90497400 & $\mathrm{H}$ & -2.60997200 & -1.85630000 & 1.82954400 \\
\hline $\mathrm{H}$ & 2.35958700 & 2.07589600 & 1.05972100 & & & & \\
\hline $\mathrm{C}$ & 4.21206700 & 2.83862200 & 0.23274300 & $\mathrm{R}_{6 \mathrm{~b}}$ & & & \\
\hline $\mathrm{C}$ & 4.86814900 & 2.82226500 & 1.62235200 & -23 & 0.75321217 & & \\
\hline $\mathrm{C}$ & 4.75917400 & 3.99934700 & -0.61282000 & $\mathrm{Pd}$ & 0.62661700 & -0.78007300 & -0.65144100 \\
\hline $\mathrm{H}$ & 4.47404400 & 1.89480000 & -0.26625100 & $\mathrm{C}$ & 3.60772000 & 1.08930100 & -0.17156600 \\
\hline $\mathrm{H}$ & 4.51023800 & 1.97907700 & 2.22355100 & $\mathrm{C}$ & 3.32536100 & 0.37923400 & -1.49531000 \\
\hline $\mathrm{H}$ & 5.95739100 & 2.73847300 & 1.53716700 & $\mathrm{H}$ & 4.29864500 & 1.89410400 & -0.43574400 \\
\hline $\mathrm{H}$ & 4.64659900 & 3.74635400 & 2.17205300 & $\mathrm{~N}$ & 2.41526800 & 1.69834000 & 0.38192200 \\
\hline $\mathrm{H}$ & 4.38894800 & 3.97585600 & -1.64295700 & $\mathrm{H}$ & 1.92884300 & 1.23857900 & 1.14607300 \\
\hline $\mathrm{H}$ & 4.48500900 & 4.96740300 & -0.17284900 & $\mathrm{C}$ & 1.89107900 & 2.79809000 & -0.25347400 \\
\hline $\mathrm{H}$ & 5.85334800 & 3.95650500 & -0.65906600 & $\mathrm{O}$ & 2.31562900 & 3.12323900 & -1.36537600 \\
\hline $\mathrm{O}$ & 1.56743000 & 0.48842700 & -1.60802900 & $\mathrm{C}$ & 0.84422000 & 3.59551400 & 0.46019600 \\
\hline $\mathrm{O}$ & 3.01987600 & 1.78105600 & -2.66805100 & $\mathrm{C}$ & 0.52654100 & 3.42195100 & 1.81508500 \\
\hline $\mathrm{S}$ & -1.11449400 & 0.27575500 & 2.59375700 & $\mathrm{C}$ & 0.19497000 & 4.59938800 & -0.27450700 \\
\hline $\mathrm{F}$ & -2.80260500 & 1.04905000 & 0.67772100 & $\mathrm{C}$ & -0.43565900 & 4.23177900 & 2.41836600 \\
\hline $\mathrm{F}$ & -3.78020300 & 0.16495700 & 2.40705800 & $\mathrm{H}$ & 1.01974100 & 2.66212900 & 2.41140900 \\
\hline $\mathrm{F}$ & -2.95976300 & 2.17675300 & 2.53672700 & $\mathrm{C}$ & -0.76340900 & 5.40774600 & 0.33051500 \\
\hline $\mathrm{O}$ & -0.17518800 & 1.38693900 & 2.34100000 & $\mathrm{H}$ & 0.46715100 & 4.73204800 & -1.31619800 \\
\hline $\mathrm{O}$ & -1.39955200 & -0.18089400 & 3.95282900 & $\mathrm{C}$ & -1.08156500 & 5.22422800 & 1.67938100 \\
\hline $\mathrm{N}$ & -0.70260400 & -0.94025400 & 1.60755500 & $\mathrm{H}$ & -0.68082900 & 4.08229600 & 3.46573300 \\
\hline $\mathrm{C}$ & -1.65665200 & -2.04818200 & 1.32411900 & $\mathrm{H}$ & -1.26077000 & 6.18254300 & -0.24666900 \\
\hline $\mathrm{C}$ & -1.88139900 & -2.01032300 & -0.17023500 & $\mathrm{H}$ & -1.82912300 & 5.85487200 & 2.15337800 \\
\hline $\mathrm{C}$ & -0.93812500 & -1.33740600 & -0.97196600 & $\mathrm{C}$ & 4.26327000 & 0.16292100 & 0.88002300 \\
\hline $\mathrm{C}$ & -1.06982100 & -1.23406600 & -2.37291400 & $\mathrm{H}$ & 4.42231100 & 0.79066100 & 1.76728800 \\
\hline $\mathrm{C}$ & -2.23774900 & -1.72733800 & -2.95642600 & $\mathrm{H}$ & 3.54337900 & -0.61334300 & 1.15871700 \\
\hline $\mathrm{C}$ & -3.20781600 & -2.36556200 & -2.18250200 & $\mathrm{C}$ & 5.59835000 & -0.49969900 & 0.48713500 \\
\hline $\mathrm{C}$ & -3.03260900 & -2.51344600 & -0.79137200 & $\mathrm{C}$ & 6.03825300 & -1.44405500 & 1.61708100 \\
\hline $\mathrm{C}$ & -2.77464000 & 0.96044000 & 2.01635000 & $\mathrm{C}$ & 6.70132300 & 0.52225600 & 0.16955200 \\
\hline $\mathrm{H}$ & 3.15490500 & 0.92691900 & -3.12526800 & $\mathrm{H}$ & 5.43106100 & -1.11462800 & -0.40854500 \\
\hline $\mathrm{H}$ & -0.30352400 & -0.74790700 & -2.96324900 & $\mathrm{H}$ & 5.27493200 & -2.20290600 & 1.82063200 \\
\hline $\mathrm{H}$ & -2.37999400 & -1.64442700 & -4.02995100 & $\mathrm{H}$ & 6.96871200 & -1.95987400 & 1.35352700 \\
\hline $\mathrm{H}$ & -4.09623000 & -2.75767100 & -2.66413600 & $\mathrm{H}$ & 6.21777600 & -0.88698700 & 2.54589300 \\
\hline $\mathrm{O}$ & -3.93488700 & -3.10940000 & 0.03255700 & $\mathrm{H}$ & 6.45430800 & 1.14736400 & -0.69486300 \\
\hline $\mathrm{C}$ & -5.17994400 & -3.54541400 & -0.50228900 & $\mathrm{H}$ & 6.88107200 & 1.18319700 & 1.02792500 \\
\hline $\mathrm{H}$ & -5.03921000 & -4.34190700 & -1.24336300 & $\mathrm{H}$ & 7.64461500 & 0.01156400 & -0.05614500 \\
\hline $\mathrm{H}$ & -5.74338700 & -3.93728800 & 0.34546300 & $\mathrm{O}$ & 2.32170900 & -0.26204300 & -1.82050500 \\
\hline $\mathrm{H}$ & -5.73352900 & -2.71390200 & -0.95570800 & $\mathrm{O}$ & 4.33435100 & 0.47870300 & -2.35428800 \\
\hline I & 2.86485900 & -1.12107300 & 1.29860600 & $\mathrm{~S}$ & -0.84773000 & -0.68939300 & 2.03795800 \\
\hline I & 1.47155200 & -3.17840300 & -1.39836200 & $\mathrm{~F}$ & -2.01070200 & 1.59528800 & 1.30867700 \\
\hline
\end{tabular}




\begin{tabular}{|c|c|c|c|}
\hline $\mathrm{F}$ & -3.34000500 & 0.18907600 & 2.30330000 \\
\hline $\mathrm{F}$ & -1.86039700 & 1.29520500 & 3.45840700 \\
\hline $\mathrm{O}$ & 0.45431800 & -0.02062600 & 2.21033500 \\
\hline $\mathrm{O}$ & -1.29659600 & -1.71517700 & 2.98089000 \\
\hline $\mathrm{N}$ & -0.99727900 & -1.10702900 & 0.47728800 \\
\hline $\mathrm{C}$ & -2.18509200 & -1.84808400 & 0.02875300 \\
\hline $\mathrm{C}$ & -3.25195100 & -0.94573100 & -0.61377100 \\
\hline $\mathrm{C}$ & -3.05057600 & 0.14721300 & -1.45543000 \\
\hline $\mathrm{C}$ & -4.08974800 & 0.91939000 & -1.98355200 \\
\hline $\mathrm{C}$ & -5.39992400 & 0.58072600 & -1.66950100 \\
\hline $\mathrm{C}$ & -5.66794000 & -0.51344300 & -0.84889600 \\
\hline $\mathrm{C}$ & -4.61043000 & -1.26761900 & -0.33601000 \\
\hline $\mathrm{C}$ & -2.09611300 & 0.68915700 & 2.28953800 \\
\hline $\mathrm{H}$ & 4.09712600 & -0.03098400 & -3.15443400 \\
\hline $\mathrm{H}$ & -3.88078300 & 1.76641200 & -2.62694700 \\
\hline $\mathrm{H}$ & -6.21942400 & 1.17038100 & -2.07008400 \\
\hline I & -6.69375700 & -0.77216700 & -0.61659600 \\
\hline $\mathrm{O}$ & -4.79239700 & -2.36287500 & 0.45393300 \\
\hline $\mathrm{C}$ & -6.10631400 & -2.70118100 & 0.87619000 \\
\hline I & -6.74324700 & -2.97305800 & 0.02487800 \\
\hline $\mathrm{H}$ & -5.99102100 & -3.56477000 & 1.53260500 \\
\hline $\mathrm{H}$ & -6.57052500 & -1.87796800 & 1.43324500 \\
\hline I & 1.89003300 & -2.96029600 & 0.24129000 \\
\hline I & -1.10627400 & 0.83577000 & -2.11170600 \\
\hline $\mathrm{C}$ & -1.82474000 & -3.01441200 & -0.90467700 \\
\hline $\mathrm{H}$ & -2.73649900 & -3.56306600 & -1.16253700 \\
\hline $\mathrm{H}$ & -1.35910400 & -2.65943400 & -1.83036600 \\
\hline $\mathrm{H}$ & -1.12608100 & -3.68981800 & -0.40615800 \\
\hline $\mathrm{H}$ & -2.64635700 & -2.28671600 & 0.91643100 \\
\hline
\end{tabular}

$\mathrm{R}_{5 \mathrm{c}}$

$-2529.83658895$

$\begin{array}{lrrr}\mathrm{Pd} & 0.90406900 & 0.15054300 & 0.23489900 \\ \mathrm{O} & 3.49546800 & 1.06959100 & -1.97628200 \\ \mathrm{C} & 2.92494200 & 1.80929800 & -4.14271800 \\ \mathrm{C} & 2.51718100 & 1.12102400 & -2.86446600 \\ \mathrm{O} & 1.40117300 & 0.64356000 & -2.67546100 \\ \mathrm{C} & -3.16155700 & 1.53710300 & -1.09310700 \\ \mathrm{C} & -1.72222400 & 1.06213500 & -1.13882500 \\ \mathrm{H} & -3.69086900 & 1.14367900 & -1.96362600 \\ \mathrm{H} & 3.34162300 & 2.79648700 & -3.91997300 \\ \mathrm{H} & 3.71153400 & 1.22662800 & -4.63434300 \\ \mathrm{H} & 2.06643200 & 1.90128100 & -4.80850100 \\ \mathrm{~N} & -3.79195400 & 1.00111600 & 0.09917400\end{array}$

\begin{tabular}{|c|c|c|c|}
\hline $\mathrm{H}$ & -3.20075100 & 0.83037500 & 0.89981500 \\
\hline $\mathrm{C}$ & -5.15441800 & 0.89455900 & 0.18301300 \\
\hline & 5.88246600 & 1.25302000 & -0.74217100 \\
\hline $\mathrm{C}$ & -5.70758600 & 0.33424000 & 1.46149900 \\
\hline C & -4.95193800 & -0.42062500 & 2.37053900 \\
\hline $\mathrm{C}$ & -7.06162800 & 0.57686700 & 1.73068000 \\
\hline $\mathrm{C}$ & -5.53938300 & -0.90860900 & 3.53813100 \\
\hline 11 & -3.91496600 & -0.66772300 & 2. 16008700 \\
\hline C & -7.64530300 & 0.09444300 & 2.89977700 \\
\hline $\mathrm{H}$ & -7.63563800 & 1.14422700 & 1.00565800 \\
\hline $\mathrm{C}$ & -6.88422100 & -0.64674500 & 3.80783300 \\
\hline $\mathrm{H}$ & -4.94837900 & -1.50075000 & 4.23122000 \\
\hline $\mathrm{H}$ & -8.69408900 & 0.29333200 & 3.10276200 \\
\hline $\mathrm{H}$ & -7.33955500 & -1.02627700 & 4.71865900 \\
\hline 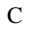 & -3.23280100 & 3.09412800 & -1.11720900 \\
\hline 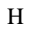 & -4.29423200 & 3.32541300 & -0.97236000 \\
\hline $\mathrm{H}$ & -2.70012400 & 3.46849100 & -0.23201400 \\
\hline C & -2.72898000 & 3.82019800 & -2.38011900 \\
\hline $\mathrm{C}$ & -2.86755200 & 5.33792200 & -2.17554000 \\
\hline $\mathrm{C}$ & -3.47128100 & 3.37292500 & -3.64918800 \\
\hline $\mathrm{H}$ & -1.66109000 & 3.59933900 & -2.51563600 \\
\hline $\mathrm{H}$ & -2.32001700 & 5.67700500 & -1.28765100 \\
\hline $\mathrm{H}$ & -2.47698500 & 5.88611300 & -3.04060500 \\
\hline $\mathrm{H}$ & -3.91972900 & 5.62242300 & -2.04778700 \\
\hline $\mathrm{H}$ & -3.27592900 & 2.32334700 & -3.89131900 \\
\hline $\mathrm{H}$ & -4.55531500 & 3.49801900 & -3.53437100 \\
\hline $\mathrm{H}$ & -3.15404200 & 3.97121900 & -4.51124400 \\
\hline $\mathrm{O}$ & -1.11740900 & 0.78677100 & -0.08888700 \\
\hline 0 & -1.20916600 & 1.03483100 & -2.34131900 \\
\hline $\mathrm{S}$ & 3.44404900 & -1.77502300 & 0.46361600 \\
\hline $\mathrm{F}$ & 5.38324700 & -0.88139400 & -1.12983300 \\
\hline $\mathrm{F}$ & 5.96262600 & -1.12793300 & 0.95469400 \\
\hline$\Gamma$ & 5.69882800 & -2.87474700 & -0.31768400 \\
\hline , & 2.79346100 & -2.34787400 & -0.74832100 \\
\hline $\mathrm{O}$ & 3.46823900 & -2.57736600 & 1.68890300 \\
\hline $\mathrm{N}$ & 2.93511500 & -0.22832400 & 0.58075100 \\
\hline C & 3.46882500 & 0.63958600 & 1.69108100 \\
\hline$C$ & 2.74754900 & 1.94724000 & 1.49648700 \\
\hline $\mathrm{C}$ & 1.49370000 & 1.93501300 & 0.87862200 \\
\hline$c$ & 0.75847700 & 3.10969900 & 0.70301200 \\
\hline $\mathrm{C}$ & 1.29850800 & 4.31225400 & 1.16190600 \\
\hline $\mathrm{C}$ & 2.54929300 & 4.35532900 & 1.78243500 \\
\hline $\mathrm{C}$ & 3.27410900 & 3.17106800 & 1.95378900 \\
\hline $\mathrm{C}$ & 5.24690300 & -1.65209900 & -0.0505710 \\
\hline
\end{tabular}




$\begin{array}{lrrr}\mathrm{H} & -0.23004700 & 0.83299100 & -2.36949700 \\ \mathrm{H} & -0.21844400 & 3.10018600 & 0.23536600 \\ \mathrm{H} & 0.73573400 & 5.23429100 & 1.03872100 \\ \mathrm{H} & 2.94467200 & 5.30379500 & 2.12664900 \\ \mathrm{H} & 3.20792500 & 0.59988700 & -1.14312000 \\ \mathrm{O} & 4.50184700 & 3.09630100 & 2.55214300 \\ \mathrm{C} & 5.10639300 & 4.29671100 & 3.00942700 \\ \mathrm{H} & 4.50180000 & 4.78052700 & 3.78744600 \\ \mathrm{H} & 6.06963900 & 4.00390100 & 3.43082400 \\ \mathrm{H} & 5.26952600 & 5.00361000 & 2.18566000 \\ \mathrm{I} & -2.62963400 & -2.93965600 & -0.55479100 \\ \mathrm{I} & 0.20753800 & -2.65593900 & -0.63271700 \\ \mathrm{C} & 3.23778400 & 0.10666800 & 3.11686000 \\ \mathrm{H} & 3.57339900 & 0.86565800 & 3.83047100 \\ \mathrm{H} & 2.17162200 & -0.08320600 & 3.28058300 \\ \mathrm{H} & 3.78862200 & -0.81722300 & 3.30099900 \\ \mathrm{H} & 4.54444000 & 0.77936200 & 1.53887400\end{array}$

$\mathrm{TS}_{\mathrm{R} 3 \mathrm{C}}$

$-2529.78797316$

\begin{tabular}{|c|c|c|c|}
\hline d & 0.72178700 & 0.15727100 & -0.25261400 \\
\hline $\mathrm{O}$ & 3.35987100 & 2.48976100 & -0.90182500 \\
\hline $\mathrm{C}$ & 2.63837800 & 4.47996400 & -1.95961300 \\
\hline C & 2.31713500 & 3.11916000 & -1.40281200 \\
\hline 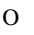 & 1.19053200 & 2.62076000 & -1.42093400 \\
\hline $\mathrm{C}$ & -3.44573200 & 1.93102400 & -0.64430600 \\
\hline $\mathrm{C}$ & -1.94297400 & 1.81878500 & -0.81180100 \\
\hline $\mathrm{H}$ & -3.88528700 & 2.23594700 & -1.59591100 \\
\hline 11 & 3.34875000 & 5.00096200 & -1.31254800 \\
\hline $\mathrm{H}$ & 3.11295300 & 4.35320500 & -2.93974900 \\
\hline $\mathrm{H}$ & 1.72589100 & 5.06537700 & -2.08030700 \\
\hline $\mathrm{N}$ & -3.97286900 & 0.61902900 & -0.31212100 \\
\hline $\mathrm{H}$ & -3.39116500 & 0.00622200 & 0.24357700 \\
\hline $\mathrm{C}$ & -5.28406200 & 0.31479100 & -0.57121400 \\
\hline $\mathrm{O}$ & -6.04133800 & 1.14034100 & -1.08160500 \\
\hline $\mathrm{C}$ & -5.74393800 & -1.06276400 & -0.19333300 \\
\hline $\mathrm{C}$ & -4.87228200 & -2.14142300 & 0.01618600 \\
\hline $\mathrm{C}$ & -7.12613000 & -1.26197700 & -0.06887100 \\
\hline $\mathrm{C}$ & -5.37823100 & -3.39466400 & 0.36284000 \\
\hline $\mathrm{H}$ & -3.80051700 & -2.02720600 & -0.11923600 \\
\hline $\mathrm{C}$ & -7.62867800 & -2.51233600 & 0.28304900 \\
\hline $\mathrm{H}$ & -7.78598400 & -0.42129400 & -0.25598100 \\
\hline $\mathrm{C}$ & -6.75490400 & -3.58092400 & 0.50282700 \\
\hline $\mathrm{H}$ & -4.69517400 & -4.22523400 & 0.51652000 \\
\hline
\end{tabular}

\begin{tabular}{|c|c|c|c|}
\hline $\mathrm{H}$ & -8.70114100 & -2.65574000 & 0.38386300 \\
\hline$H$ & -7.14614900 & -4.55765500 & 0.77504200 \\
\hline & 3.80110400 & 2.98610100 & 0.44250300 \\
\hline$H$ & -4.89125900 & 2.93579700 & 0.54369100 \\
\hline $\mathrm{H}$ & -3.37262400 & 2.64323900 & 1.39432300 \\
\hline Y & -3.38286600 & 4.44552900 & 0.17478900 \\
\hline 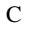 & -3.76797700 & 5.31243700 & 1.38462900 \\
\hline $\mathrm{C}$ & -4.00285200 & 5.00647000 & -1.11483700 \\
\hline . & -2.28840500 & 4.48754200 & 0.07134000 \\
\hline $\mathrm{H}$ & -3.30798400 & 4.94187000 & 2.30886100 \\
\hline $\mathrm{H}$ & -3.44697200 & 6.35071900 & 1.24163800 \\
\hline $\mathrm{H}$ & -4.85547100 & 5.31715000 & 1.53192900 \\
\hline $\mathrm{H}$ & -3.64391400 & 4.48196800 & -2.00609500 \\
\hline $\mathrm{H}$ & -5.09665300 & 4.92372000 & -1.09120000 \\
\hline $\mathrm{H}$ & -3.74957500 & 6.06640700 & -1.23481900 \\
\hline $\mathrm{O}$ & -1.29300500 & 1.04199100 & -0.09448600 \\
\hline $\mathrm{O}$ & -1.43621700 & 2.62430200 & -1.71119100 \\
\hline S & 3.34518900 & -1.00265600 & -1.70804500 \\
\hline F & 5.42032200 & 0.64499600 & -1.28931900 \\
\hline F & 5.78081700 & -1.41382900 & -0.69129000 \\
\hline $\mathrm{F}$ & 5.72578200 & -0.88864300 & -2.80348800 \\
\hline $\mathrm{O}$ & 2.84688500 & -0.20792700 & -2.83105600 \\
\hline $\mathrm{O}$ & 3.28166700 & -2.46301500 & -1.72287100 \\
\hline $\mathrm{N}$ & 2.76833100 & -0.25211100 & -0.32338400 \\
\hline $\mathrm{C}$ & 3.30373500 & -0.66255700 & 1.01324900 \\
\hline $\mathrm{C}$ & 2.48357500 & 0.13297300 & 1.99434700 \\
\hline $\mathrm{C}$ & 1.17264700 & 0.49852000 & 1.65239700 \\
\hline $\mathrm{C}$ & 0.33651800 & 1.22560500 & 2.52093600 \\
\hline $\mathrm{C}$ & 0.88203100 & 1.67819500 & 3.72023100 \\
\hline $\mathrm{C}$ & 2.19853900 & 1.36853100 & 4.07299200 \\
\hline $\mathrm{C}$ & 3.00158300 & 0.59648800 & 3.21644000 \\
\hline $\mathrm{C}$ & 5.19321300 & -0.63435400 & -1.61058700 \\
\hline $\mathrm{H}$ & -0.43659800 & 2.62896500 & -1.69237500 \\
\hline $\mathrm{H}$ & -0.68883900 & 1.43818900 & 2.24949100 \\
\hline $\mathrm{H}$ & 0.26796200 & 2.25355000 & 4.40717600 \\
\hline $\mathrm{H}$ & 2.58983100 & 1.71840400 & 5.02111700 \\
\hline 11 & 3.11242600 & 1.54374400 & -0.66554600 \\
\hline 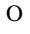 & 4.29142300 & 0.25316900 & 3.47858800 \\
\hline $\mathrm{C}$ & 4.91705800 & 0.76520600 & 4.65040000 \\
\hline $\mathrm{H}$ & 4.42731100 & 0.39405200 & 5.55904600 \\
\hline$\Pi$ & 5.94440300 & 0.40010900 & 4.61933600 \\
\hline $\mathrm{H}$ & 4.91875600 & 1.86225900 & 4.65517400 \\
\hline I & 71783300 & -1.93943400 & 1.79663700 \\
\hline & .41983400 & -1.79006200 & -1.85468700 \\
\hline
\end{tabular}




$\begin{array}{llll}\mathrm{C} & 3.28641600 & -2.16687700 & 1.34963500 \\ \mathrm{H} & 3.65449700 & -2.29360300 & 2.37292700 \\ \mathrm{H} & 2.27261500 & -2.56769300 & 1.28675800 \\ \mathrm{H} & 3.93131000 & -2.72967900 & 0.67386300 \\ \mathrm{H} & 4.34450700 & -0.31758200 & 1.07173100\end{array}$

$\mathrm{R}_{6 \mathrm{c}}$

$-2529.84575900$

Pd $\quad 0.20103600 \quad-0.29392000 \quad-0.17751900$

$\begin{array}{llll}\text { O } & 2.75629500 & 0.17152800 & -2.34350800\end{array}$

$\begin{array}{llll}\text { C } & 1.98177700 & 1.89942700 & -3.77003700\end{array}$

$\begin{array}{llll}\text { C } & 1.79454300 & 1.00097200 & -2.58017700\end{array}$

$\begin{array}{llll}\text { O } & 0.77680500 & 1.08009200 & -1.85335200\end{array}$

$\begin{array}{llll}\text { C } & -3.81280000 & 1.53520800 & -1.03947400\end{array}$

$\begin{array}{llll}\text { C } & -2.32086100 & 1.29568800 & -1.15263700\end{array}$

$\begin{array}{llll}\mathrm{H} & -4.21235600 & 1.77904700 & -2.02595400\end{array}$

$\mathrm{H} \quad 2.68054300 \quad 2.69840200 \quad-3.49566000$

$\begin{array}{llll}\mathrm{H} & 2.42379500 & 1.33722400 & -4.59635000\end{array}$

$\begin{array}{llll}\mathrm{H} & 1.03383600 & 2.34474600 & -4.07554300\end{array}$

N $\quad-4.43120700 \quad 0.29413500 \quad-0.60994000$

$\begin{array}{llll}\mathrm{H} & -3.92067200 & -0.27236600 & 0.05438000\end{array}$

$\begin{array}{llll}\text { C } & -5.71978700 & -0.00542000 & -0.96552700\end{array}$

$\begin{array}{llll}\text { O } & -6.39312600 & 0.77207200 & -1.64192300\end{array}$

$\begin{array}{llll}\text { C } & -6.26024400 & -1.32235900 & -0.48825800\end{array}$

$\begin{array}{llll}\text { C } & -5.45393600 & -2.37334500 & -0.02579500\end{array}$

$\begin{array}{llll}\text { C } & -7.65055700 & -1.49538500 & -0.53986500\end{array}$

$\begin{array}{llll}\text { C } & -6.03467900 & -3.57128200 & 0.39168200\end{array}$

H $\quad-4.37080900 \quad-2.28620400 \quad-0.01085800$

$\begin{array}{llll}\text { C } & -8.22854300 & -2.69019500 & -0.11785900\end{array}$

$\begin{array}{llll}\mathrm{H} & -8.25593700 & -0.67852900 & -0.91839700\end{array}$

$\begin{array}{llll}\text { C } & -7.42134100 & -3.73023700 & 0.35121200\end{array}$

H $\quad-5.40081200 \quad-4.38099100 \quad 0.74183400$

$\begin{array}{llll}\mathrm{H} & -9.30763000 & -2.81257200 & -0.15622100\end{array}$

$\begin{array}{llll}\mathrm{H} & -7.87088500 & -4.66404900 & 0.67841800\end{array}$

$\begin{array}{llll}\text { C } & -4.12193700 & 2.70229200 & -0.05835600\end{array}$

$\begin{array}{llll}\mathrm{H} & -5.21540800 & 2.72483100 & 0.02066600\end{array}$

$\begin{array}{llll}\mathrm{H} & -3.73211200 & 2.42207500 & 0.92928900\end{array}$

$\begin{array}{llll}\text { C } & -3.61152100 & 4.10483800 & -0.44436900\end{array}$

$\begin{array}{llll}\text { C } & -3.97037100 & 5.09933000 & 0.67165300\end{array}$

$\begin{array}{llll}\text { C } & -4.16841400 & 4.58065600 & -1.79545100\end{array}$

$\begin{array}{llll}\mathrm{H} & -2.51528900 & 4.07280000 & -0.52060300\end{array}$

$\begin{array}{llll}\mathrm{H} & -3.55158400 & 4.78995600 & 1.63662600\end{array}$

$\begin{array}{llll}\text { H } & -3.58538400 & 6.09989900 & 0.44308700\end{array}$

$\begin{array}{llll}\mathrm{H} & -5.05853100 & 5.17993400 & 0.78967500\end{array}$
$\begin{array}{llll}\mathrm{H} & -3.82625700 & 3.95467700 & -2.62617500\end{array}$

$\begin{array}{llll}\mathrm{H} & -5.26564400 & 4.56829600 & -1.79333600\end{array}$

$\begin{array}{llll}\mathrm{H} & -3.84565800 & 5.60726100 & -2.00468300\end{array}$

$\begin{array}{llll}\text { O } & -1.77200100 & 0.47294900 & -0.40877100\end{array}$

$\begin{array}{llll}\text { O } & -1.69697400 & 2.03167200 & -2.04591100\end{array}$

S $\quad 2.17446600 \quad-2.70569500 \quad-0.25032800$

F $\quad 4.04649000 \quad-2.21896700 \quad-2.12681600$

F $\quad 4.83862900 \quad-3.02511700 \quad-0.26263900$

F $\quad 3.72085900 \quad-4.30512100 \quad-1.61451900$

$\begin{array}{llll}\text { O } & 1.17038100 & -3.00975900 & -1.26896100\end{array}$

$\begin{array}{llll}\text { O } & 2.29510900 & -3.46602800 & 0.99258800\end{array}$

$\begin{array}{llll}\mathrm{N} & 2.15256700 & -1.02267200 & -0.03594100\end{array}$

$\begin{array}{llll}\text { C } & 3.06916700 & -0.49300000 & 1.05322800\end{array}$

$\begin{array}{llll}\text { C } & 3.55372300 & 0.91712300 & 0.66652400\end{array}$

$\begin{array}{llll}\text { C } & 2.95997200 & 2.15887400 & 0.94039100\end{array}$

$\begin{array}{llll}\text { C } & 3.53918200 & 3.37517300 & 0.55214500\end{array}$

$\begin{array}{llll}\text { C } & 4.74701100 & 3.37642900 & -0.13227400\end{array}$

$\begin{array}{llll}\text { C } & 5.37651700 & 2.17426100 & -0.44283900\end{array}$

$\begin{array}{llll}\text { C } & 4.78613600 & 0.96989700 & -0.05543000\end{array}$

$\begin{array}{llll}\text { C } & 3.81112100 & -3.07023400 & -1.12024900\end{array}$

$\begin{array}{llll}\mathrm{H} & -0.72291800 & 1.81495800 & -2.03417200\end{array}$

$\begin{array}{llll}\text { H } & 3.04737900 & 4.31050500 & 0.79054300\end{array}$

$\begin{array}{llll}\text { H } & 5.20179600 & 4.31867900 & -0.42536300\end{array}$

$\begin{array}{llll}\mathrm{H} & 6.31706500 & 2.17905400 & -0.97949300\end{array}$

$\begin{array}{llll}\mathrm{H} & 2.56497500 & -0.36143700 & -1.46236200\end{array}$

$\begin{array}{llll}\text { O } & 5.34277400 & -0.23714000 & -0.34696300\end{array}$

$\begin{array}{llll}\text { C } & 6.52475400 & -0.30011400 & -1.13228900\end{array}$

$\begin{array}{llll}\mathrm{H} & 7.36865500 & 0.18540600 & -0.62689600\end{array}$

$\begin{array}{llll}\mathrm{H} & 6.73308000 & -1.36312700 & -1.25271000\end{array}$

$\begin{array}{llll}\mathrm{H} & 6.37101900 & 0.15731100 & -2.11719500\end{array}$

I $\quad 1.07857300 \quad 2.43955900 \quad 1.95165800$

I $\quad-0.91847700 \quad-1.83266700 \quad 1.67082000$

$\begin{array}{llll}\text { C } & 2.57662000 & -0.67846900 & 2.49348700\end{array}$

$\begin{array}{llll}\mathrm{H} & 3.28680400 & -0.19843100 & 3.17684000\end{array}$

$\begin{array}{llll}\mathrm{H} & 1.58586800 & -0.26914800 & 2.67454700\end{array}$

$\begin{array}{llll}\mathrm{H} & 2.54044600 & -1.74610600 & 2.71832800\end{array}$

$\begin{array}{llll}\text { H } & 3.96782100 & -1.10719300 & 0.98228400\end{array}$

$\mathrm{R}_{8 \mathrm{a}}$

\section{$-2529.84575896$}

$\begin{array}{llll}\text { Pd } & -0.20092200 & -0.29412900 & 0.17748000 \\ \text { O } & -2.75629100 & 0.17183400 & 2.34320500 \\ \text { C } & -1.98153500 & 1.89956500 & 3.76978300 \\ \text { C } & -1.79436300 & 1.00104000 & 2.57999700\end{array}$ 


\begin{tabular}{|c|c|c|c|c|c|c|c|}
\hline $\mathrm{O}$ & -0.77651100 & 1.07992500 & 1.85329100 & $\mathrm{C}$ & -3.06923000 & -0.49300900 & -1.05313200 \\
\hline $\mathrm{C}$ & 3.81286700 & 1.53540200 & 1.03902600 & $\mathrm{C}$ & -3.55370200 & 0.91716300 & -0.66651800 \\
\hline $\mathrm{C}$ & 2.32097200 & 1.29567200 & 1.15227100 & $\mathrm{C}$ & -2.95979700 & 2.15885400 & -0.94031500 \\
\hline $\mathrm{H}$ & 4.21245300 & 1.77907900 & 2.02554000 & $\mathrm{C}$ & -3.53886500 & 3.37521700 & -0.55206300 \\
\hline $\mathrm{H}$ & -2.67990200 & 2.69883100 & 3.49513900 & $\mathrm{C}$ & -4.74673600 & 3.37659200 & 0.13228200 \\
\hline $\mathrm{H}$ & -2.42413000 & 1.33759700 & 4.59594000 & $\mathrm{C}$ & -5.37642400 & 2.17449000 & 0.44273600 \\
\hline $\mathrm{H}$ & -1.03352600 & 2.34451900 & 4.07559100 & $\mathrm{C}$ & -4.78617200 & 0.97006700 & 0.05532100 \\
\hline $\mathrm{N}$ & 4.43144500 & 0.29453800 & 0.60915400 & $\mathrm{C}$ & -3.81131000 & -3.06992500 & 1.12056200 \\
\hline $\mathrm{H}$ & 3.92078400 & -0.27217500 & -0.05487900 & $\mathrm{H}$ & 0.72303000 & 1.81478800 & 2.03392000 \\
\hline $\mathrm{C}$ & 5.71973800 & -0.00537200 & 0.96542900 & $\mathrm{H}$ & -3.04692000 & 4.31049600 & -0.79037100 \\
\hline $\mathrm{O}$ & 6.39286800 & 0.77188700 & 1.64230400 & $\mathrm{H}$ & -5.20141800 & 4.31888400 & 0.42540200 \\
\hline $\mathrm{C}$ & 6.26021900 & -1.32233600 & 0.48824900 & $\mathrm{H}$ & -6.31701900 & 2.17937700 & 0.97931100 \\
\hline $\mathrm{C}$ & 5.45397800 & -2.37317000 & 0.02532100 & $\mathrm{H}$ & -2.56491800 & -0.36127900 & 1.46202300 \\
\hline $\mathrm{C}$ & 7.65048400 & -1.49555700 & 0.54047000 & $\mathrm{O}$ & -5.34302100 & -0.23689500 & 0.34672500 \\
\hline $\mathrm{C}$ & 6.03474900 & -3.57114300 & -0.39201600 & $\mathrm{C}$ & -6.52493800 & -0.29973000 & 1.13215900 \\
\hline $\mathrm{H}$ & 4.37086700 & -2.28590200 & 0.00991600 & $\mathrm{H}$ & -7.36882100 & 0.18594200 & 0.62688000 \\
\hline $\mathrm{C}$ & 8.22850100 & -2.69039900 & 0.11859600 & $\mathrm{H}$ & -6.73340900 & -1.36272000 & 1.25253600 \\
\hline $\mathrm{H}$ & 8.25580200 & -0.67882400 & 0.91936700 & $\mathrm{H}$ & -6.37102200 & 0.15762000 & 2.11707000 \\
\hline $\mathrm{C}$ & 7.42137100 & -3.73028400 & -0.35094600 & I & -1.07840700 & 2.43935800 & -1.95166200 \\
\hline $\mathrm{H}$ & 5.40093100 & -4.38073400 & -0.74252900 & I & 0.91836900 & -1.83299600 & -1.67086900 \\
\hline $\mathrm{H}$ & 9.30755400 & -2.81292500 & 0.15743000 & $\mathrm{C}$ & -2.57671500 & -0.67860400 & -2.49338300 \\
\hline $\mathrm{H}$ & 7.87093900 & -4.66412000 & -0.67805300 & $\mathrm{H}$ & -3.28684600 & -0.19848700 & -3.17673300 \\
\hline $\mathrm{C}$ & 4.12184100 & 2.70272000 & 0.05814200 & $\mathrm{H}$ & -1.58590900 & -0.26942900 & -2.67447700 \\
\hline $\mathrm{H}$ & 5.21530700 & 2.72532300 & -0.02097700 & $\mathrm{H}$ & -2.54071200 & -1.74625100 & -2.71820400 \\
\hline $\mathrm{H}$ & 3.73195700 & 2.42270400 & -0.92953700 & $\mathrm{H}$ & -3.96789100 & -1.10718100 & -0.98210900 \\
\hline $\mathrm{C}$ & 3.61137500 & 4.10514700 & 0.44450500 & & & & \\
\hline $\mathrm{C}$ & 3.96965300 & 5.09983700 & -0.67151900 & \multicolumn{4}{|c|}{$\mathrm{TS}_{\mathrm{R} 5 \mathrm{a}}$} \\
\hline $\mathrm{C}$ & 4.16870000 & 4.58088000 & 1.79544400 & \multicolumn{4}{|c|}{-2529.84528531} \\
\hline $\mathrm{H}$ & 2.51518300 & 4.07294200 & 0.52115400 & $\mathrm{Pd}$ & -0.17076700 & -0.24397800 & 0.17964200 \\
\hline $\mathrm{H}$ & 3.55047900 & 4.79056600 & -1.63635700 & $\mathrm{O}$ & -2.74279300 & 0.19299700 & 2.23703200 \\
\hline $\mathrm{H}$ & 3.58467100 & 6.10033500 & -0.44263400 & $\mathrm{C}$ & -1.92247900 & 1.80318200 & 3.79429400 \\
\hline $\mathrm{H}$ & 5.05775400 & 5.18056800 & -0.78999900 & $\mathrm{C}$ & -1.78820400 & 0.98263900 & 2.53860700 \\
\hline $\mathrm{H}$ & 3.82695100 & 3.95474800 & 2.62622300 & $\mathrm{O}$ & -0.73920400 & 1.08754300 & 1.83696800 \\
\hline $\mathrm{H}$ & 5.26593100 & 4.56868400 & 1.79292800 & $\mathrm{C}$ & 3.83302700 & 1.53375800 & 1.07794300 \\
\hline $\mathrm{H}$ & 3.84587100 & 5.60741100 & 2.00492800 & $\mathrm{C}$ & 2.34118100 & 1.29027800 & 1.18865500 \\
\hline $\mathrm{O}$ & 1.77216400 & 0.47276900 & 0.40852400 & $\mathrm{H}$ & 4.23720200 & 1.73662500 & 2.07140800 \\
\hline $\mathrm{O}$ & 1.69706700 & 2.03168500 & 2.04549800 & $\mathrm{H}$ & -2.95722100 & 1.80777900 & 4.13914400 \\
\hline $\mathrm{S}$ & -2.17461500 & -2.70565000 & 0.25060800 & $\mathrm{H}$ & -1.28932600 & 1.36336700 & 4.57399600 \\
\hline $\mathrm{F}$ & -4.04657600 & -2.21850000 & 2.12700500 & $\mathrm{H}$ & -1.57506600 & 2.82443000 & 3.61622200 \\
\hline $\mathrm{F}$ & -4.83882000 & -3.02480700 & 0.26295000 & $\mathrm{~N}$ & 4.44767100 & 0.30920500 & 0.59644100 \\
\hline $\mathrm{F}$ & -3.72117000 & -4.30476700 & 1.61497000 & $\mathrm{H}$ & 3.94175800 & -0.21881100 & -0.10235600 \\
\hline $\mathrm{O}$ & -1.17056300 & -3.00966700 & 1.26928100 & $\mathrm{C}$ & 5.73429300 & -0.01125600 & 0.94222600 \\
\hline $\mathrm{O}$ & -2.29528400 & -3.46609200 & -0.99223000 & $\mathrm{O}$ & 6.40593100 & 0.72849300 & 1.66103900 \\
\hline $\mathrm{N}$ & -2.15259900 & -1.02265400 & 0.03604600 & $\mathrm{C}$ & 6.27504300 & -1.30273700 & 0.39974800 \\
\hline
\end{tabular}




\begin{tabular}{|c|c|c|c|c|c|c|c|}
\hline $\mathrm{C}$ & 5.47116100 & -2.32264200 & -0.13151200 & $\mathrm{H}$ & -2.50627500 & -0.38484900 & 1.27655000 \\
\hline $\mathrm{C}$ & 7.66394600 & -1.48448700 & 0.45948500 & $\mathrm{O}$ & -5.38207400 & -0.32909400 & 0.30613700 \\
\hline $\mathrm{C}$ & 6.05324400 & -3.49771000 & -0.60795700 & $\mathrm{C}$ & -6.57675900 & -0.41228800 & 1.07014400 \\
\hline $\mathrm{H}$ & 4.38858900 & -2.23063000 & -0.15603700 & $\mathrm{H}$ & -7.42952800 & 0.01643200 & 0.52944100 \\
\hline $\mathrm{C}$ & 8.24324600 & -2.65631200 & -0.02105300 & $\mathrm{H}$ & -6.74322600 & -1.47777000 & 1.22863600 \\
\hline $\mathrm{H}$ & 8.26704200 & -0.69252600 & 0.89084300 & $\mathrm{H}$ & -6.46332400 & 0.08940000 & 2.03870300 \\
\hline $\mathrm{C}$ & 7.43862900 & -3.66488900 & -0.55831000 & I & -1.16067800 & 2.44000000 & -1.96220200 \\
\hline $\mathrm{H}$ & 5.42114500 & -4.28376500 & -1.01121900 & I & 0.95623100 & -1.79927600 & -1.65721300 \\
\hline $\mathrm{H}$ & 9.32127700 & -2.78532300 & 0.02462800 & $\mathrm{C}$ & -2.55240200 & -0.74604800 & -2.46333100 \\
\hline $\mathrm{H}$ & 7.88906600 & -4.58089900 & -0.93142100 & $\mathrm{H}$ & -3.28410500 & -0.33266600 & -3.16705900 \\
\hline $\mathrm{C}$ & 4.13567300 & 2.73712300 & 0.14161600 & $\mathrm{H}$ & -1.58655800 & -0.28456900 & -2.65242400 \\
\hline $\mathrm{H}$ & 5.22921800 & 2.77714500 & 0.06912500 & $\mathrm{H}$ & -2.45209500 & -1.81657500 & -2.65076600 \\
\hline $\mathrm{H}$ & 3.75383900 & 2.48780400 & -0.85722600 & $\mathrm{H}$ & -3.94339100 & -1.18413200 & -0.95359700 \\
\hline $\mathrm{C}$ & 3.60172800 & 4.11660000 & 0.57541300 & & & & \\
\hline $\mathrm{C}$ & 3.93997500 & 5.15549700 & -0.50591100 & $\mathrm{R}_{9 \mathrm{a}}$ & & & \\
\hline $\mathrm{C}$ & 4.15080100 & 4.55613300 & 1.94202400 & -252 & .86481400 & & \\
\hline $\mathrm{H}$ & 2.50593500 & 4.06194400 & 0.65109800 & $\mathrm{Pd}$ & 0.42887300 & 0.79316700 & -0.09378300 \\
\hline $\mathrm{H}$ & 3.52665000 & 4.87085200 & -1.48079900 & $\mathrm{O}$ & -1.56981400 & 3.46146600 & 0.53749200 \\
\hline $\mathrm{H}$ & 3.53565500 & 6.13999000 & -0.24346700 & $\mathrm{C}$ & 0.12948500 & 5.10969500 & 0.96863000 \\
\hline $\mathrm{H}$ & 5.02623300 & 5.26144300 & -0.62113700 & $\mathrm{C}$ & -0.36625400 & 3.72953000 & 0.57329000 \\
\hline $\mathrm{H}$ & 3.82189300 & 3.89563500 & 2.75110800 & $\mathrm{O}$ & 0.57919000 & 2.87667400 & 0.28230900 \\
\hline $\mathrm{H}$ & 5.24809800 & 4.56507000 & 1.93852900 & $\mathrm{C}$ & 4.82289800 & 1.29142400 & -0.16973600 \\
\hline $\mathrm{H}$ & 3.80891000 & 5.56864400 & 2.18648100 & $\mathrm{C}$ & 3.35964700 & 1.64982900 & -0.00222200 \\
\hline $\mathrm{O}$ & 1.78385900 & 0.54690400 & 0.36767600 & $\mathrm{H}$ & 5.40525400 & 1.82316400 & 0.58501200 \\
\hline $\mathrm{O}$ & 1.73112100 & 1.92896100 & 2.15821700 & $\mathrm{H}$ & -0.71534900 & 5.78739400 & 1.09996500 \\
\hline S & -2.11665400 & -2.69072300 & 0.35126900 & $\mathrm{H}$ & 0.68913100 & 5.04334700 & 1.90860500 \\
\hline $\mathrm{F}$ & -4.04594500 & -2.21550500 & 2.17141800 & $\mathrm{H}$ & 0.80903400 & 5.50491700 & 0.20647600 \\
\hline $\mathrm{F}$ & -4.76446400 & -3.10168900 & 0.31401100 & $\mathrm{~N}$ & 4.96911500 & -0.13156800 & 0.08364700 \\
\hline $\mathrm{F}$ & -3.63306100 & -4.30194000 & 1.72712500 & $\mathrm{H}$ & 4.23033000 & -0.73900200 & -0.24490500 \\
\hline $\mathrm{O}$ & -1.12058800 & -2.92332300 & 1.39421800 & $\mathrm{C}$ & 6.14604200 & -0.63872500 & 0.56871700 \\
\hline $\mathrm{O}$ & -2.18228300 & -3.47318500 & -0.87959200 & $\mathrm{O}$ & 7.11217300 & 0.08972700 & 0.79356800 \\
\hline $\mathrm{N}$ & -2.13789200 & -0.99931300 & 0.07679200 & $\mathrm{C}$ & 6.19049100 & -2.12034600 & 0.80770300 \\
\hline $\mathrm{C}$ & -3.07097200 & -0.53574500 & -1.03741400 & $\mathrm{C}$ & 5.04647500 & -2.92591000 & 0.91066800 \\
\hline $\mathrm{C}$ & -3.61563400 & 0.86404400 & -0.70262700 & $\mathrm{C}$ & 7.45672000 & -2.70349000 & 0.95577600 \\
\hline $\mathrm{C}$ & -3.06380500 & 2.11851900 & -1.00545300 & $\mathrm{C}$ & 5.17250500 & -4.29597600 & 1.14262900 \\
\hline $\mathrm{C}$ & -3.70487900 & 3.32163600 & -0.68061400 & $\mathrm{H}$ & 4.05025700 & -2.49698800 & 0.84194400 \\
\hline $\mathrm{C}$ & -4.93128800 & 3.29596500 & -0.03049400 & $\mathrm{C}$ & 7.58030500 & -4.07222100 & 1.18181300 \\
\hline $\mathrm{C}$ & -5.51819500 & 2.08092300 & 0.31019600 & $\mathrm{H}$ & 8.32858400 & -2.06085600 & 0.89306000 \\
\hline $\mathrm{C}$ & -4.86746800 & 0.88957500 & -0.01450900 & $\mathrm{C}$ & 6.43775200 & -4.87199700 & 1.27328200 \\
\hline $\mathrm{C}$ & -3.76037500 & -3.08473800 & 1.19856900 & $\mathrm{H}$ & 4.28018900 & -4.90972200 & 1.22769400 \\
\hline $\mathrm{H}$ & 0.74913500 & 1.72667200 & 2.12998000 & $\mathrm{H}$ & 8.56632100 & -4.51614700 & 1.28952700 \\
\hline $\mathrm{H}$ & -3.24568000 & 4.26811200 & -0.93892800 & $\mathrm{H}$ & 6.53285100 & -5.93969900 & 1.45263500 \\
\hline $\mathrm{H}$ & -5.43248200 & 4.22810000 & 0.21513900 & $\mathrm{C}$ & 5.33030500 & 1.68393800 & -1.58565000 \\
\hline $\mathrm{H}$ & -6.47183500 & 2.06569900 & 0.82295100 & $\mathrm{H}$ & 6.36869600 & 1.33408700 & -1.62866000 \\
\hline
\end{tabular}




\begin{tabular}{|c|c|c|c|c|c|c|c|}
\hline $\mathrm{H}$ & 4.75995100 & 1.09828600 & -2.31871100 & $\mathrm{H}$ & -2.16539400 & -1.02281400 & -0.87766500 \\
\hline $\mathrm{C}$ & 5.27752200 & 3.17915500 & -1.95735600 & & & & \\
\hline $\mathrm{C}$ & 5.73961300 & 3.35831800 & -3.41229600 & $\mathrm{R}_{8 \mathrm{~b}}$ & & & \\
\hline $\mathrm{C}$ & 6.11522400 & 4.05114300 & -1.00865000 & -23 & .72698869 & & \\
\hline $\mathrm{H}$ & 4.23301600 & 3.51897900 & -1.89664900 & $\mathrm{Pd}$ & 0.51520400 & -1.53726400 & -0.16045000 \\
\hline $\mathrm{H}$ & 5.12665300 & 2.76893000 & -4.10461100 & $\mathrm{C}$ & -2.85453400 & 1.42782200 & -0.47626100 \\
\hline $\mathrm{H}$ & 5.67609700 & 4.40928500 & -3.71733200 & $\mathrm{C}$ & -1.46751200 & 0.81897400 & -0.47413700 \\
\hline $\mathrm{H}$ & 6.78253400 & 3.03855800 & -3.53379400 & $\mathrm{H}$ & -3.03438300 & 1.85712800 & -1.46716300 \\
\hline $\mathrm{H}$ & 5.73169000 & 4.03119300 & 0.01664600 & $\mathrm{~N}$ & -3.82746300 & 0.37085200 & -0.25697300 \\
\hline $\mathrm{H}$ & 7.15907500 & 3.71348900 & -0.98579900 & $\mathrm{H}$ & -3.47894800 & -0.51326300 & 0.08545600 \\
\hline $\mathrm{H}$ & 6.10907000 & 5.09615100 & -1.34006100 & $\mathrm{C}$ & -5.08485600 & 0.47242000 & -0.79340900 \\
\hline $\mathrm{O}$ & 2.49762200 & 0.82697000 & -0.37379400 & $\mathrm{O}$ & -5.43452400 & 1.47324900 & -1.41730800 \\
\hline $\mathrm{O}$ & 3.10593200 & 2.82559700 & 0.49275500 & $\mathrm{C}$ & -6.00318800 & -0.69504700 & -0.57804700 \\
\hline S & -1.93441300 & 0.07770700 & 1.81952700 & $\mathrm{C}$ & -5.81429100 & -1.65555100 & 0.42644100 \\
\hline $\mathrm{F}$ & -3.54249900 & 2.18828900 & 2.19941400 & $\mathrm{C}$ & -7.11480300 & -0.79882100 & -1.42543400 \\
\hline $\mathrm{F}$ & -4.58000300 & 0.27176800 & 2.16726600 & $\mathrm{C}$ & -6.71451100 & -2.71195300 & 0.56776100 \\
\hline $\mathrm{F}$ & -3.34889800 & 0.80965200 & 3.87145600 & $\mathrm{H}$ & -4.98796100 & -1.56909400 & 1.12722000 \\
\hline $\mathrm{O}$ & -0.83921900 & 0.63911500 & 2.60096800 & $\mathrm{C}$ & -8.00876900 & -1.85809600 & -1.28823600 \\
\hline $\mathrm{O}$ & -2.22744100 & -1.34327800 & 1.73622600 & $\mathrm{H}$ & -7.25647900 & -0.03586800 & -2.18362500 \\
\hline $\mathrm{N}$ & -1.71926900 & 0.70991000 & 0.17862900 & $\mathrm{C}$ & -7.80908000 & -2.81847700 & -0.29279800 \\
\hline $\mathrm{C}$ & -2.49183800 & 0.01264900 & -0.93477500 & $\mathrm{H}$ & -6.56443500 & -3.44678200 & 1.35381300 \\
\hline $\mathrm{C}$ & -4.01166700 & 0.04178900 & -0.80341000 & $\mathrm{H}$ & -8.86301100 & -1.93467600 & -1.95525900 \\
\hline $\mathrm{C}$ & -4.79613300 & -1.12001600 & -0.85811100 & $\mathrm{H}$ & -8.50777900 & -3.64346900 & -0.18318900 \\
\hline $\mathrm{C}$ & -6.19251800 & -1.07875300 & -0.79398300 & $\mathrm{C}$ & -3.00895000 & 2.55388200 & 0.58650800 \\
\hline $\mathrm{C}$ & -6.83353700 & 0.15039400 & -0.68914400 & $\mathrm{H}$ & -4.08295100 & 2.77315400 & 0.60286600 \\
\hline $\mathrm{C}$ & -6.09898200 & 1.33419500 & -0.66059100 & $\mathrm{H}$ & -2.75256900 & 2.13569500 & 1.56906900 \\
\hline $\mathrm{C}$ & -4.70538300 & 1.27749000 & -0.72353300 & $\mathrm{C}$ & -2.23798300 & 3.86859800 & 0.35728300 \\
\hline $\mathrm{C}$ & -3.47857600 & 0.90520600 & 2.55075800 & $\mathrm{C}$ & -2.55202700 & 4.83617800 & 1.51017800 \\
\hline $\mathrm{H}$ & 2.09246800 & 2.98852100 & 0.47902400 & $\mathrm{C}$ & -2.56473100 & 4.51495200 & -0.99848800 \\
\hline $\mathrm{H}$ & -6.76864500 & -1.99550900 & -0.83129300 & $\mathrm{H}$ & -1.16198500 & 3.65600100 & 0.38571700 \\
\hline $\mathrm{H}$ & -7.91808200 & 0.18914600 & -0.63813200 & $\mathrm{H}$ & -2.29849100 & 4.39677400 & 2.48251300 \\
\hline $\mathrm{H}$ & -6.60918700 & 2.28736400 & -0.59627800 & $\mathrm{H}$ & -1.98507100 & 5.76863700 & 1.40397100 \\
\hline $\mathrm{H}$ & -1.89726300 & 1.74690300 & 0.21562900 & $\mathrm{H}$ & -3.61805200 & 5.09659600 & 1.52785500 \\
\hline $\mathrm{O}$ & -3.91136300 & 2.38162400 & -0.70515300 & $\mathrm{H}$ & -2.20630100 & 3.91131500 & -1.83896600 \\
\hline $\mathrm{C}$ & -4.49635000 & 3.66407000 & -0.49743400 & $\mathrm{H}$ & -3.64617400 & 4.65427900 & -1.12076600 \\
\hline $\mathrm{H}$ & -5.07967600 & 3.68287800 & 0.43039800 & $\mathrm{H}$ & -2.08943000 & 5.49985000 & -1.07977600 \\
\hline $\mathrm{H}$ & -3.65371700 & 4.34707300 & -0.40825600 & $\mathrm{O}$ & -1.26570700 & -0.31851300 & -0.00902200 \\
\hline $\mathrm{H}$ & -5.13608000 & 3.94584100 & -1.34273400 & $\mathrm{O}$ & -0.52460600 & 1.56997800 & -0.96957000 \\
\hline I & -3.95426700 & -3.08010800 & -1.11336700 & $\mathrm{~S}$ & 2.34239500 & -0.28718000 & -2.30126900 \\
\hline I & 0.68372200 & -1.81302100 & -0.57279300 & $\mathrm{~F}$ & 1.52405600 & 2.23169900 & -2.75728400 \\
\hline $\mathrm{C}$ & -2.02631500 & 0.59407800 & -2.27893500 & $\mathrm{~F}$ & 3.68634500 & 2.01117400 & -2.59082900 \\
\hline $\mathrm{H}$ & -2.53279400 & 0.04846900 & -3.08148300 & $\mathrm{~F}$ & 2.65262000 & 1.28914600 & -4.35910300 \\
\hline $\mathrm{H}$ & -2.27318600 & 1.65399300 & -2.36518500 & $\mathrm{O}$ & 1.24255400 & -0.86580900 & -3.06915100 \\
\hline $\mathrm{H}$ & -0.94808500 & 0.45789000 & -2.40574000 & $\mathrm{O}$ & 3.69252700 & -0.84796700 & -2.31837300 \\
\hline
\end{tabular}




\begin{tabular}{|c|c|c|c|}
\hline $\mathrm{N}$ & 1.73649200 & 0.02965500 & -0.74110800 \\
\hline $\mathrm{C}$ & 2.73742800 & 0.56490000 & 0.25463900 \\
\hline 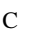 & 2.01964300 & 1.47887500 & 1.26156200 \\
\hline 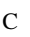 & 1.36248200 & 1.12716100 & 2.45042400 \\
\hline $\mathrm{C}$ & 0.80194400 & 2.07864300 & 3.31203600 \\
\hline 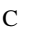 & 0.88983100 & 3.42849900 & 2.99719800 \\
\hline 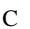 & 1.51328300 & 3.83486600 & 1.82061200 \\
\hline $\mathrm{C}$ & 2.05809900 & 2.87535300 & 0.96461500 \\
\hline C & 2.56320700 & 1.43870600 & -3.03927600 \\
\hline $\mathrm{H}$ & 0.36721300 & 1.07611000 & -0.91862300 \\
\hline $\mathrm{H}$ & 0.30587100 & 1.76226500 & 4.22148500 \\
\hline $\mathrm{H}$ & 0.46626200 & 4.16931600 & 3.66924100 \\
\hline I & 1.57272100 & 4.88816700 & 1.57626200 \\
\hline $\mathrm{O}$ & 2.66701900 & 3.20726100 & -0.20684800 \\
\hline $\mathrm{C}$ & 2.70530400 & 4.56464200 & -0.62669600 \\
\hline $\mathrm{H}$ & 3.27823000 & 5.18467900 & 0.07361300 \\
\hline $\mathrm{H}$ & 3.20448900 & 4.55103200 & -1.59582200 \\
\hline $\mathrm{H}$ & 1.69433800 & 4.97410200 & -0.74057200 \\
\hline I & 2.05295400 & -3.61599100 & -0.34187700 \\
\hline I & 1.06196500 & -0.89073100 & 3.14711200 \\
\hline $\mathrm{C}$ & 3.73217400 & -0.44397600 & 0.84589200 \\
\hline $\mathrm{H}$ & 4.36653200 & -0.83167000 & 0.04649800 \\
\hline $\mathrm{H}$ & 4.36519700 & 0.07239300 & 1.57634900 \\
\hline $\mathrm{H}$ & 3.25343900 & -1.28891000 & 1.33700800 \\
\hline $\mathrm{H}$ & 3.35387100 & 1.24890300 & -0.33213400 \\
\hline
\end{tabular}

$\begin{array}{lrrr}\mathrm{TS}_{\mathrm{R} 5 \mathrm{~b}} & & & \\ -2300.72331847 & & \\ \mathrm{Pd} & -0.59800300 & -1.51425300 & 0.47645800 \\ \mathrm{C} & 2.68272500 & 1.42796000 & 0.66849800 \\ \mathrm{C} & 1.28637300 & 0.82604000 & 0.59639400 \\ \mathrm{H} & 2.82073400 & 1.79503100 & 1.69292400 \\ \mathrm{~N} & 3.66899400 & 0.38527900 & 0.42441100 \\ \mathrm{H} & 3.30834300 & -0.51826900 & 0.15065700 \\ \mathrm{C} & 4.92401500 & 0.49058500 & 0.96341900 \\ \mathrm{O} & 5.27745300 & 1.49461900 & 1.58114400 \\ \mathrm{C} & 5.84304800 & -0.67886600 & 0.75460700 \\ \mathrm{C} & 5.64723000 & -1.65003500 & -0.23826400 \\ \mathrm{C} & 6.96042200 & -0.77415600 & 1.59525700 \\ \mathrm{C} & 6.54630200 & -2.70823300 & -0.37444000 \\ \mathrm{H} & 4.81517000 & -1.56960800 & -0.93274000 \\ \mathrm{C} & 7.85366500 & -1.83478300 & 1.46297300 \\ \mathrm{H} & 7.10708600 & -0.00295500 & 2.34416000 \\ \mathrm{C} & 7.64708100 & -2.80578200 & 0.47925800\end{array}$

\begin{tabular}{|c|c|c|c|}
\hline $\mathrm{H}$ & 6.39064100 & -3.45155100 & -1.15150500 \\
\hline 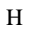 & 8.71276500 & -1.90425000 & 2.12462800 \\
\hline & 8.34514700 & -3.63188800 & 0.37343900 \\
\hline $\mathrm{C}$ & 2.87736400 & 2.61988000 & -0.30817700 \\
\hline 1 & 3.96067500 & 2.78020000 & -0.34864900 \\
\hline $\mathrm{H}$ & 2.56268000 & 2.30412300 & -1.31185600 \\
\hline & 2.20214800 & 3.95730100 & 0.05675600 \\
\hline 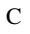 & 2.55097800 & 5.00115200 & -1.01709500 \\
\hline $\mathrm{C}$ & 2.60741300 & 4.46076900 & 1.45137900 \\
\hline $\mathrm{H}$ & 1.11540700 & 3.81150900 & 0.04828100 \\
\hline $\mathrm{H}$ & 2.24680200 & 4.66619500 & -2.01639000 \\
\hline $\mathrm{H}$ & 2.05076000 & 5.95601300 & -0.81425600 \\
\hline $\mathrm{H}$ & 3.63119300 & 5.19380100 & -1.04410800 \\
\hline $\mathrm{H}$ & 2.21447700 & 3.82110900 & 2.24901300 \\
\hline $\mathrm{H}$ & 3.69854400 & 4.49649400 & 1.55855400 \\
\hline $\mathrm{H}$ & 2.21696900 & 5.47070200 & 1.62520600 \\
\hline $\mathrm{O}$ & 1.17172300 & -0.40770100 & 0.32518000 \\
\hline $\mathrm{O}$ & 0.29732800 & 1.59704100 & 0.81071900 \\
\hline$S$ & -2.67500800 & 0.24270900 & 2.03002800 \\
\hline $\mathrm{F}$ & -1.85810400 & 2.79227500 & 2.15677500 \\
\hline F & -3.94430700 & 2.55057100 & 1.57329000 \\
\hline $\mathrm{F}$ & -3.34091100 & 2.19024700 & 3.63005100 \\
\hline $\mathrm{O}$ & -1.73758100 & -0.17490400 & 3.06534100 \\
\hline $\mathrm{O}$ & -4.01142200 & -0.32362800 & 1.88513700 \\
\hline $\mathrm{N}$ & -1.79138300 & 0.22856600 & 0.54427000 \\
\hline $\mathrm{C}$ & -2.58104900 & 0.58621100 & -0.70494100 \\
\hline $\mathrm{C}$ & -1.63921700 & 1.22970800 & -1.73261100 \\
\hline $\mathrm{C}$ & -0.82942900 & 0.60293300 & -2.69211300 \\
\hline $\mathrm{C}$ & -0.05665300 & 1.32491800 & -3.60960000 \\
\hline $\mathrm{C}$ & -0.07819100 & 2.71405700 & -3.58413600 \\
\hline $\mathrm{C}$ & -0.84892000 & 3.38812900 & -2.64149700 \\
\hline $\mathrm{C}$ & -1.60671500 & 2.65680000 & -1.72466100 \\
\hline $\mathrm{C}$ & -2.96720000 & 2.08241800 & 2.35752400 \\
\hline $\mathrm{H}$ & -0.75234800 & 0.99159400 & 0.69938700 \\
\hline $\mathrm{H}$ & 0.55403900 & 0.80340400 & -4.33663500 \\
\hline $\mathrm{H}$ & 0.51305300 & 3.27594500 & -4.30157500 \\
\hline $\mathrm{H}$ & -0.85279900 & 4.47070800 & -2.62104900 \\
\hline $\mathrm{O}$ & -2.36578600 & 3.25325100 & -0.76489900 \\
\hline $\mathrm{C}$ & -2.35993900 & 4.66937200 & -0.63640500 \\
\hline $\mathrm{H}$ & -2.76042300 & 5.15382200 & -1.53524700 \\
\hline $\mathrm{H}$ & -3.00828800 & 4.88322800 & 0.21374100 \\
\hline $\mathrm{H}$ & -1.35061600 & 5.04536700 & -0.43186900 \\
\hline I & 26810300 & 47592100 & 0.83703300 \\
\hline & 00000 & 1.52875400 & 940800 \\
\hline
\end{tabular}




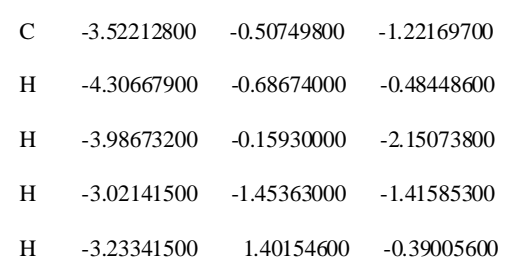

$\mathrm{R}_{9 \mathrm{~b}}$

$-2300.75503140$

$\begin{array}{llll}\mathrm{Pd} & 0.60096300 & -0.53767900 & -0.24648400\end{array}$

$\begin{array}{llll}\text { C } & 4.04327800 & 1.46172400 & -0.24727300\end{array}$

$\begin{array}{llll}\text { C } & 2.71336700 & 0.74396800 & -0.19787300\end{array}$

$\mathrm{H} \quad 4.09519200 \quad 2.16012800 \quad 0.59092400$

$\begin{array}{llll}\mathrm{N} & 5.10080100 & 0.47831400 & -0.07062600\end{array}$

$\mathrm{H} \quad 4.94553600 \quad-0.43838500 \quad-0.46636600$

$\begin{array}{llll}\text { C } & 6.32339500 & 0.83450300 & 0.43041200\end{array}$

$\begin{array}{llll}\text { O } & 6.57779900 & 1.99672700 & 0.74500100\end{array}$

$\begin{array}{llll}\text { C } & 7.33491500 & -0.26783300 & 0.56766000\end{array}$

$\begin{array}{llll}\text { C } & 6.99679500 & -1.62812700 & 0.61865300\end{array}$

$\begin{array}{llll}\text { C } & 8.68133000 & 0.10779700 & 0.67019400\end{array}$

$\begin{array}{llll}\text { C } & 7.99300600 & -2.59579700 & 0.75197000\end{array}$

$\mathrm{H} \quad 5.95704100 \quad-1.94324100 \quad 0.59008300$

$\begin{array}{llll}\text { C } & 9.67561700 & -0.85961300 & 0.79624600\end{array}$

$\begin{array}{llll}\mathrm{H} & 8.92236000 & 1.16549800 & 0.65116400\end{array}$

$\begin{array}{llll}\text { C } & 9.33367600 & -2.21419400 & 0.83448000\end{array}$

H $\quad 7.71995100 \quad-3.64633800 \quad 0.79870400$

$\begin{array}{llll}\text { H } & 10.71733700 & -0.55859900 & 0.86738300\end{array}$

$\mathrm{H} \quad 10.10845300 \quad-2.96950400 \quad 0.93576500$

$\begin{array}{llll}\text { C } & 4.20424000 & 2.24355400 & -1.58078500\end{array}$

$\begin{array}{llll}\mathrm{H} & 5.20698400 & 2.68598100 & -1.54655400\end{array}$

$\begin{array}{llll}\mathrm{H} & 4.19893100 & 1.51164500 & -2.39921200\end{array}$

$\begin{array}{llll}\text { C } & 3.16585300 & 3.34531600 & -1.86865600\end{array}$

$\begin{array}{llll}\text { C } & 3.42121100 & 3.93688800 & -3.26398300\end{array}$

$\begin{array}{llll}\text { C } & 3.17008200 & 4.44996000 & -0.79986100\end{array}$

$\begin{array}{llll}\mathrm{H} & 2.16652200 & 2.88702800 & -1.87639100\end{array}$

$\begin{array}{llll}\mathrm{H} & 3.38490300 & 3.16345800 & -4.04038700\end{array}$

$\begin{array}{llll}\mathrm{H} & 2.67167800 & 4.69771600 & -3.51214200\end{array}$

$\begin{array}{llll}\mathrm{H} & 4.40851300 & 4.41384800 & -3.31345300\end{array}$

$\begin{array}{llll}\mathrm{H} & 2.88005300 & 4.06738500 & 0.18462300\end{array}$

$\begin{array}{llll}\mathrm{H} & 4.16548800 & 4.90208600 & -0.70440800\end{array}$

$\begin{array}{llll}\mathrm{H} & 2.46648700 & 5.24824200 & -1.06625100\end{array}$

$\begin{array}{llll}\mathrm{O} & 2.62565200 & -0.45381700 & -0.64984100\end{array}$

$\begin{array}{llll}\mathrm{O} & 1.65920900 & 1.30749600 & 0.24004700\end{array}$

$\begin{array}{llll}\mathrm{S} & -1.81126300 & -1.00463900 & 1.81366600\end{array}$

$\begin{array}{llll}\text { F } & -1.92735400 & 1.40538200 & 2.92028600\end{array}$

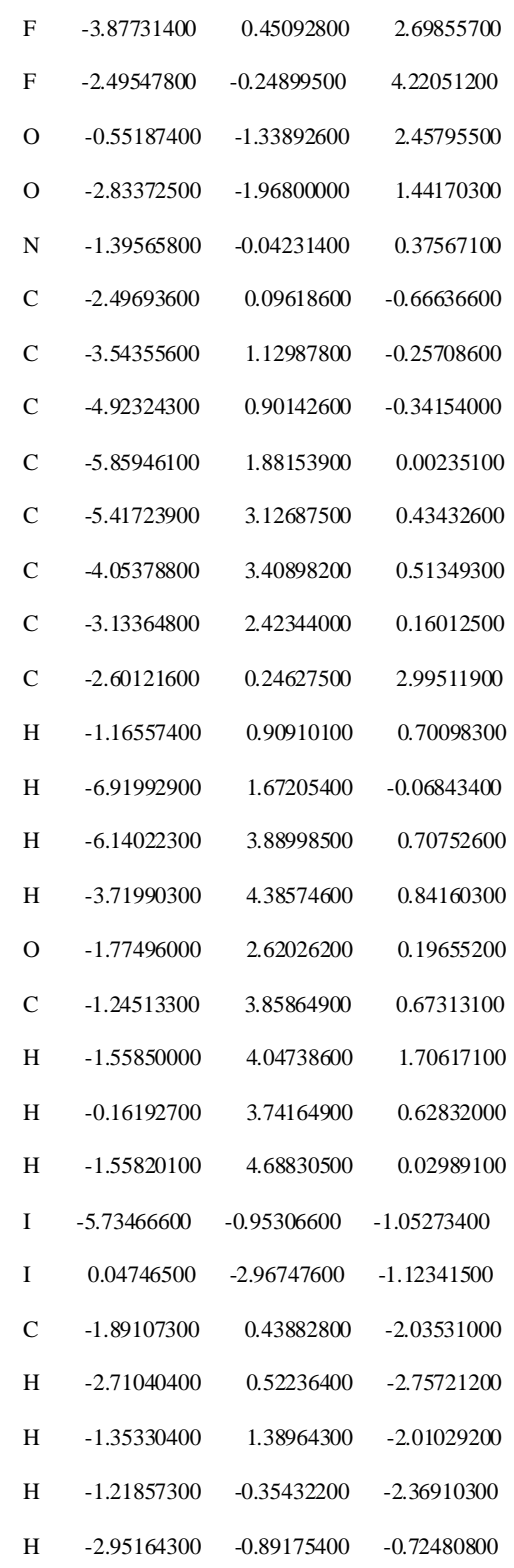

11

$-1152.75301376$

$\begin{array}{llll}\text { Pd } & 1.91915400 & -0.21078700 & -0.12453900\end{array}$

$\begin{array}{llll}\text { C } & -1.69936000 & 1.45274900 & -0.03738900\end{array}$

$\begin{array}{llll}\text { C } & -0.31470800 & 0.86044900 & -0.12928800\end{array}$

$\begin{array}{llll}\mathrm{H} & -1.89337000 & 2.02022500 & -0.95309000\end{array}$

$\begin{array}{llll}\mathrm{N} & -2.66759000 & 0.37001400 & 0.04001600\end{array}$

$\begin{array}{llll}\mathrm{H} & -2.31775200 & -0.54482700 & 0.28870900\end{array}$

$\begin{array}{llll}\text { C } & -3.93198500 & 0.53149100 & -0.46106100\end{array}$

$\begin{array}{llll}\text { O } & -4.29354900 & 1.59888500 & -0.95493400\end{array}$

$\begin{array}{llll}\text { C } & -4.84639000 & -0.65674400 & -0.37347100\end{array}$

$\begin{array}{llll}\text { C } & -4.62443200 & -1.74650500 & 0.48119200\end{array}$

$\begin{array}{llll}\text { C } & -5.98977300 & -0.64427100 & -1.18417000\end{array}$

$\begin{array}{llll}\text { C } & -5.52333400 & -2.81287900 & 0.51009400\end{array}$ 


\begin{tabular}{|c|c|c|c|}
\hline $\mathrm{H}$ & -3.77121700 & -1.76101800 & 1.15402100 \\
\hline $\mathrm{C}$ & -6.88359100 & -1.71229800 & -1.15895200 \\
\hline $\mathrm{H}$ & -6.15565100 & 0.21581800 & -1.82426500 \\
\hline $\mathrm{C}$ & -6.65087100 & -2.80045200 & -0.31348100 \\
\hline $\mathrm{H}$ & -5.34543000 & -3.64921700 & 1.18032800 \\
\hline $\mathrm{H}$ & -7.76344200 & -1.69633800 & -1.79635800 \\
\hline $\mathrm{H}$ & -7.34911100 & -3.63285600 & -0.29126700 \\
\hline $\mathrm{C}$ & -1.83312300 & 2.41009300 & 1.18256000 \\
\hline $\mathrm{H}$ & -2.88813200 & 2.70883900 & 1.19478600 \\
\hline $\mathrm{H}$ & -1.65574600 & 1.82120800 & 2.09198200 \\
\hline $\mathrm{C}$ & -0.94841400 & 3.67131900 & 1.19605200 \\
\hline $\mathrm{C}$ & -1.20525300 & 4.45480800 & 2.49380100 \\
\hline $\mathrm{C}$ & -1.17677200 & 4.56563300 & -0.03292400 \\
\hline $\mathrm{H}$ & 0.10461500 & 3.35875200 & 1.19703100 \\
\hline $\mathrm{H}$ & -1.01967900 & 3.83659000 & 3.38036200 \\
\hline $\mathrm{H}$ & -0.55393400 & 5.33436600 & 2.55522700 \\
\hline $\mathrm{H}$ & -2.24413300 & 4.80567200 & 2.54224400 \\
\hline $\mathrm{H}$ & -0.87665700 & 4.07002500 & -0.96231000 \\
\hline $\mathrm{H}$ & -2.23370700 & 4.84587000 & -0.12600700 \\
\hline $\mathrm{H}$ & -0.59202900 & 5.48945400 & 0.04876700 \\
\hline $\mathrm{O}$ & -0.10369100 & -0.34819700 & 0.23234500 \\
\hline $\mathrm{O}$ & 0.68091800 & 1.55677700 & -0.53005000 \\
\hline I & 2.75173100 & -2.60227400 & 0.54637300 \\
\hline $\mathrm{C}$ & 4.12147800 & 1.46617500 & -1.08106400 \\
\hline $\mathrm{O}$ & 3.85603600 & 0.34919300 & -0.60540500 \\
\hline $\mathrm{O}$ & 3.24088700 & 2.41775900 & -1.31696800 \\
\hline $\mathrm{H}$ & 2.31927400 & 2.15962100 & -1.06862200 \\
\hline $\mathrm{C}$ & 5.52928000 & 1.83356900 & -1.44290700 \\
\hline $\mathrm{H}$ & 5.58080600 & 2.05984700 & -2.51319500 \\
\hline $\mathrm{H}$ & 5.82219300 & 2.73870200 & -0.90122400 \\
\hline $\mathrm{H}$ & 6.20208700 & 1.01171500 & -1.20008800 \\
\hline
\end{tabular}

$\mathrm{TS}_{6}$

$-1152.72960942$

$\begin{array}{lrrr}\mathrm{Pd} & 2.09637300 & -0.22700200 & -0.19854700 \\ \mathrm{C} & -1.61710400 & 1.62481800 & -0.08614800 \\ \mathrm{C} & -0.19137000 & 1.14615400 & -0.26800600 \\ \mathrm{H} & -1.89167400 & 2.23021100 & -0.95408900 \\ \mathrm{~N} & -2.50433600 & 0.47404800 & -0.03778800 \\ \mathrm{H} & -2.09414400 & -0.41775800 & 0.19967500 \\ \mathrm{C} & -3.79645500 & 0.57011400 & -0.47951500 \\ \mathrm{O} & -4.24775300 & 1.62927400 & -0.91376100 \\ \mathrm{C} & -4.62604300 & -0.68046800 & -0.41146300 \\ \mathrm{C} & -4.29916600 & -1.79055500 & 0.38113800\end{array}$

\begin{tabular}{|c|c|c|c|}
\hline $\mathrm{C}$ & -5.79962500 & -0.70874900 & -1.17743900 \\
\hline $\mathrm{C}$ & -5.12428700 & -2.91538300 & 0.39238200 \\
\hline $\mathrm{H}$ & -3.42033100 & -1.77957600 & 1.02039700 \\
\hline $\mathrm{C}$ & -6.61962900 & -1.83468200 & -1.17039000 \\
\hline $\mathrm{H}$ & -6.04724800 & 0.16609700 & -1.76929100 \\
\hline C & -6.28235600 & -2.94189200 & -0.38727600 \\
\hline $\mathrm{I}$ & -4.86479900 & -3.76724900 & 1.01473400 \\
\hline $\mathrm{H}$ & -7.52348200 & -1.84916800 & -1.77332600 \\
\hline $\mathrm{H}$ & -6.92286700 & -3.81973700 & -0.37917000 \\
\hline $\mathrm{C}$ & -1.75726600 & 2.48990100 & 1.20142900 \\
\hline $\mathrm{H}$ & -2.83000400 & 2.70390300 & 1.28076600 \\
\hline $\mathrm{H}$ & -1.49270700 & 1.85730800 & 2.05918600 \\
\hline $\mathrm{C}$ & -0.97079200 & 3.81344800 & 1.26359000 \\
\hline $\mathrm{C}$ & -1.21385900 & 4.47989900 & 2.62760400 \\
\hline $\mathrm{C}$ & -1.33641000 & 4.76938900 & 0.11743800 \\
\hline $\mathrm{H}$ & 0.10200600 & 3.58869400 & 1.18817200 \\
\hline $\mathrm{H}$ & -0.93000900 & 3.81883000 & 3.45528800 \\
\hline $\mathrm{H}$ & -0.63136200 & 5.40373200 & 2.72009500 \\
\hline $\mathrm{H}$ & -2.27272000 & 4.73929900 & 2.75485600 \\
\hline $\mathrm{H}$ & -1.05479900 & 4.36379600 & -0.85967100 \\
\hline $\mathrm{H}$ & -2.41497200 & 4.97154700 & 0.10321000 \\
\hline $\mathrm{H}$ & -0.81770900 & 5.72791200 & 0.23557600 \\
\hline $\mathrm{O}$ & 0.10034400 & -0.02921100 & 0.12098100 \\
\hline $\mathrm{O}$ & 0.64924800 & 1.97339000 & -0.77096000 \\
\hline I & 2.33210900 & -2.65531900 & 0.70215900 \\
\hline $\mathrm{C}$ & 4.07306500 & 1.26158600 & -1.12773900 \\
\hline $\mathrm{O}$ & 4.04453900 & 0.07993400 & -0.66709400 \\
\hline $\mathrm{O}$ & 3.04175600 & 2.02695900 & -1.22963200 \\
\hline $\mathrm{H}$ & 1.86444900 & 1.89319400 & -0.96016100 \\
\hline $\mathrm{C}$ & 5.38476700 & 1.82459900 & -1.59526000 \\
\hline $\mathrm{H}$ & 5.31185900 & 2.06472500 & -2.66130300 \\
\hline $\mathrm{H}$ & 5.58902000 & 2.75834900 & -1.06174000 \\
\hline & 6.19102900 & 1.10972500 & -1.42830300 \\
\hline
\end{tabular}

12

$-1152.75282178$

$\begin{array}{lrrr}\text { Pd } & 2.27736200 & -0.25621300 & -0.19683100 \\ \mathrm{C} & -1.55995400 & 1.83296600 & -0.14075400 \\ \mathrm{C} & -0.11703100 & 1.44597700 & -0.37378700 \\ \mathrm{H} & -1.91302800 & 2.41957100 & -0.99247600 \\ \mathrm{~N} & -2.35607200 & 0.62100400 & -0.07495900 \\ \mathrm{H} & -1.89396800 & -0.22568300 & 0.22592700 \\ \mathrm{C} & -3.63490800 & 0.60221200 & -0.57084500 \\ \mathrm{O} & -4.14110000 & 1.60967900 & -1.06238000\end{array}$




\begin{tabular}{|c|c|c|c|c|c|c|c|}
\hline $\mathrm{C}$ & -4.37192300 & -0.70283300 & -0.48831500 & $\mathrm{O}$ & 0.07026000 & -3.00993300 & -1.51176200 \\
\hline $\mathrm{C}$ & -3.97534700 & -1.77486400 & 0.32494700 & $\mathrm{~N}$ & -0.40959600 & -0.81558900 & -0.08819000 \\
\hline $\mathrm{C}$ & -5.52922900 & -0.82806700 & -1.26964600 & $\mathrm{C}$ & -0.76059600 & -0.04782100 & -1.34093400 \\
\hline $\mathrm{C}$ & -4.71612300 & -2.95669400 & 0.34174600 & $\mathrm{C}$ & -1.43534000 & 1.27590700 & -0.94228100 \\
\hline $\mathrm{H}$ & -3.10663700 & -1.69574400 & 0.97294800 & $\mathrm{C}$ & -0.83813100 & 2.50914800 & -0.63900400 \\
\hline $\mathrm{C}$ & -6.26511400 & -2.01046100 & -1.25580000 & $\mathrm{C}$ & -1.58652700 & 3.64991600 & -0.32007500 \\
\hline $\mathrm{H}$ & -5.83049900 & 0.01674500 & -1.87983400 & $\mathrm{C}$ & -2.97313600 & 3.57942800 & -0.29445200 \\
\hline $\mathrm{C}$ & -5.85858600 & -3.07862300 & -0.45173200 & $\mathrm{C}$ & -3.61918700 & 2.37852800 & -0.57213700 \\
\hline $\mathrm{H}$ & -4.40180300 & -3.77908500 & 0.97805200 & $\mathrm{C}$ & -2.86203900 & 1.24765700 & -0.88345200 \\
\hline $\mathrm{H}$ & -7.15665200 & -2.09965800 & -1.87043900 & $\mathrm{C}$ & -2.25769500 & -2.93555400 & -0.25555800 \\
\hline $\mathrm{H}$ & -6.43269600 & -4.00116100 & -0.43901100 & $\mathrm{H}$ & -1.08478700 & 4.58318200 & -0.09463800 \\
\hline $\mathrm{C}$ & -1.70609100 & 2.67659500 & 1.16101500 & $\mathrm{H}$ & -3.55484300 & 4.46490300 & -0.05385400 \\
\hline $\mathrm{H}$ & -2.78694600 & 2.81939500 & 1.28099700 & $\mathrm{H}$ & -4.70057300 & 2.32741400 & -0.54472100 \\
\hline $\mathrm{H}$ & -1.37067500 & 2.05416200 & 2.00094400 & $\mathrm{O}$ & -3.43386500 & 0.04086400 & -1.14983800 \\
\hline $\mathrm{C}$ & -1.00338600 & 4.04738800 & 1.20788500 & $\mathrm{C}$ & -4.84405600 & -0.10855300 & -1.06347400 \\
\hline $\mathrm{C}$ & -1.22564700 & 4.67938500 & 2.59158700 & $\mathrm{H}$ & -5.35975800 & 0.52280800 & -1.79746600 \\
\hline $\mathrm{C}$ & -1.48217400 & 4.99211500 & 0.09438500 & $\mathrm{H}$ & -5.03592300 & -1.15846800 & -1.28566900 \\
\hline $\mathrm{H}$ & 0.07807700 & 3.89443700 & 1.08297700 & $\mathrm{H}$ & -5.20756900 & 0.12529300 & -0.05555700 \\
\hline $\mathrm{H}$ & -0.85891700 & 4.02932600 & 3.39476000 & I & 3.22775900 & -1.24828900 & -0.33685900 \\
\hline $\mathrm{H}$ & -0.70306900 & 5.63937000 & 2.67057900 & I & 1.28481200 & 2.87287000 & -0.56099800 \\
\hline $\mathrm{H}$ & -2.29237100 & 4.86458800 & 2.77115600 & $\mathrm{C}$ & 0.32546300 & 0.02842500 & -2.42260800 \\
\hline $\mathrm{H}$ & -1.23276700 & 4.61599000 & -0.90329300 & $\mathrm{H}$ & -0.03852500 & 0.64930600 & -3.24903600 \\
\hline $\mathrm{H}$ & -2.56951300 & 5.13262600 & 0.14013800 & $\mathrm{H}$ & 1.26655200 & 0.44128500 & -2.06489800 \\
\hline $\mathrm{H}$ & -1.01307900 & 5.97729300 & 0.19801500 & $\mathrm{H}$ & 0.52119100 & -0.97720000 & -2.79977600 \\
\hline $\mathrm{O}$ & 0.30694000 & 0.35343000 & 0.04397500 & $\mathrm{H}$ & -1.56472600 & -0.63128400 & -1.79482700 \\
\hline $\mathrm{O}$ & 0.60825600 & 2.34844500 & -0.99487700 & $\mathrm{C}$ & -1.08481900 & 0.61290400 & 2.82006700 \\
\hline I & 1.70507300 & -2.58086200 & 0.87647000 & $\mathrm{O}$ & 0.12570600 & 0.72093600 & 2.55969300 \\
\hline $\mathrm{C}$ & 4.33529900 & 0.73956200 & -1.11991800 & $\mathrm{O}$ & -1.93545300 & -0.02055400 & 2.05642400 \\
\hline $\mathrm{O}$ & 4.28038900 & -0.43295100 & -0.61978800 & $\mathrm{H}$ & -1.45319200 & -0.38071000 & 1.23688200 \\
\hline $\mathrm{O}$ & 3.25973900 & 1.44072300 & -1.13248200 & $\mathrm{C}$ & -1.67570800 & 1.21955100 & 4.06094700 \\
\hline $\mathrm{H}$ & 1.55931800 & 2.08314100 & -1.07814900 & $\mathrm{H}$ & -2.15238800 & 0.43481400 & 4.65741900 \\
\hline $\mathrm{C}$ & 5.61290600 & 1.27868600 & -1.68459500 & $\mathrm{H}$ & -0.90034200 & 1.71580200 & 4.64435300 \\
\hline $\mathrm{H}$ & 5.50434700 & 1.40330800 & -2.76780900 & $\mathrm{H}$ & -2.45278700 & 1.93760700 & 3.77906700 \\
\hline $\mathrm{H}$ & 5.81710900 & 2.26471800 & -1.25592900 & & & & \\
\hline $\mathrm{H}$ & 6.43882600 & 0.59649100 & -1.47612500 & \multicolumn{4}{|c|}{$\mathrm{TS}_{\mathrm{R} 5 \mathrm{c}}$} \\
\hline & & & & \multicolumn{4}{|c|}{-1743.73163990} \\
\hline \multicolumn{4}{|l|}{$\mathrm{R}_{8 \mathrm{c}}$} & $\mathrm{Pd}$ & 1.30987500 & -0.23154700 & 0.97199200 \\
\hline \multicolumn{4}{|c|}{-1743.73608710} & $\mathrm{~S}$ & -0.52139600 & -2.50217500 & -0.17115700 \\
\hline $\mathrm{Pd}$ & 1.25191700 & -0.20936900 & 0.98627200 & $\mathrm{~F}$ & -3.05381900 & -2.18811800 & 0.65562800 \\
\hline S & -0.41765500 & -2.50920900 & -0.22727000 & $\mathrm{~F}$ & -2.85634100 & -2.65234500 & -1.46464300 \\
\hline $\mathrm{F}$ & -2.94123200 & -2.23596000 & 0.65772600 & $\mathrm{~F}$ & -2.49739900 & -4.19369700 & 0.02417600 \\
\hline $\mathrm{F}$ & -2.78559800 & -2.71196500 & -1.46452900 & $\mathrm{O}$ & -0.08612400 & -3.01593100 & 1.12213300 \\
\hline $\mathrm{F}$ & -2.36205000 & -4.23341200 & 0.02550900 & $\mathrm{O}$ & 0.00714900 & -2.98329000 & -1.44257700 \\
\hline $\mathrm{O}$ & 0.04628000 & -3.04322600 & 1.05093900 & $\mathrm{~N}$ & -0.46730400 & -0.78161000 & -0.05243500 \\
\hline
\end{tabular}




\begin{tabular}{|c|c|c|c|}
\hline $\mathrm{C}$ & -0.84177200 & -0.01334100 & -1.31127500 \\
\hline $\mathrm{C}$ & -1.46929000 & 1.32872200 & -0.90896200 \\
\hline C & -0.82688800 & 2.53673400 & -0.59820600 \\
\hline 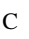 & -1.53416000 & 3.70333500 & -0.28387300 \\
\hline $\mathrm{C}$ & -2.92303700 & 3.68270200 & -0.26836400 \\
\hline $\mathrm{C}$ & -3.61166500 & 2.50665000 & -0.54900600 \\
\hline 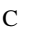 & -2.89523100 & 1.34842700 & -0.85544500 \\
\hline $\mathrm{C}$ & -2.37021000 & -2.89560400 & -0.24284500 \\
\hline $\mathrm{H}$ & -1.00058500 & 4.61651500 & -0.04998800 \\
\hline 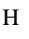 & -3.47324400 & 4.58848200 & -0.02960300 \\
\hline $\mathrm{H}$ & -4.69424400 & 2.49502600 & -0.52464900 \\
\hline $\mathrm{O}$ & -3.50702900 & 0.16176100 & -1.12387200 \\
\hline $\mathrm{C}$ & -4.91911500 & 0.05301700 & -1.00787800 \\
\hline $\mathrm{H}$ & -5.43174100 & 0.69463100 & -1.73509000 \\
\hline $\mathrm{H}$ & -5.14577700 & -0.99211600 & -1.22073500 \\
\hline $\mathrm{H}$ & -5.25398100 & 0.30195100 & 0.00604100 \\
\hline I & 3.24039600 & -1.25843600 & -0.45259200 \\
\hline I & 1.30841900 & 2.81087100 & -0.49106300 \\
\hline $\mathrm{C}$ & 0.23342100 & 0.02552900 & -2.40228700 \\
\hline $\mathrm{H}$ & -0.13678600 & 0.63533900 & -3.23392600 \\
\hline 11 & 1.18275400 & 0.43645700 & -2.06560000 \\
\hline $\mathrm{H}$ & 0.41459900 & -0.98746700 & -2.76624800 \\
\hline $\mathrm{H}$ & -1.66722400 & -0.58027500 & -1.74328000 \\
\hline $\mathrm{C}$ & -0.97222100 & 0.50981000 & 2.75820200 \\
\hline $\mathrm{O}$ & 0.27596800 & 0.61748200 & 2.55191900 \\
\hline $\mathrm{O}$ & -1.78639200 & -0.02619900 & 1.94189600 \\
\hline $\mathrm{H}$ & -1.19333100 & -0.43396400 & 0.93250900 \\
\hline $\mathrm{C}$ & -1.51909500 & 1.03654600 & 4.06247800 \\
\hline $\mathrm{H}$ & -1.76456500 & 0.18580000 & 4.70849800 \\
\hline $\mathrm{H}$ & -0.78583700 & 1.66851900 & 4.56518100 \\
\hline $\mathrm{H}$ & -2.44299100 & 1.59101100 & 3.87679500 \\
\hline
\end{tabular}

$\mathrm{R}_{\mathrm{9c}}$

$-1743.74590645$

$\begin{array}{lrrr}\text { Pd } & 0.44855900 & -0.95767500 & 0.98656800 \\ \text { C } & 2.17466500 & -0.05677900 & 4.43439800 \\ \text { C } & 1.50648100 & -0.33809300 & 3.12099400 \\ \text { O } & 1.86366200 & -1.35365300 & 2.42373000 \\ \text { O } & 0.56596800 & 0.39844600 & 2.67518300 \\ \text { S } & -2.52072200 & -1.16006900 & -0.37970000 \\ \text { F } & -4.01228800 & 0.59512000 & 0.93557700 \\ \text { F } & -4.08807700 & 0.83866900 & -1.23228500 \\ \text { F } & -5.11085100 & -0.83235700 & -0.28925800 \\ \text { O } & -2.58394100 & -2.00298900 & 0.80131300\end{array}$

\begin{tabular}{|c|c|c|c|}
\hline $\mathrm{O}$ & -2.49459400 & -1.64648800 & -1.74745800 \\
\hline $\mathrm{N}$ & -1.17059400 & -0.03358700 & -0.10812400 \\
\hline $\mathrm{C}$ & -0.81972600 & 0.96021700 & -1.23878700 \\
\hline $\mathrm{C}$ & -0.05551700 & 2.11960800 & -0.58578200 \\
\hline $\mathrm{C}$ & 1.32245300 & 2.39911000 & -0.59731300 \\
\hline $\mathrm{C}$ & 1.83672100 & 3.57408100 & -0.03274300 \\
\hline $\mathrm{C}$ & 0.98854100 & 4.48893100 & 0.57600700 \\
\hline $\mathrm{C}$ & -0.37859300 & 4.23838000 & 0.65024100 \\
\hline $\mathrm{C}$ & -0.88167600 & 3.06678100 & 0.08899900 \\
\hline $\mathrm{C}$ & -4.03999700 & -0.04980200 & -0.23749200 \\
\hline $\mathrm{H}$ & -1.50425600 & 0.53995700 & 0.67752400 \\
\hline $\mathrm{H}$ & 2.90247800 & 3.76505700 & -0.06588100 \\
\hline $\mathrm{H}$ & 1.39609100 & 5.39802800 & 1.00876000 \\
\hline $\mathrm{H}$ & -1.03381500 & 4.94287900 & 1.14686700 \\
\hline $\mathrm{O}$ & -2.20764700 & 2.72914700 & 0.15777100 \\
\hline $\mathrm{C}$ & -3.13398200 & 3.65483000 & 0.71935200 \\
\hline $\mathrm{H}$ & -3.11613000 & 4.60748800 & 0.17759100 \\
\hline $\mathrm{H}$ & -4.11294400 & 3.18868300 & 0.61175000 \\
\hline $\mathrm{H}$ & -2.92669800 & 3.82938300 & 1.78157000 \\
\hline I & 0.89948600 & -3.03945600 & -0.58400000 \\
\hline I & 2.86231600 & 1.12369500 & -1.37947000 \\
\hline $\mathrm{C}$ & -0.23196000 & 0.27303500 & -2.46793900 \\
\hline $\mathrm{H}$ & -1.02094800 & -0.26766600 & -2.99272000 \\
\hline $\mathrm{H}$ & 0.16799100 & 1.03707000 & -3.14117000 \\
\hline $\mathrm{H}$ & 0.55639500 & -0.43748100 & -2.22855300 \\
\hline $\mathrm{H}$ & -1.77333100 & 1.39579000 & -1.54123400 \\
\hline $\mathrm{H}$ & 3.25495900 & -0.19998500 & 4.34225200 \\
\hline $\mathrm{H}$ & 1.94968500 & 0.96042500 & 4.76190200 \\
\hline $\mathrm{H}$ & 1.80585700 & -0.76541600 & 5.18494800 \\
\hline
\end{tabular}

$\mathrm{S}_{1 \mathrm{~A}}$

$-2507.05391265$

$\begin{array}{lrrr}\mathrm{Pd} & 0.52338600 & -0.07280500 & -0.20229600 \\ \mathrm{O} & -1.91023500 & 1.54109800 & 1.34668200 \\ \mathrm{C} & -0.65739400 & 2.34738300 & 3.23004500 \\ \mathrm{C} & -0.81573800 & 1.56541100 & 1.93567100 \\ \mathrm{O} & 0.26394000 & 0.96511900 & 1.54717600 \\ \mathrm{C} & 3.13515600 & -1.25952600 & -0.57452800 \\ \mathrm{C} & 2.26109400 & -1.62796600 & -1.79059700 \\ \mathrm{H} & 4.14595100 & -1.07565200 & -0.94287700 \\ \mathrm{H} & -0.30402800 & 1.68407200 & 4.02646300 \\ \mathrm{H} & 0.09319800 & 3.13345600 & 3.09420400 \\ \mathrm{H} & -1.60932800 & 2.79631100 & 3.51803800 \\ \mathrm{~N} & 2.62841400 & 0.01363300 & 0.05725500\end{array}$




\begin{tabular}{|c|c|c|c|c|c|c|c|}
\hline $\mathrm{H}$ & 2.78549700 & 0.01850400 & 1.06458000 & $\mathrm{H}$ & -0.80419900 & -3.20047200 & 1.22480500 \\
\hline $\mathrm{C}$ & 3.14729600 & 1.22319900 & -0.57540400 & $\mathrm{H}$ & -1.06603400 & -3.50294000 & 3.66402400 \\
\hline $\mathrm{O}$ & 3.46644100 & 1.17056600 & -1.74327100 & $\mathrm{H}$ & -2.85887100 & -2.26408500 & 4.87315000 \\
\hline $\mathrm{C}$ & 3.31098300 & 2.44347800 & 0.26418900 & $\mathrm{H}$ & -4.37110300 & -0.75323300 & 3.64455000 \\
\hline $\mathrm{C}$ & 2.96192100 & 2.53092800 & 1.62171600 & $\mathrm{H}$ & -1.88429800 & 0.45848800 & 0.08953800 \\
\hline $\mathrm{C}$ & 3.89863100 & 3.55242200 & -0.36943400 & $\mathrm{O}$ & -4.24339400 & -0.32458600 & 0.96388700 \\
\hline $\mathrm{C}$ & 3.20903700 & 3.70697700 & 2.32989800 & $\mathrm{C}$ & -5.10889300 & 0.60127700 & 1.60994900 \\
\hline $\mathrm{H}$ & 2.44965500 & 1.71790800 & 2.12297400 & $\mathrm{H}$ & -4.53608600 & 1.27114000 & 2.25971600 \\
\hline $\mathrm{C}$ & 4.13816200 & 4.72398200 & 0.34091900 & $\mathrm{H}$ & -5.57035200 & 1.17831400 & 0.80806300 \\
\hline $\mathrm{H}$ & 4.16101100 & 3.46930500 & -1.41837400 & $\mathrm{H}$ & -5.88997000 & 0.08738100 & 2.18405200 \\
\hline $\mathrm{C}$ & 3.79774300 & 4.80201000 & 1.69447800 & $\mathrm{C}$ & -1.59878500 & -2.71865700 & -1.22284000 \\
\hline $\mathrm{H}$ & 2.93717500 & 3.76742600 & 3.37992900 & $\mathrm{H}$ & -0.56483900 & -2.86191500 & -0.90590300 \\
\hline $\mathrm{H}$ & 4.59129800 & 5.57541400 & -0.15840800 & $\mathrm{H}$ & -2.15957800 & -3.64239400 & -1.04313300 \\
\hline $\mathrm{H}$ & 3.98828500 & 5.71518100 & 2.25169900 & $\mathrm{H}$ & -1.58427500 & -2.51728300 & -2.29388700 \\
\hline $\mathrm{C}$ & 3.14448800 & -2.36631600 & 0.49754400 & $\mathrm{H}$ & -3.29358300 & -1.45738100 & -0.87544000 \\
\hline $\mathrm{H}$ & 3.78029200 & -2.02597200 & 1.33114100 & & & & \\
\hline $\mathrm{H}$ & 2.12795900 & -2.47484500 & 0.90178700 & \multicolumn{4}{|c|}{$\mathrm{TS}_{\mathrm{SIA}}$} \\
\hline $\mathrm{C}$ & 3.65205900 & -3.74731200 & 0.03128100 & \multicolumn{4}{|c|}{-2507.04677199} \\
\hline $\mathrm{C}$ & 3.57231800 & -4.73941800 & 1.20172300 & $\mathrm{Pd}$ & -0.45843500 & -0.07980200 & -0.18087700 \\
\hline $\mathrm{C}$ & 5.07328300 & -3.68637200 & -0.54745500 & $\mathrm{O}$ & 1.93953100 & -0.93355500 & 1.87351200 \\
\hline $\mathrm{H}$ & 2.98557900 & -4.09834400 & -0.76520500 & $\mathrm{C}$ & 0.69628600 & -1.79006400 & 3.70981200 \\
\hline $\mathrm{H}$ & 2.55487600 & -4.80733800 & 1.60649500 & $\mathrm{C}$ & 0.77200800 & -1.16934400 & 2.33759600 \\
\hline $\mathrm{H}$ & 3.87127300 & -5.74330700 & 0.88003900 & $\mathrm{O}$ & -0.30446900 & -0.93744300 & 1.71966300 \\
\hline $\mathrm{H}$ & 4.23941500 & -4.44164400 & 2.02183800 & $\mathrm{C}$ & -2.87934100 & 1.44912200 & -0.62965900 \\
\hline $\mathrm{H}$ & 5.09640200 & -3.11301300 & -1.47869900 & $\mathrm{C}$ & -1.95038700 & 1.63022500 & -1.84511200 \\
\hline $\mathrm{H}$ & 5.77664300 & -3.23484000 & 0.16609600 & $\mathrm{H}$ & -3.90119700 & 1.40005600 & -1.01016100 \\
\hline $\mathrm{H}$ & 5.43660200 & -4.69511500 & -0.77486200 & $\mathrm{H}$ & 0.80535200 & -2.87588000 & 3.60327700 \\
\hline $\mathrm{O}$ & 1.03177100 & -1.15369300 & -1.79129500 & $\mathrm{H}$ & 1.51626000 & -1.42798200 & 4.33398300 \\
\hline $\mathrm{O}$ & 2.69966200 & -2.36660500 & -2.65323500 & $\mathrm{H}$ & -0.26609200 & -1.57918500 & 4.17954500 \\
\hline S & -1.85571100 & 0.57626500 & -2.19046800 & $\mathrm{~N}$ & -2.56873200 & 0.14066600 & 0.04825500 \\
\hline $\mathrm{F}$ & -4.50304700 & 0.33371200 & -1.94674100 & $\mathrm{H}$ & -2.75044800 & 0.18830700 & 1.05050100 \\
\hline $\mathrm{F}$ & -3.66897200 & 2.07068000 & -0.92845000 & $\mathrm{C}$ & -3.24340200 & -1.00142500 & -0.56693200 \\
\hline $\mathrm{F}$ & -3.80933200 & 2.04272100 & -3.10033600 & $\mathrm{O}$ & -3.56652000 & -0.91912400 & -1.73191800 \\
\hline $\mathrm{O}$ & -1.94822800 & -0.39866400 & -3.26673000 & $\mathrm{C}$ & -3.54485900 & -2.18574100 & 0.28424500 \\
\hline $\mathrm{O}$ & -0.96092600 & 1.72525600 & -2.21465200 & $\mathrm{C}$ & -3.16290900 & -2.31950200 & 1.62864000 \\
\hline $\mathrm{N}$ & -1.58519400 & -0.23227300 & -0.66390900 & $\mathrm{C}$ & -4.29482700 & -3.20713800 & -0.32492000 \\
\hline $\mathrm{C}$ & -2.28743000 & -1.58415400 & -0.46897800 & $\mathrm{C}$ & -3.53511700 & -3.45423000 & 2.34867000 \\
\hline $\mathrm{C}$ & -2.42423000 & -1.80544200 & 1.02870400 & $\mathrm{H}$ & -2.53543700 & -1.57836100 & 2.11003000 \\
\hline $\mathrm{C}$ & -1.58352900 & -2.65701600 & 1.74837100 & $\mathrm{C}$ & -4.66222300 & -4.33669600 & 0.39813000 \\
\hline $\mathrm{C}$ & -1.72968600 & -2.83175800 & 3.12717800 & $\mathrm{H}$ & -4.57816700 & -3.09062000 & -1.36515100 \\
\hline $\mathrm{C}$ & -2.73313500 & -2.13968400 & 3.80081000 & $\mathrm{C}$ & -4.28594200 & -4.46076300 & 1.73877800 \\
\hline $\mathrm{C}$ & -3.59274900 & -1.28361700 & 3.10847000 & $\mathrm{H}$ & -3.23153300 & -3.55455200 & 3.38679300 \\
\hline $\mathrm{C}$ & -3.44427100 & -1.12045300 & 1.72788900 & $\mathrm{H}$ & -5.24054500 & -5.12079400 & -0.08172300 \\
\hline $\mathrm{C}$ & -3.58826200 & 1.30465500 & -2.01384300 & $\mathrm{H}$ & -4.57326300 & -5.34251500 & 2.30497100 \\
\hline
\end{tabular}




\begin{tabular}{|c|c|c|c|c|c|c|c|}
\hline $\mathrm{C}$ & -2.74572900 & 2.58353300 & 0.40418000 & $\mathrm{H}$ & 3.50305900 & 0.54697200 & -0.97439800 \\
\hline $\mathrm{H}$ & -3.42652100 & 2.35453900 & 1.24069900 & & & & \\
\hline $\mathrm{H}$ & -1.72624900 & 2.57650500 & 0.81381900 & \multicolumn{4}{|c|}{$\mathrm{S}_{2 \mathrm{~A}}$} \\
\hline $\mathrm{C}$ & -3.06890400 & 4.00348500 & -0.10810800 & \multicolumn{4}{|c|}{-2507.04749863} \\
\hline $\mathrm{C}$ & -2.90378600 & 5.00473000 & 1.04578900 & $\mathrm{Pd}$ & 0.54775400 & -0.07746200 & -0.22726300 \\
\hline $\mathrm{C}$ & -4.46730000 & 4.10561400 & -0.73442100 & $\mathrm{O}$ & -2.04354800 & 1.53163300 & 1.08035900 \\
\hline $\mathrm{H}$ & -2.33929600 & 4.25068100 & -0.88827000 & $\mathrm{C}$ & -0.99129500 & 2.72368000 & 2.84000600 \\
\hline $\mathrm{H}$ & -1.90332700 & 4.94591000 & 1.49169700 & $\mathrm{C}$ & -0.92337200 & 1.76246900 & 1.68525500 \\
\hline $\mathrm{H}$ & -3.05600600 & 6.03143900 & 0.69424200 & $\mathrm{O}$ & 0.16411800 & 1.22424100 & 1.38138900 \\
\hline $\mathrm{H}$ & -3.63567000 & 4.81575900 & 1.84265200 & $\mathrm{C}$ & 3.27535100 & -1.09835400 & -0.49518000 \\
\hline $\mathrm{H}$ & -4.53164500 & 3.51798300 & -1.65473500 & $\mathrm{C}$ & 2.41165000 & -1.65823500 & -1.64274400 \\
\hline $\mathrm{H}$ & -5.24466900 & 3.76254500 & -0.03745800 & $\mathrm{H}$ & 4.24727800 & -0.83967500 & -0.91861600 \\
\hline $\mathrm{H}$ & -4.69389500 & 5.14621100 & -0.99365100 & $\mathrm{H}$ & -1.32507200 & 2.17657500 & 3.72974800 \\
\hline $\mathrm{O}$ & -0.84210600 & 0.91404800 & -1.85402500 & $\mathrm{H}$ & -0.00716900 & 3.15279300 & 3.03240900 \\
\hline $\mathrm{O}$ & -2.23157800 & 2.43135500 & -2.71773400 & $\mathrm{H}$ & -1.72116800 & 3.50974500 & 2.63285200 \\
\hline S & 1.79329300 & -1.77636800 & -1.38777400 & $\mathrm{~N}$ & 2.65898600 & 0.15775800 & 0.05918600 \\
\hline $\mathrm{F}$ & 4.37934500 & -1.46675200 & -1.96147300 & $\mathrm{H}$ & 2.78345400 & 0.22217400 & 1.06800500 \\
\hline $\mathrm{F}$ & 3.99239100 & -2.01689000 & 0.10979900 & $\mathrm{C}$ & 3.05521000 & 1.37755400 & -0.62242800 \\
\hline $\mathrm{F}$ & 3.76990100 & -3.49688100 & -1.47136300 & $\mathrm{O}$ & 3.41042400 & 1.31454000 & -1.77922000 \\
\hline $\mathrm{O}$ & 1.60906900 & -1.68298000 & -2.83325300 & $\mathrm{C}$ & 3.00100600 & 2.65645200 & 0.14505700 \\
\hline $\mathrm{O}$ & 1.06599300 & -2.77184300 & -0.59526000 & $\mathrm{C}$ & 2.95631300 & 2.74200400 & 1.54542500 \\
\hline $\mathrm{N}$ & 1.63438100 & -0.30857700 & -0.55583600 & $\mathrm{C}$ & 3.05747200 & 3.83801900 & -0.61297700 \\
\hline $\mathrm{C}$ & 2.46220400 & 0.87328900 & -1.04708200 & $\mathrm{C}$ & 2.97182200 & 3.98728600 & 2.17318100 \\
\hline $\mathrm{C}$ & 2.29583600 & 1.98655400 & -0.02381900 & $\mathrm{H}$ & 2.91472100 & 1.85313500 & 2.16721900 \\
\hline $\mathrm{C}$ & 1.35943200 & 3.01192300 & -0.17632700 & $\mathrm{C}$ & 3.05391900 & 5.07955400 & 0.01569700 \\
\hline $\mathrm{C}$ & 1.23203100 & 4.03028700 & 0.77391600 & $\mathrm{H}$ & 3.10085400 & 3.75695200 & -1.69363600 \\
\hline $\mathrm{C}$ & 2.05212700 & 4.02377000 & 1.89958600 & $\mathrm{C}$ & 3.01449200 & 5.15665500 & 1.41056900 \\
\hline $\mathrm{C}$ & 3.00381500 & 3.01618700 & 2.07654800 & $\mathrm{H}$ & 2.95580600 & 4.04313200 & 3.25804000 \\
\hline $\mathrm{C}$ & 3.13194800 & 2.00625600 & 1.11633000 & $\mathrm{H}$ & 3.08628500 & 5.98650600 & -0.58078600 \\
\hline $\mathrm{C}$ & 3.61179500 & -2.21168300 & -1.15675200 & $\mathrm{H}$ & 3.02165000 & 6.12523100 & 1.90284900 \\
\hline $\mathrm{H}$ & 0.72529700 & 3.01796700 & -1.05645700 & $\mathrm{C}$ & 3.43880300 & -2.10996800 & 0.65620200 \\
\hline $\mathrm{H}$ & 0.50782200 & 4.82559100 & 0.62385600 & $\mathrm{H}$ & 4.05372600 & -1.63432600 & 1.43813800 \\
\hline $\mathrm{H}$ & 1.96633800 & 4. 80943600 & 2.64576900 & $\mathrm{H}$ & 2.45202300 & -2.29999200 & 1.10256800 \\
\hline $\mathrm{H}$ & 3.64453600 & 3.03127000 & 2.95085100 & $\mathrm{C}$ & 4.08822100 & -3.45915100 & 0.28173200 \\
\hline $\mathrm{H}$ & 1.88500800 & -0.61407000 & 0.77197600 & $\mathrm{C}$ & 4.16032900 & -4.35061900 & 1.53091600 \\
\hline $\mathrm{O}$ & 4.05044000 & 0.99955100 & 1.19200800 & $\mathrm{C}$ & 5.47329200 & -3.28734900 & -0.35879400 \\
\hline $\mathrm{C}$ & 4.82842400 & 0.86252700 & 2.36929600 & $\mathrm{H}$ & 3.44130500 & -3.94864400 & -0.45535200 \\
\hline $\mathrm{H}$ & 4.19144400 & 0.73171000 & 3.25271200 & $\mathrm{H}$ & 3.17077200 & -4.49631700 & 1.98173600 \\
\hline $\mathrm{H}$ & 5.43016900 & -0.03567500 & 2.22185300 & $\mathrm{H}$ & 4.56077100 & -5.33899500 & 1.27950900 \\
\hline $\mathrm{H}$ & 5.49104900 & 1.72501900 & 2.51837200 & $\mathrm{H}$ & 4.81570400 & -3.91201100 & 2.29547900 \\
\hline $\mathrm{C}$ & 2.20086300 & 1.31062100 & -2.49350500 & $\mathrm{H}$ & 5.39935600 & -2.79561100 & -1.33306800 \\
\hline $\mathrm{H}$ & 2.52444300 & 0.53833400 & -3.19290700 & $\mathrm{H}$ & 6.14345400 & -2.70137500 & 0.28566000 \\
\hline $\mathrm{H}$ & 1.14578200 & 1.50915100 & -2.68073100 & $\mathrm{H}$ & 5.94266300 & -4.26445800 & -0.52033800 \\
\hline $\mathrm{H}$ & 2.78330700 & 2.21782100 & -2.69001200 & $\mathrm{O}$ & 1.13922900 & -1.31323900 & 52719 \\
\hline
\end{tabular}




\begin{tabular}{|c|c|c|c|}
\hline $\mathrm{O}$ & 2.90040700 & -2.42680900 & -2.44993200 \\
\hline S & -1.79856900 & 0.12791000 & -2.21473000 \\
\hline F & -4.44772700 & -0.20168500 & -1.96684700 \\
\hline $\mathrm{F}$ & -3.70999600 & 1.67956800 & -1.15496900 \\
\hline $\mathrm{F}$ & -3.81657200 & 1.38906100 & -3.30732800 \\
\hline $\mathrm{O}$ & -1.87593000 & -0.94516500 & -3.20503800 \\
\hline $\mathrm{O}$ & -0.98290700 & 1.32677200 & -2.42592200 \\
\hline $\mathrm{N}$ & -1.49890400 & -0.39389400 & -0.64496300 \\
\hline $\mathrm{C}$ & -2.09347500 & -1.73763200 & -0.26882000 \\
\hline $\mathrm{C}$ & -2.25923800 & -1.76157800 & 1.24693300 \\
\hline $\mathrm{C}$ & -1.34273500 & -2.38013000 & 2.09924600 \\
\hline $\mathrm{C}$ & -1.51220000 & -2.37956200 & 3.48808400 \\
\hline $\mathrm{C}$ & -2.62502000 & -1.75168000 & 4.04030600 \\
\hline- & -3.56510200 & -1.12617100 & 3.21596700 \\
\hline C & -3.38394400 & -1.13122800 & 1.82910300 \\
\hline $\mathrm{C}$ & -3.56201000 & 0.78724200 & -2.14381700 \\
\hline H & -0.48113300 & -2.88249900 & 1.67169400 \\
\hline $\mathrm{H}$ & -0.78480600 & -2.87409700 & 4.12532800 \\
\hline $\mathrm{H}$ & -2.77644300 & -1.74875200 & 5.11666400 \\
\hline $\mathrm{H}$ & -4.43240200 & -0.64765500 & 3.65649000 \\
\hline [ & -1.91970700 & 0.78479300 & 0.35182100 \\
\hline $\mathrm{O}$ & -4.24283400 & -0.54281500 & 0.94911400 \\
\hline $\mathrm{C}$ & -5.32323600 & 0.23176700 & 1.44430600 \\
\hline $\mathrm{H}$ & -4.96387700 & 1.04852100 & 2.08235200 \\
\hline 1 & -5.81097300 & 0.64797400 & 0.56204900 \\
\hline $\mathrm{H}$ & -6.03870700 & -0.38502000 & 2.00323400 \\
\hline $\mathrm{C}$ & -1.33916900 & -2.94700600 & -0.82924200 \\
\hline$\Pi$ & -0.30904800 & -2.99970800 & -0.47158400 \\
\hline $\mathrm{H}$ & -1.86408400 & -3.86259700 & -0.53258500 \\
\hline $\mathrm{H}$ & -1.30083200 & -2.89415900 & -1.91773100 \\
\hline $\mathrm{H}$ & -3.10007100 & -1.75182500 & -0.69580200 \\
\hline
\end{tabular}

$\mathrm{S}_{3 \mathrm{~A}}$

$-2507.03580714$

$\begin{array}{llll}\mathrm{Pd} & -0.53042800 & -0.60360000 & -0.24498400 \\ \mathrm{C} & 3.65919000 & -0.90760500 & -1.10347800 \\ \mathrm{C} & 2.11867800 & -0.91601300 & -1.23261500 \\ \mathrm{H} & 4.02913800 & -0.52063000 & -2.05496400 \\ \mathrm{~N} & 4.14830900 & -0.02000100 & -0.05620800 \\ \mathrm{H} & 4.18517300 & -0.40707200 & 0.87742800 \\ \mathrm{C} & 3.98047900 & 1.33531000 & -0.15632700 \\ \mathrm{O} & 3.52596200 & 1.86727200 & -1.17046100 \\ \mathrm{C} & 4.40842200 & 2.14516500 & 1.03457000 \\ \mathrm{C} & 5.33245500 & 1.68310200 & 1.98313200\end{array}$

\begin{tabular}{|c|c|c|c|}
\hline $\mathrm{C}$ & 3.86607500 & 3.42931800 & 1.17725000 \\
\hline $\mathrm{C}$ & 5.69161900 & 2.48618300 & 3.06608600 \\
\hline I & 5.80083600 & 0.71032800 & 1.86084400 \\
\hline C & 4.21893300 & 4.22781600 & 2.26283800 \\
\hline $\mathrm{H}$ & 3.16989600 & 3.78022200 & 0.42285200 \\
\hline $\mathrm{C}$ & 5.13076600 & 3.75668000 & 3.21149600 \\
\hline $\mathrm{H}$ & 6.41545400 & 2.12322500 & 3.79074600 \\
\hline $\mathrm{H}$ & 3.78566500 & 5.21860800 & 2.36980000 \\
\hline $\mathrm{H}$ & 5.40913500 & 4.38055600 & 4.05666700 \\
\hline $\mathrm{C}$ & 4.21419000 & -2.32483300 & -0.85084600 \\
\hline $\mathrm{H}$ & 5.30619200 & -2.23394400 & -0.76106600 \\
\hline $\mathrm{H}$ & 3.84590800 & -2.66857100 & 0.12807400 \\
\hline$c$ & 3.88362000 & -3.39731400 & -1.90815100 \\
\hline $\mathrm{C}$ & 4.46577500 & -4.74875100 & -1.46502800 \\
\hline $\mathrm{C}$ & 4.38675500 & -3.01510300 & -3.30803400 \\
\hline$\pi$ & 2.79256300 & -3.49680100 & -1.96426400 \\
\hline $\mathrm{H}$ & 4.09230800 & -5.04499000 & -0.47675200 \\
\hline $\mathrm{H}$ & 4.19932100 & -5.54082600 & -2.17437600 \\
\hline $\mathrm{H}$ & 5.56189700 & -4.70895000 & -1.40888800 \\
\hline $\mathrm{H}$ & 3.86338800 & -2.13369500 & -3.68923900 \\
\hline $\mathrm{H}$ & 5.46566900 & -2.80778100 & -3.29974500 \\
\hline $\mathrm{H}$ & 4.21240500 & -3.83396400 & -4.01605500 \\
\hline $\mathrm{O}$ & 1.48604200 & -0.62404100 & -0.13330600 \\
\hline $\mathrm{O}$ & 1.60111800 & -1.20332100 & -2.31402700 \\
\hline $\mathrm{S}$ & -3.31130700 & 0.11130800 & 0.92460800 \\
\hline $\mathrm{F}$ & -5.28862500 & -1.62273600 & 0.60221500 \\
\hline $\mathrm{F}$ & -3.66984200 & -2.33199200 & 1.87740800 \\
\hline $\mathrm{F}$ & -5.07543000 & -0.84863500 & 2.62510100 \\
\hline $\mathrm{O}$ & -4.21401800 & 1.23693000 & 0.66722000 \\
\hline $\mathrm{O}$ & -2.31406100 & 0.26450700 & 2.01783800 \\
\hline $\mathrm{N}$ & -2.59940700 & -0.57405500 & -0.37024700 \\
\hline $\mathrm{C}$ & -2.93023700 & -0.08705800 & -1.75444900 \\
\hline C & -2.07781600 & 1.16888400 & -1.92856400 \\
\hline 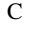 & -0.67354000 & 0.92369600 & -1.94872100 \\
\hline C & 0.26531400 & 1.97026500 & -1.80797200 \\
\hline$c$ & -0.20265500 & 3.27054300 & -1.67322400 \\
\hline$c$ & 1.57518700 & 3.53295200 & -1.71355600 \\
\hline $\mathrm{C}$ & -2.51992700 & 2.49988300 & -1.84640100 \\
\hline $\mathrm{C}$ & -4.41208600 & -1.26679000 & 1.54512600 \\
\hline $\mathrm{H}$ & -0.30083100 & -0.02045800 & -2.38227500 \\
\hline $\mathrm{H}$ & 1.32980700 & 1.76040900 & -1.83187400 \\
\hline $\mathrm{H}$ & 0.49551700 & 4.09480200 & -1.56267900 \\
\hline $\mathrm{H}$ & -1.91692800 & 4.55884400 & -1.63025100 \\
\hline 0 & & & \\
\hline
\end{tabular}




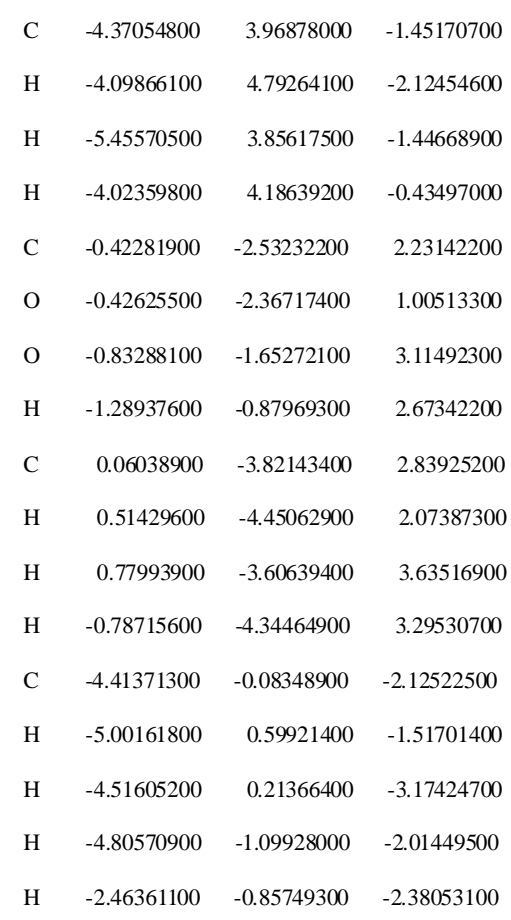

$\mathrm{TS}_{\mathrm{S} 2 \mathrm{~A}}$

$-2507.02296212$

\begin{tabular}{|c|c|c|c|}
\hline $\mathrm{Pd}$ & 0.49634300 & -0.28724100 & 0.44366200 \\
\hline $\mathrm{C}$ & -3.49985100 & 1.11060300 & 1.31120800 \\
\hline C & -1.99195500 & 0.98186700 & 1.03598500 \\
\hline $\mathrm{H}$ & -3.74763700 & 2.16399400 & 1.16860300 \\
\hline $\mathrm{N}$ & -4.28961000 & 0.33474600 & 0.36653900 \\
\hline $\mathrm{H}$ & -4.49942400 & -0.61896400 & 0.63063900 \\
\hline $\mathrm{C}$ & -4.19402200 & 0.60409300 & -0.97803800 \\
\hline $\mathrm{O}$ & -3.54113800 & 1.55886300 & -1.39722300 \\
\hline $\mathrm{C}$ & -4.94397200 & -0.31389500 & -1.89650200 \\
\hline $\mathrm{C}$ & -6.03399600 & -1.09102800 & -1.47800500 \\
\hline $\mathrm{C}$ & -4.53571500 & -0.36815200 & -3.23620300 \\
\hline $\mathrm{C}$ & -6.69355900 & -1.92225900 & -2.38367900 \\
\hline $\mathrm{H}$ & -6.39292800 & -1.02254800 & -0.45471600 \\
\hline $\mathrm{C}$ & -5.18986700 & -1.20476800 & -4.13757200 \\
\hline $\mathrm{H}$ & -3.70375000 & 0.25431900 & -3.54826500 \\
\hline $\mathrm{C}$ & -6.26868500 & -1.98522500 & -3.71252400 \\
\hline $\mathrm{H}$ & -7.54360800 & -2.51337500 & -2.05402600 \\
\hline $\mathrm{H}$ & -4.86081800 & -1.24794000 & -5.17216000 \\
\hline $\mathrm{H}$ & -6.78154900 & -2.63492600 & -4.41665700 \\
\hline $\mathrm{C}$ & -3.84598100 & 0.66207700 & 2.74585000 \\
\hline $\mathrm{H}$ & -4.93720800 & 0.73943900 & 2.85271100 \\
\hline $\mathrm{H}$ & -3.59089300 & -0.40361100 & 2.84089100 \\
\hline $\mathrm{C}$ & -3.17101700 & 1.44083500 & 3.89280000 \\
\hline & -3.56579600 & 0.81217800 & 5.23830600 \\
\hline
\end{tabular}

\begin{tabular}{|c|c|c|c|}
\hline $\mathrm{C}$ & -3.51223000 & 2.93837800 & 3.86544700 \\
\hline $\mathrm{H}$ & -2.08305900 & 1.34296000 & 3.78186100 \\
\hline $\mathrm{H}$ & 3.29556600 & -0.24997400 & 5.28141100 \\
\hline $\mathrm{H}$ & 3.06264500 & 1.31987800 & 6.06908100 \\
\hline $\mathrm{H}$ & -4.64786700 & 0.88969600 & 5.40831800 \\
\hline $\mathrm{H}$ & -3.12253400 & 3.42514300 & 2.96623100 \\
\hline $\mathrm{H}$ & -4.59845500 & 3.09687300 & 3.90326700 \\
\hline $\mathrm{H}$ & -3.07292000 & 3.44818800 & 4.73073900 \\
\hline $\mathrm{O}$ & -1.57423500 & -0.12756800 & 0.58159000 \\
\hline $\mathrm{O}$ & -1.26084700 & 1.98316000 & 1.30131500 \\
\hline $\mathrm{S}$ & 3.29708300 & -0.95117900 & -0.72355700 \\
\hline $\mathrm{F}$ & 5.39589100 & -1.38175100 & 0.85693800 \\
\hline $\mathrm{F}$ & 3.86678100 & -2.93092500 & 0.93495400 \\
\hline $\mathrm{F}$ & 5.18524700 & -2.78551200 & -0.79057600 \\
\hline $\mathrm{O}$ & 4.10428000 & -0.09616800 & -1.59897000 \\
\hline $\mathrm{O}$ & 2.36412500 & -1.92280300 & -1.35470900 \\
\hline N & 2.55051200 & -0.21633000 & 0.52249900 \\
\hline $\mathrm{C}$ & 2.86462800 & 1.23445800 & 0.80061000 \\
\hline $\mathrm{C}$ & 2.10593200 & 2.02010800 & -0.26428500 \\
\hline $\mathrm{C}$ & 0.74661500 & 1.61580500 & -0.40393500 \\
\hline $\mathrm{C}$ & -0.02004700 & 2.04436300 & -1.51213100 \\
\hline $\mathrm{C}$ & 0.53439400 & 2.93788600 & -2.41898200 \\
\hline $\mathrm{C}$ & 1.84298200 & 3.39516400 & -2.24702200 \\
\hline $\mathrm{C}$ & 2.63589800 & 2.94767300 & -1.17757000 \\
\hline $\mathrm{C}$ & 4.51994700 & -2.08232900 & 0.12913500 \\
\hline $\mathrm{H}$ & 0.08567400 & 1.76818800 & 0.61732900 \\
\hline $\mathrm{H}$ & -1.05765500 & 1.73821500 & -1.60675500 \\
\hline $\mathrm{H}$ & -0.05100400 & 3.30097400 & -3.25902400 \\
\hline $\mathrm{H}$ & 2.25114000 & 4.09966900 & -2.96278400 \\
\hline $\mathrm{O}$ & 3.92004800 & 3.36197600 & -0.98526800 \\
\hline $\mathrm{C}$ & 4.54438100 & 4.15310900 & -1.98427000 \\
\hline $\mathrm{H}$ & 4.06408200 & 5.13576500 & -2.08278900 \\
\hline $\mathrm{H}$ & 5.57443000 & 4.29110000 & -1.65083500 \\
\hline 11 & 4.54226000 & 3.64570200 & -2.95715600 \\
\hline $\mathrm{C}$ & 0.40756900 & -3.40499800 & 1.05720000 \\
\hline $\mathrm{O}$ & 0.25190200 & -2.23081900 & 1.40985500 \\
\hline $\mathrm{O}$ & 1.01114800 & -3.79555100 & -0.04142800 \\
\hline $\mathrm{H}$ & 1.43783700 & -3.03277800 & -0.53195000 \\
\hline$C$ & -0.09839700 & -4.54756800 & 1.89836900 \\
\hline $\mathrm{H}$ & -0.67938400 & -4.16700200 & 2.73838000 \\
\hline$\Pi$ & -0.70912800 & -5.21649900 & 1.28415000 \\
\hline $\mathrm{H}$ & 0.75297400 & -5.12912100 & 2.26823600 \\
\hline $\mathrm{C}$ & 4.33672900 & 1.52412300 & 1.09843100 \\
\hline & & & \\
\hline
\end{tabular}




\begin{tabular}{|c|c|c|c|c|c|c|c|}
\hline $\mathrm{H}$ & 4.44490900 & 2.56498700 & 1.41443600 & $\mathrm{H}$ & -3.50726600 & 5.13882200 & 2.20104000 \\
\hline $\mathrm{H}$ & 4.65926600 & 0.87478800 & 1.91778100 & $\mathrm{O}$ & -1.23971400 & 0.14998300 & 0.17579000 \\
\hline \multirow[t]{2}{*}{$\mathrm{H}$} & 2.32659300 & 1.39870600 & 1.74323600 & $\mathrm{O}$ & -1.10124300 & 2.39752700 & 0.21727300 \\
\hline & & & & $\mathrm{S}$ & 3.51095400 & -1.71364900 & 0.69535100 \\
\hline \multicolumn{4}{|c|}{$\mathrm{S}_{4 \mathrm{~A}}$} & $\mathrm{~N}$ & 2.86939400 & -0.24358000 & 0.89691000 \\
\hline \multicolumn{4}{|c|}{-2507.04962476} & $\mathrm{C}$ & 3.53371600 & 1.02513300 & 0.41490200 \\
\hline $\mathrm{Pd}$ & 0.86576700 & -0.12875500 & 0.55658400 & $\mathrm{C}$ & 2.82556800 & 1.38923800 & -0.89086600 \\
\hline $\mathrm{O}$ & 1.35150000 & -2.37761800 & 3.56973700 & $\mathrm{C}$ & 1.52442300 & 0.86529300 & -1.02348600 \\
\hline $\mathrm{C}$ & -0.41815300 & -1.45154800 & 4.81877100 & $\mathrm{C}$ & 0.76961000 & 1.05326300 & -2.18659700 \\
\hline $\mathrm{C}$ & 0.34331100 & -1.53815000 & 3.51922800 & $\mathrm{C}$ & 1.30647800 & 1.81828500 & -3.22250500 \\
\hline $\mathrm{O}$ & 0.01760700 & -0.87671700 & 2.52903700 & $\mathrm{C}$ & 2.56832000 & 2.39710700 & -3.09653200 \\
\hline $\mathrm{C}$ & -3.27973600 & 1.42030000 & 0.27742000 & $\mathrm{C}$ & 3.32771900 & 2.19756100 & -1.93331100 \\
\hline $\mathrm{C}$ & -1.77704500 & 1.26329700 & 0.20829100 & $\mathrm{H}$ & -0.13886000 & 2.18335600 & 0.13965500 \\
\hline $\mathrm{H}$ & -3.58150400 & 2.26688100 & -0.34485800 & $\mathrm{H}$ & -0.21450600 & 0.60683000 & -2.29060400 \\
\hline $\mathrm{H}$ & 0.26304600 & -1.15730400 & 5.62387600 & $\mathrm{H}$ & 0.73677200 & 1.97471400 & -4.13509100 \\
\hline $\mathrm{H}$ & -1.23012600 & -0.72903200 & 4.73352900 & $\mathrm{H}$ & 2.95812600 & 3.00092400 & -3.90719600 \\
\hline $\mathrm{H}$ & -0.81884500 & -2.43733200 & 5.07692900 & $\mathrm{H}$ & 1.82133600 & -2.44294700 & 2.68233000 \\
\hline $\mathrm{N}$ & -3.88745600 & 0.22045000 & -0.26993900 & $\mathrm{O}$ & 4.55703600 & 2.76640200 & -1.74918200 \\
\hline $\mathrm{H}$ & -3.32809500 & -0.62059200 & -0.27637500 & $\mathrm{C}$ & 5.13359300 & 3.51486200 & -2.80715700 \\
\hline $\mathrm{C}$ & -5.09150900 & 0.28326500 & -0.92165500 & $\mathrm{H}$ & 4.53405300 & 4.40215400 & -3.05001300 \\
\hline $\mathrm{O}$ & -5.69551200 & 1.34730400 & -1.04668100 & $\mathrm{H}$ & 6.11385700 & 3.83166200 & -2.44615400 \\
\hline $\mathrm{C}$ & -5.62347000 & -1.00669300 & -1.47615700 & $\mathrm{H}$ & 5.25962300 & 2.90520800 & -3.71121200 \\
\hline $\mathrm{C}$ & -5.18135800 & -2.27245100 & -1.06396700 & $\mathrm{C}$ & 3.56023800 & -2.20937000 & -1.11958900 \\
\hline $\mathrm{C}$ & -6.63139700 & -0.91598100 & -2.44615900 & $\mathrm{~F}$ & 2.32663800 & -2.28231800 & -1.62956600 \\
\hline $\mathrm{C}$ & -5.72700000 & -3.42639500 & -1.62662000 & $\mathrm{~F}$ & 4.14697600 & -3.40242400 & -1.23429400 \\
\hline $\mathrm{H}$ & -4.43496900 & -2.37376900 & -0.28038400 & $\mathrm{~F}$ & 4.26611600 & -1.30852000 & -1.81994200 \\
\hline $\mathrm{C}$ & -7.17094000 & -2.06868300 & -3.01206400 & $\mathrm{O}$ & 2.52601900 & -2.69143800 & 1.23316500 \\
\hline $\mathrm{H}$ & -6.97536100 & 0.07045800 & -2.73872000 & $\mathrm{O}$ & 4.90598200 & -1.81518000 & 1.12446300 \\
\hline $\mathrm{C}$ & -6.71799600 & -3.32662400 & -2.60536400 & $\mathrm{C}$ & 5.06579800 & 1.01109500 & 0.43767900 \\
\hline $\mathrm{H}$ & -5.38216700 & -4.40204100 & -1.29580700 & $\mathrm{H}$ & 5.41393800 & 0.62424900 & 1.39720700 \\
\hline $\mathrm{H}$ & -7.94656600 & -1.98753100 & -3.76861500 & $\mathrm{H}$ & 5.48996500 & 0.39297300 & -0.35574800 \\
\hline $\mathrm{H}$ & -7.14030300 & -4.22643500 & -3.04442200 & $\mathrm{H}$ & 5.42850700 & 2.03085200 & 0.30664800 \\
\hline $\mathrm{C}$ & -3.75099500 & 1.67043600 & 1.74108200 & $\mathrm{H}$ & 3.22050200 & 1.75425000 & 1.17455100 \\
\hline $\mathrm{H}$ & -4.84619200 & 1.64252500 & 1.69025000 & & & & \\
\hline $\mathrm{H}$ & -3.43462400 & 0.80965100 & 2.34480500 & $\mathrm{~S}_{3 \mathrm{~A}}$ & & & \\
\hline $\mathrm{C}$ & -3.31029900 & 2.98090600 & 2.42217600 & -22 & 7.92667460 & & \\
\hline $\mathrm{C}$ & -3.84618200 & 3.00255200 & 3.86312400 & $\mathrm{Pd}$ & 1.28388100 & 0.00257000 & -0.66399600 \\
\hline $\mathrm{C}$ & -3.77119200 & 4.22795000 & 1.65144400 & $\mathrm{C}$ & -2.85503900 & 1.02687100 & -0.71792200 \\
\hline $\mathrm{H}$ & -2.21329000 & 2.99975800 & 2.47556600 & $\mathrm{C}$ & -1.47755700 & 0.49666300 & -0.30198400 \\
\hline $\mathrm{H}$ & -3.50009400 & 2.13258300 & 4.43436500 & $\mathrm{H}$ & -2.98921300 & 0.89370600 & -1.79380300 \\
\hline $\mathrm{H}$ & -3.51324800 & 3.90385900 & 4.39029100 & $\mathrm{~N}$ & -3.84530200 & 0.21641100 & -0.02663400 \\
\hline $\mathrm{H}$ & -4.94365200 & 2.99563400 & 3.87484800 & $\mathrm{H}$ & -3.51660700 & -0.23415400 & 0.81727100 \\
\hline $\mathrm{H}$ & -3.30453400 & 4.29784400 & 0.66332000 & $\mathrm{C}$ & -5.13864200 & 0.12358100 & -0.44290700 \\
\hline $\mathrm{H}$ & -4.85959900 & 4.22418300 & 1.51155300 & $\mathrm{O}$ & -5.56554200 & 0.76219200 & -1.40629200 \\
\hline
\end{tabular}




\begin{tabular}{|c|c|c|c|c|c|c|c|}
\hline $\mathrm{C}$ & -6.03048900 & -0.79719500 & 0.34782600 & $\mathrm{H}$ & 3.52517500 & 4.21239900 & 1.54906000 \\
\hline $\mathrm{C}$ & -7.41367500 & -0.61326300 & 0.21894700 & $\mathrm{O}$ & 5.13336400 & 2.11395500 & 1.06924600 \\
\hline $\mathrm{C}$ & -5.55188400 & -1.82614200 & 1.17160000 & $\mathrm{C}$ & 5.81031000 & 3.36489200 & 1.05220700 \\
\hline $\mathrm{C}$ & -8.30316500 & -1.42608200 & 0.91824800 & $\mathrm{H}$ & 5.74047400 & 3.87311900 & 2.02219200 \\
\hline $\mathrm{H}$ & -7.76521900 & 0.17274500 & -0.44120300 & $\mathrm{H}$ & 6.85453200 & 3.12918600 & 0.84279400 \\
\hline $\mathrm{C}$ & -6.44320100 & -2.64481900 & 1.86633600 & $\mathrm{H}$ & 5.42110200 & 4.02122800 & 0.26401500 \\
\hline $\mathrm{H}$ & -4.48545900 & -2.01957500 & 1.25164900 & $\mathrm{C}$ & 5.29796300 & -0.67583000 & 0.84092300 \\
\hline $\mathrm{C}$ & -7.81964500 & -2.44280400 & 1.74625900 & $\mathrm{H}$ & 5.74135600 & -0.05986900 & 0.05896800 \\
\hline $\mathrm{H}$ & -9.37384700 & -1.27010700 & 0.81597900 & $\mathrm{H}$ & 5.73717200 & -0.39637800 & 1.80324400 \\
\hline $\mathrm{H}$ & -6.06197100 & -3.44507200 & 2.49504100 & $\mathrm{H}$ & 5.53818300 & -1.72478100 & 0.64248900 \\
\hline $\mathrm{H}$ & -8.51249100 & -3.08014400 & 2.28948100 & $\mathrm{H}$ & 3.38489000 & -1.24920400 & 1.60314400 \\
\hline $\mathrm{C}$ & -3.01853700 & 2.52958800 & -0.36480000 & & & & \\
\hline $\mathrm{H}$ & -4.06016500 & 2.77986600 & -0.59942300 & \multicolumn{4}{|c|}{$\mathrm{TS}_{\mathrm{S}_{2 \mathrm{~A}^{\prime}}}$} \\
\hline $\mathrm{H}$ & -2.90231000 & 2.63029800 & 0.72366900 & \multicolumn{4}{|c|}{-2277.90652150} \\
\hline $\mathrm{C}$ & -2.08497500 & 3.52644400 & -1.07914300 & $\mathrm{Pd}$ & 1.37720200 & 0.28705900 & -1.11210300 \\
\hline $\mathrm{C}$ & -2.36400600 & 4.94735100 & -0.56283100 & $\mathrm{C}$ & -2.87442700 & 0.79820800 & -0.91819500 \\
\hline $\mathrm{C}$ & -2.22221600 & 3.46802400 & -2.60837900 & $\mathrm{C}$ & -1.38225000 & 0.64541900 & -0.63628700 \\
\hline $\mathrm{H}$ & -1.04607000 & 3.27301500 & -0.82718800 & $\mathrm{H}$ & -3.08930400 & 0.39340400 & -1.90853000 \\
\hline $\mathrm{H}$ & -2.24748400 & 5.01193800 & 0.52658100 & $\mathrm{~N}$ & -3.61029400 & 0.01031000 & 0.05880000 \\
\hline $\mathrm{H}$ & -1.68042300 & 5.67389100 & -1.01866700 & $\mathrm{H}$ & -3.18675200 & -0.08128900 & 0.97132600 \\
\hline $\mathrm{H}$ & -3.38843900 & 5.25891600 & -0.80458400 & $\mathrm{C}$ & -4.88905900 & -0.40975100 & -0.17630900 \\
\hline $\mathrm{H}$ & -1.89615600 & 2.50190400 & -3.00575800 & $\mathrm{O}$ & -5.47977000 & -0.14117300 & -1.22205300 \\
\hline $\mathrm{H}$ & -3.26289100 & 3.63123000 & -2.91716200 & $\mathrm{C}$ & -5.53800900 & -1.21482500 & 0.91606200 \\
\hline $\mathrm{H}$ & -1.60694500 & 4.24285600 & -3.08186800 & $\mathrm{C}$ & -6.93586800 & -1.31232800 & 0.89504200 \\
\hline $\mathrm{O}$ & -0.52621000 & 0.73959000 & -1.15248300 & $\mathrm{C}$ & -4.82088300 & -1.87766100 & 1.92258100 \\
\hline $\mathrm{O}$ & -1.32091600 & -0.07894400 & 0.78339500 & $\mathrm{C}$ & -7.60753100 & -2.03857600 & 1.87599600 \\
\hline $\mathrm{S}$ & 3.00702900 & -2.14488800 & -1.21943800 & $\mathrm{H}$ & -7.47260000 & -0.81184300 & 0.09589300 \\
\hline $\mathrm{F}$ & 3.44144000 & -3.69785800 & 0.87177200 & $\mathrm{C}$ & -5.49375000 & -2.61025900 & 2.90103700 \\
\hline $\mathrm{F}$ & 1.39735700 & -2.96277200 & 0.72464600 & $\mathrm{H}$ & -3.73425100 & -1.85781800 & 1.93172500 \\
\hline $\mathrm{F}$ & 2.08014900 & -4.55006000 & -0.60334400 & $\mathrm{C}$ & -6.88793800 & -2.68696200 & 2.88339700 \\
\hline $\mathrm{O}$ & 4.22466500 & -2.69143800 & -1.80278500 & $\mathrm{H}$ & -8.69213200 & -2.10222600 & 1.85446000 \\
\hline $\mathrm{O}$ & 1.80190500 & -1.88610200 & -2.07069600 & $\mathrm{H}$ & -4.92835400 & -3.12736500 & 3.67156600 \\
\hline $\mathrm{N}$ & 3.16000200 & -0.71912700 & -0.45032600 & $\mathrm{H}$ & -7.41094200 & -3.25648200 & 3.64708000 \\
\hline $\mathrm{C}$ & 3.77823500 & -0.51340900 & 0.88588300 & $\mathrm{C}$ & -3.29238400 & 2.29405700 & -0.87337000 \\
\hline $\mathrm{C}$ & 3.15715900 & 0.83021400 & 1.28582200 & $\mathrm{H}$ & -4.38245500 & 2.29722100 & -0.99167800 \\
\hline $\mathrm{C}$ & 1.75036100 & 0.79172800 & 1.50573800 & $\mathrm{H}$ & -3.07606200 & 2.67203400 & 0.13558100 \\
\hline $\mathrm{C}$ & 0.99779400 & 1.96490100 & 1.71739700 & $\mathrm{C}$ & -2.66635000 & 3.22979400 & -1.92640700 \\
\hline $\mathrm{C}$ & 1.65300000 & 3.18948200 & 1.72705600 & $\mathrm{C}$ & -3.15185600 & 4.66790400 & -1.68248800 \\
\hline $\mathrm{C}$ & 3.03526900 & 3.24588500 & 1.53598900 & $\mathrm{C}$ & -2.97532200 & 2.78580300 & -3.36460600 \\
\hline $\mathrm{C}$ & 3.79390200 & 2.08530200 & 1.29687800 & $\mathrm{H}$ & -1.57416000 & 3.21821100 & -1.80256700 \\
\hline $\mathrm{C}$ & 2.43639500 & -3.43600500 & 0.02312200 & $\mathrm{H}$ & -2.91269400 & 5.00849300 & -0.66724600 \\
\hline $\mathrm{H}$ & 1.27496200 & -0.16622400 & 1.73017200 & $\mathrm{H}$ & -2.68580200 & 5.36451000 & -2.38912800 \\
\hline $\mathrm{H}$ & -0.06940100 & 1.87838300 & 1.88263400 & $\mathrm{H}$ & -4.23947400 & 4.74235800 & -1.81018500 \\
\hline $\mathrm{H}$ & 1.10058300 & 4.10973100 & 1.89051100 & $\mathrm{H}$ & -2.51835200 & 1.81899900 & -3.59749900 \\
\hline
\end{tabular}




\begin{tabular}{|c|c|c|c|}
\hline $\mathrm{H}$ & -4.05750100 & 2.70011400 & -3.52630600 \\
\hline $\mathrm{H}$ & -2.58494700 & 3.51452800 & -4.08476100 \\
\hline 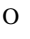 & 0.58468300 & 0.78087300 & -1.61754000 \\
\hline $\mathrm{O}$ & -1.02083600 & 0.44582300 & 0.56645400 \\
\hline $\mathrm{S}$ & 3.29528200 & -1.68690000 & -1.48209200 \\
\hline $\mathrm{F}$ & 3.30407500 & -3.53740400 & 0.41059700 \\
\hline F & 1.31195900 & -2.87160600 & -0.16251200 \\
\hline $\mathrm{F}$ & 2.43834400 & -4.18991400 & -1.48061800 \\
\hline $\mathrm{O}$ & 4.64913000 & -2.10872600 & -1.81681900 \\
\hline $\mathrm{O}$ & 2.31666300 & -1.35218600 & -2.56831500 \\
\hline$N$ & 3.16257200 & -0.37824300 & -0.52220400 \\
\hline C & 3.32887800 & -0.37776300 & 0.96203300 \\
\hline C & 2.69597600 & 0.95534500 & 1.37936600 \\
\hline C & 1.52433300 & 1.36854900 & 0.67188200 \\
\hline C & 1.02840000 & 2.68154800 & 0.81472200 \\
\hline C & 1.61118100 & 3.55123400 & 1.72575300 \\
\hline C & 2.68514700 & 3.12167400 & 2.50293300 \\
\hline C & 3.22436100 & 1.83323600 & 2.34819400 \\
\hline C & 2.53134000 & -3.17830400 & -0.62474000 \\
\hline $\mathrm{H}$ & 0.39095400 & 0.66223000 & 0.62577900 \\
\hline $\mathrm{H}$ & 0.15180300 & 2.98413100 & 0.25234100 \\
\hline $\mathrm{H}$ & 1.22442200 & 4.55807800 & 1.85389500 \\
\hline $\mathrm{H}$ & 3.11398800 & 3.80099200 & 3.22986800 \\
\hline $\mathrm{O}$ & 4.26600300 & 1.38804100 & 3.10555200 \\
\hline C & 4.87466300 & 2.26784600 & 4.04050500 \\
\hline $\mathrm{H}$ & 4.16869200 & 2.57657500 & 4.82195900 \\
\hline $\mathrm{H}$ & 5.68635700 & 1.69738600 & 4.49472200 \\
\hline $\mathrm{H}$ & 5.28768000 & 3.15750200 & 3.54836600 \\
\hline C & 4.79817900 & -0.64870300 & 1.31191100 \\
\hline$\Pi$ & 5.44117000 & 0.15591400 & 0.94727700 \\
\hline $\mathrm{H}$ & 4.93066800 & -0.75116800 & 2.38887400 \\
\hline $\mathrm{H}$ & 5.11004300 & -1.58029300 & 0.83241800 \\
\hline & 2.71582500 & -1.17676600 & 1.40932100 \\
\hline
\end{tabular}

$\mathrm{S}_{4 \mathrm{~A}^{\prime}}$

$-2277.94577511$

$\begin{array}{lrrr}\mathrm{Pd} & 1.26651000 & 0.09155400 & -1.02232300 \\ \mathrm{C} & -3.02895300 & 1.09999400 & -0.61099200 \\ \mathrm{C} & -1.57873200 & 0.71849600 & -0.40632400 \\ \mathrm{H} & -3.26272400 & 0.96244100 & -1.66733200 \\ \mathrm{~N} & -3.88710600 & 0.19235700 & 0.13308400 \\ \mathrm{H} & -3.65960300 & 0.01543200 & 1.10112400 \\ \mathrm{C} & -5.14905900 & -0.10417600 & -0.33248300 \\ \mathrm{O} & -5.56181100 & 0.34656200 & -1.39723600\end{array}$

$\begin{array}{llll}\text { C } & -5.97833900 & -1.00432000 & 0.53457800\end{array}$

$\begin{array}{llll}\text { C } & -7.36463200 & -0.99626700 & 0.32743200\end{array}$

$\begin{array}{llll}\text { C } & -5.43375300 & -1.85363400 & 1.50915800\end{array}$

$\begin{array}{llll}\text { C } & -8.19570300 & -1.80482100 & 1.09929200\end{array}$

$\begin{array}{llll}\mathrm{H} & -7.76668200 & -0.35015900 & -0.44586700\end{array}$

$\begin{array}{llll}\text { C } & -6.26683100 & -2.66759100 & 2.27683100\end{array}$

$\begin{array}{llll}\mathrm{H} & -4.35777800 & -1.91614900 & 1.64906400\end{array}$

$\begin{array}{llll}\text { C } & -7.64880700 & -2.63950900 & 2.07780900\end{array}$

$\begin{array}{llll}\mathrm{H} & -9.26978900 & -1.78751600 & 0.93671900\end{array}$

$\begin{array}{llll}\mathrm{H} & -5.83577000 & -3.32999400 & 3.02223900\end{array}$

$\begin{array}{llll}\mathrm{H} & -8.29672900 & -3.27310200 & 2.67737800\end{array}$

$\begin{array}{llll}\text { C } & -3.26945100 & 2.58786400 & -0.22687000\end{array}$

$\begin{array}{llll}\mathrm{H} & -4.34834200 & 2.74362900 & -0.34720100\end{array}$

$\begin{array}{llll}\mathrm{H} & -3.04350300 & 2.70766200 & 0.84159100\end{array}$

$\begin{array}{llll}\text { C } & -2.50938800 & 3.64961800 & -1.04733500\end{array}$

$\begin{array}{llll}\text { C } & -2.82024900 & 5.04502600 & -0.48228700\end{array}$

$\begin{array}{llll}\text { C } & -2.84183700 & 3.58018000 & -2.54616000\end{array}$

$\begin{array}{llll}\text { H } & -1.42901900 & 3.47827600 & -0.93510900\end{array}$

$\begin{array}{llll}\mathrm{H} & -2.56140400 & 5.11746800 & 0.58108400\end{array}$

$\begin{array}{llll}\mathrm{H} & -2.25722700 & 5.81697800 & -1.01870200\end{array}$

$\begin{array}{llll}\mathrm{H} & -3.88741900 & 5.28025400 & -0.58350300\end{array}$

$\begin{array}{llll}\mathrm{H} & -2.50876200 & 2.64073200 & -2.99934100\end{array}$

$\begin{array}{llll}\mathrm{H} & -3.92226800 & 3.67358900 & -2.71356100\end{array}$

$\begin{array}{llll}\mathrm{H} & -2.34665600 & 4.39484200 & -3.08641500\end{array}$

$\begin{array}{llll}\text { O } & -0.72485000 & 0.86280400 & -1.28790300\end{array}$

$\begin{array}{llll}\text { O } & -1.29104200 & 0.27265800 & 0.80856200\end{array}$

$\begin{array}{llll}\text { S } & 2.94877700 & -2.29685000 & -1.25556400\end{array}$

F $\quad 3.46568600 \quad-3.15825800 \quad 1.19601000$

F $\quad 1.35165800 \quad-2.80841100 \quad 0.80975600$

F $\quad 2.32971300 \quad-4.56540500 \quad-0.02068000$

$\begin{array}{llll}\text { O } & 4.21410000 & -2.89133700 & -1.68407100\end{array}$

$\begin{array}{llll}\text { O } & 1.72754300 & -2.36595800 & -2.09251400\end{array}$

$\begin{array}{llll}\mathrm{N} & 3.04443700 & -0.76055300 & -0.82340800\end{array}$

$\begin{array}{llll}\text { C } & 4.08113500 & -0.13360100 & 0.03059200\end{array}$

$\begin{array}{llll}\text { C } & 3.37437300 & 1.04262700 & 0.67112600\end{array}$

$\begin{array}{llll}\text { C } & 2.06208100 & 1.35694000 & 0.27473600\end{array}$

$\begin{array}{llll}\text { C } & 1.39048800 & 2.47062900 & 0.78921200\end{array}$

$\begin{array}{llll}\text { C } & 2.03100500 & 3.26505100 & 1.74086000\end{array}$

$\begin{array}{llll}\text { C } & 3.31754200 & 2.95535200 & 2.18518200\end{array}$

$\begin{array}{llll}\text { C } & 3.98756600 & 1.84661900 & 1.65420800\end{array}$

$\begin{array}{llll}\text { C } & 2.49313200 & -3.27593000 & 0.27632000\end{array}$

$\begin{array}{llll}\mathrm{H} & -0.31871400 & 0.08688000 & 0.84948000\end{array}$

$\begin{array}{llll}\mathrm{H} & 0.39249400 & 2.72979800 & 0.45233300\end{array}$

$\begin{array}{llll}\mathrm{H} & 1.52315800 & 4.13629000 & 2.14677300\end{array}$ 


\begin{tabular}{|c|c|c|c|c|c|c|c|}
\hline $\mathrm{H}$ & 3.78774600 & 3.57740800 & 2.93764900 & $\mathrm{H}$ & 6.34007100 & -1.33270500 & -0.68088100 \\
\hline $\mathrm{O}$ & 5.23992200 & 1.45595900 & 2.03830000 & $\mathrm{H}$ & 6.37257900 & -2.94938200 & -1.40083700 \\
\hline $\mathrm{C}$ & 5.91479600 & 2.21149700 & 3.03273000 & $\mathrm{O}$ & 2.58038900 & -2.12528400 & -2.53294100 \\
\hline $\mathrm{H}$ & 5.36166700 & 2.21335600 & 3.98089500 & $\mathrm{O}$ & 0.83140900 & -1.40621100 & -1.35187500 \\
\hline $\mathrm{H}$ & 6.87799700 & 1.71928100 & 3.17754000 & $\mathrm{~S}$ & -1.89670700 & -0.15608000 & 1.77572700 \\
\hline $\mathrm{H}$ & 6.08109500 & 3.24684000 & 2.70816000 & $\mathrm{~F}$ & -2.45089400 & -2.25467500 & 3.29910100 \\
\hline $\mathrm{C}$ & 5.29627900 & 0.26924000 & -0.82250100 & $\mathrm{~F}$ & -0.32187200 & -1.89555700 & 3.02529800 \\
\hline $\mathrm{H}$ & 4.98498600 & 0.96882200 & -1.60523500 & $\mathrm{~F}$ & -1.47962400 & -0.61195900 & 4.34864600 \\
\hline $\mathrm{H}$ & 6.05968600 & 0.74304200 & -0.20021900 & $\mathrm{O}$ & -3.19926500 & 0.40020600 & 2.11590400 \\
\hline $\mathrm{H}$ & 5.72149000 & -0.61904500 & -1.29789700 & $\mathrm{O}$ & -0.67154000 & 0.76493700 & 1.80911200 \\
\hline $\mathrm{H}$ & 4.41046500 & -0.83409100 & 0.80892800 & $\mathrm{~N}$ & -1.62437100 & -0.91418100 & 0.42194000 \\
\hline & & & & $\mathrm{C}$ & -2.47904700 & -1.72889100 & -0.47896100 \\
\hline $\mathrm{S}_{3 \mathrm{~A}}$ & & & & $\mathrm{C}$ & -2.68881600 & -1.02389000 & -1.81261900 \\
\hline & 94522267 & & & $\mathrm{C}$ & -2.14865400 & -1.58142700 & -2.97249500 \\
\hline $\mathrm{Pd}$ & 0.27916900 & -0.22745800 & 0.13434100 & $\mathrm{C}$ & -2.32577900 & -0.98717400 & -4.22507900 \\
\hline $\mathrm{C}$ & 3.04221200 & -0.57859600 & -0.76203100 & $\mathrm{C}$ & -3.05131000 & 0.19630900 & -4.31910300 \\
\hline $\mathrm{C}$ & 2.11567000 & -1.43167700 & -1.64657200 & $\mathrm{C}$ & -3.59403900 & 0.78766100 & -3.17424400 \\
\hline $\mathrm{H}$ & 3.78142100 & -0.11594900 & -1.41753100 & $\mathrm{C}$ & -3.41370600 & 0.18354100 & -1.92600200 \\
\hline $\mathrm{N}$ & 2.26385800 & 0.52519100 & -0.09171200 & $\mathrm{C}$ & -1.50965900 & -1.30761400 & 3.21072400 \\
\hline $\mathrm{H}$ & 2.61947400 & 0.69530700 & 0.84905300 & $\mathrm{H}$ & -1.55682100 & -2.48864100 & -2.88813200 \\
\hline $\mathrm{C}$ & 2.19998100 & 1.76724600 & -0.85161200 & $\mathrm{H}$ & -1.88919400 & -1.44125000 & -5.10929700 \\
\hline $\mathrm{O}$ & 2.37685800 & 1.73430400 & -2.05033100 & $\mathrm{H}$ & -3.19753700 & 0.67567500 & -5.28353900 \\
\hline $\mathrm{C}$ & 1.95594600 & 3.02215900 & -0.08592000 & $\mathrm{H}$ & -4.15391200 & 1.71184600 & -3.26212500 \\
\hline $\mathrm{C}$ & 1.58427000 & 3.06269800 & 1.26817500 & $\mathrm{O}$ & -3.90030400 & 0.69481600 & -0.75843700 \\
\hline $\mathrm{C}$ & 2.12973600 & 4.22550100 & -0.79085600 & $\mathrm{C}$ & -4.56636400 & 1.94610500 & -0.76997100 \\
\hline $\mathrm{C}$ & 1.39903700 & 4.28996800 & 1.90367000 & $\mathrm{H}$ & -5.49490000 & 1.90336100 & -1.35493200 \\
\hline $\mathrm{H}$ & 1.38739400 & 2.15498100 & 1.82856700 & $\mathrm{H}$ & -4.79909400 & 2.16092300 & 0.27382600 \\
\hline $\mathrm{C}$ & 1.94903400 & 5.44684000 & -0.15017200 & $\mathrm{H}$ & -3.92274000 & 2.73923400 & -1.17233000 \\
\hline $\mathrm{H}$ & 2.40951100 & 4.17678500 & -1.83765600 & $\mathrm{C}$ & -3.77882100 & -2.20695900 & 0.18817600 \\
\hline $\mathrm{C}$ & 1.58532200 & 5.48105400 & 1.19941100 & $\mathrm{H}$ & -4.43953200 & -1.37228900 & 0.42480500 \\
\hline $\mathrm{H}$ & 1.09823200 & 4.31221100 & 2.94677300 & $\mathrm{H}$ & -4.29657300 & -2.87893800 & -0.50364200 \\
\hline $\mathrm{H}$ & 2.08983400 & 6.37217900 & -0.70122200 & $\mathrm{H}$ & -3.56016000 & -2.76364400 & 1.10470100 \\
\hline $\mathrm{H}$ & 1.44106200 & 6.43481600 & 1.69947100 & $\mathrm{H}$ & -1.87121200 & -2.61487600 & -0.68964700 \\
\hline $\mathrm{C}$ & 3.73515900 & -1.41820700 & 0.33020500 & & & & \\
\hline $\mathrm{H}$ & 4.36689400 & -0.73845800 & 0.92532900 & \multicolumn{4}{|c|}{$\mathrm{TS}_{\mathrm{S} 2 \mathrm{~A}^{\prime \prime}}$} \\
\hline $\mathrm{H}$ & 2.96840700 & -1.81669500 & 1.00970200 & \multicolumn{4}{|c|}{-2277.87060096} \\
\hline $\mathrm{C}$ & 4.61549700 & -2.58250200 & -0.17162300 & $\mathrm{Pd}$ & -0.07940800 & 0.07412000 & 0.31508500 \\
\hline $\mathrm{C}$ & 5.21272900 & -3.32169600 & 1.03559500 & $\mathrm{C}$ & 2.71372400 & -0.30665700 & -1.26637700 \\
\hline $\mathrm{C}$ & 5.71489500 & -2.11351800 & -1.13597900 & $\mathrm{C}$ & 1.68404100 & -0.65739700 & -2.37738300 \\
\hline $\mathrm{H}$ & 3.96974400 & -3.27949800 & -0.71803600 & $\mathrm{H}$ & 3.41995700 & 0.40560000 & -1.69282500 \\
\hline $\mathrm{H}$ & 4.43133500 & -3.67789300 & 1.71808000 & $\mathrm{~N}$ & 2.09114200 & 0.37796400 & -0.06218000 \\
\hline $\mathrm{H}$ & 5.79346300 & -4.19153000 & 0.70875900 & $\mathrm{H}$ & 2.43379500 & -0.07493500 & 0.78562500 \\
\hline $\mathrm{H}$ & 5.88609900 & -2.66951500 & 1.60798800 & $\mathrm{C}$ & 2.29566700 & 1.80050700 & 0.02526200 \\
\hline $\mathrm{H}$ & 5.28663500 & -1.72677000 & -2.06530500 & $\mathrm{O}$ & 2.44766400 & 2.45726000 & -0.98704700 \\
\hline
\end{tabular}




\begin{tabular}{|c|c|c|c|c|c|c|c|}
\hline $\mathrm{C}$ & 2.30149900 & 2.41002300 & 1.39253600 & $\mathrm{H}$ & -3.47734200 & 3.29072600 & -3.11916100 \\
\hline $\mathrm{C}$ & 2.01896400 & 1.70870600 & 2.57761500 & $\mathrm{O}$ & -4.69823500 & 1.38126400 & -1.69462100 \\
\hline $\mathrm{C}$ & 2.61874400 & 3.77648400 & 1.46473000 & $\mathrm{C}$ & -5.58819100 & 2.31822600 & -2.28386400 \\
\hline $\mathrm{C}$ & 2.05559600 & 2.36630200 & 3.80695900 & $\mathrm{H}$ & -5.51227000 & 2.31450200 & -3.37873300 \\
\hline $\mathrm{H}$ & 1.73792000 & 0.65962800 & 2.57028900 & $\mathrm{H}$ & -6.58899900 & 1.99584400 & -1.99198900 \\
\hline $\mathrm{C}$ & 2.66049500 & 4.42705100 & 2.69411000 & $\mathrm{H}$ & -5.41121000 & 3.33441800 & -1.90877800 \\
\hline $\mathrm{H}$ & 2.83137600 & 4.30606300 & 0.54246800 & $\mathrm{C}$ & -4.35044700 & -0.53403500 & 0.26641200 \\
\hline $\mathrm{C}$ & 2.37873100 & 3.72305300 & 3.86837600 & $\mathrm{H}$ & -4.61269100 & 0.39427200 & 0.77789600 \\
\hline $\mathrm{H}$ & 1.82477000 & 1.81598800 & 4.71414800 & $\mathrm{H}$ & -5.05291100 & -0.69847600 & -0.55165100 \\
\hline $\mathrm{H}$ & 2.91097100 & 5.48312300 & 2.73789300 & $\mathrm{H}$ & -4.44396000 & -1.36045100 & 0.97618900 \\
\hline $\mathrm{H}$ & 2.40796300 & 4.23108100 & 4.82833000 & $\mathrm{H}$ & -2.70754900 & -1.43993300 & -0.77917000 \\
\hline $\mathrm{C}$ & 3.42906700 & -1.57431500 & -0.76854500 & & & & \\
\hline $\mathrm{H}$ & 4.18820100 & -1.28435500 & -0.02338400 & $\mathrm{~S}_{4 \mathrm{~A}^{\prime \prime}}$ & & & \\
\hline $\mathrm{H}$ & 2.69068200 & -2.20797500 & -0.25858500 & -227 & 7.94046890 & & \\
\hline $\mathrm{C}$ & 4.11524900 & -2.40965700 & -1.87026300 & $\mathrm{Pd}$ & -0.14609500 & -0.26914000 & -0.33661900 \\
\hline $\mathrm{C}$ & 4.76186600 & -3.65410700 & -1.24367500 & $\mathrm{C}$ & 2.38977200 & 1.26351800 & 0.60286600 \\
\hline $\mathrm{C}$ & 5.13937400 & -1.59636400 & -2.67606400 & $\mathrm{C}$ & 1.47969800 & 2.37742200 & 0.09757500 \\
\hline $\mathrm{H}$ & 3.33361300 & -2.74649300 & -2.56370900 & $\mathrm{H}$ & 2.13423100 & 1.08446800 & 1.64489600 \\
\hline $\mathrm{H}$ & 4.02853600 & -4.25461700 & -0.69181900 & $\mathrm{~N}$ & 2.06621400 & 0.01010900 & -0.14782900 \\
\hline $\mathrm{H}$ & 5.20568400 & -4.29319700 & -2.01554900 & $\mathrm{H}$ & 2.25560700 & 0.15402000 & -1.14049500 \\
\hline $\mathrm{H}$ & 5.56131200 & -3.37476100 & -0.54420100 & $\mathrm{C}$ & 2.68060500 & -1.19853600 & 0.32847400 \\
\hline $\mathrm{H}$ & 4.65277400 & -0.80000500 & -3.24698200 & $\mathrm{O}$ & 3.05634700 & -1.27256600 & 1.48473200 \\
\hline $\mathrm{H}$ & 5.89670600 & -1.14811000 & -2.01790200 & $\mathrm{C}$ & 2.82026500 & -2.31713900 & -0.65015100 \\
\hline $\mathrm{H}$ & 5.66358900 & -2.24215000 & -3.39028400 & $\mathrm{C}$ & 2.26489800 & -2.31199100 & -1.94159200 \\
\hline $\mathrm{O}$ & 1.97181000 & -0.35152400 & -3.53286700 & $\mathrm{C}$ & 3.56893700 & -3.42768400 & -0.22813200 \\
\hline $\mathrm{O}$ & 0.62115400 & -1.27803900 & -1.96752500 & $\mathrm{C}$ & 2.46338900 & -3.39805800 & -2.79234400 \\
\hline $\mathrm{S}$ & -1.66208000 & -1.38981700 & 2.04983400 & $\mathrm{H}$ & 1.64655600 & -1.49042000 & -2.29273100 \\
\hline $\mathrm{F}$ & -2.78665700 & -3.47370900 & 0.88166600 & $\mathrm{C}$ & 3.76741300 & -4.50840600 & -1.08240400 \\
\hline $\mathrm{F}$ & -0.69665000 & -3.17964800 & 0.34413800 & $\mathrm{H}$ & 3.98591900 & -3.42053200 & 0.77307300 \\
\hline $\mathrm{F}$ & -1.21180200 & -3.98044600 & 2.30260400 & $\mathrm{C}$ & 3.21618100 & -4.49487700 & -2.36650200 \\
\hline $\mathrm{O}$ & -2.65823100 & -1.45864900 & 3.11042900 & $\mathrm{H}$ & 2.02094400 & -3.38869500 & -3.78385500 \\
\hline $\mathrm{O}$ & -0.23125200 & -1.06695100 & 2.38579100 & $\mathrm{H}$ & 4.34985600 & -5.36178000 & -0.74737500 \\
\hline$N$ & -1.95619300 & -0.31048300 & 0.86533600 & $\mathrm{H}$ & 3.36807200 & -5.33948500 & -3.03278400 \\
\hline $\mathrm{C}$ & -2.91374500 & -0.48802800 & -0.26505100 & $\mathrm{C}$ & 3.87977500 & 1.65334300 & 0.46779300 \\
\hline $\mathrm{C}$ & -2.49519800 & 0.62105800 & -1.23379400 & $\mathrm{H}$ & 4.47145800 & 0.81586800 & 0.85516300 \\
\hline $\mathrm{C}$ & -1.08846100 & 0.74036500 & -1.47212000 & $\mathrm{H}$ & 4.11659000 & 1.75627800 & -0.60065000 \\
\hline $\mathrm{C}$ & -0.56752500 & 1.81332800 & -2.22816900 & $\mathrm{C}$ & 4.29708500 & 2.94498300 & 1.20449600 \\
\hline $\mathrm{C}$ & -1.43672900 & 2.72347400 & -2.81337700 & $\mathrm{C}$ & 5.78950100 & 3.21157200 & 0.95435600 \\
\hline $\mathrm{C}$ & -2.81355100 & 2.57785200 & -2.64404900 & $\mathrm{C}$ & 3.99098500 & 2.88822900 & 2.70944400 \\
\hline $\mathrm{C}$ & -3.35419700 & 1.53655600 & -1.86705700 & $\mathrm{H}$ & 3.73026300 & 3.78409000 & 0.77808900 \\
\hline $\mathrm{C}$ & -1.57517300 & -3.13320700 & 1.34345500 & $\mathrm{H}$ & 6.01435700 & 3.27640400 & -0.11713600 \\
\hline $\mathrm{H}$ & -0.43263200 & -0.27927500 & -1.70949800 & $\mathrm{H}$ & 6.09912800 & 4.15405500 & 1.41969000 \\
\hline $\mathrm{H}$ & 0.50188500 & 1.88943900 & -2.39168400 & $\mathrm{H}$ & 6.40898400 & 2.41126700 & 1.37935300 \\
\hline $\mathrm{H}$ & -1.05486500 & 3.54126800 & -3.41725400 & $\mathrm{H}$ & 2.91430300 & 2.85191300 & 2.90305300 \\
\hline
\end{tabular}




\begin{tabular}{|c|c|c|c|c|c|c|c|}
\hline $\mathrm{H}$ & 4.45721500 & 2.01028400 & 3.17542200 & $\mathrm{O}$ & -1.13527200 & -0.29978500 & -2.27425500 \\
\hline $\mathrm{H}$ & 4.38260700 & 3.77979300 & 3.21222300 & $\mathrm{C}$ & -3.46328300 & -0.43759800 & -1.83647500 \\
\hline $\mathrm{O}$ & 0.99437100 & 3.23831400 & 0.79000200 & $\mathrm{C}$ & -4.40179900 & -1.25696900 & -1.19498900 \\
\hline $\mathrm{O}$ & 1.33046800 & 2.31325100 & -1.25068000 & $\mathrm{C}$ & -3.84502900 & 0.26203600 & -2.99151200 \\
\hline S & -2.32879100 & 0.34344400 & -2.20030100 & $\mathrm{C}$ & -5.70352000 & -1.36122200 & -1.68873400 \\
\hline $\mathrm{F}$ & -3.76235100 & 2.28867000 & -1.11210800 & $\mathrm{H}$ & -4.10921400 & -1.83552900 & -0.32537500 \\
\hline $\mathrm{F}$ & -1.64610700 & 2.76378000 & -1.30606300 & $\mathrm{C}$ & -5.14932800 & 0.17166500 & -3.47277900 \\
\hline $\mathrm{F}$ & -2.92922700 & 2.78638000 & -3.06327000 & $\mathrm{H}$ & -3.10353800 & 0.86706900 & -3.50299500 \\
\hline $\mathrm{O}$ & -3.51092900 & -0.12601100 & -2.92352000 & $\mathrm{C}$ & -6.08269900 & -0.64025000 & -2.82203400 \\
\hline $\mathrm{O}$ & -0.98872500 & 0.37040200 & -2.84155500 & $\mathrm{H}$ & -6.41899900 & -2.00881100 & -1.18896900 \\
\hline $\mathrm{N}$ & -2.08396000 & -0.32562500 & -0.77939700 & $\mathrm{H}$ & -5.43702200 & 0.72861500 & -4.36051800 \\
\hline $\mathrm{C}$ & -3.08162500 & -0.68831100 & 0.25259600 & $\mathrm{H}$ & -7.09826900 & -0.71589800 & -3.20153400 \\
\hline $\mathrm{C}$ & -2.26591500 & -0.78461400 & 1.52517200 & $\mathrm{C}$ & -2.75973500 & -0.88718000 & 2.07885600 \\
\hline $\mathrm{C}$ & -0.86374500 & -0.68977500 & 1.46523100 & $\mathrm{H}$ & -3.20362200 & -1.75604000 & 1.57468400 \\
\hline $\mathrm{C}$ & -0.06915000 & -0.82530900 & 2.60456500 & $\mathrm{H}$ & -1.76719300 & -1.21157700 & 2.42110800 \\
\hline $\mathrm{C}$ & -0.69313200 & -1.03226100 & 3.83585900 & $\mathrm{C}$ & -3.62552700 & -0.51726400 & 3.30069800 \\
\hline $\mathrm{C}$ & -2.08405800 & -1.09295600 & 3.93809000 & $\mathrm{C}$ & -3.71779200 & -1.72529600 & 4.24632000 \\
\hline $\mathrm{C}$ & -2.86876500 & -0.96638100 & 2.78669000 & $\mathrm{C}$ & -5.02477600 & -0.02102100 & 2.90502100 \\
\hline $\mathrm{C}$ & -2.69331900 & 2.15936900 & -1.90959200 & $\mathrm{H}$ & -3.12696200 & 0.29875900 & 3.83778600 \\
\hline $\mathrm{H}$ & 0.63254800 & 2.94527700 & -1.51094300 & $\mathrm{H}$ & -2.72378500 & -2.07411400 & 4.55315000 \\
\hline $\mathrm{H}$ & 1.01372800 & -0.78977200 & 2.55022900 & $\mathrm{H}$ & -4.27840100 & -1.47130000 & 5.15370700 \\
\hline $\mathrm{H}$ & -0.08788600 & -1.14334300 & 4.73202200 & $\mathrm{H}$ & -4.23161300 & -2.56763400 & 3.76367500 \\
\hline $\mathrm{H}$ & -2.54396900 & -1.23788600 & 4.90858300 & $\mathrm{H}$ & -4.97069100 & 0.93148600 & 2.36936400 \\
\hline $\mathrm{O}$ & -4.23798600 & -0.99414000 & 2.78745300 & $\mathrm{H}$ & -5.54320600 & -0.75100100 & 2.26822700 \\
\hline $\mathrm{C}$ & -4.91229300 & -1.12966200 & 4.02814900 & $\mathrm{H}$ & -5.64306200 & 0.13822300 & 3.79652300 \\
\hline $\mathrm{H}$ & -4.67005200 & -0.30412600 & 4.71001100 & $\mathrm{~S}$ & 3.07749000 & 1.89159400 & -0.55040900 \\
\hline $\mathrm{H}$ & -5.97792000 & -1.10343400 & 3.79298900 & $\mathrm{~F}$ & 0.78213700 & 3.11206000 & -1.11931900 \\
\hline $\mathrm{H}$ & -4.67239800 & -2.08341400 & 4.51638300 & $\mathrm{~F}$ & 1.61308000 & 3.57500800 & 0.84759300 \\
\hline $\mathrm{C}$ & -3.79656400 & -1.99955500 & -0.11597800 & $\mathrm{~F}$ & 2.52504800 & 4.40071000 & -0.95284500 \\
\hline $\mathrm{H}$ & -3.06270600 & -2.80475600 & -0.22739800 & $\mathrm{O}$ & 3.01820500 & 1.32706400 & -1.88935900 \\
\hline $\mathrm{H}$ & -4.51062300 & -2.27204700 & 0.66531700 & $\mathrm{O}$ & 4.33013200 & 2.32432200 & 0.06157600 \\
\hline $\mathrm{H}$ & -4.33053200 & -1.87991300 & -1.06272800 & $\mathrm{~N}$ & 2.27808100 & 0.77262500 & 0.55719900 \\
\hline \multirow[t]{2}{*}{$\mathrm{H}$} & -3.82986500 & 0.10883200 & 0.35301700 & $\mathrm{C}$ & 3.09505300 & -0.48926500 & 0.78129600 \\
\hline & & & & $\mathrm{C}$ & 2.68809900 & -1.58801100 & -0.19318900 \\
\hline \multicolumn{4}{|l|}{$\mathrm{S}_{3 \mathrm{~B}}$} & $\mathrm{C}$ & 1.62849500 & -1.47241800 & -1.10537700 \\
\hline \multicolumn{4}{|c|}{-2277.91497058} & $\mathrm{C}$ & 1.19084700 & -2.55361900 & -1.87971500 \\
\hline$P d$ & 0.19780400 & 0.22345500 & 0.17218900 & $\mathrm{C}$ & 1.85598600 & -3.76970900 & -1.78024900 \\
\hline $\mathrm{C}$ & -2.55082700 & 0.21673900 & 1.01053800 & $\mathrm{C}$ & 2.95867000 & -3.89984600 & -0.93154500 \\
\hline $\mathrm{C}$ & -1.86652300 & 1.43299000 & 1.66069800 & $\mathrm{C}$ & 3.37804600 & -2.81948800 & -0.14757300 \\
\hline $\mathrm{O}$ & -0.54464200 & 1.49639400 & 1.49475000 & $\mathrm{C}$ & 1.88932600 & 3.35471600 & -0.43619900 \\
\hline $\mathrm{O}$ & -2.46100200 & 2.25367800 & 2.33258600 & $\mathrm{H}$ & 1.22768400 & -0.49473000 & -1.42160100 \\
\hline $\mathrm{H}$ & -3.51741300 & 0.54303400 & 0.61811700 & $\mathrm{H}$ & 0.35638100 & -2.40233300 & -2.55467600 \\
\hline $\mathrm{N}$ & -1.69950400 & -0.27105000 & -0.08435100 & $\mathrm{H}$ & 1.53903000 & -4.62093700 & -2.37570000 \\
\hline $\mathrm{C}$ & -2.02672200 & -0.32189100 & -1.40236700 & $\mathrm{H}$ & 3.48685600 & -4.84499900 & -0.88478100 \\
\hline
\end{tabular}




$\begin{array}{lrrr}\mathrm{H} & 2.22833500 & 1.28077400 & 1.44635200 \\ \mathrm{O} & 4.45678100 & -2.86609300 & 0.69345500 \\ \mathrm{C} & 5.24473200 & -4.04927400 & 0.73200700 \\ \mathrm{H} & 5.66200500 & -4.28262300 & -0.25525300 \\ \mathrm{H} & 6.05771800 & -3.84114500 & 1.42933900 \\ \mathrm{H} & 4.66454000 & -4.90687000 & 1.09521700 \\ \mathrm{H} & 4.15818400 & -0.25045800 & 0.66075900 \\ \mathrm{C} & 2.85502900 & -0.88390200 & 2.25016600 \\ \mathrm{H} & 1.78979000 & -1.05885700 & 2.43360000 \\ \mathrm{H} & 3.40844500 & -1.79472500 & 2.47809000 \\ \mathrm{H} & 3.20882800 & -0.09201700 & 2.92152200\end{array}$

$\mathrm{TS}_{\mathrm{S} 2 \mathrm{~B}}$

$-2277.89422606$

Pd $\quad 0.27143400 \quad-0.15416800 \quad-0.45648900$

$\begin{array}{llll}\text { C } & -2.57987000 & -0.29924200 & -0.90234500\end{array}$

$\begin{array}{llll}\text { C } & -1.96970100 & -1.66494700 & -1.31190200\end{array}$

O $\quad-0.64955400 \quad-1.73582100 \quad-1.29041400$

$\begin{array}{llll}\text { O } & -2.68161500 & -2.58639100 & -1.67372800\end{array}$

H $\quad-3.49059600 \quad-0.49691000 \quad-0.33166100$

$\begin{array}{llll}\mathrm{N} & -1.60163900 & 0.40560100 & -0.06420800\end{array}$

$\begin{array}{llll}\text { C } & -1.73461100 & 0.81006800 & 1.19609700\end{array}$

$\begin{array}{llll}\text { O } & -0.70132400 & 1.07872400 & 1.90414600\end{array}$

$\begin{array}{llll}\text { C } & -3.06157900 & 0.98886100 & 1.86246900\end{array}$

$\begin{array}{llll}\text { C } & -4.17696400 & 1.52645900 & 1.20459700\end{array}$

$\begin{array}{llll}\text { C } & -3.16372500 & 0.64812700 & 3.22055500\end{array}$

$\begin{array}{lrrr}\text { C } & -5.37957100 & 1.70381100 & 1.88967900\end{array}$

$\begin{array}{llll}\text { H } & -4.10006000 & 1.83132900 & 0.16664200\end{array}$

$\begin{array}{llll}\text { C } & -4.37081000 & 0.81065300 & 3.89651900\end{array}$

$\begin{array}{llll}\mathrm{H} & -2.28929900 & 0.25961700 & 3.73136400\end{array}$

$\begin{array}{lrrr}\text { C } & -5.48165900 & 1.33855200 & 3.23296500\end{array}$

$\begin{array}{llll}\mathrm{H} & -6.23485800 & 2.13008100 & 1.37281400\end{array}$

H $\quad-4.44324700 \quad 0.53194700 \quad 4.94414700$

$\begin{array}{llll}\mathrm{H} & -6.42099300 & 1.47070600 & 3.76304200\end{array}$

$\begin{array}{llll}\text { C } & -2.91142000 & 0.53714400 & -2.16238600\end{array}$

$\begin{array}{llll}\mathrm{H} & -3.31291700 & 1.50419100 & -1.82595300\end{array}$

$\begin{array}{llll}\mathrm{H} & -1.96443700 & 0.76475500 & -2.67079100\end{array}$

$\begin{array}{llll}\text { C } & -3.89343400 & -0.09092600 & -3.17207500\end{array}$

$\begin{array}{llll}\text { C } & -4.08576800 & 0.86397700 & -4.36050900\end{array}$

$\begin{array}{llll}\text { C } & -5.24242000 & -0.45665700 & -2.53480700\end{array}$

$\begin{array}{llll}\mathrm{H} & -3.44629400 & -1.01907300 & -3.54754100\end{array}$

$\mathrm{H} \quad-3.12928000 \quad 1.11156500 \quad-4.83740100$

H $\quad-4.73378600 \quad 0.41518200 \quad-5.12250100$

$\mathrm{H} \quad-4.55242800 \quad 1.80581300 \quad-4.04127900$
$\mathrm{H} \quad-5.12547500 \quad-1.25623400 \quad-1.79738700$

$\begin{array}{llll}\mathrm{H} & -5.70232100 & 0.41241700 & -2.04360700\end{array}$

H $\quad-5.94463000 \quad-0.81320900 \quad-3.29778700$

$\begin{array}{llll}\text { S } & 2.70479300 & -1.89400200 & 0.93086600\end{array}$

F $\quad 0.24421100 \quad-2.82074200 \quad 1.36958400$

F $\quad 1.29011700 \quad-3.72721800 \quad-0.32505700$

F $\quad 1.86755600 \quad-4.21968200 \quad 1.71783300$

$\begin{array}{llll}\text { O } & 2.50130400 & -1.04049700 & 2.08960500\end{array}$

$\begin{array}{llll}\text { O } & 3.98095800 & -2.54669500 & 0.65180900\end{array}$

$\begin{array}{llll}\mathrm{N} & 2.24998200 & -0.97361600 & -0.51436300\end{array}$

$\begin{array}{llll}\text { C } & 3.23072500 & 0.15886400 & -0.74721400\end{array}$

$\begin{array}{llll}\text { C } & 2.68311800 & 1.46025800 & -0.18589900\end{array}$

$\begin{array}{llll}\text { C } & 1.32579200 & 1.64913200 & 0.17639500\end{array}$

$\begin{array}{llll}\text { C } & 0.87379200 & 2.96956000 & 0.38490600\end{array}$

$\begin{array}{llll}\text { C } & 1.74407300 & 4.05213700 & 0.32973800\end{array}$

$\begin{array}{llll}\text { C } & 3.09792100 & 3.85605200 & 0.06270200\end{array}$

$\begin{array}{llll}\text { C } & 3.56104000 & 2.56470300 & -0.20243500\end{array}$

$\begin{array}{llll}\text { C } & 1.40347500 & -3.26464000 & 0.91632900\end{array}$

$\begin{array}{llll}\mathrm{H} & 0.45621700 & 1.05193900 & 1.04176500\end{array}$

$\begin{array}{llll}\mathrm{H} & -0.15918500 & 3.13136500 & 0.66774000\end{array}$

$\begin{array}{llll}\text { H } & 1.37431800 & 5.05668900 & 0.51558500\end{array}$

$\begin{array}{llll}\mathrm{H} & 3.77516200 & 4.70180500 & 0.04821000\end{array}$

H $\quad 2.32052200 \quad-1.64304700 \quad-1.28696200$

$\begin{array}{llll}\text { O } & 4.86309000 & 2.27748500 & -0.52197500\end{array}$

$\begin{array}{llll}\text { C } & 5.82310400 & 3.32465000 & -0.48483300\end{array}$

$\begin{array}{llll}\mathrm{H} & 5.88018500 & 3.77929500 & 0.51195100\end{array}$

$\begin{array}{llll}\mathrm{H} & 6.78012400 & 2.85875100 & -0.72539700\end{array}$

$\begin{array}{llll}\mathrm{H} & 5.60064100 & 4.10175400 & -1.22734400\end{array}$

$\begin{array}{llll}\mathrm{H} & 4.19169600 & -0.09174900 & -0.28621700\end{array}$

$\begin{array}{llll}\text { C } & 3.43668500 & 0.23577100 & -2.27061900\end{array}$

$\begin{array}{llll}\mathrm{H} & 2.48310900 & 0.41636000 & -2.77866300\end{array}$

$\begin{array}{llll}\mathrm{H} & 4.12103100 & 1.05227900 & -2.50454600\end{array}$

$\begin{array}{llll}\mathrm{H} & 3.87674800 & -0.69563500 & -2.64793300\end{array}$

$\mathrm{S}_{4 \mathrm{~B}}$

$-2277.93735005$

$\begin{array}{llll}\text { Pd } & 0.28706400 & 0.12840100 & 0.24163200\end{array}$

$\begin{array}{llll}\text { C } & -2.50047500 & 0.28660400 & 0.94681800\end{array}$

$\begin{array}{llll}\text { C } & -1.88705600 & 1.67159100 & 1.32893500\end{array}$

$\begin{array}{llll}\text { O } & -0.62129500 & 1.82510200 & 1.06538800\end{array}$

$\begin{array}{llll}\text { O } & -2.61344200 & 2.49649900 & 1.86911700\end{array}$

$\begin{array}{llll}\mathrm{H} & -3.51935500 & 0.46521400 & 0.59918000\end{array}$

$\begin{array}{llll}\mathrm{N} & -1.68474500 & -0.28050300 & -0.14785600\end{array}$

$\begin{array}{llll}\text { C } & -2.13041900 & -0.52778000 & -1.33688800\end{array}$ 


\begin{tabular}{|c|c|c|c|c|c|c|c|}
\hline $\mathrm{O}$ & -1.27641200 & -0.72678400 & -2.35129400 & $\mathrm{H}$ & 2.26677300 & 1.43086300 & 1.37745600 \\
\hline $\mathrm{C}$ & -3.54610200 & -0.62466000 & -1.76899400 & $\mathrm{O}$ & 4.68806200 & -2.61683800 & 0.33841200 \\
\hline $\mathrm{C}$ & -4.50593700 & -1.29909200 & -0.99917000 & $\mathrm{C}$ & 5.57917600 & -3.68928000 & 0.06973700 \\
\hline $\mathrm{C}$ & -3.91739700 & -0.06450700 & -3.00201200 & $\mathrm{H}$ & 5.67213500 & -3.87242600 & -1.00825400 \\
\hline $\mathrm{C}$ & -5.82183300 & -1.39445500 & -1.44902500 & $\mathrm{H}$ & 6.54717200 & -3.38163200 & 0.46949600 \\
\hline $\mathrm{H}$ & -4.21855200 & -1.76759700 & -0.06393600 & $\mathrm{H}$ & 5.25794000 & -4.61344700 & 0.56749400 \\
\hline $\mathrm{C}$ & -5.23705000 & -0.15132200 & -3.43984300 & $\mathrm{H}$ & 4.17615600 & -0.18285400 & 0.54482200 \\
\hline $\mathrm{H}$ & -3.17045100 & 0.44038500 & -3.60502100 & $\mathrm{C}$ & 3.17584600 & -0.60088700 & 2.39494400 \\
\hline $\mathrm{C}$ & -6.19100600 & -0.81539800 & -2.66496900 & $\mathrm{H}$ & 2.16546700 & -0.77805800 & 2.77887600 \\
\hline $\mathrm{H}$ & -6.55697600 & -1.92447300 & -0.85054000 & $\mathrm{H}$ & 3.79282400 & -1.47243100 & 2.62777100 \\
\hline $\mathrm{H}$ & -5.51964900 & 0.29611500 & -4.38832600 & $\mathrm{H}$ & 3.61060900 & 0.26963600 & 2.90216900 \\
\hline $\mathrm{H}$ & -7.21858900 & -0.88629100 & -3.01048200 & & & & \\
\hline $\mathrm{C}$ & -2.51098300 & -0.67640800 & 2.15423300 & $\mathrm{~S}_{1 \mathrm{C}}$ & & & \\
\hline $\mathrm{H}$ & -2.93858200 & -1.63543000 & 1.82363400 & -25 & 03512848 & & \\
\hline $\mathrm{H}$ & -1.46835100 & -0.88938500 & 2.42735700 & $\mathrm{Pd}$ & 0.58814300 & -0.18408100 & 0.24785100 \\
\hline $\mathrm{C}$ & -3.27378400 & -0.19164300 & 3.40405000 & $\mathrm{C}$ & 2.85126300 & -1.90410900 & -0.19578300 \\
\hline $\mathrm{C}$ & -3.15458700 & -1.24733900 & 4.51411900 & $\mathrm{C}$ & 1.59412400 & -2.69158800 & -0.60510100 \\
\hline $\mathrm{C}$ & -4.74469000 & 0.13795900 & 3.10896400 & $\mathrm{H}$ & 3.64768500 & -2.17716700 & -0.89077800 \\
\hline $\mathrm{H}$ & -2.79597300 & 0.73168700 & 3.75265100 & $\mathrm{~N}$ & 2.58391600 & -0.43345800 & -0.34916800 \\
\hline $\mathrm{H}$ & -2.10596700 & -1.46964500 & 4.74806900 & $\mathrm{H}$ & 3.02595900 & 0.15461800 & 0.38203200 \\
\hline $\mathrm{H}$ & -3.63750700 & -0.90243800 & 5.43582100 & $\mathrm{C}$ & 2.70004700 & 0.03701400 & -1.72089300 \\
\hline $\mathrm{H}$ & -3.63660200 & -2.18942800 & 4.21943900 & $\mathrm{O}$ & 2.56738400 & -0.76855100 & -2.62046500 \\
\hline $\mathrm{H}$ & -4.82517300 & 1.00253300 & 2.44397300 & $\mathrm{C}$ & 2.96707900 & 1.48149900 & -1.97501700 \\
\hline $\mathrm{H}$ & -5.26262000 & -0.71595100 & 2.64937600 & $\mathrm{C}$ & 3.56497900 & 2.35221500 & -1.05107400 \\
\hline $\mathrm{H}$ & -5.27696300 & 0.38441800 & 4.03540200 & $\mathrm{C}$ & 2.64993800 & 1.95046700 & -3.26215500 \\
\hline S & 2.92982600 & 1.75056900 & -0.76802700 & $\mathrm{C}$ & 3.83180400 & 3.67295700 & -1.41351900 \\
\hline $\mathrm{F}$ & 0.68075600 & 3.09136000 & -1.25581000 & $\mathrm{H}$ & 3.82521300 & 2.02008600 & -0.05267500 \\
\hline $\mathrm{F}$ & 1.69152800 & 3.63761700 & 0.60597200 & $\mathrm{C}$ & 2.90393500 & 3.27246300 & -3.61124700 \\
\hline $\mathrm{F}$ & 2.51365500 & 4.25374600 & -1.31522400 & $\mathrm{H}$ & 2.20464300 & 1.26117000 & -3.97122300 \\
\hline $\mathrm{O}$ & 2.65725500 & 1.05996200 & -2.01892100 & $\mathrm{C}$ & 3.49765400 & 4.13697800 & -2.68665900 \\
\hline $\mathrm{O}$ & 4.27521400 & 2.17226100 & -0.38715900 & $\mathrm{H}$ & 4.30351300 & 4.33899200 & -0.69656900 \\
\hline $\mathrm{N}$ & 2.26415900 & 0.79392900 & 0.57337000 & $\mathrm{H}$ & 2.64274900 & 3.62921300 & -4.60339300 \\
\hline $\mathrm{C}$ & 3.15676300 & -0.40872900 & 0.87315400 & $\mathrm{H}$ & 3.70189000 & 5.16868600 & -2.96020700 \\
\hline $\mathrm{C}$ & 2.58472000 & -1.59039200 & 0.12937900 & $\mathrm{C}$ & 3.28528500 & -2.19422200 & 1.25492500 \\
\hline $\mathrm{C}$ & 1.23446700 & -1.57816200 & -0.26919300 & $\mathrm{H}$ & 4.17133100 & -1.57956000 & 1.47006700 \\
\hline $\mathrm{C}$ & 0.70723300 & -2.72600300 & -0.88104600 & $\mathrm{H}$ & 2.49989100 & -1.84520000 & 1.93883900 \\
\hline $\mathrm{C}$ & 1.51633200 & -3.83990900 & -1.10895100 & $\mathrm{C}$ & 3.61342600 & -3.66909500 & 1.56850500 \\
\hline $\mathrm{C}$ & 2.86062100 & -3.84703400 & -0.73198500 & $\mathrm{C}$ & 4.01108400 & -3.79387600 & 3.04742900 \\
\hline $\mathrm{C}$ & 3.39280000 & -2.71866100 & -0.10190700 & $\mathrm{C}$ & 4.70695000 & -4.23902200 & 0.65238500 \\
\hline $\mathrm{C}$ & 1.84663100 & 3.29870100 & -0.67133500 & $\mathrm{H}$ & 2.70500600 & -4.26149700 & 1.40542100 \\
\hline $\mathrm{H}$ & -0.35937500 & -0.58482900 & -2.02072800 & $\mathrm{H}$ & 3.22645900 & -3.40867400 & 3.71035900 \\
\hline $\mathrm{H}$ & -0.33307100 & -2.76576000 & -1.18555300 & $\mathrm{H}$ & 4.19180800 & -4.84133100 & 3.31482700 \\
\hline $\mathrm{H}$ & 1.09684700 & -4.72164300 & -1.58747500 & $\mathrm{H}$ & 4.93084500 & -3.23279000 & 3.25940200 \\
\hline $\mathrm{H}$ & 3.47166000 & -4.72193000 & -0.92078000 & $\mathrm{H}$ & 4.35976300 & -4.30867700 & -0.38277400 \\
\hline
\end{tabular}




\begin{tabular}{|c|c|c|c|c|c|c|c|}
\hline $\mathrm{H}$ & 5.61432400 & -3.61994600 & 0.68049300 & $\mathrm{Pd}$ & 0.26014900 & -0.20480700 & -0.02407900 \\
\hline $\mathrm{H}$ & 4.98678200 & -5.24937800 & 0.97270700 & $\mathrm{C}$ & 3.13826400 & -0.11092200 & -1.11173400 \\
\hline $\mathrm{O}$ & 1.65729700 & -3.86145900 & -0.93004400 & $\mathrm{C}$ & 2.47729900 & 0.05345400 & -2.51754900 \\
\hline $\mathrm{O}$ & 0.45017100 & -2.02375500 & -0.52555500 & $\mathrm{H}$ & 4.04094700 & 0.50162700 & -1.13706900 \\
\hline S & -2.34716600 & 1.38480600 & 0.33829500 & $\mathrm{~N}$ & 2.33169400 & 0.39897000 & 0.06807400 \\
\hline $\mathrm{F}$ & -3.18034300 & 3.06940300 & 2.17183000 & $\mathrm{H}$ & 2.54637200 & -0.14610100 & 0.92506800 \\
\hline $\mathrm{F}$ & -1.53121600 & 1.84952900 & 2.87684800 & $\mathrm{C}$ & 2.44857900 & 1.82459800 & 0.26917500 \\
\hline $\mathrm{F}$ & -1.17692600 & 3.50833200 & 1.48741700 & $\mathrm{O}$ & 2.75524100 & 2.52610700 & -0.67890200 \\
\hline $\mathrm{O}$ & -3.78928300 & 1.18369500 & 0.39460000 & $\mathrm{C}$ & 2.16890300 & 2.40161000 & 1.61886100 \\
\hline $\mathrm{O}$ & -1.67059600 & 1.92238800 & -0.83021800 & $\mathrm{C}$ & 1.78556200 & 1.65504500 & 2.74467200 \\
\hline$N$ & -1.51623000 & -0.12816800 & 0.81892200 & $\mathrm{C}$ & 2.31329900 & 3.79634700 & 1.73410500 \\
\hline $\mathrm{C}$ & -2.31680700 & -1.45157600 & 0.55162400 & $\mathrm{C}$ & 1.54251200 & 2.30121600 & 3.95728100 \\
\hline $\mathrm{C}$ & -3.08519100 & -1.45189700 & -0.76699200 & $\mathrm{H}$ & 1.69081300 & 0.57633800 & 2.69604200 \\
\hline $\mathrm{C}$ & -2.33047000 & -1.59021300 & -1.94200200 & $\mathrm{C}$ & 2.07111800 & 4.43344200 & 2.94614600 \\
\hline $\mathrm{C}$ & -2.92172900 & -1.61694100 & -3.20467100 & $\mathrm{H}$ & 2.62304600 & 4.35895500 & 0.86016100 \\
\hline $\mathrm{C}$ & -4.30598800 & -1.52434800 & -3.30798000 & $\mathrm{C}$ & 1.68092700 & 3.68596300 & 4.06166500 \\
\hline $\mathrm{C}$ & -5.08913400 & -1.41414500 & -2.15918800 & $\mathrm{H}$ & 1.24575200 & 1.71513300 & 4.82223700 \\
\hline $\mathrm{C}$ & -4.49484300 & -1.38060900 & -0.89115400 & $\mathrm{H}$ & 2.18767400 & 5.51083600 & 3.02347800 \\
\hline $\mathrm{C}$ & -2.00806900 & 2.54343500 & 1.83025200 & $\mathrm{H}$ & 1.48990300 & 4.18220400 & 5.00948600 \\
\hline $\mathrm{H}$ & -1.25474600 & -1.69901600 & -1.85400900 & $\mathrm{C}$ & 3.51025500 & -1.58722900 & -0.86212600 \\
\hline $\mathrm{H}$ & -2.30241000 & -1.72132700 & -4.09011300 & $\mathrm{H}$ & 3.97217400 & -1.66846500 & 0.13179500 \\
\hline $\mathrm{H}$ & -4.79042600 & -1.54752000 & -4.28065200 & $\mathrm{H}$ & 2.58852900 & -2.18194000 & -0.83066200 \\
\hline $\mathrm{H}$ & -6.16659800 & -1.35576300 & -2.25573200 & $\mathrm{C}$ & 4.47039500 & -2.21654100 & -1.89492000 \\
\hline $\mathrm{O}$ & -5.22859200 & -1.29770100 & 0.25460400 & $\mathrm{C}$ & 4.70539100 & -3.69057900 & -1.52881200 \\
\hline $\mathrm{C}$ & -6.62181500 & -1.04763900 & 0.16424400 & $\mathrm{C}$ & 5.80148000 & -1.45828200 & -2.00564300 \\
\hline $\mathrm{H}$ & -7.15500700 & -1.88089000 & -0.31207500 & $\mathrm{H}$ & 3.98765600 & -2.17794700 & -2.87812200 \\
\hline $\mathrm{H}$ & -6.97128400 & -0.94181100 & 1.19309900 & $\mathrm{H}$ & 3.76156300 & -4.24766800 & -1.47052100 \\
\hline $\mathrm{H}$ & -6.82520000 & -0.12109400 & -0.38637100 & $\mathrm{H}$ & 5.33812400 & -4.18278600 & -2.27677500 \\
\hline $\mathrm{H}$ & -1.42036800 & -0.05095200 & 1.83680800 & $\mathrm{H}$ & 5.20855700 & -3.78260400 & -0.55668400 \\
\hline $\mathrm{C}$ & 1.85923500 & 1.86261400 & 1.91423700 & $\mathrm{H}$ & 5.64676300 & -0.46410600 & -2.43336200 \\
\hline $\mathrm{O}$ & 2.92857500 & 1.23295200 & 1.85102800 & $\mathrm{H}$ & 6.28605900 & -1.35550400 & -1.02436000 \\
\hline $\mathrm{O}$ & 0.77269600 & 1.57988200 & 1.25855800 & $\mathrm{H}$ & 6.49614000 & -1.99650800 & -2.66182500 \\
\hline $\mathrm{C}$ & 1.74610300 & 3.10174800 & 2.78956700 & $\mathrm{O}$ & 3.26685200 & 0.20372400 & -3.44836000 \\
\hline $\mathrm{H}$ & 0.86287900 & 3.03802200 & 3.43102300 & $\mathrm{O}$ & 1.19673100 & -0.09474000 & -2.61712300 \\
\hline $\mathrm{H}$ & 2.64561400 & 3.21136900 & 3.39725400 & $\mathrm{~S}$ & -2.76689200 & -0.76869800 & 1.06266500 \\
\hline $\mathrm{H}$ & 1.62405100 & 3.98564300 & 2.15377800 & $\mathrm{~F}$ & -4.50421000 & -2.63028100 & 0.26683400 \\
\hline $\mathrm{C}$ & -3.04437600 & -1.82974100 & 1.84333900 & $\mathrm{~F}$ & -2.65308200 & -3.41253000 & 1.10573500 \\
\hline $\mathrm{H}$ & -3.77811000 & -1.08230100 & 2.14591400 & $\mathrm{~F}$ & -4.16051400 & -2.52985900 & 2.41293400 \\
\hline $\mathrm{H}$ & -3.57238300 & -2.77685600 & 1.70526400 & $\mathrm{O}$ & -3.78523200 & 0.19529800 & 0.67429900 \\
\hline $\mathrm{H}$ & -2.30927700 & -1.97387300 & 2.64674200 & $\mathrm{O}$ & -1.95730600 & -0.62789600 & 2.26006100 \\
\hline \multirow[t]{2}{*}{$\mathrm{H}$} & -1.49004900 & -2.14918700 & 0.40706600 & $\mathrm{~N}$ & -1.68154400 & -1.03241000 & -0.30663800 \\
\hline & & & & $\mathrm{C}$ & -2.08503200 & -0.53705100 & -1.70072200 \\
\hline \multicolumn{4}{|c|}{$\mathrm{TS}_{\mathrm{SIC}}$} & $\mathrm{C}$ & -1.93383000 & 0.97696500 & -1.64719300 \\
\hline \multicolumn{4}{|c|}{-2506.97249304} & $\mathrm{C}$ & -0.62671800 & 1.43752600 & -1.32126600 \\
\hline
\end{tabular}




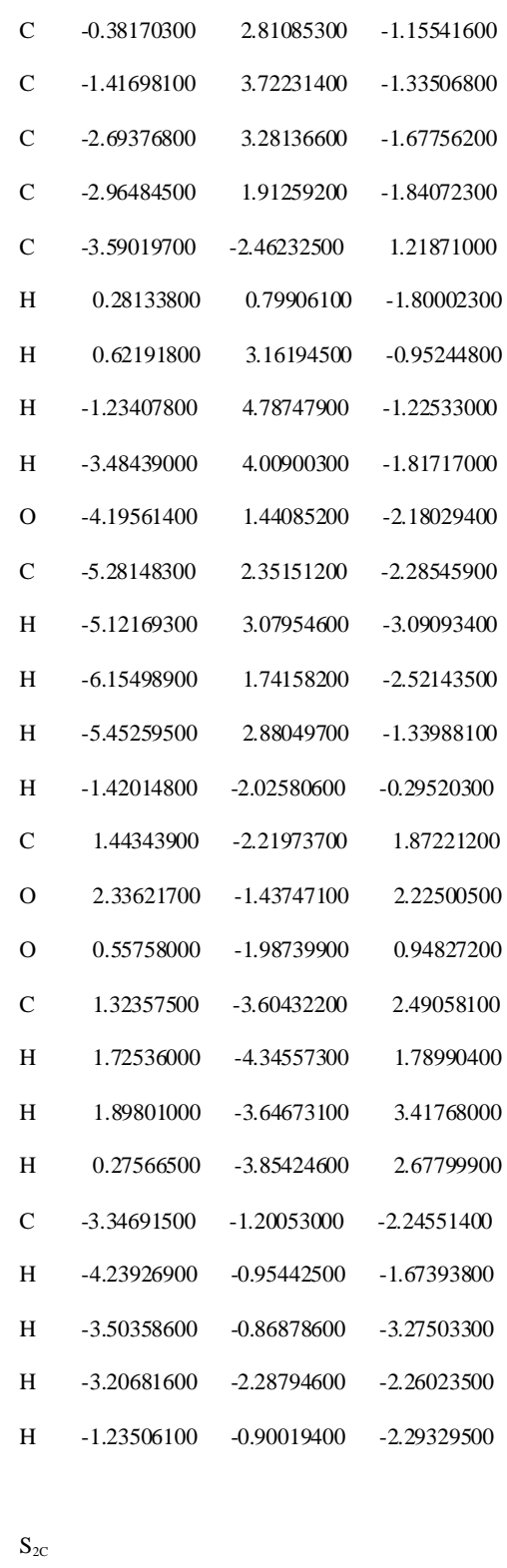

$-2507.01965996$

$\begin{array}{llll}\mathrm{Pd} & 0.18185700 & -0.14538700 & -0.21194100 \\ \mathrm{C} & 3.29549800 & 0.65888300 & -0.48108900 \\ \mathrm{C} & 3.24925000 & 2.19152500 & -0.40809700 \\ \mathrm{H} & 4.28748100 & 0.39446700 & -0.10695500 \\ \mathrm{~N} & 2.30450700 & -0.08230200 & 0.38190200 \\ \mathrm{H} & 2.39897800 & -1.10322400 & 0.14710000 \\ \mathrm{C} & 2.48613800 & 0.18228600 & 1.79055100 \\ \mathrm{O} & 3.01440800 & 1.22842300 & 2.12583800 \\ \mathrm{C} & 1.98550800 & -0.80607900 & 2.78981400 \\ \mathrm{C} & 1.53937100 & -2.09985600 & 2.47551800 \\ \mathrm{C} & 1.99312600 & -0.38530200 & 4.13226700 \\ \mathrm{C} & 1.09661000 & -2.94690100 & 3.49184400 \\ \mathrm{H} & 1.56213400 & -2.46783500 & 1.45610100 \\ \mathrm{C} & 1.54546600 & -1.23340400 & 5.13907200\end{array}$

\begin{tabular}{|c|c|c|c|}
\hline $\mathrm{H}$ & 2.35672300 & 0.61032200 & 4.36215900 \\
\hline & 1.09197200 & -2.51694600 & 4.81942300 \\
\hline & 0.75516500 & -3.94673800 & 3.24039400 \\
\hline $\mathrm{H}$ & 1.55033800 & -0.89664100 & 6.17187800 \\
\hline $\mathrm{H}$ & 0.74105400 & -3.18104500 & 5.60485800 \\
\hline $\mathrm{C}$ & 3.15594400 & 0.19905400 & -1.94956400 \\
\hline $\mathrm{H}$ & 3.25839000 & -0.89137700 & -1.96480100 \\
\hline $\mathrm{H}$ & 2.13619200 & 0.42573000 & -2.28866800 \\
\hline $\mathrm{C}$ & 4.16744100 & 0.80622500 & -2.94564300 \\
\hline $\mathrm{C}$ & 3.84711500 & 0.29123900 & -4.35801700 \\
\hline $\mathrm{C}$ & 5.62502200 & 0.50064400 & -2.56773900 \\
\hline $\mathrm{H}$ & 4.04560200 & 1.89678800 & -2.94949300 \\
\hline $\mathrm{H}$ & 2.81729600 & 0.53097400 & -4.65114300 \\
\hline $\mathrm{H}$ & 4.51900500 & 0.74093300 & -5.09812500 \\
\hline $\mathrm{H}$ & 3.96673300 & -0.79850400 & -4.41874300 \\
\hline $\mathrm{H}$ & 5.90638000 & 0.99697000 & -1.63453500 \\
\hline $\mathrm{H}$ & 5.78754400 & -0.58001800 & -2.45635100 \\
\hline $\mathrm{H}$ & 6.30609900 & 0.85964300 & -3.34826800 \\
\hline $\mathrm{O}$ & 4.26722400 & 2.83717900 & -0.49227000 \\
\hline 0 & 2.04783400 & 2.79700600 & -0.42094100 \\
\hline$S$ & 2.79536200 & -1.21393300 & 0.13482600 \\
\hline 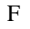 & -5.05610800 & -1.40796800 & -1.28048400 \\
\hline$F$ & -3.32197700 & -2.32293000 & -2.23136800 \\
\hline $\mathrm{F}$ & -4.27075300 & -3.28568100 & -0.52112300 \\
\hline $\mathrm{O}$ & -3.59709500 & -0.29344700 & 0.92413500 \\
\hline $\mathrm{O}$ & -1.97280300 & -2.26418700 & 0.70994600 \\
\hline $\mathrm{N}$ & -1.76662200 & -0.28372000 & -0.96391000 \\
\hline $\mathrm{C}$ & -2.16024400 & 1.15260700 & -1.31706200 \\
\hline $\mathrm{C}$ & -1.75204900 & 1.97981100 & -0.10271000 \\
\hline $\mathrm{C}$ & -0.60920400 & 1.52985800 & 0.60508500 \\
\hline $\mathrm{C}$ & -0.19792200 & 2.18954400 & 1.76502700 \\
\hline $\mathrm{C}$ & -0.88955000 & 3.32118700 & 2.19957300 \\
\hline C & -1.98479900 & 3.80464100 & 1.49082900 \\
\hline $\mathrm{C}$ & -2.41900000 & 3.14252600 & 0.33492600 \\
\hline $\mathrm{C}$ & -3.95583500 & -2.11984000 & -1.06589900 \\
\hline 11 & 1.31886000 & 2.17354000 & -0.22989500 \\
\hline 1 & 0.67114300 & 1.86265600 & 2.32140700 \\
\hline $\mathrm{H}$ & -0.56029900 & 3.84281500 & 3.09429500 \\
\hline $\mathrm{H}$ & -2.49677000 & 4.69368500 & 1.83806300 \\
\hline $\mathrm{O}$ & 3.47773200 & 3.57993900 & -0.41267900 \\
\hline $\mathrm{C}$ & 4.20760600 & 4.71494000 & 0.02973000 \\
\hline 1 & -3.57887700 & 5.61402900 & 0.05481300 \\
\hline $\mathrm{H}$ & -5.00736600 & 4.85550900 & -0.69989700 \\
\hline $\mathrm{H}$ & & & 20110 \\
\hline
\end{tabular}




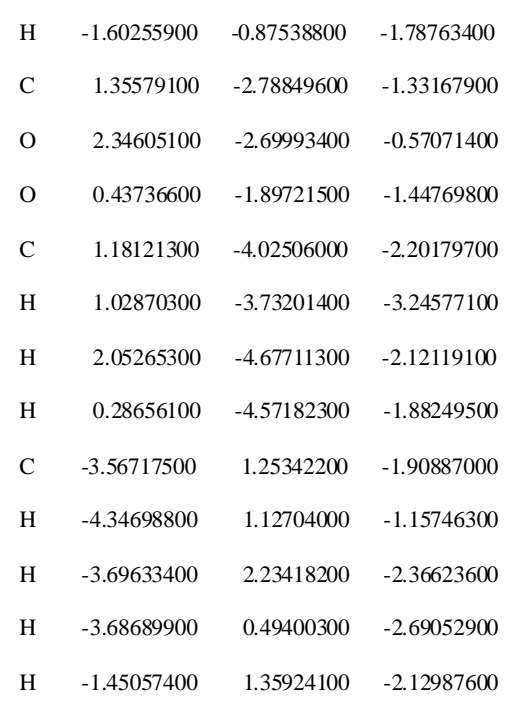

$\mathrm{S}_{1 \mathrm{D}}$

$-2507.05027079$

$\begin{array}{llll}\text { Pd } & -0.36814600 & 0.26097100 & 0.20188300\end{array}$

$\begin{array}{llll}\text { O } & 2.08241100 & -1.05491500 & -1.61213800\end{array}$

$\begin{array}{llll}\text { C } & 0.97537200 & -2.92887600 & -2.55278600\end{array}$

$\begin{array}{llll}\text { C } & 0.97855500 & -1.73332800 & -1.64144800\end{array}$

$\begin{array}{llll}\text { O } & -0.03677600 & -1.45117100 & -0.97006900\end{array}$

$\begin{array}{llll}\text { C } & -3.05009900 & 1.33603700 & 0.29444000\end{array}$

$\begin{array}{llll}\text { C } & -2.16112700 & 2.17366900 & 1.24128000\end{array}$

$\begin{array}{llll}\mathrm{H} & -2.91235200 & 1.71628700 & -0.72091800\end{array}$

$\mathrm{H} \quad 1.21385300 \quad-2.60846600 \quad-3.57209800$

$\mathrm{H} \quad 1.75665500 \quad-3.62664900 \quad-2.23412200$

$\begin{array}{llll}\mathrm{H} & 0.00217300 & -3.41916800 & -2.53138800\end{array}$

$\begin{array}{llll}\mathrm{N} & -2.49404900 & -0.06538600 & 0.25880400\end{array}$

$\begin{array}{llll}\mathrm{H} & -2.66467500 & -0.52241500 & 1.15488700\end{array}$

$\begin{array}{llll}\text { C } & -2.97074200 & -0.89484500 & -0.83351100\end{array}$

$\begin{array}{llll}\text { O } & -3.28170900 & -0.37878000 & -1.88550000\end{array}$

$\begin{array}{llll}\text { C } & -3.07764600 & -2.35836200 & -0.57153100\end{array}$

$\begin{array}{llll}\text { C } & -3.80655200 & -3.11429400 & -1.50372200\end{array}$

$\begin{array}{llll}\text { C } & -2.50544200 & -2.99990300 & 0.53896600\end{array}$

$\begin{array}{llll}\text { C } & -3.97540000 & -4.48381700 & -1.32009000\end{array}$

$\mathrm{H} \quad-4.23596800 \quad-2.60581100 \quad-2.36036200$

$\begin{array}{llll}\text { C } & -2.67289400 & -4.37212500 & 0.71709800\end{array}$

$\mathrm{H} \quad-1.89261800 \quad-2.45141500 \quad 1.24826700$

C $\quad-3.41120100 \quad-5.11454300 \quad-0.20763600$

$\mathrm{H} \quad-4.54761500 \quad-5.05950900 \quad-2.04184600$

$\mathrm{H} \quad-2.22066400 \quad-4.86227900 \quad 1.57425500$

$\begin{array}{llll}\mathrm{H} & -3.54323500 & -6.18328900 & -0.06322600\end{array}$

$\begin{array}{llll}\text { C } & -4.53551400 & 1.35693900 & 0.68928300\end{array}$

$\begin{array}{llll}\mathrm{H} & -5.03927200 & 0.49202000 & 0.23511300\end{array}$

\begin{tabular}{|c|c|c|c|}
\hline $\mathrm{H}$ & -4.60394700 & 1.24222800 & 1.77863200 \\
\hline $\mathrm{C}$ & -5.29026500 & 2.63775000 & 0.26606500 \\
\hline 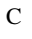 & -6.64073000 & 2.70782800 & 0.99427800 \\
\hline $\mathrm{C}$ & -5.49140000 & 2.71302000 & -1.25534600 \\
\hline $\mathrm{H}$ & -4.68658700 & 3.49238800 & 0.58971400 \\
\hline $\mathrm{H}$ & -6.50581000 & 2.72182100 & 2.08201500 \\
\hline $\mathrm{H}$ & -7.19046400 & 3.61353700 & 0.71314900 \\
\hline $\mathrm{H}$ & -7.27285000 & 1.84521800 & 0.74239000 \\
\hline $\mathrm{H}$ & -4.54614700 & 2.67975000 & -1.80836900 \\
\hline $\mathrm{H}$ & -6.11124700 & 1.87978600 & -1.61232400 \\
\hline $\mathrm{H}$ & -5.99680600 & 3.64609700 & -1.53002800 \\
\hline $\mathrm{O}$ & -0.89268000 & 1.82076300 & 1.30500200 \\
\hline $\mathrm{O}$ & -2.62287900 & 3.10096400 & 1.88030200 \\
\hline S & 1.81006200 & 2.34974900 & -0.41506400 \\
\hline $\mathrm{F}$ & 4.48490300 & 2.25198800 & -0.42867600 \\
\hline $\mathrm{F}$ & 3.55611000 & 1.26794100 & -2.13595500 \\
\hline $\mathrm{F}$ & 3.57910700 & 3.43886900 & -2.00902500 \\
\hline $\mathrm{O}$ & 1.98055200 & 3.41065200 & 0.57737700 \\
\hline $\mathrm{O}$ & 0.83043000 & 2.44960000 & -1.50051700 \\
\hline $\mathrm{N}$ & 1.66805900 & 0.80642400 & 0.23556400 \\
\hline $\mathrm{C}$ & 2.41876700 & 0.56674400 & 1.53107400 \\
\hline $\mathrm{C}$ & 2.70090800 & -0.92824700 & 1.63555100 \\
\hline $\mathrm{C}$ & 1.93423100 & -1.79077900 & 2.42118000 \\
\hline $\mathrm{C}$ & 2.21156300 & -3.16012800 & 2.49639200 \\
\hline $\mathrm{C}$ & 3.28165200 & -3.68139700 & 1.77484600 \\
\hline $\mathrm{C}$ & 4.07303400 & -2.84560500 & 0.98149800 \\
\hline C & 3.78554400 & -1.47857200 & 0.91272300 \\
\hline $\mathrm{C}$ & 3.47064000 & 2.31284700 & -1.30144000 \\
\hline $\mathrm{H}$ & 1.10941800 & -1.38500500 & 2.99803400 \\
\hline $\mathrm{H}$ & 1.60182500 & -3.80550400 & 3.12219500 \\
\hline $\mathrm{H}$ & 3.51560400 & -4.74154100 & 1.82762600 \\
\hline $\mathrm{H}$ & 4.90847500 & -3.26126500 & 0.42962100 \\
\hline $\mathrm{H}$ & 2.01137900 & -0.27672000 & -0.91953800 \\
\hline $\mathrm{O}$ & 4.49718900 & -0.58932600 & 0.16444100 \\
\hline C & 5.53064000 & -1.06268000 & -0.68445600 \\
\hline $\mathrm{H}$ & 5.14322300 & -1.78261400 & -1.41584000 \\
\hline $\mathrm{H}$ & 5.90494700 & -0.18122200 & -1.20619900 \\
\hline $\mathrm{H}$ & 6.34512700 & -1.52261800 & -0.11022000 \\
\hline $\mathrm{C}$ & 1.73922800 & 1.15790300 & 2.76999500 \\
\hline $\mathrm{H}$ & 0.74027200 & 0.74697200 & 2.93320100 \\
\hline $\mathrm{H}$ & 2.35617200 & 0.94817100 & 3.65146500 \\
\hline $\mathrm{H}$ & 1.62998600 & 2.23714800 & 2.65881100 \\
\hline $\mathrm{H}$ & 3.38667700 & 1.06324700 & 1.41646400 \\
\hline
\end{tabular}

$\mathrm{H} \quad-4.60394700 \quad 1.24222800 \quad 1.77863200$

$\begin{array}{lll}-5.29026500 & 2.63775000 & 0.26606500\end{array}$

$\begin{array}{llll}\text { C } & -6.64073000 & 2.70782800 & 0.99427800\end{array}$

$\begin{array}{lll}-5.49140000 & 2.71302000 & -1.25534600\end{array}$

$\begin{array}{llll}-6.50581000 & 2.72182100 & 2.08201500\end{array}$

$\begin{array}{llll}-7.19046400 & 3.61353700 & 0.71314900\end{array}$

$-7.27285000-1.84521800-0.74239000$

$\begin{array}{llll}-5.99680600 & 3.64609700 & -1.53002800\end{array}$

$\begin{array}{lll}-0.89268000 & 1.82076300 & 1.30500200\end{array}$

$-2.62287900-3.10096400-1.88030200$
0

$\begin{array}{lll}4.48490300 & 2.25198800 & -0.42867600\end{array}$

$\begin{array}{lll}3.55611000 & 1.26794100 & -2.13595500\end{array}$

$1.98055200-3.41065200-0.57737700$

$\begin{array}{llll}\mathrm{O} & 0.83043000 & 2.44960000 & -1.50051700\end{array}$

$\begin{array}{lll}1.66805900 & 0.80642400 & 0.23556400\end{array}$

$2.41876700 \quad 0.56674400 \quad 1.53107400$

$\begin{array}{lll}\text { C } & 1.93423100 & -1.79077900 \quad 2.42118000\end{array}$

$\begin{array}{llll}\text { C } & 2.21156300 & -3.16012800 & 2.49639200\end{array}$

$\begin{array}{llll}\text { C } & 3.28165200 & -3.68139700 & 1.77484600\end{array}$

$\begin{array}{llll}4.07303400 & -2.84560500 & 0.98149800\end{array}$

$3.78554400 \quad-1.47857200 \quad 0.91272300$

$\begin{array}{lll}3.47064000 & 2.31284700 & -1.30144000\end{array}$

$1.10941800-1.38500500-2.99803400$

$3.51560400-4.74154100 \quad 1.82762600$

$\begin{array}{llll}H & 4.90847500 & -3.26126500 & 0.42962100\end{array}$

$4.49718900-0.58932600-0.16444100$

$\begin{array}{llll}\text { C } & 5.53064000 & -1.06268000 & -0.68445600\end{array}$

$\begin{array}{lll}5.14322300 & -1.78261400 & -1.41584000\end{array}$

$5.90494700-0.18122200-1.20619900$ 


\begin{tabular}{|c|c|c|c|c|c|c|c|}
\hline \multicolumn{4}{|c|}{$\mathrm{TS}_{\mathrm{SID}}$} & F & 4.94941700 & 1.26837800 & 0.16219500 \\
\hline \multicolumn{4}{|c|}{-2506.98609244} & $\mathrm{~F}$ & 4.59570300 & -0.19592600 & -1.40758400 \\
\hline $\mathrm{Pd}$ & -0.25787600 & 0.36560800 & -0.15452200 & $\mathrm{~F}$ & 5.08487800 & 1.86183700 & -1.92897600 \\
\hline $\mathrm{O}$ & 2.26947600 & -1.82992400 & -1.72792500 & $\mathrm{O}$ & 2.55323700 & 2.81373600 & -0.32768900 \\
\hline $\mathrm{C}$ & 1.35767100 & -3.24653500 & -3.41997400 & $\mathrm{O}$ & 2.18441900 & 1.44543500 & -2.46686000 \\
\hline $\mathrm{C}$ & 1.22866600 & -2.40020400 & -2.16261400 & $\mathrm{~N}$ & 1.86770600 & 0.26026000 & -0.22751500 \\
\hline $\mathrm{O}$ & 0.07079800 & -2.30069600 & -1.64058600 & $\mathrm{C}$ & 2.33327400 & -0.09344600 & 1.16700900 \\
\hline $\mathrm{C}$ & -2.74334500 & 1.93243400 & -0.13191200 & $\mathrm{C}$ & 1.41222900 & -1.21125000 & 1.63938800 \\
\hline $\mathrm{C}$ & -1.65975200 & 2.73695000 & -0.87162800 & $\mathrm{C}$ & 0.20286000 & -1.50420900 & 0.96739400 \\
\hline $\mathrm{H}$ & -3.67522500 & 2.04160100 & -0.69057800 & $\mathrm{C}$ & -0.70475900 & -2.42464300 & 1.53366400 \\
\hline $\mathrm{H}$ & 1.20276100 & -2.60083400 & -4.29304900 & $\mathrm{C}$ & -0.40117300 & -3.06846400 & 2.72577900 \\
\hline $\mathrm{H}$ & 2.36166700 & -3.67192900 & -3.49062700 & $\mathrm{C}$ & 0.81668600 & -2.82324000 & 3.36644100 \\
\hline $\mathrm{H}$ & 0.60292500 & -4.03652200 & -3.43991600 & $\mathrm{C}$ & 1.72568300 & -1.90700200 & 2.82430200 \\
\hline $\mathrm{N}$ & -2.38929400 & 0.46390200 & -0.10850600 & $\mathrm{C}$ & 4.44443000 & 1.07905900 & -1.06537900 \\
\hline $\mathrm{H}$ & -2.67107400 & 0.02928200 & 0.76919600 & $\mathrm{H}$ & 0.16216300 & -1.64050300 & -0.25213600 \\
\hline $\mathrm{C}$ & -2.87244000 & -0.29628100 & -1.25792400 & $\mathrm{H}$ & -1.60843400 & -2.68125700 & 0.99162600 \\
\hline $\mathrm{O}$ & -2.85153100 & 0.20771600 & -2.35592800 & $\mathrm{H}$ & -1.09349300 & -3.78717200 & 3.15552200 \\
\hline $\mathrm{C}$ & -3.37727200 & -1.67039300 & -0.98015500 & $\mathrm{H}$ & 1.05356000 & -3.35194300 & 4.28253000 \\
\hline $\mathrm{C}$ & -3.12007600 & -2.66766300 & -1.93285100 & $\mathrm{H}$ & 2.02175600 & -0.63042600 & -0.87640300 \\
\hline $\mathrm{C}$ & -4.15769200 & -1.96923000 & 0.14842800 & $\mathrm{O}$ & 2.93810900 & -1.61974500 & 3.38402800 \\
\hline $\mathrm{C}$ & -3.62016700 & -3.95324500 & -1.74336500 & $\mathrm{C}$ & 3.34100400 & -2.32634300 & 4.54975300 \\
\hline $\mathrm{H}$ & -2.50177900 & -2.42724900 & -2.78924700 & $\mathrm{H}$ & 3.38828800 & -3.40696900 & 4.36588300 \\
\hline $\mathrm{C}$ & -4.67368100 & -3.25354300 & 0.32192700 & $\mathrm{H}$ & 4.33861600 & -1.95595100 & 4.79030500 \\
\hline $\mathrm{H}$ & -4.40384700 & -1.19945800 & 0.87667900 & $\mathrm{H}$ & 2.66699700 & -2.12835600 & 5.39305400 \\
\hline $\mathrm{C}$ & -4.39858800 & -4.24734500 & -0.62021900 & $\mathrm{C}$ & 2.27783000 & 1.09749600 & 2. 13925900 \\
\hline $\mathrm{H}$ & -3.40140900 & -4.72805200 & -2.47209600 & $\mathrm{H}$ & 1.27397100 & 1.53328400 & 2.15202400 \\
\hline $\mathrm{H}$ & -5.29290900 & -3.47468300 & 1.18646100 & $\mathrm{H}$ & 2.52730000 & 0.74338800 & 3.14108800 \\
\hline $\mathrm{H}$ & -4.79338900 & -5.24985500 & -0.48033500 & $\mathrm{H}$ & 2.98589500 & 1.87755100 & 1.85921700 \\
\hline $\mathrm{C}$ & -2.92762000 & 2.41069200 & 1.32153600 & $\mathrm{H}$ & 3.36214800 & -0.46483000 & 1.10865000 \\
\hline $\mathrm{H}$ & -3.70679900 & 1.78504800 & 1.78786800 & & & & \\
\hline $\mathrm{H}$ & -1.99585700 & 2.22178200 & 1.87323500 & $\mathrm{~S}_{2 \mathrm{D}}$ & & & \\
\hline $\mathrm{C}$ & -3.32317400 & 3.89195900 & 1.49864000 & -25 & .02655180 & & \\
\hline $\mathrm{C}$ & -3.44168600 & 4.20821300 & 2.99739400 & $\mathrm{Pd}$ & -0.25328100 & 0.40060900 & -0.01862000 \\
\hline $\mathrm{C}$ & -4.61671800 & 4.24889500 & 0.75170500 & $\mathrm{O}$ & 2.16240900 & -1.74007300 & -1.99969800 \\
\hline $\mathrm{H}$ & -2.51772500 & 4.50499700 & 1.07862000 & $\mathrm{C}$ & 1.30281200 & -2.83637900 & -3.96932200 \\
\hline $\mathrm{H}$ & -2.51244100 & 3.98031700 & 3.53392900 & $\mathrm{C}$ & 1.26628200 & -2.37261000 & -2.53758900 \\
\hline $\mathrm{H}$ & -3.66420500 & 5.26987500 & 3.15216800 & $\mathrm{O}$ & 0.12882900 & -2.71528300 & -1.91698200 \\
\hline $\mathrm{H}$ & -4.24990200 & 3.62886100 & 3.46403900 & $\mathrm{C}$ & -2.75267300 & 1.93767600 & -0.06766100 \\
\hline $\mathrm{H}$ & -4.47871100 & 4.18318800 & -0.33144000 & $\mathrm{C}$ & -1.73247500 & 2.70160300 & -0.94834900 \\
\hline $\mathrm{H}$ & -5.44530400 & 3.58940000 & 1.04604100 & $\mathrm{H}$ & -3.73155600 & 2.01040300 & -0.54750000 \\
\hline $\mathrm{H}$ & -4.91768900 & 5.27743400 & 0.98107800 & $\mathrm{H}$ & 0.66003600 & -2.17968500 & -4.56744700 \\
\hline $\mathrm{O}$ & -0.46458700 & 2.19373300 & -0.92108500 & $\mathrm{H}$ & 2.32320100 & -2.77432400 & -4.34850700 \\
\hline $\mathrm{O}$ & -1.92060500 & 3.83840200 & -1.32011500 & $\mathrm{H}$ & 0.91638400 & -3.85509400 & -4.05968600 \\
\hline $\mathrm{S}$ & 2.62361900 & 1.57681600 & -1.08964000 & $\mathrm{~N}$ & -2.39884700 & 0.46312000 & 0.02404500 \\
\hline
\end{tabular}




\begin{tabular}{|c|c|c|c|c|c|c|c|}
\hline $\mathrm{H}$ & -2.68232300 & 0.07961800 & 0.92404400 & $\mathrm{~F}$ & 4.93736800 & 1.47250300 & 0.41203400 \\
\hline $\mathrm{C}$ & -2.91605900 & -0.33144300 & -1.07476900 & $\mathrm{~F}$ & 4.60352300 & 0.03151600 & -1.18636200 \\
\hline $\mathrm{O}$ & -2.83345900 & 0.07835400 & -2.21084100 & $\mathrm{~F}$ & 5.09500900 & 2.10055200 & -1.66564400 \\
\hline $\mathrm{C}$ & -3.56059700 & -1.63670000 & -0.73540700 & $\mathrm{O}$ & 2.46708400 & 2.93851500 & -0.02814500 \\
\hline $\mathrm{C}$ & -3.52020200 & -2.64557100 & -1.71096400 & $\mathrm{O}$ & 2.24732500 & 1.69811300 & -2.27543100 \\
\hline $\mathrm{C}$ & -4.26115700 & -1.86164500 & 0.46060300 & $\mathrm{~N}$ & 1.87998000 & 0.37458300 & -0.10360400 \\
\hline $\mathrm{C}$ & -4.14320900 & -3.86860600 & -1.47984800 & $\mathrm{C}$ & 2.37626500 & -0.09944700 & 1.25534200 \\
\hline $\mathrm{H}$ & -2.98805100 & -2.45345800 & -2.63613100 & $\mathrm{C}$ & 1.47509700 & -1.27436700 & 1.56750300 \\
\hline $\mathrm{C}$ & -4.90135800 & -3.08157900 & 0.68015900 & $\mathrm{C}$ & 0.15725200 & -1.26227900 & 1.06911800 \\
\hline $\mathrm{H}$ & -4.34455600 & -1.08309300 & 1.21498500 & $\mathrm{C}$ & -0.67958800 & -2.35001900 & 1.36895300 \\
\hline $\mathrm{C}$ & -4.83561300 & -4.08818700 & -0.28517500 & $\mathrm{C}$ & -0.21095400 & -3.40814300 & 2.15357700 \\
\hline $\mathrm{H}$ & -4.09372000 & -4.65035300 & -2.23221000 & $\mathrm{C}$ & 1.09016500 & -3.41202600 & 2.65675600 \\
\hline $\mathrm{H}$ & -5.45384000 & -3.24227000 & 1.60130100 & $\mathrm{C}$ & 1.93823600 & -2.33686700 & 2.36354000 \\
\hline $\mathrm{H}$ & -5.32834300 & -5.04042500 & -0.10947700 & $\mathrm{C}$ & 4.44657200 & 1.30664200 & -0.82069000 \\
\hline $\mathrm{C}$ & -2.81393200 & 2.50145600 & 1.36345600 & $\mathrm{H}$ & 0.14674500 & -2.36624800 & -0.99725600 \\
\hline $\mathrm{H}$ & -3.54847700 & 1.91169100 & 1.93847200 & $\mathrm{H}$ & -1.69682300 & -2.38863400 & 0.99429900 \\
\hline $\mathrm{H}$ & -1.83661300 & 2.34321500 & 1.84111600 & $\mathrm{H}$ & -0.86871700 & -4.24411600 & 2.37942600 \\
\hline $\mathrm{C}$ & -3.19191500 & 3.99301000 & 1.48005800 & $\mathrm{H}$ & 1.43197100 & -4.24128500 & 3.26508800 \\
\hline $\mathrm{C}$ & -3.17928100 & 4.40603500 & 2.95972600 & $\mathrm{H}$ & 2.01678000 & -0.40238600 & -0.78188600 \\
\hline $\mathrm{C}$ & -4.54406900 & 4.31042800 & 0.82482600 & $\mathrm{O}$ & 3.22834100 & -2.22971400 & 2.80746700 \\
\hline $\mathrm{H}$ & -2.42592600 & 4.57123600 & 0.95124400 & $\mathrm{C}$ & 3.77182100 & -3.29056200 & 3.57958100 \\
\hline $\mathrm{H}$ & -2.20688100 & 4.20663000 & 3.42679900 & $\mathrm{H}$ & 3.76960300 & -4.23553700 & 3.02134900 \\
\hline $\mathrm{H}$ & -3.38690800 & 5.47692600 & 3.06549200 & $\mathrm{H}$ & 4.80136000 & -3.00272100 & 3.79943100 \\
\hline $\mathrm{H}$ & -3.94391400 & 3.86252600 & 3.53166100 & $\mathrm{H}$ & 3.22377300 & -3.42433800 & 4.52111200 \\
\hline $\mathrm{H}$ & -4.49177700 & 4.17826400 & -0.25953800 & $\mathrm{C}$ & 2.29786300 & 0.98004700 & 2.33989500 \\
\hline $\mathrm{H}$ & -5.34461500 & 3.67272800 & 1.22633900 & $\mathrm{H}$ & 1.27816200 & 1.36879300 & 2.42051200 \\
\hline $\mathrm{H}$ & -4.82644500 & 5.35226200 & 1.01635900 & $\mathrm{H}$ & 2.57338400 & 0.51531100 & 3.29136000 \\
\hline $\mathrm{O}$ & -0.54259300 & 2.18780100 & -1.01217700 & $\mathrm{H}$ & 2.97622100 & 1.81356500 & 2.15034700 \\
\hline $\mathrm{O}$ & -2.09778100 & 3.75067600 & -1.46224200 & $\mathrm{H}$ & 3.41181800 & -0.43427900 & 1.14813400 \\
\hline S & 2.61706100 & 1.76825700 & -0.87447100 & & & & \\
\hline $\mathrm{S}_{1 \mathrm{E}}$ & & & & $\mathrm{C}$ & -3.93053600 & 2.26964000 & 2.75485000 \\
\hline-250 & 7.05992055 & & & $\mathrm{H}$ & -2.95760000 & 0.38650500 & 2.47132700 \\
\hline $\mathrm{Pd}$ & 0.27108300 & -0.11188800 & 0.19493900 & $\mathrm{C}$ & -4.19003500 & 3.66665800 & 0.79783100 \\
\hline $\mathrm{C}$ & -1.79353500 & -1.87752300 & -0.60738500 & $\mathrm{H}$ & -3.43716000 & 2.82836900 & -1.04547700 \\
\hline $\mathrm{C}$ & -0.87671100 & -1.68629100 & -1.84091000 & $\mathrm{C}$ & -4.37084400 & 3.45694300 & 2.16860900 \\
\hline $\mathrm{O}$ & 0.19155100 & -0.94285200 & -1.61978000 & $\mathrm{H}$ & -4.07460100 & 2.09968700 & 3.81801700 \\
\hline $\mathrm{O}$ & -1.09675300 & -2.23414000 & -2.90560900 & $\mathrm{H}$ & -4.53398500 & 4.58824200 & 0.33658500 \\
\hline $\mathrm{H}$ & -1.28824200 & -2.60706000 & 0.03590400 & $\mathrm{H}$ & -4.85651800 & 4.21603200 & 2.77600000 \\
\hline $\mathrm{N}$ & -1.76818800 & -0.61401900 & 0.21558200 & $\mathrm{C}$ & -3.20675400 & -2.37410500 & -0.94242300 \\
\hline $\mathrm{C}$ & -2.52828200 & 0.49421300 & -0.32793900 & $\mathrm{H}$ & -3.87034000 & -2.14922400 & -0.09338100 \\
\hline $\mathrm{O}$ & -2.66144700 & 0.57248300 & -1.53355700 & $\mathrm{H}$ & -3.57578900 & -1.81159400 & -1.80372800 \\
\hline $\mathrm{C}$ & -3.11926500 & 1.49603500 & 0.60673900 & $\mathrm{C}$ & -3.28162200 & -3.88743500 & -1.24591300 \\
\hline $\mathrm{C}$ & -3.30410300 & 1.29005200 & 1.98289400 & $\mathrm{C}$ & -4.63745300 & -4.22043100 & -1.88641400 \\
\hline $\mathrm{C}$ & -3.57520700 & 2.69070500 & 0.02111500 & $\mathrm{C}$ & -3.05296700 & -4.75102800 & 0.00450900 \\
\hline
\end{tabular}




\begin{tabular}{|c|c|c|c|c|c|c|c|}
\hline $\mathrm{H}$ & -2.49883700 & -4.11039300 & -1.98022700 & $\mathrm{H}$ & 2.39412600 & 2.05474300 & 0.95270300 \\
\hline $\mathrm{H}$ & -4.78516500 & -3.65715800 & -2.81475200 & & & & \\
\hline $\mathrm{H}$ & -4.70782200 & -5.28820000 & -2.12509000 & \multicolumn{4}{|c|}{$\mathrm{TS}_{\mathrm{SIE}}$} \\
\hline $\mathrm{H}$ & -5.46652500 & -3.97666700 & -1.20828100 & \multicolumn{4}{|c|}{-2506.98367539} \\
\hline $\mathrm{H}$ & -2.07891600 & -4.56788100 & 0.47479500 & $\mathrm{Pd}$ & -0.39395300 & 0.13415200 & -0.40698900 \\
\hline $\mathrm{H}$ & -3.82683200 & -4.56127900 & 0.76047500 & $\mathrm{C}$ & 2.16921400 & 1.38642300 & -0.48067700 \\
\hline $\mathrm{H}$ & -3.09152400 & -5.81700100 & -0.24852500 & $\mathrm{C}$ & 1.34505000 & 2.00407000 & -1.63021100 \\
\hline S & 3.54684400 & -0.63555700 & 0.80880700 & $\mathrm{O}$ & 0.08755700 & 1.60863400 & -1.67756900 \\
\hline $\mathrm{F}$ & 1.72878500 & -2.17081100 & 2.03430100 & $\mathrm{O}$ & 1.80856700 & 2.84315100 & -2.37951200 \\
\hline $\mathrm{F}$ & 2.17228100 & -2.75055200 & -0.02236600 & $\mathrm{H}$ & 1.87178500 & 1.91683700 & 0.43099400 \\
\hline $\mathrm{F}$ & 3.62772700 & -3.10996900 & 1.55734500 & $\mathrm{~N}$ & 1.69539900 & -0.02998200 & -0.22324500 \\
\hline $\mathrm{O}$ & 3.70735700 & 0.02759900 & 2.09503100 & $\mathrm{C}$ & 2.25752100 & -1.01490200 & -1.13499900 \\
\hline $\mathrm{O}$ & 4.67447000 & -0.96325300 & -0.05851100 & $\mathrm{O}$ & 2.19348000 & -0.81377700 & -2.33048100 \\
\hline$N$ & 2.36822100 & 0.26939100 & -0.13197400 & $\mathrm{C}$ & 2.92556900 & -2.21814800 & -0.55879300 \\
\hline $\mathrm{C}$ & 2.61292100 & 1.78006300 & -0.08049600 & $\mathrm{C}$ & 3.32441000 & -2.33021400 & 0.78455200 \\
\hline $\mathrm{C}$ & 1.58611000 & 2.43374400 & -0.98910400 & $\mathrm{C}$ & 3.22054300 & -3.25896300 & -1.45712500 \\
\hline $\mathrm{C}$ & 0.78681300 & 3.46643000 & -0.49131900 & $\mathrm{C}$ & 4.00117200 & -3.47346100 & 1.21318600 \\
\hline $\mathrm{C}$ & -0.14243700 & 4.12127300 & -1.30170500 & $\mathrm{H}$ & 3.11009500 & -1.54941400 & 1.50704000 \\
\hline $\mathrm{C}$ & -0.28819500 & 3.72304400 & -2.62891600 & $\mathrm{C}$ & 3.88657900 & -4.39885700 & -1.02004000 \\
\hline $\mathrm{C}$ & 0.49099600 & 2.68819000 & -3.15088400 & $\mathrm{H}$ & 2.92127300 & -3.14753100 & -2.49379700 \\
\hline $\mathrm{C}$ & 1.43660100 & 2.05372400 & -2.34199000 & $\mathrm{C}$ & 4.27979300 & -4.50755100 & 0.31796200 \\
\hline $\mathrm{C}$ & 2.68130200 & -2.28501600 & 1.11658200 & $\mathrm{H}$ & 4.31078800 & -3.55206500 & 2.25133300 \\
\hline $\mathrm{H}$ & 0.88944000 & 3.74952800 & 0.55304500 & $\mathrm{H}$ & 4.10505300 & -5.20017000 & -1.72028800 \\
\hline $\mathrm{H}$ & -0.75249700 & 4.92065800 & -0.89279300 & $\mathrm{H}$ & 4.80572900 & -5.39504900 & 0.65969700 \\
\hline $\mathrm{H}$ & -1.01672800 & 4.21058700 & -3.27073000 & $\mathrm{C}$ & 3.68847200 & 1.49354300 & -0.67418700 \\
\hline $\mathrm{H}$ & 0.35305500 & 2.37879800 & -4.17974200 & $\mathrm{H}$ & 4.18336400 & 0.76193100 & -0.01845400 \\
\hline $\mathrm{O}$ & 2.25981500 & 1.04830000 & -2.78022800 & $\mathrm{H}$ & 3.92407100 & 1.21962800 & -1.70735800 \\
\hline $\mathrm{C}$ & 1.93990300 & 0.38019000 & -4.00765500 & $\mathrm{C}$ & 4.26524300 & 2.89578600 & -0.37389800 \\
\hline $\mathrm{H}$ & 2.02520600 & 1.06614600 & -4.85789900 & $\mathrm{C}$ & 5.69362100 & 2.99726600 & -0.92935100 \\
\hline $\mathrm{H}$ & 2.68470800 & -0.41037000 & -4.10888000 & $\mathrm{C}$ & 4.24746900 & 3.22578500 & 1.12665700 \\
\hline $\mathrm{H}$ & 0.94149200 & -0.06189900 & -3.95215800 & $\mathrm{H}$ & 3.64416500 & 3.62400000 & -0.90728300 \\
\hline $\mathrm{H}$ & 2.48858300 & -0.02256500 & -1.11441700 & $\mathrm{H}$ & 5.70962400 & 2.82662000 & -2.01170200 \\
\hline $\mathrm{C}$ & -0.19021000 & 0.16097100 & 3.03961900 & $\mathrm{H}$ & 6.11965900 & 3.98922400 & -0.73845300 \\
\hline $\mathrm{O}$ & -1.05557800 & -0.72630900 & 2.93700600 & $\mathrm{H}$ & 6.35494600 & 2.25602200 & -0.46035900 \\
\hline $\mathrm{O}$ & 0.44050100 & 0.71483900 & 2.04715200 & $\mathrm{H}$ & 3.23974000 & 3.18596500 & 1.55744900 \\
\hline $\mathrm{C}$ & 0.21402900 & 0.70964600 & 4.39832300 & $\mathrm{H}$ & 4.87546800 & 2.52361300 & 1.69116900 \\
\hline $\mathrm{H}$ & 1.29459800 & 0.59971400 & 4.53448700 & $\mathrm{H}$ & 4.63546000 & 4.23552900 & 1.30528000 \\
\hline $\mathrm{H}$ & -0.01356400 & 1.78023300 & 4.44673700 & $\mathrm{~S}$ & -3.14009900 & 2.16805400 & -0.23918600 \\
\hline $\mathrm{H}$ & -0.31951300 & 0.18372200 & 5.19197300 & $\mathrm{~F}$ & -0.85072700 & 2.70112800 & 1.08935800 \\
\hline $\mathrm{H}$ & -1.86314900 & -0.78155200 & 1.23061800 & $\mathrm{~F}$ & -1.14024900 & 3.86744600 & -0.73640600 \\
\hline $\mathrm{C}$ & 4.05182000 & 2.18157500 & -0.44209900 & $\mathrm{~F}$ & -2.34947300 & 4.26935500 & 1.01708600 \\
\hline $\mathrm{H}$ & 4.32216200 & 1.83723900 & -1.44315300 & $\mathrm{O}$ & -3.97429000 & 2.03671600 & 0.94936700 \\
\hline $\mathrm{H}$ & 4.11143900 & 3.27451400 & -0.42591300 & $\mathrm{O}$ & -3.63242800 & 2.68171700 & -1.51226600 \\
\hline $\mathrm{H}$ & 4.77842800 & 1.79728700 & 0.27806100 & $\mathrm{~N}$ & -2.48000200 & 0.52616200 & -0.60454000 \\
\hline
\end{tabular}




\begin{tabular}{|c|c|c|c|}
\hline $\mathrm{C}$ & -3.31174300 & -0.57144400 & 0.07120400 \\
\hline $\mathrm{C}$ & -2.42867300 & -1.81874600 & 0.15418600 \\
\hline $\mathrm{C}$ & -1.11611300 & -1.61867100 & 0.66300700 \\
\hline $\mathrm{C}$ & -0.26145600 & -2.71584000 & 0.86605500 \\
\hline $\mathrm{C}$ & -0.68276800 & -4.00357200 & 0.55862600 \\
\hline $\mathrm{C}$ & -1.95642300 & -4.20657600 & 0.03318500 \\
\hline 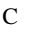 & -2.83638700 & -3.13009500 & -0.17290400 \\
\hline $\mathrm{C}$ & -1.72626700 & 3.32814200 & 0.31029000 \\
\hline $\mathrm{H}$ & -0.89114500 & -0.71916900 & 1.54690000 \\
\hline I & 0.71416300 & -2.54554400 & 1.30562800 \\
\hline $\mathrm{H}$ & -0.02776900 & -4.85489200 & 0.72170100 \\
\hline $\mathrm{H}$ & -2.27235700 & -5.21357500 & -0.21121000 \\
\hline $\mathrm{O}$ & -4.09090700 & -3.31382400 & -0.68026300 \\
\hline $\mathrm{C}$ & -4.51193600 & -4.62095400 & -1.04364000 \\
\hline $\mathrm{H}$ & -4.53575500 & -5.29396700 & -0.17714900 \\
\hline $\mathrm{H}$ & -5.52350700 & -4.50471200 & -1.43633100 \\
\hline I & -3.86828600 & -5.05042300 & -1.82178600 \\
\hline $\mathrm{H}$ & -2.60515400 & 0.47674400 & -1.62280200 \\
\hline $\mathrm{C}$ & 0.48301200 & 0.05704600 & 3.16972300 \\
\hline $\mathrm{O}$ & 1.50354600 & -0.27640600 & 2.51692100 \\
\hline $\mathrm{O}$ & -0.73002900 & -0.01147400 & 2.75785500 \\
\hline $\mathrm{C}$ & 0.65420900 & 0.57941100 & 4.59172500 \\
\hline $\mathrm{H}$ & 0.08443400 & 1.50463700 & 4.72322000 \\
\hline $\mathrm{H}$ & 0.24582000 & -0.15255100 & 5.29814500 \\
\hline $\mathrm{H}$ & 1.70868100 & 0.75043900 & 4.81765400 \\
\hline $\mathrm{H}$ & 1.77646700 & -0.25294100 & 0.79001200 \\
\hline $\mathrm{C}$ & -4.68743900 & -0.66059300 & -0.59435600 \\
\hline $\mathrm{H}$ & -4.61525800 & -0.97279700 & -1.64044200 \\
\hline $\mathrm{H}$ & -5.30857400 & -1.38948100 & -0.07349900 \\
\hline $\mathrm{H}$ & -5.18951800 & 0.30849100 & -0.54218300 \\
\hline $\mathrm{H}$ & -3.44375800 & -0.21109500 & 1.09603500 \\
\hline
\end{tabular}

$\mathrm{S}_{2 \mathrm{E}}$

$-2507.03396526$

$\begin{array}{llll}\mathrm{Pd} & 0.25672400 & -0.28332400 & -0.58243800 \\ \mathrm{C} & -2.50842600 & -1.14236400 & -0.37130300 \\ \mathrm{C} & -1.83585600 & -2.09185600 & -1.39987200 \\ \mathrm{O} & -0.54605200 & -1.94804600 & -1.51454300 \\ \mathrm{O} & -2.50741600 & -2.94390900 & -1.96370500 \\ \mathrm{H} & -2.29389800 & -1.57414100 & 0.61297600 \\ \mathrm{~N} & -1.79500300 & 0.19474600 & -0.31031000 \\ \mathrm{C} & -2.16470200 & 1.14587500 & -1.33850400 \\ \mathrm{O} & -2.28662500 & 0.77430300 & -2.48732300 \\ \mathrm{C} & -2.38524600 & 2.56400200 & -0.92514800\end{array}$

$\begin{array}{llll}\text { C } & -2.77140000 & 2.94590100 & 0.36971200\end{array}$

$\begin{array}{llll}\text { C } & -2.27289100 & 3.54105000 & -1.92773600\end{array}$

$\begin{array}{llll}\text { C } & -3.03000300 & 4.28778600 & 0.65269000\end{array}$

$\begin{array}{llll}\mathrm{H} & -2.89464200 & 2.21254100 & 1.16077100\end{array}$

$\begin{array}{llll}\text { C } & -2.51469700 & 4.87969100 & -1.63597000\end{array}$

$\begin{array}{llll}\mathrm{H} & -1.99735300 & 3.22675900 & -2.92887000\end{array}$

$\begin{array}{llll}\text { C } & -2.89523200 & 5.25548900 & -0.34396700\end{array}$

$\begin{array}{llll}\mathrm{H} & -3.34006400 & 4.57472600 & 1.65349200\end{array}$

H $\quad-2.41322800 \quad 5.63009600 \quad-2.41484400$

$\begin{array}{llll}\mathrm{H} & -3.09205500 & 6.29983600 & -0.11713600\end{array}$

$\begin{array}{llll}\text { C } & -4.02772300 & -1.00473500 & -0.55621800\end{array}$

$\begin{array}{llll}\text { H } & -4.36892500 & -0.06537600 & -0.09443600\end{array}$

$\mathrm{H} \quad-4.23795200 \quad-0.93871400 \quad-1.62720400$

$\begin{array}{llll}\text { C } & -4.82885100 & -2.17553500 & 0.05517500\end{array}$

$\begin{array}{llll}\text { C } & -6.24642300 & -2.21080600 & -0.53345600\end{array}$

$\begin{array}{llll}\text { C } & -4.88499800 & -2.09803700 & 1.58910900\end{array}$

H $\quad-4.32016600 \quad-3.10023800 \quad-0.24014400$

H $\quad-6.21651100 \quad-2.34400100 \quad-1.62054500$

$\begin{array}{llll}\mathrm{H} & -6.83010200 & -3.03612300 & -0.10810900\end{array}$

$\mathrm{H} \quad-6.78743900 \quad-1.27829800 \quad-0.32147200$

$\begin{array}{llll}\mathrm{H} & -3.88886600 & -2.04126400 & 2.04485700\end{array}$

$\mathrm{H} \quad-5.44460300 \quad-1.21056300 \quad 1.91492800$

$\begin{array}{llll}\mathrm{H} & -5.38719000 & -2.97876700 & 2.00676500\end{array}$

$\begin{array}{llll}\text { S } & 2.90854000 & -1.97568300 & 0.29407900\end{array}$

F $\quad 0.75862200 \quad-3.52651100 \quad 0.55541600$

F $\quad 1.81338500 \quad-3.73334800 \quad-1.35159700$

F $\quad 2.66760100 \quad-4.55686700 \quad 0.47490300$

$\begin{array}{llll}\text { O } & 2.53530900 & -1.46245300 & 1.60465700\end{array}$

$\begin{array}{llll}\text { O } & 4.29590800 & -2.25981400 & -0.06178100\end{array}$

N $\quad 2.22834400 \quad-0.91273000 \quad-0.95768600$

$\begin{array}{llll}\text { C } & 3.09594700 & 0.32812700 & -1.16469600\end{array}$

$\begin{array}{llll}\text { C } & 2.56422300 & 1.38627600 & -0.22953600\end{array}$

$\begin{array}{llll}\text { C } & 1.21965800 & 1.31902800 & 0.18667400\end{array}$

$\begin{array}{llll}\text { C } & 0.73987800 & 2.30966900 & 1.05507400\end{array}$

$\begin{array}{llll}\text { C } & 1.58003700 & 3.33978400 & 1.48454500\end{array}$

$\begin{array}{llll}\text { C } & 2.90842400 & 3.41393600 & 1.06295900\end{array}$

$\begin{array}{llll}\text { C } & 3.40092900 & 2.43269100 & 0.19603700\end{array}$

$\begin{array}{llll}\text { C } & 1.93790500 & -3.56889100 & -0.03687800\end{array}$

$\begin{array}{llll}\text { H } & 0.49826900 & -0.03519800 & 2.33852800\end{array}$

$\begin{array}{llll}\mathrm{H} & -0.28766500 & 2.29437500 & 1.40188800\end{array}$

$\begin{array}{llll}\mathrm{H} & 1.19587900 & 4.10392800 & 2.15627100\end{array}$

$\begin{array}{llll}\mathrm{H} & 3.54101100 & 4.22433800 & 1.40588700\end{array}$

$\begin{array}{llll}\text { O } & 4.68045300 & 2.40471900 & -0.29501600\end{array}$

$\begin{array}{llll}\text { C } & 5.60189900 & 3.38436400 & 0.16083000\end{array}$ 


\begin{tabular}{|c|c|c|c|c|c|c|c|}
\hline $\mathrm{H}$ & 5.72900000 & 3.33901800 & 1.24993000 & $\mathrm{C}$ & 2.17191300 & 6.49742500 & -0.52355700 \\
\hline $\mathrm{H}$ & 6.55133400 & 3.15093100 & -0.32444600 & $\mathrm{H}$ & 2.70435900 & 4.78560000 & 0.65944100 \\
\hline $\mathrm{H}$ & 5.28753200 & 4.39648900 & -0.12527300 & $\mathrm{H}$ & 2.16860800 & 5.77169700 & 2.89502500 \\
\hline $\mathrm{H}$ & 2.21532700 & -1.47850300 & -1.81386100 & $\mathrm{H}$ & 3.29610600 & 6.79752400 & 1.99003800 \\
\hline $\mathrm{C}$ & -1.05661600 & 0.02507400 & 3.45655500 & $\mathrm{H}$ & 1.58812200 & 7.24897400 & 2.10683300 \\
\hline $\mathrm{O}$ & -1.81002500 & 0.44366400 & 2.58781800 & $\mathrm{H}$ & 2.08137600 & 5.93100400 & -1.45634000 \\
\hline $\mathrm{O}$ & 0.23551100 & -0.24356400 & 3.26134900 & $\mathrm{H}$ & 1.43000400 & 7.30547000 & -0.54622100 \\
\hline $\mathrm{C}$ & -1.47921600 & -0.24851000 & 4.87771800 & $\mathrm{H}$ & 3.16708900 & 6.95646000 & -0.51641800 \\
\hline $\mathrm{H}$ & -1.27848500 & -1.29491000 & 5.12964800 & $\mathrm{O}$ & 0.71197800 & 1.86371200 & 0.14026100 \\
\hline $\mathrm{H}$ & -0.89110300 & 0.36883200 & 5.56482600 & $\mathrm{O}$ & 2.00119700 & 3.01649500 & -1.30188500 \\
\hline $\mathrm{H}$ & -2.54113600 & -0.03242300 & 4.99551900 & $\mathrm{~S}$ & 1.48104600 & -2.87492300 & -0.94173000 \\
\hline $\mathrm{H}$ & -1.86794900 & 0.56627100 & 0.64014700 & $\mathrm{~F}$ & 2.55315800 & -4.31200300 & 0.98469500 \\
\hline $\mathrm{C}$ & 3.00788100 & 0.71316200 & -2.64616500 & $\mathrm{~F}$ & 0.97962800 & -2.96349500 & 1.65793800 \\
\hline $\mathrm{H}$ & 1.97255800 & 0.92185900 & -2.93524400 & $\mathrm{~F}$ & 0.46224000 & -4.78862200 & 0.58983500 \\
\hline $\mathrm{H}$ & 3.60257200 & 1.61703000 & -2.80391300 & $\mathrm{O}$ & 1.85805300 & -3.90158900 & -1.91179000 \\
\hline $\mathrm{H}$ & 3.41233400 & -0.07718300 & -3.29100600 & $\mathrm{O}$ & 0.18319700 & -2.14454800 & -1.05710000 \\
\hline $\mathrm{H}$ & 4.13419400 & 0.07270600 & -0.93299700 & $\mathrm{~N}$ & 2.54703400 & -1.71709200 & -0.71365800 \\
\hline & & & & $\mathrm{C}$ & 4.02407200 & -1.82585800 & -0.73794400 \\
\hline $\mathrm{S}_{5 \mathrm{~A}}$ & & & & $\mathrm{C}$ & 4.48452200 & -0.59502500 & 0.01339700 \\
\hline & .73463239 & & & $\mathrm{C}$ & 3.54985700 & 0.40379500 & 0.33711900 \\
\hline Pd & 1.69413500 & 0.00562300 & -0.22190100 & $\mathrm{C}$ & 3.91233000 & 1.53848000 & 1.07028000 \\
\hline $\mathrm{C}$ & 0.09024900 & 4.12674100 & -0.38552500 & $\mathrm{C}$ & 5.24391500 & 1.68615600 & 1.46345200 \\
\hline C & 0.95776600 & 2.89368700 & -0.50445700 & $\mathrm{C}$ & 6.19742200 & 0.71655600 & 1.15010000 \\
\hline $\mathrm{H}$ & 0.15395500 & 4.69951700 & -1.31214800 & $\mathrm{C}$ & 5.81857900 & -0.42702500 & 0.43544700 \\
\hline $\mathrm{N}$ & -1.28759600 & 3.69374100 & -0.23795200 & $\mathrm{C}$ & 1.35044300 & -3.79919500 & 0.68134200 \\
\hline $\mathrm{H}$ & -1.46887000 & 2.91656900 & 0.38189900 & $\mathrm{H}$ & 2.53869800 & 2.18853700 & -1.25193800 \\
\hline $\mathrm{C}$ & -2.31810800 & 4.50460500 & -0.65633400 & $\mathrm{H}$ & 3.17612300 & 2.28032100 & 1.36171400 \\
\hline $\mathrm{O}$ & -2.09706300 & 5.59226900 & -1.18369900 & $\mathrm{H}$ & 5.54350400 & 2.56295400 & 2.03163300 \\
\hline $\mathrm{C}$ & -3.70522000 & 3.98356200 & -0.43184200 & $\mathrm{H}$ & 7.22210000 & 0.84979700 & 1.47633000 \\
\hline $\mathrm{C}$ & -3.99596400 & 2.62670700 & -0.22477100 & $\mathrm{O}$ & 6.66923800 & -1.44559900 & 0.11387800 \\
\hline $\mathrm{C}$ & -4.75264800 & 4.91505500 & -0.45742200 & $\mathrm{C}$ & 8.02064600 & -1.36858500 & 0.54561100 \\
\hline $\mathrm{C}$ & -5.31320500 & 2.21161500 & -0.02841300 & $\mathrm{H}$ & 8.09010900 & -1.31884200 & 1.63970500 \\
\hline $\mathrm{H}$ & -3.20809800 & 1.87872300 & -0.25347400 & $\mathrm{H}$ & 8.49727400 & -2.28497600 & 0.19389100 \\
\hline $\mathrm{C}$ & -6.06671600 & 4.50022700 & -0.25464100 & $\mathrm{H}$ & 8.53314400 & -0.50186900 & 0.10881700 \\
\hline $\mathrm{H}$ & -4.51232000 & 5.95713700 & -0.64041900 & I & -2.49067800 & -2.44581900 & -0.19959300 \\
\hline $\mathrm{C}$ & -6.34872100 & 3.14869300 & -0.03666600 & I & -5.18972000 & -2.52704600 & 0.65300500 \\
\hline $\mathrm{H}$ & -5.52879200 & 1.15745600 & 0.12125200 & $\mathrm{C}$ & 4.55836200 & -1.90230300 & -2.17779700 \\
\hline $\mathrm{H}$ & -6.87218900 & 5.22930400 & -0.26922700 & $\mathrm{H}$ & 4.16303300 & -2.79130000 & -2.67639500 \\
\hline $\mathrm{H}$ & -7.37429700 & 2.82457500 & 0.11795300 & $\mathrm{H}$ & 4.24456700 & -1.01716100 & -2.74169900 \\
\hline $\mathrm{C}$ & 0.55775600 & 5.01758600 & 0.80108500 & $\mathrm{H}$ & 5.65060800 & -1.95238700 & -2.17136500 \\
\hline $\mathrm{H}$ & -0.17295600 & 5.83363800 & 0.84934200 & $\mathrm{H}$ & 4.34162800 & -2.72358200 & -0.19217800 \\
\hline $\mathrm{H}$ & 0.45142600 & 4.43215800 & 1.72429400 & & & & \\
\hline $\mathrm{C}$ & 1.97864800 & 5.61064800 & 0.71679900 & \multicolumn{4}{|c|}{$\mathrm{TS}_{\mathrm{S3A}}$} \\
\hline $\mathrm{C}$ & 2.27506100 & 6.39966800 & 2.00234600 & \multicolumn{4}{|c|}{-2300.70981238} \\
\hline
\end{tabular}




\begin{tabular}{|c|c|c|c|c|c|c|c|}
\hline $\mathrm{Pd}$ & -0.38953500 & 0.41574800 & -0.44040800 & $\mathrm{C}$ & -2.12565500 & -3.04712300 & 0.76220300 \\
\hline $\mathrm{S}$ & 0.83704600 & 0.64345200 & 2.24797700 & $\mathrm{H}$ & -1.77200800 & -3.68113100 & 1.58631900 \\
\hline $\mathrm{F}$ & 0.44194200 & 2.66173800 & 3.92235600 & $\mathrm{H}$ & -2.08466600 & -2.01363700 & 1.12412400 \\
\hline $\mathrm{F}$ & -1.36553700 & 1.56563900 & 3.40365600 & $\mathrm{C}$ & -3.58395400 & -3.42384000 & 0.43193200 \\
\hline $\mathrm{F}$ & 0.08974500 & 0.68489500 & 4.76077500 & $\mathrm{C}$ & -4.46984700 & -3.12043000 & 1.65064700 \\
\hline $\mathrm{O}$ & 2.24258900 & 0.58492700 & 2.66254400 & $\mathrm{C}$ & -3.73628300 & -4.89193100 & 0.00364600 \\
\hline $\mathrm{O}$ & 0.09353900 & -0.63249800 & 2.06831500 & $\mathrm{H}$ & -3.93188300 & -2.78947100 & -0.39578800 \\
\hline $\mathrm{N}$ & 0.47946300 & 1.62341900 & 0.98107400 & $\mathrm{H}$ & -4.39712200 & -2.06777400 & 1.94769800 \\
\hline $\mathrm{C}$ & 1.54562500 & 2.47420900 & 0.34388100 & $\mathrm{H}$ & -5.52168900 & -3.33718600 & 1.43279000 \\
\hline $\mathrm{C}$ & 2.24432700 & 1.58803900 & -0.67580600 & $\mathrm{H}$ & -4.17446100 & -3.73245900 & 2.51258500 \\
\hline $\mathrm{C}$ & 1.49134700 & 0.50669900 & -1.15443600 & $\mathrm{H}$ & -3.20450200 & -5.11121300 & -0.92781700 \\
\hline $\mathrm{C}$ & 1.95329100 & -0.38529200 & -2.11608700 & $\mathrm{H}$ & -3.35599900 & -5.56779300 & 0.78087300 \\
\hline $\mathrm{C}$ & 3.23259400 & -0.17216700 & -2.63345800 & $\mathrm{H}$ & -4.79230800 & -5.13635300 & -0.15887300 \\
\hline $\mathrm{C}$ & 4.00861600 & 0.90677700 & -2.21210100 & $\mathrm{O}$ & -1.11319100 & -1.18687400 & -1.79518300 \\
\hline $\mathrm{C}$ & 3.51736000 & 1.79794300 & -1.24812900 & $\mathrm{O}$ & -2.14832500 & -3.01574000 & -2.50002600 \\
\hline $\mathrm{C}$ & -0.06681800 & 1.44986600 & 3.67643200 & $\mathrm{H}$ & -2.34308200 & -2.39440800 & -3.22972700 \\
\hline $\mathrm{H}$ & 1.35538100 & -1.21794800 & -2.46508500 & $\mathrm{C}$ & 2.38083500 & 3.28655900 & 1.34171100 \\
\hline $\mathrm{H}$ & 3.62682400 & -0.85416700 & -3.38209100 & $\mathrm{H}$ & 3.12408000 & 2.67662500 & 1.85500400 \\
\hline $\mathrm{H}$ & 4.99342100 & 1.05028500 & -2.64033200 & $\mathrm{H}$ & 2.89275700 & 4.08875700 & 0.80865500 \\
\hline $\mathrm{O}$ & 4.21020300 & 2.89674100 & -0.82381700 & $\mathrm{H}$ & 1.71301000 & 3.73206600 & 2.08450600 \\
\hline $\mathrm{C}$ & 5.51973900 & 3.11312700 & -1.32444200 & $\mathrm{H}$ & 0.94461100 & 3.20059500 & -0.21874000 \\
\hline $\mathrm{H}$ & 5.51617500 & 3.26925700 & -2.41114900 & & & & \\
\hline $\mathrm{H}$ & 5.87985000 & 4.01841300 & -0.83209300 & $\mathrm{~S}_{6 \mathrm{~A}}$ & & & \\
\hline $\mathrm{H}$ & 6.18813500 & 2.27718900 & -1.08083300 & -23 & 0.71259513 & & \\
\hline I & -3.21443000 & 0.92957900 & 0.17841200 & $\mathrm{Pd}$ & 0.41372800 & -0.65342500 & -0.40666000 \\
\hline I & -1.67177100 & 2.50980600 & -2.00970600 & $\mathrm{~S}$ & -0.80784100 & -0.71810500 & 2.16615000 \\
\hline $\mathrm{C}$ & -1.09089300 & -3.22114700 & -0.37431500 & $\mathrm{~F}$ & -0.58308500 & -2.65272200 & 3.93252200 \\
\hline $\mathrm{C}$ & -1.42180500 & -2.36284600 & -1.59607500 & $\mathrm{~F}$ & 1.32430100 & -1.78997100 & 3.33564500 \\
\hline $\mathrm{H}$ & -1.10756600 & -4.25101900 & -0.73867000 & $\mathrm{~F}$ & -0.00584600 & -0.67643000 & 4.64851700 \\
\hline $\mathrm{N}$ & 0.24242100 & -2.94682300 & 0.11740500 & $\mathrm{O}$ & -2.16907600 & -0.45236900 & 2.63010000 \\
\hline $\mathrm{H}$ & 0.34774100 & -2.35451500 & 0.93424100 & $\mathrm{O}$ & 0.05877500 & 0.44253100 & 1.78186800 \\
\hline $\mathrm{C}$ & 1.31405300 & -3.39950600 & -0.61149700 & $\mathrm{~N}$ & -0.59874900 & -1.82630900 & 0.94831400 \\
\hline $\mathrm{O}$ & 1.12315400 & -3.92858400 & -1.71039400 & $\mathrm{C}$ & -1.82966700 & -2.42465300 & 0.32376800 \\
\hline $\mathrm{C}$ & 2.68602800 & -3.26650200 & -0.02452700 & $\mathrm{C}$ & -2.42627200 & -1.34804600 & -0.56525800 \\
\hline $\mathrm{C}$ & 3.73667800 & -3.85878200 & -0.74148100 & $\mathrm{C}$ & -1.52364200 & -0.38461900 & -1.03230200 \\
\hline $\mathrm{C}$ & 2.96682400 & -2.60011100 & 1.17708300 & $\mathrm{C}$ & -1.87560600 & 0.64637300 & -1.89443100 \\
\hline $\mathrm{C}$ & 5.04260200 & -3.79284500 & -0.26365800 & $\mathrm{C}$ & -3.20857600 & 0.71283900 & -2.30471400 \\
\hline $\mathrm{H}$ & 3.50105800 & -4.36730000 & -1.67015700 & $\mathrm{C}$ & -4.14160800 & -0.23423500 & -1.88270200 \\
\hline $\mathrm{C}$ & 4.27773400 & -2.52429500 & 1.64731600 & $\mathrm{C}$ & -3.75758300 & -1.27489400 & -1.02624800 \\
\hline $\mathrm{H}$ & 2.18666000 & -2.11748400 & 1.75447200 & $\mathrm{C}$ & 0.05964200 & -1.52550300 & 3.62076400 \\
\hline $\mathrm{C}$ & 5.31649400 & -3.12326800 & 0.93245300 & $\mathrm{H}$ & -1.15539400 & 1.37200900 & -2.24964500 \\
\hline $\mathrm{H}$ & 5.84775400 & -4.26095300 & -0.82377000 & $\mathrm{H}$ & -3.52211900 & 1.51181300 & -2.97096600 \\
\hline $\mathrm{H}$ & 4.48037000 & -1.98838700 & 2.56983900 & $\mathrm{H}$ & -5.16527700 & -0.16048500 & -2.22954500 \\
\hline $\mathrm{H}$ & 6.33634200 & -3.06625200 & 1.30406000 & $\mathrm{O}$ & -4.60787900 & -2.25593100 & -0.60488500 \\
\hline
\end{tabular}




\begin{tabular}{|c|c|c|c|c|c|c|c|}
\hline $\mathrm{C}$ & -5.97164200 & -2.19061500 & -0.99504700 & $\mathrm{H}$ & -1.39902100 & -3.19240800 & -0.32973700 \\
\hline $\mathrm{H}$ & -6.08258400 & -2.26122900 & -2.08471700 & & & & \\
\hline $\mathrm{H}$ & -6.45547300 & -3.04965400 & -0.52695400 & \multicolumn{4}{|c|}{$\mathrm{TS}_{\mathrm{S4A}}$} \\
\hline $\mathrm{H}$ & -6.44698300 & -1.26717200 & -0.64040300 & \multicolumn{4}{|c|}{-2300.70868254} \\
\hline I & 3.10967200 & -1.27289900 & 0.23643500 & $\mathrm{Pd}$ & -0.75847100 & -0.42800100 & -0.22593700 \\
\hline I & 0.80636100 & -2.51398600 & -2.30300600 & $\mathrm{C}$ & 3.59697800 & -1.60096700 & 0.32903600 \\
\hline $\mathrm{C}$ & 1.58809300 & 3.00845800 & -0.42309800 & $\mathrm{C}$ & 2.16859600 & -1.66388300 & -0.19882400 \\
\hline $\mathrm{C}$ & 1.76885200 & 2.03370900 & -1.58608700 & $\mathrm{H}$ & 4.27410400 & -1.81049700 & -0.50182500 \\
\hline $\mathrm{H}$ & 1.74341400 & 3.99898400 & -0.85666500 & $\mathrm{~N}$ & 3.90210400 & -0.25832200 & 0.78328100 \\
\hline $\mathrm{N}$ & 0.23781700 & 2.96923700 & 0.09801700 & $\mathrm{H}$ & 3.26685500 & 0.14671100 & 1.45643600 \\
\hline $\mathrm{H}$ & 0.05682800 & 2.42342200 & 0.93008000 & $\mathrm{C}$ & 4.73576100 & 0.57161300 & 0.06669900 \\
\hline $\mathrm{C}$ & -0.76664100 & 3.60675000 & -0.58574100 & $\mathrm{O}$ & 5.33749000 & 0.18749900 & -0.93076200 \\
\hline $\mathrm{O}$ & -0.53760100 & 4.11785600 & -1.68410100 & $\mathrm{C}$ & 4.86447600 & 1.97883900 & 0.57881100 \\
\hline $\mathrm{C}$ & -2.11843700 & 3.69326800 & 0.05950800 & $\mathrm{C}$ & 4.53544800 & 2.36469400 & 1.88721000 \\
\hline $\mathrm{C}$ & -3.04744500 & 4.55330100 & -0.54479400 & $\mathrm{C}$ & 5.35444400 & 2.94022600 & -0.31629100 \\
\hline $\mathrm{C}$ & -2.49511800 & 2.97414300 & 1.20316000 & $\mathrm{C}$ & 4.67783100 & 3.69501500 & 2.28554600 \\
\hline $\mathrm{C}$ & -4.32414300 & 4.70318900 & -0.00917000 & $\mathrm{H}$ & 4.20508600 & 1.62952100 & 2.61614200 \\
\hline $\mathrm{H}$ & -2.74174100 & 5.09461000 & -1.43371900 & $\mathrm{C}$ & 5.48970000 & 4.26863900 & 0.08027000 \\
\hline $\mathrm{C}$ & -3.77803400 & 3.11699600 & 1.73151000 & $\mathrm{H}$ & 5.62303900 & 2.62037800 & -1.31750400 \\
\hline $\mathrm{H}$ & -1.81625600 & 2.27877000 & 1.68421500 & $\mathrm{C}$ & 5.14875700 & 4.64980500 & 1.38114400 \\
\hline $\mathrm{C}$ & -4.69274400 & 3.98443700 & 1.13129400 & $\mathrm{H}$ & 4.43410800 & 3.98114100 & 3.30499400 \\
\hline $\mathrm{H}$ & -5.03248300 & 5.37895700 & -0.48093000 & $\mathrm{H}$ & 5.86426600 & 5.00713900 & -0.62304600 \\
\hline $\mathrm{H}$ & -4.05916400 & 2.54229300 & 2.60924900 & $\mathrm{H}$ & 5.26028900 & 5.68481400 & 1.69288600 \\
\hline $\mathrm{H}$ & -5.68990100 & 4.09749800 & 1.54868300 & $\mathrm{C}$ & 3.80850600 & -2.62463700 & 1.47915100 \\
\hline $\mathrm{C}$ & 2.60844700 & 2.76340900 & 0.71480300 & $\mathrm{H}$ & 4.82607900 & -2.44222700 & 1.84795600 \\
\hline $\mathrm{H}$ & 2.35011000 & 3.48348500 & 1.50316100 & $\mathrm{H}$ & 3.12619800 & -2.36076300 & 2.29955100 \\
\hline $\mathrm{H}$ & 2.44461200 & 1.76162200 & 1.12736400 & $\mathrm{C}$ & 3.65049000 & -4.12196800 & 1.14612300 \\
\hline $\mathrm{C}$ & 4.09874100 & 2.92788100 & 0.35571500 & $\mathrm{C}$ & 3.85866300 & -4.94633700 & 2.42691900 \\
\hline $\mathrm{C}$ & 4.95050500 & 2.57759300 & 1.58604500 & $\mathrm{C}$ & 4.61162700 & -4.58052200 & 0.03903600 \\
\hline $\mathrm{C}$ & 4.43465900 & 4.33759500 & -0.15537200 & $\mathrm{H}$ & 2.62358800 & -4.30022400 & 0.80193900 \\
\hline $\mathrm{H}$ & 4.34812100 & 2.20555600 & -0.43335400 & $\mathrm{H}$ & 3.15838800 & -4.64740600 & 3.21586900 \\
\hline $\mathrm{H}$ & 4.75027800 & 1.55705000 & 1.93037000 & $\mathrm{H}$ & 3.70566300 & -6.01338700 & 2.23080000 \\
\hline $\mathrm{H}$ & 6.01876600 & 2.65160800 & 1.35318600 & $\mathrm{H}$ & 4.87770300 & -4.82172700 & 2.81598700 \\
\hline $\mathrm{H}$ & 4.74214700 & 3.26428400 & 2.41710400 & $\mathrm{H}$ & 4.41040300 & -4.08177900 & -0.91454800 \\
\hline $\mathrm{H}$ & 3.92470400 & 4.57424300 & -1.09508300 & $\mathrm{H}$ & 5.65533200 & -4.38070000 & 0.31551700 \\
\hline $\mathrm{H}$ & 4.15595500 & 5.09971500 & 0.58454600 & $\mathrm{H}$ & 4.51392000 & -5.65870700 & -0.13092600 \\
\hline $\mathrm{H}$ & 5.51133900 & 4.43274400 & -0.33727000 & $\mathrm{O}$ & 1.31381100 & -0.86208000 & 0.21148400 \\
\hline $\mathrm{O}$ & 1.24738300 & 0.92772300 & -1.73492900 & $\mathrm{O}$ & 1.94714100 & -2.62856800 & -1.05152600 \\
\hline $\mathrm{O}$ & 2.60138000 & 2.49639400 & -2.51413200 & $\mathrm{~S}$ & -3.06235400 & 0.17925000 & 1.44957200 \\
\hline $\mathrm{H}$ & 2.70564600 & 1.80047500 & -3.19389400 & $\mathrm{~F}$ & -5.52628300 & -0.50671200 & 0.82764800 \\
\hline $\mathrm{C}$ & -2.70690100 & -3.15998400 & 1.34724300 & $\mathrm{~F}$ & -4.22217700 & -2.20815700 & 1.20417200 \\
\hline $\mathrm{H}$ & -3.30998200 & -2.47954000 & 1.94839600 & $\mathrm{~F}$ & -4.91609000 & -0.98777000 & 2.86399600 \\
\hline $\mathrm{H}$ & -3.37415500 & -3.84058000 & 0.81617000 & $\mathrm{O}$ & -3.56520000 & 1.46301200 & 1.94766700 \\
\hline $\mathrm{H}$ & -2.06211800 & -3.74832700 & 2.00767700 & $\mathrm{O}$ & -1.91921500 & -0.47077700 & 2.12432400 \\
\hline
\end{tabular}




\begin{tabular}{|c|c|c|c|}
\hline $\mathrm{N}$ & -2.74078800 & 0.12674900 & -0.20672100 \\
\hline $\mathrm{C}$ & -2.88547300 & 1.43225400 & -0.91957500 \\
\hline $\mathrm{C}$ & -1.74839700 & 2.31656900 & -0.40736800 \\
\hline 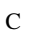 & -0.50108700 & 1.67231300 & -0.32246400 \\
\hline $\mathrm{C}$ & 0.65844700 & 2.26172000 & 0.19161100 \\
\hline $\mathrm{C}$ & 0.54292400 & 3.55070600 & 0.69956600 \\
\hline $\mathrm{C}$ & -0.67420700 & 4.23572400 & 0.63603400 \\
\hline $\mathrm{C}$ & -1.82057500 & 3.63448400 & 0.08814200 \\
\hline $\mathrm{C}$ & -4.53179600 & -0.97978700 & 1.58503800 \\
\hline I & 0.96150100 & -2.76269900 & -1.18373500 \\
\hline $\mathrm{H}$ & 1.60351100 & 1.73459100 & 0.19092600 \\
\hline $\mathrm{H}$ & 1.41330700 & 4.03900800 & 1.12902400 \\
\hline $\mathrm{H}$ & -0.72555300 & 5.25020400 & 1.01277200 \\
\hline $\mathrm{O}$ & -3.01849900 & 4.26446100 & -0.00074700 \\
\hline $\mathrm{C}$ & -3.19183900 & 5.51678100 & 0.65076400 \\
\hline H & -2.56917900 & 6.29726700 & 0.19502600 \\
\hline H & -4.24367800 & 5.77366800 & 0.51764500 \\
\hline $\mathrm{H}$ & -2.96639700 & 5.44238400 & 1.72141700 \\
\hline I & -1.36887700 & -3.14924100 & -0.56863400 \\
\hline I & 0.09058000 & 0.59567400 & -2.66954300 \\
\hline $\mathrm{C}$ & -4.33030300 & 1.94394300 & -0.95992600 \\
\hline $\mathrm{H}$ & -4.69593300 & 2.26993500 & 0.01116800 \\
\hline $\mathrm{H}$ & -4.38882000 & 2.78531800 & -1.65549300 \\
\hline $\mathrm{H}$ & -4.96511200 & 1.13573100 & -1.33543400 \\
\hline $\mathrm{H}$ & 2.63787900 & 1.16643000 & -1.95195400 \\
\hline
\end{tabular}

$\mathrm{S}_{7 \mathrm{~A}}$

$-2300.73812221$

$\begin{array}{llll}\text { Pd } & 0.93337600 & -0.30442700 & -0.26804400\end{array}$

$\begin{array}{llll}\text { C } & -3.33978300 & -1.71603900 & 0.08137700\end{array}$

$\begin{array}{llll}\text { C } & -1.83401100 & -1.68947300 & 0.25088900\end{array}$

$\mathrm{H} \quad-3.80163100 \quad-1.76791200 \quad 1.07242500$

N $\quad-3.75819000 \quad-0.47401200 \quad-0.54481700$

$\begin{array}{llll}\mathrm{H} & -3.05385500 & 0.04410600 & -1.05112900\end{array}$

$\begin{array}{llll}\text { C } & -4.96080100 & 0.10157500 & -0.23063500\end{array}$

$\begin{array}{llll}\text { O } & -5.73446200 & -0.41020000 & 0.57541400\end{array}$

$\begin{array}{llll}\text { C } & -5.27209700 & 1.39951600 & -0.92178600\end{array}$

$\begin{array}{llll}\text { C } & -4.70117200 & 1.77619000 & -2.14735500\end{array}$

$\begin{array}{llll}\text { C } & -6.19813400 & 2.25271000 & -0.30567200\end{array}$

$\begin{array}{llll}\text { C } & -5.03775000 & 2.99669000 & -2.73485000\end{array}$

$\begin{array}{llll}\mathrm{H} & -4.02523300 & 1.10332400 & -2.66842000\end{array}$

$\begin{array}{llll}\text { C } & -6.52449300 & 3.47614300 & -0.88817100\end{array}$

$\begin{array}{llll}\mathrm{H} & -6.65188900 & 1.93349700 & 0.62691400\end{array}$

$\begin{array}{llll}\text { C } & -5.94311700 & 3.85199900 & -2.10228400\end{array}$

\begin{tabular}{|c|c|c|c|}
\hline $\mathrm{H}$ & -4.60429300 & 3.27278600 & -3.69226200 \\
\hline & -7.23696300 & 4.13460900 & -0.39913900 \\
\hline & 6.20313700 & 4.80263900 & -2.55971600 \\
\hline & -3.81118800 & -2.94200200 & -0.75748000 \\
\hline & -4.88119700 & -2.76604100 & -0.92341800 \\
\hline & -3.32469500 & -2.88633900 & -1.74035400 \\
\hline G & -3.62565400 & -4.34998200 & -0.15838400 \\
\hline 0 & -4.16269800 & -5.38927800 & -1.15654000 \\
\hline 0 & -4.31280200 & -4.50314100 & 1.20740000 \\
\hline $\mathrm{H}$ & -2.55311400 & -4.53974300 & -0.02548800 \\
\hline $\mathrm{H}$ & -3.66319300 & -5.30908700 & -2.12937100 \\
\hline H & -4.00385200 & -6.40649600 & -0.78131200 \\
\hline t & -5.24025100 & -5.25851500 & -1.32004700 \\
\hline $\mathrm{H}$ & -3.86201100 & -3.86077400 & 1.97134700 \\
\hline $\mathrm{H}$ & -5.37989300 & -4.25474100 & 1.14236600 \\
\hline H & -4.23131300 & -5.53634100 & 1.56370500 \\
\hline $\mathrm{O}$ & -1.13367300 & -0.87957900 & -0.37480200 \\
\hline $\mathrm{O}$ & -1.36451600 & -2.59746500 & 1.07289800 \\
\hline S & 3.56701300 & 0.73643100 & -1.59206300 \\
\hline $\mathrm{F}$ & 5.70480000 & 0.43961500 & -0.08272300 \\
\hline $\mathrm{F}$ & 4.97034000 & -1.43222000 & -0.91586500 \\
\hline $\mathrm{F}$ & 6.00485200 & -0.01241000 & -2.19355000 \\
\hline $\mathrm{O}$ & 4.02124600 & 2.10156200 & -1.88163400 \\
\hline $\mathrm{O}$ & 2.90967500 & -0.05774700 & -2.63389900 \\
\hline N & 2.74576500 & 0.56350300 & -0.16428700 \\
\hline c & 2.41385600 & 1.67683000 & 0.76314900 \\
\hline C & 0.88281100 & 1.90022600 & 0.63841900 \\
\hline C & -0.01706600 & 1.76475900 & 1.73043100 \\
\hline C & -1.36910400 & 2.06598300 & 1.62100400 \\
\hline C & -1.87448200 & 2.52275100 & 0.39871000 \\
\hline C & -1.04403300 & 2.69984200 & -0.69914200 \\
\hline C & 0.32943800 & 2.42586300 & -0.58133100 \\
\hline C & 5.16206200 & -0.14132100 & -1.16345400 \\
\hline $\mathrm{H}$ & -0.37399000 & -2.66480700 & 0.97243600 \\
\hline $\mathrm{H}$ & -2.02962100 & 1.95306700 & 2.47268300 \\
\hline $\mathrm{H}$ & -2.93225200 & 2.75242100 & 0.31156400 \\
\hline $\mathrm{H}$ & -1.44731400 & 3.08360300 & -1.62845200 \\
\hline $\mathrm{O}$ & 1.22264600 & 2.69409100 & -1.54909700 \\
\hline C & 0.79079400 & 3.00382200 & -2.87349300 \\
\hline $\mathrm{H}$ & 0.26563000 & 3.96570400 & -2.90130700 \\
\hline $\mathrm{H}$ & 1.70765100 & 3.06105600 & -3.45877600 \\
\hline $\mathrm{H}$ & 0.14767600 & 2.20938600 & -3.26855400 \\
\hline I & 1.80245200 & -2.84106700 & -0.27584900 \\
\hline 1 & & Tr & 3.67269000 \\
\hline
\end{tabular}




\begin{tabular}{|c|c|c|c|c|c|c|c|}
\hline $\mathrm{C}$ & 3.24268500 & 2.96726700 & 0.73101400 & $\mathrm{~F}$ & -1.22073700 & -3.51898400 & 2.58395200 \\
\hline $\mathrm{H}$ & 3.09289900 & 3.54880000 & -0.17538900 & $\mathrm{~F}$ & -1.23312600 & -1.92235400 & 4.06507100 \\
\hline $\mathrm{H}$ & 2.95495100 & 3.56343900 & 1.60511100 & $\mathrm{O}$ & -1.12043800 & 0.24388600 & 1.99424800 \\
\hline $\mathrm{H}$ & 4.30543100 & 2.72455900 & 0.81541600 & $\mathrm{O}$ & 0.76250200 & -1.46520400 & 1.91198900 \\
\hline $\mathrm{H}$ & 2.56857400 & 1.23920600 & 1.75072000 & $\mathrm{~N}$ & -1.20555300 & -1.48717200 & 0.16697100 \\
\hline & & & & $\mathrm{C}$ & -0.74035200 & -2.77710600 & -0.44878000 \\
\hline $\mathrm{S}_{5 \mathrm{~B}}$ & & & & $\mathrm{C}$ & -0.04575100 & -2.42137500 & -1.73723000 \\
\hline-23 & 0.74947383 & & & $\mathrm{C}$ & -0.20349900 & -1.12877100 & -2.24564900 \\
\hline $\mathrm{Pd}$ & -1.29348800 & 0.09089600 & -1.15711600 & $\mathrm{C}$ & 0.40175800 & -0.72767500 & -3.43926700 \\
\hline $\mathrm{C}$ & -1.71056800 & 3.05385900 & -0.48128500 & $\mathrm{C}$ & 1.18071800 & -1.65408400 & -4.13342900 \\
\hline $\mathrm{C}$ & -1.46428200 & 2.87843500 & -1.96955000 & $\mathrm{C}$ & 1.36228200 & -2.95302500 & -3.64969600 \\
\hline $\mathrm{H}$ & -2.30899400 & 3.96007200 & -0.35638600 & $\mathrm{C}$ & 0.74839800 & -3.33803300 & -2.45302200 \\
\hline $\mathrm{N}$ & -2.48305900 & 1.88038500 & -0.02944000 & $\mathrm{C}$ & -1.55431100 & -2.24172800 & 2.81186000 \\
\hline $\mathrm{H}$ & -2.26065200 & 1.54890100 & 0.90976100 & $\mathrm{H}$ & -1.24194400 & 3.78356000 & -3.59548100 \\
\hline $\mathrm{C}$ & -3.82836000 & 1.88383600 & -0.46032600 & $\mathrm{H}$ & 0.26902100 & 0.27852700 & -3.82176400 \\
\hline $\mathrm{O}$ & -4.12014100 & 2.58327300 & -1.42487700 & $\mathrm{H}$ & 1.65898800 & -1.36461400 & -5.06596800 \\
\hline $\mathrm{C}$ & -4.83188200 & 1.04732400 & 0.25359600 & $\mathrm{H}$ & 1.98142900 & -3.64862500 & -4.20407400 \\
\hline $\mathrm{C}$ & -4.54002800 & 0.26131400 & 1.37795400 & $\mathrm{O}$ & 0.86607400 & -4.58024800 & -1.88887900 \\
\hline $\mathrm{C}$ & -6.14661700 & 1.08694400 & -0.24122100 & $\mathrm{C}$ & 1.71524600 & -5.52873700 & -2.51542300 \\
\hline $\mathrm{C}$ & -5.55241900 & -0.47238700 & 1.99580000 & $\mathrm{H}$ & 2.74842400 & -5.16345600 & -2.57716500 \\
\hline $\mathrm{H}$ & -3.53401800 & 0.19791600 & 1.77545700 & $\mathrm{H}$ & 1.68371500 & -6.42016200 & -1.88620300 \\
\hline $\mathrm{C}$ & -7.15285800 & 0.35467400 & 0.37999600 & $\mathrm{H}$ & 1.36084400 & -5.78347400 & -3.52301800 \\
\hline $\mathrm{H}$ & -6.35382300 & 1.70047800 & -1.11111000 & I & 3.10570900 & -0.22912300 & 1.16268500 \\
\hline $\mathrm{C}$ & -6.85693800 & -0.42642500 & 1.50136700 & I & 5.56941700 & 0.97780800 & 0.42493800 \\
\hline $\mathrm{H}$ & -5.31423400 & -1.08531900 & 2.85935800 & $\mathrm{C}$ & -1.95600800 & -3.69161500 & -0.66741500 \\
\hline $\mathrm{H}$ & -8.16675800 & 0.39092100 & -0.00828700 & $\mathrm{H}$ & -2.43126000 & -3.93339800 & 0.28788000 \\
\hline $\mathrm{H}$ & -7.64199200 & -0.99983000 & 1.98698400 & $\mathrm{H}$ & -2.69140500 & -3.19278500 & -1.30731900 \\
\hline $\mathrm{C}$ & -0.38358300 & 3.17144400 & 0.30399100 & $\mathrm{H}$ & -1.64065300 & -4.62214200 & -1.14858900 \\
\hline $\mathrm{H}$ & -0.64583500 & 3.18917300 & 1.37019700 & $\mathrm{H}$ & -0.03432700 & -3.28116200 & 0.22018900 \\
\hline $\mathrm{H}$ & 0.20106600 & 2.25725500 & 0.14805400 & & & & \\
\hline $\mathrm{C}$ & 0.48156200 & 4.40787000 & -0.02014500 & \multicolumn{4}{|c|}{$\mathrm{TS}_{\mathrm{S3B}}$} \\
\hline $\mathrm{C}$ & 1.78558000 & 4.33557400 & 0.78994200 & \multicolumn{4}{|c|}{-2300.67904254} \\
\hline $\mathrm{C}$ & -0.24975800 & 5.73365100 & 0.24439700 & $\mathrm{Pd}$ & 0.28182700 & -0.41961100 & -0.12061400 \\
\hline $\mathrm{H}$ & 0.75057000 & 4.37574700 & -1.08582700 & $\mathrm{C}$ & 2.69128400 & 1.97906700 & -1.13474600 \\
\hline $\mathrm{H}$ & 2.33818900 & 3.41240600 & 0.58499900 & $\mathrm{C}$ & 2.61245000 & 0.63696300 & -1.86288300 \\
\hline $\mathrm{H}$ & 2.43925900 & 5.18084600 & 0.54769600 & $\mathrm{H}$ & 3.14867900 & 2.66010600 & -1.85773400 \\
\hline $\mathrm{H}$ & 1.58072500 & 4.36994700 & 1.86751300 & $\mathrm{~N}$ & 1.36425400 & 2.47562300 & -0.82764100 \\
\hline $\mathrm{H}$ & -1.12214100 & 5.86746500 & -0.40423200 & $\mathrm{H}$ & 1.04852300 & 2.48063800 & 0.13632400 \\
\hline $\mathrm{H}$ & -0.58661600 & 5.79363100 & 1.28766200 & $\mathrm{C}$ & 0.57101800 & 2.88663100 & -1.87291400 \\
\hline $\mathrm{H}$ & 0.42059900 & 6.58172700 & 0.06532500 & $\mathrm{O}$ & 0.93649700 & 2.66870600 & -3.03007600 \\
\hline $\mathrm{O}$ & -1.24719600 & 1.78913600 & -2.50760100 & $\mathrm{C}$ & -0.69422600 & 3.62615300 & -1.56744800 \\
\hline $\mathrm{O}$ & -1.42763700 & 4.00770400 & -2.66209000 & $\mathrm{C}$ & -1.06808700 & 4.04104400 & -0.28130900 \\
\hline S & -0.64772300 & -1.10909700 & 1.62319900 & $\mathrm{C}$ & -1.51440200 & 3.95119700 & -2.65896100 \\
\hline $\mathrm{F}$ & -2.88067800 & -2.11580300 & 2.65905200 & $\mathrm{C}$ & -2.24514300 & 4.76565300 & -0.09317400 \\
\hline
\end{tabular}




\begin{tabular}{|c|c|c|c|c|c|c|c|}
\hline $\mathrm{H}$ & -0.45978800 & 3.80833700 & 0.58648300 & $\mathrm{H}$ & -6.63910600 & -2.44515100 & -0.41380800 \\
\hline $\mathrm{C}$ & -2.68721200 & 4.67628900 & -2.46870600 & $\mathrm{H}$ & -6.17034700 & -1.41359000 & -1.79343600 \\
\hline $\mathrm{H}$ & -1.20297000 & 3.63128800 & -3.64741800 & I & 2.22510600 & -1.60623300 & 1.58205300 \\
\hline $\mathrm{C}$ & -3.05450000 & 5.08670400 & -1.18383000 & I & 0.40690400 & -3.65662300 & -0.78771600 \\
\hline $\mathrm{H}$ & -2.52784800 & 5.07125800 & 0.90952100 & $\mathrm{C}$ & -3.50433200 & -1.08467400 & 1.89848600 \\
\hline $\mathrm{H}$ & -3.31395000 & 4.92485600 & -3.32083300 & $\mathrm{H}$ & -3.23442100 & -0.92587500 & 2.94516200 \\
\hline $\mathrm{H}$ & -3.96873300 & 5.65478200 & -1.03358500 & $\mathrm{H}$ & -4.07560000 & -0.22725400 & 1.53659000 \\
\hline $\mathrm{C}$ & 3.54875000 & 1.93104100 & 0.15204800 & $\mathrm{H}$ & -4.14009100 & -1.96863900 & 1.84368600 \\
\hline $\mathrm{H}$ & 3.52577200 & 2.95028700 & 0.56104600 & $\mathrm{H}$ & -1.76569200 & -2.24479300 & 1.44059400 \\
\hline $\mathrm{H}$ & 3.05239300 & 1.28422100 & 0.88321300 & & & & \\
\hline $\mathrm{C}$ & 5.01761000 & 1.49071800 & -0.00618400 & $\mathrm{~S}_{6 \mathrm{~B}}$ & & & \\
\hline $\mathrm{C}$ & 5.67321200 & 1.43104100 & 1.38280700 & -230 & 0.74258499 & & \\
\hline $\mathrm{C}$ & 5.81568300 & 2.40743700 & -0.94644000 & $\mathrm{Pd}$ & -0.34125200 & 0.45976900 & -0.48753900 \\
\hline $\mathrm{H}$ & 5.03436500 & 0.47394100 & -0.42289300 & $\mathrm{C}$ & -3.52926000 & 1.70650200 & -0.28739200 \\
\hline $\mathrm{H}$ & 5.13143800 & 0.75277600 & 2.05144300 & $\mathrm{C}$ & -2.64346000 & 2.15002400 & -1.44818000 \\
\hline $\mathrm{H}$ & 6.70836400 & 1.07888200 & 1.30994300 & $\mathrm{H}$ & -4.55300600 & 1.90616800 & -0.61496300 \\
\hline $\mathrm{H}$ & 5.69080300 & 2.42343000 & 1.85174400 & $\mathrm{~N}$ & -3.39016300 & 0.27753100 & -0.06942400 \\
\hline $\mathrm{H}$ & 5.43250800 & 2.38603500 & -1.97184600 & $\mathrm{H}$ & -2.91734600 & -0.04602900 & 0.76925300 \\
\hline $\mathrm{H}$ & 5.79072100 & 3.44704400 & -0.59373600 & $\mathrm{C}$ & -3.84051300 & -0.56837200 & -1.05272300 \\
\hline $\mathrm{H}$ & 6.86619200 & 2.09715700 & -0.98397400 & $\mathrm{O}$ & -4.21732000 & -0.09983900 & -2.13016600 \\
\hline $\mathrm{O}$ & 1.78572100 & -0.26265300 & -1.69502600 & $\mathrm{C}$ & -3.87440900 & -2.04008000 & -0.77777200 \\
\hline $\mathrm{O}$ & 3.58714100 & 0.47465000 & -2.75129400 & $\mathrm{C}$ & -3.60836400 & -2.60831800 & 0.47600200 \\
\hline $\mathrm{S}$ & -0.89738200 & 0.69186400 & 2.48573000 & $\mathrm{C}$ & -4.22635200 & -2.87520500 & -1.85033000 \\
\hline $\mathrm{F}$ & -2.94002400 & 2.06314400 & 1.38898800 & $\mathrm{C}$ & -3.68504900 & -3.99061700 & 0.64864800 \\
\hline $\mathrm{F}$ & -3.20381300 & 1.60960900 & 3.49837600 & $\mathrm{H}$ & -3.33789000 & -1.99243700 & 1.32696600 \\
\hline $\mathrm{F}$ & -1.79835700 & 3.15007100 & 2.88757000 & $\mathrm{C}$ & -4.30363500 & -4.25427800 & -1.67428600 \\
\hline $\mathrm{O}$ & 0.26631600 & 1.51795400 & 2.09889700 & $\mathrm{H}$ & -4.44702000 & -2.41525000 & -2.80778100 \\
\hline $\mathrm{O}$ & -0.94139700 & 0.02668100 & 3.78900600 & $\mathrm{C}$ & -4.03258800 & -4.81492000 & -0.42261900 \\
\hline $\mathrm{N}$ & -1.23430100 & -0.21970300 & 1.18434700 & $\mathrm{H}$ & -3.46513800 & -4.41827500 & 1.62185000 \\
\hline $\mathrm{C}$ & -2.23329400 & -1.31748400 & 1.07371100 & $\mathrm{H}$ & -4.57927600 & -4.89215600 & -2.50965100 \\
\hline $\mathrm{C}$ & -2.37690700 & -1.45868100 & -0.44216100 & $\mathrm{H}$ & -4.09316500 & -5.89090200 & -0.28303100 \\
\hline $\mathrm{C}$ & -1.20289900 & -1.19659200 & -1.18551800 & $\mathrm{C}$ & -3.23131100 & 2.47656200 & 1.02000400 \\
\hline $\mathrm{C}$ & -1.13055900 & -1.27345800 & -2.58711300 & $\mathrm{H}$ & -3.90870800 & 2.05725800 & 1.77651300 \\
\hline $\mathrm{C}$ & -2.31407600 & -1.51718900 & -3.28113800 & $\mathrm{H}$ & -2.21093700 & 2.24290100 & 1.34199300 \\
\hline $\mathrm{C}$ & -3.49623600 & -1.76779200 & -2.58819800 & $\mathrm{C}$ & -3.41943800 & 4.00606600 & 0.97346300 \\
\hline $\mathrm{C}$ & -3.53622400 & -1.76299100 & -1.17685900 & $\mathrm{C}$ & -2.99152800 & 4.60217400 & 2.32377400 \\
\hline $\mathrm{C}$ & -2.31460400 & 1.95658000 & 2.56656700 & $\mathrm{C}$ & -4.85765300 & 4.42033000 & 0.62464900 \\
\hline $\mathrm{H}$ & 3.49069500 & -0.41750800 & -3.14029300 & $\mathrm{H}$ & -2.74899800 & 4.41688600 & 0.20534600 \\
\hline $\mathrm{H}$ & -0.19002200 & -1.12022100 & -3.10090600 & $\mathrm{H}$ & -1.95427100 & 4.34277600 & 2.56201600 \\
\hline $\mathrm{H}$ & -2.31025900 & -1.55021300 & -4.36669500 & $\mathrm{H}$ & -3.07401900 & 5.69492100 & 2.31066500 \\
\hline $\mathrm{H}$ & -4.39712800 & -1.98269800 & -3.14992500 & $\mathrm{H}$ & -3.62856900 & 4.22943800 & 3.13636900 \\
\hline $\mathrm{O}$ & -4.67002000 & -2.04322400 & -0.48048500 & $\mathrm{H}$ & -5.15428000 & 4.09711200 & -0.37892600 \\
\hline $\mathrm{C}$ & -5.88353300 & -2.27913200 & -1.18311200 & $\mathrm{H}$ & -5.57214000 & 3.99813300 & 1.34372000 \\
\hline $\mathrm{H}$ & -5.81352900 & -3.17038700 & -1.81906500 & $\mathrm{H}$ & -4.96129500 & 5.51091100 & 0.65879500 \\
\hline
\end{tabular}




\begin{tabular}{|c|c|c|c|}
\hline $\mathrm{O}$ & -1.51272800 & 1.73292900 & -1.71817800 \\
\hline $\mathrm{O}$ & -3.18612300 & 3.10694500 & -2.19006400 \\
\hline S & 0.06839500 & -1.04745000 & 2.11492500 \\
\hline & -0.57984600 & -3.48592400 & 1.14662200 \\
\hline $\mathrm{F}$ & 0.56381600 & -3.53404100 & 2.99678200 \\
\hline $\mathrm{F}$ & -1.52626400 & -2.95317500 & 3.03066700 \\
\hline $\mathrm{O}$ & -1.25446300 & -0.39124700 & 2. 15493400 \\
\hline $\mathrm{O}$ & 1.06169600 & -0.81023900 & 3.16347400 \\
\hline $\mathrm{N}$ & 0.62665400 & -0.91714400 & 0.57284900 \\
\hline $\mathrm{C}$ & 2.06764900 & -1.18722900 & 0.31406500 \\
\hline $\mathrm{C}$ & 2.50824700 & -0.71954600 & -1.07537300 \\
\hline $\mathrm{C}$ & 3.78462700 & -0.17469100 & -1.28412800 \\
\hline C & 4.26040600 & 0.18088200 & -2.54924900 \\
\hline $\mathrm{C}$ & 3.45011800 & -0.01622000 & -3.65956000 \\
\hline $\mathrm{C}$ & 2.17767300 & -0.56333500 & -3.51031100 \\
\hline $\mathrm{C}$ & 1.71736800 & -0.91281700 & -2.24019800 \\
\hline C & -0.38966200 & -2.88398300 & 2.32400000 \\
\hline $\mathrm{H}$ & -2.53658900 & 3.35748600 & -2.87680100 \\
\hline $\mathrm{H}$ & 5.25077200 & 0.60538100 & -2.66093800 \\
\hline I & 3.80781400 & 0.25781100 & -4.64788400 \\
\hline $\mathrm{H}$ & 1.55061600 & -0.70916300 & -4.38099800 \\
\hline $\mathrm{O}$ & 0.45472700 & -1.43902600 & -2.04793100 \\
\hline $\mathrm{C}$ & -0.30553500 & -1.85085900 & -3.18565400 \\
\hline $\mathrm{H}$ & 0.25943500 & -2.57097700 & -3.78834000 \\
\hline $\mathrm{H}$ & -1.19934100 & -2.32690300 & -2.78051200 \\
\hline $\mathrm{H}$ & -0.59753700 & -0.99430700 & -3.80403600 \\
\hline I & 0.86127300 & 2.53913700 & 0.61927100 \\
\hline I & 5.18459300 & 0.14087500 & 0.31994700 \\
\hline $\mathrm{C}$ & 2.39962100 & -2.68976200 & 0.45261500 \\
\hline $\mathrm{H}$ & 2.24437800 & -3.03972700 & 1.47488400 \\
\hline $\mathrm{H}$ & 1.78442900 & -3.28355000 & -0.22839600 \\
\hline $\mathrm{H}$ & 3.45485500 & -2.84732300 & 0.20958800 \\
\hline & 2.65680000 & -0.63545500 & 1.04989600 \\
\hline
\end{tabular}

$\mathrm{S}_{5 \mathrm{C}}$

$-2529.85222073$

$\begin{array}{lrrr}\mathrm{Pd} & 1.16917500 & 1.00946300 & -0.48595900 \\ \mathrm{O} & 3.43606600 & 0.42720700 & -2.84045100 \\ \mathrm{C} & 2.21951100 & -0.14063600 & -4.79128900 \\ \mathrm{C} & 2.24949000 & 0.21428000 & -3.33157800 \\ \mathrm{O} & 1.20641800 & 0.27347300 & -2.64894700 \\ \mathrm{C} & -3.13871100 & 1.81459500 & -1.56925800 \\ \mathrm{C} & -1.70426000 & 1.33213500 & -1.67277400 \\ \mathrm{H} & -3.74913600 & 1.28139800 & -2.29903300\end{array}$

\begin{tabular}{|c|c|c|c|}
\hline $\mathrm{H}$ & 1.26183100 & 0.13865100 & -5.23430800 \\
\hline & 3.04585100 & 0.33764700 & -5.32093100 \\
\hline & 2.33965300 & 1.22696900 & -4.88285000 \\
\hline & 3.63605200 & 1.46708500 & -0.24775700 \\
\hline & -2.99705600 & 1.57082900 & 0.52787000 \\
\hline & 4.98383700 & 1.33106000 & -0.02015400 \\
\hline $\mathrm{O}$ & -5.80507100 & 1.46996300 & -0.92384100 \\
\hline C & -5.38707700 & 1.00757800 & 1.38844400 \\
\hline $\mathrm{C}$ & -4.51945400 & 0.42541900 & 2.32426000 \\
\hline C & -6.70943900 & 1.28850000 & 1.75881900 \\
\hline $\mathrm{C}$ & -4.96470400 & 0.14728300 & 3.61679600 \\
\hline H & -3.50759500 & 0.14695400 & 2.04220500 \\
\hline C & -7.15032800 & 1.01679200 & 3.05185300 \\
\hline $\mathrm{H}$ & -7.37324400 & 1.71669400 & 1.01498600 \\
\hline C & -6.27777600 & 0.44876000 & 3.98434200 \\
\hline $\mathrm{H}$ & -4.28918800 & -0.31457100 & 4.33127900 \\
\hline $\mathrm{H}$ & -8.17504300 & 1.24402300 & 3.33293200 \\
\hline $\mathrm{H}$ & -6.62300200 & 0.23241300 & 4.99178600 \\
\hline C & -3.21571000 & 3.34228300 & -1.83937200 \\
\hline $\mathrm{H}$ & -4.26864300 & 3.61330500 & -1.69896600 \\
\hline $\mathrm{H}$ & -2.63904100 & 3.84865200 & -1.05408900 \\
\hline $\mathrm{C}$ & -2.74087800 & 3.82578700 & -3.22441000 \\
\hline C & -2.81626700 & 5.35987700 & -3.28188300 \\
\hline C & -3.54283300 & 3.19683900 & -4.37489200 \\
\hline $\mathrm{H}$ & -1.68487800 & 3.54199700 & -3.35139400 \\
\hline $\mathrm{H}$ & -2.21975100 & 5.82186400 & -2.48625100 \\
\hline $\mathrm{H}$ & -2.44508600 & 5.73541200 & -4.24241200 \\
\hline $\mathrm{H}$ & -3.85197300 & 5.70434900 & -3.16728000 \\
\hline $\mathrm{H}$ & -3.41323500 & 2.11069100 & -4.42282300 \\
\hline $\mathrm{H}$ & -4.61448800 & 3.40482100 & -4.26422600 \\
\hline $\mathrm{H}$ & -3.22064800 & 3.60814300 & -5.33865200 \\
\hline $\mathrm{O}$ & -0.92141900 & 1.49227700 & -0.72449100 \\
\hline $\mathrm{O}$ & -1.39059100 & 0.79735100 & -2.82939300 \\
\hline S & 3.33567700 & -0.97615200 & 0.38677700 \\
\hline $\mathrm{F}$ & 5.31474500 & -1.22683000 & -1.36912900 \\
\hline $\mathrm{F}$ & 5.96276100 & -0.59074200 & 0.60837400 \\
\hline $\mathrm{F}$ & 5.35563000 & -2.65470500 & 0.27311500 \\
\hline $\mathrm{O}$ & 2.55708700 & -1.83196100 & -0.53283200 \\
\hline $\mathrm{O}$ & 3.25271100 & -1.15986100 & 1.83475300 \\
\hline $\mathrm{N}$ & 3.18170900 & 0.58913900 & -0.14114500 \\
\hline $\mathrm{C}$ & 3.74899800 & 1.62889900 & 0.79973900 \\
\hline $\mathrm{C}$ & 2.62594300 & 2.11772900 & 1.67856400 \\
\hline $\mathrm{C}$ & 1.30692000 & 1.89118500 & 1.26483000 \\
\hline$C$ & & & 2.0458460 \\
\hline
\end{tabular}




\begin{tabular}{|c|c|c|c|}
\hline C & 0.47132700 & 2.94509700 & 3.25496000 \\
\hline $\mathrm{C}$ & 1.77706300 & 3.19198900 & 3.68855900 \\
\hline $\mathrm{C}$ & 2.85574100 & 2.78036100 & 2.89853200 \\
\hline $\mathrm{C}$ & 5.11379200 & -1.38762500 & -0.05728200 \\
\hline $\mathrm{H}$ & -0.42740500 & 0.53414600 & -2.86152100 \\
\hline $\mathrm{H}$ & -0.79563200 & 2.11779400 & 1.71707700 \\
\hline 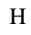 & -0.36160600 & 3.27052400 & 3.87347200 \\
\hline $\mathrm{H}$ & 1.94073400 & 3.69683500 & 4.63358400 \\
\hline $\mathrm{H}$ & 3.41115200 & 0.55244400 & -1.82010000 \\
\hline $\mathrm{O}$ & 4.17237800 & 2.96835000 & 3.22556200 \\
\hline $\mathrm{C}$ & 4.47939500 & 3.53575200 & 4.48968400 \\
\hline $\mathrm{H}$ & 4.07520200 & 2.92916100 & 5.31017100 \\
\hline 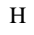 & 5.56895900 & 3.55181700 & 4.55397800 \\
\hline $\mathrm{H}$ & 4.09665300 & 4.56133800 & 4.57530100 \\
\hline $\mathrm{C}$ & 4.38743100 & 2.74011500 & -0.04233000 \\
\hline $\mathrm{H}$ & 4.78340000 & 3.51770300 & 0.61748000 \\
\hline $\mathrm{H}$ & 5.21049800 & 2.34136900 & -0.64598400 \\
\hline $\mathrm{H}$ & 3.64645100 & 3.19601600 & -0.70889800 \\
\hline 11 & 4.53137300 & 1.18038400 & 1.41799200 \\
\hline I & -2.47703900 & -4.33025000 & 0.18938800 \\
\hline I & 0.04636200 & -3.07562800 & -0.08724800 \\
\hline
\end{tabular}

$\mathrm{TS}_{\mathrm{S} 3 \mathrm{C}}$

$-2529.77931385$

$\begin{array}{llll}\mathrm{Pd} & 0.68364900 & -0.07050900 & -0.28021100\end{array}$

$\begin{array}{llll}\text { O } & 3.42090300 & 1.85660900 & -1.51697400\end{array}$

$\begin{array}{llll}\text { C } & 2.70652800 & 3.65355600 & -2.88377000\end{array}$

$\begin{array}{llll}\text { C } & 2.39860200 & 2.36258100 & -2.17257800\end{array}$

$\begin{array}{llll}\text { O } & 1.29768800 & 1.80637500 & -2.21348600\end{array}$

$\begin{array}{llll}\text { C } & -3.38926800 & 1.76648000 & -1.16768500\end{array}$

$\begin{array}{llll}\text { C } & -1.90062700 & 1.50515100 & -1.29038200\end{array}$

$\begin{array}{llll}\mathrm{H} & -3.80270000 & 1.94964400 & -2.16086200\end{array}$

$\mathrm{H} \quad 1.78271500 \quad 4.16930100 \quad-3.14954900$

$\begin{array}{llll}\mathrm{H} & 3.34032600 & 4.29191500 & -2.26285900\end{array}$

$\begin{array}{llll}\mathrm{H} & 3.26203800 & 3.42597100 & -3.80113400\end{array}$

$\begin{array}{llll}\mathrm{N} & -4.01720000 & 0.56861000 & -0.63677000\end{array}$

$\begin{array}{llll}\mathrm{H} & -3.48225700 & 0.01429300 & 0.01868400\end{array}$

$\begin{array}{llll}\text { C } & -5.35297700 & 0.33937300 & -0.84287700\end{array}$

$\begin{array}{llll}\text { O } & -6.04217400 & 1.12923100 & -1.48835000\end{array}$

$\begin{array}{llll}\text { C } & -5.92501100 & -0.90892900 & -0.23788600\end{array}$

$\begin{array}{llll}\text { C } & -5.14558600 & -1.99745300 & 0.18059200\end{array}$

$\begin{array}{llll}\text { C } & -7.31983500 & -0.97276000 & -0.11106500\end{array}$

$\begin{array}{llll}\text { C } & -5.75560600 & -3.12438000 & 0.73248900\end{array}$

$\begin{array}{llll}\mathrm{H} & -4.06628300 & -1.99606600 & 0.05280300\end{array}$

\begin{tabular}{|c|c|c|c|}
\hline $\mathrm{C}$ & -7.92593300 & -2.09642400 & 0.44530600 \\
\hline $\mathrm{H}$ & -7.90674000 & -0.12908300 & -0.45872800 \\
\hline C & -7.14416300 & -3.17395500 & 0.87111200 \\
\hline H & -5.14380300 & -3.96517500 & 1.04697100 \\
\hline 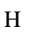 & -9.00733900 & -2.13422400 & 0.54547300 \\
\hline $\mathrm{H}$ & -7.61613800 & -4.05239400 & 1.30324300 \\
\hline C & -3.65312000 & 3.00374300 & -0.26351900 \\
\hline H & -4.74388800 & 3.07759500 & -0.18611900 \\
\hline $\mathrm{H}$ & -3.27266900 & 2.76940900 & 0.73990500 \\
\hline C & -3.08185100 & 4.35482100 & -0.73771200 \\
\hline $\mathrm{C}$ & -3.38233300 & 5.43270600 & 0.31643500 \\
\hline $\mathrm{C}$ & -3.62486800 & 4.77376900 & -2.11324000 \\
\hline $\mathrm{H}$ & -1.98753000 & 4.26699500 & -0.81868400 \\
\hline $\mathrm{H}$ & -2.97613900 & 5.16117800 & 1.29849200 \\
\hline $\mathrm{H}$ & -2.94883200 & 6.39707600 & 0.02687000 \\
\hline $\mathrm{H}$ & -4.46409700 & 5.57537000 & 0.43207800 \\
\hline $\mathrm{H}$ & -3.31574300 & 4.08426300 & -2.90532600 \\
\hline $\mathrm{H}$ & -4.72140200 & 4.81038700 & -2.10540200 \\
\hline $\mathrm{H}$ & -3.25864100 & 5.77085800 & -2.38474300 \\
\hline $\mathrm{O}$ & -1.31636000 & 0.85097900 & -0.41312700 \\
\hline $\mathrm{O}$ & -1.33595600 & 2.05737000 & -2.33547300 \\
\hline S & 3.00555500 & -1.93051100 & -1.26446300 \\
\hline $\mathrm{F}$ & 4.50486700 & -0.38244600 & -2.82681700 \\
\hline F & 5.68032000 & -1.51063900 & -1.38711100 \\
\hline $\mathrm{F}$ & 4.77790100 & -2.52660500 & -3.08023400 \\
\hline $\mathrm{O}$ & 2.02647300 & -1.88205500 & -2.35028900 \\
\hline $\mathrm{O}$ & 3.27583100 & -3.14642200 & -0.49559500 \\
\hline $\mathrm{N}$ & 2.71858800 & -0.57740000 & -0.30141700 \\
\hline $\mathrm{C}$ & 3.19769300 & -0.57490500 & 1.13072800 \\
\hline C & 2.64449600 & 0.74812600 & 1.65365500 \\
\hline $\mathrm{C}$ & 1.32256600 & 1.04281400 & 1.25366400 \\
\hline 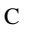 & 0.63225700 & 2.20574300 & 1.63835400 \\
\hline C & 1.33024100 & 3.16021500 & 2.37126500 \\
\hline $\mathrm{C}$ & 2.64705600 & 2.92345600 & 2.76280700 \\
\hline $\mathrm{C}$ & 3.30928000 & 1.72719400 & 2.42162500 \\
\hline C & 4.60982700 & -1.55263400 & -2.18614500 \\
\hline $\mathrm{H}$ & -0.33994000 & 1.95834200 & -2.33758600 \\
\hline $\mathrm{H}$ & -0.40471300 & 2.34791400 & 1.36476500 \\
\hline $\mathrm{H}$ & 0.83851400 & 4.08091800 & 2.67214200 \\
\hline $\mathrm{H}$ & 3.16088700 & 3.67225000 & 3.35295300 \\
\hline $\mathrm{H}$ & 3.16753300 & 0.96200000 & -1.11756600 \\
\hline $\mathrm{O}$ & 4.58625900 & 1.47337500 & 2.80908100 \\
\hline C & 5.30003000 & 2.44863100 & 3.55851100 \\
\hline $\mathrm{H}$ & 4.81888900 & 2.64408800 & 4.52486600 \\
\hline
\end{tabular}




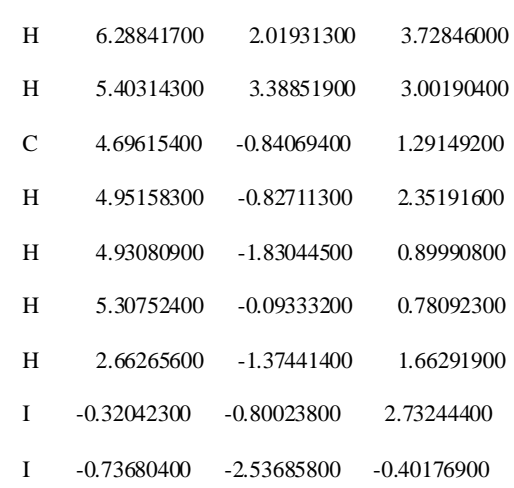

$\mathrm{S}_{6 \mathrm{C}}$

$-2529.84904409$

$\begin{array}{llll}\mathrm{Pd} & 0.20260200 & -0.27433100 & -0.43197300\end{array}$

$\begin{array}{llll}\mathrm{O} & 2.89515200 & 0.47074900 & -2.32374100\end{array}$

$\begin{array}{llll}\text { C } & 1.99321700 & 1.84985500 & -4.03057200\end{array}$

$\begin{array}{llll}\text { C } & 1.83678100 & 1.06837600 & -2.75642800\end{array}$

$\begin{array}{lllll}\mathrm{O} & 0.72800300 & 0.99473000 & -2.17519900\end{array}$

$\begin{array}{llll}\text { C } & -3.86343100 & 1.36488900 & -1.35748500\end{array}$

$\begin{array}{llll}\text { C } & -2.38054000 & 1.10172300 & -1.51516900\end{array}$

$\begin{array}{llll}\mathrm{H} & -4.32228200 & 1.43683500 & -2.34545300\end{array}$

$\begin{array}{llll}\mathrm{H} & 1.26127500 & 2.65833800 & -4.07831700\end{array}$

$\begin{array}{llll}\mathrm{H} & 3.00760900 & 2.24536100 & -4.11117100\end{array}$

$\begin{array}{llll}\mathrm{H} & 1.82180600 & 1.17368100 & -4.87697900\end{array}$

$\begin{array}{llll}\mathrm{N} & -4.45029800 & 0.22252800 & -0.67976800\end{array}$

$\begin{array}{llll}\mathrm{H} & -3.89924400 & -0.21917000 & 0.04432900\end{array}$

$\begin{array}{llll}\text { C } & -5.76996500 & -0.09645500 & -0.86845100\end{array}$

$\begin{array}{llll}\text { O } & -6.48606800 & 0.56836400 & -1.61693600\end{array}$

$\begin{array}{llll}\text { C } & -6.28862700 & -1.28804300 & -0.11745900\end{array}$

$\begin{array}{llll}\text { C } & -5.46291700 & -2.26191500 & 0.46404500\end{array}$

$\begin{array}{llll}\text { C } & -7.68094600 & -1.42244200 & -0.02231400\end{array}$

$\begin{array}{llll}\text { C } & -6.02556200 & -3.34336400 & 1.14259300\end{array}$

$\begin{array}{llll}\mathrm{H} & -4.38134300 & -2.20855700 & 0.37355800\end{array}$

$\begin{array}{lllll}\text { C } & -8.24030500 & -2.50009400 & 0.65991300\end{array}$

$\begin{array}{llll}\mathrm{H} & -8.30292100 & -0.66923500 & -0.49441700\end{array}$

$\begin{array}{llll}\text { C } & -7.41299700 & -3.46189500 & 1.24625800\end{array}$

$\begin{array}{llll}\mathrm{H} & -5.37751500 & -4.09535000 & 1.58371500\end{array}$

$\begin{array}{llll}\mathrm{H} & -9.32056600 & -2.59233300 & 0.73327200\end{array}$

$\begin{array}{llll}\mathrm{H} & -7.84803600 & -4.30478600 & 1.77674500\end{array}$

$\begin{array}{llll}\text { C } & -4.10928100 & 2.68789400 & -0.57640200\end{array}$

$\begin{array}{llll}\mathrm{H} & -5.19582600 & 2.74083100 & -0.43931800\end{array}$

$\begin{array}{llll}\mathrm{H} & -3.66115700 & 2.57553700 & 0.41973800\end{array}$

$\begin{array}{llll}\text { C } & -3.61472700 & 3.99432600 & -1.22823400\end{array}$

$\begin{array}{llll}\text { C } & -3.88618800 & 5.17029500 & -0.27607600\end{array}$

$\begin{array}{lrrr}\text { C } & -4.25781800 & 4.24493200 & -2.60127500\end{array}$

\begin{tabular}{|c|c|c|c|}
\hline & -2.52594900 & 3.92824500 & -1.36983800 \\
\hline $\mathrm{H}$ & -3.39916400 & 5.02163200 & 0.6949370 \\
\hline & -3.51434600 & 6.11069600 & -0.69915200 \\
\hline & -4.96239100 & 5.28838100 & -0.09628000 \\
\hline & -3.98250600 & 3.47702400 & -3.33169500 \\
\hline & -5.35224800 & 4.25979200 & -2.52420200 \\
\hline & -3.93759700 & 5.21215700 & -3.00610000 \\
\hline & -1.77237800 & 0.46795100 & -0.64213300 \\
\hline & -1.82669500 & 1.61877500 & -2.58919300 \\
\hline & 2.21393100 & -2.57254000 & -0.61065500 \\
\hline $\mathrm{F}$ & 4.12282100 & -1.99421300 & -2.40572300 \\
\hline $\mathrm{F}$ & 4.89811900 & -2.87151700 & -0.56916100 \\
\hline & 3.80555400 & -4.10082500 & -1.98240800 \\
\hline & 1.28797800 & -2.68426400 & -1.74197700 \\
\hline & 2.19947000 & -3.52435500 & 0.4968390 \\
\hline $\mathrm{N}$ & 2.18068800 & -0.93476300 & -0.17073700 \\
\hline & 2.74501400 & -0.43049500 & 1.156479 \\
\hline & 3.33651400 & 0.96519200 & 0.939289 \\
\hline & 2.81035200 & 2.12731900 & 1.521373 \\
\hline & 3.38343100 & 3.39150700 & 1.342460 \\
\hline & 4.52553900 & 3.51265300 & 0.5614330 \\
\hline & 5.09709400 & 2.38868800 & -0.0318260 \\
\hline & 4.51411600 & 1.13351000 & 0.159760 \\
\hline & 3.88045100 & -2.88594900 & -1.43611200 \\
\hline & -0.84291700 & 1.45457200 & -2.5701510 \\
\hline & 2.94389700 & 4.26348200 & 1.8116180 \\
\hline & 4.97999900 & 4.48884900 & 0.416219 \\
\hline & 5.99128600 & 2.49464300 & -0.6334310 \\
\hline & 2.68583600 & -0.10125100 & -1.4691810 \\
\hline & 5.02153900 & -0.00500300 & -0.3901220 \\
\hline & 6.17626400 & 0.08383900 & -1.21569900 \\
\hline & 7.03212400 & 0.48689800 & -0.6607450 \\
\hline & 6.39113100 & -0.93700100 & -1.52758600 \\
\hline & 5.98196000 & 0.70366900 & -2.0990830 \\
\hline & 3.70874800 & -1.34836100 & 1.93116700 \\
\hline & 3.98723900 & -0.81645600 & 2.8473810 \\
\hline & 3.21101100 & -2.27717200 & 2.2107530 \\
\hline 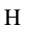 & 4.61721400 & -1.57723000 & $1.3776060 \mathrm{c}$ \\
\hline & 1.87762800 & -0.31478400 & 1.80318500 \\
\hline 1 & 1.07626600 & 2.10643600 & 2.79528300 \\
\hline I & -0.80681500 & -1.72859200 & 1.53986000 \\
\hline
\end{tabular}

$\begin{array}{llll}\mathrm{H} & -2.52594900 & 3.92824500 & -1.36983800\end{array}$

$\begin{array}{llll}H & -3.39916400 & 5.02163200 & 0.69493700\end{array}$

H $\quad-3.51434600 \quad 6.11069600 \quad-0.69915200$

$\begin{array}{lll}-4.96239100 & 5.28838100 & -0.09628000\end{array}$

$-3.98250600-3.47702400-3.33169500$

$-3.93759700 \quad 5.21215700-3.00610000$

$\begin{array}{llll}\text { O } & -1.77237800 & 0.46795100 & -0.64213300\end{array}$

$\begin{array}{llll}\text { O } & -1.82669500 & 1.61877500 & -2.58919300\end{array}$

$\begin{array}{lll}2.21393100 & -2.57254000 & -0.61065500\end{array}$

$\begin{array}{lll}4.12282100 & -1.99421300 & -2.40572300\end{array}$

$\begin{array}{lll}4.89811900 & -2.87151700 & -0.56916100\end{array}$

$\begin{array}{llll}3.80555400 & -4.10082500 & -1.98240800\end{array}$

$\begin{array}{lll}2.19947000 & -3.52435500 & 0.49683900\end{array}$

N $\quad 2.18068800 \quad-0.93476300 \quad-0.17073700$

$\begin{array}{llll}\text { C } & 2.74501400 & -0.43049500 & 1.15647900\end{array}$

$\begin{array}{llll} & 3.33651400 & 0.96519200 & 0.93928900\end{array}$

$\begin{array}{llll}\text { C } & 2.81035200 & 2.12731900 & 1.52137300\end{array}$

$\begin{array}{lll}3.38343100 & 3.39150700 & 1.34246000\end{array}$

$\begin{array}{llll}\text { C } & 5.09709400 & 2.38868800 & -0.03182600\end{array}$

$\begin{array}{lll}4.51411600 & 1.13351000 & 0.15976000\end{array}$

$\begin{array}{llll}\text { C } & 3.88045100 & -2.88594900 & -1.43611200\end{array}$

$2.94389700-4.26348200-1.81161800$

$\begin{array}{llll} & 4.97999900 & 4.48884900 & 0.41621900\end{array}$

$\begin{array}{lll}5.99128600 & 2.49464300 & -0.63343100\end{array}$

$\begin{array}{llll}5.02153900 & -0.00500300 & -0.39012200\end{array}$

$\begin{array}{lll}6.17626400 & 0.08383900 & -1.21569900\end{array}$

$\begin{array}{llll}7.03212400 & 0.48689800 & -0.66074500\end{array}$

$5.98196000-0.70366900--209908300$

$\begin{array}{llll}\text { C } & 3.70874800 & -1.34836100 & 1.93116700\end{array}$

C. $3.98723900,-0.81645600,2.84738100$

H $\quad 4.61721400 \quad-1.57723000 \quad 1.37760600$

$\begin{array}{lll}1.87762800 & -0.31478400 & 1.80318500\end{array}$

$-2529.84904439$ 


\begin{tabular}{|c|c|c|c|c|c|c|c|}
\hline $\mathrm{Pd}$ & -0.20276500 & -0.27567900 & 0.43222200 & $\mathrm{~F}$ & -3.80769000 & -4.10103000 & 1.97984600 \\
\hline $\mathrm{O}$ & -2.89542800 & 0.46957900 & 2.32363100 & $\mathrm{O}$ & -1.28964700 & -2.68551200 & 1.74043200 \\
\hline $\mathrm{C}$ & -1.99335900 & 1.84758500 & 4.03139200 & $\mathrm{O}$ & -2.20116200 & -3.52388300 & -0.49903500 \\
\hline $\mathrm{C}$ & -1.83702400 & 1.06676200 & 2.75682800 & $\mathrm{~N}$ & -2.18119200 & -0.93465600 & 0.17004600 \\
\hline $\mathrm{O}$ & -0.72815900 & 0.99322300 & 2.17573900 & $\mathrm{C}$ & -2.74509900 & -0.42928700 & -1.15688500 \\
\hline $\mathrm{C}$ & 3.86319000 & 1.36387900 & 1.35809300 & $\mathrm{C}$ & -3.33616200 & 0.96646100 & -0.93892700 \\
\hline $\mathrm{C}$ & 2.38040300 & 1.10029900 & 1.51588200 & $\mathrm{C}$ & -2.80946500 & 2.12877600 & -1.52015300 \\
\hline $\mathrm{H}$ & 4.32195600 & 1.43661100 & 2.34605300 & $\mathrm{C}$ & -3.38211200 & 3.39306300 & -1.34055000 \\
\hline $\mathrm{H}$ & -1.81759500 & 1.17191800 & 4.87731900 & $\mathrm{C}$ & -4.52433700 & 3.51411200 & -0.55967400 \\
\hline $\mathrm{H}$ & -1.26389200 & 2.65841800 & 4.07759900 & $\mathrm{C}$ & -5.09640900 & 2.38997100 & 0.03275500 \\
\hline $\mathrm{H}$ & -3.00882400 & 2.23976900 & 4.11433800 & $\mathrm{C}$ & -4.51385400 & 1.13470500 & -0.15952700 \\
\hline $\mathrm{N}$ & 4.45052400 & 0.22132500 & 0.68110200 & $\mathrm{C}$ & -3.88213900 & -2.88587200 & 1.43410300 \\
\hline $\mathrm{H}$ & 3.89963500 & -0.22094400 & -0.04277900 & $\mathrm{H}$ & 0.84239400 & 1.45401800 & 2.57000900 \\
\hline $\mathrm{C}$ & 5.77072700 & -0.09601600 & 0.86884000 & $\mathrm{H}$ & -2.94217300 & 4.26517100 & -1.80907400 \\
\hline $\mathrm{O}$ & 6.48670700 & 0.57005100 & 1.61633600 & $\mathrm{H}$ & -4.97847700 & 4.49037300 & -0.41390100 \\
\hline $\mathrm{C}$ & 6.29008900 & -1.28749200 & 0.11816000 & $\mathrm{H}$ & -5.99065700 & 2.49587300 & 0.63428700 \\
\hline $\mathrm{C}$ & 5.46496200 & -2.26266800 & -0.46198000 & $\mathrm{H}$ & -2.68610300 & -0.10197200 & 1.46873600 \\
\hline $\mathrm{C}$ & 7.68247300 & -1.42034900 & 0.02182200 & $\mathrm{O}$ & -5.02177500 & -0.00395900 & 0.38956300 \\
\hline $\mathrm{C}$ & 6.02822200 & -3.34390200 & -1.14036500 & $\mathrm{C}$ & -6.17671300 & 0.08483200 & 1.21485400 \\
\hline $\mathrm{H}$ & 4.38341800 & -2.21044800 & -0.37052400 & $\mathrm{H}$ & -7.03224900 & 0.48854500 & 0.65987600 \\
\hline $\mathrm{C}$ & 8.24243900 & -2.49778800 & -0.66024100 & $\mathrm{H}$ & -6.39205500 & -0.93610600 & 1.52608300 \\
\hline $\mathrm{H}$ & 8.30401900 & -0.66612900 & 0.49287300 & $\mathrm{H}$ & -5.98244200 & 0.70407800 & 2.09865500 \\
\hline $\mathrm{C}$ & 7.41569500 & -3.46090700 & -1.24522300 & $\mathrm{C}$ & -3.70899800 & -1.34633300 & -1.93236200 \\
\hline $\mathrm{H}$ & 5.38063300 & -4.09690100 & -1.58043200 & $\mathrm{H}$ & -3.98729900 & -0.81368900 & -2.84820200 \\
\hline $\mathrm{H}$ & 9.32273600 & -2.58883900 & -0.73454000 & $\mathrm{H}$ & -3.21144900 & -2.27505900 & -2.21257500 \\
\hline $\mathrm{H}$ & 7.85122000 & -4.30362600 & -1.77558200 & $\mathrm{H}$ & -4.61758300 & -1.57543200 & -1.37908000 \\
\hline $\mathrm{C}$ & 4.10864800 & 2.68653600 & 0.57624000 & $\mathrm{H}$ & -1.87759000 & -0.31342700 & -1.80340900 \\
\hline $\mathrm{H}$ & 5.19515300 & 2.73949100 & 0.43888900 & I & -1.07505900 & 2.10810100 & -2.79367100 \\
\hline $\mathrm{H}$ & 3.66031400 & 2.57357800 & -0.41974300 & I & 0.80607100 & -1.72916200 & -1.54046500 \\
\hline $\mathrm{C}$ & 3.61419500 & 3.99333700 & 1.22741000 & & & & \\
\hline $\mathrm{C}$ & 3.88565600 & 5.16874400 & 0.27455500 & \multicolumn{4}{|c|}{$\mathrm{TS}_{\mathrm{S} 5 \mathrm{~A}}$} \\
\hline $\mathrm{C}$ & 4.25743300 & 4.24465000 & 2.60024800 & \multicolumn{4}{|c|}{-2529.84861715} \\
\hline $\mathrm{H}$ & 2.52544200 & 3.92739500 & 1.36916700 & $\mathrm{Pd}$ & -0.17550200 & -0.27964100 & 0.40677400 \\
\hline $\mathrm{H}$ & 3.39859000 & 5.01952600 & -0.69635300 & $\mathrm{O}$ & -2.84801900 & 0.35715400 & 2.25154400 \\
\hline $\mathrm{H}$ & 3.51384800 & 6.10940300 & 0.69708800 & $\mathrm{C}$ & -1.98528700 & 1.70862600 & 4.01227300 \\
\hline $\mathrm{H}$ & 4.96185400 & 5.28669500 & 0.09464600 & $\mathrm{C}$ & -1.82311200 & 0.95589700 & 2.71819700 \\
\hline $\mathrm{H}$ & 3.98203100 & 3.47719900 & 3.33111500 & $\mathrm{O}$ & -0.69036400 & 0.92271100 & 2.15466500 \\
\hline $\mathrm{H}$ & 5.35186000 & 4.25928900 & 2.52310100 & $\mathrm{C}$ & 3.87099700 & 1.35540800 & 1.34838900 \\
\hline $\mathrm{H}$ & 3.93738900 & 5.21215500 & 3.00454900 & $\mathrm{C}$ & 2.38959000 & 1.07777500 & 1.49915700 \\
\hline $\mathrm{O}$ & 1.77267100 & 0.46474000 & 0.64384900 & $\mathrm{H}$ & 4.32429100 & 1.42943300 & 2.33864700 \\
\hline $\mathrm{O}$ & 1.82609800 & 1.61884200 & 2.58894900 & $\mathrm{H}$ & -1.68737400 & 1.05169400 & 4.83844100 \\
\hline S & -2.21536700 & -2.57270700 & 0.60901000 & $\mathrm{H}$ & -1.34068600 & 2.59051300 & 4.02802300 \\
\hline $\mathrm{F}$ & -4.12427200 & -1.99449200 & 2.40408800 & $\mathrm{H}$ & -3.02837700 & 1.99465300 & 4.15690200 \\
\hline $\mathrm{F}$ & -4.89972600 & -2.87071200 & 0.56708600 & $\mathrm{~N}$ & 4.47231700 & 0.21949000 & 0.67212300 \\
\hline
\end{tabular}




\begin{tabular}{|c|c|c|c|c|c|c|c|}
\hline $\mathrm{H}$ & 3.93441900 & -0.21746900 & -0.06454700 & $\mathrm{H}$ & 0.84177600 & 1.39724300 & 2.55630900 \\
\hline $\mathrm{C}$ & 5.79059900 & -0.09568700 & 0.87685900 & $\mathrm{H}$ & -3.04484800 & 4.31039700 & -1.62538200 \\
\hline $\mathrm{O}$ & 6.49426800 & 0.56665300 & 1.63901700 & $\mathrm{H}$ & -5.09484300 & 4.44511200 & -0.23903100 \\
\hline $\mathrm{C}$ & 6.32347400 & -1.28141100 & 0.12634000 & $\mathrm{H}$ & -6.07443400 & 2.39666800 & 0.73080800 \\
\hline $\mathrm{C}$ & 5.50903500 & -2.25494300 & -0.47135400 & $\mathrm{H}$ & -2.60463600 & -0.24019700 & 1.30944300 \\
\hline $\mathrm{C}$ & 7.71736900 & -1.41131500 & 0.04999500 & $\mathrm{O}$ & -5.05828400 & -0.07523700 & 0.39936900 \\
\hline $\mathrm{C}$ & 6.08424400 & -3.33153000 & -1.14709600 & $\mathrm{C}$ & -6.19636700 & -0.03387100 & 1.25155300 \\
\hline $\mathrm{H}$ & 4.42621100 & -2.20518500 & -0.39544600 & $\mathrm{H}$ & -7.06924700 & 0.37781600 & 0.73048700 \\
\hline $\mathrm{C}$ & 8.28942200 & -2.48410600 & -0.62940000 & $\mathrm{H}$ & -6.39220700 & -1.06858900 & 1.52817800 \\
\hline $\mathrm{H}$ & 8.33044000 & -0.65870000 & 0.53452800 & $\mathrm{H}$ & -5.99006400 & 0.55424100 & 2.15344400 \\
\hline $\mathrm{C}$ & 7.47332900 & -3.44556700 & -1.23178100 & $\mathrm{C}$ & -3.70667600 & -1.31381100 & -1.94514900 \\
\hline $\mathrm{H}$ & 5.44470300 & -4.08331900 & -1.60080300 & $\mathrm{H}$ & -4.00193600 & -0.75753300 & -2.84136500 \\
\hline $\mathrm{H}$ & 9.37087200 & -2.57289000 & -0.68795600 & $\mathrm{H}$ & -3.18670300 & -2.21895900 & -2.25956700 \\
\hline $\mathrm{H}$ & 7.91829300 & -4.28471700 & -1.75995700 & $\mathrm{H}$ & -4.60711500 & -1.58393500 & -1.39733200 \\
\hline $\mathrm{C}$ & 4.10755400 & 2.68149200 & 0.57044300 & $\mathrm{H}$ & -1.89969600 & -0.24344700 & -1.78725300 \\
\hline $\mathrm{H}$ & 5.19454900 & 2.74881800 & 0.44293100 & I & -1.12368500 & 2.22579200 & -2.66483300 \\
\hline $\mathrm{H}$ & 3.66969100 & 2.56420000 & -0.42969200 & I & 0.80321900 & -1.66743300 & -1.63168900 \\
\hline $\mathrm{C}$ & 3.58974700 & 3.98067100 & 1.21866200 & & & & \\
\hline $\mathrm{C}$ & 3.85161100 & 5.16070000 & 0.26888600 & $\mathrm{~S}_{9 \mathrm{~A}}$ & & & \\
\hline $\mathrm{C}$ & 4.21864300 & 4.23979000 & 2.59669900 & -25 & 9.85952970 & & \\
\hline $\mathrm{H}$ & 2.50097400 & 3.89909800 & 1.35209400 & $\mathrm{Pd}$ & 0.39701900 & 0.85663900 & -0.16715800 \\
\hline $\mathrm{H}$ & 3.37381200 & 5.00554900 & -0.70568800 & $\mathrm{O}$ & 0.21155300 & 4.08461200 & -0.51562800 \\
\hline $\mathrm{H}$ & 3.46342400 & 6.09559900 & 0.68942000 & $\mathrm{C}$ & 2.01484700 & 4.76219400 & 0.89078800 \\
\hline $\mathrm{H}$ & 4.92735600 & 5.29402000 & 0.09699200 & $\mathrm{C}$ & 1.04525900 & 3.72743400 & 0.34102300 \\
\hline $\mathrm{H}$ & 3.95009800 & 3.46666300 & 3.32410200 & $\mathrm{O}$ & 1.18949000 & 2.54041900 & 0.82198600 \\
\hline $\mathrm{H}$ & 5.31327100 & 4.27235900 & 2.52776700 & $\mathrm{C}$ & -3.85537100 & 1.89222900 & -0.15385600 \\
\hline $\mathrm{H}$ & 3.88004600 & 5.20125500 & 3.00028800 & $\mathrm{C}$ & -2.36173800 & 2.06527900 & -0.35258600 \\
\hline $\mathrm{O}$ & 1.79375100 & 0.44875900 & 0.61155100 & $\mathrm{H}$ & -4.37548500 & 2.36811100 & -0.98768600 \\
\hline $\mathrm{O}$ & 1.82758300 & 1.57307200 & 2.57584100 & $\mathrm{H}$ & 1.97046300 & 4.77716300 & 1.98444800 \\
\hline $\mathrm{S}$ & -2.18852200 & -2.60259300 & 0.56207000 & $\mathrm{H}$ & 3.03595700 & 4.48852900 & 0.60412900 \\
\hline F & -4.10617300 & -2.10213200 & 2.36762800 & $\mathrm{H}$ & 1.77747200 & 5.75082500 & 0.49594200 \\
\hline F & -4.86394500 & -2.94701400 & 0.50844600 & $\mathrm{~N}$ & -4.16928900 & 0.47252400 & -0.17257800 \\
\hline F & -3.75045100 & -4.19200500 & 1.89185100 & $\mathrm{H}$ & -3.43427500 & -0.17191000 & 0.08461400 \\
\hline $\mathrm{O}$ & -1.25645100 & -2.72943100 & 1.68406600 & $\mathrm{C}$ & -5.43851600 & 0.04113400 & -0.44279700 \\
\hline $\mathrm{O}$ & -2.15600400 & -3.50138400 & -0.58592800 & $\mathrm{O}$ & -6.35856700 & 0.83634500 & -0.63697200 \\
\hline $\mathrm{N}$ & -2.17604000 & -0.93703400 & 0.16513100 & $\mathrm{C}$ & -5.65440100 & -1.44533500 & -0.47335000 \\
\hline $\mathrm{C}$ & -2.76378600 & -0.39745000 & -1.14521400 & $\mathrm{C}$ & -4.62240100 & -2.37709800 & -0.65724400 \\
\hline $\mathrm{C}$ & -3.38291800 & 0.97599200 & -0.87874500 & $\mathrm{C}$ & -6.97295100 & -1.89979300 & -0.33177300 \\
\hline $\mathrm{C}$ & -2.87244000 & 2.16805200 & -1.41250400 & $\mathrm{C}$ & -4.90765800 & -3.74299200 & -0.68063300 \\
\hline $\mathrm{C}$ & -3.47101100 & 3.41341900 & -1.19257000 & $\mathrm{H}$ & -3.59839700 & -2.05177600 & -0.82103700 \\
\hline $\mathrm{C}$ & -4.62101600 & 3.48359500 & -0.41659800 & $\mathrm{C}$ & -7.25440000 & -3.26380600 & -0.34863500 \\
\hline $\mathrm{C}$ & -5.17530900 & 2.32859200 & 0.13128000 & $\mathrm{H}$ & -7.76018400 & -1.16246300 & -0.21421200 \\
\hline $\mathrm{C}$ & -4.56838700 & 1.09205100 & -0.10192800 & $\mathrm{C}$ & -6.22097600 & -4.18911100 & -0.52024600 \\
\hline $\mathrm{C}$ & -3.85072700 & -2.96465000 & 1.37911100 & $\mathrm{H}$ & -4.10244700 & -4.45621900 & -0.83387500 \\
\hline
\end{tabular}




\begin{tabular}{|c|c|c|c|c|c|c|c|}
\hline $\mathrm{H}$ & -8.27905700 & -3.60656200 & -0.23174000 & $\mathrm{H}$ & 4.95141900 & -1.10755600 & -0.62047900 \\
\hline $\mathrm{H}$ & -6.43981400 & -5.25363500 & -0.53773300 & $\mathrm{H}$ & 4.87664900 & -0.44879000 & 1.04087300 \\
\hline $\mathrm{C}$ & -4.29729100 & 2.56541500 & 1.17728500 & $\mathrm{H}$ & 2.55660700 & -1.70727100 & -0.54280500 \\
\hline $\mathrm{H}$ & -5.36608000 & 2.34324000 & 1.27365900 & I & 1.75416500 & -4.22407600 & 0.42791300 \\
\hline $\mathrm{H}$ & -3.78074700 & 2.04998300 & 1.99781400 & I & -0.41830000 & -1.19044800 & -1.65080400 \\
\hline $\mathrm{C}$ & -4.07805800 & 4.08695600 & 1.29404100 & & & & \\
\hline $\mathrm{C}$ & -4.50717800 & 4.55480600 & 2.69407100 & $\mathrm{~S}_{8 \mathrm{~B}}$ & & & \\
\hline $\mathrm{C}$ & -4.82469300 & 4.87356300 & 0.20502400 & -23 & .72787661 & & \\
\hline $\mathrm{H}$ & -3.00423800 & 4.30000000 & 1.18731700 & $\mathrm{Pd}$ & -0.39708600 & -1.32086200 & -0.58554800 \\
\hline $\mathrm{H}$ & -3.95917600 & 4.02354100 & 3.48188100 & $\mathrm{C}$ & 3.10808700 & 1.42402100 & -0.04282000 \\
\hline $\mathrm{H}$ & -4.32472100 & 5.62821800 & 2.82195300 & $\mathrm{C}$ & 1.74473200 & 0.78163400 & 0.13307500 \\
\hline $\mathrm{H}$ & -5.57834400 & 4.37732500 & 2.85456600 & $\mathrm{H}$ & 3.42647200 & 1.84342000 & 0.91222100 \\
\hline $\mathrm{H}$ & -4.44678000 & 4.64547100 & -0.79661600 & $\mathrm{~N}$ & 4.05225100 & 0.37152300 & -0.38558600 \\
\hline $\mathrm{H}$ & -5.89814300 & 4.64609000 & 0.22381400 & $\mathrm{H}$ & 3.74769100 & -0.32581200 & -1.05042100 \\
\hline $\mathrm{H}$ & -4.70739200 & 5.95244500 & 0.36061800 & $\mathrm{C}$ & 5.38638400 & 0.50933800 & -0.08708400 \\
\hline $\mathrm{O}$ & -1.59548800 & 1.23636500 & 0.19701000 & $\mathrm{O}$ & 5.81283200 & 1.50185400 & 0.49900900 \\
\hline $\mathrm{O}$ & -1.99531000 & 3.09360000 & -1.03308500 & $\mathrm{C}$ & 6.28178100 & -0.61494900 & -0.51672500 \\
\hline S & 3.12550600 & 0.88089100 & -1.82445700 & $\mathrm{C}$ & 5.81928800 & -1.91336500 & -0.77699200 \\
\hline $\mathrm{F}$ & 4.55046900 & 2.38159600 & -0.13562200 & $\mathrm{C}$ & 7.65051900 & -0.33652700 & -0.63425500 \\
\hline $\mathrm{F}$ & 5.76590600 & 1.04802200 & -1.35818400 & $\mathrm{C}$ & 6.71219000 & -2.91191800 & -1.16674000 \\
\hline $\mathrm{F}$ & 4.86000500 & 2.80720100 & -2.25027900 & $\mathrm{H}$ & 4.77087300 & -2.16486100 & -0.63968700 \\
\hline $\mathrm{O}$ & 2.23016200 & 1.91374400 & -2.31701300 & $\mathrm{C}$ & 8.53957200 & -1.33270900 & -1.03086500 \\
\hline $\mathrm{O}$ & 3.51661500 & -0.29204900 & -2.58989100 & $\mathrm{H}$ & 7.99387700 & 0.66730300 & -0.40696600 \\
\hline$N$ & 2.49052900 & 0.35598300 & -0.25681400 & $\mathrm{C}$ & 8.07156700 & -2.62169700 & -1.30093800 \\
\hline $\mathrm{C}$ & 2.93118000 & -1.02658600 & 0.21957300 & $\mathrm{H}$ & 6.34705500 & -3.91750000 & -1.35561600 \\
\hline $\mathrm{C}$ & 2.23502700 & -1.33952500 & 1.53805500 & $\mathrm{H}$ & 9.59796400 & -1.10642000 & -1.12643700 \\
\hline $\mathrm{C}$ & 1.62456100 & -2.57542000 & 1.79549700 & $\mathrm{H}$ & 8.76536400 & -3.40026400 & -1.60635800 \\
\hline $\mathrm{C}$ & 0.96652800 & -2.83915400 & 2.99966800 & $\mathrm{C}$ & 3.06555600 & 2.54244000 & -1.11788300 \\
\hline $\mathrm{C}$ & 0.93484000 & -1.86025900 & 3.98694500 & $\mathrm{H}$ & 4.10313600 & 2.87706500 & -1.23520900 \\
\hline $\mathrm{C}$ & 1.59443000 & -0.64581500 & 3.80455400 & $\mathrm{H}$ & 2.76061100 & 2.08510300 & -2.06841900 \\
\hline $\mathrm{C}$ & 2.26240900 & -0.40468000 & 2.60344800 & $\mathrm{C}$ & 2.16668400 & 3.75672800 & -0.81388300 \\
\hline $\mathrm{C}$ & 4.70136200 & 1.84143200 & -1.35822000 & $\mathrm{C}$ & 2.17232900 & 4.71051900 & -2.01901300 \\
\hline $\mathrm{H}$ & -0.98179100 & 3.36884100 & -0.88159500 & $\mathrm{C}$ & 2.59295700 & 4.49530200 & 0.46503200 \\
\hline $\mathrm{H}$ & 0.48959800 & -3.79806000 & 3.16292800 & $\mathrm{H}$ & 1.13396100 & 3.40652000 & -0.67557000 \\
\hline $\mathrm{H}$ & 0.41182100 & -2.05253900 & 4.91924300 & $\mathrm{H}$ & 1.83803300 & 4.20479300 & -2.93255800 \\
\hline $\mathrm{H}$ & 1.59475200 & 0.09360900 & 4.59557600 & $\mathrm{H}$ & 1.50744700 & 5.56466200 & -1.84580700 \\
\hline $\mathrm{H}$ & 2.77302700 & 1.05593900 & 0.44774900 & $\mathrm{H}$ & 3.17983400 & 5.10495100 & -2.20321400 \\
\hline $\mathrm{O}$ & 3.00353500 & 0.72598200 & 2.37430200 & $\mathrm{H}$ & 2.49152900 & 3.86901700 & 1.35830100 \\
\hline $\mathrm{C}$ & 2.97471400 & 1.77897400 & 3.33635000 & $\mathrm{H}$ & 3.63924900 & 4.81968300 & 0.40216300 \\
\hline $\mathrm{H}$ & 3.38843100 & 1.44227200 & 4.29381600 & $\mathrm{H}$ & 1.97593500 & 5.38890200 & 0.61727500 \\
\hline $\mathrm{H}$ & 3.60473900 & 2.56749700 & 2.92441600 & $\mathrm{O}$ & 1.35376400 & -0.08373700 & -0.67004700 \\
\hline $\mathrm{H}$ & 1.95690900 & 2.15733300 & 3.46884700 & $\mathrm{O}$ & 1.03549200 & 1.21304900 & 1.13810500 \\
\hline $\mathrm{C}$ & 4.45485100 & -1.18182800 & 0.34783700 & $\mathrm{~S}$ & -1.08126000 & -1.55059400 & 2.34002600 \\
\hline $\mathrm{H}$ & 4.65915100 & -2.18066900 & 0.74527400 & $\mathrm{~F}$ & 0.29078500 & 0.30781400 & 3.67941800 \\
\hline
\end{tabular}




\begin{tabular}{|c|c|c|c|}
\hline $\mathrm{F}$ & -1.79707700 & 0.18170800 & 4.28445200 \\
\hline $\mathrm{F}$ & -0.41016900 & -1.38954600 & 4.84246200 \\
\hline $\mathrm{O}$ & 0.20551200 & -2.21214600 & 2. 11458900 \\
\hline $\mathrm{O}$ & -2.29041200 & -2.30682700 & 2.65716200 \\
\hline $\mathrm{N}$ & -1.24117400 & -0.41264100 & 1.07811300 \\
\hline$C$ & -2.58446800 & 0.21787500 & 0.73076700 \\
\hline $\mathrm{C}$ & -2.34088400 & 1.61356800 & 0.15697800 \\
\hline $\mathrm{C}$ & -2.72005800 & 1.99040800 & -1.13907300 \\
\hline 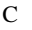 & -2.55844300 & 3.29257500 & -1.62417500 \\
\hline $\mathrm{C}$ & -2.00811900 & 4.26112000 & -0.79401800 \\
\hline C & -1.62327500 & 3.94173500 & 0.50748300 \\
\hline $\mathrm{C}$ & -1.79058800 & 2.63647000 & 0.97659200 \\
\hline C & -0.73684300 & -0.52373800 & 3.88394600 \\
\hline $\mathrm{H}$ & 0.15266300 & 0.71558200 & 1.19149400 \\
\hline $\mathrm{H}$ & -2.86517700 & 3.54389700 & -2.63218600 \\
\hline $\mathrm{H}$ & -1.88109100 & 5.27640200 & -1.15910700 \\
\hline $\mathrm{H}$ & -1.20267000 & 4.70712300 & 1.14758700 \\
\hline $\mathrm{O}$ & -1.44175900 & 2.25427000 & 2.23813900 \\
\hline $\mathrm{C}$ & -0.89612200 & 3.22311300 & 3.12837500 \\
\hline $\mathrm{H}$ & -1.60267700 & 4.04363400 & 3.30160700 \\
\hline $\mathrm{H}$ & -0.71598100 & 2.69102900 & 4.06128100 \\
\hline $\mathrm{H}$ & 0.05026500 & 3.62170500 & 2.74533100 \\
\hline $\mathrm{C}$ & -3.66884500 & 0.25744400 & 1.82768000 \\
\hline $\mathrm{H}$ & -4.55664900 & 0.70803000 & 1.37144400 \\
\hline $\mathrm{H}$ & -3.92833000 & -0.74755100 & 2.16037200 \\
\hline $\mathrm{H}$ & -3.37771900 & 0.86268000 & 2.68392100 \\
\hline $\mathrm{H}$ & -2.99792600 & -0.39482000 & -0.06728600 \\
\hline I & -3.64118900 & 0.62119900 & -2.51807600 \\
\hline I & -2.07436700 & -3.24978600 & -1.02619700 \\
\hline
\end{tabular}

$\begin{array}{llll}\mathrm{TS}_{\mathrm{SSB}} & & & \\ -2300.72365059 & & \\ \mathrm{Pd} & -0.37821700 & -1.46346200 & -0.51160200 \\ \mathrm{C} & 2.93334900 & 1.37706100 & 0.00483200 \\ \mathrm{C} & 1.57059900 & 0.71183600 & 0.14592100 \\ \mathrm{H} & 3.19665600 & 1.82496900 & 0.96415700 \\ \mathrm{~N} & 3.92118700 & 0.34685600 & -0.28005300 \\ \mathrm{H} & 3.63442200 & -0.41030700 & -0.88385500 \\ \mathrm{C} & 5.24182200 & 0.53206100 & 0.03294600 \\ \mathrm{O} & 5.63826600 & 1.56322400 & 0.57412500 \\ \mathrm{C} & 6.17407600 & -0.59012700 & -0.32374100 \\ \mathrm{C} & 5.75140400 & -1.91209100 & -0.52569600 \\ \mathrm{C} & 7.53701600 & -0.28190700 & -0.43217900 \\ \mathrm{C} & 6.67707400 & -2.90471600 & -0.84899400\end{array}$

\begin{tabular}{|c|c|c|c|}
\hline $\mathrm{H}$ & 4.70735900 & -2.18466400 & -0.39505600 \\
\hline & 8.45926800 & -1.27218000 & -0.76266800 \\
\hline & 7.84916300 & 0.74110500 & -0.24979400 \\
\hline & 8.03069500 & -2.58542800 & -0.97477100 \\
\hline & 6.34162200 & -3.92798500 & -0.99324100 \\
\hline & 9.51294000 & -1.02207100 & -0.85181400 \\
\hline & 8.75010400 & -3.35934200 & -1.22861200 \\
\hline & 2.91622800 & 2.47548500 & -1.09186700 \\
\hline & 3.95257800 & 2.82440000 & -1.17397200 \\
\hline H & 2.65761500 & 1.99697100 & -2.04601600 \\
\hline C & 1.98952100 & 3.68197100 & -0.84783200 \\
\hline C & 2.05901300 & 4.63084800 & -2.05474100 \\
\hline$C$ & 2.33061900 & 4.42871200 & 0.45126000 \\
\hline $\mathrm{H}$ & 0.95546800 & 3.32095900 & -0.76824800 \\
\hline $\mathrm{H}$ & 1.78880100 & 4.11757400 & -2.98529900 \\
\hline $\mathrm{H}$ & 1.37436700 & 5.47775700 & -1.92750600 \\
\hline $\mathrm{H}$ & 3.07118100 & 5.03708400 & -2.17818700 \\
\hline $\mathrm{H}$ & 2.16813100 & 3.80397800 & 1.33612500 \\
\hline $\mathrm{H}$ & 3.37865400 & 4.75390000 & 0.45654400 \\
\hline $\mathrm{H}$ & 1.70356800 & 5.32229200 & 0.55749200 \\
\hline $\mathrm{O}$ & 1.31409800 & -0.26175400 & -0.62538600 \\
\hline $\mathrm{O}$ & 0.75430200 & 1.17948100 & 1.00160200 \\
\hline S & -1.14397500 & -1.34301400 & 2.43983400 \\
\hline $\mathrm{F}$ & 0.02437100 & 0.70792000 & 3.68986600 \\
\hline $\mathrm{F}$ & -2.07277800 & 0.44808300 & 4.22352700 \\
\hline $\mathrm{F}$ & -0.58863100 & -0.96180500 & 4.94016800 \\
\hline $\mathrm{O}$ & 0.17093800 & -1.97111800 & 2.33814600 \\
\hline O & -2.35981900 & -2.10158300 & 2.70273100 \\
\hline $\mathbb{N}$ & -1.26113900 & -0.32538300 & 1.03584600 \\
\hline $\mathrm{C}$ & -2.58690900 & 0.28838800 & 0.56162300 \\
\hline C & -2.31409100 & 1.67111600 & -0.02489100 \\
\hline C & -2.47876400 & 1.97021100 & -1.38498700 \\
\hline C & -2.32160300 & 3.26133400 & -1.89758500 \\
\hline C & -1.98334900 & 4.29475200 & -1.03185100 \\
\hline C & 1.79226000 & 4.04830100 & 0.32627900 \\
\hline C & 1.95138400 & 2.75225800 & 0.82333500 \\
\hline $\mathrm{C}$ & 0.93831000 & -0.18352900 & 3.91760700 \\
\hline $\mathrm{H}$ & -0.26279700 & 0.50699500 & 1.06281300 \\
\hline H & -2.46370900 & 3.45435000 & -2.95380200 \\
\hline $\mathrm{H}$ & -1.86268400 & 5.30316100 & -1.41744900 \\
\hline $\mathrm{H}$ & -1.52004000 & 4.86186700 & 0.98650000 \\
\hline $\mathrm{O}$ & -1.77267900 & 2.43600300 & 2.13475400 \\
\hline C & -1.35422700 & 3.45563700 & 3.03735500 \\
\hline $\mathrm{H}$ & & & 3.0883440 \\
\hline
\end{tabular}




$\begin{array}{llll}\mathrm{H} & -1.27809900 & 2.97207000 & 4.00989400 \\ \mathrm{H} & -0.37639300 & 3.85779700 & 2.74916400 \\ \mathrm{C} & -3.75981800 & 0.29890600 & 1.55849000 \\ \mathrm{H} & -4.60845200 & 0.75313100 & 1.03640200 \\ \mathrm{H} & -4.03769400 & -0.71578900 & 1.84380500 \\ \mathrm{H} & -3.55205100 & 0.88521200 & 2.45056000 \\ \mathrm{H} & -2.90545600 & -0.35257400 & -0.25654200 \\ \mathrm{I} & -3.00348000 & 0.47591200 & -2.84042100 \\ \mathrm{I} & -2.09784800 & -3.39755700 & -0.81360700\end{array}$

$\mathrm{S}_{9 \mathrm{~B}}$

$-2300.75399951$

$\begin{array}{llll}\mathrm{Pd} & -0.01445500 & -0.52239900 & -0.13309900\end{array}$

$\begin{array}{llll}\text { C } & 3.49055300 & 1.10891300 & 0.84837100\end{array}$

$\begin{array}{llll}\text { C } & 2.13481900 & 0.50285200 & 0.55573400\end{array}$

$\begin{array}{llll}\mathrm{H} & 3.71511400 & 0.96860800 & 1.90761100\end{array}$

$\begin{array}{llll}\mathrm{N} & 4.48921200 & 0.37203900 & 0.08732800\end{array}$

$\begin{array}{llll}\mathrm{H} & 4.22584900 & 0.06700900 & -0.83932600\end{array}$

$\begin{array}{llll}\text { C } & 5.78749300 & 0.29299900 & 0.51410400\end{array}$

$\begin{array}{llll}\text { O } & 6.15828200 & 0.83545500 & 1.55457000\end{array}$

$\begin{array}{llll}\text { C } & 6.73133500 & -0.48599800 & -0.35757500\end{array}$

$\begin{array}{llll}\text { C } & 6.31180300 & -1.42709100 & -1.30935200\end{array}$

$\begin{array}{llll}\text { C } & 8.10253600 & -0.25859500 & -0.17686100\end{array}$

$\begin{array}{llll}\text { C } & 7.25061700 & -2.11508100 & -2.07870400\end{array}$

$\begin{array}{llll}\mathrm{H} & 5.25722100 & -1.65729600 & -1.43594600\end{array}$

$\begin{array}{llll}\text { C } & 9.03860600 & -0.94154500 & -0.94986800\end{array}$

$\begin{array}{llll}\mathrm{H} & 8.40872600 & 0.45714000 & 0.57883500\end{array}$

$\begin{array}{llll}\text { C } & 8.61422800 & -1.86954900 & -1.90484200\end{array}$

$\begin{array}{llll}\mathrm{H} & 6.91597100 & -2.84783700 & -2.80770100\end{array}$

$\begin{array}{llll}\text { H } & 10.09924300 & -0.75317300 & -0.80707300\end{array}$

$\begin{array}{llll}\mathrm{H} & 9.34371600 & -2.40519200 & -2.50649500\end{array}$

$\begin{array}{llll}\text { C } & 3.51267700 & 2.62307800 & 0.50622900\end{array}$

$\begin{array}{llll}\mathrm{H} & 4.54017900 & 2.95870100 & 0.69131600\end{array}$

$\begin{array}{llll}\mathrm{H} & 3.32533600 & 2.72508100 & -0.57071900\end{array}$

$\begin{array}{llll}\text { C } & 2.52993100 & 3.51679200 & 1.28716400\end{array}$

$\begin{array}{llll}\text { C } & 2.58942100 & 4.95013300 & 0.73558000\end{array}$

$\begin{array}{llll}\text { C } & 2.80444700 & 3.50556700 & 2.79944700\end{array}$

$\mathrm{H} \quad 1.51076200 \quad 3.13815700 \quad 1.12284000$

$\begin{array}{llll}\mathrm{H} & 2.35334300 & 4.97714000 & -0.33477000\end{array}$

$\begin{array}{llll}\mathrm{H} & 1.87662900 & 5.60261100 & 1.25438200\end{array}$

$\begin{array}{llll}\text { H } & 3.58995000 & 5.38155900 & 0.86821200\end{array}$

$\begin{array}{llll}\mathrm{H} & 2.66985500 & 2.50951700 & 3.23508300\end{array}$

$\begin{array}{llll}\mathrm{H} & 3.83124700 & 3.82739700 & 3.01425900\end{array}$

$\begin{array}{llll}\mathrm{H} & 2.12629700 & 4.19131100 & 3.32260200\end{array}$ $\begin{array}{llll}\text { O } & 1.87931300 & 0.10030600 & -0.63655400\end{array}$

$\begin{array}{llll}\text { O } & 1.22803600 & 0.40426300 & 1.44055800\end{array}$

$\begin{array}{llll}\text { S } & -2.03620300 & -2.76939600 & 0.97799400\end{array}$

F $\quad-1.74593700 \quad-2.17123100 \quad 3.54548900$

F $\quad-3.76454300 \quad-2.81962400 \quad 3.04110400$

F $\quad-2.15253800 \quad-4.27153600 \quad 3.12166500$

$\begin{array}{llll}\text { O } & -0.70449200 & -3.34011600 & 0.87459700\end{array}$

$\begin{array}{llll}\text { O } & -3.17141400 & -3.17813700 & 0.16671200\end{array}$

N $\quad-1.84742900 \quad-1.00928200 \quad 0.87731100$

$\begin{array}{llll}\text { C } & -3.05801100 & -0.18157200 & 0.45633500\end{array}$

$\begin{array}{llll}\text { C } & -2.64709900 & 1.28856100 & 0.48674200\end{array}$

$\begin{array}{llll}\text { C } & -2.95902900 & 2.17977200 & -0.55060700\end{array}$

$\begin{array}{llll}\text { C } & -2.59078900 & 3.52724600 & -0.50633800\end{array}$

$\begin{array}{llll}\text { C } & -1.90772300 & 4.01110100 & 0.60379100\end{array}$

$\begin{array}{llll}\text { C } & -1.61722200 & 3.17337100 & 1.67959800\end{array}$

$\begin{array}{llll}\text { C } & -2.00244100 & 1.83424900 & 1.62722800\end{array}$

$\begin{array}{llll}\text { C } & -2.47298300 & -3.02556100 & 2.80614800\end{array}$

$\begin{array}{llll}\mathrm{H} & -1.58077800 & -0.68344600 & 1.81956800\end{array}$

$\begin{array}{llll}\mathrm{H} & -2.83694500 & 4.18793700 & -1.32894500\end{array}$

H $\quad-1.60909500 \quad 5.05469000 \quad 0.64008400$

$\begin{array}{llll}\mathrm{H} & -1.10253500 & 3.56588100 & 2.54760600\end{array}$

$\begin{array}{llll}\text { O } & -1.79769200 & 0.96236500 & 2.67069800\end{array}$

$\begin{array}{llll}\text { C } & -0.98542700 & 1.37020100 & 3.77710400\end{array}$

$\begin{array}{llll}\text { H } & -1.44834400 & 2.20745000 & 4.31053700\end{array}$

H $\quad-0.94025800 \quad 0.50145700 \quad 4.43496800$

$\begin{array}{llll}\mathrm{H} & 0.02096900 & 1.63066300 & 3.43831000\end{array}$

$\begin{array}{llll}\text { C } & -4.32060900 & -0.41395800 & 1.29937800\end{array}$

$\begin{array}{llll}\text { H } & -5.09831300 & 0.26114900 & 0.92927500\end{array}$

$\begin{array}{llll}\mathrm{H} & -4.69196800 & -1.43437100 & 1.19565700\end{array}$

H $\quad-4.14842300 \quad-0.18645200 \quad 2.35492400$

H $\quad-3.24713500 \quad-0.47862000 \quad-0.57388200$

I $\quad-4.10438400 \quad 1.58606000 \quad-2.26334800$

I $\quad-0.76631000 \quad-1.45158000 \quad-2.48327200$

$\mathrm{S}_{8 \mathrm{C}}$

$-1743.73723144$

$\begin{array}{llll}\text { Pd } & 0.35269600 & -1.00883800 & 1.27667100\end{array}$

$\begin{array}{llll}\text { S } & -1.91273800 & -1.55622700 & -0.68069300\end{array}$

$\begin{array}{llll}\text { F } & -3.93323300 & 0.06639600 & -0.03425200\end{array}$

$\begin{array}{llll}\text { F } & -3.51129400 & 0.03383000 & -2.16918900\end{array}$

F $\quad-4.45213200 \quad-1.67722300 \quad-1.22490800$

$\begin{array}{llll}\text { O } & -2.21785900 & -2.28564900 & 0.55068300\end{array}$

O $\quad-1.55631000 \quad-2.24614500 \quad-1.91878800$

N $\quad-0.87675200 \quad-0.28328000 \quad-0.22986800$ 


\begin{tabular}{|c|c|c|c|}
\hline $\mathrm{C}$ & -0.03902900 & 0.46867500 & -1.25743100 \\
\hline $\mathrm{C}$ & 0.22336200 & 1.87331500 & -0.71409400 \\
\hline C & 1.50283800 & 2.36289400 & -0.41880000 \\
\hline $\mathrm{C}$ & 1.72426500 & 3.66146800 & 0.05250200 \\
\hline C & 0.63925200 & 4.51031800 & 0.23258200 \\
\hline C & -0.65308700 & 4.07954300 & -0.06367900 \\
\hline $\mathrm{C}$ & -0.85645300 & 2.78212000 & -0.53993500 \\
\hline $\mathrm{C}$ & -3.55332300 & -0.70717300 & -1.05945200 \\
\hline $\mathrm{H}$ & 2.72941800 & 4.00069300 & 0.27180300 \\
\hline $\mathrm{H}$ & 0.79914600 & 5.52003200 & 0.60051200 \\
\hline $\mathrm{H}$ & -1.48942900 & 4.75449900 & 0.07030000 \\
\hline $\mathrm{O}$ & -2.08995200 & 2.29753100 & -0.86078900 \\
\hline $\mathrm{C}$ & -3.22864500 & 3.13555000 & -0.70021000 \\
\hline $\mathrm{H}$ & -3.15007300 & 4.03655700 & -1.32048000 \\
\hline $\mathrm{H}$ & -4.07729200 & 2.53679800 & -1.02916500 \\
\hline $\mathrm{H}$ & -3.36594000 & 3.41676500 & 0.35030800 \\
\hline I & 1.59699000 & -2.91726300 & 0.03585300 \\
\hline I & 3.28005900 & 1.18492900 & -0.69789300 \\
\hline 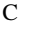 & -1.34269300 & 1.02287800 & 2.82434500 \\
\hline $\mathrm{O}$ & -0.31552100 & 0.32619400 & 2.79411100 \\
\hline $\mathrm{O}$ & -2.13092100 & 1.19525100 & 1.79520900 \\
\hline $\mathrm{H}$ & -1.77043400 & 0.70251600 & 0.98835900 \\
\hline $\mathrm{C}$ & -1.76898500 & 1.73790800 & 4.07431200 \\
\hline $\mathrm{H}$ & -2.76247300 & 1.38544800 & 4.37118900 \\
\hline $\mathrm{H}$ & -1.05324900 & 1.55435300 & 4.87539900 \\
\hline $\mathrm{H}$ & -1.84533300 & 2.81148400 & 3.87440900 \\
\hline $\mathrm{C}$ & -0.54943700 & 0.52207900 & -2.71223000 \\
\hline $\mathrm{H}$ & 0.21637100 & 1.05063500 & -3.29004200 \\
\hline $\mathrm{H}$ & -0.65913500 & -0.47962900 & -3.12780100 \\
\hline $\mathrm{H}$ & -1.48734500 & 1.06437000 & -2.80822900 \\
\hline $\mathrm{H}$ & 0.91530900 & -0.05363800 & -1.30090200 \\
\hline
\end{tabular}

$\mathrm{TS}_{\mathrm{SSC}}$

\section{$-1743.73216824$}

$\begin{array}{lrrr}\text { Pd } & 0.56579100 & -1.03141300 & 1.24016300 \\ \text { S } & -1.86488600 & -1.68577800 & -0.53386000 \\ \text { F } & -3.97484600 & -0.21626200 & 0.17310200 \\ \text { F } & -3.64216100 & -0.26176100 & -1.97784100 \\ \text { F } & -4.39980100 & -2.02254900 & -0.96159600 \\ \text { O } & -2.03915300 & -2.39917500 & 0.72773900 \\ \text { O } & -1.47488900 & -2.35168300 & -1.77013000 \\ \text { N } & -0.86420600 & -0.32136100 & -0.15647200 \\ \text { C } & -0.15744600 & 0.50418900 & -1.24461700 \\ \text { C } & -0.01517600 & 1.93600700 & -0.73587400\end{array}$

\begin{tabular}{|c|c|c|c|}
\hline $\mathrm{C}$ & 1.22056400 & 2.53343000 & -0.45155800 \\
\hline $\mathrm{C}$ & 1.33498600 & 3.85909600 & -0.02213700 \\
\hline C & 0.18259500 & 4.62008600 & 0.13502000 \\
\hline $\mathrm{C}$ & -1.07030100 & 4.07487900 & -0.13802500 \\
\hline $\mathrm{C}$ & -1.16815700 & 2.75216000 & -0.57633000 \\
\hline $\mathrm{C}$ & -3.58513300 & -0.97410000 & -0.85203700 \\
\hline $\mathrm{H}$ & 2.30819100 & 4.28615900 & 0.18678300 \\
\hline $\mathrm{H}$ & 0.25893900 & 5.65023000 & 0.47173100 \\
\hline $\mathrm{H}$ & -1.95912300 & 4.68060400 & -0.01299300 \\
\hline $\mathrm{O}$ & -2.35551800 & 2.15603600 & -0.87231200 \\
\hline $\mathrm{C}$ & -3.56481900 & 2.84868800 & -0.58170300 \\
\hline $\mathrm{H}$ & -3.64664500 & 3.77130600 & -1.16878300 \\
\hline $\mathrm{H}$ & -4.36611100 & 2.16694800 & -0.86358800 \\
\hline $\mathrm{H}$ & -3.63469700 & 3.07476600 & 0.48799400 \\
\hline I & 1.85789800 & -2.81852200 & -0.15191100 \\
\hline I & 3.08403800 & 1.48108100 & -0.65656300 \\
\hline $\mathrm{C}$ & -1.16072500 & 0.87832700 & 2.73564000 \\
\hline $\mathrm{O}$ & -0.08789000 & 0.20233300 & 2.75838300 \\
\hline $\mathrm{O}$ & -1.91671500 & 0.98069800 & 1.71749200 \\
\hline $\mathrm{H}$ & -1.46040900 & 0.36573500 & 0.74319700 \\
\hline $\mathrm{C}$ & -1.56717300 & 1.60643500 & 3.99320400 \\
\hline $\mathrm{H}$ & -2.52183000 & 1.20153900 & 4.34588100 \\
\hline $\mathrm{H}$ & -0.81017900 & 1.49520900 & 4.77014700 \\
\hline $\mathrm{H}$ & -1.72129800 & 2.66551400 & 3.76441900 \\
\hline $\mathrm{C}$ & -0.73000200 & 0.45350300 & -2.67307200 \\
\hline $\mathrm{H}$ & -0.05778900 & 1.05441700 & -3.29487100 \\
\hline $\mathrm{H}$ & -0.72981900 & -0.56533600 & -3.06074900 \\
\hline $\mathrm{H}$ & -1.72989100 & 0.87463600 & -2.74310100 \\
\hline $\mathrm{H}$ & 0.83862900 & 0.07287900 & -1.31083800 \\
\hline
\end{tabular}

$\mathrm{S}_{9 \mathrm{C}}$

$-1743.76060113$

$\begin{array}{lrrr}\text { Pd } & -0.73675900 & -1.35985300 & -0.42549400 \\ \text { S } & -2.13381000 & 1.52451300 & -0.86614800 \\ \text { F } & -3.52360100 & 1.53230900 & 1.39364900 \\ \text { F } & -2.80981900 & 3.51024600 & 0.82005900 \\ \text { F } & -4.45036800 & 2.59602400 & -0.26943200 \\ \text { O } & -2.97049900 & 0.50680100 & -1.47776100 \\ \text { O } & -1.41965700 & 2.55485700 & -1.60290900 \\ \text { N } & -1.00435200 & 0.68415000 & 0.20563800 \\ \text { C } & 0.28876800 & 1.40014300 & 0.58226300 \\ \text { C } & 1.07558600 & 0.47716800 & 1.50908400 \\ \text { C } & 2.44922700 & 0.24250800 & 1.34955500 \\ \text { C } & 3.16034900 & -0.60452100 & 2.20372600\end{array}$




\begin{tabular}{|c|c|c|c|}
\hline C & 2.49659900 & -1.22507600 & 3.25609000 \\
\hline $\mathrm{C}$ & 1.14307600 & -0.98206700 & 3.48365100 \\
\hline $\mathrm{C}$ & 0.44983000 & -0.12246000 & 2.63267400 \\
\hline $\mathrm{C}$ & -3.30810200 & 2.36609100 & 0.36179700 \\
\hline $\mathrm{H}$ & 4.21840600 & -0.77552900 & 2.04616700 \\
\hline $\mathrm{H}$ & 3.04060100 & -1.89426700 & 3.91638800 \\
\hline $\mathrm{H}$ & 0.64145400 & -1.45479400 & 4.31861000 \\
\hline $\mathrm{O}$ & -0.86994600 & 0.21125200 & 2.82934300 \\
\hline $\mathrm{C}$ & -1.63141400 & -0.49739600 & 3.81228600 \\
\hline $\mathrm{H}$ & -1.23424600 & -0.31549600 & 4.81699500 \\
\hline $\mathrm{H}$ & -2.64042800 & -0.08866000 & 3.74273900 \\
\hline $\mathrm{H}$ & -1.64860700 & -1.56643100 & 3.58363100 \\
\hline I & 0.46167500 & -0.76346600 & -2.69768600 \\
\hline I & 3.60958600 & 1.22321500 & -0.16393400 \\
\hline $\mathrm{C}$ & -1.28312900 & -3.55986300 & 0.56161000 \\
\hline $\mathrm{O}$ & -0.67962800 & -3.40923500 & -0.55889900 \\
\hline $\mathrm{O}$ & -1.60738300 & -2.51743200 & 1.21985000 \\
\hline $\mathrm{H}$ & -1.50702100 & 0.49423000 & 1.08620800 \\
\hline $\mathrm{C}$ & -1.61737500 & -4.93935400 & 1.05008100 \\
\hline $\mathrm{H}$ & -2.53649900 & -5.27956600 & 0.55869000 \\
\hline $\mathrm{H}$ & -0.81711600 & -5.63612000 & 0.78751200 \\
\hline $\mathrm{H}$ & -1.78027800 & -4.93004000 & 2.13019400 \\
\hline $\mathrm{C}$ & 0.09717300 & 2.78494500 & 1.21756500 \\
\hline $\mathrm{H}$ & 1.08696200 & 3.17400700 & 1.47560200 \\
\hline $\mathrm{H}$ & -0.36222100 & 3.48691400 & 0.52052000 \\
\hline $\mathrm{H}$ & -0.49543500 & 2.73087700 & 2.13493100 \\
\hline $\mathrm{H}$ & 0.82746200 & 1.50230400 & -0.35844000 \\
\hline
\end{tabular}

$\mathrm{S}_{\mathrm{R}-\mathrm{Me}}$

$-520.05279242$

$\begin{array}{lrrr}\mathrm{N} & -2.33932400 & -0.05078200 & -0.49273200 \\ \mathrm{C} & -1.50045900 & -0.67450800 & 0.53789600 \\ \mathrm{C} & 0.00880900 & -0.65689800 & 0.26429800 \\ \mathrm{C} & 0.72939400 & -1.85216800 & 0.23528700 \\ \mathrm{C} & 2.10758500 & -1.88908600 & 0.00257800 \\ \mathrm{C} & 2.78925100 & -0.69751700 & -0.21758100 \\ \mathrm{C} & 2.10069300 & 0.51934900 & -0.20490000 \\ \mathrm{C} & 0.72345500 & 0.54046000 & 0.03367000 \\ \mathrm{H} & 0.19108400 & -2.78263700 & 0.40466500 \\ \mathrm{H} & 2.63501000 & -2.83863000 & -0.00960500 \\ \mathrm{H} & 3.85999600 & -0.70031400 & -0.40355800 \\ \mathrm{H} & 2.64501900 & 1.43994500 & -0.38100000 \\ \mathrm{C} & -1.84502700 & -0.06865200 & 1.90780700 \\ \mathrm{H} & -1.57840800 & 0.99192400 & 1.94535800\end{array}$

$\begin{array}{rrrr}\mathrm{H} & -2.92056500 & -0.16229100 & 2.08847600 \\ \mathrm{H} & -1.30245600 & -0.58374900 & 2.70807400 \\ \mathrm{H} & -1.79359400 & -1.73353700 & 0.56273600 \\ \mathrm{H} & -2.07849100 & 0.93043300 & -0.56056200 \\ \mathrm{O} & -0.01657300 & 1.69984700 & 0.05840000 \\ \mathrm{C} & 0.64990800 & 2.93721700 & -0.13619800 \\ \mathrm{H} & 1.11844400 & 2.99291600 & -1.12756500 \\ \mathrm{H} & -0.12017100 & 3.70704300 & -0.05802000 \\ \mathrm{H} & 1.41345500 & 3.10870700 & 0.63351400 \\ \mathrm{C} & -2.23880900 & -0.67099900 & -1.80989100 \\ \mathrm{H} & -2.88682600 & -0.13141800 & -2.50917100 \\ \mathrm{H} & -1.22053100 & -0.70040500 & -2.23634500 \\ \mathrm{H} & -2.60291900 & -1.70448100 & -1.75285700\end{array}$

$\mathrm{S}_{\mathrm{S}-\mathrm{Me}}$

$-520.05279242$

$\begin{array}{llll}\mathrm{N} & 2.33932000 & -0.05081200 & -0.49272300\end{array}$

$\begin{array}{llll}\text { C } & 1.50044200 & -0.67453900 & 0.53789600\end{array}$

$\begin{array}{llll}\text { C } & -0.00882200 & -0.65690400 & 0.26429600\end{array}$

$\begin{array}{llll}\text { C } & -0.72942600 & -1.85216300 & 0.23527800\end{array}$

$\begin{array}{llll}\text { C } & -2.10761800 & -1.88905900 & 0.00256700\end{array}$

$\begin{array}{llll}\text { C } & -2.78926500 & -0.69747800 & -0.21758700\end{array}$

$\begin{array}{llll}\text { C } & -2.10068600 & 0.51937700 & -0.20489900\end{array}$

$\begin{array}{llll}\text { C } & -0.72344900 & 0.54046700 & 0.03367300\end{array}$

H $\quad-0.19113000 \quad-2.78264100 \quad 0.40465100$

$\begin{array}{llll}\mathrm{H} & -2.63505700 & -2.83859500 & -0.00962400\end{array}$

$\begin{array}{llll}\mathrm{H} & -3.86000900 & -0.70025800 & -0.40356500\end{array}$

$\begin{array}{llll}\mathrm{H} & -2.64500000 & 1.43998200 & -0.38099300\end{array}$

$\begin{array}{llll}\mathrm{H} & 2.07852500 & 0.93041200 & -0.56052900\end{array}$

$\begin{array}{llll}\text { O } & 0.01660000 & 1.69984000 & 0.05841300\end{array}$

$\begin{array}{llll}\text { C } & -0.64985100 & 2.93722500 & -0.13619200\end{array}$

$\begin{array}{llll}\mathrm{H} & -1.41339800 & 3.10873600 & 0.63351400\end{array}$

$\begin{array}{llll}\mathrm{H} & 0.12024600 & 3.70703300 & -0.05801300\end{array}$

$\begin{array}{llll}\mathrm{H} & -1.11837900 & 2.99293200 & -1.12756200\end{array}$

$\begin{array}{llll}\mathrm{H} & 1.79356700 & -1.73357000 & 0.56272700\end{array}$

$\begin{array}{llll}\text { C } & 1.84502000 & -0.06868700 & 1.90780400\end{array}$

$\begin{array}{llll}\mathrm{H} & 1.57842000 & 0.99189400 & 1.94535400\end{array}$

$\begin{array}{llll}\mathrm{H} & 1.30244000 & -0.58377200 & 2.70807300\end{array}$

$\begin{array}{llll}\mathrm{H} & 2.92055700 & -0.16234500 & 2.08847500\end{array}$

$\begin{array}{llll}\text { C } & 2.23880700 & -0.67100100 & -1.80989500\end{array}$

$\begin{array}{llll}\mathrm{H} & 1.22052700 & -0.70041000 & -2.23633800\end{array}$

$\begin{array}{llll}\mathrm{H} & 2.88680400 & -0.13138800 & -2.50916700\end{array}$

$\begin{array}{llll}\mathrm{H} & 2.60293700 & -1.70447800 & -1.75289600\end{array}$ 


\begin{tabular}{|c|c|c|c|c|c|c|c|}
\hline \multicolumn{4}{|c|}{$\mathrm{R}-\mathrm{Me}_{3}$} & $\mathrm{H}$ & 2.43233100 & -0.36521400 & -2.68100700 \\
\hline \multicolumn{4}{|c|}{-1660.77115016} & $\mathrm{H}$ & 0.11529600 & -0.03270700 & -3.51588300 \\
\hline $\mathrm{Pd}$ & 2.09782000 & 0.17949600 & 0.00216500 & $\mathrm{H}$ & -1.73428200 & -1.39032700 & -2.54925200 \\
\hline $\mathrm{C}$ & -1.96015800 & 1.80435000 & -0.58646700 & $\mathrm{H}$ & -1.30642100 & -2.98528200 & -0.73627800 \\
\hline $\mathrm{C}$ & -0.42490200 & 1.70707700 & -0.65900300 & $\mathrm{O}$ & 1.12820400 & -3.42715200 & 0.37905100 \\
\hline $\mathrm{H}$ & -2.34886600 & 1.70798200 & -1.60194000 & $\mathrm{C}$ & 0.08544300 & -4.17778900 & 0.99162100 \\
\hline $\mathrm{N}$ & -2.49454000 & 0.68548400 & 0.18788900 & $\mathrm{H}$ & -0.39237300 & -4.85520800 & 0.27291100 \\
\hline $\mathrm{H}$ & -1.84759300 & 0.32911600 & 0.88010000 & $\mathrm{H}$ & 0.56625700 & -4.76305200 & 1.77697100 \\
\hline $\mathrm{C}$ & -3.44131400 & -0.16008500 & -0.31387800 & $\mathrm{H}$ & -0.67483600 & -3.52185300 & 1.43257100 \\
\hline $\mathrm{O}$ & -4.01559400 & 0.02996000 & -1.38694800 & $\mathrm{C}$ & 4.19242100 & -2.66700400 & -1.87200900 \\
\hline $\mathrm{C}$ & -3.74981900 & -1.37388200 & 0.52805600 & $\mathrm{H}$ & 4.21171700 & -2.01497000 & -2.75042500 \\
\hline $\mathrm{C}$ & -3.41317200 & -1.48671700 & 1.88518500 & $\mathrm{H}$ & 5.22512500 & -2.81633100 & -1.53728900 \\
\hline $\mathrm{C}$ & -4.41893700 & -2.43493800 & -0.09780400 & $\mathrm{H}$ & 3.78194700 & -3.64171000 & -2.16319400 \\
\hline $\mathrm{C}$ & -3.71881200 & -2.65009900 & 2.59437700 & $\mathrm{H}$ & 3.34802100 & -2.74094300 & 0.10798400 \\
\hline $\mathrm{H}$ & -2.94332100 & -0.65676400 & 2.40525900 & $\mathrm{C}$ & 2.31987100 & 2.33023000 & 2.23827800 \\
\hline $\mathrm{C}$ & -4.71795500 & -3.59921000 & 0.60806800 & $\mathrm{O}$ & 2.93621300 & 1.54770200 & 1.49707300 \\
\hline $\mathrm{H}$ & -4.69921300 & -2.31763700 & -1.13954900 & $\mathrm{O}$ & 1.01923100 & 2.47026600 & 2.25448000 \\
\hline $\mathrm{C}$ & -4.36481600 & -3.71150300 & 1.95582200 & $\mathrm{H}$ & 0.59431700 & 1.89633100 & 1.52008900 \\
\hline $\mathrm{H}$ & -3.46435000 & -2.72114600 & 3.64860800 & $\mathrm{C}$ & 3.05236900 & 3.22033800 & 3.20725100 \\
\hline $\mathrm{H}$ & -5.23271400 & -4.41673000 & 0.11024500 & $\mathrm{H}$ & 2.59006600 & 3.15616700 & 4.19665600 \\
\hline $\mathrm{H}$ & -4.60370900 & -4.61578400 & 2.50942100 & $\mathrm{H}$ & 4.10451300 & 2.93950000 & 3.25752600 \\
\hline $\mathrm{C}$ & -2.42624200 & 3.15234200 & 0.01838700 & $\mathrm{H}$ & 2.96673800 & 4.25982500 & 2.87127000 \\
\hline $\mathrm{H}$ & -3.52358300 & 3.11523900 & 0.05499100 & $\mathrm{C}$ & 4.72950800 & -0.77222300 & 0.79485900 \\
\hline $\mathrm{H}$ & -2.08358600 & 3.19787200 & 1.06233000 & $\mathrm{H}$ & 4.98936100 & 0.23788000 & 1.11514100 \\
\hline $\mathrm{C}$ & -1.98458100 & 4.43326100 & -0.71713900 & $\mathrm{H}$ & 4.31149100 & -1.32583400 & 1.65647200 \\
\hline $\mathrm{C}$ & -2.51509800 & 5.66378600 & 0.03541400 & $\mathrm{H}$ & 5.65697800 & -1.28543400 & 0.49612400 \\
\hline $\mathrm{C}$ & -2.43155400 & 4.45155800 & -2.18666800 & & & & \\
\hline $\mathrm{H}$ & -0.88780400 & 4.47418500 & -0.70535100 & $\mathrm{TS}_{2}$ & & & \\
\hline $\mathrm{H}$ & -2.18051400 & 5.67180800 & 1.08030400 & -16 & .73625126 & & \\
\hline $\mathrm{H}$ & -2.16879000 & 6.59167600 & -0.43506300 & $\mathrm{Pd}$ & -1.27121100 & 0.46850500 & -0.98043300 \\
\hline $\mathrm{H}$ & -3.61306300 & 5.68217900 & 0.03749600 & $\mathrm{C}$ & 2.19307800 & 0.85021700 & 1.73058600 \\
\hline $\mathrm{H}$ & -1.93202100 & 3.66596200 & -2.76100700 & $\mathrm{C}$ & 0.84566700 & 0.57988600 & 1.04724400 \\
\hline $\mathrm{H}$ & -3.51738100 & 4.31114600 & -2.27434600 & $\mathrm{H}$ & 2. 10907800 & 0.45298100 & 2.74396200 \\
\hline $\mathrm{H}$ & -2.18123400 & 5.41160200 & -2.65402000 & $\mathrm{~N}$ & 3.28308300 & 0.15622300 & 1.06169800 \\
\hline $\mathrm{O}$ & 0.12230200 & 1.01987500 & 0.33224700 & $\mathrm{H}$ & 3.82798400 & 0.68029500 & 0.39056900 \\
\hline $\mathrm{O}$ & 0.19458700 & 2.24710200 & -1.56428500 & C & 3.23991000 & -1.21176000 & 0.93529700 \\
\hline $\mathrm{N}$ & 3.84030400 & -0.71671300 & -0.35497200 & $\mathrm{O}$ & 2.33495400 & -1.86974300 & 1.44435800 \\
\hline C & 3.35075600 & -2.04948600 & -0.74632000 & $\mathrm{C}$ & 4.35690100 & -1.84967300 & 0.16441800 \\
\hline C & 1.88943400 & -1.79150400 & -1.15148100 & C & 5.60064900 & -1.23316900 & -0.03565800 \\
\hline $\mathrm{C}$ & 1.60637500 & -0.89023100 & -2.21240900 & C & 4.13550100 & -3.13343500 & -0.35276500 \\
\hline $\mathrm{C}$ & 0.30749900 & -0.74504000 & -2.72187600 & C & 6.60084100 & -1.88525000 & -0.75718900 \\
\hline $\mathrm{C}$ & -0.71904300 & -1.50382200 & -2.17936300 & $\mathrm{H}$ & 5.80663600 & -0.25942100 & 0.40063000 \\
\hline C & -0.47848300 & -2.41069800 & -1.13360700 & C & 5.13244500 & -3.78036300 & -1.07939100 \\
\hline $\mathrm{C}$ & 0.80485900 & -2.55901100 & -0.61442800 & $\mathrm{H}$ & 3.17488900 & -3.60387200 & -0.17089900 \\
\hline
\end{tabular}




\begin{tabular}{|c|c|c|c|c|c|c|c|}
\hline $\mathrm{C}$ & 6.36627000 & -3.15659300 & -1.28518800 & $\mathrm{H}$ & 1.41508900 & 1.32889000 & -1.50673200 \\
\hline $\mathrm{H}$ & 7.56490800 & -1.40459100 & -0.89917100 & $\mathrm{C}$ & 0.81742700 & 2.88105100 & -4.29261900 \\
\hline $\mathrm{H}$ & 4.94970300 & -4.77218500 & -1.48368700 & $\mathrm{H}$ & 1.51608700 & 2.33362100 & -4.93440600 \\
\hline $\mathrm{H}$ & 7.14535600 & -3.66302200 & -1.84865200 & $\mathrm{H}$ & -0.11595400 & 3.06046700 & -4.82608400 \\
\hline $\mathrm{C}$ & 2.50002800 & 2.36211600 & 1.76442400 & $\mathrm{H}$ & 1.29142800 & 3.83263500 & -4.03105900 \\
\hline $\mathrm{H}$ & 3.49451300 & 2.48120400 & 2.21710600 & $\mathrm{C}$ & -3.56857100 & -0.08878800 & -2.59179900 \\
\hline $\mathrm{H}$ & 2.57893400 & 2.71939500 & 0.72664900 & $\mathrm{H}$ & -2.87917800 & 0.07672200 & -3.42567500 \\
\hline $\mathrm{C}$ & 1.49267900 & 3.25049200 & 2.52253800 & $\mathrm{H}$ & -3.60309100 & -1.17114500 & -2.37605000 \\
\hline $\mathrm{C}$ & 1.90415900 & 4.72412200 & 2.37947400 & $\mathrm{H}$ & -4.57256300 & 0.21320600 & -2.93303500 \\
\hline $\mathrm{C}$ & 1.36114000 & 2.85898500 & 4.00212600 & & & & \\
\hline $\mathrm{H}$ & 0.50628400 & 3.13031100 & 2.05492400 & \multicolumn{4}{|c|}{$\mathrm{R}-\mathrm{Me}_{4}$} \\
\hline $\mathrm{H}$ & 1.96806900 & 5.02449100 & 1.32649900 & \multicolumn{4}{|c|}{-1660.75238028} \\
\hline $\mathrm{H}$ & 1.17814000 & 5.38119900 & 2.87130800 & $\mathrm{Pd}$ & 1.32139700 & 0.48668500 & 0.95482800 \\
\hline $\mathrm{H}$ & 2.88394000 & 4.90746500 & 2.84013800 & $\mathrm{C}$ & -2.29579400 & 0.74152300 & -1.79410500 \\
\hline $\mathrm{H}$ & 0.94514300 & 1.85398100 & 4.12132300 & $\mathrm{C}$ & -0.93736900 & 0.42516300 & -1.17164200 \\
\hline $\mathrm{H}$ & 2.33590500 & 2.89316600 & 4.50733900 & $\mathrm{H}$ & -2.27566100 & 0.34982100 & -2.81368500 \\
\hline $\mathrm{H}$ & 0.69297000 & 3.55358200 & 4.52400900 & $\mathrm{~N}$ & -3.36023600 & 0.07857800 & -1.06062600 \\
\hline $\mathrm{O}$ & 0.80617400 & 0.47652700 & -0.21780600 & $\mathrm{H}$ & -3.91543800 & 0.63400400 & -0.42435200 \\
\hline $\mathrm{O}$ & -0.17899000 & 0.50953600 & 1.79440800 & $\mathrm{C}$ & -3.29715000 & -1.28495800 & -0.88149600 \\
\hline $\mathrm{N}$ & -3.18004900 & 0.75751200 & -1.46516000 & $\mathrm{O}$ & -2.37582100 & -1.93303000 & -1.37375700 \\
\hline $\mathrm{C}$ & -4.01396700 & 0.49309100 & -0.26481800 & $\mathrm{C}$ & -4.39071100 & -1.91517100 & -0.07823600 \\
\hline $\mathrm{C}$ & -3.46016600 & -0.73687600 & 0.41865800 & $\mathrm{C}$ & -5.64091500 & -1.31073900 & 0.11972600 \\
\hline $\mathrm{C}$ & -2.04579400 & -0.85048900 & 0.44851800 & $\mathrm{C}$ & -4.13957200 & -3.17746700 & 0.47752600 \\
\hline $\mathrm{C}$ & -1.45864700 & -2.07414300 & 0.84412100 & $\mathrm{C}$ & -6.61940600 & -1.95411000 & 0.87723400 \\
\hline $\mathrm{C}$ & -2.26096400 & -3.12560000 & 1.27384400 & $\mathrm{H}$ & -5.86971100 & -0.35521600 & -0.34470000 \\
\hline $\mathrm{C}$ & -3.65465700 & -2.99953500 & 1.28660800 & $\mathrm{C}$ & -5.11565500 & -3.81499900 & 1.23969200 \\
\hline $\mathrm{C}$ & -4.25568000 & -1.81296800 & 0.84829300 & $\mathrm{H}$ & -3.17358000 & -3.63819400 & 0.29944000 \\
\hline $\mathrm{H}$ & -1.17176600 & 0.07648700 & 1.09127100 & $\mathrm{C}$ & -6.35604800 & -3.20361900 & 1.44290100 \\
\hline $\mathrm{H}$ & -0.37652600 & -2.17141400 & 0.87281000 & $\mathrm{H}$ & -7.58868700 & -1.48419300 & 1.01863700 \\
\hline $\mathrm{H}$ & -1.80944700 & -4.05416800 & 1.61379900 & $\mathrm{H}$ & -4.91095600 & -4.78935200 & 1.67432700 \\
\hline $\mathrm{H}$ & -4.25964000 & -3.83001300 & 1.63391100 & $\mathrm{H}$ & -7.11835500 & -3.70284100 & 2.03483600 \\
\hline $\mathrm{O}$ & -5.61259400 & -1.61904700 & 0.79219900 & $\mathrm{C}$ & -2.54946900 & 2.26523300 & -1.79853500 \\
\hline $\mathrm{C}$ & -6.46359700 & -2.68839500 & 1.16407400 & $\mathrm{H}$ & -3.56021300 & 2.41874500 & -2.20139900 \\
\hline $\mathrm{H}$ & -6.32929700 & -2.96688900 & 2.21821500 & $\mathrm{H}$ & -2.56435200 & 2.60933300 & -0.75451600 \\
\hline $\mathrm{H}$ & -7.48297700 & -2.32569000 & 1.01713500 & $\mathrm{C}$ & -1.55276200 & 3.12795200 & -2.59926200 \\
\hline $\mathrm{H}$ & -6.30105300 & -3.57493500 & 0.53589000 & $\mathrm{C}$ & -1.91133300 & 4.61175400 & -2.42211500 \\
\hline $\mathrm{C}$ & -4.04825500 & 1.72331800 & 0.65121200 & $\mathrm{C}$ & -1.50849400 & 2.75020300 & -4.08787700 \\
\hline $\mathrm{H}$ & -3.04653900 & 1.95961500 & 1.02733800 & $\mathrm{H}$ & -0.54798600 & 2.97671300 & -2.18062300 \\
\hline $\mathrm{H}$ & -4.41531900 & 2.59031900 & 0.09159800 & $\mathrm{H}$ & -1.91733900 & 4.90179400 & -1.36465500 \\
\hline $\mathrm{H}$ & -4.70663900 & 1.54680400 & 1.50990100 & $\mathrm{H}$ & -1.18817500 & 5.25100400 & -2.94022400 \\
\hline $\mathrm{H}$ & -5.04036700 & 0.29488800 & -0.60102400 & $\mathrm{H}$ & -2.90457900 & 4.82869600 & -2.83645800 \\
\hline $\mathrm{C}$ & 0.54931000 & 2.08361300 & -3.04314600 & $\mathrm{H}$ & -1.13868400 & 1.73194300 & -4.24451500 \\
\hline $\mathrm{O}$ & -0.57636000 & 1.67919300 & -2.75245200 & $\mathrm{H}$ & -2.50467100 & 2.82767300 & -4.54367100 \\
\hline $\mathrm{O}$ & 1.63750500 & 1.87092100 & -2.31681700 & $\mathrm{H}$ & -0.84095400 & 3.42609000 & 3391000 \\
\hline
\end{tabular}




\begin{tabular}{|c|c|c|c|}
\hline $\mathrm{O}$ & -0.76543500 & 0.39357900 & 0.05537800 \\
\hline $\mathrm{O}$ & 0.03479100 & 0.26866100 & -2.03780400 \\
\hline$N$ & 3.16434600 & 0.82124100 & 1.64157800 \\
\hline 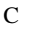 & 4.16796100 & 0.39593600 & 0.62936200 \\
\hline 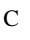 & 3.63693900 & -0.81406800 & -0.10164600 \\
\hline $\mathrm{C}$ & 2.23337500 & -0.95348300 & -0.08575700 \\
\hline $\mathrm{C}$ & 1.63682700 & -2.07847700 & -0.68253100 \\
\hline $\mathrm{C}$ & 2.44094500 & -3.02964200 & -1.31729800 \\
\hline $\mathrm{C}$ & 3.83021100 & -2.88933600 & -1.35510900 \\
\hline $\mathrm{C}$ & 4.42956600 & -1.77952100 & -0.74265500 \\
\hline $\mathrm{H}$ & 0.87438600 & 0.04896100 & -1.54635900 \\
\hline $\mathrm{H}$ & 0.55891300 & -2.22152000 & -0.65873700 \\
\hline $\mathrm{H}$ & 1.98305600 & -3.89636400 & -1.78870800 \\
\hline $\mathrm{H}$ & 4.43039300 & -3.64377800 & -1.85201700 \\
\hline $\mathrm{O}$ & 5.78480600 & -1.55728800 & -0.71333600 \\
\hline $\mathrm{C}$ & 6.63611100 & -2.52157200 & -1.30372000 \\
\hline I & 6.44990100 & -2.62371700 & -2.38185000 \\
\hline $\mathrm{H}$ & 7.65493400 & -2.15955500 & -1.14919500 \\
\hline $\mathrm{H}$ & 6.52625700 & -3.50607700 & -0.82859100 \\
\hline C & 4.47655200 & 1.55976600 & -0.32526600 \\
\hline $\mathrm{H}$ & 3.56452400 & 1.86986000 & -0.84909700 \\
\hline $\mathrm{H}$ & 4.85339800 & 2.41901000 & 0.24005600 \\
\hline $\mathrm{H}$ & 5.22514400 & 1.26356300 & -1.06910000 \\
\hline $\mathrm{H}$ & 5.10228900 & 0.14016300 & 1.15133100 \\
\hline $\mathrm{C}$ & -0.80999700 & 2.34089800 & 2.68630800 \\
\hline $\mathrm{O}$ & 0.34498700 & 2.05232200 & 2.40061000 \\
\hline $\mathrm{O}$ & -1.87962800 & 1.83876600 & 2.06181200 \\
\hline $\mathrm{H}$ & -1.56921000 & 1.22071900 & 1.35168300 \\
\hline $\mathrm{C}$ & -1.17333600 & 3.30032200 & 3.78908200 \\
\hline $\mathrm{H}$ & -1.75223500 & 2.77494900 & 4.55645100 \\
\hline $\mathrm{H}$ & -0.26703700 & 3.71675400 & 4.22866700 \\
\hline $\mathrm{H}$ & -1.80581200 & 4.10246800 & 3.39516700 \\
\hline $\mathrm{C}$ & 3.36568200 & 0.11892400 & 2.90730800 \\
\hline $\mathrm{H}$ & 2.56672200 & 0.38318000 & 3.60844100 \\
\hline $\mathrm{H}$ & 3.40812300 & -0.98247200 & 2.82746200 \\
\hline $\mathrm{H}$ & 4.31598600 & 0.45443800 & 3.35657000 \\
\hline
\end{tabular}

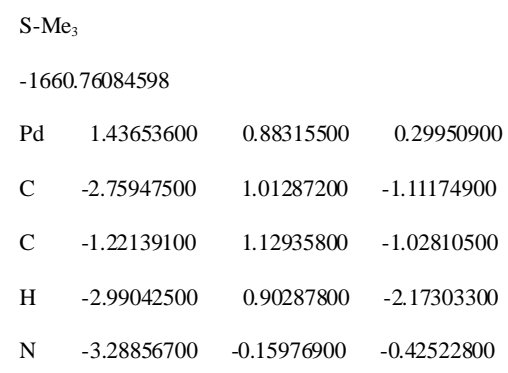

\begin{tabular}{|c|c|c|c|}
\hline $\mathrm{H}$ & -3.55962600 & -0.04324100 & 0.54177500 \\
\hline $\mathrm{C}$ & -2.96424600 & -1.42203600 & -0.84564100 \\
\hline $\mathrm{O}$ & -2.30170100 & -1.62363600 & -1.86281700 \\
\hline C & -3.48731700 & -2.55498300 & -0.00591800 \\
\hline C & -4.58860500 & -2.43037500 & 0.85340300 \\
\hline $\mathrm{C}$ & -2.84787000 & -3.79650100 & -0.12027400 \\
\hline $\mathrm{C}$ & -5.02855100 & -3.52432200 & 1.59964100 \\
\hline $\mathrm{H}$ & -5.12926600 & -1.49000000 & 0.91703500 \\
\hline $\mathrm{C}$ & -3.28257300 & -4.88681000 & 0.63001000 \\
\hline $\mathrm{H}$ & -2.01266100 & -3.88203200 & -0.80735400 \\
\hline $\mathrm{C}$ & -4.37276900 & -4.75250700 & 1.49430900 \\
\hline $\mathrm{H}$ & -5.88868500 & -3.41934500 & 2.25540200 \\
\hline $\mathrm{H}$ & -2.77486100 & -5.84343400 & 0.53954100 \\
\hline $\mathrm{H}$ & -4.71523300 & -5.60375500 & 2.07673900 \\
\hline $\mathrm{C}$ & -3.45128800 & 2.27038600 & -0.54423500 \\
\hline $\mathrm{H}$ & -4.53621800 & 2.11802000 & -0.63808600 \\
\hline $\mathrm{H}$ & -3.23487000 & 2.32920600 & 0.53360000 \\
\hline $\mathrm{C}$ & -3.07402700 & 3.61607800 & -1.19587200 \\
\hline $\mathrm{C}$ & -3.82121200 & 4.75559400 & -0.48548000 \\
\hline $\mathrm{C}$ & -3.34770700 & 3.63554300 & -2.70717200 \\
\hline $\mathrm{H}$ & -1.99730900 & 3.77471300 & -1.05288300 \\
\hline $\mathrm{H}$ & -3.61547700 & 4.76334400 & 0.59223000 \\
\hline $\mathrm{H}$ & -3.52429600 & 5.73013100 & -0.89003100 \\
\hline $\mathrm{H}$ & -4.90724800 & 4.65878800 & -0.61704300 \\
\hline $\mathrm{H}$ & -2.70204400 & 2.92739600 & -3.23409800 \\
\hline $\mathrm{H}$ & -4.39550900 & 3.38643000 & -2.92435400 \\
\hline $\mathrm{H}$ & -3.15186800 & 4.63235500 & -3.11986500 \\
\hline $\mathrm{O}$ & -0.67785500 & 0.64825300 & 0.06365600 \\
\hline $\mathrm{O}$ & -0.60452300 & 1.66385700 & -1.94484600 \\
\hline $\mathrm{N}$ & 3.38766500 & 1.28482100 & 0.55764600 \\
\hline $\mathrm{C}$ & 4.07367200 & 0.86016100 & -0.68689300 \\
\hline $\mathrm{C}$ & 3.28853200 & -0.38018700 & -1.11035700 \\
\hline C & 2.02569300 & -0.10552700 & -1.70805600 \\
\hline $\mathrm{C}$ & 1.10371700 & -1.14164400 & -1.99780200 \\
\hline $\mathrm{C}$ & 1.45266100 & -2.44744000 & -1.71222800 \\
\hline $\mathrm{C}$ & 2.69930500 & -2.74426900 & -1.13476100 \\
\hline $\mathrm{C}$ & 3.59618300 & -1.72956300 & -0.80051900 \\
\hline $\mathrm{H}$ & 1.84959600 & 0.87837800 & -2.14439600 \\
\hline $\mathrm{H}$ & 0.14197200 & -0.90253200 & -2.43544000 \\
\hline $\mathrm{H}$ & 0.76516200 & -3.25673200 & -1.93944900 \\
\hline $\mathrm{H}$ & 2.95248000 & -3.78019700 & -0.93957900 \\
\hline $\mathrm{O}$ & 4.80274200 & -1.96325500 & -0.19748200 \\
\hline C & 5.17539900 & -3.30330500 & 0.08181200 \\
\hline $\mathrm{H}$ & 5.24171000 & -3.90271600 & -0.83563300 \\
\hline
\end{tabular}




$\begin{array}{lrrr}\mathrm{H} & 6.16098300 & -3.24692000 & 0.54732300 \\ \mathrm{H} & 4.47274200 & -3.78223500 & 0.77636000 \\ \mathrm{C} & -0.07481300 & 1.77930100 & 2.92501600 \\ \mathrm{O} & 1.00774700 & 1.74142900 & 2.32326300 \\ \mathrm{O} & -1.21389000 & 1.33905300 & 2.44507500 \\ \mathrm{H} & -1.08617100 & 1.00654300 & 1.47834200 \\ \mathrm{C} & -0.17577400 & 2.35976600 & 4.31276300 \\ \mathrm{H} & -0.62589000 & 1.62570000 & 4.98877500 \\ \mathrm{H} & 0.81152500 & 2.64928500 & 4.67306300 \\ \mathrm{H} & -0.83504700 & 3.23429000 & 4.29429800 \\ \mathrm{C} & 3.89787400 & 0.65700900 & 1.77454200 \\ \mathrm{H} & 3.25470000 & 0.94518600 & 2.60781400 \\ \mathrm{H} & 3.96954100 & -0.44196800 & 1.73487500 \\ \mathrm{H} & 4.90550000 & 1.04505700 & 1.98312800 \\ \mathrm{C} & 5.60086600 & 0.80735800 & -0.61509600 \\ \mathrm{H} & 5.97606700 & 0.07806600 & 0.10129900 \\ \mathrm{H} & 6.00256700 & 0.54946500 & -1.60143600 \\ \mathrm{H} & 5.97710700 & 1.80082900 & -0.34540800 \\ \mathrm{H} & 3.82881900 & 1.62921400 & -1.43067600\end{array}$

$\mathrm{TS}_{2 \mathrm{~S}-\mathrm{Me}}$

$-1660.72851022$

\begin{tabular}{lrrr}
$\mathrm{Pd}$ & 1.23052500 & 0.53657200 & 0.94341900 \\
$\mathrm{C}$ & -2.24631300 & 0.78564400 & -1.77467500 \\
$\mathrm{C}$ & -0.89344300 & 0.56268700 & -1.08486200 \\
$\mathrm{H}$ & -2.16107900 & 0.34165100 & -2.76832700 \\
$\mathrm{~N}$ & -3.32633400 & 0.11224600 & -1.06967600 \\
$\mathrm{H}$ & -3.87216600 & 0.66107800 & -0.41943700 \\
$\mathrm{C}$ & -3.26962500 & -1.24821300 & -0.88216900 \\
$\mathrm{O}$ & -2.36146100 & -1.92014000 & -1.36666900 \\
$\mathrm{C}$ & -4.37638100 & -1.86106700 & -0.07685400 \\
$\mathrm{C}$ & -5.62372700 & -1.24635200 & 0.10553400 \\
$\mathrm{C}$ & -4.14126900 & -3.11977600 & 0.49320400 \\
$\mathrm{C}$ & -6.61368400 & -1.87463500 & 0.86147000 \\
$\mathrm{H}$ & -5.84068500 & -0.29385100 & -0.37051700 \\
$\mathrm{C}$ & -5.12800300 & -3.74276000 & 1.25388200 \\
$\mathrm{H}$ & -3.17815600 & -3.59015600 & 0.32489900 \\
$\mathrm{H}$ & -6.36539500 & -3.12040600 & 1.44162600 \\
$\mathrm{H}$ & -7.58057800 & -1.39576900 & 0.98962500 \\
$\mathrm{H}$ & -4.93446200 & -4.71490100 & 1.69891200 \\
$\mathrm{H}$ & -7.13654100 & -3.60824600 & 2.03177300 \\
$\mathrm{H}$ & -2.57146000 & 2.29069800 & -1.87904500 \\
$\mathrm{H}$ & -3.56966300 & 2.37560500 & -2.33115800 \\
$\mathrm{H}$ & -2.64970400 & 2.69598200 & -0.85897900 \\
\hline
\end{tabular}

\begin{tabular}{|c|c|c|c|}
\hline $\mathrm{C}$ & -1.57942100 & 3.15438600 & -2.68427400 \\
\hline & -2.00575800 & 4.62869000 & -2.60499200 \\
\hline $\mathrm{C}$ & 1.45475300 & 2.69749800 & -4.14559100 \\
\hline $\mathrm{H}$ & 0.58848900 & 3.06607300 & -2.21933300 \\
\hline $\mathrm{H}$ & -2.06337400 & 4.97615700 & -1.56625000 \\
\hline $\mathrm{H}$ & 1.29129400 & 5.27010900 & -3.13311500 \\
\hline $\mathrm{H}$ & -2.99157000 & 4.78101100 & -3.06411300 \\
\hline $\mathrm{H}$ & -1.03096100 & 1.69162700 & -4.22241700 \\
\hline $\mathrm{H}$ & -2.43335400 & 2.70085900 & -4.64437000 \\
\hline $\mathrm{H}$ & -0.79630900 & 3.37334200 & -4.70326400 \\
\hline $\mathrm{O}$ & -0.84902200 & 0.50680300 & 0.18254000 \\
\hline $\mathrm{O}$ & 0.12975300 & 0.47997200 & -1.83322400 \\
\hline $\mathrm{N}$ & 3.13720500 & 0.84304700 & 1.41101700 \\
\hline $\mathrm{C}$ & 3.90349700 & 0.66649900 & 0.14234800 \\
\hline $\mathrm{C}$ & 3.44444500 & -0.64986200 & -0.47731600 \\
\hline 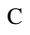 & 2.02922000 & -0.80127200 & -0.45353000 \\
\hline $\mathrm{C}$ & 1.44279500 & -2.04660200 & -0.76631800 \\
\hline $\mathrm{C}$ & 2.24319600 & -3.10693100 & -1.17510900 \\
\hline $\mathrm{C}$ & 3.62898100 & -2.95106800 & -1.25407000 \\
\hline $\mathrm{C}$ & 4.23472200 & -1.73545300 & -0.90126300 \\
\hline $\mathrm{H}$ & 1.13006500 & 0.08955500 & -1.12187100 \\
\hline $\mathrm{H}$ & 0.36154300 & -2.15533400 & -0.75128500 \\
\hline $\mathrm{H}$ & 1.79728700 & -4.05906200 & -1.45109600 \\
\hline $\mathrm{H}$ & 4.23456300 & -3.78521000 & -1.59009200 \\
\hline $\mathrm{O}$ & 5.59238100 & -1.54812600 & -0.94684300 \\
\hline $\mathrm{C}$ & 6.41952500 & -2.63023700 & -1.33234100 \\
\hline $\mathrm{H}$ & 6.21182600 & -2.95696400 & -2.36050700 \\
\hline $\mathrm{H}$ & 7.44412200 & -2.25658300 & -1.27657900 \\
\hline $\mathrm{H}$ & 6.31113900 & -3.48867100 & -0.65529200 \\
\hline $\mathrm{C}$ & 0.61265900 & 2.18149800 & 2.97049600 \\
\hline $\mathrm{O}$ & 0.51647800 & 1.78128300 & 2.68919900 \\
\hline $\mathrm{O}$ & 69895300 & 1.94197200 & 2.24885100 \\
\hline $\mathrm{H}$ & 1.47020500 & 1.38399600 & 1.45199200 \\
\hline C & 0.88946200 & 3.00533900 & 4.20095300 \\
\hline $\mathrm{H}$ & -1.58728500 & 2.46887500 & 4.85278700 \\
\hline $\mathrm{H}$ & 0.04125100 & 3.20208700 & 4.73294900 \\
\hline $\mathrm{H}$ & 1.36773500 & 3.94819800 & 3.91639200 \\
\hline$C$ & 3.56243200 & -0.06259400 & 2.47916500 \\
\hline $\mathrm{H}$ & 2.85784500 & 0.02034700 & 3.31270500 \\
\hline п & 3.64085500 & -1.12571500 & 2.19398200 \\
\hline $\mathrm{H}$ & 4.54444900 & 0.25667000 & 2.86044300 \\
\hline 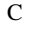 & 5.39809500 & 0.93961100 & 0.35446600 \\
\hline $\mathrm{H}$ & 5.88880700 & 0.16972600 & 0.95346100 \\
\hline $\mathrm{H}$ & & & -0.6048790 \\
\hline
\end{tabular}




\begin{tabular}{|c|c|c|c|c|c|c|c|}
\hline $\mathrm{H}$ & 5.49863400 & 1.90212700 & 0.86861900 & $\mathrm{C}$ & 2.41664000 & -2.99154900 & -1.27559900 \\
\hline \multirow[t]{2}{*}{$\mathrm{H}$} & 3.53533900 & 1.46487800 & -0.51755200 & $\mathrm{C}$ & 3.79362300 & -2.81228200 & -1.39868600 \\
\hline & & & & $\mathrm{C}$ & 4.40578200 & -1.66824000 & -0.86086700 \\
\hline \multicolumn{2}{|c|}{ S-Me ${ }_{4}$} & & & $\mathrm{H}$ & 0.82511000 & 0.03290800 & -1.58399600 \\
\hline \multicolumn{2}{|c|}{-1660.74444740} & & & $\mathrm{H}$ & 0.55571900 & -2.19151300 & -0.54792300 \\
\hline $\mathrm{Pd}$ & 1.29358700 & 0.56773400 & 0.89899300 & $\mathrm{H}$ & 1.95631400 & -3.88488200 & -1.69157400 \\
\hline $\mathrm{C}$ & -2.35795100 & 0.66712100 & -1.82253100 & $\mathrm{H}$ & 4.38332900 & -3.56336500 & -1.91208700 \\
\hline $\mathrm{C}$ & -0.98749100 & 0.39829000 & -1.20395800 & $\mathrm{O}$ & 5.75433400 & -1.43102800 & -0.96843700 \\
\hline $\mathrm{H}$ & -2.34308500 & 0.23429500 & -2.82547400 & $\mathrm{C}$ & 6.57107000 & -2.41229300 & -1.57725100 \\
\hline $\mathrm{N}$ & -3.40284500 & 0.01812700 & -1.04923200 & $\mathrm{H}$ & 6.30711600 & -2.56788700 & -2.63259600 \\
\hline $\mathrm{H}$ & -3.96008700 & 0.59108100 & -0.43048400 & $\mathrm{H}$ & 7.59289200 & -2.03074000 & -1.51774600 \\
\hline $\mathrm{C}$ & -3.31235300 & -1.33483100 & -0.81100800 & $\mathrm{H}$ & 6.51400600 & -3.37467000 & -1.05033300 \\
\hline $\mathrm{O}$ & -2.38541800 & -1.98735000 & -1.28661700 & $\mathrm{C}$ & -0.85745800 & 2.45153700 & 2.58237700 \\
\hline $\mathrm{C}$ & -4.38276900 & -1.94857500 & 0.03501100 & $\mathrm{O}$ & 0.29876000 & 2.17272000 & 2.29293000 \\
\hline $\mathrm{C}$ & -5.64173800 & -1.35969900 & 0.22392900 & $\mathrm{O}$ & -1.92552600 & 1.91058500 & 1.98767300 \\
\hline $\mathrm{C}$ & -4.09917000 & -3.17937500 & 0.64332800 & $\mathrm{H}$ & -1.61123800 & 1.27503100 & 1.29493800 \\
\hline $\mathrm{C}$ & -6.59669000 & -1.98657000 & 1.02416400 & $\mathrm{C}$ & -1.22506300 & 3.44215900 & 3.65587100 \\
\hline $\mathrm{H}$ & -5.89563900 & -0.43068200 & -0.27958900 & $\mathrm{H}$ & -1.79247600 & 2.93567800 & 4.44419300 \\
\hline $\mathrm{C}$ & -5.05168800 & -3.80009000 & 1.44797400 & $\mathrm{H}$ & -0.32088700 & 3.88370800 & 4.07482100 \\
\hline $\mathrm{H}$ & -3.12683400 & -3.62914700 & 0.47194100 & $\mathrm{H}$ & -1.86980300 & 4.22343600 & 3.24031500 \\
\hline $\mathrm{C}$ & -6.30091400 & -3.20384800 & 1.64173200 & $\mathrm{C}$ & 3.35226500 & 0.13094200 & 2.79004600 \\
\hline $\mathrm{H}$ & -7.57297300 & -1.52927400 & 1.15859500 & $\mathrm{H}$ & 2.52383600 & 0.31038400 & 3.48342200 \\
\hline $\mathrm{H}$ & -4.82172400 & -4.74956600 & 1.92314100 & $\mathrm{H}$ & 3.44153000 & -0.95869000 & 2.63377600 \\
\hline $\mathrm{H}$ & -7.04489200 & -3.69004200 & 2.26689600 & $\mathrm{H}$ & 4.26777800 & 0.47328300 & 3.29635300 \\
\hline $\mathrm{C}$ & -2.63620800 & 2.18530700 & -1.88577200 & $\mathrm{C}$ & 5.55435800 & 0.66367700 & 0.98494500 \\
\hline $\mathrm{H}$ & -3.65322500 & 2.30680400 & -2.28374100 & $\mathrm{H}$ & 5.82865300 & -0.18782300 & 1.61503600 \\
\hline $\mathrm{H}$ & -2.64619600 & 2.57146700 & -0.85640600 & $\mathrm{H}$ & 6.24895900 & 0.69093000 & 0.14331600 \\
\hline $\mathrm{C}$ & -1.66121900 & 3.03010200 & -2.73106700 & $\mathrm{H}$ & 5.67237200 & 1.58317400 & 1.57012900 \\
\hline $\mathrm{C}$ & -2.03888400 & 4.51454200 & -2.60772100 & $\mathrm{H}$ & 3.98553700 & 1.39078100 & -0.25257000 \\
\hline $\mathrm{C}$ & -1.62836100 & 2.59464100 & -4.20409200 & & & & \\
\hline $\mathrm{H}$ & -0.64970400 & 2.90941400 & -2.31866500 & DM & & & \\
\hline $\mathrm{H}$ & -2.03466000 & 4.84585600 & -1.56246900 & & 18693590 & & \\
\hline $\mathrm{H}$ & -1.33211800 & 5.14283700 & -3.16070700 & $\mathrm{O}$ & 0.00271200 & 1.50827000 & 0.38700100 \\
\hline $\mathrm{H}$ & -3.04074300 & 4.70145800 & -3.01602200 & $\mathrm{C}$ & 1.36201200 & -0.82427400 & 0.18011100 \\
\hline $\mathrm{H}$ & -1.24754200 & 1.57555400 & -4.32461800 & $\mathrm{H}$ & 1.32984400 & -1.79849900 & -0.31748100 \\
\hline $\mathrm{H}$ & -2.63043400 & 2.64207000 & -4.65099100 & $\mathrm{H}$ & 2.29954400 & -0.31689500 & -0.05986100 \\
\hline $\mathrm{H}$ & -0.97545900 & 3.25662300 & -4.78383400 & $\mathrm{H}$ & 1.27391500 & -0.93564100 & 1.26495600 \\
\hline $\mathrm{O}$ & -0.80134600 & 0.42041500 & 0.02101900 & $\mathrm{C}$ & -1.36488700 & -0.81999300 & 0.18020200 \\
\hline $\mathrm{O}$ & -0.02265000 & 0.22167900 & -2.07448500 & $\mathrm{H}$ & -1.33534300 & -1.79428700 & -0.31744000 \\
\hline $\mathrm{N}$ & 3.13382000 & 0.91820200 & 1.57360300 & $\mathrm{H}$ & -1.27722500 & -0.93164500 & 1.26504100 \\
\hline $\mathrm{C}$ & 4.10352500 & 0.58343400 & 0.48564000 & $\mathrm{H}$ & -2.30088900 & -0.30996200 & -0.06007600 \\
\hline $\mathrm{C}$ & 3.63512000 & -0.70240000 & -0.18716800 & S & 0.00035700 & 0.24289800 & -0.43956400 \\
\hline $\mathrm{C}$ & 2.23359200 & -0.88029200 & -0.10716700 & & & & \\
\hline $\mathrm{C}$ & 1.62856200 & -2.03416400 & -0.63146000 & $\mathrm{TS}_{\mathrm{I}}$ & DMSO & & \\
\hline
\end{tabular}




\begin{tabular}{|c|c|c|c|}
\hline \multicolumn{4}{|c|}{-2831.13559494} \\
\hline $\mathrm{Pd}$ & 0.66591800 & -0.21059200 & 0.25848000 \\
\hline $\mathrm{C}$ & -3.22659400 & 1.31484500 & 1.41517800 \\
\hline $\mathrm{C}$ & -1.75708400 & 1.10789200 & 1.01189500 \\
\hline $\mathrm{H}$ & -3.43281500 & 2.37732800 & 1.27111100 \\
\hline N & -4.13578600 & 0.56055600 & 0.56554600 \\
\hline $\mathrm{H}$ & -4.46082000 & -0.33134100 & 0.91181800 \\
\hline $\mathrm{C}$ & -4.14686900 & 0.77221100 & -0.79012800 \\
\hline $\mathrm{O}$ & -3.46652800 & 1.65708900 & -1.30623300 \\
\hline $\mathrm{C}$ & -5.03557600 & -0.12449900 & -1.60159500 \\
\hline $\mathrm{C}$ & -6.13137300 & -0.81442200 & -1.06219000 \\
\hline $\mathrm{C}$ & -4.75065700 & -0.25061000 & -2.96832600 \\
\hline $\mathrm{C}$ & -6.91733500 & -1.63192700 & -1.87497200 \\
\hline $\mathrm{H}$ & -6.39977300 & -0.68660600 & -0.01679400 \\
\hline $\mathrm{C}$ & -5.53134800 & -1.07346300 & -3.77724600 \\
\hline $\mathrm{H}$ & -3.91450700 & 0.30916500 & -3.37432600 \\
\hline $\mathrm{C}$ & -6.61470600 & -1.76798100 & -3.23160900 \\
\hline $\mathrm{H}$ & -7.77122500 & -2.15363500 & -1.45152200 \\
\hline $\mathrm{H}$ & -5.29884400 & -1.17072900 & -4.83412700 \\
\hline $\mathrm{H}$ & -7.22698600 & -2.40569100 & -3.86349300 \\
\hline $\mathrm{C}$ & -3.46684200 & 0.91592800 & 2.88626300 \\
\hline $\mathrm{H}$ & -4.54048200 & 1.04584300 & 3.08378700 \\
\hline $\mathrm{H}$ & -3.25200700 & -0.15828300 & 2.98845200 \\
\hline $\mathrm{C}$ & -2.66380800 & 1.68845100 & 3.95214300 \\
\hline $\mathrm{C}$ & -2.96658200 & 1.10408600 & 5.34084000 \\
\hline C & -2.94618100 & 3.19803300 & 3.91892000 \\
\hline $\mathrm{H}$ & -1.59480400 & 1.54250900 & 3.75031700 \\
\hline $\mathrm{H}$ & -2.73630300 & 0.03265900 & 5.38695800 \\
\hline $\mathrm{H}$ & -2.37276400 & 1.60657500 & 6.11256000 \\
\hline $\mathrm{H}$ & -4.02565800 & 1.23130300 & 5.60155400 \\
\hline $\mathrm{H}$ & -2.61265700 & 3.65184300 & 2.98074000 \\
\hline $\mathrm{H}$ & -4.01823700 & 3.40292200 & 4.04250000 \\
\hline $\mathrm{H}$ & -2.41627400 & 3.70465400 & 4.73365600 \\
\hline $\mathrm{O}$ & -1.41731600 & -0.01471500 & 0.52517900 \\
\hline $\mathrm{O}$ & -0.96391500 & 2.07585300 & 1.21753900 \\
\hline$S$ & 3.52414100 & -1.27896600 & -0.64440100 \\
\hline $\mathrm{F}$ & 5.16628900 & -1.40109000 & 1.41827000 \\
\hline $\mathrm{F}$ & 3.56288700 & -2.87321800 & 1.48422800 \\
\hline $\mathrm{F}$ & 5.22928500 & -3.14616700 & 0.11495500 \\
\hline $\mathrm{O}$ & 4.60691100 & -0.68650300 & -1.44108000 \\
\hline $\mathrm{O}$ & 2.62366300 & -2.27047400 & -1.25385000 \\
\hline $\mathrm{N}$ & 2.69474900 & -0.18459100 & 0.22194200 \\
\hline $\mathrm{C}$ & 3.20876800 & 1.21276100 & 0.26429100 \\
\hline $\mathrm{C}$ & 2.28692600 & 2.01723600 & -0.62488900 \\
\hline
\end{tabular}

\begin{tabular}{|c|c|c|c|}
\hline $\mathrm{C}$ & 0.90955900 & 1.68037700 & -0.62571600 \\
\hline $\mathrm{C}$ & 0.06250200 & 2.20593100 & -1.62999200 \\
\hline $\mathrm{C}$ & 0.56862400 & 3.11239000 & -2.55408300 \\
\hline 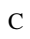 & 1.91280500 & 3.49788200 & -2.51248800 \\
\hline & 2.77410700 & 2.95558300 & -1.54933800 \\
\hline C & 4.42123400 & -2.24059000 & 0.68052700 \\
\hline $\mathrm{H}$ & 0.14640900 & 1.81936600 & 0.48159200 \\
\hline $\mathrm{H}$ & -0.99487800 & 1.95759500 & -1.63612800 \\
\hline $\mathrm{H}$ & -0.08351300 & 3.54028900 & -3.31066100 \\
\hline $\mathrm{H}$ & 2.28292200 & 4.21289900 & -3.23904500 \\
\hline $\mathrm{O}$ & 4.10182400 & 3.26059800 & -1.45070100 \\
\hline $\mathrm{C}$ & 4.69046500 & 4.06930700 & -2.45693700 \\
\hline $\mathrm{H}$ & 4.27261300 & 5.08500000 & -2.45497400 \\
\hline $\mathrm{H}$ & 5.75366200 & 4.11797100 & -2.21575300 \\
\hline $\mathrm{H}$ & 4.56385800 & 3.62601400 & -3.45310700 \\
\hline C & 3.26458400 & 1.71678500 & 1.71214500 \\
\hline $\mathrm{H}$ & 2.27044100 & 1.70825600 & 2.17255700 \\
\hline $\mathrm{H}$ & 3.92406400 & 1.07122000 & 2.30050400 \\
\hline $\mathrm{H}$ & 3.65210700 & 2.74159700 & 1.73909500 \\
\hline $\mathrm{H}$ & 4.21613200 & 1.23715500 & -0.15677200 \\
\hline $\mathrm{O}$ & 0.61815600 & -2.19049200 & 1.11489900 \\
\hline c & -0.38884400 & -3.08175900 & -1.27041700 \\
\hline $\mathrm{H}$ & -0.91163400 & -3.94484300 & -1.69512400 \\
\hline $\mathrm{H}$ & -0.97525500 & -2.16623800 & -1.38947300 \\
\hline $\mathrm{H}$ & 0.60004400 & -2.96046500 & -1.71531100 \\
\hline S & -0.11028900 & -3.40742100 & 0.50134200 \\
\hline $\mathrm{C}$ & -1.84786400 & -3.27189700 & 1.05226200 \\
\hline $\mathrm{H}$ & -2.20770100 & -2.26623600 & 0.82134600 \\
\hline $\mathrm{H}$ & -2.44303900 & -4.04238500 & 0.55228400 \\
\hline $\mathrm{H}$ & 1.85466900 & -3.43989300 & 2.13192500 \\
\hline
\end{tabular}

$\mathrm{TS}_{\text {S2A-DMSO }}$

$-2831.12446895$

$\begin{array}{lrrr}\mathrm{Pd} & -0.60966500 & -0.09703900 & -0.49664900 \\ \mathrm{C} & 3.32147400 & 1.75559500 & -0.78924100 \\ \mathrm{C} & 1.83307800 & 1.41906200 & -0.59717800 \\ \mathrm{H} & 3.48768700 & 2.69800000 & -0.26342000 \\ \mathrm{~N} & 4.18724500 & 0.75004100 & -0.19283900 \\ \mathrm{H} & 4.55279600 & 0.03251500 & -0.80335200 \\ \mathrm{C} & 4.09578300 & 0.47306000 & 1.14856600 \\ \mathrm{O} & 3.36158200 & 1.12445400 & 1.88879100 \\ \mathrm{C} & 4.94270400 & -0.65644800 & 1.65816500 \\ \mathrm{C} & 6.08901900 & -1.11799800 & 0.99437500 \\ \mathrm{C} & 4.56300900 & -1.25362900 & 2.86819900\end{array}$




\begin{tabular}{|c|c|c|c|c|c|c|c|}
\hline $\mathrm{C}$ & 6.83230000 & -2.17297800 & 1.52468200 & $\mathrm{H}$ & -4.58919000 & 4.07737000 & 3.43624700 \\
\hline $\mathrm{H}$ & 6.42876200 & -0.63147100 & 0.08387900 & $\mathrm{H}$ & -6.01620600 & 3.30582000 & 2.69486600 \\
\hline $\mathrm{C}$ & 5.30164200 & -2.31211400 & 3.39277500 & $\mathrm{H}$ & -4.95478400 & 2.35506500 & 3.77108300 \\
\hline $\mathrm{H}$ & 3.68759900 & -0.86842700 & 3.38066400 & $\mathrm{O}$ & -0.47478000 & -1.77462600 & -1.87190400 \\
\hline $\mathrm{C}$ & 6.43609600 & -2.77578700 & 2.72061200 & $\mathrm{C}$ & 0.55518800 & -3.27145200 & 0.16973200 \\
\hline $\mathrm{H}$ & 7.72558000 & -2.51653200 & 1.01024700 & $\mathrm{H}$ & -0.44471600 & -3.28803800 & 0.60733400 \\
\hline $\mathrm{H}$ & 4.99621000 & -2.77373600 & 4.32772900 & $\mathrm{H}$ & 1.08019400 & -4.21629000 & 0.34309900 \\
\hline $\mathrm{H}$ & 7.01509300 & -3.59800700 & 3.13240000 & $\mathrm{H}$ & 1.12255600 & -2.42480900 & 0.56579700 \\
\hline $\mathrm{C}$ & 3.67765700 & 1.90460500 & -2.28302100 & $\mathrm{C}$ & -4.53930400 & 1.59882000 & -0.71215000 \\
\hline $\mathrm{H}$ & 4.75964400 & 2.09129400 & -2.33810700 & $\mathrm{H}$ & -5.18515800 & 1.14963900 & 0.03961200 \\
\hline $\mathrm{H}$ & 3.49633400 & 0.93793200 & -2.77636400 & $\mathrm{H}$ & -4.71231400 & 2.67852600 & -0.71912800 \\
\hline $\mathrm{C}$ & 2.93836100 & 3.00944600 & -3.06451400 & $\mathrm{H}$ & -4.79856600 & 1.19612600 & -1.69586600 \\
\hline $\mathrm{C}$ & 3.36447700 & 2.96070600 & -4.54011900 & $\mathrm{H}$ & -2.51634700 & 1.80349200 & -1.28317100 \\
\hline $\mathrm{C}$ & 3.17583300 & 4.40667000 & -2.47179300 & $\mathrm{~S}$ & 0.32229700 & -3.07400300 & -1.62705600 \\
\hline $\mathrm{H}$ & 1.86141700 & 2.80325300 & -3.01641600 & $\mathrm{C}$ & 2.05631100 & -2.69492900 & -2.06287500 \\
\hline $\mathrm{H}$ & 3.17095000 & 1.97655500 & -4.98412500 & $\mathrm{H}$ & 2.35370400 & -1.78424100 & -1.53710400 \\
\hline $\mathrm{H}$ & 2.81751700 & 3.70589400 & -5.12850600 & $\mathrm{H}$ & 2.68689100 & -3.54702600 & -1.79031900 \\
\hline $\mathrm{H}$ & 4.43629300 & 3.17334800 & -4.64894600 & $\mathrm{H}$ & 2.08855400 & -2.53954500 & -3.14398600 \\
\hline $\mathrm{H}$ & 2.75944200 & 4.49508100 & -1.46379300 & & & & \\
\hline $\mathrm{H}$ & 4.24819100 & 4.64045700 & -2.42697900 & & & & \\
\hline $\mathrm{H}$ & 2.69588400 & 5.17225300 & -3.09214700 & & & & \\
\hline $\mathrm{O}$ & 1.48887200 & 0.19971700 & -0.54450500 & & & & \\
\hline $\mathrm{O}$ & 1.03148500 & 2.40154600 & -0.52810900 & & & & \\
\hline S & -3.37106500 & -1.26565800 & 0.29641000 & & & & \\
\hline $\mathrm{F}$ & -5.29484500 & -1.35377800 & -1.54062200 & & & & \\
\hline $\mathrm{F}$ & -3.61442900 & -2.68042100 & -1.93457400 & & & & \\
\hline $\mathrm{F}$ & -5.04903400 & -3.17546500 & -0.37671800 & & & & \\
\hline $\mathrm{O}$ & -4.34453200 & -0.74849200 & 1.26846800 & & & & \\
\hline $\mathrm{O}$ & -2.39813800 & -2.28605000 & 0.72090700 & & & & \\
\hline $\mathrm{N}$ & -2.64286700 & -0.11517200 & -0.61993500 & & & & \\
\hline $\mathrm{C}$ & -3.05592300 & 1.32371600 & -0.45582600 & & & & \\
\hline $\mathrm{C}$ & -2.37059600 & 1.79618400 & 0.82224100 & & & & \\
\hline $\mathrm{C}$ & -0.99445100 & 1.44081300 & 0.88513300 & & & & \\
\hline $\mathrm{C}$ & -0.27901700 & 1.56357000 & 2.09684900 & & & & \\
\hline $\mathrm{C}$ & -0.90390400 & 2.11590800 & 3.20795100 & & & & \\
\hline $\mathrm{C}$ & -2.23538300 & 2.53134900 & 3.13326900 & & & & \\
\hline $\mathrm{C}$ & -2.97559900 & 2.37839600 & 1.95040400 & & & & \\
\hline $\mathrm{C}$ & -4.39551800 & -2.17237000 & -0.97783700 & & & & \\
\hline $\mathrm{H}$ & -0.10740500 & 1.92810300 & -0.03002400 & & & & \\
\hline $\mathrm{H}$ & 0.77234400 & 1.29316200 & 2.13830700 & & & & \\
\hline $\mathrm{H}$ & -0.35712100 & 2.24321800 & 4.13834800 & & & & \\
\hline $\mathrm{H}$ & -2.70074700 & 2.96902000 & 4.00913700 & & & & \\
\hline $\mathrm{O}$ & -4.27952100 & 2.76253100 & 1.83964300 & & & & \\
\hline $\mathrm{C}$ & -4.98353900 & 3.14491000 & 3.00984600 & & & & \\
\hline
\end{tabular}


11. The relative free energies and electronic energies in gas phase and the single-point energies in DMSO solvent for complexes calculated in this study

Table S2. The relative free energies and electronic energies in gas phase and the single-point energies in DMSO solvent for complexes calculated in this study

\begin{tabular}{|c|c|c|c|c|c|c|c|c|}
\hline & \multicolumn{4}{|c|}{$R$} & \multicolumn{4}{|c|}{$S$} \\
\hline & Structure & $\begin{array}{c}\Delta \mathrm{G}_{\mathrm{gas}} \\
(\mathrm{kcal} / \mathrm{mol})\end{array}$ & $\begin{array}{c}\Delta \mathrm{E}_{\mathrm{gas}} \\
(\mathrm{kcal} / \mathrm{mol})\end{array}$ & $\begin{array}{c}\Delta \mathrm{E}_{\mathrm{DMSO}} \\
(\mathrm{kcal} / \mathrm{mol})\end{array}$ & Structure & $\begin{array}{c}\Delta \mathrm{G}_{\mathrm{gas}} \\
(\mathrm{kcal} / \mathrm{mol})\end{array}$ & $\begin{array}{c}\Delta \mathrm{E}_{\mathrm{gas}} \\
(\mathrm{kcal} / \mathrm{mol})\end{array}$ & $\begin{array}{c}\Delta \mathrm{E}_{\mathrm{DMSO}} \\
(\mathrm{kcal} / \mathrm{mol})\end{array}$ \\
\hline \multirow{27}{*}{$\begin{array}{c}\text { C-H } \\
\text { Activation }\end{array}$} & $\mathrm{R}_{\mathrm{la}}$ & 39.5 & 31.8 & 4.0 & $\mathrm{~S}_{1 \mathrm{~A}}$ & 40.4 & 32.6 & 2.9 \\
\hline & $\mathrm{TS}_{\mathrm{R} 1 \mathrm{a}}$ & 42.6 & 37.4 & 6.6 & $\mathrm{TS}_{\mathrm{S}_{1 \mathrm{~A}}}$ & 42.1 & 37.1 & 5.6 \\
\hline & $\mathrm{R}_{2 \mathrm{a}}$ & 43.5 & 37.1 & 4.4 & $\mathrm{~S}_{2 \mathrm{~A}}$ & 42.3 & 36.6 & 1.2 \\
\hline & $\mathrm{R}_{3 \mathrm{a}}$ & 41.9 & 38.3 & 10.7 & $\mathrm{~S}_{3 \mathrm{~A}}$ & 48.5 & 44.0 & 16.3 \\
\hline & $\mathrm{TS}_{\mathrm{R} 2 \mathrm{a}}$ & 47.3 & 45.9 & 19.1 & $\mathrm{TS}_{\mathrm{S} 2 \mathrm{~A}}$ & 54.8 & 52.0 & 25.0 \\
\hline & $\mathrm{R}_{4 \mathrm{a}}$ & 31.3 & 27.2 & -3.5 & $\mathrm{~S}_{4 \mathrm{~A}}$ & 38.7 & 35.3 & 5.1 \\
\hline & $\mathrm{R}_{3 \mathrm{a}^{\prime}}$ & 44.2 & 56.1 & 28.9 & $\mathrm{~S}_{3 \mathrm{~A}^{\prime}}$ & 51.3 & 61.1 & 33.2 \\
\hline & $\mathrm{TS}_{\mathrm{R} 2 \mathrm{a}^{\prime}}$ & 58.6 & 72.0 & 43.4 & $\mathrm{TS}_{{\mathrm{S} 2 \mathrm{~A}^{\prime}}}$ & 61.4 & 73.8 & 47.6 \\
\hline & $\mathrm{R}_{4 \mathrm{a}^{\prime}}$ & 39.1 & 49.7 & 18.4 & $\mathrm{~S}_{4 \mathrm{~A}^{\prime}}$ & 38.2 & 49.1 & 16.8 \\
\hline & $R_{3 a^{\prime \prime}}$ & 38.4 & 46.7 & 21.1 & $\mathrm{~S}_{3 \mathrm{~A}^{\prime \prime}}$ & 40.9 & 49.5 & 24.0 \\
\hline & $\mathrm{TS}_{\mathrm{R} 2 \mathrm{a}^{\prime \prime}}$ & 83.0 & 94.1 & 58.8 & $\mathrm{TS}_{\mathrm{S}_{2} \mathrm{~A}^{\prime \prime}}$ & 86.3 & 96.3 & 60.5 \\
\hline & $\mathrm{R}_{4 \mathrm{a}^{\prime \prime}}$ & 44.7 & 53.3 & 19.1 & $\mathrm{~S}_{4 \mathrm{~A}^{\prime \prime}}$ & 44.2 & 52.5 & 17.5 \\
\hline & $\mathrm{L}_{1}$ & 15.2 & 10.3 & 2.2 & $\mathrm{~L}_{1}$ & 15.2 & 10.3 & 2.2 \\
\hline & $\mathrm{TS}_{\mathrm{L}}$ & 21.3 & 19.5 & 11.2 & $\mathrm{TS}_{\mathrm{L}}$ & 21.3 & 19.5 & 11.2 \\
\hline & $\mathrm{L}_{2}$ & 21.5 & 17.6 & 7.4 & $\mathrm{~L}_{2}$ & 21.5 & 17.6 & 7.4 \\
\hline & $\mathrm{R}_{3 \mathrm{~b}}$ & 48.5 & 55.8 & 31.5 & $\mathrm{~S}_{3 \mathrm{~B}}$ & 59.9 & 68.5 & 39.3 \\
\hline & $\mathrm{TS}_{\mathrm{R} 2 \mathrm{~b}}$ & 66.6 & 75.4 & 46.0 & $\mathrm{TS}_{\mathrm{S} 2 \mathrm{~B}}$ & 72.3 & 81.5 & 50.6 \\
\hline & $\mathrm{R}_{4 \mathrm{~b}}$ & 50.2 & 56.0 & 21.7 & $\mathrm{~S}_{4 \mathrm{~B}}$ & 47.9 & 54.4 & 21.2 \\
\hline & $\mathrm{R}_{\mathrm{lc}}$ & 41.0 & 35.1 & 9.0 & $S_{1 C}$ & 52.1 & 44.4 & 19.2 \\
\hline & $\mathrm{TS}_{\mathrm{R} 1 \mathrm{c}}$ & 82.7 & 76.7 & 38.9 & $\mathrm{TS}_{\mathrm{S} I \mathrm{C}}$ & 89.8 & 83.7 & 45.5 \\
\hline & $\mathrm{R}_{2 \mathrm{c}}$ & 53.2 & 46.5 & 10.0 & $\mathrm{~S}_{2 \mathrm{C}}$ & 61.2 & 54.1 & 17.2 \\
\hline & $\mathrm{R}_{\mathrm{ld}}$ & 58.0 & 52.7 & 8.2 & $S_{1 D}$ & 40.9 & 34.9 & 1.2 \\
\hline & $\mathrm{TS}_{\mathrm{R} I \mathrm{~d}}$ & 77.0 & 73.6 & 37.2 & $\mathrm{TS}_{\mathrm{S} 1 \mathrm{D}}$ & 78.9 & 75.1 & 37.3 \\
\hline & $\mathrm{R}_{2 \mathrm{~d}}$ & 38.6 & 33.1 & -2.4 & $S_{2 D}$ & 55.8 & 49.8 & 8.0 \\
\hline & $\mathrm{R}_{\mathrm{le}}$ & 38.2 & 29.9 & 6.5 & $S_{1 E}$ & 35.9 & 28.8 & 4.4 \\
\hline & $\mathrm{TS}_{\text {Rle }}$ & 74.7 & 70.8 & 39.4 & $\mathrm{TS}_{\mathrm{S} 1 \mathrm{E}}$ & 80.1 & 76.7 & 46.8 \\
\hline & $\mathrm{R}_{2 \mathrm{e}}$ & 55.2 & 49.5 & 12.1 & $S_{2 E}$ & 50.3 & 45.1 & 9.2 \\
\hline \multirow{7}{*}{$\begin{array}{l}\text { Oxidative addition } \\
\text { and Reductive } \\
\text { elimination }\end{array}$} & $\mathrm{R}_{5 \mathrm{a}}$ & 35.9 & 34.3 & -5.0 & $\mathrm{~S}_{5 \mathrm{~A}}$ & 42.0 & 43.7 & 8.6 \\
\hline & $\mathrm{TS}_{\mathrm{R} 3 \mathrm{a}}$ & 59.2 & 54.8 & 11.1 & $\mathrm{TS}_{\mathrm{S} 3 \mathrm{~A}}$ & 63.3 & 59.2 & 14.1 \\
\hline & $\mathrm{R}_{6 \mathrm{a}}$ & 57.8 & 54.7 & 12.5 & $S_{6 A}$ & 61.3 & 57.5 & 15.8 \\
\hline & $\mathrm{TS}_{\mathrm{R} 4 \mathrm{a}}$ & 58.5 & 58.3 & 19.3 & $\mathrm{TS}_{\mathrm{S} 4 \mathrm{~A}}$ & 61.7 & 59.9 & 21.5 \\
\hline & $\mathrm{R}_{7 \mathrm{a}}$ & 43.7 & 42.0 & -6.0 & $\mathrm{~S}_{7 \mathrm{~A}}$ & 43.6 & 41.5 & -5.3 \\
\hline & $\mathrm{R}_{5 \mathrm{~b}}$ & 38.9 & 41.2 & 4.2 & $\mathrm{~S}_{5 \mathrm{~B}}$ & 36.8 & 34.3 & -5.1 \\
\hline & $\mathrm{TS}_{\mathrm{R} 3 \mathrm{~b}}$ & 74.1 & 71.4 & 26.5 & $\mathrm{TS}_{\mathrm{S} 3 \mathrm{~B}}$ & 82.2 & 78.5 & 35.8 \\
\hline
\end{tabular}




\begin{tabular}{|c|c|c|c|c|c|c|c|c|}
\hline & $\mathrm{R}_{6 \mathrm{~b}}$ & 36.6 & 32.0 & -21.4 & $\mathrm{~S}_{6 \mathrm{~B}}$ & 43.7 & 38.7 & -8.1 \\
\hline & $\mathrm{R}_{5 \mathrm{c}}$ & 43.6 & 31.0 & -11.0 & $\mathrm{~S}_{5 \mathrm{C}}$ & 33.6 & 21.2 & -14.0 \\
\hline & $\mathrm{TS}_{\mathrm{R} 3 \mathrm{c}}$ & 76.2 & 61.5 & 21.0 & $\mathrm{TS}_{\mathrm{S}_{3 \mathrm{C}}}$ & 81.8 & 66.9 & 26.9 \\
\hline & $\mathrm{R}_{6 \mathrm{c}}$ & 41.5 & 25.2 & -19.1 & $\mathrm{~S}_{6 \mathrm{C}}$ & 40.0 & 23.2 & -18.6 \\
\hline \multirow{14}{*}{$\begin{array}{c}\text { Regeneration of the } \\
\text { catalyst }\end{array}$} & $\mathrm{R}_{8 \mathrm{a}}$ & 41.5 & 25.2 & -19.1 & $\mathrm{~S}_{8 \mathrm{~A}}$ & 40.0 & 23.2 & -18.6 \\
\hline & $\mathrm{TS}_{\mathrm{R} 5 \mathrm{a}}$ & 40.1 & 25.5 & -16.3 & $\mathrm{TS}_{\mathrm{S} 5 \mathrm{~A}}$ & 38.6 & 23.4 & -16.5 \\
\hline & $\mathrm{R}_{9 \mathrm{a}}$ & 30.1 & 13.3 & -21.9 & $\mathrm{~S}_{9 \mathrm{~A}}$ & 33.3 & 16.6 & -20.4 \\
\hline & $\mathrm{P}_{\mathrm{RI}}$ & 25.2 & 27.8 & 8.5 & $\mathrm{P}_{\mathrm{SI}}$ & 25.2 & 27.8 & 8.5 \\
\hline & $\mathrm{R}_{8 \mathrm{~b}}$ & 51.5 & 48.4 & 0.4 & $\mathrm{~S}_{8 \mathrm{~B}}$ & 51.2 & 47.9 & 1.2 \\
\hline & $\mathrm{TS}_{\mathrm{R} 5 \mathrm{~b}}$ & 51.6 & 50.8 & 4.4 & $\mathrm{TS}_{\mathrm{S} 5 \mathrm{~B}}$ & 51.0 & 50.5 & 5.4 \\
\hline & $\mathrm{R}_{9 \mathrm{~b}}$ & 33.2 & 30.9 & -3.7 & $\mathrm{~S}_{9 \mathrm{~B}}$ & 34.6 & 31.5 & -4.7 \\
\hline & 10 & -0.2 & -3.2 & -12.9 & 10 & -0.2 & -3.2 & -12.9 \\
\hline & $\mathrm{TS}_{6}$ & 11.6 & 11.5 & 1.4 & $\mathrm{TS}_{6}$ & 11.6 & 11.5 & 1.4 \\
\hline & 11 & -0.6 & -3.1 & -12.1 & 11 & -0.6 & -3.1 & -12.1 \\
\hline & $\mathrm{R}_{8 \mathrm{c}}$ & 46.5 & 44.0 & 2.8 & $\mathrm{~S}_{8 \mathrm{C}}$ & 46.2 & 43.2 & 2.7 \\
\hline & $\mathrm{TS}_{\mathrm{R} 5 \mathrm{c}}$ & 46.6 & 46.7 & 9.1 & $\mathrm{TS}_{\mathrm{S} 5 \mathrm{C}}$ & 47.3 & 46.4 & 9.4 \\
\hline & $\mathrm{R}_{9 \mathrm{c}}$ & 40.5 & 37.8 & 5.3 & $\mathrm{~S}_{9 \mathrm{C}}$ & 31.5 & 28.6 & -1.5 \\
\hline & $\mathrm{TS}_{\mathrm{R} 2 \mathrm{a}-\mathrm{DMSO}}$ & 49.5 & 47.3 & 14.2 & $\mathrm{TS}_{\mathrm{S} 2 \mathrm{~A}-\mathrm{DMSO}}$ & 57.0 & 54.3 & 20.3 \\
\hline
\end{tabular}

